\title{
The effectiveness and cost-effectiveness of hospital-based specialist palliative care for adults with advanced illness and their caregivers
} (Review)

Bajwah S, Oluyase AO, Yi D, Gao W, Evans CJ, Grande G, Todd C, Costantini M, Murtagh FE, Higginson IJ

Bajwah S, Oluyase AO, Yi D, Gao W, Evans CJ, Grande G, Todd C, Costantini M, Murtagh FE, Higginson IJ.

The effectiveness and cost-effectiveness of hospital-based specialist palliative care for adults with advanced illness and their caregivers.

Cochrane Database of Systematic Reviews 2020, Issue 9. Art. No.: CD012780.

DOI: 10.1002/14651858.CD012780.pub2. 
TABLE OF CONTENTS

HEADER

ABSTRACT

PLAIN LANGUAGE SUMMARY

SUMMARY OF FINDINGS

BACKGROUND

OBJECTIVES

METHODS

Figure 1.

RESULTS

Figure 2.

Figure 3.

Figure 4.

Figure 5.

Figure 6.

Figure 7.

DISCUSSION

AUTHORS' CONCLUSIONS

ACKNOWLEDGEMENTS

REFERENCES

CHARACTERISTICS OF STUDIES

DATA AND ANALYSES

Analysis 1.1. Comparison 1: Patient health-related quality of life, Outcome 1: HSPC versus usual care on patient HRQoL: adjusted endpoint values

Analysis 1.2. Comparison 1: Patient health-related quality of life, Outcome 2: HSPC versus usual care on patient HRQoL: adjusted endpoint values (excluding McCorkle 2015)

Analysis 1.3. Comparison 1: Patient health-related quality of life, Outcome 3: HSPC versus usual care on patient HRQoL: unadjusted endpoint values

Analysis 1.4. Comparison 1: Patient health-related quality of life, Outcome 4: HSPC versus usual care on patient HRQoL: unadjusted endpoint values (excluding McCorkle 2015)

Analysis 1.5. Comparison 1: Patient health-related quality of life, Outcome 5: HSPC versus usual care on patient HRQoL: unadjusted change values

Analysis 2.1. Comparison 2: Patient symptom burden, Outcome 1: HSPC versus usual care on patient symptom burden: adjusted endpoint values

Analysis 2.2. Comparison 2: Patient symptom burden, Outcome 2: HSPC versus usual care on patient symptom burden: unadjusted endpoint values

Analysis 2.3. Comparison 2: Patient symptom burden, Outcome 3: HSPC versus usual care on patient symptom burden: unadjusted endpoint values (excluding McCorkle 2015)

Analysis 2.4. Comparison 2: Patient symptom burden, Outcome 4: HSPC versus usual care on patient symptom burden: adjusted change values

Analysis 2.5. Comparison 2: Patient symptom burden, Outcome 5: HSPC versus usual care on patient symptom burden: adjusted change values (excluding McCorkle 2015)

Analysis 2.6. Comparison 2: Patient symptom burden, Outcome 6: HSPC versus usual care on patient symptom burden: unadjusted change values

Analysis 3.1. Comparison 3: Patient satisfaction with care, Outcome 1: HSPC versus usual care on patient satisfaction with care: adjusted endpoint values

Analysis 4.1. Comparison 4: Achieving patient preferred place of death, Outcome 1: HSPC versus usual care on home deaths .. Analysis 5.1. Comparison 5: Pain, Outcome 1: HSPC versus usual care on pain: adjusted endpoint values .............................. Analysis 5.2. Comparison 5: Pain, Outcome 2: HSPC versus usual care on pain: adjusted change values ...................................

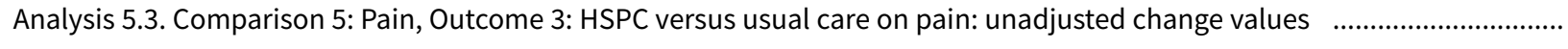
Analysis 6.1. Comparison 6: Patient anxiety, Outcome 1: HSPC versus usual care on patient anxiety: adjusted endpoint values . Analysis 6.2. Comparison 6: Patient anxiety, Outcome 2: HSPC versus usual care on patient anxiety: adjusted endpoint values (excluding McCorkle 2015)

The effectiveness and cost-effectiveness of hospital-based specialist palliative care for adults with advanced illness and their caregivers 
Analysis 6.3. Comparison 6: Patient anxiety, Outcome 3: HSPC versus usual care on patient anxiety: unadjusted endpoint values

Analysis 6.4. Comparison 6: Patient anxiety, Outcome 4: HSPC versus usual care on patient anxiety: unadjusted endpoint values (excluding McCorkle 2015)

Analysis 6.5. Comparison 6: Patient anxiety, Outcome 5: HSPC versus usual care on patient anxiety: unadjusted change values . Analysis 6.6. Comparison 6: Patient anxiety, Outcome 6: HSPC versus usual care on patient anxiety in different populations: adjusted endpoint values

Analysis 6.7. Comparison 6: Patient anxiety, Outcome 7: HSPC versus usual care on patient anxiety in different populations: adjusted endpoint values (excluding McCorkle 2015)

Analysis 6.8. Comparison 6: Patient anxiety, Outcome 8: EPC vs LPC on patient anxiety: adjusted endpoint values

Analysis 6.9. Comparison 6: Patient anxiety, Outcome 9: Effect of MDT-led services on patient anxiety: adjusted endpoint values

Analysis 6.10. Comparison 6: Patient anxiety, Outcome 10: Effect of MDT-led services on patient anxiety: adjusted endpoint values (excluding McCorkle 2015)

Analysis 6.11. Comparison 6: Patient anxiety, Outcome 11: HSPC versus usual care on patient anxiety in different countries: adjusted endpoint values

Analysis 6.12. Comparison 6: Patient anxiety, Outcome 12: HSPC versus usual care on patient anxiety in different countries: adjusted endpoint values (excluding McCorkle 2015)

Analysis 7.1. Comparison 7: Unpaid caregiver anxiety, Outcome 1: HSPC versus usual care on unpaid caregiver anxiety: unadjusted endpoint values

Analysis 8.1. Comparison 8: Patient depression, Outcome 1: HSPC versus usual care on patient depression: adjusted endpoint values

Analysis 8.2. Comparison 8: Patient depression, Outcome 2: HSPC versus usual care on patient depression: unadjusted endpoint values

Analysis 8.3. Comparison 8: Patient depression, Outcome 3: HSPC versus usual care on patient depression: unadjusted endpoint values (excluding McCorkle 2015)

Analysis 8.4. Comparison 8: Patient depression, Outcome 4: HSPC versus usual care on patient depression: adjusted change values

Analysis 8.5. Comparison 8: Patient depression, Outcome 5: HSPC versus usual care on patient depression: unadjusted change values

Analysis 8.6. Comparison 8: Patient depression, Outcome 6: HSPC versus usual care on patient depression as a binary outcome

Analysis 9.1. Comparison 9: Unpaid caregiver depression, Outcome 1: HSPC versus usual care on unpaid caregiver depression: adjusted endpoint values

Analysis 9.2. Comparison 9: Unpaid caregiver depression, Outcome 2: HSPC versus usual care on unpaid caregiver depression: unadjusted endpoint values

Analysis 10.1. Comparison 10: Unpaid caregiver quality of life, Outcome 1: HSPC versus usual care on unpaid caregiver quality of life: unadjusted endpoint values

Analysis 11.1. Comparison 11: Unpaid caregiver burden, Outcome 1: HSPC versus usual care on unpaid caregiver burden: adjusted change values

Analysis 12.1. Comparison 12: Patient breathlessness, Outcome 1: HSPC versus usual care on patient breathlessness: adjusted endpoint values

Analysis 12.2. Comparison 12: Patient breathlessness, Outcome 2: HSPC versus usual care on patient breathlessness: unadjusted endpoint values

Analysis 12.3. Comparison 12: Patient breathlessness, Outcome 3: HSPC versus usual care on patient breathlessness: unadjusted change values

ADDITIONAL TABLES

APPENDICES

HISTORY

CONTRIBUTIONS OF AUTHORS

DECLARATIONS OF INTEREST

SOURCES OF SUPPORT

DIFFERENCES BETWEEN PROTOCOL AND REVIEW 
[Intervention Review]

\section{The effectiveness and cost-effectiveness of hospital-based specialist palliative care for adults with advanced illness and their caregivers}

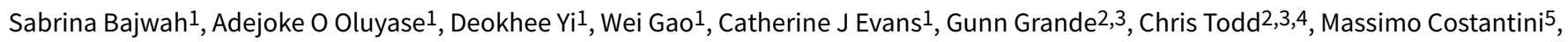
Fliss E Murtagh1,6, Irene J Higginson ${ }^{1}$

${ }^{1}$ Cicely Saunders Institute of Palliative Care, Policy and Rehabilitation, King's College London, London, UK. 2School of Health Sciences, University of Manchester, Manchester, UK. ${ }^{3}$ Manchester Academic Health Science Centre, Manchester, UK. ${ }^{4}$ Manchester University NHS Foundation Trust, Manchester, UK. ${ }^{5}$ Palliative Care Unit, Azienda USL-IRCCS, Reggio Emilia, Italy. ${ }^{6}$ Wolfson Palliative Care Research Centre, Hull York Medical School, University of Hull, Hull, UK

Contact address: Sabrina Bajwah, sabrina.bajwah@kcl.ac.uk.

Editorial group: Cochrane Pain, Palliative and Supportive Care Group.

Publication status and date: New, published in Issue 9, 2020.

Citation: Bajwah S, Oluyase AO, Yi D, Gao W, Evans CJ, Grande G, Todd C, Costantini M, Murtagh FE, Higginson IJ. The effectiveness and cost-effectiveness of hospital-based specialist palliative care for adults with advanced illness and their caregivers. Cochrane Database of Systematic Reviews 2020, Issue 9. Art. No.: CD012780. DOI: 10.1002/14651858.CD012780.pub2.

Copyright ( 2020 The Cochrane Collaboration. Published by John Wiley \& Sons, Ltd.

\section{A B S T R A C T}

\section{Background}

Serious illness is often characterised by physical/psychological problems, family support needs, and high healthcare resource use. Hospital-based specialist palliative care (HSPC) has developed to assist in better meeting the needs of patients and their families and potentially reducing hospital care expenditure. There is a need for clarity on the effectiveness and optimal models of HSPC, given that most people still die in hospital and also to allocate scarce resources judiciously.

\section{Objectives}

To assess the effectiveness and cost-effectiveness of HSPC compared to usual care for adults with advanced illness (hereafter patients) and their unpaid caregivers/families.

\section{Search methods}

We searched CENTRAL, CDSR, DARE and HTA database via the Cochrane Library; MEDLINE; Embase; CINAHL; PsycINFO; CareSearch; National Health Service Economic Evaluation Database (NHS EED) and two trial registers to August 2019, together with checking of reference lists and relevant systematic reviews, citation searching and contact with experts to identify additional studies.

\section{Selection criteria}

We included randomised controlled trials (RCTs) evaluating the impact of HSPC on outcomes for patients or their unpaid caregivers/ families, or both. HSPC was defined as specialist palliative care delivered by a palliative care team that is based in a hospital providing holistic care, co-ordination by a multidisciplinary team, and collaboration between HSPC providers and generalists. HSPC was provided to patients while they were admitted as inpatients to acute care hospitals, outpatients or patients receiving care from hospital outreach teams at home. The comparator was usual care, defined as inpatient or outpatient hospital care without specialist palliative care input at the point of entry into the study, community care or hospice care provided outside of the hospital setting.

\section{Data collection and analysis}

We used standard methodological procedures expected by Cochrane. We assessed risk of bias and extracted data. To account for use of different scales across studies, we calculated standardised mean differences (SMDs) with 95\% confidence intervals (Cls) for continuous 
data. We used an inverse variance random-effects model. For binary data, we calculated odds ratio (ORs) with $95 \% \mathrm{Cls}$. We assessed the evidence using GRADE and created a 'Summary of findings' table.

Our primary outcomes were patient health-related quality of life (HRQoL) and symptom burden (a collection of two or more symptoms). Key secondary outcomes were pain, depression, satisfaction with care, achieving preferred place of death, mortality/survival, unpaid caregiver burden, and cost-effectiveness. Qualitative data was analysed where available.

\section{Main results}

We identified 42 RCTs involving 7779 participants (6678 patients and 1101 caregivers/family members). Twenty-one studies were with cancer populations, 14 were with non-cancer populations (of which six were with heart failure patients), and seven with mixed cancer and non-cancer populations (mixed diagnoses).

HSPC was offered in different ways and included the following models: ward-based, inpatient consult, outpatient, hospital-at-home or hospital outreach, and service provision across multiple settings which included hospital. For our main analyses, we pooled data from studies reporting adjusted endpoint values. Forty studies had a high risk of bias in at least one domain.

Compared with usual care, HSPC improved patient HRQoL with a small effect size of $0.26 \mathrm{SMD}$ over usual care $\left(95 \% \mathrm{Cl} 0.15\right.$ to $0.37 ; \mathrm{I}^{2}=$ 3\%, 10 studies, 1344 participants, low-quality evidence, higher scores indicate better patient HRQoL). HSPC also improved other personcentred outcomes. It reduced patient symptom burden with a small effect size of $-0.26 \mathrm{SMD}$ over usual care $(95 \% \mathrm{Cl}-0.41$ to $-0.12 ; \mathrm{I}=0 \%$, 6 studies, 761 participants, very low-quality evidence, lower scores indicate lower symptom burden). HSPC improved patient satisfaction with care with a small effect size of $0.36 \mathrm{SMD}$ over usual care $\left(95 \% \mathrm{Cl} 0.41\right.$ to $0.57 ; \mathrm{I}^{2}=0 \%, 2$ studies, 337 participants, low-quality evidence, higher scores indicate better patient satisfaction with care). Using home death as a proxy measure for achieving patient's preferred place of death, patients were more likely to die at home with HSPC compared to usual care (OR $1.63,95 \% \mathrm{Cl} 1.23$ to $2.16 ; \mathrm{I}^{2}=0 \%, 7$ studies, 861 participants, low-quality evidence). Data on pain (4 studies, 525 participants) showed no evidence of a difference between HSPC and usual care (SMD $-0.16,95 \% \mathrm{Cl}-0.33$ to $0.01 ; \mathrm{I}^{2}=0 \%$, very low-quality evidence). Eight studies ( $\mathrm{N}=1252$ participants) reported on adverse events and very low-quality evidence did not demonstrate an effect of HSPC on serious harms. Two studies (170 participants) presented data on caregiver burden and both found no evidence of effect of HSPC (very low-quality evidence). We included 13 economic studies (2103 participants). Overall, the evidence on cost-effectiveness of HSPC compared to usual care was inconsistent among the four full economic studies. Other studies that used only partial economic analysis and those that presented more limited resource use and cost information also had inconsistent results (very low-quality evidence).

\section{Quality of the evidence}

The quality of the evidence assessed using GRADE was very low to low, downgraded due to a high risk of bias, inconsistency and imprecision.

\section{Authors' conclusions}

Very low- to low-quality evidence suggests that when compared to usual care, HSPC may offer small benefits for several person-centred outcomes including patient HRQoL, symptom burden and patient satisfaction with care, while also increasing the chances of patients dying in their preferred place (measured by home death). While we found no evidence that HSPC causes serious harms, the evidence was insufficient to draw strong conclusions. Although these are only small effect sizes, they may be clinically relevant at an advanced stage of disease with limited prognosis, and are person-centred outcomes important to many patients and families. More well conducted studies are needed to study populations with non-malignant diseases and mixed diagnoses, ward-based models of HSPC, 24 hours access (out-ofhours care) as part of HSPC, pain, achieving patient preferred place of care, patient satisfaction with care, caregiver outcomes (satisfaction with care, burden, depression, anxiety, grief, quality of life), and cost-effectiveness of HSPC. In addition, research is needed to provide validated person-centred outcomes to be used across studies and populations.

\section{PLAIN LANGUAGE SUMMARY}

\section{Effectiveness and cost-effectiveness of hospital-based specialist palliative care (HSPC) for adults with advanced illness and their unpaid caregivers}

\section{Review question}

How effective is hospital-based specialist palliative care for adults with a terminal illness and their unpaid caregivers, and is it cost-effective?

\section{Why is this question important?}

Palliative care aims to improve the quality of life of people who have a terminal illness (a disease that cannot be cured and is likely to lead to death). It seeks to help patients, their unpaid caregivers and families manage symptoms that cause distress (for example, pain) and to meet patients' and unpaid caregivers' needs for psychological, social and spiritual support. Palliative care is known as an 'holistic' approach, because it considers the 'whole' person and their support network - not just the illness and its symptoms. It usually involves a team of people that can include physicians, nurses, pharmacists, other allied health professionals, social workers, chaplains or volunteers.

The effectiveness and cost-effectiveness of hospital-based specialist palliative care for adults with advanced illness and their caregivers 
A growing number of hospitals are setting up specialist palliative care services (known as hospital-based specialist palliative care (HSPC)). HSPC can be provided:

- either in the hospital itself - for inpatients or outpatients;

- or as 'hospital-at-home' - which means that the hospital team visits patients in the community;

- or across multiple settings (for example, hospital and home).

To find out whether HSPC benefits patients and their unpaid caregivers, and how cost-effective it is, we reviewed the evidence from research.

\section{How did we identify and evaluate the evidence?}

First, we searched for all relevant studies in the medical literature. We specifically looked for:

- randomised controlled studies: these are studies where people are randomly divided into different treatment groups. This type of study provides the most robust evidence about the effects of a treatment;

- studies that compared HSPC to either hospital care without specialist palliative care; care received in the community; or hospice care outside hospital.

We compared the results, and summarised the evidence from all the studies. Finally, we assessed how certain the evidence was. We considered factors such as the way studies were conducted, study sizes, and consistency of findings across studies. Based on our assessments, we categorised the evidence as being of very low, low, moderate or high certainty.

\section{What did we find?}

We found 42 studies that involved a total of 6678 patients and 1101 caregivers or family members. The patients were suffering from: cancer ( 21 studies); an advanced illness that was not cancer (14 studies); and a combination of cancer and non-cancer (mixed) diagnoses ( 7 studies). Patients in six of the 14 non-cancer studies had heart failure. Almost half (19) of the studies were set in the USA. Thirteen studies reported information on the costs of HSPC.

The evidence from the studies we found suggests that, when compared to usual care:

- HSPC may slightly improve patients' health-related quality of life, their overall symptom burden and their satisfaction with care;

- HSPC may increase the chances of people dying in their preferred place of death.

It is unclear what the effects of HSPC are on pain, caregivers' burden, or unwanted events. This is because the evidence we found was not robust (very low-certainty evidence). Similarly, because the evidence relating to costs was of very low certainty, it is unclear how costeffective HSPC is.

\section{What does this mean?}

When compared with usual care, HSPC may slightly improve a patient's quality of life, symptom burden and their satisfaction with care. It may also increase their chances of dying at home. However, future research is likely to change these findings, since they are based on lowcertainty evidence. We need further studies to evaluate the effect of HSPC on other outcomes, such as pain, caregivers' burden, unwanted events and cost-effectiveness.

\section{How-up-to date is this review?}

The evidence in this Cochrane Review is current to August 2019. 


\begin{tabular}{|c|c|c|c|c|c|c|}
\hline 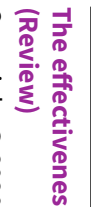 & \multirow{2}{*}{\multicolumn{6}{|c|}{$\begin{array}{l}\text { Summary of findings } 1 \text {. Hospital-based specialist palliative care compared to usual care for adults with advanced illness and thei } \\
\text { families } \\
\text { Hospital-based specialist palliative care compared to usual care for adults with advanced illness and their unpaid caregivers/families }\end{array}$}} \\
\hline 旁 & & & & & & \\
\hline 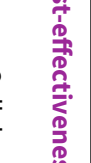 & \multicolumn{6}{|c|}{$\begin{array}{l}\text { Patient or population: adults with advanced illness and their unpaid caregivers/families } \\
\text { Setting: hospital and home } \\
\text { Intervention: hospital-based specialist palliative care } \\
\text { Comparison: usual care }\end{array}$} \\
\hline$\stackrel{\circ}{\frac{0}{\sigma}}$ & \multirow[t]{2}{*}{ Outcomes } & \multicolumn{2}{|c|}{ Anticipated absolute effects ${ }^{\star}(95 \% \mathrm{Cl})$} & \multirow{2}{*}{$\begin{array}{l}\text { Relative effect } \\
(95 \% \mathrm{Cl})\end{array}$} & \multirow{2}{*}{$\begin{array}{l}\text { № of participants } \\
\text { (studies) }\end{array}$} & \multirow{2}{*}{$\begin{array}{l}\text { Certainty of the } \\
\text { evidence } \\
\text { (GRADE) }\end{array}$} \\
\hline 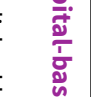 & & Risk with usual care & $\begin{array}{l}\text { Risk with hospital-based } \\
\text { specialist palliative care }\end{array}$ & & & \\
\hline 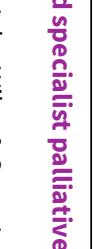 & $\begin{array}{l}\text { Patient health-related quality of life } \\
\text { (HRQOL) i, SD units } \\
\text { (higher scores indicate better quality } \\
\text { of life) } \\
\text { Follow-up: range two weeks after hos- } \\
\text { pitalisation to } 13 \text { months }\end{array}$ & $\begin{array}{l}\text { Mean }(\mathrm{SD}) \text { ranging from } \\
-45.4(26.83) \text { to } 131.14 \\
(26.62)\end{array}$ & $\begin{array}{l}\text { SMD } 0.26 \text { SDs higher } \\
\text { ( } 0.15 \text { higher to } 0.37 \text { higher) }\end{array}$ & - & $\begin{array}{l}1344 \\
(10 \mathrm{RCTs})\end{array}$ & $\begin{array}{l}\oplus \oplus \oplus \ominus \\
\mathrm{LOW} a\end{array}$ \\
\hline 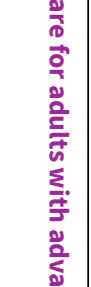 & $\begin{array}{l}\text { Patient symptom burden assessed } \\
\text { with generalised measuresii, SD units } \\
\text { (lower scores indicate lower symptom } \\
\text { burden) } \\
\text { Follow-up: range two weeks after hos- } \\
\text { pitalisation to } 13 \text { months }\end{array}$ & $\begin{array}{l}\text { Mean (SD) ranging from } \\
-19.3(4.2) \text { to } 268.59(201.65)\end{array}$ & $\begin{array}{l}\text { SMD } 0.26 \text { SDs lower } \\
\text { ( } 0.41 \text { lower to } 0.12 \text { lower) }\end{array}$ & - & $\begin{array}{l}761 \\
\text { (6 RCTs) }\end{array}$ & $\begin{array}{l}\oplus \odot \ominus \ominus \\
\text { VERY LOWa,b }\end{array}$ \\
\hline 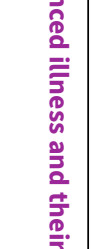 & $\begin{array}{l}\text { Patient satisfaction with care iii, SD } \\
\text { units } \\
\text { (higher scores indicate better patient } \\
\text { satisfaction) } \\
\text { Follow-up: range } 3 \text { months to } 6 \\
\text { months }\end{array}$ & $\begin{array}{l}\text { Mean (SD) ranging from } 6.4 \\
(1.1) \text { to } 68.37(9.03)\end{array}$ & $\begin{array}{l}\text { SMD } 0.36 \text { SDs higher } \\
\text { (0.41 higher to } 0.57 \text { higher) }\end{array}$ & - & $\begin{array}{l}337 \\
(2 \text { RCTs })\end{array}$ & $\begin{array}{l}\oplus \oplus \odot \odot \\
\mathrm{LOW} a\end{array}$ \\
\hline 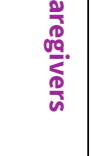 & $\begin{array}{l}\text { Achieving patient preferred place } \\
\text { of death (measured by number of pa- } \\
\text { tients with home death) }\end{array}$ & 462 per 1000 & $\begin{array}{l}583 \text { per } 1000 \\
\text { (513 to } 649)\end{array}$ & $\begin{array}{l}\text { OR } 1.63 \text { higher } \\
\text { (1.23 higher to } 2.16 \\
\text { higher) }\end{array}$ & $\begin{array}{l}861 \\
(7 \mathrm{RCTs})\end{array}$ & $\begin{array}{l}\oplus \oplus \oplus \ominus \\
\mathrm{LOW} a\end{array}$ \\
\hline
\end{tabular}

Patient or population: adults with advanced illness and their unpaid caregivers/families

hospital and home 


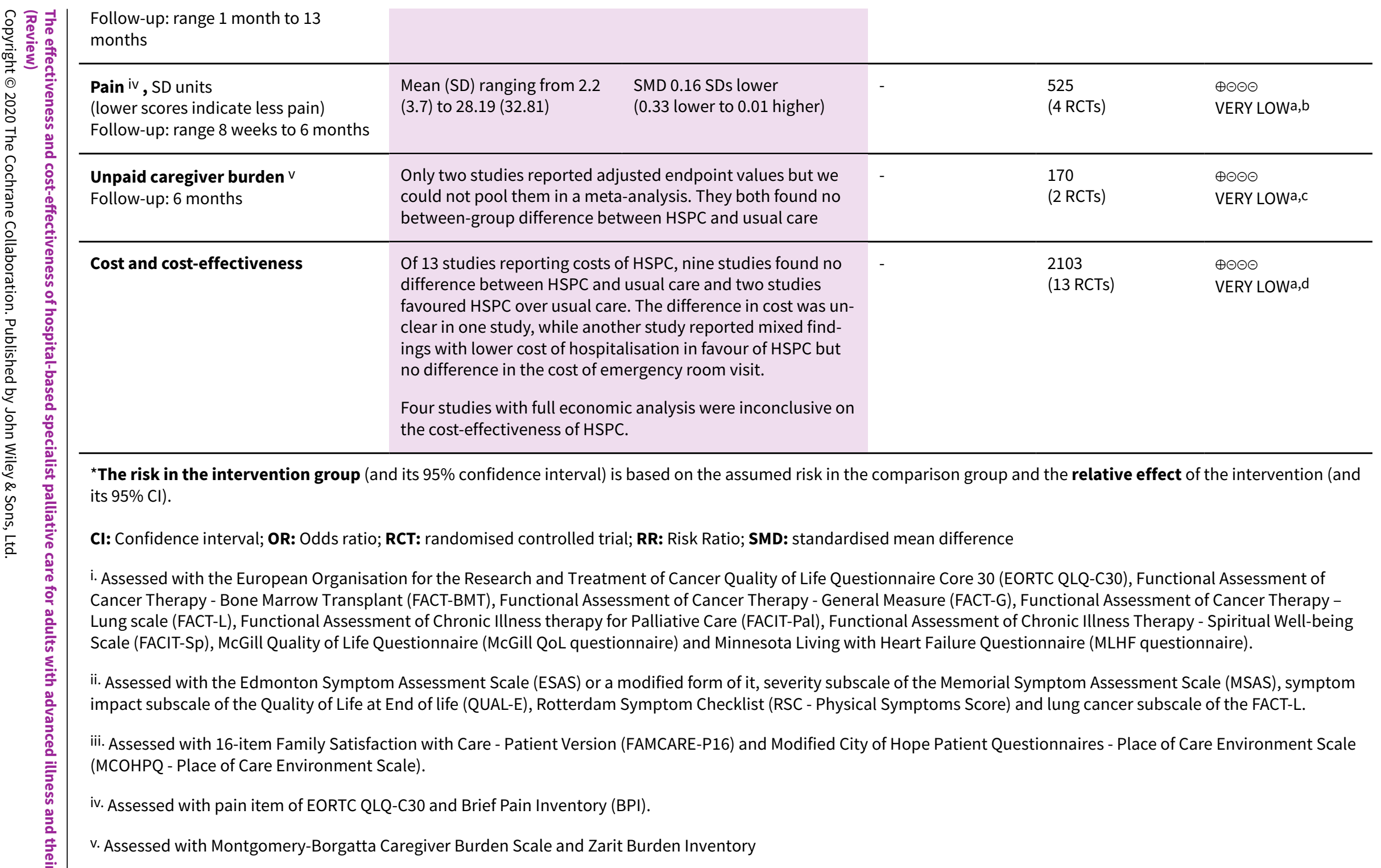

\section{GRADE Working Group grades of evidence}

High quality: We are very confident that the true effect lies close to that of the estimate of the effect

Moderate quality: We are moderately confident in the effect estimate: The true effect is likely to be close to the estimate of the effect, but there is a possibility that it is substantially different

Low quality: Our confidence in the effect estimate is limited: The true effect may be substantially different from the estimate of the effect 
Very low quality: We have very little confidence in the effect estimate: The true effect is likely to be substantially different from the estimate of effect

a We downgraded by 2 levels for very serious study limitations due to a high risk of bias in studies.

$b$ We downgraded by 1 level due to inconsistency between our main meta-analysis and sensitivity analyses.

c We downgraded by 1 level for imprecision due to the small number of participants.

$d$ We downgraded by 1 level for inconsistency because the results were inconsistent across studies. 


\section{B A C K G R O U N D}

The global burden of disease has increased, and this change is placing considerable strain on healthcare systems internationally (Bloom 2016). Most adults develop one or more chronic illnesses with which they may live for many years before they die. For a minority of patients with serious illness, the time following diagnosis is characterised by a stable period of relatively good functional and cognitive performance, followed by a predictable and short period of functional and clinical decline. The time following diagnosis may also be characterised by months to years of physical and psychological symptom distress, progressive functional dependence and frailty, considerable family support needs and high healthcare resource use (Evans 2019; Prince 2015). In addition to increased clinical complexity, the rise of ageing populations has led to considerable healthcare costs globally. This has occurred despite efforts to reduce acute hospital care expenditure in many high-income countries, including, for example, in the USA (Kashihara 2012), and the UK (Imison 2017; Lafond 2014), by shifting care from the hospital setting to primary care and the community.

It could be argued that increased staffing costs and the introduction or expansion of novel services in hospitals and the community, such as specialist palliative care, plays a role in this increased expenditure. Hospital-based palliative care encompasses palliative care interventions that are delivered by specialist palliative care teams based in a hospital to patients with advanced (C-TAC 2015), life-limiting (Palliative Care Australia 2005), or life-threatening illness (NCP 2013), which is likely to compromise their quality of life (WHOQOL Group 1995). The care is provided to the patient while they are admitted as inpatients to acute care hospitals, outpatients or patients receiving care from hospital outreach teams at home. Between 2000 and 2016, the prevalence of specialist palliative care in hospitals with 50 or more beds increased by $178 \%$ in USA, from $25 \%$ of hospitals in 2000 to $75 \%$ in 2016 (CAPC 2018). Furthermore, the growth of specialist palliative care in acute hospitals is likely to continue in the foreseeable future as most older adults ( $\geq 65$ years old) die in hospitals (Broad 2013), most deaths in hospital occur due to terminal illness (Pivodic 2016), and also because deaths in institutional care persist into older stages of life, with one in five centenarians dying in hospital (Evans 2014). In the UK, it has been estimated that by 2040 about 160,000 more people yearly are likely to have palliative care needs, including pain management in chronic illnesses and end-of-life care in hospitals, hospices and at home (Etkind 2017). Cost-effective commissioning of end-of-life resources has been highlighted as a priority (PHE 2017). Preliminary evidence shows that palliative care improves clinical outcomes and quality of care (Higginson 2003). Furthermore, palliative care, which includes bereavement care and preparatory grief work, has the potential to help unpaid caregivers access the care they need related to the death of a loved one (Grande 2017).

The numbers of inpatient hospital palliative care teams are increasing (CAPC 2018; Meier 2011). This is occurring in response to unmet palliative care needs of inpatients and their unpaid caregivers (Meier 2011), yet clarity around effective models of care are needed. This Cochrane Review will provide muchneeded clarity regarding the effectiveness and cost-effectiveness of hospital-based specialist palliative care. In the review, five different models of hospital-based specialist palliative care were specified due to its evolving nature and also to make the findings more relevant to clinical practice. The models of hospital-based specialist palliative care eligible are ward-based models, inpatient consulting models, outpatient models, hospital-at-home or hospital outreach models (hereafter outreach model) and service provision across multiple settings which included hospital. The review findings will have the potential to aid the future development, funding and implementation of hospital-based specialist palliative care. This may help transform services, which have mostly developed locally in culturally responsive ways in relation to local needs and populations (Higginson 2003; Kamal 2013). Therefore, the review will help deliver hospital-based specialist palliative care services in the midst of increased ageing populations that present with complex clinical needs against a backdrop of fiscal constraint and increased healthcare utilisation.

\section{Description of the condition}

Population-based estimates of palliative care have indicated which populations require this service (Murtagh 2014), including those with malignant neoplasms and non-malignant and other health-related conditions, specifically: heart disease, including cerebrovascular disease, renal disease, liver disease, respiratory disease, neurodegenerative disease (Huntington's disease, Parkinson's disease, multiple sclerosis, motor neuron disease, multi-system degeneration, progressive supranuclear ophthalmoplegia, Alzheimer's dementia and senility) and HIV/ AIDS. Patients with any of these conditions and their unpaid caregivers were considered for inclusion in this review.

\section{Description of the intervention}

The intervention of interest is hospital-based specialist palliative care (HSPC). In this review, hospital-based specialist palliative care encompasses the following essential components:

- care co-ordinated by a multiprofessional or multidisciplinary team;

- collaboration between specialist palliative care providers and generalist providers; and

- holistic care (NCP 2013).

HSPC refers to care that is provided with the input of specialist palliative care providers to patients while they are admitted as inpatients to acute care hospitals, outpatients or patients receiving care from hospital outreach teams at home. The models of HSPC eligible for inclusion include ward-based models, inpatient consulting models, outpatient models, hospital outreach models and service provision across multiple settings which included hospital. Ward-based models encompassed care provision to patients and their families on a palliative care ward in hospital. Inpatient consulting models encompassed care provision to patients and their families by an inpatient consult team while they are admitted as inpatients to acute hospitals. Outpatient models comprised care provision to hospital outpatients and their families. Hospital-at-home or hospital outreach into the community involved care provision by hospital outreach teams in the patient's home as well as service provision across multiple settings including hospital.

The intervention aims to prevent or relieve physical, psychological, social and spiritual problems. It is provided to patients who have a malignant and/or non-malignant condition who may or may not be at the end of their life (Dixon 2015). Recognising the importance 
of the informal unpaid caregiver, palliative care also aims to meet the psychological, social and spiritual needs of unpaid caregivers. (Grande 2017).

At the heart of palliative care is the belief that every person is unique, autonomous and that they have the right to continue to live and enjoy quality of life even though they are diagnosed with an advanced, life-limiting or life-threatening illness. Specialist palliative care is differentiated from generalist palliative care. Specialists are likely to have received higher specialist training in palliative care work and services focus mainly or exclusively on patients with palliative care needs; whereas for generalists, provision of palliative care is a component of their service provision (Dixon 2015) and they will not have received higher specialist traing in palliative care. Specialist care is mostly provided to patients with advanced, life-limiting or life-threatening illness who present with complex needs (NHS England 2016). Complexity, although sometimes difficult to define, involves clinical complexity and its interaction with the confidence or ability of the lead clinical team (generalists) to address the presenting need. Complexity may involve intertwined and multiple factors which may include related age, the serious nature of illness, social or familial backgrounds, and/or the nature of a symptom (e.g. the usualness or intractable nature of the symptom) (Palliative Care Australia 2005; Quill 2013).

Pre-bereavement interventions are also specialist palliative care interventions administered to prevent or manage bereavementrelated physical, psychological, social and spiritual problems experienced by unpaid caregivers prior to the death of the patient (Aoun 2017; Breen 2014). We included specialist palliative care interventions involving pre-bereavement interventions either to the unpaid caregiver alone or together with the patient.

\section{How the intervention might work}

Although positive outcomes, such as symptom reduction, improved quality of care and care co-ordination, and reduced hospital costs, can result from hospital-based specialist palliative care, qualitative methods such as interviews and empirical testing using randomised controlled trials have yet to definitively establish how hospital-based specialist palliative care might work. Therefore, any descriptions of how hospital-based specialist palliative care may work are speculative. That acknowledged, hospital-based specialist palliative care may work with patients by the following:

- directly improving symptoms (including physical and psychological symptoms, such as uncertainty and feelings of loss) through specialist interventions and holistic care (Temel 2010);

- improving care quality by delivering or facilitating improved care co-ordination and person-centred holistic care (Daveson 2014; Pinnock 2011);

- reducing futile medical interventions by mitigating against disease-modifying priorities through optimal communication and shared decision-making practice (Harris 2013

- addressing holistic needs that span multimorbidity (Burge 2012); and

- reducing unnecessary hospital costs through significant reduction in pharmaceutical, laboratory and intensive care unit costs (May 2014);
In addition, findings from published a systematic review (Harding 2012), RCTs (Allen 2008; Hudson 2005), and a before-and-after study (Lichtenthal 2011), indicated that the intervention may work for unpaid caregivers prior to the death of the patient through the following mechanisms:

- emphasising the positive aspects of caregiving by providing relevant information, guidance and instruction. The intervention may also work by providing unpaid caregivers with individual support to see problems differently, draw out their optimism, helping them to plan and by providing them with access to expert information;

- improving the unpaid caregiver's understanding of their experiences and role to result in increased caregiving competencies and knowledge;

- aiding their interpretation of their circumstance and normalising their emotional responses to caregiving demands;

- enabling their involvement in care planning, where possible;

- engaging both patients and unpaid caregivers in a life review within consultations which may work to reduce unpaid caregivers' stress; and

- ensuring timely assessment of needs, adaptive coping and access to needs-based care through pre-bereavement work.

The intervention may therefore also work via a preventive mechanism.

\section{Why it is important to do this review}

A previous systematic review by Higginson 2002 showed that hospital-based palliative care improved clinical outcomes and quality of care and can reduce hospital costs. However, this review was small (nine studies) and only included cancer patients. A recent review in hospital, hospice or community settings by Gaertner 2017 showed that specialist palliative care led to improvement in quality of life with significant benefits for patients with cancer receiving specialist palliative care early. The results for pain and other outcomes were inconclusive. Another review by Haun 2017 showed that early palliative care interventions resulted in improved quality of life and lower symptom intensity compared with the control condition. Survival and levels of depression did not differ significantly between the early palliative care group and control group.

Since the publication of these systematic reviews, there have been at least six newly published RCTs on hospital-based specialist palliative care and no review on its different models. In addition, the models of palliative care are continuously evolving. Recent UK government (DoH 2008), and commissioning guidance (NCPC 2012), have recommended that there ought to be delivery of a 24/7 palliative care service. However, the End of Life Care Audit 2016 showed that of the 142 acute NHS trusts in England participating, only $37 \%$ had specialist palliative care services available out-ofhours and this service varied with level of contact (telephone or on-site visiting) and health professional involved (specialist nurse, junior doctor or consultant) (RCP 2016). The research priorities identified by the James Lind Alliance highlighted the need for research into identifying the core palliative care services needed and the best way of providing palliative care outside of working hours (JLA 2015). This Cochrane Review addresses these priorities. It is important that, following the Liverpool Care Pathway and Neuberger review, we examine the most effective methods and

The effectiveness and cost-effectiveness of hospital-based specialist palliative care for adults with advanced illness and their caregivers 
models of hospital-based specialist palliative care in order to ensure that there is an evidence-based approach to its delivery (Crown 2013)

A Cochrane Review has provided valuable evidence synthesis on the effectiveness and cost-effectiveness of home palliative care services (Gomes 2013). However, there is no such available evidence for specialist palliative care in hospital inpatient, outpatient, outreach and services provided across multiple settings. Furthermore, the numbers of hospital-based specialist palliative care teams are increasing (CAPC 2018; Meier 2011). This is occurring in response to unmet palliative needs of patients and their unpaid caregivers (Meier 2011), yet clarity regarding the effective components of the intervention is needed. This review may therefore assist with providing much-needed solutions to problems, and clarity regarding the effectiveness and costeffectiveness of the component parts of hospital-based specialist palliative care. In essence, the review may address some of the problems encountered by contemporary healthcare systems and services, service-users, clinicians, policy-makers, researchers and commissioners.

\section{O B J E C T IVES}

To assess the effectiveness and cost-effectiveness of hospital-based specialist palliative care compared to usual care for adults with advanced illness and their unpaid caregivers/families.

\section{METHODS}

\section{Criteria for considering studies for this review}

\section{Types of studies}

Due to the increasing numbers of RCTs in palliative and end-oflife care, and also because they are the most robust experimental design, this review only included RCTs (including cluster-unit randomised trials). We used established approaches to include and analyse RCTs following the Cochrane Handbook for Systematic Reviews of Interventions (Higgins 2011a).

All studies evaluated effectiveness regarding one or more of our primary or secondary outcomes. In the economic component of the review, we included studies conducted alongside (or as part of) the main effectiveness trial and ones that also met the eligibility criteria for the effectiveness component. Full economic evaluation (i.e. cost-effectiveness analyses, cost-utility analyses, cost-benefit analyses); partial economic evaluations (i.e. cost analyses, costdescription studies, cost-outcome descriptions); and studies that reported more limited information, such as estimates of resource use or costs associated with service use, were eligible for inclusion.

\section{Types of participants}

- Adult ( $\geq 18$ years) patients receiving hospital-based specialist palliative care:

* these patients were diagnosed with advanced, life-limiting or life-threatening illness (malignant or non-malignant), which was likely to compromise their quality of life in some way;

* diseases and health-related conditions included (with the corresponding International Classification of Diseases (ICD-10)) malignant neoplasms (ICD-10 codes: C00-C97) and non-malignant and other health-related conditions, specifically: heart disease, including cerebrovascular disease
(ICD-10 codes: 100-152, 160-69), renal disease (ICD-10 codes: N17, N18, N28, I12, I13), liver disease (ICD-10 codes: K70-K77), respiratory disease (ICD-10 codes: J06-J18, J20-22, J40-47, J96), neurodegenerative disease (Huntington's disease (ICD-10 code: G10), Parkinson's disease (ICD-10 code: G20), multiple sclerosis (ICD-10 code: G35), motor neuron disease (ICD-10 code: G12.2)), multi-system degeneration (ICD-10 code: G90.3), progressive supranuclear ophthalmoplegia (ICD-10 code: G23.1), Alzheimer's dementia and senility (ICD-10 codes: F01, F03, G20, R54), and HIV/AIDS (ICD-10 codes: B20-B24)); and

- unpaid caregivers, including those who had received a prebereavement intervention from one or more hospital-based specialist palliative care staff in order to manage or alleviate bereavement-related problems prior to the death of the inpatient: unpaid caregivers are likely to be family, friends or significant others associated with the patient (Payne 2010a; Payne 2010b).

\section{Types of interventions}

Hospital-based Specialist Palliative Care (herein HSPC) varies between settings and countries. In order to allow for these differences, we included studies that described HSPC as "palliative care, generic palliative care, hospice care (provided in hospital settings) or specialist palliative care". It was delivered by a specialist palliative care team or by a "specialist palliative care", "palliative care" or "hospice outreach (based in hospital settings)" staff member. In order to account for differences in specialist palliative care between countries, and also because of the sometimes limited details provided on the specialist training of palliative care teams, we decided to include studies where training/clinical experience in specialist palliative care was made explicit as well as those that simply stated the involvement of a palliative care team; eligibility was informed by activity of delivering specialist palliative care rather than level of specialist training (Luckett 2014). Higher specialist training in palliative care was also accepted if the authors described the professionals as palliative care experts or specialists (for example, palliative care physician or nurse) or if they had obtained clinical competencies and professional characteristics required for the delivery of specialist palliative care through clinical experience (NCPC 2012). The intervention was provided to adults receiving hospital inpatient, outpatient, outreach or HSPC as part of wider services, and their unpaid caregivers/families.

We included studies of HSPC compared with usual care. Usual care was defined as inpatient or outpatient hospital care without specialist palliative care input (e.g. oncological care) at the point of entry into the study, community care (e.g. primary or specialist care provided in the patient's place of residence) or hospice care provided outside of the hospital setting. Usual care patients may receive specialist palliative care after entry into the study if requested by the patient, their families or clinicians, however specialist palliative care should not be a routine part of usual care. We extracted descriptive data on what was involved in each intervention.

Similar to a Cochrane Review that examined home palliative care (Gomes 2013), we excluded trials that evaluated hospital palliative care practitioners' provision of only a biomedical component of palliative care (e.g. oxygen therapy) as this does not encompass the holistic nature of palliative care assessment or treatment.

The effectiveness and cost-effectiveness of hospital-based specialist palliative care for adults with advanced illness and their caregivers 


\section{Types of outcome measures}

We developed the primary and secondary outcomes for this review from previous reviews regarding the effectiveness of palliative care and those that we thought to be clinically relevant (Gomes 2013; Gysels 2004; Higginson 2003; Higginson 2010). The outcomes reflect the multicomponent nature of palliative care and the provision of both direct (e.g. face-to-face delivery of patient care) and indirect (e.g. concerning practitioners' prescribing rationale) patient care, and care for unpaid caregivers/families while the patient is still alive. We chose to measure health-related quality of life and symptom burden reported as adjusted endpoint values as our primary outcomes. We selected health-related quality of life and symptom burden as primary outcomes because the major focus of palliative care is to improve quality of life while providing optimal management of symptoms (Dixon 2015).

\section{Primary outcomes}

- Patient health-related quality of life, measured using validated assessment scales which may be generic and disease/conditionspecific health-related quality of life measures; and

- Patient symptom burden, specifically, a collection of two or more symptoms which could be physical (e.g. pain), psychological (e.g. anxiety, depression), social or spiritual domains, either patient or proxy-reported through validated generalised assessment scales.

\section{Secondary outcomes}

- Patient satisfaction with care through validated assessment scales;

- Caregiver/family satisfaction with care through validated assessment scales;

- Achieving patient's preferred place of care;

- Achieving patient's preferred place of death;

- Patient mortality/survival;

- Pain measured using validated assessment scales;

- Patient anxiety and depression measured using validated assessment scales;

- Breathlessness measured using validated assessment scales;

- Adverse events in participants and unpaid caregivers;

- Unpaid caregiver symptom control, specifically physical, psychological (e.g. anxiety and depression), social or spiritual domains, reported through validated assessment scales and burden, including emotional strain, burden, distress, mastery or positive aspects of caregiving through validated assessment scales;

- Unpaid caregiver pre- and post-bereavement outcomes, reported using validated outcome scales of multidimensional caregiving experiences (strain, distress, positive appraisals, and family well-being), caregiver prolonged grief, multidimensional grief responses (despair, panic behaviour, blame and anger, detachment, disorganisation and personal growth), quality of life.

- Resource use: institutional care services use (e.g. emergency department (ED) or accident and emergency (A\&E), intensive care unit use, inpatient stay, care in nursing homes (or skilled nursing homes) etc.), outpatient clinic services use (e.g. palliative care visits in outpatient settings, consultation with experts in outpatient settings), community care services use (e.g. contact with general practitioners, district nurses, home care, hospice care at home etc.), unpaid caregiver's care, and medications and other resources;

- Costs and cost-effectiveness: costs were calculated based on resource use and unit costs of services, while cost-effectiveness was measured using e.g. incremental cost-effectiveness ratios of costs and condition-specific outcome measures or qualityadjusted life years (QALYS) or an equivalent.

\section{Search methods for identification of studies}

We identified studies through electronic searches, handsearching, electronic citation tracking, personal contact and searching of grey literature. We did not place restrictions on language; we assessed non-English papers with the assistance of a native speaker.

\section{Electronic searches}

We identified studies by searching the databases listed below, using a combination of key terms and MeSH terms:

- Cochrane Library:

* Cochrane Central Register of Controlled Trials (CENTRAL); Issue 8 of 12, 2019

* Cochrane Database of Systematic Reviews (CDSR); Issue 8 of 12, 2019

* Database of Abstracts of Reviews of Effects (DARE), Issue 2 of 4, 2015;

* Health Technology Assessment (HTA), Issue 4 of 4, 2016;

* National Health Service Economic Evaluation Database (NHS EED), Issue 2 of 4, 2015;

- MEDLINE \& MEDLINE-in-Process (OVID), 1947 to 27 August 2019;

- Embase (OVID), 1974 to 27 August 2019;

- CINAHL (EBSCO),1982 to 28 August 2019;

- PsycINFO (OVID), 1806 to 28 August 2019;

- CareSearch, Australian Government's Department of Health and Ageing (http://www.caresearch.com.au/) (from inception to 12 September 2019).

We could not carry out more recent searches in DARE, HTA and NHS EED because they are no longer updated. We also could not carry out a search of the health economic database EURONHEED as it is no longer available. We refined our search strategies with the assistance of the Information Specialist of the Cochrane Pain, Palliative and Supportive Care Review Group. Please see Appendix 1 for the MEDLINE search strategy in OVID and Appendix 2, Appendix 3, Appendix 4, Appendix 5 and Appendix 6 for all other search strategies.

\section{Searching other resources}

We searched clinicaltrials.gov (www.clinicaltrials.gov) and the World Health Organisation (WHO) International Clinical Trials Registry Platform (ICTRP) (http://apps.who.int/trialsearch/) for ongoing trials on 12 September 2019 (search term: palliative).

\section{Handsearching}

We screened the reference lists of all included studies, and three relevant systematic reviews (Haun 2017; Gaertner 2017; Gomes 2013), for additional studies.

The effectiveness and cost-effectiveness of hospital-based specialist palliative care for adults with advanced illness and their caregivers 


\section{Electronic citation tracking}

We used the "Citation tacking" option in MEDLINE for lateral searching on the included studies, as recommended for palliative care reviews (Payne 2010a).

\section{Personal contact}

We contacted 15 experts in the field for unpublished and ongoing trials. We also contacted study authors for additional information where necessary.

\section{Data collection and analysis}

\section{Selection of studies}

Two review authors (AO and SB) independently screened all titles and abstracts identified in our electronic searches. If, after reading the abstract, doubt persisted regarding the eligibility of the study, we retrieved the full-text articles for further assessment and again the two reviewers independently assessed these full-text articles. We resolved disagreements by discussion and consensus. We reported our study selection process using a Preferred Reporting Items for Systematic Reviews and Meta-Analyses (PRISMA) flow diagram (Liberati 2009) in Figure 1 , as recommended in the Cochrane Handbook for Systematic Reviews of Interventions (Higgins 2011a). 
Figure 1. PRISMA flow diagram.

Electronic search $(n=15,927)$
From MEDLINE $(n=6474)$
From Cochrane library (inclu
$1606)$
From Embase $(n=4302)$
From PsycINFO $(n=349)$
From CINAHL $(n=2170)$
From CareSearch $(n=1026)$

Other sources $(n=55)$

From trial registers $(n=34)$

From searching systematic reviews ( $\mathrm{n}$ $=3$ )

From searching reference list $(n=13)$

From contacting experts $(n=5)$

\section{0,774 records after \\ duplicates removed}

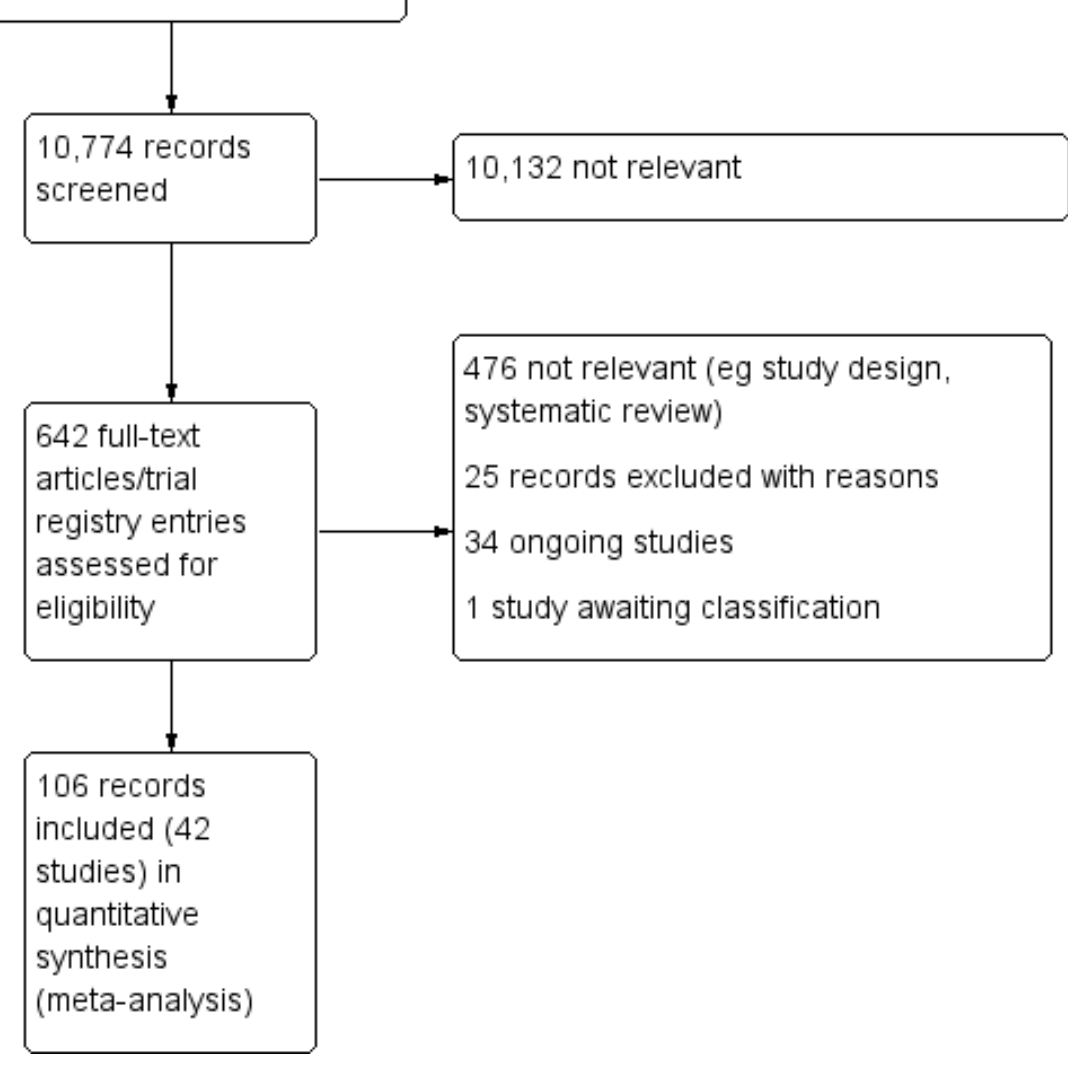

\section{Data extraction and management}

Two reviewers (AO and $\mathrm{SB}$ ) independently extracted data from all included studies using a piloted data extraction form (Appendix 7), that we further developed for economic evaluation based on the format and guidelines used to produce structured abstracts of economic evaluations for inclusion in the NHS EED. We entered data into Review Manager (RevMan) (RevMan 2014). We resolved any disagreements by discussion and consensus. Given that the review included some studies by the review authors, we did not involve these authors in the assessment of or extraction of data from their studies. The data extraction form has been used previously for a review on the effectiveness of home palliative care (Gomes 2013). We adapted the form for this review regarding HSPC.

We collated multiple reports of the same study, so that each study rather than each report was the unit of interest in the review. We collected characteristics of the included studies in sufficient detail to populate a 'Characteristics of included studies' table.

\section{Assessment of risk of bias in included studies}

Two reviewers (AO and $\mathrm{SB}$ )independently assessed risk of bias for each included study, using the criteria outlined in

The effectiveness and cost-effectiveness of hospital-based specialist palliative care for adults with advanced illness and their caregivers 
the Cochrane Handbook for Systematic Reviews of Interventions (Chapter 12, Schunemann 2011), with any disagreements resolved by discussion. We completed a 'Risk of bias' table for each included study using the 'Risk of bias' tool for randomised controlled studies in RevMan (RevMan 2014).

We assessed the following for each included study:

- Random sequence generation (checking for possible selection bias). We assessed the method used to generate the allocation sequence as:

* low risk of bias (any truly random process, e.g. random number table; computer random number generator); or

* unclear risk of bias (method used to generate sequence not clearly stated);

* we excluded studies that used a non-random process (e.g. odd or even date of birth; hospital or clinic record number).

- Allocation concealment (checking for possible selection bias). The method used to conceal allocation to interventions prior to assignment determines whether intervention allocation could have been foreseen in advance of, or during recruitment, or changed after assignment. We assessed the methods as:

* low risk of bias (e.g. telephone or central randomisation; consecutively numbered sealed opaque envelopes); or

* unclear risk of bias (method not clearly stated);

* we excluded studies that did not conceal allocation.

- Blinding of participants and personnel (checking for possible performance bias) (subjective). We assessed the methods used to blind study participants and personnel from knowledge of which intervention a participant received for subjective outcomes (e.g. quality of life, pain, breathlessness). We grouped all subjective outcomes as being at high risk of bias if blinding was unsuccessful. When the study did not include subjective outcomes, we left this domain blank. We assessed the methods as:

* low risk of bias (blinding of participants and key study personnel ensured, and unlikely that the blinding could have been broken);

* unclear risk of bias (insufficient information to permit judgement of 'low risk' or 'high risk');

* high risk of bias (no blinding or incomplete blinding; blinding of key study participants and personnel attempted, but likely that the blinding could have been broken, and the outcome is likely to be influenced by lack of blinding).

- Blinding of participants and personnel (checking for possible performance bias) (objective). We assessed the methods used to blind study participants and personnel from knowledge of which intervention a participant received for objective outcomes (e.g. mortality, length of stay in hospital, number of readmissions). When the study did not include objective outcomes, we left this domain blank. We assessed the methods as:

- low risk of bias (objective outcomes are unlikely to be influenced by lack of blinding and we treated these outcomes as a 'low risk of bias' even if blinding was unsuccessful or not carried out);

- unclear risk of bias (insufficient information to permit judgement of 'low risk' or 'high risk'); or

- we did not rate a high risk of bias for an objective outcome.
- Blinding of outcome assessment (checking for possible detection bias) (subjective). We assessed the methods used to blind outcome assessors from knowledge of which intervention a participant received for subjective outcomes. We grouped all subjective outcomes as being at high risk of bias if blinding was unsuccessful. When the study did not include subjective outcomes, we left this domain blank. We assessed the methods as:

* low risk of bias (blinding of outcome assessment ensured, and unlikely that the blinding could have been broken);

* unclear risk of bias (insufficient information to permit judgement of 'low risk' or 'high risk'); or

* high risk of bias (no blinding of outcome assessment; blinding of outcome assessment, but likely that the blinding could have been broken, and the outcome measurement is likely to be influenced by lack of blinding).

- Blinding of outcome assessment (checking for possible detection bias) (objective). We assessed the methods used to blind outcome assessors from knowledge of which intervention a participant received for objective outcomes. Objective outcomes are unlikely to be influenced by lack of blinding and we rated these outcomes as a 'low risk of bias' even when blinding was unsuccessful or not carried out. When the study did not include objective outcomes, we left this domain blank. We assessed the methods as:

- low risk of bias (e.g. no blinding of outcome assessment, but the review authors judge that the outcome measurement is not likely to be influenced by lack of blinding; blinding of outcome assessment ensured, and unlikely that the blinding could have been broken);

- unclear risk of bias (insufficient information to permit judgement of 'low risk' or 'high risk'); or

- we did not rate a high risk of bias for an objective outcome.

- Selective reporting (checking for reporting bias). We assessed whether primary and secondary outcome measures were prespecified and whether these were consistent with those reported. We assessed the methods as:

* low risk of bias (protocol is available and all of the study's prespecified primary and secondary outcomes that are of interest in the review have been reported in the prespecified way);

* unclear risk of bias (insufficient information to permit judgement of 'low risk' or 'high risk', including, when the protocol is not available); or

* high risk of bias (protocol is available and some prespecified outcomes were not reported; one or more primary outcomes were reported using measurements, analysis methods or subsets of the data (e.g. subscales) that were not prespecified; one or more reported primary outcomes were not prespecified).

- Incomplete outcome data (checking for possible attrition bias due to the amount, nature and handling of incomplete outcome data). We assessed the methods used to deal with incomplete data as:

* low risk ( $<10 \%$ of participants did not complete the study or used 'baseline observation-carried-forward' analysis); 
* unclear risk of bias (used 'last-observation-carried-forward' analysis or when the number of dropouts was not reported); or

* high risk of bias (used 'completer' analysis).

- Other bias (other sources of bias). We also assessed whether groups were balanced at baseline and whether differences at baseline were controlled for. We assessed the studies as:

* low risk of bias (e.g. if there were no baseline differences or if observed differences were controlled for);

* unclear risk of bias (e.g. if there were baseline differences and it was unclear if the differences were significant and also if they were controlled for); or

* high risk of bias (e.g. if there were differences that were not controlled for).

- Size of study (checking for possible biases confounded by small size). We assessed studies as being at:

* low risk of bias ( $\geq 200$ participants per treatment arm);

* unclear risk of bias (50 to 199 participants per treatment arm; 50 to 199 participants in one treatment arm and $\geq 200$ participants in another treatment arm; $<50$ participants in one treatment arm and 50 to 199 participants in another treatment arm); or

* high risk of bias (< 50 participants per treatment arm).

\section{Quality assessment in studies with a cost/cost-effectiveness component}

We classified health economics studies per the design of the health economic study (e.g. full economic evaluation, partial economic evaluation) and the design of the study generating the effectiveness data of the health economic study (e.g. a single study design, a synthesis of several studies). For full economic evaluations, we assessed the risk of bias in results of the single effectiveness study on which the full economic evaluation study was based and methodological quality of the full economic evaluation study. We used as checklists the BMJ Checklist for authors and peer reviewers of economic submissions (Drummond 1996), and the Consensus on Health Economic Criteria (CHEC) list for assessment of methodological quality of economic evaluations (Evers 2005).

For assessment of the quality of relevant economic modelling studies, we planned to use tools such as the Consolidated Health Economic Evaluation Reporting Standards (CHEERS) statement (Husereau 2013), and the Quality Appraisal Checklist for Economic Evaluations (NICE 2012), supplemented by the Philips Checklist (Philips 2004). We could not apply these planned methods in this review as we did not identify any relevant economic modelling studies for inclusion; we plan to use these tools for future updates of the review, where appropriate.

\section{Measures of treatment effect}

If appropriate, we undertook meta-analysis of the primary and secondary outcomes using RevMan (RevMan 2014). Given that eligible studies were conducted with different populations, countries and years, and that they included different models of HSPC, we had to incorporate the assumption of heterogeneity in the meta-analysis of our outcomes. We used the inverse variance random-effects model for meta-analysis. This method summarises effect sizes from studies by calculating the weighted mean of the effect sizes using the inverse variance of the individual studies as weights (Lee 2016).
We combined data from the RCTs for the primary outcomes (patient health-related quality of life and patient symptom burden) and expressed the pooled effect as standardised mean difference (SMD) for HSPC compared to usual care; values greater than 0 indicated better patient health-related quality of life with HSPC, and less than 0 indicated worse health-related quality of life. By contrast, for symptom burden, values greater than 0 indicated higher symptom burden and less than 0 reduced symptom burden.

We used a P value of 0.05 as the cut-off value to determine statistical significance and we presented data as effect size with $95 \% \mathrm{Cls}$. We did not combine change values with endpoint values in our meta-analysis because we pooled the data using SMD (Deeks 2011). Furthermore, we pooled adjusted endpoint values presented for patient health-related quality of life and patient symptom burden as our main analyses because adjusted endpoint values control for differences and provide the most precise and least biased estimates of treatment effects (Deeks 2011). Where possible, we conducted similar meta-analyses for the other outcomes with the exception of achieving preferred place of death (measured as home deaths) where we expressed the pooled effect as an odds ratio (OR) for HSPC compared to usual care; values greater than 1 indicated increased odds of achieving preferred place of death with HSPC, and less than 1 indicated decreased odds. Even though we used ORs to detect treatment effect, we also presented findings as risk ratios (RRs) (or relative risk) in order to aid the use and interpretation of the findings by end users. We used the Mantel-Haenszel (M-H) method in the meta-analysis for achieving preferred place of death.

In order to combine different instruments in which an increase in score indicates improvement or an increase in score is worse in the same meta-analysis, we multiplied the mean values from one set of studies by -1 to ensure that all the scales were in the same direction.

In order to interpret subgroup differences in our subgroup analyses, we considered the test for subgroup differences and also checked for confidence interval overlap. Where $P$ values were $<0.05$ in the test for subgroup differences, we considered this to be evidence of a subgroup effect. However, we were cautious in the interpretation of our subgroup analyses where there were a small number of studies and participants.

We considered that a SMD of 0.2 to $<0.5$ constituted a small effect, 0.5 to $<0.8$ a moderate effect and $\geq 0.8$ constituted a large effect (Cohen 1988).

\section{Economic data}

We presented characteristics of the included health economics studies, such as year of study; details of interventions and comparators; study design; data sources; jurisdiction and setting; analytic perspective and time horizon, in the 'Characteristics of included studies' table as recommended in the Cochrane Handbook for Systematic Reviews of Interventions (Higgins 2011a). We summarised characteristics and results of included economic evaluations using additional tables, supplemented by a narrative summary that compared and evaluated methods used and principal results between studies. Where possible, we presented point estimates of measures of items of resource use and cost with associated measures of uncertainty for both the intervention and its comparators, as well as point estimates of incremental costs and cost-effectiveness, again with associated measures of uncertainty. We converted costs to Great British Pounds (GBP) (2018) based on 
Purchasing Power Parities (PPP) and gross domestic product (GDP) deflators.

\section{Unit of analysis issues}

We addressed issues in the analysis of studies with particular characteristics, for example cluster-randomised trials, in our meta-analysis. We highlighted whether cluster-randomised trials presented their intra-cluster correlation coefficient (ICC) and if they made adjustment for clustering. Where studies adjusted for clustering, we used the data they presented in the meta-analysis. However, where the authors did not present their ICC or adjust for clustering, we contacted the authors for an estimate of the ICC. Where authors did not respond, we estimated an ICC from a previous Cochrane review (Shepperd 2011) and used it to adjust for clustering in order to allow for inclusion of the study in our metaanalysis. We carried out sensitivity analysis to test the estimate we used for clustering. The Cochrane Handbook for Systematic Reviews of Interventions suggests that decisions that may be somewhat unclear should be tested using sensitivity analysis (Higgins 2011a).

\section{Dealing with missing data}

When sample sizes and mean (SD) were missing, we did not carry out imputations or estimate the missing values for metaanalysis. Rather, as recommended by the Cochrane Handbook for Systematic Reviews of Interventions (Higgins 2011a), we contacted study authors to request additional data. Where studies had missing intervention data (such as number of staff involved and skills and so on), we assessed the potential impact of these missing data on the findings of the review in the 'Discussion' section of the review. We sought clarity from study authors regarding study population, analysis and interventions, where required.

\section{Assessment of heterogeneity}

We examined and assessed heterogeneity through the following three measures:

- inspecting the studies to examine for plausible areas of heterogeneity based on clinical factors that may influence findings of our meta-analysis;

- inspecting the forest plots;

- using the $1^{2}$ statistics to examine the extent and impact of heterogeneity between included studies (Higgins 2011a).

\section{Assessment of reporting biases}

In order to detect and manage reporting bias, we took the following steps to attend to:

- multiple (publication) bias by contacting study authors to ascertain whether duplication had occurred;

- location bias by searching relevant national and international trial registries for all relevant studies (e.g. CENTRAL);

- language bias by including studies published in languages other than English; and

- outcomes reporting (including non-publication of economic evaluation outlined in the protocol) through comparing the findings in eligible studies with published protocols, where available. Where published protocols were unavailable, we asked study authors to supply them.
In addition, where there were more than 10 included studies in our meta-analysis, we used funnel plots and visually inspected them for asymmetry/symmetry as a means of exploring whether there was evidence that study size (precision) was associated with effect size. Where possible, we also conducted relevant tests for asymmetry influenced by data type (e.g. continuous or dichotomous), to assist with examining publication bias and to overcome any reliance on visual inspection (Lau 2006). When we observed asymmetry, we considered publication bias as one (of several) plausible explanations (Sterne 2001).

\section{Data synthesis}

Where eligible studies were not sufficiently homogenous to permit meta-analysis, we extracted quantitative data (means, standard deviations, frequencies and proportions, test coefficients, 95\% Cls and effects sizes, where available) and we employed techniques used in narrative synthesis to analyse the data, including:

- tabulation, which involved inserting the main elements of extracted data into a table format;

- textual descriptions, which involved collating a summary description of each included study (part of Characteristics of included studies);

- clustering of group textual descriptions according to attributes; and

- vote counting to determine how often certain attributes were reported (Rodgers 2009).

Where possible, we included qualitative data from nested or embedded qualitative studies where qualitative data were used as part of the trial to explore stakeholder views and experiences of the intervention. We analysed these through narrative synthesis methods.

\section{Quality of the evidence}

Two review authors independently rated the quality of the outcomes. We used the Grading of Recommendations, Assessment, Development and Evaluation (GRADE) system to rank the quality of the evidence using the GRADEprofiler Guideline Development Tool software (GRADEpro GDT 2015) and the guidelines provided in the Cochrane Handbook (Chapter 12, Higgins 2011a).

The GRADE approach uses five considerations (study limitations, consistency of effect, imprecision, indirectness and publication bias) to assess the quality of the body of evidence for each outcome (Chapter 12, Higgins 2011a). The GRADE system uses the following criteria for assigning grades of evidence:

- high: we are very confident that the true effect lies close to that of the estimate of the effect.

- moderate: we are moderately confident in the effect estimate; the true effect is likely to be close to the estimate of effect, but there is a possibility that it is substantially different.

- low: our confidence in the effect estimate is limited; the true effect may be substantially different from the estimate of the effect.

- very low: we have very little confidence in the effect estimate; the true effect is likely to be substantially different from the estimate of effect. 
The GRADE system uses the following criteria for assigning a quality level to a body of evidence (Chapter 12, Higgins 2011a):

- high: randomised trials; or double-upgraded observational studies;

- moderate: downgraded randomised trials; or upgraded observational studies;

- low: double-downgraded randomised trials; or observational studies;

- very low: triple-downgraded randomised trials; or downgraded observational studies; or case series/case reports.

Factors that may decrease the quality level of a body of evidence are:

- limitations in the design and implementation of available studies suggesting high likelihood of bias;

- indirectness of evidence (indirect population, intervention,control, outcomes);

- unexplained heterogeneity or inconsistency of results (including problems with subgroup analyses);

- imprecision of results (wide Cls);

- high probability of publication bias.

Factors that may increase the quality level of a body of evidence are:

- large magnitude of effect;

- all plausible confounding would reduce a demonstrated effect or suggest a spurious effect when results show no effect;

- dose-response gradient.

We downgraded the quality of the evidence by one $(-1)$ or two $(-2)$ if we identified:

- serious $(-1)$ or very serious $(-2)$ limitation to study quality;

- important inconsistency $(-1)$;

- some $(-1)$ or major $(-2)$ uncertainty about directness;

- imprecise or sparse data $(-1)$

- high probability of reporting bias $(-1)$.

\section{'Summary of findings' table}

We included a 'Summary of findings' table to present the main findings in a transparent and simple tabular format. The table summarised the comparison of HSPC versus usual care (which could be inpatient or outpatient hospital care without specialist palliative care input (e.g. oncological care) at the point of entry to the study, community care (e.g. primary or specialist care provided in the patient's place of residence), and hospice care provided outside of the hospital setting). The table included key information concerning the quality of the evidence, the magnitude of effect of the interventions examined, and the sum of available data on the outcomes patient health-related quality of life, patient symptom burden, patient satisfaction with care, achieving patient preferred place of death (measured by number of patients with home death), pain, unpaid caregiver burden, and cost/cost-effectiveness.

\section{Subgroup analysis and investigation of heterogeneity}

As part of our primary objective, we identified the effective components and determined the comparative effectiveness of HSPC for adults with advanced illness and their unpaid caregivers/ families. We compared the resources and costs associated with these services and determined their cost-effectiveness; compared effectiveness by disease type (e.g. malignant and non-malignant groups), and country; and we examined other sources of heterogeneity and the applicability of meta-analysis.

Where possible, we performed subgroup analysis using the following components known to influence the effectiveness of specialist palliative care:

- disease type, including malignant, non-malignant and mixed malignant and non-malignant disease (mixed diagnoses);

- frailty associated with advanced age;

- HSPC team composition (e.g. physician-led, nurse-led versus multidisciplinary team-led palliative care services and organisation (e.g. 24-hour access (out-of-hours) versus temporally restricted access)) and taxonomy of the components;

- models of HSPC (ward-based model, inpatient consult model, outpatient model, outreach model and service provision across multiple settings);

- early palliative care versus late palliative care to assess the effectiveness of hospital-based palliative care applied early in the course of a life-threatening disease from palliative care delivered mainly with high symptom burden or in the terminal phase of illness. To be classified as early palliative care, early palliative care intent had to be stated explicitly or reflected in the sample composition, i.e. most participants had to be enrolled shortly after diagnosis of advanced disease (Haun 2017). Anything besides this, we classified as late palliative care; and

- country of origin.

\section{Sensitivity analysis}

We carried out sensitivity analyses to explore a number of our methodological decisions.

We conducted sensitivity analysis to assess our decision to use an estimate of intracluster-correlation coefficient (ICC) we had obtained from a previous Cochrane review (Shepperd 2011) to adjust for clustering in one of the cluster-RCTs (McCorkle 2015). The authors did not respond to our request for the ICC from their study.

Given that combining endpoint scores and change scores is not recommended when using standardised mean differences (SMDs) and also that Cochrane does not recommend pooling adjusted and unadjusted estimates together

(Deeks 2011), we pooled studies presenting adjusted endpoint scores as our main meta-analysis while we carried out sensitivity analyses with studies reporting unadjusted endpoint scores, adjusted change scores and unadjusted change scores.

\section{RES U LTS}

\section{Description of studies}

Also see the Characteristics of included studies; Characteristics of excluded studies; Characteristics of studies awaiting classification; Characteristics of ongoing studies tables.

\section{Results of the search}

We identified 15,927 records from our electronic searches and an additional 55 records from other sources. After removing

The effectiveness and cost-effectiveness of hospital-based specialist palliative care for adults with advanced illness and their caregivers 16 (Review)

Copyright (c) 2020 The Cochrane Collaboration. Published by John Wiley \& Sons, Ltd. 
duplicates, two authors independently screened the titles and abstracts of 10,774 records, excluded 10,132 records and selected 642 for full-text reading. We classed 476 records as not relevant (e.g. systematic reviews, study design).

We included 42 studies reported in 106 records (91 full papers and 15 abstracts), ranging from one to ten records per study (see Included studies). Of the remaining records, we excluded 25 with reasons (see Excluded studies); 34 are ongoing studies, and one study is awaiting classification (Aljohani 2015) (see Figure 1 for the PRISMA flow diagram).

\section{Included studies}

\section{Design}

All the studies we included were RCTs, comprising one cluster-RCT (McCorkle 2015), one cluster-randomised crossover trial (Ma 2019) and eight fast-track RCTs (Bajwah 2015; Bakitas 2015; Edmonds 2010; Farquhar 2014; Farquhar 2016; Higginson 2009; Higginson 2014; McWhinney 1994). The remaining 32 RCTs had a parallel design.

\section{Sample sizes}

Sample sizes in included studies ranged from 30 to 621 participants. The length of recruitment in included studies varied between 10 months and 50 months. In total, we included data from studies involving 7779 participants (6678 adults with advanced illness and 1101 unpaid caregivers/family members). Thirty-three studies had power calculations (details in 'Characteristics of included studies'). Nine studies were powered on quality of life only (Bekelman 2018; El-Jawahri 2016; Franciosi 2019; Groenvold 2017; Rogers 2017; Tattersall 2014; Temel 2010; Temel 2017; Vanbutsele 2018). Ma 2019 was powered on proportion of patients transitioning to do-notresuscitate and do-not-intubate (DNR/DNI). In addition to quality of life, Bakitas 2015 also performed calculations on depression, Solari 2018 on symptom burden, O'Riordan 2019 on pain, while Bakitas 2009 and Sidebottom 2015 included depression and symptom burden. Farquhar 2014 and Farquhar 2016 were powered on distress due to breathlessness, Brannstrom 2014 on symptom burden, Brumley 2007 on cost, Carson 2016 on depression and anxiety, Grudzen 2016 on time to palliative care, Janssens 2019 on hospital admission, Rodin 2019 on traumatic stress symptoms,
Bajwah 2015, Edmonds 2010 and Higginson 2009 on the Palliative care Outcome Scale (POS), Lowther 2015 on the African Palliative care Outcome Scale (APOS), Higginson 2014 on Chronic Respiratory Disease Questionnaire (CRDQ) mastery domain, Hopp 2016 and Ozcelik 2014 on palliative outcomes and palliative care service respectively, McWhinney 1994 on pain and nausea and Woo 2019 on pain and depression.

Eight studies were well-powered at recruitment and also at the primary point of analyses (Carson 2016; Edmonds 2010; Farquhar 2016; Higginson 2014; Ma 2019; Ozcelik 2014; Solari 2018; Temel 2017). Fourteen studies were underpowered at recruitment stage (i.e. participants enrolled) by three participants (Brumley 2007; Groenvold 2017; Hopp 2016), four (Grudzen 2016), eight (Rodin 2019), 19 (Nottelmann 2018), 25 (O'Riordan 2019), 30 (Tattersall 2014), 50 (Rogers 2017), 74 (McWhinney 1994), 78 (Bakitas 2009), 111 (Janssens 2019), 153 (Bakitas 2015) and 268 (Sidebottom 2015). In one of the underpowered studies (Rogers 2017), the data and safety monitoring board in consultation with the sponsoring agency recommended a sample size reduction due to enrollment rates, a mortality rate that was lower than predicted and observed outcomes differences at the intermediate time point. Reasons provided for underpowered studies included slower than anticipated accrual, resource constraints, early deaths, problems with recruitment and low compliance rate for completion of questionnaires. The remaining 11 studies included the numbers that they had planned to recruit but dropped below the required numbers by the first time point of analyses (i.e. following baseline assessment and after receiving the intervention or control). These studies were underpowered by two participants (Brannstrom 2014), three participants (El-Jawahri 2016), five participants each (Bajwah 2015; Higginson 2009), six participants each (Lowther 2015; Farquhar 2014), 13 participants (Temel 2010), 22 participants (Vanbutsele 2018), 29 participants (Franciosi 2019), 60 participants (Woo 2019) and 70 participants (Bekelman 2018). Nine studies did not report any power calculation (Ahronheim 2000; Cheung 2010; Gade 2008; Jingfen 2017; Kane 1984; Mendoza-Galindo 2018 (abstract only); McCaffrey 2013; McCorkle 2015; Wallen 2012) (see Figure 2 for a graphical representation of the power of included studies at recruitment and follow-up). Overall, 14 studies examined post-intervention assessments in more than 100 participants. 
Figure 2. A figure describing the power of included studies at recruitment and follow-up

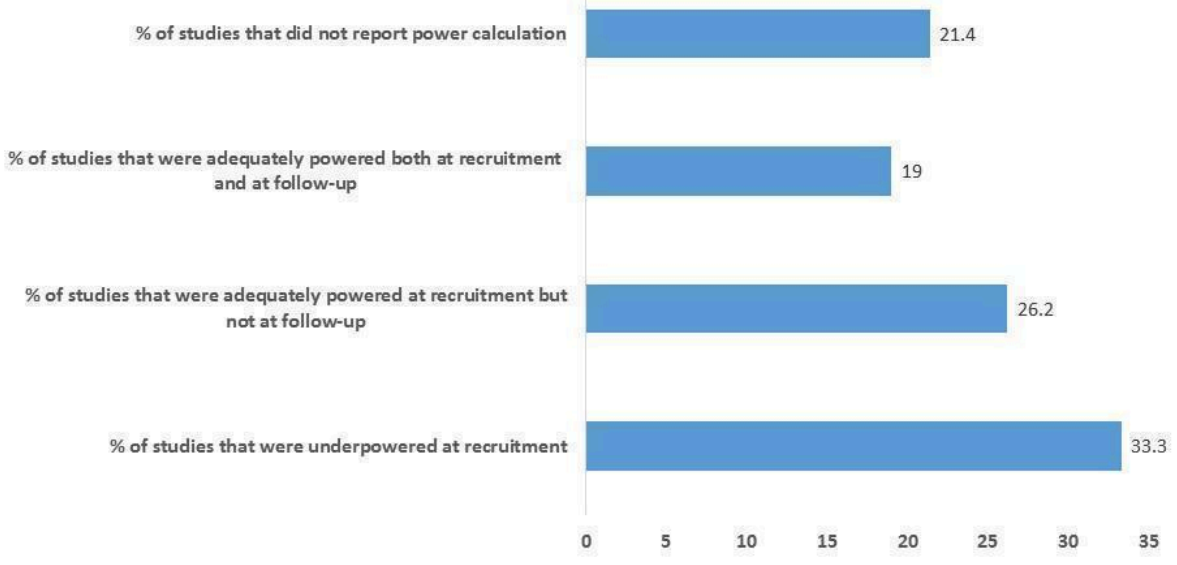

\section{Setting}

Nineteen studies were carried out in USA. Six studies took place in the UK (Bajwah 2015; Edmonds 2010; Farquhar 2014; Farquhar 2016; Higginson 2009; Higginson 2014), and three studies occurred in Australia (Cheung 2010; McCaffrey 2013; Tattersall 2014). One study was conducted in Sweden (Brannstrom 2014), two in Denmark (Groenvold 2017; Nottelmann 2018), one in Switzerland (Janssens 2019), one in Belgium (Vanbutsele 2018), two in Italy (Franciosi 2019; Solari 2018), and one in Turkey (Ozcelik 2014). McWhinney 1994 and Rodin 2019 were carried out in Canada, while Woo 2019 was undertaken in South Korea. Lowther 2015 took place in Kenya and Jingfen 2017 was conducted in China. MendozaGalindo 2018 (abstract only) was carried out in Mexico.

Thirty studies recruited from hospital settings. Three of these studies recruited from intensive care units (ICU) (Carson 2016; Cheung 2010; Ma 2019). Of these 30 studies, Ahronheim 2000 recruited patients with advanced dementia from Mount Sinai Hospital, Bajwah 2015 recruited from a specialist interstitial lung disease centre, Janssens 2019 from patients followed by Geneva University Hospitals on long-term oxygen therapy (LTOT) and/or home non-invasive ventilation (NIV) as well as those hospitalised for acute exacerbation of Chronic Obstructive Oulmonary Disease (COPD) in the general internal medicine and geriatric wards, Lowther 2015 from outpatient HIV clinics in a community hospital, McCorkle 2015 from disease-specific multidisciplinary clinics at a cancer hospital, O'Riordan 2019 from new inpatient admissions to the medicine and cardiology services, Solari 2018 from three Italian multiple sclerosis centres and Franciosi 2019 from outpatient and inpatient settings at five Italian cancer centres. Seven studies recruited from oncology centres or clinics (Groenvold 2017; Rodin 2019; Tattersall 2014; Temel 2010; Temel 2017; Vanbutsele 2018; Woo 2019). Bakitas 2009 and Bakitas 2015 recruited from oncology clinics of a cancer centre and affiliated outreach clinics and the Veterans Affairs Medical Centre (VAMC).

Eleven studies recruited from primary care and/or secondary care. For example, Gade 2008 recruited from community medical services and inpatient units, while McWhinney 1994 recruited through family physicians and home care nurses. Brumley 2007 received referrals from discharge planners, primary care physicians and other specialty physicians, whereas Rogers 2017 enrolled both hospitalised patients and recently discharged patients who were at high risk of rehospitalisation. Higginson 2009 received referrals from local health and social care professionals. Edmonds 2010 received referrals from health and social care professionals and, in a few instances, through voluntary organisations and self-referral.

Mendoza-Galindo 2018 (abstract only) did not present the setting where recruitment took place..

\section{Participants}

Twenty-one studies were carried out with patients who had severe/ advanced cancer or their unpaid caregivers/family members or both (Bakitas 2009; Bakitas 2015; El-Jawahri 2016; Farquhar 2014; Franciosi 2019; Groenvold 2017; Grudzen 2016; Jingfen 2017; Kane 1984; McCorkle 2015; McWhinney 1994; MendozaGalindo 2018 (abstract only); Nottelmann 2018; Ozcelik 2014; Rodin 2019; Tattersall 2014; Temel 2010; Temel 2017; Vanbutsele 2018; Wallen 2012; Woo 2019). A range of cancers were included in these studies comprising solid and non-solid tumour cancers. Seven studies involved both cancer and non-cancer populations (mixed populations) (Brumley 2007; Carson 2016; Cheung 2010; Gade 2008; Higginson 2014; Ma 2019; McCaffrey 2013), while the remaining 14 studies had only non-cancer populations. The non-cancer populations were those with interstitial lung disease (Bajwah 2015), heart failure (Bekelman 2018; Brannstrom 2014;

The effectiveness and cost-effectiveness of hospital-based specialist palliative care for adults with advanced illness and their caregivers 
Hopp 2016; O'Riordan 2019; Rogers 2017; Sidebottom 2015), HIV (Lowther 2015), dementia (Ahronheim 2000), multiple sclerosis (Edmonds 2010; Higginson 2009; Solari 2018), COPD (Janssens 2019) and a combination of COPD (83\%) and other non-malignant diseases (Farquhar 2016). Two studies were with rural populations (Bakitas 2009; Bakitas 2015), while Hopp 2016 was with a predominantly African-American population (92\%). Thirty-five studies were conducted or first published from 2010 onwards, with $89 \%$ taking place within the last six years (see Characteristics of included studies for details).

Mean/median age ranged from 38.3 to 85.6 years. About the same number of males and females were included in most studies. However, five studies had between $69 \%$ and $82 \%$ females (Ahronheim 2000; Edmonds 2010; Higginson 2009; Lowther 2015; Ozcelik 2014), whereas nine studies had $60 \%$ to $98 \%$ males (Bajwah 2015; Bakitas 2009; Bekelman 2018; Brannstrom 2014; Farquhar 2016; Franciosi 2019; Kane 1984; Rodin 2019; Vanbutsele 2018). Ahronheim 2000 had the highest percentage of females (82\%). Kane 1984 who recruited at a Veterans Administration hospital included predominantly male veterans. Wallen 2012 did not provide the gender distribution in their population. Unpaid caregivers/family members included in studies tended to be mainly females. Nine of the 16 studies involving unpaid caregivers/families described one or more of their characteristics: they were mainly spouses and women, and had a median/mean age ranging from 51 to 65.6 years. In five studies, between $16 \%$ and $43 \%$ of patients lived alone (Farquhar 2014; Farquhar 2016; Higginson 2009; McCorkle 2015; Vanbutsele 2018).

Sixteen studies had survival as an inclusion criterion. Life expectancy specified in these studies ranged from $>72$ hours to 24 months. Eight studies specifically stated that they included newly diagnosed patients (Bakitas 2015; Franciosi 2019; McCorkle 2015; Nottelmann 2018; Rodin 2019; Temel 2010; Temel 2017; Woo 2019). Exclusion criteria included the presence of severe mental illness (Bakitas 2009; Bakitas 2015; Hopp 2016; Jingfen 2017; Temel 2017), and palliative care/hospice involvement previously or at present/request for palliative care involvement (Bajwah 2015; Carson 2016; Cheung 2010; Franciosi 2019; Grudzen 2016; Ma 2019; Nottelmann 2018; Rodin 2019; Sidebottom 2015; Solari 2018; Tattersall 2014; Temel 2010; Temel 2017; Vanbutsele 2018). In three studies, patients without surrogate decision-makers were excluded (Carson 2016; Cheung 2010; Solari 2018), while Gade 2008 excluded patients if they had impaired cognitive status and no surrogate. Janssens 2019 and Rodin 2019 excluded patients with moderate or severe cognitive impairment.

\section{Intervention}

\section{Hospital Based Specialist Palliative Care (HSPC)}

We included different models of HSPC in this review. Some were new interventions evaluated through feasibility/pilot studies or early phase trials (e.g. Bajwah 2015; Cheung 2010; Edmonds 2010; Higginson 2009; Nottelmann 2018; Rodin 2019); others had existed for some time. Services were based in hospitals, with three studies in hospital ICUs (Carson 2016; Cheung 2010; Ma 2019), and three in palliative care centres/units of hospitals (Groenvold 2017; Jingfen 2017; McWhinney 1994). The hospice programme in Kane 1984 was located in a Veterans Administration hospital. Most served urban and suburban populations. Both Bakitas 2009 and Bakitas

The effectiveness and cost-effectiveness of hospital-based specialist palliative care for adults with advanced illness and their caregivers (Review)

Copyright (c) 2020 The Cochrane Collaboration. Published by John Wiley \& Sons, Ltd.
2015 evaluated telephone-based hospital interventions for rural populations.

The HSPC models in the 42 included studies were:

- ward-based services. This was provided by only Jingfen 2017;

- inpatient consult or advisory services. This was provided by 10 studies: Ahronheim 2000; Carson 2016; Cheung 2010; El-Jawahri 2016; Gade 2008; Grudzen 2016; Hopp 2016; Ma 2019; Ozcelik 2014; Sidebottom 2015;

- outpatient services. This was provided by six studies: Lowther 2015; Mendoza-Galindo 2018 (abstract only); Nottelmann 2018; Tattersall 2014, Temel 2010; Woo 2019;

- hospital outreach services. This was provided by five studies: Bajwah 2015; Brannstrom 2014; Janssens 2019; McWhinney 1994; Solari 2018; and

- service provision across multiple settings including hospital. This was provided by 20 studies: Bakitas 2009; Bakitas 2015; Bekelman 2018; Brumley 2007; Edmonds 2010; Farquhar 2014; Farquhar 2016; Franciosi 2019; Groenvold 2017; Higginson 2009; Higginson 2014; Kane 1984; McCaffrey 2013; McCorkle 2015; O'Riordan 2019; Rodin 2019; Rogers 2017; Temel 2017; Vanbutsele 2018; Wallen 2012.

In order to be included in this review, one of the criteria was that care should be co-ordinated by a multidisciplinary team. Consequently, all the studies included a multidisciplinary HSPC team either as the core team providing the intervention or a multidisciplinary team was included as needed. Seven studies included HSPC teams led by nurses (Bajwah 2015; Bakitas 2009; Lowther 2015; McCaffrey 2013; Nottelmann 2018; Tattersall 2014; Vanbutsele 2018), while none of the studies included HSPC teams that were physician-led. In one study, it was unclear who was leading the HSPC (Mendoza-Galindo 2018 (abstract only)). Multidisciplinary team members ranged from two to eight professionals, mainly comprising nurses, physicians and sometimes social workers.

Five studies had HSPC that had 24 hours access (out-of-hours care). The hospital outreach service provided by McWhinney 1994 included 24 hours on-call service, while another hospital outreach service organised the intervention in close co-operation with outof-hours palliative advanced home care (Brannstrom 2014). In McCaffrey 2013, services traversed multiple settings, including hospital and nursing services, and were provided up to 24 hours a day at home for up to five days. Brumley 2007 involved service provision across multiple settings including hospital and also included 24 hours on-call service. The inpatient consult service provided by Gade 2008 included a palliative care physician on call after hours.

Thirty-one studies either included certified experts in palliative care or those described as palliative care clinicians (without being explicit about their training). For example, Bakitas 2015 included a board-certified palliative care clinician and advanced practice palliative care nurse specialists, while Gade 2008 included a multiprofessional team comprising a palliative care physician, nurse, hospital social worker and chaplain. Janssens 2019 included a palliative care team comprising nurses with experience in palliative care and a physician specialised in palliative care. Furthermore, Higginson 2009 evaluated a new short-term specialist palliative care intervention involving one to three contacts 
provided by a core team of a part-time consultant in palliative medicine, part-time palliative care nurse, psychosocial worker and administrator comprising consultation and shared care with other care providers. Bajwah 2015, Edmonds 2010 and Nottelmann 2018 were also new palliative care services. Bajwah 2015 was developed for people with interstitial lung disease and involved a hospitalto-home case conference attended by the palliative care nurse who organised it and different healthcare professionals, while the service in Edmonds 2010 comprised a part-time consultant in palliative medicine with a special interest in neurological conditions, a part-time clinical nurse specialist and a full time administrator. Nottelmann 2018 was a palliative rehabilitation service delivered by a specialised palliative care team consisting of physicians, nurses, physiotherapists, psychologists, a part time social worker, dietician, occupational therapist, and chaplain. In Franciosi 2019 , the palliative care intervention was provided across multiple settings and involved nurses working full time in palliative care as well as double-boarded certified oncologists and palliative care physicians. A palliative care physician and nurse that were separate from the haematology team provided the intervention in Rodin 2019. Other multidisciplinary team members were involved as needed. Sidebottom 2015 assessed inpatient palliative care for patients with heart failure. The inpatient palliative care team included four physicians who were board-certified in hospice and palliative medicine, two clinical nurse specialists board-certified in advanced practice palliative care nursing, a chaplain and a social worker. The remaining 11 studies only stated the involvement of professionals who delivered specialist level interventions without any details on their training or whether they were palliative care clinicians (Ahronheim 2000; Cheung 2010; Groenvold 2017; Grudzen 2016; Hopp 2016; Jingfen 2017; McCaffrey 2013; MendozaGalindo 2018 (abstract only); O'Riordan 2019; Ozcelik 2014; Woo 2019).

Early palliative care was evaluated in 19 studies (Bakitas 2009; Bakitas 2015; El-Jawahri 2016; Franciosi 2019; Groenvold 2017; Grudzen 2016; Higginson 2014; Janssens 2019; Ma 2019; McCorkle 2015; Mendoza-Galindo 2018 (abstract only); Nottelmann 2018; Rodin 2019; Tattersall 2014; Temel 2010; Temel 2017; Vanbutsele 2018; Wallen 2012; Woo 2019). Early palliative care intent either had to be stated explicitly or most participants had to enrolled shortly after diagnosis of advanced disease. Bakitas 2009 included patients who were within eight to 12 weeks of a new diagnosis of advanced cancer, while Bakitas 2015 included patients with advanced cancer who were within 30 and 60 days of diagnosis. McCorkle 2015 recruited patients with a late-stage cancer diagnosis within 100 days, whereas Temel 2010 included patients with metastatic lung cancer diagnosed within the previous eight weeks. Similarly, four studies included patients who were within eight weeks of diagnosis of advanced cancer (Franciosi 2019; Nottelmann 2018; Temel 2017; Woo 2019). Temel 2017 recruited patients with incurable lung or non-colorectal Gastrointestinal cancer, while Franciosi 2019 recruited patients with non-small cell lung cancer, pancreatic, gastric or biliary tract cancer. Nottelmann 2018 involved patients diagnosed with non-resectable solid cancer, and Woo 2019 recruited those with a diagnosis of advanced or metastatic pancreatic or biliary tract cancer. Vanbutsele 2018 included patients who were within the first 12 weeks of a new primary tumour or had a diagnosis progression. In El-Jawahri 2016, the intention was early palliative care and the intervention was delivered during hospitalisation for haematopoietic stem cell transplantation (HCT) care. Groenvold 2017 initiated their palliative care intervention earlier than would otherwise have been the case among patients with advanced cancer, while Grudzen 2016 assessed early referral to palliative care for emergency department patients with advanced cancer. Rodin 2019 delivered early palliative care interventions to patients newly diagnosed with acute leukaemia. Wallen 2012 began an early palliative care intervention postoperatively for patients with advanced cancer. Tattersall 2014 included ambulatory patients with newlydetected incurable metastatic cancer. Higginson 2014 evaluated early palliative care integrated with respiratory services for patients with a range of malignant and non-malignant advanced diseases (mixed populations) and refractory breathlessness. Janssens 2019 assessed early palliative care for patients with severe and very severe COPD over a one-year period while Mendoza-Galindo 2018 (abstract only) stated that their intervention was an early palliative care intervention for patients with newly-diagnosed or relapsed metastatic breast cancer. Ma 2019 involved early triggered palliative care consultation within 48 hours of ICU admission.

Eleven studies were theoretically grounded: case conference/ management (Bajwah 2015; Ozcelik 2014), chronic care model (Bakitas 2009), person-centred palliative care (Brannstrom 2014), palliative care approach (Farquhar 2014; Farquhar 2016), hospice (Brumley 2007; Kane 1984), knowledge-belief-action model (Jingfen 2017), trauma-focussed cognitive behavioural therapy (Rodin 2019), and palliative care and physiotherapy approach (Higginson 2014). Two studies were modelled after hospice programmes (Brumley 2007; Kane 1984).

Twenty-three studies included HSPC that provided some level of unpaid caregiver/family support ranging from meeting with unpaid caregivers/families to discuss care options to education/ counselling or provision of psychological interventions aimed at supporting patient and unpaid caregiver/family dyads.

\section{Taxonomy of the components of HSPC}

We assessed the components of HSPC in the studies included in this review using the principles and domains of palliative care highlighted by Zimmermann 2019. Zimmermann 2019 developed a conceptual framework highlighting the domains and principles of team-based outpatient early palliative care for patients with cancer. This framework is based on palliative care theory (Doyle 1998; WHO 2002; Zimmermann 2004; Zimmermann 2012), review of previous palliative care interventions (Zimmermann 2008) and practice guidelines (Cancer Care Ontario 2016; NCCN 2016). This framework was chosen above others such as the Holistic Common Assessment (National End of Life Care Programme 2010), which is used for comprehensive palliative care assessment, because the essential elements of the framework are consistent with the need for early provision of palliative care in collaboration with the multidisciplinary team, and also because it is based on the needs of the patient and their family, rather than on prognosis.

The four domains are coping and support, decision-making, symptom control and future planning, while the four principles are that care is flexible, attentive, patient- and family-centred.

\section{Components of HSPC in studies that either included certified experts} in palliative care or those described as palliative care clinicians

Thirty-one studies either included certified experts in palliative care or those described as palliative care clinicians. Eight studies were only patient-centred (Brannstrom 2014; Rodin 2019; Rogers

The effectiveness and cost-effectiveness of hospital-based specialist palliative care for adults with advanced illness and their caregivers 
2017; Sidebottom 2015; Tattersall 2014; Temel 2017; Vanbutsele 2018; Wallen 2012), while Carson 2016 was only family-centred because the intervention was a palliative care-led meeting for families of patients in the medical intensive care unit. Twentytwo studies were both patient-centred and family-centred (Bajwah 2015; Bakitas 2009; Bakitas 2015; Bekelman 2018; Brumley 2007; Edmonds 2010; El-Jawahri 2016; Farquhar 2014; Farquhar 2016; Franciosi 2019; Gade 2008; Higginson 2009; Higginson 2014; Janssens 2019; Kane 1984; Lowther 2015; Ma 2019; McCorkle 2015; McWhinney 1994; Nottelmann 2018; Solari 2018; Temel 2010). For instance, the HSPC intervention in Bajwah 2015 was individualised to each patient and carer, while Vanbutsele 2018 described the use of semi-structured monthly consultations by palliative care nurses that allowed for individualised care. Bekelman 2018 described

\section{Figure 3. A figure showing the domains of HSPC in the studies that either included certified experts in palliative care or those described as palliative care clinicians}

collaboration between patients and the nurse as they both agreed on the symptom to focus on.

Palliative care in all 31 studies except Kane 1984 involved provision of care that was flexible and attentive to the needs of patients and/or their families as they allowed for the involvement of other members of the healthcare team in order to address these needs.

We mapped the 31 studies to the four domains highlighted above. We added care co-ordination as an additional domain because the need for co-ordinated care for those with advanced disease is not always delivered and this can result in increased hospitalisations and suboptimal clinical outcomes (Higginson 2003; Walsh 2011) (see Table 1 under Additional tables for the domains covered in the studies and Figure 3 for the percentage of studies assessing different domains).

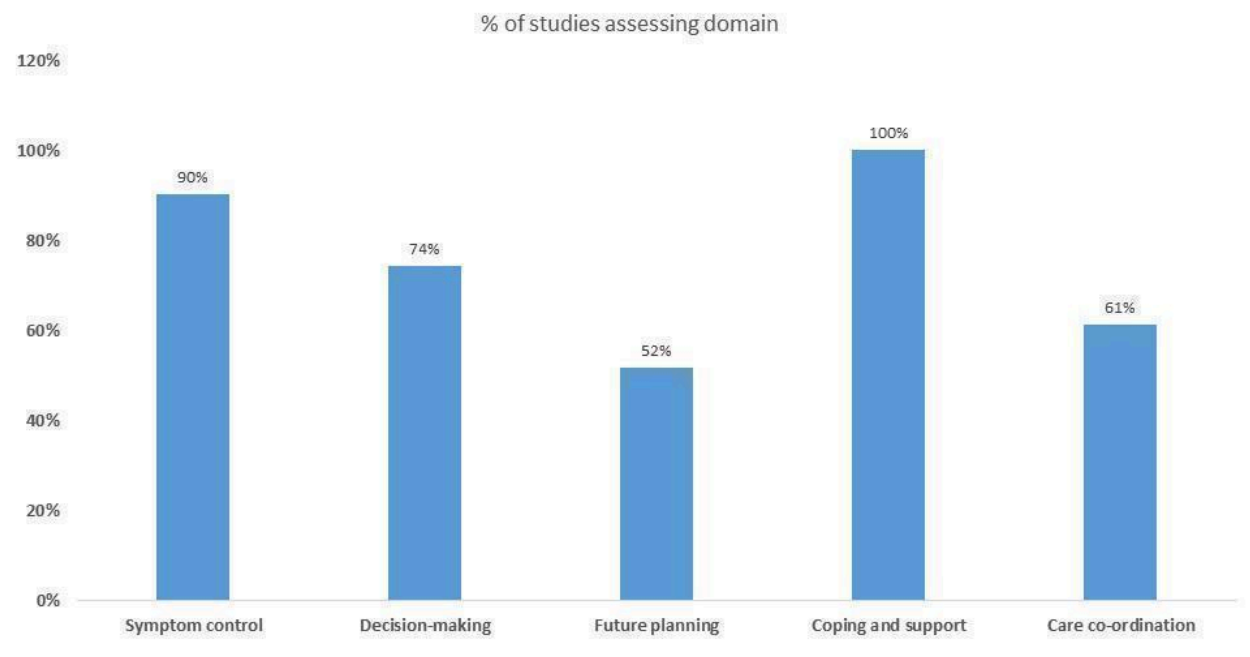

\section{Symptom control}

This involved assessment and management of symptoms. Twentyeight studies highlighted that the HSPC intervention included symptom or needs assessment and management. In two studies, this was unclear (McWhinney 1994; Solari 2018), while it appeared that Carson 2016 did not address this domain.

\section{Decision-making}

This domain involved assessing patient and their family's understanding of illness, assessing individual and cultural values/ beliefs or assessing goals of care and regularly reviewing them. Twenty-three studies included HSPC that addressed one or more aspects of decision-making. One study included HSPC that did not focus on decision-making as the intervention was targeted at managing patients' physical and psychological symptoms during hospitalisation (El-Jawahri 2016). A further five studies of HSPC did not involve this domain (Higginson 2009; Kane 1984; Rodin 2019; Tattersall 2014; Wallen 2012). In two studies, this was unclear (McWhinney 1994; Solari 2018).

\section{Future planning}

Future planning involved discussing concerns and preferences for end-of-life care, making a will, power of attorney and decisions about resuscitation. Half of the studies $(n=16)$ included HSPC that involved planning for the future, while this was unclear in two studies (McWhinney 1994; Solari 2018). The remaining 13 studies did not include this domain in delivery of HSPC (Bekelman 2018; Brannstrom 2014; Carson 2016; El-Jawahri 2016; Franciosi 2019; Ma 2019; McCorkle 2015; Rodin 2019; Tattersall 2014; Temel 2010; Temel 2017; Vanbutsele 2018; Wallen 2012).

The effectiveness and cost-effectiveness of hospital-based specialist palliative care for adults with advanced illness and their caregivers 


\section{Coping and support}

This involved establishing a therapeutic relationship, facilitating coping with advanced illness and spiritual support, providing emotional and practical support, addressing family needs and bereavement care.

All 31 studies involved HSPC that had one or more elements of this domain. In particular, three studies included HSPC that specifically highlighted bereavement care or involved a bereavement coordinator as needed (Bakitas 2009; Brumley 2007; Higginson 2009). Bakitas 2009 provided a bereavement follow-up call to the unpaid caregiver as part of the HSPC intervention, while Higginson 2009 described HSPC that provided bereavement support when needed. Brumley 2007 described HSPC that included a bereavement coordinator, as needed. Furthermore, Bekelman 2018 included a topic on grief and loss as part of the counselling session in their HSPC intervention.

In addition to the areas described above, we assessed the provision of spiritual care. Thirteen studies include HSPC provided spiritual care or support (Bajwah 2015; Brannstrom 2014; Brumley 2007; Carson 2016; Higginson 2014; Janssens 2019; Kane 1984; Lowther 2015; Nottelmann 2018; Rogers 2017; Sidebottom 2015; Vanbutsele 2018; Wallen 2012).

\section{Care co-ordination}

Although Zimmermann 2019 did not include care co-ordination as a domain in their conceptual framework, we decided to include this domain. Over half of the studies $(n=19)$ involved HSPC that provided care co-ordination (Bajwah 2015; Bakitas 2009; Bakitas
2015; Bekelman 2018; Brannstrom 2014; Brumley 2007; Edmonds 2010; Franciosi 2019; Higginson 2009; Higginson 2014; Janssens 2019; Ma 2019; McCorkle 2015; Nottelmann 2018; Rogers 2017; Sidebottom 2015; Temel 2010; Temel 2017; Vanbutsele 2018), while this was unclear in two studies (McWhinney 1994; Solari 2018). In 10 studies, it appeared that the HSPC intervention did not include this domain.

The main domains of care in the HSPC intervention in the 31 studies were symptom control, coping and support, and decisionmaking. At least half of the studies included HSPC that provided care co-ordination and future planning. Besides McWhinney 1994 and Solari 2018, the remaining studies addressed at least two domains.

\section{Components of HSPC in studies that were unclear about palliative care training}

Eleven studies were unclear about the palliative care training of those who delivered the HSPC intervention (Ahronheim 2000; Cheung 2010; Groenvold 2017; Grudzen 2016; Hopp 2016; Jingfen 2017; McCaffrey 2013; Mendoza-Galindo 2018 (abstract only); O'Riordan 2019; Ozcelik 2014; Woo 2019) (see Table 2 under Additional tables for the domains covered in the studies and Figure 4 for the percentage of studies assessing different domains). Four of these studies were only patient-centred (Groenvold 2017; Hopp 2016; O'Riordan 2019; Woo 2019), while Ahronheim 2000 was only family-centred. Three studies were both patient- and family-centred (Grudzen 2016; Jingfen 2017; Ozcelik 2014). In three studies, this was unclear (Cheung 2010; McCaffrey 2013; MendozaGalindo 2018 (abstract only)).

\section{Figure 4. A figure showing the domains of HSPC in studies that were unclear about palliative care training}

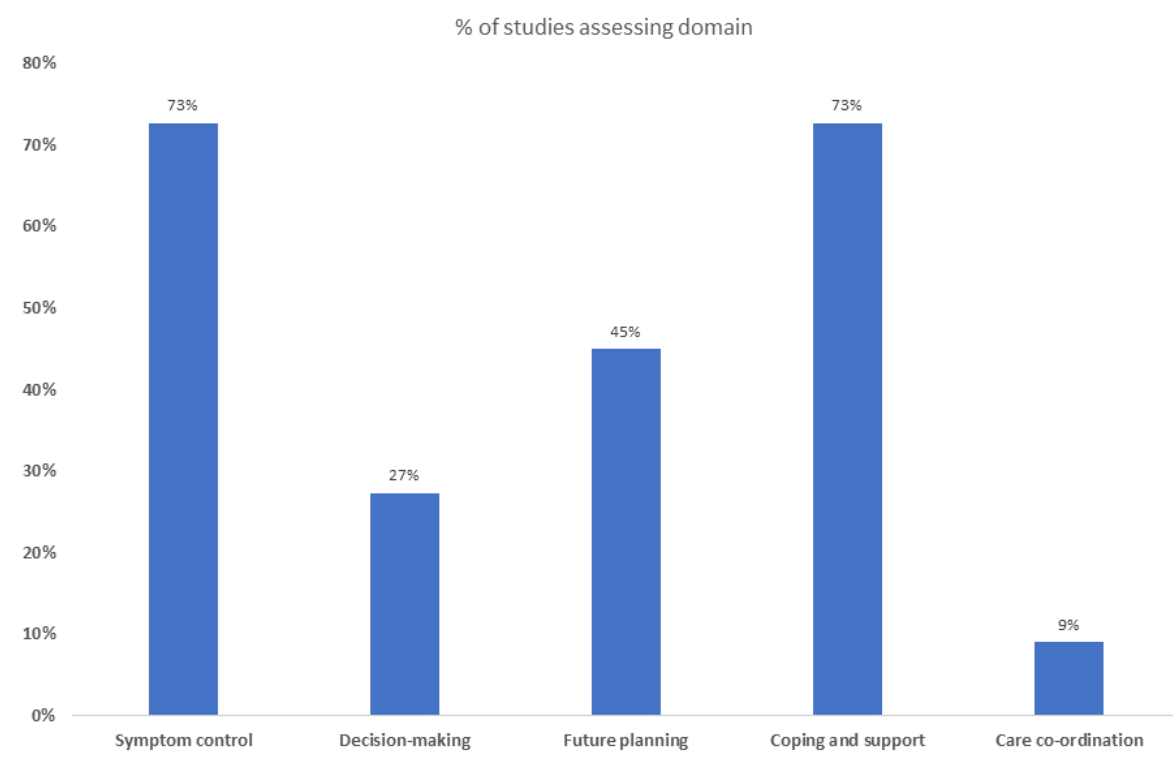

Palliative care provision was flexible in all the 11 studies, with the involvement of the multidisciplinary team as required to address the needs of patients and/or their families. In 10 studies, the palliative care providers were attentive to the needs of patients and their families, while this was unclear in Mendoza-Galindo 2018 (abstract only). Mendoza-Galindo 2018 (abstract only) only

The effectiveness and cost-effectiveness of hospital-based specialist palliative care for adults with advanced illness and their caregivers 
reported that the intervention was provided by a palliative team, which included psychological, nutritional and symptom support.

We assessed the domains of HSPC included in these studies as follows:

\section{Symptom control}

Eight studies highlighted the issue that the HSPC intervention included symptom or needs assessment and management. In three studies, this was unclear (Cheung 2010; Groenvold 2017; McCaffrey 2013).

\section{Decision-making}

Three studies involved one or more aspects of decision-making (Grudzen 2016; Hopp 2016; Jingfen 2017), while this was unclear in three studies (Cheung 2010; Groenvold 2017; McCaffrey 2013). It appeared five studies did not involve this domain (Ahronheim 2000; Mendoza-Galindo 2018 (abstract only); O'Riordan 2019; Ozcelik 2014; Woo 2019).

\section{Future planning}

Five studies involved planning for the future (Ahronheim 2000; Grudzen 2016; Hopp 2016; O'Riordan 2019; Ozcelik 2014), while this was unclear in three studies (Cheung 2010; Groenvold 2017; McCaffrey 2013). Three studies did not include this domain (Jingfen 2017; Mendoza-Galindo 2018 (abstract only); Woo 2019).

\section{Coping and support}

Eight studies involved one or more elements of this domain, while three studies were unclear (Cheung 2010; Groenvold 2017; McCaffrey 2013). O'Riordan 2019 further involved the provision of spiritual care.

\section{Care co-ordination}

Only McCaffrey 2013 involved care co-ordination, while eight studies did not. It was unclear whether two studies included this domain (Cheung 2010; Groenvold 2017).

The main domains of care in the HSPC intervention in studies with unclear training were symptom control, coping and support, and future planning. Very few studies involved decision-making and care co-ordination. Besides three studies where the domains were unclear, the remaining eight studies addressed at least two domains.

When compared to studies that included experts or those described as palliative care clinicians, studies with unclear palliative care training often did not include decision-making and care coordination. There was also less focus on symptom control, and coping and support in studies with unclear palliative care training. Both groups were similar in the extent to which they focussed on future planning.

\section{Controls}

HSPC was compared with usual care. Overall, there was poor description of usual care in most studies with no information or very little information provided. For example, Ahronheim 2000 only stated that the control group was treated by the primary care team without palliative care input, and Cheung 2010 stated that control group received usual ICU care without palliative care consultation. Among studies providing some level of detail on usual care, it appeared usual care was varied, probably reflecting the local context as well as differences in health systems. For example, in the Kenyan study by Lowther 2015, those in the usual care group received care from nurses without experience in palliative care from the HIV clinic, consisting of monthly clinical assessments once antiretroviral therapy (ART) was established. In the Swiss study by Janssens 2019, patients receiving usual care had no contact with the palliative care team. Specialised nurses provided regular home visits to patients under long-term oxygen therapy (LTOT) and/or home non-invasive ventilation (NIV). In the Belgian study by Vanbutsele 2018, usual oncology care in all the participating departments was provided by a multidisciplinary team, including oncologists, other medical specialists, social workers, psychologists, dieticians and specialist nurses. All patients with advanced cancer usually have an introductory consultation with a specialist nurse trained in oncological care, a dietician, and a psychologist at the start of their treatment. Follow-up consultations were at the patient's discretion. The palliative care team was only involved on demand and often late in the disease trajectory, and their services were not systematically offered to all patients from oncology departments. Usual care in the South Korean study by Woo 2019 comprised anticancer and symptom control treatments and consultation with psychiatric and pain specialists. In Bajwah 2015, a UK study, the control group remained under interstitial lung disease (ILD) specialist care which included input from ILD physicians, ILD clinical nurse specialist, occupational therapists, physiotherapists and oxygen assessment and treatment services. All patients were also able to access inpatient ILD treatment, as needed. The control group received the intervention after four weeks. In Higginson 2014, the control group received usual care services according to UK guidance. After six weeks, the control group was offered the intervention. Similarly, in Solari 2018 , usual care involved health and social services provided by the Italian National Health Service and dyads were offered the intervention at the end of the study.

In 20 studies, usual care included involvement of palliative care professionals if needed (Bajwah 2015; Bakitas 2009; Bakitas 2015; Bekelman 2018; Carson 2016; El-Jawahri 2016; Franciosi 2019; Groenvold 2017; Grudzen 2016; Ma 2019; McCaffrey 2013; McWhinney 1994; Rodin 2019; Rogers 2017; Tattersall 2014; Temel 2010; Temel 2017; Vanbutsele 2018; Wallen 2012; Woo 2019), and, in one study, usual care incorporated hospice care (Brumley 2007). Wallen 2012 reported that the usual care group was permitted to cross over to the intervention arm if standard pain and symptom management was inadequate to meet their needs.

\section{Outcomes}

Our primary outcomes of patient health-related quality of life (HRQOL) and their symptom burden (assessed using generalised measures) were assessed by 10 and six studies reporting adjusted endpoint values, respectively. Of the 10 studies assessing patient HRQoL, nine were with cancer populations, and one with noncancer populations. Nine of the 10 studies involved early palliative care (Bakitas 2009; Bakitas 2015; El-Jawahri 2016; McCorkle 2015; Rodin 2019; Tattersall 2014; Temel 2010; Temel 2017; Vanbutsele 2018). All six studies that reported symptom burden were with cancer populations and they involved early palliative care (Bakitas 2009; Bakitas 2015; El-Jawahri 2016; Rodin 2019; Tattersall 2014; Temel 2010). 
Other patient outcomes reported included individual symptoms (pain, anxiety, depression, post-traumatic stress disorder, breathlessness, fatigue, nausea/vomiting, appetite loss, sleep disturbance), traumatic stress symptoms, mortality (death at home, hospital and ICU), survival, advanced care planning, functional independence, achieving preferred place of care or death, satisfaction with care, physical function, psychological, social and spiritual well-being, nutrition, and cognitive status.

Unpaid caregiver outcomes assessed in studies included satisfaction with care, symptom control (e.g. anxiety, depression), HRQoL, burden, coping, distress with patients' symptoms, and grief.

\section{Economic dato}

We included 31 studies in the economic component of this review as they compared the resource use and/or costs/cost-effectiveness between HSPC and usual care alongside clinical effectiveness. We restricted the economic component of the review to economic analyses conducted alongside the studies meeting eligibility criteria for the effectiveness component of the review. Of the 31 studies, four studies were full economic evaluations that compared the costs and effects of the intervention and control group between baseline and follow-up (Farquhar 2014; Farquhar 2016; Higginson 2009; McCaffrey 2013), five partial economic evaluations that compared only costs and outcomes without reporting incremental changes or decision criteria (Brumley 2007; Gade 2008; Higginson 2014; Kane 1984; Temel 2010), and 22 studies reported more limited resource use/cost information.

The studies measured resource use associated with care received in the intervention and the control group. Use of the following resources was assessed: institutional care services use (e.g. emergency department (ED) or accident and emergency (A\&E), intensive care unit use, inpatient stay, care in nursing homes (or skilled nursing homes)); outpatient clinic use (e.g. palliative care visits in outpatient settings, consultation with experts in outpatient settings); community care services use (e.g. GP contacts, nurse visits, home care, hospice care at home); unpaid caregiver care; medications and other resource use. Thirteen studies calculated the costs associated with resource utilisation (Brannstrom 2014; Brumley 2007; Farquhar 2014; Farquhar 2016; Gade 2008; Higginson
2009; Higginson 2014; Kane 1984; Ma 2019; McCaffrey 2013; Mendoza-Galindo 2018 (abstract only); Ozcelik 2014; Temel 2010). Four studies reported the results of cost-effectiveness analysis using relevant outcome measures (palliative outcome, unpaid caregiver burden, quality-adjusted life years (QALYs)) (Farquhar 2014; Farquhar 2016; Higginson 2009; McCaffrey 2013), and hospital costs or total costs. Results of cost-effectiveness analyses were reported by incremental cost-effectiveness ratios (ICERs) and/or costs per QALY (point estimates or cost-effectiveness planes). The four studies reported ICERs, cost/QALY, or cost-effectiveness planes from cost-effectiveness analysis.

\section{Excluded studies}

We excluded 25 studies for the following reasons: studies were not RCTs $(n=8)$, usual care included palliative care as part of routine care $(n=5)$, studies did not conceal their allocation sequence ( $n$ $=3$ ), intervention was not delivered by a multidisciplinary team $(n=7)$, intervention was not HSPC $(n=1)$, and study included hospices based outside hospital settings $(n=1)$ (see Characteristics of excluded studies).

\section{Studies awaiting classification}

We could not classify one study due to insufficient information to clarify the nature of the palliative care team and setting (see Characteristics of studies awaiting classification).

\section{Ongoing studies}

We identified 34 ongoing studies (see Characteristics of ongoing studies).

\section{Risk of bias in included studies}

We assessed risk of bias using the Cochrane 'Risk of bias' tool (see Figure 5 and Figure 6) (Higgins 2011b). Across trials, we assessed risk of bias for all outcomes in all the domains specified for RCTs in the Cochrane Handbook (Higgins 2011b). The domains we covered were selection bias (random sequence generation and allocation concealment), performance, detection, attrition and reporting biases. We also assessed 'size of study' as a potential risk of bias. Under the 'other bias' domain, we assessed whether groups were balanced at baseline and also if differences at baseline were adjusted for. 
Figure 5. Risk of bias summary: review authors' judgements about each risk of bias item for each included study.

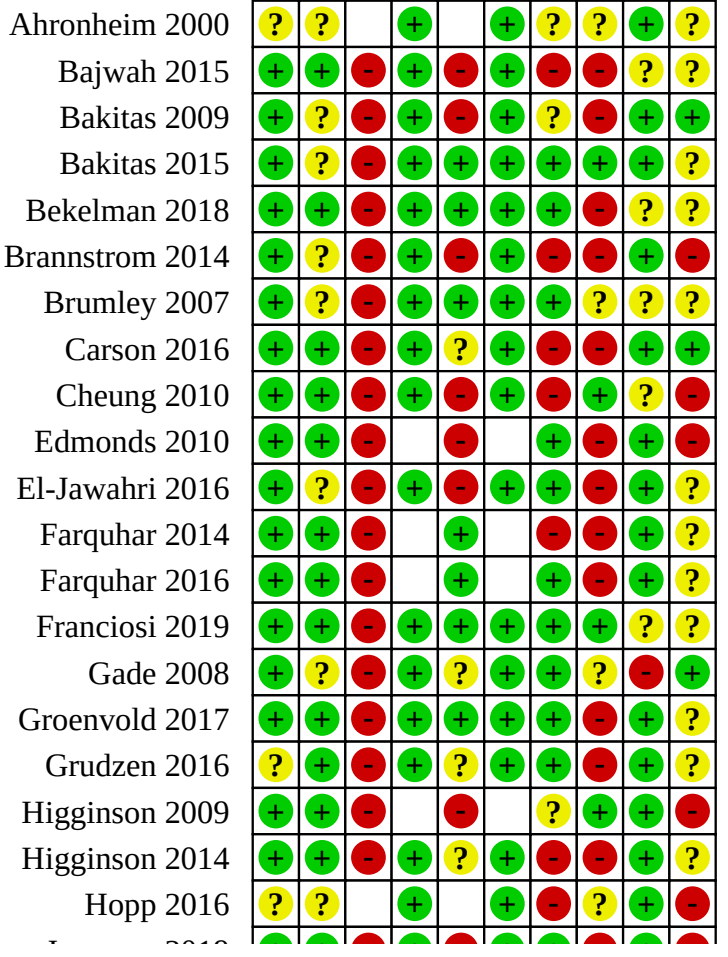


Figure 5. (Continued)

\begin{tabular}{|c|c|c|c|c|c|c|c|c|c|c|}
\hline Hopp 2016 & \begin{tabular}{|l|}
$?$ \\
\end{tabular} & $?$ & & + & & + & - & $?$ & + & \\
\hline Janssens 2019 & + & + & 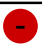 & + & - & + & + & - & + & \\
\hline Jingfen 2017 & + & $?$ & $?$ & & $?$ & $?$ & $?$ & $?$ & + & $?$ \\
\hline Kane 1984 & ? & $?$ & (- & + & $?$ & + & O & $?$ & + & $?$ \\
\hline Lowther 2015 & $?$ & $?$ & 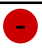 & & - & & $?$ & - & $?$ & ? \\
\hline Ма 2019 & \begin{tabular}{|l|}
$?$ \\
\end{tabular} & + & & + & & + & - & - & + & ? \\
\hline McCaffrey 2013 & \begin{tabular}{|l|}
$?$ \\
\end{tabular} & $?$ & & + & & + & + & - & $?$ & - \\
\hline McCorkle 2015 & + & $?$ & 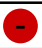 & & $?$ & & - & - & $?$ & $?$ \\
\hline McWhinney 1994 & + & + & - & & + & & - & $?$ & $?$ & $?$ \\
\hline Mendoza-Galindo 2018 (abstract only) & $?$ & $?$ & $?$ & $?$ & $?$ & $?$ & $?$ & $?$ & $?$ & - \\
\hline Nottelmann 2018 & + & + & & + & $?$ & + & $?$ & $?$ & $?$ & $?$ \\
\hline O'Riordan 2019 & $?$ & $?$ & & & $?$ & & - & - & - & G \\
\hline Ozcelik 2014 & $?$ & $?$ & & & $?$ & & + & $?$ & $?$ & E \\
\hline Rodin 2019 & + & + & & + & - & + & + & E & + & E \\
\hline Rogers 2017 & $?$ & $?$ & & + & $\overline{7}$ & + & - & - & + & $?$ \\
\hline Sidebottom 2015 & $?$ & $?$ & & + & $?$ & + & - & $?$ & + & $?$ \\
\hline Solari 2018 & + & + & & + & + & + & + & - & $?$ & $?$ \\
\hline Tattersall 2014 & + & + & & + & & + & 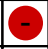 & + & + & $?$ \\
\hline Temel 2010 & $?$ & $?$ & & + & & + & + & E & + & $?$ \\
\hline Temel 2017 & + & + & & & 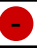 & & 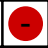 & - & + & $?$ \\
\hline Vanbutsele 2018 & + & + & & + & - & + & + & $?$ & + & $?$ \\
\hline Wallen 2012 & \begin{tabular}{|l|}
$?$ \\
\end{tabular} & $?$ & 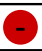 & & $?$ & & - & - & + & $?$ \\
\hline Woo 2019 & $?$ & $?$ & & + & $?$ & + & + & $?$ & + & $?$ \\
\hline
\end{tabular}

Figure 6. Risk of bias graph: review authors' judgements about each risk of bias item presented as percentages across all included studies.

Random sequence generation (selection bias) Allocation concealment (selection bias) Blinding of participants and personnel (performance bias), subjective outcomes Blinding of participants and personnel (performance bias), objective outcomes Blinding of outcome assessment (detection bias), subjective outcomes Blinding of outcome assessment (detection bias), objective outcomes Incomplete outcome data (attrition bias): All outcomes Selective reporting (reporting bias) Other bias Size of study

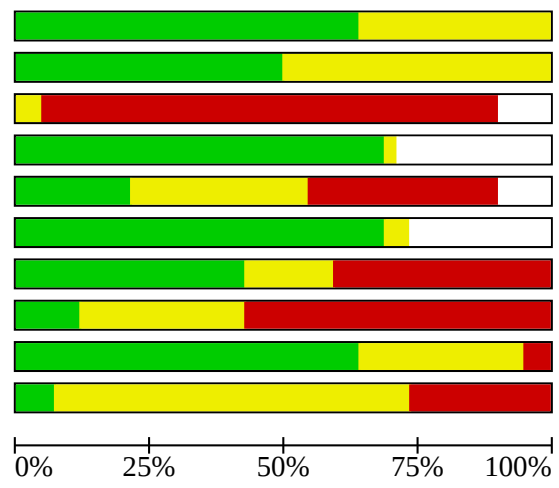

\section{Allocation}

\section{Random sequence generation}

Twenty-seven studies were randomised and provided adequate description of the sequence generation process. We therefore judged them to be at low risk of bias. However, we judged 15 studies to be at unclear risk of bias due to insufficient description of the sequence generation process (Ahronheim 2000; Grudzen 2016; Hopp 2016; Kane 1984; Lowther 2015; Ma 2019; McCaffrey 2013; Mendoza-Galindo 2018 (abstract only); O'Riordan 2019; Ozcelik 2014; Rogers 2017; Sidebottom 2015; Temel 2010; Wallen 2012; Woo 2019). 


\section{Allocation concealment}

Authors of 21 studies did not provide adequate information on how they concealed the allocation and so we judged them to be at unclear risk of bias (Ahronheim 2000; Bakitas 2009; Bakitas 2015; Brannstrom 2014; Brumley 2007; El-Jawahri 2016; Gade 2008; Hopp 2016; Jingfen 2017; Kane 1984; Lowther 2015; McCaffrey 2013; McCorkle 2015; Mendoza-Galindo 2018 (abstract only); O'Riordan 2019; Ozcelik 2014; Rogers 2017; Sidebottom 2015; Temel 2010; Wallen 2012; Woo 2019). We judged 21 studies as having a low risk of bias because the methods used to conceal the allocation sequence were described.

\section{Blinding}

As stated in the methods, we assessed blinding for subjective and objective outcomes separately.

\section{Blinding of participants and personnel (subjective outcomes)}

None of the studies that reported on subjective outcomes blinded participants. We judged two studies as having an unclear risk of bias because they did not state whether participants and personnel were blinded (Jingfen 2017; Mendoza-Galindo 2018 (abstract only)), while we gave the remaining 36 studies a high risk of bias because they did not carry out blinding. Four studies did not include subjective outcomes and we therefore did not assess this domain in these studies (Ahronheim 2000; Hopp 2016; Ma 2019; McCaffrey 2013) and we left the domain blank. Generally, in palliative care research, blinding of participants and personnel is not possible or feasible due to the nature of palliative care interventions which involve service provision by a multidisciplinary team (Piggott 2004), and also because of ethical considerations as patients need to be informed about the intervention.

\section{Blinding of participants and personnel (objective outcomes)}

We judged 29 studies to be at low risk of bias because we considered that lack of blinding of participants and personnel was unlikely to affect the objective outcomes they assessed. We could not assess this domain in 12 studies because they did not include objective outcomes (Edmonds 2010; Farquhar 2014; Farquhar 2016; Higginson 2009; Jingfen 2017; Lowther 2015; McCorkle 2015; McWhinney 1994; O'Riordan 2019; Ozcelik 2014; Temel 2017; Wallen 2012) and so we therefore left this domain blank. We gave MendozaGalindo 2018 (abstract only) an unclear risk of bias because the authors did not state whether blinding of participants and personnel occurred.

\section{Blinding of outcome assessment (subjective outcomes)}

We judged nine studies as having a low risk of bias because they blinded outcome assessors (Bakitas 2015; Bekelman 2018; Brumley 2007; Farquhar 2014; Farquhar 2016; Franciosi 2019; Groenvold 2017; McWhinney 1994; Solari 2018). We assessed 14 studies as having an unclear risk of bias rating (Carson 2016; Gade 2008; Grudzen 2016; Higginson 2014; Jingfen 2017; Kane 1984; McCorkle 2015; Mendoza-Galindo 2018 (abstract only); Nottelmann 2018; O'Riordan 2019; Ozcelik 2014; Sidebottom 2015; Wallen 2012; Woo 2019) because it was unclear whether outcome assessors were blinded, while we gave 15 studies a high risk of bias rating because they did not blind outcome assessors. Some authors of studies with a high risk of bias stated explicitly that they did not blind outcome assessors (e.g. Vanbutsele 2018), while others stated that they were open-label or non-blinded studies (e.g. Bakitas 2009; Janssens 2019; Rodin 2019; Temel 2017).

We could not assess this domain in four studies because they did not include subjective outcomes (Ahronheim 2000; Hopp 2016; Ma 2019; McCaffrey 2013) so we therefore left this domain blank.

\section{Blinding of outcome assessment (objective outcomes)}

We assessed 29 studies as having a low risk of bias because they blinded outcome assessors, while we rated two studies as having an unclear risk of bias (Jingfen 2017; Mendoza-Galindo 2018 (abstract only)) due to lack of clarity on whether outcome assessors were blinded. We could not rate the remaining 11 studies because they did not include objective outcomes (Edmonds 2010; Farquhar 2014; Farquhar 2016; Higginson 2009; Lowther 2015; McCorkle 2015; McWhinney 1994; O'Riordan 2019; Ozcelik 2014; Temel 2017; Wallen 2012) and so we left this domain blank.

\section{Incomplete outcome data}

Twenty-nine of the 42 included studies reported almost identical attrition rates in the intervention and control groups (Bajwah 2015; Bakitas 2009; Bekelman 2018; Brumley 2007; Carson 2016; Edmonds 2010; El-Jawahri 2016; Farquhar 2014; Farquhar 2016; Franciosi 2019; Gade 2008; Groenvold 2017; Grudzen 2016; Higginson 2009; Higginson 2014; Hopp 2016; Janssens 2019; Kane 1984; Lowther 2015; Ma 2019; McCaffrey 2013; McCorkle 2015; O'Riordan 2019; Rogers 2017; Sidebottom 2015; Solari 2018; Temel 2017; Wallen 2012; Woo 2019). The level of attrition ranged from $1 \%$ to $93 \%$. Reasons given for attrition included clinical staff missed patient ( $n=1$, note all ' $n$ 's are studies), death $(n=27)$, deterioration/ severe illness $(n=4)$, did not receive intervention $(n=1)$, did not complete $(n=3)$, feeling too well $(n=1)$, form mailed but not returned $(n=3)$, hospitalised or too ill/hospitalised/hospice $(n=2)$, lack of interest $(n=1)$, lost to follow-up $(n=2)$, migrated $(n=1)$, not eligible after enrollment $(n=1)$, overwhelmed $(n=1)$, patients could not be reached $(n=2)$, passive withdrawal $(n=1)$, protocol violation $(n=1)$, refused to participate $(n=5)$, transfer of care $(n=$ $3)$, treated at another facility $(n=1)$, unable to attend appointments and unavailable $(n=1)$, unknown reason $(n=2)$, withdrawal of consent $(n=16)$ and went on holiday $(n=1)$.

We judged 17 studies as having a high risk of bias. For example, we assessed Brannstrom 2014 as having a high risk of bias because attrition was not balanced across the intervention and control groups with $77.8 \%$ completers in the intervention and $88.9 \%$ completers in the control group. Missing data were also excluded from the analysis in this study. Similarly, in McCorkle 2015, missing data were not included in the analysis. McCorkle 2015 had 55\% completers in the intervention and $70 \%$ completers in the control group. We gave Tattersall 2014 a high risk of bias rating due to high attrition as only $18.3 \%$ of intervention group and $30 \%$ of control completed the study and reasons for non-completion were not stated. In McWhinney 1994, a high attrition rate was reported at one month (36\%). However, the attrition rate in each of the treatment arms (intervention and control) was not stated. Janssens 2019 had a $16 \%$ death rate at 12 months but did not indicate the number of deaths in each of the treatment arms.

We judged 18 studies as having a low risk of bias (Bakitas 2015; Bekelman 2018; Brumley 2007; Edmonds 2010; El-Jawahri 2016; Farquhar 2016; Franciosi 2019; Gade 2008; Groenvold 2017; Grudzen 2016; Janssens 2019; McCaffrey 2013; Ozcelik 2014; Rodin

The effectiveness and cost-effectiveness of hospital-based specialist palliative care for adults with advanced illness and their caregivers 27 (Review) 
2019; Solari 2018; Temel 2010; Vanbutsele 2018; Woo 2019). In Bekelman 2018, there were $79 \%$ completers in both intervention and control groups with 14 (8.9\%) and 12 (7.6\%) being unaccounted for in the intervention and control groups, respectively. Given that missing data were included in the analysis using maximum likelihood estimates, we gave a low risk of bias rating. Franciosi 2019 had $63.4 \%$ completers in the intervention group and 62.6\% completers in the control group. We rated it as having a low risk of bias because an imputation method was used for missing data as described in the FACIT Administration and Scoring Guidelines. Rodin 2019 had $59 \%$ completers in the intervention group and $95 \%$ completers in the control group. In spite of this difference, we gave a low risk of bias rating because missing data were included in the analysis.

We judged the remaining seven studies as having an unclear risk of bias (Ahronheim 2000; Bakitas 2009; Higginson 2009; Jingfen 2017; Lowther 2015; Mendoza-Galindo 2018 (abstract only); Nottelmann 2018). Examples of reasons for unclear risk of bias ratings were inclusion of missing data in primary outcome analysis but not secondary outcome analysis (Bakitas 2009) and study was an abstract and provided no information on attrition (MendozaGalindo 2018 (abstract only).

\section{Selective reporting}

We judged only five studies as having a low risk of bias (Bakitas 2015; Cheung 2010; Franciosi 2019; Higginson 2009; Tattersall 2014) because all prespecified outcomes were reported, while we gave 13 studies an unclear risk of bias either because their study protocols were not available or study protocols were available but only an abstract had been published. We gave 24 studies a high risk of bias for a number of reasons (for example. Bajwah 2015; Bekelman 2018; Carson 2016; Edmonds 2010; Janssens 2019; Rodin 2019; Solari 2018; Temel 2010; Vanbutsele 2018; Wallen 2012): some prespecified outcomes were not reported (for example, Edmonds 2010; Wallen 2012; Rodin 2019; Solari 2018); some outcomes in published papers were not stated a priori in the protocol/trial registry (for example, Brannstrom 2014; Janssens 2019); or because outcomes specified as primary outcomes in the protocol/trial registry were reported as secondary outcomes in published papers (for example, Bakitas 2009). We gave Temel 2017 a high risk of bias because it used a terminal decline joint modelling approach in modelling the trend in outcomes backward from death. This approach was not prespecified in the protocol.

\section{Other potential sources of bias}

Overall, we judged 27 studies as having a low risk of bias in this domain because the studies either appeared free of other biases or controlled for confounders in their analyses. For example, in Bakitas 2015, although the intervention group had significantly less education, higher weekly alcoholic beverage use, and higher clinical trial enrollment, the intention to treat analyses were adjusted for baseline values. Similarly, in Brannstrom 2014, the intervention and control groups were balanced with respect to baseline characteristics except for mean age. However, we gave a low risk of bias rating because the authors controlled for age in their analysis. We gave two studies a high risk of bias rating because the authors stated that there were baseline differences which were not adjusted for (Gade 2008; O'Riordan 2019). In 13 studies, we rated an unclear risk of bias because there were baseline differences and it was unclear if any adjustment was carried out for the differences (e.g. Bajwah 2015; Bekelman 2018; Brumley 2007; Cheung 2010; Franciosi 2019; McCorkle 2015). We gave McWhinney 1994 an unclear risk of bias because the sample characteristics at baseline were not reported.

\section{Size of study}

We assessed the size of studies in order to check for possible biases confounded by small size. We assessed 11 studies as having a high risk of bias because they included fewer than 50 participants in each treatment group (Brannstrom 2014; Cheung 2010; Edmonds 2010; Higginson 2009; Hopp 2016; Janssens 2019; McCaffrey 2013; Mendoza-Galindo 2018 (abstract only); O'Riordan 2019; Ozcelik 2014; Rodin 2019). Three studies included greater than 200 participants in each treatment group and we rated them as having a low risk of bias (Bakitas 2009; Carson 2016; Gade 2008). We gave the remaining 28 studies an unclear risk of bias rating because they had between 50 and 199 participants in one of the treatment groups or both groups. For example, Bekelman 2018 had 157 participants in the intervention group and 157 participants in the control group.

\section{Quality assessment for cost-effectiveness studies}

For full economic evaluations (Farquhar 2014; Farquhar 2016; Higginson 2009; McCaffrey 2013), we assessed risk of bias in results of the single effectiveness study on which the full economic evaluation study was based (see Figure 5 and Figure 6 for 'Risk of bias' assessment). We judged Farquhar 2014, Farquhar 2016 and Higginson 2009 to be at low risk of selection bias due to adequate description of the sequence generation process and allocation concealment. We gave McCaffrey 2013 an unclear risk of bias rating because there was insufficient information about the random sequence generation process and allocation concealment. Three of the studies reported on subjective outcomes but did not blind participants (Farquhar 2014; Farquhar 2016; Higginson 2009). Consequently, we gave the three studies a high risk of bias rating under 'blinding of participants and personnel (subjective outcomes)'. McCaffrey 2013 did not include subjective outcomes; we therefore left this domain blank. Besides McCaffrey 2013, the remaining three studies did not include objective outcomes and we left the domain 'blinding of participants and personnel (objective outcomes)' blank. We gave McCaffrey 2013 a low of risk under 'blinding of participants and personnel (objective outcomes)' because lack of blinding was unlikely to lead to bias in objective outcomes such as place of death.

We judged Farquhar 2014 and Farquhar 2016 to be at a low risk of bias for blinding of outcome assessment (subjective outcomes) because they blinded outcome assessors, while we gave Higginson 2009 a high risk of bias due to lack of blinding. McCaffrey 2013 did not include subjective outcomes and we therefore left this domain blank. McCaffrey 2013 included objective outcomes and we rated a low risk of bias for blinding of outcome assessment (objective outcomes) because lack of blinding was unlikely to affect objective outcomes. We left this domain blank in Farquhar 2014, Farquhar 2016 and Higginson 2009 because they did not include objective outcomes.

We judged Farquhar 2016 and McCaffrey 2013 as having a low risk of bias for incomplete outcome data (attrition bias), while we assessed Higginson 2009 as having an unclear risk of bias because the number of patients analysed differed from the number of patients randomly assigned to the intervention and control

The effectiveness and cost-effectiveness of hospital-based specialist palliative care for adults with advanced illness and their caregivers $\mathbf{2 8}$ (Review)

Copyright @ 2020 The Cochrane Collaboration. Published by John Wiley \& Sons, Ltd. 
groups. We assessed Farquhar 2014 as having a high risk of bias in this domain due to exclusion of missing data from the analysis. With the exception of Higginson 2009, we rated a high risk of bias for selective reporting (reporting bias) in the remaining three studies because all outcomes in the protocol/trial registry were not reported in the publication.

We gave a low risk of bias rating for 'other bias' in all studies except McCaffrey 2013. In McCaffrey 2013, it was unclear whether the differences between the intervention and control groups were controlled for. We assessed Farquhar 2014 and Farquhar 2016 as having an unclear risk of bias for 'size of study', and Higginson 2009 and McCaffrey 2013 as having a high risk of bias due to sample sizes below 50 in the intervention and control groups.

\section{BMJ Checklist for authors and peer reviews of economic submissions}

The methodological quality of the 13 studies that examined total costs varied across the different areas assessed (see Appendix 8). We assessed methodological quality using the BMJ Checklist for authors and peer reviewers of economic submissions (Drummond 1996). Given that they used different methods and reported on different resources used by patients, we could not pool their data in a meta-analysis. All the studies were clear about their research question. We considered all the studies to have provided the rationale for choosing the alternatives they compared because they all compared HSPC (or HSPC in addition to usual care) with usual care. However, only eight of them stated the economic importance of the research question. Six studies stated the form of economic evaluation used. The viewpoint of the analysis was stated only in three studies (Higginson 2009; McCaffrey 2013; Sahlen 2016 (linked to Brannstrom 2014)). All studies were clear about the source of effectiveness estimates used. Besides Mendoza-Galindo 2018 (abstract only), they all provided details on the design and results of their effectiveness study. The primary outcome for the economic evaluation was clearly stated in seven studies (Farquhar 2014; Farquhar 2016; Gade 2008; Higginson 2009; Higginson 2014; McCaffrey 2013; Sahlen 2016 (linked to Brannstrom 2014)). Quantities of resources were not reported separately from their unit costs in four studies (Ma 2019; Mendoza-Galindo 2018 (abstract only); Ozcelik 2014; Sahlen 2016 (linked to Brannstrom 2014)). In Brumley 2007, this was unclear because the authors described how the costs were derived but did not present the unit costs. Details of currency of price adjustments for inflation or currency conversion were not provided in any of the studies. The relevance of productivity changes to the study question was also not discussed in any of the studies. All studies except Mendoza-Galindo 2018 (abstract only) stated the time horizon of costs and benefits. They all addressed the research question with conclusions following from their findings. Gade 2008, Higginson 2009, Higginson 2014, McCaffrey 2013 and Sahlen 2016 (linked to Brannstrom 2014) provided details of statistical tests and confidence intervals.

\section{Consensus on Health Economic Criteria (CHEC) list}

We also used the CHEC list to assess the methodological quality of economic evaluations (see Appendix 9). Overall, 13 studies met seven to 16 (out of 19) quality items on the list. Five items were considered to have been met by all studies: clear description of study population; a well-defined research question in answerable form; identification of important and relevant outcomes for each alternative; appropriate measurement of outcomes; and conclusion following the reported data. All studies but MendozaGalindo 2018 (abstract only) discussed the generalisation of results to other settings or patient group and chose the appropriate time horizon to include relevant costs and outcomes. Eleven of 13 studies used the appropriate economic study design to answer the stated objective with the exception of Brumley 2007 and Brannstrom 2014. All studies except McCaffrey 2013 and Mendoza-Galindo 2018 (abstract only) discussed the ethical and distributional issues appropriately. Only two studies clearly described the competing alternatives (Higginson 2014; Ozcelik 2014), and three studies were considered to have appropriately chosen a perspective for the study (Higginson 2014; McCaffrey 2013; Temel 2010). Valuing outcomes appropriately was achieved only in five studies (Farquhar 2014; Farquhar 2016; Kane 1984; McCaffrey 2013; Temel 2010). No study needed nor clearly stated the discounting methods.

\section{Effects of interventions}

See: Summary of findings 1 Hospital-based specialist palliative care compared to usual care for adults with advanced illness and their caregivers/families

\section{Primary outcomes}

\section{Patient Health-related Quality of Life (HRQOL)}

Ten studies contributed adjusted endpoint data to the main metaanalysis on patient HRQoL (Bakitas 2009; Bakitas 2015; El-Jawahri 2016; McCorkle 2015; O'Riordan 2019; Rodin 2019; Tattersall 2014; Temel 2010; Temel 2017; Vanbutsele 2018). We also pooled nine studies reporting unadjusted endpoint data (Bajwah 2015; Brannstrom 2014; El-Jawahri 2016; Franciosi 2019; Gade 2008; Higginson 2014; Jingfen 2017; McCorkle 2015; Rogers 2017), and nine studies presenting unadjusted change data in our sensitivity analyses (Bajwah 2015; Bekelman 2018; El-Jawahri 2016; Grudzen 2016; Ozcelik 2014; Rogers 2017; Sidebottom 2015; Temel 2010; Temel 2017). We further carried out sensitivity analyses to explore the effect of using 0.02 in adjusting for clustering in McCorkle 2015 among studies that reported adjusted endpoint data and unadjusted endpoint data. Only Solari 2018 reported adjusted change data.

Of the remaining 19 studies that were not in any of the metaanalyses, 10 did not report on patient HRQoL (Ahronheim 2000; Brumley 2007; Carson 2016; Cheung 2010; Higginson 2009; Kane 1984; Ma 2019; McCaffrey 2013; Mendoza-Galindo 2018 (abstract only); Wallen 2012), six presented data on different domains of HRQoL (Edmonds 2010; Farquhar 2014; Farquhar 2016; Groenvold 2017; Janssens 2019; Lowther 2015), one assessed HRQoL only at baseline but not at follow-up (Hopp 2016), while one study only reported that there was "no significant difference" without presenting data (McWhinney 1994). Nottelmann 2018 assessed HRQoL but did not present analysable data.

Pooled data from 10 studies reporting adjusted endpoint data (main meta-analysis) with 1344 participants showed that HSPC was beneficial at improving patient HRQoL when compared to usual care (SMD $0.26,95 \% \mathrm{Cl} 0.15$ to $0.37 ; \mathrm{I}^{2}=3 \%$; Analysis 1.1). Positive SMDs indicate better patient $\mathrm{HRQ}$ LL while negative SMDs indicate lower patient HRQoL. The effect size obtained $(0.26)$ is small based on conventional standards (Cohen 1988). 
We carried out sensitivity analysis with studies that reported adjusted endpoint data to assess the impact of using an estimate of 0.02 in adjusting for clustering in McCorkle 2015. We found similar results to the main analysis, in favour of HSPC (SMD 0.29, 95\% Cl 0.18 to $0.40 ; 1^{2}=0 \% ; n=9$ studies; $N=1280$ participants; Analysis 1.2). Sensitivity analysis using unadjusted endpoint values led to a larger difference between groups but the confidence intervals were wider and there was greater heterogeneity (SMD $0.41,95 \% \mathrm{Cl}$ 0.11 to $0.70 ; I^{2}=83 \% ; n=9$ studies; $N=1201$ participants; Analysis 1.3). When McCorkle 2015 was removed, HSPC was still better than usual care in improving HRQoL (SMD $0.46,95 \% \mathrm{Cl} 0.13$ to $0.78 ; \mathrm{I}^{2}$ $=85 \% ; \mathrm{n}=8$ studies; $\mathrm{N}=1137$ participants; Analysis 1.4). When we pooled unadjusted change values, we also found benefit with HSPC (SMD 0.67, 95\% Cl 0.16 to $1.18 ;\left.\right|^{2}=95 \% ; n=9$ studies; $\mathrm{N}=$ 1278 participants; Analysis 1.5). The results from these sensitivity analyses supported that from the main analyses.

Solari 2018 was the only study that presented adjusted change data and it assessed patient HRQoL using the Schedule for the Evaluation of Individual Quality of Life - Direct Weighting (SEIQoLDW) (range, 0 to 100, 100 = best HRQoL). It found no between-group difference between HSPC and usual care both at three months and six months. At three months, mean change in the HSPC group was $-0.9(95 \% \mathrm{Cl}-6.8$ to 5.1$)$ and $-3.7(95 \% \mathrm{Cl}-17.6$ to 10.3$)$ in the usual care group with a difference of $2.8(95 \% \mathrm{Cl}-12.2$ to 17.8$)$ between the groups. At six months, mean change in the HSPC group was 0.8 $(95 \% \mathrm{Cl}-5.3$ to 6.9$)$ and that in the usual care group was $-4.0(95 \% \mathrm{Cl}$ -21.1 to 13.1$)$ with a difference of $4.8(95 \% \mathrm{Cl}-13.2$ to 22.7$)$ between the groups.

Across the studies in the meta-analyses, we combined different scales assessing patient HRQoL by calculating SMDs. Table 3 under Additional tables describes the HRQoL scales and the dimensions they covered. The studies used different scales for measuring patient HRQoL (Kings Brief Interstitial Lung Disease in Bajwah 2015; Functional Assessment of Chronic Illness therapy for Palliative Care, FACIT-Pal, in Bakitas 2009, Bakitas 2015 and Rogers 2017; Kansas City Cardiomyopathy Questionnaire (KCCQ) in Bekelman
2018; EQ-5D in Brannstrom 2014; Functional Assessment of Cancer Therapy-Bone Marrow Transplant, FACT-BMT, in El-Jawahri 2016; Functional Assessment of Chronic Illness Therapy - Spiritual Wellbeing Scale, FACIT-Sp, in Rodin 2019; Modified City of Hope Patient Questionnaires in Gade 2008; Functional Assessment of Cancer Therapy-General Measure, FACT-G, in Franciosi 2019; Grudzen 2016 and McCorkle 2015; Chronic Respiratory Disease QuestionnaireHealth Related Quality of IIfe (CRQ HRQL) in Higginson 2014; European Organisation for the Research and Treatment of Cancer Quality of Life Questionnaire Core 30, EORTC QLQ-C30 (Chinese version), in Jingfen 2017; EORTC QLQ-C30 in Ozcelik 2014 and Vanbutsele 2018; Minnesota Living with Heart Failure Questionnaire (MLHF) in O'Riordan 2019 and Sidebottom 2015; Schedule for the Evaluation of Individual Quality of Life - Direct Weighting (SEIQoLDW) in Solari 2018; McGill Quality of Life Questionnaire in Tattersall 2014; Functional Assessment of Cancer Therapy - Lung scale, FACT-L, in Temel 2010; Functional Assessment of Cancer Therapy General scale, FACT-G, in Temel 2017).

Four studies used more than one scale to measure patient HRQoL (Bajwah 2015; Brannstrom 2014; Higginson 2014; Rogers 2017). In particular, Brannstrom 2014 only showed data obtained using the EQ-5D and not that from the KCCQ. Consequently, data from the EQ-5D was used in the meta-analysis. Higginson 2014 assessed HRQoL using the CRQ HRQL and the EQ-5D. We only used data from the CRQ HRQL in the meta-analysis because unlike the EQ-5D (Williams 1995), a generic health-related quality of life measure, it is more specific to chronic respiratory disease (Guyatt 1987). Rogers 2017 assessed HRQoL using the FACIT-Pal and the KCCQ and both were presented as primary outcomes. Given that the FACIT-Pal has more extensive validation in palliative populations, we used it in the meta-analysis.

Overall, the funnel plot suggested some asymmetry (Figure 7). Egger's test for asymmetry resulted in a P value of 0.02 . However, given evidence of publication of negative studies in the funnel plot, this asymmetry is not necessarily indicative of publication bias. We did not carry out subgroup analysis due to low heterogeneity $\left(1^{2}=\right.$ $3 \%)$ in our main meta-analysis. 
Figure 7. Funnel plot of comparison: 1 Patient health-related quality of life, outcome: 1.1 HSPC versus usual care on patient HRQoL: adjusted endpoint values.

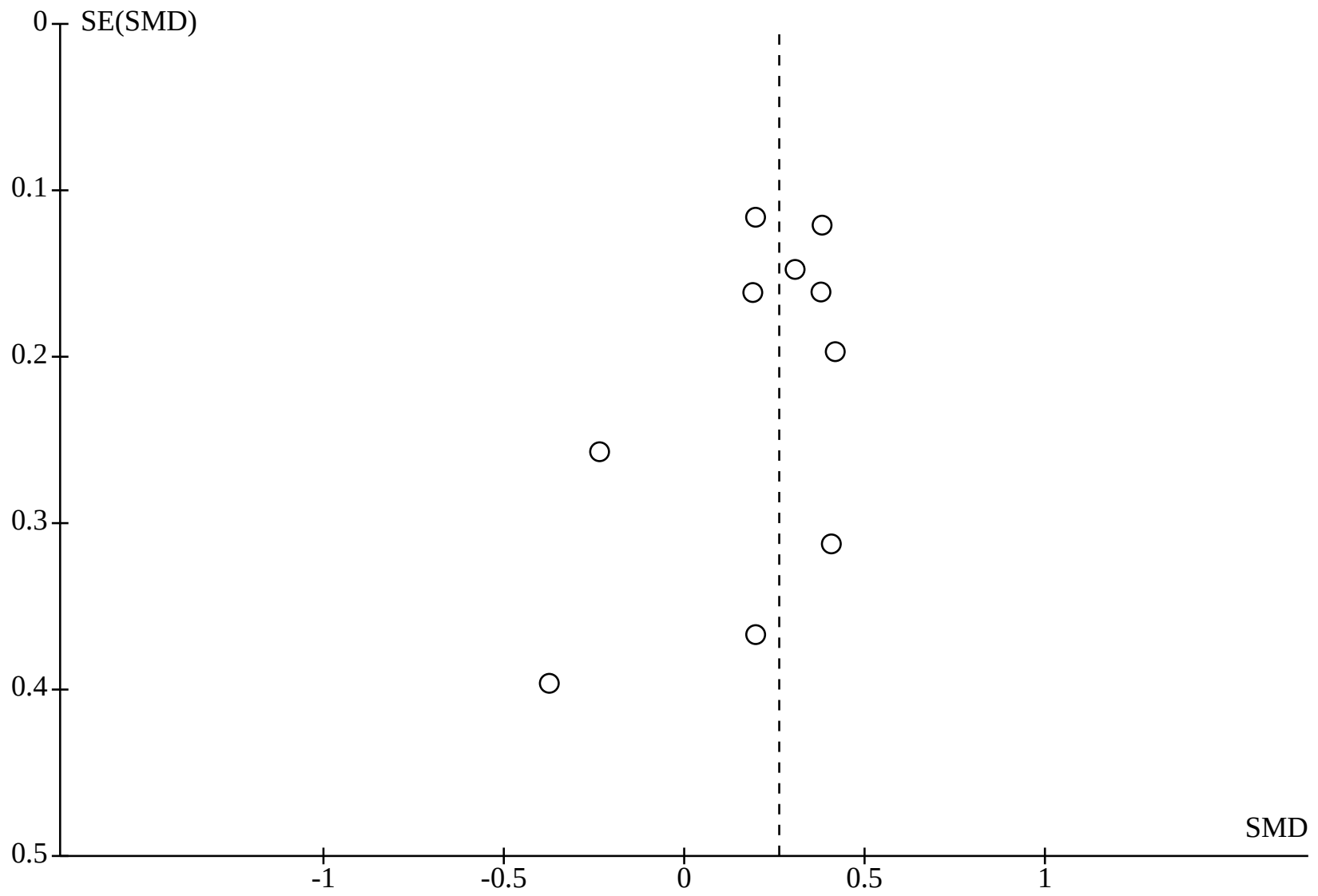

\section{Quality of the evidence}

Within the Grade approach, we downgraded the quality of the evidence on patient $\mathrm{HRQ}$ oL to low due to a high risk of bias across studies (-2 levels as a result of very serious study limitations: high risk of bias for selection, performance, detection, attrition and reporting bias) (see Summary of findings 1 ).

\section{Patient symptom burden (as a collection of two or more symptoms)}

We pooled six studies that reported adjusted endpoint data as the main meta-analysis on patient symptom burden (Bakitas 2009; Bakitas 2015; El-Jawahri 2016; Rodin 2019; Tattersall 2014; Temel 2010). We pooled six studies that reported unadjusted endpoint values (Bajwah 2015; El-Jawahri 2016; Gade 2008; Higginson 2014; Lowther 2015; McCorkle 2015), four studies presenting adjusted change values (Edmonds 2010; McCorkle 2015; Sidebottom 2015; Solari 2018), and six studies that reported unadjusted change values in our sensitivity analyses (Bajwah 2015; Bekelman 2018; El-Jawahri 2016; Higginson 2009; Ozcelik 2014; Temel 2010). We further carried out sensitivity analyses to explore the effect of using 0.02 in adjusting for clustering in McCorkle 2015 among studies that reported unadjusted endpoint data and adjusted change data.

Pooled data from six studies with 761 participants reporting adjusted endpoint values showed that HSPC was beneficial at reducing patient symptom burden compared to usual care (SMD
$-0.26,95 \% \mathrm{Cl}-0.41$ to $-0.12 ; \mathrm{I}^{2}=0 \%$; Analysis 2.1$)$. Negative SMDs indicate benefit (lower symptom burden) and positive SMDs reflect higher symptom burden.

Sensitivity analysis in the six studies ( $N=833$ participants) that reported unadjusted endpoint values showed a pooled effect of SMD $-0.17\left(95 \% \mathrm{Cl}-0.54\right.$ to $0.20 ; 1^{2}=83 \%$; Analysis 2.2). Among studies that reported unadjusted endpoint values, we carried out another sensitivity analysis to assess the impact of using 0.02 in adjusting for clustering in McCorkle 2015 and had similar findings (SMD - $0.19,95 \% \mathrm{Cl}-0.62$ to $0.24 ; \mathrm{I}^{2}=87 \% ; \mathrm{n}=5$ studies; $\mathrm{N}=769$ participants; Analysis 2.3). When we considered adjusted change values, the pooled effect was a SMD of $-1.31(95 \% \mathrm{Cl}-3.27$ to 0.64 ; $\mathrm{I}^{2}=98 \% ; \mathrm{n}=4$ studies; $\mathrm{N}=353$ participants; Analysis 2.4). When we excluded McCorkle 2015 from the studies that reported adjusted change values, we found a pooled effect of SMD -1.79 (95\% Cl -4.29 to $0.70 ; 1^{2}=98 \% ; n=3$ studies; $\mathrm{N}=289$ participants; Analysis 2.5). When we considered unadjusted change values, the pooled effect from the studies was a SMD of $-0.44\left(95 \% \mathrm{Cl}-0.94\right.$ to $0.06 ; \mathrm{I}^{2}=88 \%$; $\mathrm{n}=6$ studies; $\mathrm{N}=641$ participants; Analysis 2.6).

Of the remaining 25 studies that were not in any of the metaanalyses, 20 did not report on patient symptom burden (Ahronheim 2000; Brumley 2007; Carson 2016; Cheung 2010; Franciosi 2019; Farquhar 2014; Farquhar 2016; Groenvold 2017; Grudzen 2016; Hopp 2016; Jingfen 2017; Ma 2019; McCaffrey 2013; McWhinney

The effectiveness and cost-effectiveness of hospital-based specialist palliative care for adults with advanced illness and their caregivers 
1994; Mendoza-Galindo 2018 (abstract only); Nottelmann 2018; Rogers 2017; Temel 2017; Vanbutsele 2018; Woo 2019), two studies reported that there were "no significant differences" between intervention and control groups but they did not present data (Brannstrom 2014; Kane 1984), while O'Riordan 2019 did not present data from the Edmonton Symptom Assessment Scale (ESAS). Wallen 2012 did not present analysable data, while Janssens 2019 only assessed patient symptom burden using the Edmonton Symptom Assessment Scale (ESAS) in the intervention group.

Across the studies that we pooled in the meta-analyses, we combined different generalised measures of patient symptom burden by applying SMDs. Included studies used the following measures in assessing patient symptom burden: Palliative care Outcome Scale (POS) or a modified form of it in Bajwah 2015, Edmonds 2010, Higginson 2009, Higginson 2014 and Solari 2018; African POS in Lowther 2015; Edmonton Symptom Assessment Scale (ESAS) or a modified form of it in Bakitas 2009, El-Jawahri 2016, Ozcelik 2014 and Sidebottom 2015; symptom impact subscale of the Quality of Life at End of life (QUAL-E) in Bakitas 2015; General Symptom Distress Scale in Bekelman 2018; physical area scale of the Modified City of Hope Patient Questionnaires (MCOHPQ) in Gade 2008; Symptom Distress Scale (SDS) in McCorkle 2015 and Wallen 2012; Rotterdam Symptom Checklist (RSC - Physical Symptoms Score) in Tattersall 2014; lung cancer subscale (LCS) of the FACT-L in Temel 2010 and Memorial Symptom Assessment Scale (MSAS) in Rodin 2019. Only the severity subscale of the MSAS reported by Rodin 2019 was used in the meta-analysis.

Given that there were fewer than 10 included studies in the main meta-analysis of studies that presented adjusted endpoint values, we did not use funnel plots or carry out tests for funnel plot asymmetry. We also did not carry out subgroup analysis due to lack of heterogeneity $\mathrm{I}^{2}=0 \%$ ) in our main meta-analysis.

\section{Quality of the evidence}

Within the GRADE approach, we downgraded the quality of the evidence for patient symptom burden to very low due to a high risk of bias across studies ( -2 levels as a result of very serious study limitations: high risk of bias for selection, performance, detection, attrition and reporting bias and inconsistency: -1 level due to differences between our main meta-analysis and sensitivity analyses) (see Summary of findings 1).

\section{Secondary outcomes}

\section{Patient satisfaction with care}

Eight studies assessed the effect of HSPC on patient satisfaction with care (Brumley 2007; Gade 2008; Jingfen 2017; Kane 1984; O'Riordan 2019; Ozcelik 2014; Rodin 2019; Wallen 2012). We excluded three of the studies from the synthesis because they used measures that had not been validated (Jingfen 2017; O'Riordan 2019; Ozcelik 2014), while one study did not present analysable data (Wallen 2012). We excluded Janssens 2019 because the authors did not state the outcome measure used in assessing satisfaction with the intervention. The remaining four studies with 733 participants used validated measures (Brumley 2007; Gade 2008; Kane 1984; Rodin 2019). However, we could not include Brumley 2007 and Kane 1984 in our meta-analysis because Brumley 2007 presented odds ratio while Kane 1984 only presented P values.
Gade 2008 and Rodin 2019 reported adjusted endpoint values and found evidence in favour of HSPC (SMD $0.36,95 \% \mathrm{Cl} 0.14$ to 0.57 ; $\mathrm{I}^{2}=0 \% ; \mathrm{N}=337$ participants; Analysis 3.1). Positive SMDs indicate better patient satisfaction while negative SMDs indicate lower patient satisfaction. Gade 2008 used the Modified City of Hope Patient Questionnaires (MCOHPQ) Place of Care Environment Scale and the Doctors, Nurses/Other Care Providers Communication scale for assessing patient satisfaction with care. The MCOHPQ Place of Care Environment scale addressed experiences receiving pain management and symptom relief, psychological and social support, discharge planning, and end-of-life planning, while the Doctors, Nurses/Other Health Care Providers Communication scale addressed the level of caring and respect a patient felt from their providers, as well as the opportunity, ease, and the level of understanding the patient had with their providers. Only data from the MCOHPQ Place of Care Environment scale was used in the meta-analysis. Rodin 2019 assessed patient satisfaction with care using the 16-item Family Satisfaction with Care - Patient Version (FAMCARE-P16).

Brumley 2007 found a 3.37 higher odds of satisfaction in the HSPC group compared to control group $(P=0.03)$. Brumley 2007 used the Reid-Gundlach Satisfaction with Services instrument for assessing patient satisfaction. Kane 1984 found differences in satisfaction scores $(P<0.01)$ with HSPC patients expressing more satisfaction than control patients in two of the three areas examined. The two areas were interpersonal care and involvement in care. Kane 1984 used the Interpersonal Care scale adapted from the Ware scale (Ware 1979), a physical environment scale from McCaffree and Harkins (McCaffree 1976) and involvement-in-care questions adapted from the National Cancer Institute's Hospice Study (Baker 1981). Kane 1984 reported that these measures have been shown to be reliable and valid for patients with terminal cancer. No study reported decreased satisfaction of care in the HSPC group.

Due to small numbers in our main meta-analysis with adjusted endpoint values, we could not carry out subgroup analysis and we did not use funnel plots or carry out tests for funnel plot asymmetry.

\section{Quality of the evidence}

Within the Grade approach, we downgraded the quality of the evidence for patient satisfaction to low due to a high risk of bias in some domains in the two studies (-2 levels as a result of very serious study limitations: high risk of performance, detection, reporting, attrition, size of study and other biases) (see Summary of findings 1).

\section{Unpaid caregiver satisfaction with care}

Four studies assessed the effect of HSPC on family satisfaction with care (Carson 2016; Cheung 2010; Kane 1984; Ozcelik 2014). We excluded Cheung 2010 and Ozcelik 2014 from the synthesis because they used non-validated family satisfaction measures. Carson 2016 and Kane 1984 used validated measures with a total of 408 participants.

Carson 2016 was the only study that presented adjusted endpoint values, with family satisfaction assessed using the Family Satisfaction in the Intensive Care Unit (FS-ICU) survey (range, 0 to $100,100=$ best unpaid caregiver satisfaction). It found no betweengroup difference between HSPC and usual care. The mean $(95 \% \mathrm{CI})$ satisfaction in the HSPC group was 81.1 (78.3 to 83.9) while that in 
the usual care group was 84.3 ( 81.3 to 87.3 ), with a difference of -3.1 $(-7.3$ to 1.0$)$ between groups $(P=0.13)$.

Kane 1984 did not present their data. They only reported $\mathrm{P}$ values in favour of the HSPC group in two of the five cohorts they assessed. Kane 1984 assessed family satisfaction with care using the Interpersonal Care scale adapted from the Ware scale (Ware 1979), a physical environment scale based on that of McCaffree and Harkins (McCaffree 1976), and involvement-in-care questions adapted from the National Cancer Institute's Hospice Study (Baker 1981).

\section{Quality of the evidence}

Within the Grade approach, we downgraded the quality of the evidence for unpaid caregiver satisfaction with care to very low due to a high risk of bias across studies ( -2 levels as a result of very serious study quality limitations: high risk of bias for performance, attrition and reporting biases and inconsistency: - 1 level due to heterogeneity in study findings).

\section{Achieving patient preferred place of death (measured by number of patients with home death)}

Given that most people in developed countries prefer to die at home (Gomes 2012), we used number of home deaths as a proxy measure for achieving patient preferred place of death.

Pooled data from seven studies with 861 analysed participants showed that those receiving HSPC had higher odds of home deaths compared to those receiving usual care (OR $1.63,95 \% \mathrm{Cl} 1.23$ to $2.16 ; 1^{2}=0 \%$; Analysis 4.1$)$. The odds ratio of 1.63 translates to a risk ratio of $1.22(95 \% \mathrm{Cl} 1.08$ to 1.39$)$. This implies an increase in the relative risk of home deaths of $22 \%$ (95\% Cl $8 \%$ to $39 \%)$ when compared to usual care.

Kane 1984 reported that in the intervention group, only 3\% of deaths occurred at home with almost $60 \%$ dying in the inpatient hospice, while in the control group, $7 \%$ of deaths occurred at home with almost $80 \%$ dying in hospital. The actual number of deaths was not given but the authors stated that the difference between intervention and control group was not "statistically significant". Janssens 2019 reported two home deaths but did not state whether they occurred in the HSPC group or control group.

The remaining 33 studies did not report on home death.

Given that there were fewer than 10 included studies in the metaanalysis, we did not use funnel plots or carry out tests for funnel plot asymmetry. In addition, we could not carry out subgroup analysis due to lack of heterogeneity $\left(I^{2}=0 \%\right)$ in our meta-analysis.

\section{Quality of the evidence}

Within the Grade approach, we downgraded the quality of the evidence for achieving patient preferred place of death to low due to a high risk of bias across studies (-2 levels as a result of very serious study limitations: high risk of bias for selection, performance, detection, attrition and reporting bias) (see Summary of findings 1 ).

\section{Achieving patient preferred place of care}

Only one study by Bajwah 2015 ( $n=47$ participants) reported on this outcome. Bajwah 2015 was a fast-track RCT. Patients in the intervention group received HSPC immediately after randomisation, while the control group received HSPC four weeks after randomisation. Consequently, both the intervention and control group received HSPC. Results at the end of the study showed that all eight patients $(100 \%)$ who died in the intervention group achieved their preferred place of care, while 11 patients (84\%) in the control group who received HSPC after four weeks achieved this.

\section{Quality of the evidence}

Within the Grade approach, we downgraded the quality of the evidence for achieving preferred place of care to very low due to a high risk of bias in different domains ( -2 levels as a result of very serious study limitations: high risk of bias for performance, detection, attrition and reporting bias and imprecision: -1 level due to limited number of studies and participants).

\section{Mortality/survival}

Thirty-six studies with 7103 participants reported on mortality/ survival (Ahronheim 2000; Bajwah 2015; Bakitas 2009; Bakitas 2015; Bekelman 2018; Brannstrom 2014; Brumley 2007; Carson 2016; Cheung 2010; Edmonds 2010; El-Jawahri 2016; Farquhar 2014; Farquhar 2016; Franciosi 2019; Gade 2008; Groenvold 2017; Grudzen 2016; Higginson 2009; Higginson 2014; Hopp 2016; Janssens 2019; Kane 1984; Lowther 2015; Ma 2019; McCaffrey 2013; McCorkle 2015; McWhinney 1994; O'Riordan 2019; Rogers 2017; Sidebottom 2015; Solari 2018; Tattersall 2014; Temel 2010; Temel 2017; Vanbutsele 2018; Woo 2019) (see Table 4 under Additional tables). We decided against pooling of their hazard ratios in a metaanalysis due to methodological limitations in the included studies. Three studies did not report on the number of deaths (MendozaGalindo 2018 (abstract only); Ozcelik 2014; Wallen 2012), while Nottelmann 2018 only reported number of deaths in the HSPC group. Rodin 2019 reported that there were no deaths during the study, while this was unclear in the foreign language study because it was not described (Jingfen 2017).

Ten of these studies reported on deaths in the HSPC and control group without presenting survival time and they found no betweengroup difference in number of deaths (Ahronheim 2000; Bekelman 2018; Brannstrom 2014; Cheung 2010; Franciosi 2019; Higginson 2009; Hopp 2016; Ma 2019; McCaffrey 2013; Rogers 2017), while Sidebottom 2015 reported no association between study group assignment and death within six months after adjustment for age, gender, and marital status (Hazard Ratio: 1.90 (95\% Cl: 0.88, 4.09); P $=0.101$ ). Sidebottom 2015 reported 14 deaths $(12.1 \%)$ in the HSPC group and 5 deaths $(4.3 \%)$ in the control group.

In 11 studies, it was unclear if there was any difference in mortality because the $P$ values were not presented (Bajwah 2015; Edmonds 2010; El-Jawahri 2016; Farquhar 2014; Farquhar 2016; Lowther 2015; McCorkle 2015; McWhinney 1994; O'Riordan 2019; Solari 2018; Temel 2017). McWhinney 1994 only presented the total number of deaths at one month (36 (24.7\%)) but did not report the numbers in the HSPC and control group.

In the studies that reported survival time, there was probably little to no effect of HSPC on survival (Bakitas 2009; Bakitas 2015; Carson 2016; Gade 2008; Groenvold 2017; Grudzen 2016; Kane 1984; Janssens 2019; Vanbutsele 2018; Woo 2019).

In Bakitas 2009, median survival $(95 \% \mathrm{Cl})$ in the HSPC group was 14 months (10.6 to 18.4 ) and 8.5 months (7 to 11.1) in control with a

The effectiveness and cost-effectiveness of hospital-based specialist palliative care for adults with advanced illness and their caregivers 
$P$ value of 0.14 . There were 112 deaths (69.6\%) in the HSPC group and 119 deaths $(73.9 \%)$ in the control group. The Cox proportional hazards model estimate demonstrated a reduced relative risk of death (Hazard Ratio (HR): 0.67 (95\% Cl: 0.496 to 0.906), $\mathrm{P}=0.009$ ) in the HSPC group during the first year of the study and a greater relative risk after one year ( $\mathrm{HR}, 1.56(95 \% \mathrm{Cl}: 0.908$ to 2.655$)$ ).

In Bakitas 2015, a fast-track RCT in which the intervention group was offered HSPC immediately, while the control group received HSPC after three months, median survival by the end of data collection in the intervention group was 18.3 months and 11.8 months in the control group who began HSPC three months later. Kaplan-Meier curves illustrated a $15 \%$ difference in survival at 1 year (HSPC, 63\% versus control, 48\%; $\mathrm{P}=0.038$ ). However, the overall log-rank test $P$ value was 0.18 , suggesting a convergence in overall survival after 12 months. At one year, there were 109 deaths $(52.7 \%)$ but numbers in intervention and control groups were not reported.

Carson 2016 reported a median survival $(95 \% \mathrm{Cl})$ of 19 (12 to 37$)$ days in the HSPC group and 23 (12 to 39) days in control group ( $\mathrm{P}=$ 0.51). There was no difference in 90 -day survival $(\mathrm{HR}, 0.95(95 \% \mathrm{Cl}$ : 0.65 to 1.38$), P=0.96$ ). Post hoc adjustment for baseline activities of daily living and study site did not alter the outcome (HR,1.01 (95\% $\mathrm{Cl}: 0.69$ to 1.47$), \mathrm{P}=0.96)$.

In Grudzen 2016, median survival $(95 \% \mathrm{CI})$ in the HSPC group was 289 days ( 128 to 453 ) and 132 days ( 80 to 302 ) in control with a $P$ value of 0.2 . At one year, 41 participants $(59.4 \%)$ had died in the HSPC group and $44(65.7 \%)$ had died in the control group. However, there was no difference between the groups $(P=0.20)$.

Janssens 2019 was not clear about whether they were reporting mean or median survival. Survival in the HSPC group was 454 days (95\% Cl: 382 to 525$)$ and 425 days (95\% Cl: 339 to 509) in the control group (log-rank test, $\mathrm{P}=0.91$ ). During the follow-up period in Janssens 2019, there were four deaths (15.4\%) in the HSPC group and four deaths $(17.4 \%)$ in the control group.

Kane 1984 reported no difference in survival between HSPC and the control group as the survival curves were similar.

In Gade 2008, median survival (IQR) was 30 days (6 to 104) in the HSPC group and 36 days ( 13 to 106$)$ in the control group $(P=0.08)$. There were 173 deaths (63\%) in the HSPC group and 132 deaths $(56 \%)$ in the control group during the study period.

Groenvold 2017 reported that survival time did not differ between HSPC and the control group. Median survival in the HSPC group was 323 days and 364 days in the control group $(P=0.16$, but in the adjusted analysis $\mathrm{P}=0.39$ ). There were 25 deaths $(27 \%)$ in the HSPC group and 22 deaths $(23 \%)$ in the control group.

Woo 2019 reported that there was no difference in survival between HSPC and usual care but did not present any data.

Vanbutsele 2018 found the median survival $(95 \% \mathrm{Cl})$ in the HSPC group to be 312 days (190 to 434 ) and 343 days (253 to 433 ) in the control group $(P=0.97)$.

Sidebottom 2015 reported no association between study group assignment and death within six months after adjusting for age, gender and marital status $(P=0.10)$.
Higginson 2014 and Temel 2010 found evidence in favour of HSPC for longer survival compared to usual care. Higginson 2014 was a fast-track RCT in which the intervention group received HSPC immediately while those in the control group were offered HSPC after six weeks. Survival was calculated from the time of randomisation to the time of death, if death occurred during the study period, or to the time of censoring. Median survival (range) from randomisation to the time of censoring was 745 (338 to 1075) days in the intervention group compared to 711 (345 to 1045 ) in the control group who received HSPC after six weeks $(P=0.048)$. In subgroup analysis, this pattern was not recorded for patients with cancer $(P=0.97)$; but it became more marked for patients with diseases other than cancer $(P=0.01)$. Temel 2010 reported that median survival $(95 \% \mathrm{Cl})$ was 11.6 months (6.4 to 16.9) in the HSPC group and 8.9 months (6.3 to 11.4) in control (log rank $P=0.02$ ). After adjustment for age, sex, and baseline Eastern Cooperative Oncology Group performance status, the group assignment remained a predictor of survival (hazard ratio for death in the standard care group, $1.70 ; 95 \% \mathrm{Cl}, 1.14$ to $2.54 ; \mathrm{P}=$ $0.01)$.

By contrast, Brumley 2007 and Tattersall 2014 reported greater survival (SD) in the control group compared to the HSPC group. Brumley 2007 reported a mean (SD) survival of 242 (SD:200) days in the control group compared to 196 (SD:164) days in those receiving HSPC ( $P=0.03$ ). However, results of the Kaplan-Meier survival analysis did not show differences in survival time between study groups $(P=0.08)$. The authors also highlighted $75 \%$ death among participants but the percentages in the HSPC and control groups were not stated. In Tattersall 2014, there were 39 (65\%) deaths in the HSPC group and 31 (51.7\%) in the control group at 12 months. Tattersall 2014 found the median survival $(95 \% \mathrm{Cl})$ in the HSPC group to be 7 months (5.2 to 9.8) compared to 11.7 months (9.8 to 18.8$)$ in the control group ( $\log$ rank $P=0.014)$. The estimated hazard ratio was $1.6(95 \% \mathrm{Cl}: 1.1$ to $2.3 ; \mathrm{P}=0.015)$. This estimate changed to $1.5(95 \% \mathrm{Cl} 0.99$ to $2.2 ; \mathrm{P}=0.06)$ when adjusted for the oncologist's baseline estimate of likely survival, diagnosis, months since diagnosis, and gender.

\section{Quality of the evidence}

Within the Grade approach, we downgraded the quality of the evidence for mortality/survival to very low due to a high risk of bias across studies ( -2 levels as a result of very serious study limitations: high risk of bias for selection, performance, detection, attrition, reporting and other biases and inconsistency: -1 level due to variability in study findings).

\section{Pain}

We pooled data from four studies ( $n=525$ participants) that reported adjusted endpoint values for pain as the main metaanalysis. The meta-analysis showed that HSPC may lead to little to no difference in pain relief (SMD $-0.16,95 \% \mathrm{Cl}-0.33$ to $0.01 ;\left.\right|^{2}=0 \%$; Analysis 5.1). Positive SMDs indicate more pain while negative SMDs indicate lower pain (benefit). Only Woo 2019 reported unadjusted endpoint values and it assessed pain using the Brief Pain Inventory. It found no difference in mean pain scores between HSPC and usual care $(P=0.22)$. However, sensitivity analysis with studies reporting adjusted change values showed evidence in favour of HSPC (SMD $-0.47,95 \% \mathrm{Cl}-0.74$ to $-0.20, \mathrm{I}^{2}=0 \% ; \mathrm{n}=2$ studies; $\mathrm{N}=$ 218 participants; Analysis 5.2). 
When we carried out sensitivity analysis using unadjusted change values, we found no evidence of a difference between HSPC and usual care (SMD -0.93, 95\% Cl -3.05 to $1.19 ; \mathrm{I}^{2}=97 \% ; \mathrm{n}=2$ studies; $\mathrm{N}=291$ participants; Analysis 5.3).

Although we had initially specified that we would treat pain as a binary outcome in our published protocol (Bajwah 2017), this was not possible as most studies presented pain as a continuous outcome. Studies such as Tattersall 2014 reported on the percentage of patients with pain, while Lowther 2015 presented pain data as medians. Kane 1984 reported that there was no difference in pain between the intervention and control group over time but did not present data. Also, McWhinney 1994 stated that there were "no clinically or statistically significant differences" between the intervention and control groups but did not report their data. The remaining 30 studies did not report on pain.

We combined different scales assessing pain by calculating SMDs. Across the studies in these meta-analyses, we combined different measures for assessing pain (PEG derived from the Brief Pain Inventory (BPI) in Bekelman 2018; pain item of the EORTC QLQ-C30 in Groenvold 2017 and Vanbutsele 2018; pain item of the POS in Higginson 2009; pain severity on the BPI in O'Riordan 2019, Rodin 2019 and Woo 2019; pain item of the ESAS in Ozcelik 2014 and Sidebottom 2015).

Given that there were fewer than 10 included studies in our main meta-analysis on pain using adjusted endpoint values, we did not use funnel plots or carry out tests for funnel plot asymmetry. In addition, we could not carry out subgroup analysis due to lack of heterogeneity $(12=0 \%)$ in our main meta-analysis with adjusted endpoint values.

\section{Quality of the evidence}

Within the Grade approach, we downgraded the quality of the evidence for pain to very low due to a high risk of bias across studies ( -2 levels as a result of very serious study limitations: high risk of bias for performance, attrition and other bias and inconsistency: -1 level due to differences between our main meta-analysis and sensitivity analyses) (see Summary of findings 1 ).

\section{Patient anxiety and depression}

\section{Patient anxiety}

We pooled data from five studies ( $N=384$ participants) that reported adjusted endpoint values as the main meta-analysis. The five studies used the anxiety subscale of the Hospital Anxiety and Depression Scale (HADS-A) for assessing anxiety (seven items; 0 to 21 scale, 21 = maximum distress). HSPC showed no evidence of difference with a mean difference of -0.63 points when compared to usual care $\left(95 \% \mathrm{Cl}-2.22\right.$ to $0.96 ; \mathrm{I}^{2}=76 \%$; Analysis 6.1$)$. Negative mean difference (MD) indicates benefit (lower anxiety) and positive $M D$ reflects harm (higher anxiety).

We carried out sensitivity analysis to test the estimate we used in adjusting for clustering in McCorkle 2015 and found evidence in favour of HSPC (MD $-1.60,95 \% \mathrm{Cl}-2.56$ to $-0.65 ; \mathrm{I}^{2}=17 \% ; \mathrm{n}=4$ studies; $\mathrm{N}=320$ participants; Analysis 6.2).

Evidence from the sensitivity analysis of studies that reported unadjusted endpoint values produced a mean difference of -0.90 between HSPC and usual care $\left(95 \% \mathrm{Cl}-2.52\right.$ to $0.71 ; \mathrm{I}^{2}=67 \% ; n$
= 4 studies; $\mathrm{N}=273$ participants; Analysis 6.3). Included studies measured anxiety using the HADS-A. When we removed McCorkle 2015 , the mean difference was $-1.48\left(95 \% \mathrm{Cl}-3.52\right.$ to $0.56 ; \mathrm{I}^{2}=71 \%$; $\mathrm{n}=3$ studies; $\mathrm{N}=209$ participants; Analysis 6.4).

Sensitivity analysis with studies that presented unadjusted change values showed an effect in favour of HSPC (SMD - $0.62 ; 95 \% \mathrm{Cl}-1.02$ to $-0.21 ; 1^{2}=74 \% ; \mathrm{n}=4$ studies; $\mathrm{N}=496$ participants; Analysis 6.5). SMD was used in pooling the estimates because the four studies used different scales for measuring anxiety. Bajwah 2015 and El-Jawahri 2016 used the HADS-A, Bekelman 2018 used the Generalised Anxiety Disorder-7 (GAD-7), while Ozcelik 2014 used the anxiety subscale of the Edmonton Symptom Assessment Scale (ESAS)).

Only Sidebottom 2015 ( $n=167$ participants) reported adjusted change values and it assessed anxiety using the anxiety subscale of the ESAS (using a visual scale line, 0 to $10,10=$ worst possible). It found that anxiety scores improved by a mean of 1.27 points in the HSPC group and 0.89 in the control group at three months (difference $0.38, \mathrm{P}=0.017$ ) after adjusting for age, gender, and marital status differences between study groups. This difference was already evident at one month $(P=0.007)$.

Five studies also assessed patient anxiety but they could not be included in the meta-analysis for the following reasons: Kane 1984 did not provide data on anxiety but rather it only stated the $P$ values, Temel 2010 only presented the percentage of patients with anxiety at the primary point of analysis, Temel 2017 did not provide data but stated that scores did not differ between the intervention and control groups at 12 weeks or 24 weeks, Solari 2018 reported no difference between groups for change at three and six months but did not present usable data and Vanbutsele 2018 presented an odds ratio at 12,18 and 24 weeks. This study did not find any evidence of a difference between groups at these different time points.

The remaining 26 studies did not report on patient anxiety.

Given that there were fewer than 10 included studies in the main meta-analysis on patient anxiety using adjusted endpoint values, we did not use funnel plots or carry out tests for funnel plot asymmetry.

\section{Subgroup analysis on patient anxiety}

We carried out the following subgroup analyses on patient anxiety.

\section{Effect of HSPC on patient anxiety in different populations}

Among studies that reported adjusted endpoint values, we carried out subgroup analysis to assess the effect of HSPC on patient anxiety in different populations. Three studies with 275 participants were with cancer populations and two with noncancer populations ( $\mathrm{N}=109$ participants). Subgrouping according to patient population explained heterogeneity in the non-cancer population subgroup $\left(1^{2}=0 \%\right)$, but not the cancer population subgroup $\left(1^{2}=87 \%\right)$ (Analysis 6.6). There was no evidence of a subgroup effect $(P=0.90,12=0 \%)$. This finding may be spurious due to the small number of studies and participants in the subgroups. When McCorkle 2015 was excluded from the cancer population subgroup, heterogeneity (12) reduced to $24 \%$ (Analysis 6.7 ). No subgroup difference was observed $\left(P=0.29,1^{2}=10 \%\right)$.

The effectiveness and cost-effectiveness of hospital-based specialist palliative care for adults with advanced illness and their caregivers 


\section{Effect of different models of HSPC on patient anxiety}

Four studies ( $N=227$ participants) that involved service provision across multiple settings and one study by El-Jawahri 2016 with an inpatient consult model ( $\mathrm{N}=157$ participants) reported adjusted endpoint values. We could not carry out subgroup analysis because of the limited number of studies in the inpatient consult model subgroup.

\section{Effect of 24 hours access (out-of-hours care) on patient anxiety}

None of the studies had provision for 24 hours access.

\section{Effect of early palliative care versus late palliative care on patient anxiety}

Among studies that reported adjusted endpoint data, two studies with 221 participants provided HSPC early and three with 163 participants provided it late. Subgrouping only explained heterogeneity in the late palliative care subgroup $(12=0 \%)$, but not the early palliative care subgroup $\left(I^{2}=94 \%\right)$ (Analysis 6.8). There was no evidence of a subgroup effect $\left(P=0.90,1^{2}=0 \%\right)$. When McCorkle 2015 was removed from the early palliative care subgroup, only El-Jawahri 2016 was remaining in the subgroup and we could not carry out any further analysis.

\section{Effect of nurse led multi-disciplinary team versus multidisciplinary led} team services on patient anxiety

All five studies ( $N=384$ participants) that reported adjusted endpoint values were MDTservices not led by nurses with a pooled mean difference of -0.63 between HSPC and usual care $(95 \% \mathrm{Cl}$ -2.22 to $0.96 ; I^{2}=76 \%$; Analysis 6.9). After removal of McCorkle 2015, there was evidence in favour of HSPC when compared to usual care (MD $-1.60,95 \% \mathrm{Cl}-2.56$ to $-0.65 ; \mathrm{I}^{2}=17 \% ; \mathrm{n}=4$ studies; $\mathrm{N}=320$ participants; Analysis 6.10).

\section{Effect of HSPC on patient anxiety in different countries}

Among studies that reported adjusted endpoint values, three ( $\mathrm{N}$ $=251$ participants) were carried out in USA and two $(\mathrm{N}=133$ participants) in the UK. Subgrouping by country only explained heterogeneity in the UK studies $(12=0 \%)$, but not the USA studies $\left(I^{2}=88 \%\right)$ (Analysis 6.11). Subgroup analysis showed no difference across the two countries $(P=0.66,12=0 \%)$. This analysis is unlikely to detect a subgroup difference due to the small number of studies and participants in the subgroups. When McCorkle 2015 was removed from the USA subgroup, $1^{2}$ was $52 \%$ in the subgroup (Analysis 6.12) and there was no evidence of a subgroup effect and heterogeneity $\left(P=0.77,1^{2}=0 \%\right)$.

\section{Quality of the evidence}

Within the Grade approach, we downgraded the quality of the evidence for patient anxiety to very low due to a high risk of bias across studies (-2 levels as a result of very serious study limitations: high risk of bias for selection, performance, detection, attrition and reporting biases and inconsistency: -1 level due to unexplained heterogeneity).

\section{Patient depression}

We pooled data from eight studies ( $N=1096$ participants) reporting adjusted endpoint values for our main meta-analysis on patient depression. The results showed that HSPC improved depression when compared to usual care (SMD $-0.22,95 \% \mathrm{Cl}-0.34$ to -0.10 ;
$1^{2}=0 \%$; Analysis 8.1). Negative SMDs indicate benefit (lower depression) and positive SMDs reflect harm (higher depression).

We carried out sensitivity analysis with five studies ( $\mathrm{N}=350$ participants) presenting unadjusted endpoint values and found a pooled estimate of SMD $-0.25\left(95 \% \mathrm{Cl}-0.55\right.$ to $0.04 ; 1^{2}=47 \%$; Analysis 8.2 ). We carried out sensitivity analysis to assess the impact of using an estimate of 0.02 in adjusting for clustering in McCorkle 2015 and found evidence in favour of HSPC (SMD -0.34, $95 \% \mathrm{Cl}-0.65$ to $-0.03 ; \mathrm{I}=42 \% ; \mathrm{n}=4$ studies; $\mathrm{N}=286$ participants; Analysis 8.3).

Only two studies (McCorkle 2015, Sidebottom 2015) with 231 participants contributed data to the sensitivity analysis using adjusted change values with a pooled estimate of MD - 0.32 (95\% $\mathrm{Cl}-1.10$ to $0.45 ; \mathrm{I}^{2}=92 \%$; Analysis 8.4 ). The sensitivity analysis using unadjusted change values showed evidence in favour of HSPC (SMD $-0.38,95 \% \mathrm{Cl}-0.58$ to $-0.18 ; \mathrm{I}^{2}=12 \% ; \mathrm{n}=4$ studies; $\mathrm{N}=488$ participants; Analysis 8.5).

Three studies also presented binary data and we pooled them using odds ratio (El-Jawahri 2016; Temel 2010; Woo 2019). We found evidence of lower odds of patient depression with HSPC compared to usual care (OR $0.38,95 \% \mathrm{Cl} 0.21$ to $0.68 ; \mathrm{I}^{2}=32 \% ; \mathrm{n}=3$ studies; $\mathrm{N}$ $=338$ participants; Analysis 8.6). The odds ratio of 0.38 translates to a risk ratio of 0.55 , implying that the risk of patient depression was 0.55 times lower with HSPC compared to usual care.

Four studies assessed patient depression but we excluded them from the main meta-analysis because they did not present analysable data (Kane 1984; Solari 2018; Vanbutsele 2018; Wallen 2012). Kane 1984 described no between-group difference between intervention and control group but did not provide the data. Solari 2018 reported that they found no difference between groups at three and six months but did not present analysable data, Vanbutsele 2018 presented only odds ratios and the corresponding $95 \% \mathrm{Cls}$ for the two measures it used in assessing depression (HADS-D and PHQ-9). There was no difference between intervention and control groups at 12,18 and 24 weeks in Vanbutsele 2018. Wallen 2012 assessed depression but did not present data on it at baseline and follow-up. The remaining 21 studies did not report on patient depression.

Studies included in the meta-analyses used different scales in assessing depression (Becks Depression Inventory-II (BDI-II) in Rodin 2019; depression subscale of the Hospital Anxiety and Depression Scale (HADS-D) in Bajwah 2015, El-Jawahri 2016, Farquhar 2014, Farquhar 2016, Higginson 2014, O'Riordan 2019, Rogers 2017; Patient Health Questionnaire (PHQ-9) in Bekelman 2018, Grudzen 2016, McCorkle 2015, Sidebottom 2015 and Temel 2017; depression subscale of the Edmonton Symptom Assessment Scale (ESAS) in Ozcelik 2014; Centre for Epidemiological Studies Depression Scale (CES-D) in Bakitas 2009, Bakitas 2015 and Woo 2019). El-Jawahri 2016 and Temel 2017 also assessed depression using the PHQ-9.

Given that there was no heterogeneity in our main meta-analysis ( 12 $=0 \%$ ), we did not carry out any subgroup analysis. There were fewer than 10 studies that reported adjusted endpoint values in the main meta-analysis, and we did not use funnel plots or carry out tests for funnel plot asymmetry.

The effectiveness and cost-effectiveness of hospital-based specialist palliative care for adults with advanced illness and their caregivers 


\section{Quality of the evidence}

Within the Grade approach, we downgraded the quality of the evidence for patient depression to very low due to a high risk of bias across studies ( -2 levels as a result of very serious study limitations: high risk of bias for selection, performance, detection, attrition and reporting biases and inconsistency: -1 level due to differences between our main meta-analysis and sensitivity analyses).

\section{Patient breathlessness}

We pooled data from five studies reporting adjusted endpoint values for our main meta-analysis on patient breathlessness with a pooled estimate of SMD $-0.04\left(95 \% \mathrm{Cl}-0.19\right.$ to $0.12 ; \mathrm{I}^{2}=0 \%, \mathrm{~N}$ $=616$ participants; Analysis 12.1). Negative SMDs indicate benefit (reduced breathlessness) and positive SMDs reflect worsened breathlessness. The five studies used different instruments and reported on different breathlessness domains. For instance, Farquhar 2014 and Farquhar 2016 both assessed distress due to breathlessness and breathlessness mastery using a Numeric Rating Scale (NRS) and the mastery domain of the Chronic Respiratory Questionnaire (CRQ), respectively; Groenvold 2017 and Vanbutsele 2018 assessed breathlessness intensity using the dyspnoea item of EORTC QLQ-C30; O'Riordan 2019 assessed breathlessness intensity using the BORG scale. For Farquhar 2014 and Farquhar 2016, we used only data for distress due to breathlessness assessed with the NRS in our meta-analysis because it was the primary outcome. We did not differentiate between different breathlessness domains in our meta-analysis due to small numbers.

Sensitivity analysis carried out with the two studies ( $N=128$ participants) presenting unadjusted endpoint values showed a pooled estimate in favour of HSPC (SMD $-0.35,95 \% \mathrm{Cl}-0.70$ to -0.00 ; $\mathrm{I}^{2}=0 \%$; Analysis 12.2).

Only Sidebottom 2015 presented adjusted change values. It assessed breathlessness using the dyspnoea item of ESAS (using a visual scale line, 0 to $10,10=$ worst possible) and found that breathlessness scores improved by a mean of 2.8 points in the HSPC group and 1.7 in the control group at 3 months (difference 1.08, $\mathrm{P}<$ 0.001 ) after adjusting for age, gender, and marital status differences between study groups. This difference was evident at one month with a mean difference of $1.10(P<0.001)$.

Sensitivity analysis with the two studies that reported unadjusted change values showed a pooled estimate of SMD $-0.47(95 \% \mathrm{Cl}-1.55$ to $0.61 ; I^{2}=90 \%, \mathrm{~N}=292$ participants; Analysis 12.3).

A study by Tattersall 2014 also recorded this outcome but did not present analysable data. The remaining 31 studies did not report on breathlessness.

Studies included in the meta-analyses used different scales in assessing breathlessness: D-12 in Bajwah 2015; Memorial Symptom Assessment Scale in Bekelman 2018; Numeric Rating Scale (NRS) for distress due to breathlessness in Farquhar 2014 and Farquhar 2016; dyspnoea item of EORTC QLQ-C30 in Groenvold 2017 and Vanbutsele 2018; breathlessness mastery domain of the Chronic Respiratory Disease Questionnaire (CRQ mastery) in Higginson 2014; BORG scale in O'Riordan 2019; dyspnoea item of ESAS in Ozcelik 2014 and Sidebottom 2015.

Due to lack of heterogeneity $(12=0 \%)$ in our main meta-analysis, we could not carry out subgroup analysis. Given that there were fewer than 10 included studies in the main meta-analysis on breathlessness using adjusted endpoint values, we did not use funnel plots or carry out tests for funnel plot asymmetry.

\section{Quality of the evidence}

Within the Grade approach, we downgraded the quality of evidence for breathlessness to very low due to a high risk of bias across studies (-2 levels as a result of very serious study limitations: high risk of bias for selection, performance, detection, attrition and reporting biases, imprecision: -1 level due to wide $95 \% \mathrm{Cl}$ around the effect estimates that included both benefit and harm and inconsistency: -1 level due to differences between our main metaanalysis and sensitivity analyses).

\section{Adverse events in patients and unpaid caregivers}

Eight studies with 1252 participants reported on adverse events (Bajwah 2015; Bekelman 2018; Groenvold 2017; Higginson 2014; Lowther 2015; Rodin 2019; Solari 2018; Tattersall 2014) (see Table 5 under Additional tables). Two of these studies involved unpaid caregivers (Bajwah 2015; Higginson 2014).

Six studies ( $\mathrm{N}=976$ participants) reported no harmful effect (Bajwah 2015; Bekelman 2018; Groenvold 2017; Higginson 2014; Lowther 2015; Rodin 2019).

One study by Tattersall 2014 ( $N=120$ participants) found that more patients in the HSPC group had the mild adverse event of poorer appetite $(P=0.04)$ compared to the control group.

Solari 2018 ( $N=156$ participants $)$ reported 15 serious adverse events in 13 patients in the HSPC group and seven in seven patients in the control group $(P=0.78)$. Serious adverse events reported included aspiration pneumonia, generalised anxiety, breathing difficulty, urine retention/infection, anarthria, contact dermatitis, dysphagia, vomiting, bladder catheter malfunctioning, fever, arrhythmia, necrotising fasciitis, traumatic wound, macrohaematuria, constipation, abdominalgia and bronchitis. Three patients in the HSPC group died but this was considered to be unrelated to the intervention.

\section{Quality of the evidence}

Within the Grade approach, we downgraded the quality of the evidence for adverse events to very low due to a high risk of bias across studies (-2 levels as a result of very serious study limitations: high risk of bias for performance, detection, attrition and reporting bias and inconsistency: -1 level due to variability in the results).

\section{Unpaid caregiver symptom control}

\section{Unpaid caregiver anxiety}

Only Carson 2016 ( $\mathrm{N}=312$ participants) presented adjusted endpoint values. Carson 2016 assessed unpaid caregiver anxiety using the HADS-A (seven items; 0 to 21 scale, 21 = maximum distress). Carson 2016 reported no difference in the unpaid caregiver anxiety in the HSPC group compared to the control group at three months on adjusting for baseline and multiple respondents (mean $(95 \% \mathrm{Cl}): 7.2$ (6.6 to 7.9 ) versus 6.4 (5.7 to 7.1$)$, mean difference was 0.8 (95\% Cl: -0.1 to 1.8$), \mathrm{P}=0.09)$. Adjustments for three variables (baseline, multiple respondents and study sites) and six variables (baseline, multiple respondents, study sites, race, sex and primary/additional surrogate) also produced similar results with $P$ values of 0.11 and 0.12 , respectively.

The effectiveness and cost-effectiveness of hospital-based specialist palliative care for adults with advanced illness and their caregivers 
Only Bajwah 2015 and Carson 2016 with 351 participants provided unadjusted endpoint data with a pooled estimate of MD -0.71 (95\% $\mathrm{Cl}-4.27$ to $2.85 ; \mathrm{I}^{2}=77 \%$; Analysis 7.1 ). Both studies used the HADS$A$ in assessing unpaid caregiver anxiety. A negative MD indicates benefit (lower unpaid caregiver anxiety) and a positive MD reflects harm (higher unpaid caregiver anxiety).

Four studies recorded this outcome but did not present analysable data (El-Jawahri 2016; Farquhar 2014; Farquhar 2016; Kane 1984). El-Jawahri 2016 and Farquhar 2016 did not present the number of participants in the intervention and control group at the primary point of analysis. Farquhar 2014 reported that there was little change in carer outcomes but did not present data, while Kane 1984 found differences in favour of HSPC in three of the five cohorts examined but did not present usable data.

The remaining 36 studies did not report on unpaid caregiver anxiety.

Given that we had only one study that presented adjusted endpoint values, we could not carry out any further analysis.

\section{Quality of the evidence}

Within the Grade approach, we downgraded the quality of the evidence for unpaid caregiver anxiety to very low due to a high risk of bias (-2 levels as a result of very serious study limitations: high risk of bias for performance, attrition and reporting biases, and imprecision: -1 level due to the small number of participants).

\section{Unpaid caregiver depression}

Two studies ( $\mathrm{N}=413$ participants) reported on unpaid caregiver depression and also presented adjusted endpoint values. They found that HSPC had little to no effect on unpaid caregiver depression (SMD $-0.02,95 \% \mathrm{Cl}-0.21$ to $0.18 ; \mathrm{I}^{2}=0 \%$; Analysis 9.1 ). Negative SMDs indicate benefit (lower depression) and positive SMDs reflect harm (higher depression).

Sensitivity analysis with the three studies that reported unadjusted endpoint values resulted in a SMD of $-0.29\left(95 \% \mathrm{Cl}-0.70\right.$ to $0.12 ; \mathrm{I}^{2}=$ $63 \% ; \mathrm{n}=3$ studies; $\mathrm{N}=420$ participants; Analysis 9.2).

Bajwah 2015, ( $N=35$ unpaid caregiver participants), was the only study that presented unadjusted change values on the HADS-D (seven items; 0 to 21 scale, 21 = maximum distress). It found a $30 \%$ mean decrease in unpaid caregiver depression scores from baseline at four weeks for the HSPC group while for controls, unpaid caregiver depression increased by one point. The effect size $(95 \%$ $\mathrm{Cl}$ ) at four weeks was $-0.7(-1.3$ to 0.0$)$. Between the period when the control group received HSPC (four weeks) and eight weeks, mean (SD) depression improved in the control group from 9.6 (4.9) to 7.2 (3.9).

Four studies reported on unpaid caregiver depression but did not present usable data (El-Jawahri 2016; Farquhar 2014; Farquhar 2016; Kane 1984). In El-Jawahri 2016, the number of participants in the intervention and control groups at the primary point of analysis was not reported. Farquhar 2014, Farquhar 2016 and Kane 1984 did not present their data. The remaining 34 studies did not report on unpaid caregiver depression.

Studies included in the meta-analyses used different scales in assessing unpaid caregiver depression (Bajwah 2015 and Carson 2016 used the depression subscale of the HADS (HADS-D); Bakitas
2015 used the CES-D; Bekelman 2018 assessed depression using the Patient Health Questionnaire-8 (PHQ-8)).

We could not carry out subgroup analysis due to lack of heterogeneity in our main meta-analysis $(12=0 \%)$. Given that there were fewer than 10 included studies in the meta-analysis on unpaid caregiver depression, we did not use funnel plots or carry out tests for funnel plot asymmetry.

\section{Quality of the evidence}

Within the Grade approach, we downgraded the quality of evidence for unpaid caregiver depression to very low due to a high risk of bias ( -2 levels as a result of very serious study limitations: high risk of bias for performance, attrition and reporting bias and imprecision: -1 level due to wide $95 \% \mathrm{Cls}$ around the effect estimates that included both benefit and harm).

\section{Unpaid caregiver burden}

Two studies with 170 participants presented adjusted endpoint values (Dionne-Odom 2015a (linked to Bakitas 2015); Bekelman 2018)). However, we could not pool them together in a metaanalysis due to how they presented their data. Dionne-Odom 2015a assessed unpaid caregiver burden using the Montgomery-Borgatta Caregiver Burden (MBCB) scale and presented results for three different subscales of the MBCB, namely, the objective burden scale (range, 6 to 30; 30 indicates worst level of interference with the unpaid caregiver's private, social, recreational time and normal daily routine), stress burden scale (range, 4 to $20 ; 20$ indicates worst level of strained emotional demands related to caregiving) and the demand scale (range, 4 to 20; > 15 indicates worst level of caregiver strain by his or her caregiving demands). Bekelman 2018 assessed unpaid caregiver burden using the Zarit Burden Inventory (ZBI) (range, 0 to $88 ; 88$ indicates highest burden).

On the objective burden scale of the $M B C B$, the mean unpaid caregiver burden scores for the HSPC group was 0.3 points higher (range 6 to 30; 30 indicates worst) than that of the control group with adjustment for patient death $(P=0.64)$. On the stress burden scale of the MBCB, the mean caregiver burden scores for the HSPC group was 0.5 points lower (range, 4 to $20 ; 20$ indicates worst) than the control group with adjustment for patient death $(P=0.29)$. There was no difference in the mean caregiver burden score with adjustment for patient death on the demand scale of the MBCB (P $=0.97$ ). Bekelman 2018 reported a mean (SE) caregiver burden of 12.9 (1.3) in the HSPC group and 14.8 (1.4) in the control group at 12 months $(P=0.30)$.

Two studies ( $\mathrm{N}=108$ participants) reported unadjusted endpoint data but we could not pool them in a meta-analysis (Bajwah 2015; Dionne-Odom 2015a (linked to Bakitas 2015)). Dionne-Odom 2015a reported the following results: on the objective burden scale of the MBCB, the mean caregiver burden scores for the HSPC group was 0.3 points higher (range 6 to $30 ; 30$ indicates worst) than that of the control group $(P=0.62)$. On the stress burden scale of the MBCB, the mean caregiver burden scores for the HSPC group was 0.6 points lower (range, 4 to 20; 20 indicates worst) than that of the control group. There was no difference between HSPC and control group in the mean caregiver burden score on the demand scale of the MBCB $(P=0.99)$. Bajwah 2015 assessed unpaid caregiver burden using the $\mathrm{ZBI}$ (range, 0 to $88 ; 88$ indicates highest burden), and reported a mean (SD) unpaid caregiver burden of 22.3 (15.3) in the fast-track group and 31.7 (17.3) for the control group at four weeks. After

The effectiveness and cost-effectiveness of hospital-based specialist palliative care for adults with advanced illness and their caregivers 38 (Review)

Copyright $\odot 2020$ The Cochrane Collaboration. Published by John Wiley \& Sons, Ltd. 
the control group was offered HSPC between four weeks and eight weeks, mean (SD) unpaid caregiver burden reduced to 25.4 (13.4).

We carried out sensitivity analysis with the three studies that reported adjusted change values and found evidence in favour of HSPC (MD = $-3.88,95 \% \mathrm{Cl}-5.95$ to $-1.80 ; 1^{2}=0 \% ; \mathrm{N}=128$ participants; Analysis 11.1). All three studies assessed unpaid caregiver burden using the ZBI.

Bajwah 2015 ( $N=39$ participants) was the only study that presented unadjusted change values. Bajwah 2015 reported a 0.1 mean increase in unpaid caregiver burden score from baseline to four weeks for 16 intervention unpaid caregivers while for 23 unpaid caregivers in the control group, unpaid caregiver burden decreased by a 0.1 point. The effect size $(95 \% \mathrm{Cl})$ at four weeks was $-0.6(-1.2$ to 0.1 ).

Bakitas 2009 reported on unpaid caregiver burden but did not present usable data for the meta-analysis. The remaining 36 studies did not report on unpaid caregiver burden.

We did not carry out any further analysis on unpaid caregiver burden due to limited number of studies.

\section{Quality of the evidence}

Within the Grade approach, we downgraded the quality of the evidence for unpaid caregiver burden to very low due to a high risk of bias across studies ( -2 levels as a result of very serious study limitations: high risk of bias for performance and reporting bias and imprecision: -1 level due to small number of participants).

\section{Unpaid caregiver pre- and post-bereavement outcomes}

\section{Unpaid caregiver grief}

Only Dionne-Odom 2016 (linked to Bakitas 2015) with 44 participants provided usable data for unpaid caregiver grief. Dionne-Odom 2016 assessed unpaid caregiver grief using the Prigerson Inventory of Complicated Grief - Short Form (PG 13) and reported a non-signifacnt difference in the mean unpaid caregiver grief score in the HSPC group that was 2.2 points lower (range, 11 to $55 ; 55$ indicates highest grief) than that of the control group $(P=0.21)$. There was no evidence of a difference on adjusting for religious preference $(P=0.40)$, baseline depression levels $(P=0.51)$ and patient hospice use $(P=0.51)$.

\section{Quality of the evidence}

We downgraded the quality of the evidence on unpaid caregiver grief to low due to a high risk of bias (-1 level as a result of serious study limitations: high risk of performance bias and imprecision: -1 level due to small number of participants).

\section{Unpaid caregiver quality of life}

Only Dionne-Odom 2015a (linked to Bakitas 2015) with 69 participants reported adjusted endpoint data on unpaid caregiver quality of life with no evidence of benefit of HSPC over usual care. Dionne-Odom 2015a assessed unpaid caregiver quality of life using the unpaid caregiver Quality of Life (CQOL) Index (range, 0 to $140 ; 140$ indicates worse (QOL), and found a non-significant improvement in the mean unpaid caregiver quality of life score in the HSPC group that was two points better than that of the control group at three months with adjustment for patient death $(P$ $=0.39$ ). In decedents' unpaid caregivers, a terminal decline analysis indicated a mean difference of -4.9 points between HSPC group and control $(P=0.07)$.

Sensitivity analysis in two studies ( $N=105$ participants) that reported unadjusted endpoint values showed a pooled effect in favour of HSPC (MD $=6.11,95 \% \mathrm{Cl} 0.42$ to $11.81 ;\left.\right|^{2}=0 \%$; Analysis 10.1). A positive MD indicates better unpaid caregiver quality of life and a negative MD reflects lower unpaid caregiver quality of life. The two studies assessed unpaid caregiver quality of life using the unpaid caregiver Quality of Life (CQOL) Index (range, 0 to 140; 140 indicates worse CQOL).

In addition, Bajwah 2015 with 36 participants also presented unadjusted change values and assessed unpaid caregiver quality of life using the CQOL index. Bajwah 2015 found a non-significant 2.5 point mean improvement (range, 0 to 140; 140 indicates worse $\mathrm{CQOL}$ ) in unpaid caregiver quality of life from baseline at four weeks for the HSPC group while for controls, unpaid caregiver quality of life improved by 0.7 points. The effect size $(95 \% \mathrm{Cl})$ at four weeks was $-0.4(-1.1$ to 0.2$)$. At eight weeks, the mean (SD) score for the HSPC group was 58.3 (15.6), while that for the control group was 60.2 (23.9).

The remaining 39 studies did not report on unpaid caregiver quality of life.

We could not perform any further analysis due to the limited number of studies.

\section{Quality of the evidence}

Within the Grade approach, we downgraded the quality of the evidence for unpaid caregiver quality of life to low due to a high risk of bias (-1 level as a result of serious study limitations: high risk of bias for performance bias and imprecision: -1 level due to small number of participants).

\section{Resource use}

It was not possible to combine data for resource use or costs due to differences in measurement and reporting, such as type of analysis, tools used, assessment time points or time horizon and statistics reported. Consequently, we provided a narrative synthesis on the economic studies.

Thirty-one studies compared resource use or costs or both between the treatment groups in different ways. Three studies collected information on resource use and/or costs by chart review (Ahronheim 2000; Kane 1984; Bakitas 2009), while four studies collected resource use data from patients using either the Client Services Receipt Inventory (CSRI) or a modified form of it (Farquhar 2014; Farquhar 2016; Higginson 2009; Higginson 2014). Eight studies used medical/health records (Grudzen 2016; Ma 2019; Rogers 2017; Sidebottom 2015; Tattersall 2014; Temel 2010; O'Riordan 2019; Vanbutsele 2018). Four studies used a combination of methods (Bekelman 2018; Bakitas 2015; Janssens 2019; Rodin 2019). Bekelman 2018 collected data from medical records and supplemented these with patient or family selfreport, while Janssens 2019 collected data from medical records as well as contact with patients and their GPs. Rodin 2019 collected data from patients and their medical charts. Bakitas 2015 used patient self-report for hospital and intensive care unit days and emergency department visits, while decedents' data for the period between the last patient-reported assessment and

The effectiveness and cost-effectiveness of hospital-based specialist palliative care for adults with advanced illness and their caregivers 
death, and chemotherapy use in last 14 days were obtained from medical records. In Ozcelik 2014, a patient expenditure record form was created to capture resources and their costs. Brumley 2007 obtained resource use for each patient retrospectively from the non-profit HMO mainframe database, while Gade 2008 used standard data extract protocols to extract information from the managed care organisation's (MCO) database. Methods for collecting resource use information were unclear in nine RCTs (Brannstrom 2014; Cheung 2010; Carson 2016; El-Jawahri 2016; Groenvold 2017; McCaffrey 2013; Mendoza-Galindo 2018 (abstract only); Temel 2017; Woo 2019).

We considered resource use in the following areas: institutional care services use, outpatient clinic services use, community care services use, unpaid caregiver's care, and medications and other resources.

\section{Institutional care services use}

Thirty studies compared the effect of HSPC and usual care on institutional care use. Eight studies assessed emergency department (ED) visits (Bakitas 2009; Bakitas 2015; Brumley 2007; Janssens 2019; Ma 2019; Mendoza-Galindo 2018 (abstract only); Rogers 2017; Temel 2010), and their results were inconsistent (see Table 6 under Additional tables). Two of the studies reported fewer ED visits in favour of the HSPC group (Brumley 2007; Ma 2019). Brumley 2007 found that $20 \%$ of intervention group patients had ED visits compared to $33 \%$ of control group patients ( $P=$ $0.01)$. Linear regression adjusting for survival, age and severity of illness showed the intervention reduced ED visits by 0.35 visits $(P=0.02)$. Ma 2019 reported fewer post-discharge ED visits in the HSPC group compared to the control group (1.3\% versus $12.5 \%$; $P=0.0067)$. Four of the remaining six studies described little to no difference between HSPC and control group (Bakitas 2009; Bakitas 2015; Janssens 2019; Mendoza-Galindo 2018 (abstract only)). In particular, Janssens 2019 initially reported that patients in the HSPC group were twice as likely to be admitted to the emergency ward for respiratory failure compared to the control group (incidence rate ratio $(95 \% \mathrm{Cl}): 2.05$ (1.11 to 3.94); $\mathrm{P}=0.014$ ). However, after correction for multiple testing, there was no longer any evidence of a difference. Two studies reported fewer ED visits in the HSPC group compared to the control group but did not present their P values (Rogers 2017; Temel 2010).

Nine studies assessed ICU use (see Table 7 under Additional tables). Six studies of these studies assessed ICU days (Bakitas 2009; Bakitas 2015; Carson 2016; Cheung 2010; Kane 1984; Ma 2019), and three assessed number of ICU admissions (Gade 2008; Grudzen 2016; Janssens 2019). Five of the six studies assessing ICU days found no difference between HSPC and control group (Bakitas 2009; Bakitas 2015; Carson 2016; Cheung 2010; Ma 2019). Kane 1984 reported slightly shorter mean number of ICU days per patient in the HSPC group compared to the control group ( 0.2 versus 0.3 ) but did not report P values. Gade 2008, Grudzen 2016 and Janssens 2019 reported contrasting results regarding ICU admission. Janssens 2019 compared number of ICU admissions for respiratory failure between HSPC and control groups in the year before study inclusion ( 7 versus 7 ; incidence rate ratio $0.88,95 \%$ $\mathrm{Cl}: 0.26$ to $2.96 ; \mathrm{P}=0.82$ ) and also during the study (5 versus 1 ; incidence rate ratio $4.42,95 \% \mathrm{Cl}: 0.49$ to $20.92 ; \mathrm{P}=0.16$ ), but did not find any evidence of a difference. On the other hand, Gade 2008 found evidence in favour of HSPC in reduction in ICU admissions. The median number of ICU admissions in the HSPC group was
12 while in the control group it was $21(P=0.04)$. Grudzen 2016 reported that no difference between the treatment arms in the number of ICU admissions during the index-admission $(P>0.99)$ and also at 180 days $(P>0.99)$.

Carson 2016 and Ma 2019 provided details on resource use in the ICU and their findings were varied (see Table 8 under Additional tables). Carson 2016 found no difference in use of the following resources between HSPC and control group in the ICU: dialysis (median (IQR): $13(10)$ versus $15(12) ; P=0.64)$, mechanical ventilation (median (IQR): 40 (31) versus $33(26) ; P=0.41$ ), nutrition (median (IQR): 18 (14) versus $21(17) ; \mathrm{P}=0.60)$ and vasopressors (median (IQR): 18 (14) versus 19 (15); $\mathrm{P}=0.86$ ). Ma 2019 reported lower use of tracheostomy ( $1 \%$ versus $7.8 \% ; \mathrm{P}=0.035$ ) and fewer median (IQR) number of days on mechanical ventilation (4 (3 to 7) versus 6 ( 3 to 13 ); $P=0.042$ ) in the ICU in the HSPC group compared to the control group.

Kane 1984 further reported reduced mean number of nursing home days per patient in favour of the HSPC group (HSPC 1 and control 11.4, $\mathrm{P}<0.05)$.

Twelve studies provided mixed results on hospital admissions (Ahronheim 2000; Bekelman 2018; Brannstrom 2014; Brumley 2007; Farquhar 2014; Farquhar 2016; Janssens 2019; Ma 2019; MendozaGalindo 2018 (abstract only); Rogers 2017; Sidebottom 2015; Temel 2010) (see Table 9 under Additional tables). Four studies found no difference in the number of hospital admissions between HSPC and the control group (Ahronheim 2000; Bekelman 2018; Ma 2019; Sidebottom 2015). Ma 2019 initially described fewer hospital readmissions in the intervention group compared to the control group (17.3\% versus 33.3\%; $\mathrm{P}=0.024)$. Hospital admission for respiratory failure during the study was almost twice as often in the HSPC group compared to the control group (Incidence rate ratio $1.87,95 \% \mathrm{Cl}: 1.04$ to $3.48, \mathrm{P}=0.026$ ). However, after the Benjamini and Hochberg correction for multiple testing, there was no longer any evidence of a difference in the number of hospital admissions during the study period. Sidebottom 2015 reported no association between study group assignment and 30-day inpatient readmission (adjusting for age, gender, and marital status) ( $P$ $=0.50$ ). Janssens 2019 described a non-significant increase in hospital admissions for respiratory failure in the HSPC group compared to the control group in the year before the study ( 24 versus $18 ; P=0.60$ ) and also during the study period (38 versus 18 ; $P=0.026)$. Two studies found fewer hospital admissions in favour of the HSPC group (Brannstrom 2014; Brumley 2007). Brannstrom 2014 found fewer mean (SD) number of hospitalisations in the HSPC group compared to the control group (0.42 (0.60) versus 1.47 (1.81); $P=0.009)$. Brumley 2007 found fewer hospital admissions in the intervention group compared to the control group (36\% versus $59 \%, \mathrm{P}<0.001)$. Three studies further reported fewer hospital admissions in the HSPC group but they did not present their P values (Farquhar 2014; Mendoza-Galindo 2018 (abstract only); Temel 2010). Farquhar 2014 reported 7\% inpatient admissions in the HSPC group compared to $12 \%$ in the control group, while Mendoza-Galindo 2018 (abstract only) found that $48 \%$ of patients in HSPC group had hospital admissions compared to $51 \%$ in the control group. Temel 2010 described fewer hospital admissions in the HSPC group compared to the control group from enrollment to death $(73.5 \%$ versus $76.8 \%)$ and also within 30 days of death (36.7\% versus $53.6 \%$ ). By contrast, Farquhar 2016 reported more inpatient admissions in the HSPC group compared to the control

The effectiveness and cost-effectiveness of hospital-based specialist palliative care for adults with advanced illness and their caregivers 
group ( $15 \%$ versus $11 \%$ ), but did not report the P value. In Rogers 2017, there was more hospitalisation for heart failure during the study in the HSPC group (30.7\% versus $29.3 \%$; P value was not reported), more hospitalisation for non-heart failure cardiovascular conditions (16\% versus $13 \%$; P value was not reported) and fewer hospitalisations for non-cardiovascular conditions ( $10.7 \%$ versus $24 \%$; P value was not reported).

Length of hospital admission was assessed in 17 studies (Ahronheim 2000; Bakitas 2009; Bakitas 2015; Brannstrom 2014; Brumley 2007; Carson 2016; Cheung 2010; El-Jawahri 2016; Gade 2008; Grudzen 2016; Higginson 2009; Higginson 2014; Kane 1984; Ma 2019; Mendoza-Galindo 2018 (abstract only); Ozcelik 2014; Temel 2010) (see Table 10 under Additional tables). Nine studies found no difference in length of admission between HSPC and the control group (Ahronheim 2000; Bakitas 2009; Carson 2016; Cheung 2010; Gade 2008; Grudzen 2016; Ma 2019; Mendoza-Galindo 2018 (abstract only); Ozcelik 2014). Bakitas 2015 described fewer hospitalisation days in the HSPC group $(0.69$ (95\% $\mathrm{Cl} 0.4$ to 1.18$)$ versus $1.39(95 \% \mathrm{Cl} 0.97$ to 1.97$) ; \mathrm{P}=0.03)$ but not in decedents in the HSPC group $(0.95(95 \% \mathrm{Cl} 0.61$ to 1.46$)$ versus $1.3(95 \% \mathrm{Cl}$ 0.91 to 1.86$) ; P=0.26$ ). Brannstrom 2014 reported that the mean (SD) number of days spent in hospital was lower in the HSPC group compared to the control group (2.9 (8.3) versus 8.5 (12.4), $\mathrm{P}=0.011$ ). The number of days spent in the Department of Medicine-Geriatrics (100, range 1 - 45 versus 242, range 2 - 46) and Surgery (0 versus 56) were also lower in the HSPC group, but not in other departments (3, range 1 - 2 versus 7 range 1 - 6). Brumley 2007 reported fewer hospital days in the HSPC group. Linear regression adjusted for survival, age and severity of illness showed that the intervention reduced hospital days by $4.36(P<0.001)$. Kane 1984 reported on total inpatient days as well as general medicine, hospice, intensive care unit and intermediate care inpatient days. The mean number of total inpatient days per patient did not differ between HSPC and control group (51 versus 47.5). However, Kane 1984 found fewer mean days of general medical inpatient care (HSPC 13.2 and control 20.7, $\mathrm{P}<0.05)$ and intermediate inpatient care per patient (HSPC 8.3 and control $26.5, P<0.05)$. Four studies described fewer hospital days in the HSPC group compared to the control group but did not report their P values (El-Jawahri 2016; Higginson 2009; Higginson 2014; Temel 2010). El-Jawahri 2016 reported the median duration of hospitalisation in the HSPC group to be 20 (range: 12 to 102 days) and that in the control group to be 21 (13 to 40). Institutional days (hospital admission) was reported to be increased in the control group by Higginson 2009. Higginson 2014 reported mean hospital days of 4.5 (6.8) in the HSPC group and 4.6 (7.6) in the control group, while Temel 2010 reported the number of inpatient days from enrollment to death to be 5 (range: 0 to 50 ) in the HSPC group and 7 (range: 0 to 45 ) in the control group.

Palliative care visits during hospitalisation was further compared between HSPC and usual care in two studies (El-Jawahri 2016; Tattersall 2014) (see Table 11 under Additional tables). El-Jawahri 2016 reported that HSPC patients had at least two palliative care visits during the first two weeks of their hospitalisation (median 4; range, 2-7), while two control patients received a palliative care consultation ( $P$ values were not reported). Tattersall 2014 highlighted that $86 \%$ of patients in the HSPC group had palliative care contact during hospitalisation compared to $78 \%$ of control group patients $(P=0.37)$.
With the exception of days spent in nursing homes reported in one study to be in favour of HSPC, the overall evidence on institutional care use was inconsistent.

\section{Outpatient clinic services use}

Seven studies provided inconsistent evidence on the effect of HSPC compared to usual care on outpatient clinic visits (Brannstrom 2014; Higginson 2009; Groenvold 2017; Rogers 2017; Temel 2010; Temel 2017; Vanbutsele 2018) (see Table 12 under Additional tables). One of these studies reported fewer outpatient clinic visits in favour of HSPC (Brannstrom 2014). Brannstrom 2014 found fewer physician visits, nurse visits, phone calls and prescriptions in the HSPC group compared to the control group. Another study by Vanbutsele 2018 reported a difference in favour of the control group for number of consultations with a psychologist at 18 weeks ( $P=$ 0.02 ), but not at 24 weeks. Three studies described more contacts with palliative care teams in the HSPC group compared to the control group, but did not present $P$ values (Groenvold 2017; Temel 2010; Temel 2017). Temel 2017 highlighted more palliative care visits in the HSPC group compared to the control group (mean (range): 6.54 (0 to 14) versus 0.89 (0 to 7)). Temel 2010 reported that all the patients assigned to HSPC, except for one patient who died shortly after enrollment, had at least one visit with the palliative care service by the 12th week. The average number of visits in the palliative care group was 4 (range, 0 to 8 ). Ten patients who received usual care (14\%) had a palliative care consultation in the first 12 weeks of the study, with seven patients having one visit and three having two visits. In Groenvold 2017, 138 patients had at least one face-to-face contact with the HSPC team compared to 13 patients in the control group. Groenvold 2017 further reported no difference in mean (SD) number of specialists visits between HSPC and control group (4.9 (8.1) versus 7.0 (9.1); $\mathrm{P}=0.25)$.

Higginson 2009 described fewer hospital specialist visits in the HSPC group (8 patients (35\%)) compared to control group (16 patients $(76 \%)$ ), but $P$ values were not reported. Rogers 2017 reported more mean (SD) total number of clinic encounters in the HSPC group compared to control group (21.9 (1.99) versus 20.8 (1.92)), but did not present $P$ values. There were more visits to the rehabilitation clinic in the HSPC group compared to the control group (mean (SD): 1.4 (0.68) versus $0.9(0.48)$ ) and fewer cardiology visits in the HSPC group compared to control group (mean (SD): 2.3 (0.55) versus 3.2 (1.0)). Woo 2019 reported that similar proportions of patients in the HSPC group and control group consulted with a psychiatrist ( $12 \%$ versus $12 \%$ ), but did not present P values. Tattersall 2014 reported more contacts with palliative care physicians in the HSPC group compared to the control group by the end of the study (51 patients ( $85 \%$ ) versus 8 patients $(13.3 \%)$ ) and also in the last month of life (16 patients (26.7\%) versus 6 patients $(10 \%))$. However, the P values were not reported.

\section{Community care services use}

Fourteen studies compared community care services use between the HSPC group and control group and their findings were inconsistent (Bakitas 2009; Bakitas 2015; Brannstrom 2014; Brumley 2007; Farquhar 2014; Farquhar 2016; Gade 2008; Grudzen 2016; Higginson 2009; Kane 1984; McCaffrey 2013; Rogers 2017; Sidebottom 2015; Temel 2010) (see Table 13 under Additional tables). The studies reported on a range of community services. Two UK studies by the same author found different results for mean number (SD) of GP contacts for cancer (Farquhar 2014), and noncancer populations (Farquhar 2016). Farquhar 2014 reported the

The effectiveness and cost-effectiveness of hospital-based specialist palliative care for adults with advanced illness and their caregivers 41 (Review) 
mean number of GP contacts to be slightly higher in the control group (1.3 (0.5)) compared to the HSPC group (1.2 (0.6)) in cancer populations, while Farquhar 2016 found the mean number of GP contacts to be slightly higher in the HSPC group (1.8 (1.2)) compared to the control group (1.6 (0.7)) in non-cancer populations. However, these studies did not provide their $P$ values. Higginson 2009 described differences in contact with GPs, district/practice nurse, multiple sclerosis (MS) nurse and social services, but the $P$ values of the results were not reported.

A study set in the USA by Gade 2008 found longer median length of stay in hospice favouring the HSPC group (24 days) compared to the control group (12 days) $(P=0.04)$, while two USA studies found nobetween group differences (Brumley 2007; Temel 2010). Grudzen 2016 and Bakitas 2015 reported no between-group differences in hospice use at 180 days. Sidebottom 2015 found no evidence of an association between group assignment and hospice use within six months adjusting for age, gender and marital status in USA. Ma 2019 highlighted more transfers to hospice care in the HSPC group compared to usual care $(18.6 \%$ versus $4.9 \% ; P=0.0026)$.

Brannstrom 2014 further reported more nurse visits in the HSPC group compared to the control group (1075 versus $230, P=0.000$ ) in Sweden. By contrast, this study found that phone calls and prescriptions by doctors were more common in the control group (108 versus 231) while physician visits were similar (194 versus 201).

Kane 1984 and McCaffrey 2013 both reported more days spent at home in the HSPC group compared to the control group, but did not present $P$ values. Kane 1984 reported a mean of 44.8 days at home per patient while that in the control group was 37.9 days at home per patient. In McCaffrey 2013, the HSPC group spent a mean of 13.1 days $(95 \% \mathrm{Cl} 8.5$ to 17.7$)$ at home compared to 12.1 days $(95 \% \mathrm{Cl} 5.9$ to 18.4 ) in the control group.

Rogers 2017 reported on the frequency of interaction between patients and primary care providers and found fewer interactions in the HSPC group (mean (SD): 4.4 (0.93)) compared to the control group (mean (SD): $5.2(0.82)$ ). The authors did not present the $P$ values.

\section{Unpaid caregiver's care}

Higginson 2009 and Farquhar 2014 reported on the effect of HSPC and usual care on the support provided by informal unpaid caregivers (see Table 14 under Additional tables). Increased care by informal unpaid caregivers was reported by Higginson 2009 with more hours of informal care provided in the control group. The $P$ value was not reported. Farquhar 2014 reported more use of informal care in the control group compared to the HSPC group. However, the $P$ value was not also stated.

\section{Medication and other resources}

Seventeen studies either reported on the use of medications or other resources, or both (Ahronheim 2000; Bakitas 2009; Bakitas 2015; Brumley 2007; Carson 2016; Farquhar 2014; Farquhar 2016; Groenvold 2017; Higginson 2009; Janssens 2019; Kane 1984; Ma 2019; Markgren 2016 (linked to Brannstrom 2014); O'Riordan 2019; Rodin 2019; Rogers 2017; Temel 2010) (see Table 15 under Additional tables). Markgren 2016 (part of Brannstrom 2014) assessed the number of patients receiving the target doses of medications based on current guidelines for heart failure among HSPC and control group patients. This study found that the number of patients treated with mineralocorticoid receptor antagonists (MRAs) differed between groups, and increased from $10(28 \%)$ of 36 patients to $15(48 \%)$ of 31 patients in the HSPC arm compared with $13(35 \%)$ of 36 patients to $13(39 \%)$ of 33 patients in the control group. The change in number of patients receiving full target doses of the angiotensin-converting enzymes inhibitors (ACEIs)/ angiotensin receptor blockers (ARBs), beta-blockers and MRAs was higher in the HSPC arm than in the control arm $(P=0.009)$. Conversely, O'Riordan 2019 found no evidence of a difference in use of guideline-driven heart failure treatments such as beta-blockers and ACEIs/ARBs. Similarly, Janssens 2019 did not find any evidence of a difference between HSPC and the control group in antibiotics use $(P=0.819)$. Temel 2010 reported a difference in aggressive endof-life care among decedents with $33 \%$ (16 of 49 patients) of those in the HSPC group and 54\% (30 of 56 patients) in the control group receiving aggressive end-of-life care $(P=0.05)$. Aggressive end-oflife care was defined as chemotherapy within 14 days before death, no hospice care, or admission to hospice three days or less before death.

Kane 1984 further reported more use of chemotherapy in the HSPC group, with a mean of 1.3 patients receiving chemotherapy in the HSPC group compared to 0.49 in the control group $(P=0.03)$. More patients in the HSPC group (mean: 0.09) also received major surgical procedures compared to the control group (mean: 0.01) $(P<0.05)$. Bakitas 2015 reported no between-group difference in chemotherapy use in the last two weeks of life.

Ahronheim 2000 reported lower use of intravenous therapy for the entire admission among $61(66 \%)$ of 92 admissions in the HSPC group compared to 79 (81\%) of 98 admissions in the control group in patients with advanced dementia. On the other hand, the study reported no evidence of a difference in use of other resources such as feeding tubes, mechanical ventilation, tracheostomy, systemic antibiotics, days with restraints, mechanical restraints and cardiopulmonary resuscitation. In Ma 2019, the HSPC group had fewer ventilator days (median 4 versus $6 ; \mathrm{P}=0.042$ ) and tracheostomies performed ( $1 \%$ versus $7.8 \% ; P=0.035$ ), while there was no between-group difference in mechanical ventilation, use of vasopressors, haemodialysis, cardiopulmonary resuscitation. Carson 2016 found no between-group difference in ventilator days between the HSPC and control group.

Higginson 2009 reported differences in resource use such as primary/secondary care, use of specialist wards, occupation therapist/physiotherapist, palliative care nurse, dietician, chiropodist, day centre and respite care. However, the P values of the differences were not reported. Rogers 2017 reported more hospital encounters with the HSPC team (mean (SD): 2.5 (0.45) versus $2.4(0.35)$ ) and telephone contacts (mean (SD): 12.6 (1.2) versus $10.6(0.88)$ ) in the HSPC group compared to the control group, but did not present P values. Groenvold 2017 also highlighted the face that 116 patients in the HSPC group had at least one telephone contact with the HSPC team compared to nine patients in the control group. However, they did not report their $\mathrm{P}$ value.

Bakitas 2009 and Brumley 2007 reported no evidence of a difference in referral to palliative care/hospice care. Bakitas 2009 reported that 34 (235) of 145 patients were referred to palliative care in the HSPC group compared to $39(29 \%)$ of 134 patients in the control group $(P=0.34)$, while $6(3.7 \%)$ of 161 patients in the HSPC group and $4(2.5 \%)$ of 161 patients in the control group were referred to

The effectiveness and cost-effectiveness of hospital-based specialist palliative care for adults with advanced illness and their caregivers 42 (Review)

Copyright $\odot 2020$ The Cochrane Collaboration. Published by John Wiley \& Sons, Ltd. 
hospice care $(P=0.75)$. Brumley 2007 presented results on hospice referral for only one of the sites in their study and reported that $25 \%$ of patients in the HSPC group were referred to hospice care compared to $36 \%$ of patients in the control group $(P=0.15)$. Rodin 2019 described more referrals to palliative care (22 patients $(100 \%)$ versus 1 patient $(5 \%)$ ), but not psychiatry (1 patient $(4.5 \%)$ versus 1 patient (5\%)) in the HSPC group compared to the control group. The $P$ values for the differences were not reported. There was no difference in referral to social work between HSPC and control group (22 patients (100\%) versus 20 patients $(100 \%)$ ).

Other resource use with no between-group differences include hospital discharge disposition (Carson 2016). Farquhar 2014 and Farquhar 2016 reported differences between HSPC and the control group in use of services provided by nurses, social care, other health professionals and other hospital services but the $P$ values for differences were not reported.

\section{Certainty of the evidence}

Within the Grade approach, we downgraded the certainty of evidence for resource use to very low due to a high risk of bias across studies ( -2 levels as a result of very serious study limitations: high risk of bias for performance, detection, attrition, reporting, size of study and other bias and inconsistency: -1 level due to variability in results) (Summary of findings 1 ).

\section{Costs and cost-effectiveness of HSPC}

Thirteen economic studies with 2103 participants reported on cost. The utilisations included were: ED or A\&E visits; inpatient and outpatient hospital care; home and community care; care in nursing homes (or skilled nursing homes); inpatient stay; day care in hospice; hospice care at home; informal care; drugs and equipment. Four studies reported the results of costeffectiveness analysis using relevant outcome measures (palliative outcome, unpaid caregiver's burden, quality-adjusted-life-years) and hospital costs or total costs (Farquhar 2014; Farquhar 2016; Higginson 2009; McCaffrey 2013). Results of cost-effectiveness analyses were reported by ICERs and/or costs per QALY (point estimates or cost-effectiveness planes).

Two studies found evidence of lowered cost with HSPC (Brumley 2007; Gade 2008). When compared to usual care, MendozaGalindo 2018 (abstract only) reported a reduction in the cost of hospitalisation days in the HSPC group. However, no difference was found between groups in the cost of emergency room visits. In Brannstrom 2014, this was unclear as no $P$ value was presented for the difference in cost between HSPC and usual care. We identified four full economic studies (Farquhar 2014; Farquhar 2016; Higginson 2009; McCaffrey 2013). The evidence on the costeffectiveness of HSPC compared to usual care was inconsistent.

With the exception of Mendoza-Galindo 2018 (abstract only), all other studies had applied more robust methodology since the first relevant study we identified which compared the costs of HSPC and conventional care among cancer patients (Kane 1984). Kane 1984 provided services across multiple settings and was carried out in the USA. The HSPC group had lower total costs when compared to conventional care. However, the authors reported that the difference was "not significant". The estimated mean expenditure per patient was reported to be US dollars (USD) 15,263 (converts to Great British Pounds (GBP) 29,058 in 2018) in the HSPC group and USD 15,493 (converts to GBP 29,496 in 2018) in the conventional care group. Resource use was measured in hospital stays, hospice stays, surgical procedures, chemotherapy and radiotherapy, and costs were calculated using different assumptions. However, difference in survival (days since enrollment in the study) as well as other factors (e.g. age, severity of diseases) which might be associated with costs, were not adjusted for.

Brumley 2007 compared resource use and costs between the HSPC and usual care group versus usual care only among terminally ill patients with mixed cancer and non-cancer diagnoses in the USA involved service provision across multiple settings. A wider range of resource use was reported from the health insurance database: the number of ED visits, physician office visits, hospital days, hospice days, skilled nursing facility days, home health and palliative visits and palliative physician home visits. Service utilisation was lower in the HSPC group than usual care group even after controlling for age, survival and severity measured using the Palliative Performance Scale. Stay in hospital decreased by 4.36 days and ED visits by 0.35 . Due to the difference in the survival (days on service), mean costs per patient were adjusted using regression analysis, controlling for survival, age, severity of illness and primary disease. Mean costs per patient in the intervention group were much lower (Australian dollars (AUD) 12,670, SD AUD 12,523; converts to GBP 8383, SD GBP 8285 in 2018), compared with the usual care group (AUD 20,222, SD AUD 30,026; converts to GBP 13,379, SD GBP 19,866 in 2018). Average daily costs per patient were also lower in the intervention group (AUD 95.30, converts to GBP 63.05 in 2018) compared to the usual care group (AUD 212.80, converts to GBP 140.76 in 2018) ( $P=$ 0.02).

Gade 2008 used the health insurance database to extract resource use and unit cost of services of hospitalised patients with lifelimiting illnesses (mixed cancer and non-cancer diagnoses), who were randomly assigned to the HSPC intervention or usual care. Included utilisations were ED visits, clinic and hospital outpatient visits, home health visits, hospital admission, skilled nursing facility admissions and prescriptions. The cost of the palliative care team was calculated as the intervention cost. HSPC patients stayed longer in hospice after the index hospitalisation (24 days) than usual care patients (12 days) however this was a non-significant difference $(P=0.08)$, and had shorter ICU stays on readmission (12 times versus 21 times, $P=0.04$ ) and lower total healthcare costs (USD 14,486, converts to GBP 15,013 in 2018 versus USD 21,252, converts to GBP 22,025 in 2018, P = 0.001). Gade 2008 involved an inpatient consult model and was a USA study.

Temel 2010 examined the effectiveness of early palliative care integrated with standard oncologic care among patients with newly diagnosed metastatic non-small-cell lung cancer, where standard oncologic care alone was a comparator. It was an outpatient model of HSPC that took place in the USA. Data on health utilisations and end-of-life care were collected from the medical records: anticancer therapy, medication prescriptions, referral to hospice, hospital admissions and ED visits. Patients in standard care received more aggressive end-of-life care (54\% [30 of 56 patients] versus 33\% [16 of 49 patients], $P=0.05$ ), and had non-significant longer stays in hospice care (median 11 days versus 4 days, $P=0.09$ ) than the intervention group. Patients in the HSPC group used more palliative care and less aggressive care while there was greater improvement in quality of life and survival in this group than control. However, this was not conclusive because the sample size of the study did not allow the statistical power to test the differences in service

The effectiveness and cost-effectiveness of hospital-based specialist palliative care for adults with advanced illness and their caregivers 
utilisation. Detailed analyses of costs and cost-effectiveness were conducted and reported later although lacking in statistical power to detect the difference in Greer 2016 (linked to Temel 2010)). Comparisons of costs per day alive and costs for the last 30 days were made between HSPC and the usual care group and the costeffectiveness per life year saved was calculated. Total costs per day were on average lower in the HSPC group. However, this was a non-significant difference (mean difference USD 117, SE USD 74; converts to GBP 103, SE GBP 65 in 2018, P = 0.13). Total costs for the last 30 days were also reduced non-significantly (mean difference USD 2527, SE USD 3311; converts to GBP 2230, SE GBP 2922 in 2018, $P=0.44$ ). The cost-effectiveness ratio was USD 41,938 per life year saved. There was a non-signifant increase in use of hospice care (mean difference USD -1053, SE USD 538; converts to GBP -929, SE GBP 475 in 2018, $P=0.07$ ) and less use of chemotherapy (mean difference USD 757, SE USD 365; converts to GBP 668, SE GBP 322 in $2018, P=0.03$ ) for the last 30 days.

Higginson 2014 examined the effectiveness of the early introduction of palliative care among patients with chronic breathlessness in the UK. The intervention (HSPC) was provided across multiple settings and patients had mixed cancer and noncancer diagnoses. Patients were randomly assigned either to the HSPC group or to usual care. Resource use was collected using the Client Services Receipt Inventory (CSRI) on health, voluntary, and social care received over the past three months at baseline and since the last interview at six weeks follow-up. Limited results on resource use and costs were reported: hospital inpatient stays (mean 4.5, SD 6.8 in BSS; mean 4.6, SD 7.6 in control) and costs of formal care use (mean GBP 1422, 95\% Cl: 897 to 2101; converts to GBP 1611, 95\% Cl: 1016 to 2380 in 2018 in Breathlessness Support Service (BSS) group; mean GBP 1408, 95\% Cl: 899 to 2023; converts to GBP 1595, 95\% Cl: 1018 to 2292 in 2018 in control group). There was no between-group difference between the two groups.

Brannstrom 2014 compared service use between patients randomised to the integrated palliative advanced home care and heart failure care (PREFER) intervention and usual care among patients with severe chronic heart failure in Sweden. The PREFER intervention involved an outreach model of HSPC. Resource use collected included hospital admissions, inpatient days, physician and nurse visits, phone calls and drug prescriptions. The HSPC group had fewer hospitalisations than the control group $(0.42 \pm 0.60$ versus $1.47 \pm 1.81, \mathrm{P}=0.009$ ) and the length of stay in hospital was also shorter in patients receiving the intervention (mean 2.9, SD 8.3 versus mean $8.5, \mathrm{SD} 12.4, \mathrm{P}=0.011$ ). The total days or total contacts per study arm were compared between HSPC and the control group and additional cost analysis was reported in Sahlen 2016 (linked to Brannstrom 2014). QALY gain was 0.25 years between baseline and the end of intervention across the HSPC group $(P=0.025)$. Over six months, total cost was Swedish Krona (SEK) 1.4 million (EUR 140,000 , converts to GBP 126,132 in 2018) in the HSPC group $(n=36)$ and SEK 2.0 million (EUR 205,000, converts to GBP 180,188 in 2018) in the control group $(n=26)$, and the difference SEK 600,000 (EUR 61,000 , converts to GBP 54,056 in 2018) was the saving achieved by providing the intervention in addition to usual heart failure care.

Ozcelik 2014 compared duration of hospitalisation and direct cost between HSPC and usual care in Turkey. It was an inpatient consult model of HSPC. A patient cost record form was used to document cost. This form was created by listing direct health expenditure, which consisted of all expenses incurred while in hospital. Direct expenses assessed included medicines used from the start of the patient's stay in hospital, medical equipment, laboratory and diagnosis tests, consultations, professional care, and hospital stay expenses (including those of companions). On the patient's discharge from hospital, costs were recorded on the form by obtaining the expenses list from the clinic secretary. Mean (SD) direct cost in the HSPC group was USD 68,869 (SD 48.522) (converts to GBP 60,154 (GBP 42,382) in 2018) and USD 81,076 $(72,700)$ (converts to GBP 70,816 (GBP 63,500) in 2018) in the control group $(P=0.76)$. There was no evidence of a difference in the duration of hospitalisation ( $P=0.07)$, with a mean (SD) length of stay in hospital of 9.4 (6.27) days in the HSPC group and 13.9 (11.5) days in the control group.

The first study using robust cost-effectiveness analysis (CEA) method among papers we identified was by Higginson 2009. This was the CEA alongside a feasibility trial of a new HSPC service among patients with multiple sclerosis (MS) in the UK, randomised into either fast-track of the new intervention or control of usual care. Higginson 2009 involved service provision across multiple settings. Costs were measured in health, social and voluntary services, and informal care provided by family or friends was also included for the analysis from a broad perspective. As the usual unit costs were applied for the formal services, 'shadow price' was used for the informal care. CEA used the differences in costs and outcomes (palliative care outcome scale (POS-8) and Zarit Burden Inventory (ZBI)) between baseline and follow-up at 12 weeks. Total costs for 12 weeks measured at follow-up were lower in the fasttrack intervention group than usual care group by GBP 1789 (95\% Cl: GBP -5224 to GBP 1902); converts to GBP 2424 (GBP -7077 to GBP 2577) in 2018. When inpatient care and informal care were excluded, mean service costs for 12 weeks were GBP 1195 lower for the intervention group (95\% Cl GBP -2916 to GBP 178); converts to GBP 1619 (GBP -3950 to GBP 241) in 2018. Cost-effectiveness planes showed that $33.8 \%$ of replications for POS-8 indicated that patients in the intervention group had lower cost and better outcomes than in the control group, and $54.9 \%$ had lower cost but worse outcomes. For ZBI, $47.3 \%$ of replications showed lower costs and better outcomes while $48 \%$ indicated higher costs and better outcomes.

McCaffrey 2013 estimated incremental net monetary benefit (INMB) and cost-effectiveness acceptability curves (CEACs) for one extra day at home in an RCT among patients with mixed cancer and noncancer diagnoses with complex or unstable symptom management and high care needs in Australia. McCaffrey 2013 provided services across multiple settings. Data on resource use were prospectively collected and costed including: days at home, specialist palliative care service use, acute hospital and palliative care unit inpatient days, and outpatient visits. Intervention costs were calculated based on staff administration, travel and direct patient contact time, overheads and consumables. The analysis was conducted from a healthcare provider perspective and bootstrapping was used to calculate the confidence intervals around INMB and CEACs. Total costs were AUD 6452 (95\% CI AUD 4469 to AUD 8586) (converts to GBP 5750 (95\% Cl GBP 3983 to GBP 7652) in 2018) in the HSPC group and AUD 5425 (95\% CI AUD 2404 to AUD 8531) (converts to GBP 4835 (GBP 2142 to GBP 7602) in 2018) in the control group. The increment costs between the two groups was AUD 1027 (95\% CI AUD -2612 to AUD 4738) (converts to GBP 915.22 (95\% Cl GBP -2327.71 to 4222.32). When the INMB of one more day at home was compared with varying threshold values, HSPC was preferred

The effectiveness and cost-effectiveness of hospital-based specialist palliative care for adults with advanced illness and their caregivers 
to usual care beyond AUD 1068. Sensitivity analyses with different inclusion ranges of costs (using hospital inpatient costs only and excluding high cost outliers) indicated that HSPC was preferred above AUD 2547 (converts to GBP 2270 in 2018) and AUD 846 (converts to GBP 754 in 2018). It was concluded that HSPC had a potential to be cost-effective, especially in trials with longer followup. The meaning of the threshold value for one extra day at home remains for future research.

Farquhar 2014 and Farquhar 2016 reported the cost-effectiveness of the Breathlessness Intervention Service (BIS), a multidisciplinary complex intervention underpinned by a palliative care approach for patients with advanced cancer and advanced non-malignant disease separately. The BIS was a model of HSPC where service provision traversed multiple settings in the UK. In Farquhar 2014, data from patients with advanced cancer were analysed from a societal perspective by including costs of informal care. Total health and social costs, including informal care for eight weeks prior to the baseline assessment, in the HSPC group were GBP 6137 (SD GBP 6099) which converts to GBP 6952 (GBP 6909) in 2018 and GBP 5461 (SD GBP 6099) which converts to GBP 6186 (GBP 6909) in 2018 for usual care. Costs between baseline and follow-up at two weeks were GBP 794 (SD GBP 866) which converts to GBP 899 (SD GBP 981) in 2018 for HSPC and GBP 1121 (SD GBP 1635) which converts to GBP 1270 (SD GBP 1852) in 2018 for usual care. Intervention costs for HSPC were GBP 119 (SD GBP 62) which converts to GBP 135 (SD GBP 70) in 2018. Total costs were GBP 354 lower for HSPC (95\% Cl: GBP -1020 to GBP 246) which converts to GBP 401 (95\% Cl: GBP -1155 to GBP 279) in 2018 and incremental QALY-gain was 0.0002 years ( $95 \% \mathrm{Cl},-0.001$ to 0.002$)$, after controlling for baseline. The chance of HSPC being lower in total costs and providing better outcomes in terms of reduced distress due to breathlessness was $80.9 \%$ according to cost-effectiveness planes and $16.4 \%$ for higher costs and better outcomes. It was $50.9 \%$ for the chance of HSPC being lower in total costs and greater in QALY, and $11 \%$ for higher costs and a greater QALY gain.

An NHS perspective was taken in the analysis of data from the UK study of patients with advanced non-malignant disease in Farquhar 2016. Total health and social costs for eight weeks prior to the baseline assessment in the HSPC group was GBP 1952 (SD GBP 3290) which converts to GBP 2211 (SD GBP 3727) in 2018 and GBP 3630 (SD GBP 5588) which converts to GBP 4112 (SD GBP 6330 ) in 2018 for usual care. Costs between baseline and followup at four weeks were GBP 1371 (SD GBP 2948) which converts to GBP 1553 (SD GBP 3339) in 2018 for HSPC and GBP 659 (SD GBP 1253) which converts to GBP 746 (SD GBP 1419) in 2018 for usual care. Intervention costs for HSPC were GBP 156 (SD GBP 80) which converts to GBP 177 (SD GBP 91) in 2018. With adjusting for baseline, total costs were GBP 799 higher for HSPC $(95 \% \mathrm{Cl}$ : GBP -237 to GBP 1904) which converts to GBP 905 (95\% Cl: GBP -268 to GBP 2157) in 2018 and the HSPC group gained 0.003 extra QALYs (95\% Cl: -0.001 to 0.007 ). A cost per QALY for HSPC was GBP 266,333 (converts to GBP 301,692 in 2018). The chance of HSPC being lower in total costs and greater in QALYs was 7\% according to cost-effectiveness planes. There was an $86.5 \%$ likelihood of HSPC being higher in total costs and greater in QALY gain. The HSPC intervention appeared to be cost-effective among patients with cancer but not among those with non-malignant disease.

Mendoza-Galindo 2018 (abstract only) compared resource use and costs between the early palliative care (EPC) group and usual care in patients with cancer diagnoses in Mexico. The study involved an outpatient model of HSPC. Resource use assessed included number/days of hospitalisation and emergency room (ER) visits as well as their cost. The number of ER visits in the EPC group was 39 while that in the control group was $50(P=0.074)$. There was also no difference in the number of hospitalisations ( $48 \%$ versus $51 \%$ ) and days of hospitalisation (78 versus 90 days; $P=0.808$ ) among both groups. Median cost associated with ER visits were non-significantly lower in the EPC group (USD 21.99: converts to GBP 16.97 in 2018) compared to usual care (USD 46.35: converts to GBP 35.76 in 2018) $(P=0.081)$. The authors further reported lower median cost of hospitalisation days in favour of EPC (USD 167.57: converts to GBP 129.30 in 2018) compared to usual care (USD 295.05: converts to GBP 227.66 in 2018) $(P=0.015)$.

Ma 2019 assessed resource use and operating costs between an early palliative care intervention and usual care for patients in the ICU setting in USA. It was an inpatient consult model of HSPC. Resources used were extracted from patients' electronic medical records, including mechanical ventilation, vasopressors, haemodialysis, tracheostomy, cardiopulmonary resuscitation, ED visits, hospital readmission, hospital duration and ICU duration. Early palliative care patients had fewer ventilator days (median 4 versus $6 ; P=0.042)$, tracheostomies performed $(1 \%$ versus $7.8 \%$; $P$ $=0.035)$, postdischarge emergency department visits $(1.3 \%$ versus $12.5 \% ; P=0.007$ ), days on mechanical ventilation (median (IQR) $4(3-7)$ versus $6(3-13) ; P=0.042)$ and hospital readmissions $(17.3 \%$ versus $33.3 \% ; P=0.0024)$. There was no difference between the intervention and control group in ICU length of stay (median 5 versus 5.5 days), numbers on mechanical ventilation (53.6\% versus $56.9 \% ; P=0.64)$, numbers on vasopressors ( $48.5 \%$ versus $50 \% ; P=$ 0.83 ), days on vasopressors (median 3 versus $3 ; P=0.91$ ), numbers on haemodialysis ( $15.5 \%$ versus $23.5 \% ; \mathrm{P}=0.15)$, numbers receiving cardiopulmonary resuscitation $(5.2 \%$ versus $6.9 \% ; P=0.61)$ and hospital length of stay (median 10 versus 11 days). Analysis of operating costs was conducted though lacking in statistical power to detect the difference. Intervention patients had lower medical ICU (USD 9860 (converts to GBP 7608.08 in 2018) versus USD 15,660 (converts to GBP 12,083.42 in 2018); $P=0.004$ ) and pharmacy costs (USD 3430 (converts to GBP 2646.62 in 2018) versus USD 5850 (converts to GBP 4513.92 in 2018); $P=0.016$ ) per patient compared with the control group. However, the total operating cost per patient was not different between intervention and control group (USD 37,310 (converts to GBP 28,788.78 in 2018) versus USD 45,790 (converts to GBP 35,332.04 in 2018); $P=0.14$ ). An estimated USD 880 (converts to GBP 679.02) of the intervention group's per patient total operating cost was due to the added cost of the palliative care consultation.

\section{Quality of the evidence}

Within the Grade approach, we downgraded the quality of evidence for cost and cost-effectiveness to very low due to a high risk of bias across studies (-2 levels as a result of very serious study limitations: high risk of bias for performance, detection, attrition, reporting, size of study and other bias and inconsistency in the direction of the results: -1 level due to variability in results) (Summary of findings 1 ).

\section{Synthesis of nested or embedded qualitative studies that explored stakeholders' views and experiences of HSPC}

Ten studies with a total of 322 participants ( 245 patients, 20 carers, 9 HSPC team members, 29 physicians (including oncologists), 14

The effectiveness and cost-effectiveness of hospital-based specialist palliative care for adults with advanced illness and their caregivers 
oncology nurse practitioners, one consultant in interstitial lung disease, one clinical nurse specialist in interstitial lung disease, one community matron, one community palliative care nurse and one general practitioner) also had qualitative components that were used to explore stakeholders' views and experiences of HSPC (Bajwah 2015; Farquhar 2014; Farquhar 2016; Hopp 2016; Veron 2018 (linked to Janssens 2019); Lowther 2018 (linked to Lowther 2015); Maloney 2013 (linked to Bakitas 2009); Giovannetti 2018 (linked to Solari 2018); Talabani 2017 (linked to Brannstrom 2014); Wallen 2012) (see Table 16 under Additional tables). The number of patients interviewed by Wallen 2012 was unclear. However, a study (Slota 2014 linked to Wallen 2012) reporting the same data by the authors stated that 34 patients were involved in the qualitative analysis.

Data collection was mainly through semi-structured interviews. However, Slota 2014 (linked to Wallen 2012) collected their data using open-ended questions on a questionnaire, while Hopp 2016 qualitatively reviewed clinical records. Approaches to data analysis in these studies included content analysis, framework analysis and thematic analysis. Slota 2014 (linked to Wallen 2012) stated that they used thematic analysis while another study that reported the same data by the authors stated that they used transcriptbased analysis. Bajwah 2015 reported using a constant comparison approach within framework analysis, while Hopp 2016 did not state their approach.

Four studies were HSPC models involving service provision across multiple settings (Farquhar 2014; Farquhar 2016; Maloney 2013 (linked to Bakitas 2009); Wallen 2012), and another four were hospital outreach services (Bajwah 2015; Talabani 2017 (linked to Brannstrom 2014); Veron 2018 (linked to Janssens 2019); Solari 2018). Lowther 2018 (linked to Lowther 2015) was an outpatient HSPC model while Hopp 2016 was an inpatient consult model. Data from the studies were synthesised into two themes: valued components and challenges to HSPC provision.

Participants valued the patient and family-centredness of the HSPC intervention as it helped to address the varied needs of patients and their unpaid caregivers/families. Benefits described included better symptom control, effective communication and shared decision-making, psychosocial support and coping, respectful and compassionate care, supporting role maintenance and empowerment, reduced isolation, and improved use of devices. HSPC facilitated effective communication and shared decisionmaking as patients and their unpaid caregivers/families had control over the care the patient received. They were able to ask questions, they were listened to and were able to receive the support they needed. Shared decision-making and the psychosocial support provided as part of HSPC was therapeutic for patients and their unpaid caregivers/families, and also reassured them that they were not alone. Patients particularly valued services they received in the secure environment of their homes, and the involvement and support of their families. In addition to the care delivered, the process of delivery of care was also considered to be important. For instance, patients and their unpaid caregivers/families noted that the palliative care professionals were approachable, attentive and supportive. HSPC further facilitated care planning and the discussion of advanced care plans.

Although HSPC was viewed favourably by participants in these studies, there was also evidence that some participants questioned its usefulness. For instance, in Veron 2018 (linked to Janssens
2019), there were mixed reactions among advanced COPD patients about the value of the HSPC intervention. Authors described poor recollection of the HSPC consultation by patients who tended not to consider themselves to be sick. They ascribed their functional limitations to health problems other than COPD. Patients in this study avoided talking about the future and end-of-life issues and wanted to focus on the present. Also in Hopp 2016, participants expressed concerns that HSPC might prevent them from receiving more aggressive interventions and many did not want to discuss advanced directives.

Patients and their unpaid caregivers/families found the information provided during the HSPC intervention to be useful, as it ensured a better understanding of illness and treatment options. Patients and their unpaid caregivers/families valued the multidisciplinary nature of the HSPC team and their specialist expertise. Healthcare professionals such as oncologists tended to describe better patient care resulting from integration of palliative care with oncology at the time of diagnosis of advanced cancer.

Challenges to HSPC provision in these studies were identified, including lack of referral to HSPC by other health professionals, perception of palliative care as being synonymous with imminent death, lack of willingness to engage with palliative care, organisational barriers (e.g. insufficient services) and issues with the experimental study design (e.g. inadequate length of the HSPC intervention).

\section{ISC USSION}

\section{Summary of main results}

Studies on the effectiveness of HSPC in patients with an advanced illness have yielded evidence of low quality and very low quality indicating small benefits for patient HRQoL and symptom burden, respectively. Due to very low- to low-quality evidence, we are uncertain about the true effect of HSPC on these outcomes. The results of the 10 studies including a total of 1344 participants indicate that, when compared to usual care, HSPC may improve patient HRQoL on average by $0.26 \mathrm{SMD}\left(95 \% \mathrm{Cl} 0.15\right.$ to $0.37 ; \mathrm{I}^{2}=$ $3 \%)$. Positive SMDs indicate better patient HRQoL while negative SMDs indicate lower patient HRQoL. Data from the six studies, including a total of 761 participants, suggests that HSPC may reduce patient symptom burden on average by -0.26 SMD over usual care $\left(95 \% \mathrm{Cl}-0.41\right.$ to $\left.-0.12 ; \mathrm{I}^{2}=0 \%\right)$. Negative SMDs indicate benefit (lower symptom burden) and positive SMDs reflect higher symptom burden. Data from the two studies, including a total of 337 participants, suggests that HSPC may improve patient satisfaction with care on average by 0.36 SMD over usual care $(95 \% \mathrm{Cl} 0.14$ to $0.57 ; 1^{2}=0 \%$; low-quality evidence). Positive SMDs indicate better patient satisfaction with care while negative SMDs indicate lower patient satisfaction with care. By conventional criteria, these effects are considered small.

Very low-quality evidence from one study including 312 participants suggests that when compared to usual care, there was no evidence of effect of HSPC on unpaid caregiver satisfaction with care. We used home death as a proxy measure for achieving patient's preferred place of death and we found low-quality evidence favouring home death in those that received HSPC. Results from the seven studies ( $\mathrm{N}=861$ participants) favoured HSPC which is reflected in 1.63 higher odds of home death $(95 \% \mathrm{Cl} 1.23$ to $\left.2.16 ; 1^{2}=0 \%\right)$. Very low-quality evidence from one study of 47

The effectiveness and cost-effectiveness of hospital-based specialist palliative care for adults with advanced illness and their caregivers 46 (Review)

Copyright (c) 2020 The Cochrane Collaboration. Published by John Wiley \& Sons, Ltd. 
participants showed that when HSPC was involved, patients were more likely to achieve their preferred place of care.

We found no difference in mortality/survival between HSPC and usual care in 36 studies ( $\mathrm{N}=7103$ participants) (very low-quality evidence). Very low-quality evidence from four studies ( $N=525$ participants) measuring pain also showed no evidence of effect of HSPC (SMD $-0.16,95 \% \mathrm{Cl}-0.33$ to $0.01 ; \mathrm{I}^{2}=0 \%$ ). Positive SMDs indicate more pain while negative SMDs indicate lower pain (benefit). As a result of the very low-quality evidence, we are uncertain about the effect of HSPC on mortality/survival and pain.

Very low-quality evidence from five studies ( $N=384$ participants) suggests that there is no difference in patient anxiety when HSPC is compared to usual care (MD $-0.63,95 \% \mathrm{Cl}-2.22$ to $0.96 ; \mathrm{I}^{2}=76 \%$ ). Negative mean differences (MDs) indicate benefit (lower anxiety) and positive MDs reflect higher anxiety. However, eight studies ( $\mathrm{N}=1096$ participants) found that HSPC may improve patient depression on average with a small effect size of -0.22 SMD $(95 \% \mathrm{Cl}$ -0.34 to $-0.10 ; 12=0 \%$; very low-quality evidence). Negative SMDs indicate benefit (lower depression) and positive SMDs reflect higher depression. We found no evidence of effect on unpaid caregiver anxiety and depression. However, there was only very low-quality evidence from one study that assessed unpaid caregiver anxiety ( $\mathrm{N}=312$ participants), and two studies ( $\mathrm{N}=413$ participants) that reported on unpaid caregiver depression (SMD $-0.02,95 \% \mathrm{Cl}-0.21$ to $\left.0.18 ; 1^{2}=0 \%\right)$.

The data we pooled from five studies ( $\mathrm{N}=616$ participants) that reported adjusted endpoint values and constituted very low-quality evidence indicated no evidence of effect of HSPC on breathlessness when compared to usual care (SMD -0.04, $95 \% \mathrm{Cl}-0.19$ to $\left.0.12 ; 1^{2}=0 \%\right)$. Negative SMDs indicate benefit (reduced breathlessness) and positive SMDs reflect worsened breathlessness.

Of the eight studies ( $N=1252$ participants) that reported on adverse events, six described no adverse events while the remaining two described more adverse events in the HSPC group compared to the control group. While we found no evidence that HSPC causes serious harms, the evidence was very low quality and insufficient to draw strong conclusions.

We could not pool data from the two studies ( $n=170$ participants) that reported adjusted endpoint data for unpaid caregiver burden. Both studies suggested that HSPC may make little to no difference to unpaid caregiver burden (very low-quality evidence).

Only one study in 44 participants assessed unpaid caregiver grief and also reported adjusted endpoint values. Similarly, one study in 69 participants assessed unpaid caregiver quality of life and also presented adjusted endpoint values. There was no evidence of a difference between HSPC and usual care on unpaid caregiver grief and quality of life (low-quality evidence).

Very low-quality evidence suggests that the effect of HSPC compared to usual care on resource utilisation, cost and costeffectiveness is inconclusive. The evidence on resource use was varied across the different areas assessed. Two studies found reduced cost with HSPC when compared to usual care, while one study found a reduction in the cost of hospitalisation days but no difference in the cost of emergency room visits. The difference in cost was unclear in one study, while the remaining nine studies indicated no difference between HSPC and usual care. It was hard to tell if the costs were shifted to other settings (e.g. from acute sector to community) when data on resource utilisation were limited to hospital. Regarding cost-effectiveness, the evidence from the full economic studies was also inconsistent. One study reported cost-effectiveness planes of the palliative care outcome scale (POS-8) and unpaid caregiver burden (ZBI) against total costs, and found that $34 \%$ and $47 \%$ of bootstrapped differences in costs and outcomes indicated lower costs and better outcomes for the intervention. Another study also presented cost-effectiveness planes with bootstrapping, where $66 \%$ of replicated combinations of costs and outcomes of distress due to breathlessness (NRS) against total cost indicated lower costs and better outcomes. However, another study found that the intervention was not costeffective: the incremental cost-effective ratio (ICER) was 266,333 per QALY, and there was only about a 7\% likelihood of lower cost and higher QALYs. The last cost-effectiveness study calculated incremental net monetary benefit (INMB) of HSPC and found that the intervention was cost-effective when the willingness to pay threshold was larger than AUD 1,027 (converts to GBP 915 in 2018) for one extra day at home.

Evidence from the qualitative studies that explored views and experiences of HSPC by stakeholders suggested that HSPC was beneficial as it ensured personalised and holistic care for patients and their families, while also fostering open communication, shared decision-making, respectful and compassionate care and psychosocial support. A previous systematic review also found these areas to be important by patients and their families for endof-life care in the hospital setting (Virdun 2015). Patients found the specialist expertise and multidisciplinary nature of the HSPC teams to be helpful, and there was oncologists' support for early palliative care for patients with newly diagnosed advanced stage cancer.

The main domains of palliative care addressed in the studies that either included certified experts in palliative care, or those described as palliative care clinicians, were symptom control, coping and support, and decision-making. Some of the studies also addressed care co-ordination and future planning. With the exception of future planning, studies that were unclear about palliative care training of those delivering the HSPC intervention had less focus on symptom control, decision-making, care coordination and coping and support when compared to those that included certified experts in palliative care.

\section{Overall completeness and applicability of evidence}

We had a highly sensitive electronic search strategy in addition to contacting experts in order to locate grey literature and unpublished studies. As a result, we had a large number of references to screen. Given our intensive search strategy, we are of the opinion that we captured the breadth of evidence on HSPC so far. In particular, we were able to identify 42 RCTs including one foreign language study (Chinese). This allowed us to report on the effect of HSPC on different outcomes. It is noteworthy that the number of studies reporting on different outcomes varied, especially, as we decided to report adjusted endpoint values as our main meta-analysis. We decided to present adjusted values as our main meta-analysis because they control for differences, and also provide the most precise and least biased estimates of treatment effects. Although we had indicated that we would be carrying out subgroup analyses by disease type, HSPC team composition (e.g. physician-led versus nurse-led versus MDT-led services and 24-hour

The effectiveness and cost-effectiveness of hospital-based specialist palliative care for adults with advanced illness and their caregivers 
access versus temporarily restricted access), models of HSPC and country of origin in order to explain heterogeneity, we could only carry out subgroup analyses on one outcome (patient anxiety) due to low to no heterogeneity in the main meta-analysis in other outcomes. The results of subgroup analysis should be interpreted with caution due to the small number of studies available and the exploratory nature of this approach. We had indicated that we would be carrying out a subgroup analysis using frailty associated with advanced age. However, no study reported on frailty. In addition, there is a need for better reporting of the findings of studies. We could not include some studies in the meta-analysis because they did not present analysable data.

Most of the studies were conducted in hospitals with specialised palliative care teams and were largely carried out in the US and UK. Regulatory environment can have a significant impact on the provision and impact of HSPC on hospitals, patients and unpaid caregivers. For example, in the US, non-hospital palliative care is provided through a large number of varied private for profit and non-profit entities whose effectiveness and success may vary significantly. This aspect of the service also makes the hospital to home-based care transition difficult and lacking in continuity of care. In addition, palliative care, health policy and resources in developed countries differ from what is obtainable in low- and middle-income countries where resources are somewhat limited and palliative care at its infancy (WPCA/WHO 2014). The results obtained from these highly developed healthcare systems may not be applicable to low resource settings. The majority of our included studies were for populations of cancer patients and, importantly, this review has shown that HSPC is being extended to other patient populations.

\section{Quality of the evidence}

Besides Ahronheim 2000 and Jingfen 2017, a foreign language study, we judged all other studies as having a high risk of bias in at least one domain. Nine studies had a high risk of bias in four or more domains (Bajwah 2015; Brannstrom 2014; Cheung 2010; Edmonds 2010; Janssens 2019; O'Riordan 2019; Rodin 2019; Rogers 2017; Temel 2017). We carried out sensitivity analyses using unadjusted endpoint values and (un)adjusted changes and the results from these analyses sometimes supported the results we obtained from our main analysis.

Using the GRADE approach, the quality of the evidence ranged from very low to low across different outcomes. Generally, we downgraded the evidence mainly due to serious/very serious study limitations (high risk of bias), inconsistency resulting from unexplained heterogeneity and imprecision due to small numbers of participants.

There were differences across studies in the models of HSPC and usual care, patient population, outcome measures and time point of primary analysis. The evidence for mortality/survival was also quite varied. This difference could have resulted because of the diverse patient populations in the studies as well as the heterogeneous models of the intervention.

This review provided evidence of low quality concerning the effectiveness of HSPC on the primary outcomes of patient HRQoL and patient symptom burden. Given the low quality of the evidence, the findings should be interpreted circumspectly. Findings from ongoing studies and other future studies may assist in further strengthening the certainty of the effect estimates on the effectiveness of HSPC.

\section{Potential biases in the review process}

Given that decisions taken during the process of conducting a systematic review and meta-analysis may be affected by subjective decisions (Shrier 2008), it is important to consider potential biases that may have occurred. Generally, the methods of a systematic review provide for transparency and standardisation thereby enhancing reproducibility of the process. For most of our outcomes such as patient HRQoL and patient symptom burden, we combined studies reporting adjusted endpoint data as our main meta-analyses. We pooled these studies using standardised mean differences (SMDs) because they used different scales. Restricting our main meta-analyses to studies reporting adjusted endpoint data reduced the number of studies we could pool together.

We could not include some studies in our meta-analyses because they did not present analysable data. Outcomes that were not reported in a usable format may be systematically different from those that were included in the meta-analyses, thereby introducing selective outcome reporting bias (Higgins 2011b). We followed the GRADE approach in assessing the quality of the evidence for different outcomes. Although the GRADE approach may not always ensure consistency of conclusions, we believe it offers the advantage of a systematic and transparent process of judging the quality of the evidence (Guyatt 2011).

An important step in minimising bias in systematic reviews is to address publication bias. Publication bias affects the validity and generalisability of the findings of a meta-analysis (Lin 2017). In order to reduce the possibility of publication bias, we searched electronic databases, carried out citation tracking, handsearched relevant studies and reviews and contacted experts for grey literature and unpublished studies. We drew on a comprehensive search strategy with input from the information specialist from the PaPaS Group in order to minimise our chances of missing out relevant studies. We believe that this synthesis includes an unbiased sample that covers the populations targeted by this review. Nonetheless, we cannot rule out time-lag bias, that is, when the results of negative trials take longer to publish when compared to positive trials (Sterne 2011).

In order to include studies in this review, the intervention had to have been delivered by a multidisciplinary team. Our definition of a multidisciplinary team was quite broad encompassing studies where different professionals delivered the intervention to those where one single professional led the service and included other professionals, as needed. We excluded studies such as Maltoni 2016 and Schenker 2018 because they did not meet our definition of a multidisciplinary team. Further, we excluded studies such as Brims 2019 and Wong 2016 because palliative care was an integral part of routine usual care. Our decision to include studies where the training of the palliative care team was unclear, with eligibility informed by activity of delivering specialist palliative care rather than level of specialist training, might have implications for the effect estimates we found, with the possibility of smaller effect sizes in the review. Also, in almost half of the studies $(n=20)$, there was palliative care involvement in the control group. This could have resulted in a smaller effect of the intervention in these studies. Due to differences in the reporting of the cost-effectiveness results and also the dearth of cost-effectiveness studies in this review, we

The effectiveness and cost-effectiveness of hospital-based specialist palliative care for adults with advanced illness and their caregivers 48 
could not carry out subgroup analysis to explore differences in costeffectiveness across countries.

We included studies where the authors stated that the intervention they provided was early palliative care or where this was their intention. Given that the definition of early palliative care is still an area of ongoing debate (Haun 2017), there is a need for consensus on its definition. In order to make our review more relevant for clinical practice and policy makers, we made some changes to the protocol such as expansion of the remit of our review from only inpatient specialist palliative care to five different HSPC models, limiting eligible studies to only RCTs and change of our primary outcome from pain to two primary outcomes, patient HRQoL (previously, a secondary outcome in the protocol) and patient symptom burden. We carried out these changes before we began data extraction and analysis. Consequently, the changes were not post hoc. We have provided a full description of the changes we made to the protocol under Differences between protocol and review.

As in a previous Cochrane Review on the effectiveness of early palliative care for adults with advanced cancer (Haun 2017), we provided a description of the quality of the evidence for our outcomes rather than on providing clinical guidance. We have presented quality ratings for each outcome and have not determined the quality across outcomes. Given that it has been suggested that the lowest quality rating of the primary outcome(s) should be applied to the overall quality rating across studies (Guyatt 2013), the evidence for HSPC would be considered as very low. However, as recognised in Haun 2017 and also in this review, this rating is likely biased and we need larger and well-conducted studies to establish the effectiveness of HSPC.

\section{Agreements and disagreements with other studies or reviews}

Four relevant systematic reviews have been published prior to this review (Dalgaard 2014; Gaertner 2017; Haun 2017; Higginson 2002). Three of these reviews included HSPC while Haun 2017 assessed the effectiveness of early palliative care for cancer patients only. None of these previous reviews included all the RCTs in our review. Our Cochrane Review is the first to assess the effectiveness and cost-effectiveness of HSPC on diverse outcomes in a broad population consisting of people with cancer, non-cancer and mixed diagnoses.

Dalgaard 2014 assessed the best methods for early identification of palliative trajectories in patients with cancer, chronic heart failure and COPD, while also identifying preconditions for early integration of general palliative care in hospitals and outcomes for patients and relatives. This review included only one of the seminal papers on early palliative care by Temel 2010 which found that early integration of palliative care with standard oncology care for patients with non-small-cell lung cancer (NSCLC) led to better quality of life and mood as well as longer survival. This review concluded that evidence about outcomes was sparse and mostly related to cancer populations receiving specialised palliative care.

Gaertner 2017 assessed the effect of specialist palliative care on quality of life and other outcomes in adults with advanced illness in hospital, hospice or community settings. This review included eight RCTs that we also identified in our review and concluded that "specialist palliative care was associated with a small effect on quality of life and might have most pronounced effects for patients with cancer who received such care early". The review found that the results for pain and other secondary outcomes (fatigue, nausea, dyspnoea, psychosocial variables (distress, depression, anxiety, spiritual well-being, social well-being, and satisfaction), survival time, place of death, cost of care, and attrition (or completion rate)) were inconclusive.

Haun 2017 assessed the effectiveness of early palliative care on different outcomes such as HRQoL, depression, symptom intensity and survival among patients with advanced cancer. This review included six RCTs that were also part of our review and concluded that "early palliative care interventions may have more beneficial effects on HRQoL and symptom intensity among patients with advanced cancer than among those given usual/standard cancer care alone". The authors found only small effect sizes. The effects on mortality and depression were uncertain. The authors further stated that results should be interpreted with caution due to the very low-to low-quality of the evidence and between-study differences regarding participant populations, interventions, and methods.

Higginson 2002 is the oldest review that was relevant. Its objective was to assess whether hospital-based palliative care teams improved the process or outcomes of care for patients and families at the end-of-life through a qualitative meta-synthesis and quantitative meta-analysis. It did not include any of the studies in our review and there was only one RCT. The authors found a small positive effect for hospital-based palliative care teams. Higginson 2002 further highlighted the need for better designed studies comparing different models of HSPC as well as the use of standardised outcome measures for assessing symptoms.

Our review agrees with these past reviews in some respect especially with regards to HRQoL. We found evidence that HSPC may be effective in improving HRQoL of patients and patient symptom burden with small effect sizes. We also found that HSPC may lead to benefits on some of the secondary outcomes we assessed: better patient satisfaction, achieving patient preferred place of death (measured by number of home deaths) and improvement in patient depression. Quality of the evidence ranged from very low to low. Similar to our review, the review by Gaertner 2017 found a small effect of specialist palliative care on HRQoL (SMD $0.16,95 \% \mathrm{Cl} 0.01$ to $0.31 ; \mathrm{n}=7$ studies with 1218 participants; moderate-quality evidence). The Cochrane Review by Haun 2017 also showed a small effect of early palliative care on HRQoL (SMD $0.27,95 \% \mathrm{Cl} 0.15$ to $0.38 ; \mathrm{n}=7$ studies with 1028 participants; lowquality evidence).

\section{AUTHORS' CONCLUSIONS}

\section{Implications for practice}

\section{For patients and carers}

Available evidence of very low- to low-quality suggests that patients with advanced illness may benefit from HSPC with respect to small improvements in patient HRQOL and symptom burden, and HSPC may improve patient satisfaction, patient depression, and increase the chances of patients dying in their preferred place (measured by home death). There is limited evidence of the effect on unpaid caregiver grief as this has not been well studied. While we found no evidence that HSPC causes serious harms, the evidence was very

The effectiveness and cost-effectiveness of hospital-based specialist palliative care for adults with advanced illness and their caregivers 
low quality and insufficient to draw strong conclusions. Patients could approach their clinicians and request referral to HSPC.

\section{For clinicians}

Although we found evidence that HSPC may improve patient $\mathrm{HRQ}$ oL, symptom burden, patient depression, patient satisfaction with care and may improve the chances that patients achieve their preferred place of death (measured by home death), the certainty of the evidence was very low to low. Despite the limited quality of the evidence, HSPC may be considered in practice for patients with advanced diseases. From a practitioner's perspective, some previous reviews have reported definitive success of palliative care in prolonging life. Results from our review do not support increased survival with HSPC but HSPC may increase the chances of a home death. Very low-quality evidence did not demonstrate an effect of HSPC on serious harms. Therefore, clinicians may consider offering HSPC on a case-by-case basis to address patient HRQoL and symptom burden, but refrain from claiming these interventions will improve survival. More research is needed before solid conclusions can be drawn.

\section{For policy makers}

Given that population-based projections have indicated that palliative care needs will increase in the future (Sleeman 2019), one area that policy makers could prioritise is the further commissioning of HSPC. Importantly, our review showed that those receiving HSPC may have 1.63 higher odds of dying in their preferred place (measured by home death), in addition to benefits for patient HRQoL and symptom burden. The 1.63 higher odds translates to an increase in the relative risk of dying in the patient's preferred place of $22 \%$ ( $8 \%$ to $39 \%$ ). Very low-quality evidence did not demonstrate an effect of HSPC on serious harms. There is an urgent need for well-powered high-quality RCTs on the effect of HSPC in populations with non-cancer and mixed diagnoses, wardbased care, 24 hours access (out-of-hours care), achieving patient preferred place of care, patient satisfaction with care, unpaid caregiver outcomes (satisfaction with care, burden, depression, anxiety, grief, quality of life) and cost-effectiveness. Of note, there were no studies looking at the effectiveness of HSPC on broader effects in hospitals such as patient flow and readmission rates.

\section{For funders of the intervention}

When compared to usual care, HSPC may improve patient HRQoL, symptom burden, patient satisfaction, patient depression, while also helping patients die in their preferred place (measured by home death). We suggest that the evidence should be interpreted cautiously until more RCTs are available. Very low-quality evidence did not demonstrate an effect of HSPC on serious harms. It appears that HSPC carried no greater cost than usual care.

\section{Implications for research}

\section{General}

This review has shown that there is a need for larger and wellconducted RCTs assessing different models of HSPC in non-cancer and mixed populations. Compared with cancer studies, RCTs involving populations with non-cancer and mixed diagnoses are fewer. Also, this review found only few RCTs assessing ward-based HSPC models and 24 hours access (out-of-hours care), and no study assessing relatively new constructs such as frailty or a focus on multimorbidity. These are areas for exploration in future RCTs that are sufficiently powered to detect differences between the intervention and control groups. There is also an urgent need for studies to consider the varied regulatory environment and conduct more systems-wide research looking at HSPC spanning more than one setting and how integrated HSPC across hospital and community changes outcomes and costs. It is paramount that more RCTs are carried out in low- and middle-income countries with a good description of the intervention and usual care in order to expand the existing evidence base. More RCTs on the effectiveness of HSPC on other outcomes besides patient HRQoL and symptom burden are also needed. For instance, patient satisfaction with care, achieving patient preferred place of care, unpaid caregiver outcomes (e.g. satisfaction with care, burden, depression, anxiety, grief, quality of life) and cost-effectiveness should be further explored. There is an urgent need for more cost-effectiveness studies on HSPC as we only identified four such studies in this review. A clearer definition of early palliative care by the palliative care community would assist future RCTs evaluating it to be more focussed.

\section{Design}

Future RCTs need to be larger, well-designed and well-conducted, with high-quality reporting of their methods. Interventions should be described clearly under the different models we have proposed for HSPC. To strengthen the internal validity of effect estimates, future studies need to be rigorous in both design and delivery, and should be based on sufficient power. To ensure fidelity of delivery of the intervention, detailed descriptions of the components of the intervention should be provided in the methods, including training of staff involved in the provision of HSPC. In addition, the delivery of HSPC (including frequency and duration of treatment), receipt of HSPC, and enactment of HSPC should be clearly described. Where possible, usual care groups should not include access to HSPC and where this does happen, there should be clear documentation.

Where possible, investigators should aim to control for selection bias (i.e. to ensure adequate allocation concealment), performance bias (i.e. to blind study participants) and detection bias (i.e. to blind outcome assessors). However, this will continue to be a challenge in this area. With respect to settings, interventions that span acute and community settings are needed.

Concerning heterogeneity of samples, there is a need to investigate disease-homogenous samples to better account for diseasespecific trajectories and multimorbidity.

In addition, future studies should also consider effectivenessimplementation hybrid designs, combining elements of clinical effectiveness and implementation research to enhance public health impact (Curran 2012). In particular, strategies to encourage implementation of evaluation findings should be incorporated and be based on a scientific understanding of the behaviours that need to change, the relevant decision-making processes, and the barriers and facilitators of change. This will speed the translation of research findings into routine practice.

\section{Measurement}

Patient HRQOL and symptom burden are appropriate outcomes that appear to be sensitive to change and can be recommended for routine collection. However, most of the available quality of life measures do not include domains that have been found to be important in palliative populations such as existential or

The effectiveness and cost-effectiveness of hospital-based specialist palliative care for adults with advanced illness and their caregivers 50 (Review) 
spiritual domains (Cohen 2001; Roscoe 2010), and this could potentially underestimate the effect of HSPC. Further, many of the HRQOL measures have been validated on the assumption that scores deteriorate towards death and so exhibit floor effects in palliative care. In addition, they are not individualised. Pain, whilst an appropriate primary outcome in studies of participants with malignancies, does not appear to be an appropriate outcome for studies of participants with non-malignant diagnoses. Better outcome measures are needed, which are person-centred and can be used across studies.

It is also important that RCTs report adequately on outcomes they stated in their protocol in order to avoid selective outcome reporting bias. There is a need for more studies reporting adjusted endpoint data. It appears that consensus is needed by palliative care researchers on whether endpoint scores or change scores are the most informative for this population. The ongoing focus on improvement of outcomes may be leading to discounting of the effectiveness of HSPC in the important clinical outcome of slowing deterioration compared to usual care.

Concerning economic measurements, data sources such as health insurance database and hospital medical records are more reliable and accurate but the information on services in community and/or at home (including delivery of care by unpaid caregivers) requires different approaches. For example, hospital records (e.g. Hospital Episode Statistics) linked with community service data (e.g. Clinical Practice Research Datalink) would help understand the change of resource use and its implication on costs/cost-effectiveness. Moreover, future studies need primary data collection from patients or family members, using tools such as the Client Service Receipt Inventory providing information on delivery of care by unpaid caregivers as well as health and social care.

\section{ACKNOWLEDGEMENTS}

We acknowledge Barbara Daveson, Melinda Smith, Hamid Benalia, Emily West, Sue Hall, Barbara Gomes and Nancy Preston who contributed to earlier drafts of the protocol.

BuildCARE members: Emma Bennett, Francesca Cooper, Barbara Daveson, Susanne de Wolf-Linder, Mendwas Dzingina, Clare EllisSmith, Catherine Evans, Taja Ferguson, Lesley Henson, Irene Higginson, Bridget Johnston, Pauline Kane, Peter Lawlor, Paul McCrone, Regina McQuillan, Diane Meier, Sean Morrison, Fliss Murtagh, Charles Normand, Steve Pantilat, Ana Reison, Karen Ryan, Lucy Selman, Melinda Smith, Katy Tobin, Rowena Vohora and Gao Wei.

We are grateful to the following peer reviewers for their time and comments: Brian Duncan, Ollie Minton, Claudia Virdun, and Abhijna Vithal Yergolkar.

Cochrane Review Group funding acknowledgement: this project was funded by the National Institute for Health Research (NIHR) via Cochrane Infrastructure funding to the Cochrane Pain, Palliative and Supportive Care Review Group (PaPaS). The views expressed are those of the author(s) and not necessarily those of the NIHR or the Department of Health and Social Care.

This research was funded by the National Institute for Health Research Health Services and Delivery Research programme (Project Number 16/02/17). It was also supported by the National Institute for Health Research Collaboration for Leadership in Applied Health Research and Care South London, now recommissioned as NIHR Applied Research Collaboration South London. The views expressed in this publication are those of the authors and not necessarily those of the NIHR or the Department of Health and Social Care. 


\section{R E F E R E N C E S}

\section{References to studies included in this review}

Ahronheim 2000 \{published data only\}

Ahronheim JC, Morrison RS, Morris J, Baskin S, Meier DE. Palliative care in advanced dementia: a randomised controlled trial and descriptive analysis. Journal of Palliative Medicine 2000;3(3):265-73.

\section{Bajwah 2015 \{published data only\}}

Bajwah S, Higginson IJ, Wells AU, Koffman J, Ross JR, Birring SS, et al. Developing and evaluating a hospital2home palliative care service for patients with advanced progressive idiopathic fibrotic interstitial lung disease: phase 0 -II [Abstract]. Palliative Medicine 2012;26(4):545.

* Bajwah S, Ross JR, Wells AU, Mohammed K, Oyebode C, Birring SS, et al. Palliative care for patients with advanced fibrotic lung disease: a randomised controlled phase II and feasibility trial of a community case conference intervention. Thorax 2015;70(9):830-9.

\section{Bakitas 2009 \{published data only\}}

Bakitas M, Lyons KD, Hegel MT, Ahles T. Oncologists' perspectives on concurrent palliative care in a National Cancer Institute-designated comprehensive cancer center. Palliative \& Supportive Care 2013;11(5):415-23.

Bakitas M, Lyons KD, Hegel MT, Balan S, Barnett KN, Brokaw FC, et al. The project ENABLE II randomised controlled trial to improve palliative care for rural patients with advanced cancer: baseline findings, methodological challenges, and solutions. Palliative \& Supportive Care 2009;7(1):75-86.

* Bakitas M, Lyons KD, Hegel MT, Balan S, Brokaw FC, Seville J, et al. Effects of a palliative care intervention on clinical outcomes in patients with advanced cancer: the Project ENABLE II randomised controlled trial. Journal of the American Medical Association 2009;302(7):741-9.

Maloney C, Lyons KD, Li Z, Hegel M, Ahles TA, Bakitas M. Patient perspectives on participation in the ENABLE II randomised controlled trial of a concurrent oncology palliative care intervention: benefits and burdens. Palliative Medicine 2013;27(4):375-83.

O'Hara RE, Hull JG, Lyons KD, Bakitas M, Hegel MT, Li Z, et al. Impact on caregiver burden of a patient-focused palliative care intervention for patients with advanced cancer. Palliative \& Supportive Care 2010;8(4):395-404.

\section{Bakitas 2015 \{published data only\}}

* Bakitas MA, Tosteson TD, Li Z, Lyons KD, Hull JG, DionneOdom JN, et al. Early versus delayed initiation of concurrent palliative oncology care: patient outcomes in the ENABLE III randomised controlled trial. Journal of Clinical Oncology 2015;33(13):1438-45.

Dionne-Odom JN, Azuero A, Lyons KD, Hull JG, Prescott AT, Tosteson T, et al. Family caregiver depressive symptom and grief outcomes from the ENABLE III randomised controlled trial. Journal of Pain and Symptom Management 2016;52(3):378-85.
Dionne-Odom JN, Azuero A, Lyons KD, Hull JG, Tosteson T, Li Z, et al. Benefits of early versus delayed palliative care to informal family caregivers of patients with advanced cancer: outcomes from the ENABLE III randomised controlled trial. Journal of Clinical Oncology 2015a;33(13):1446-52.

Dionne-Odom JN, Hull JG, Martin M, Akyar I. The association between family caregiver burden and the survival of advanced cancer patients. Psycho-Oncology 2015;24:57.

Dionne-Odom JN, Hull JG, Martin MY, Lyons KD, Prescott AT, Tosteson T, et al. Associations between advanced cancer patients' survival and family caregiver presence and burden. Cancer Medicine 2016;5(5):853-62.

Dionne-Odom JN, Raju D, Hull J, Akyar I, Lyons K, Azuero A, et al. Characteristics and outcomes of persons with advanced cancer associated with having a family caregiver: a classification tree analysis. Journal of Clinical Oncology 2014;32(31):29.

Bekelman 2018 \{published data only\}

* Bekelman DB, Allen LA, McBryde CF, Hattler B, Fairclough DL, Havranek EP, et al. Effect of a collaborative care intervention vs usual care on health status of patients with chronic heart failure: the CASA randomised clinical trial. JAMA Internal Medicine 2018;178(4):511-9.

Bekelman DB, Allen LA, Peterson J, Hattler B, Havranek EP, Fairclough DL, et al. Rationale and study design of a patientcentred intervention to improve health status in chronic heart failure: the Collaborative Care to Alleviate Symptoms and Adjust to Illness (CASA) randomised trial. Contemporary Clinical Trials 2016;51:1-7.

Flint K, Johnson RA, Bekelman D. Informal (family) caregiver outcomes from a symptom and psychosocial collaborative care intervention in patients with heart failure: a randomized clinical trial. Circulation: Cardiovascular Quality and Outcomes 2018;11:A234.

\section{Brannstrom 2014 \{published data only\}}

Brännström M, Boman K. A new model for integrated heart failure and palliative advanced home care - rationale and design of a prospective randomised study. European Journal of Cardiovascular Nursing 2013;12(3):269-75.

* Brannstrom M, Boman K. Effects of person-centred and integrated chronic heart failure and palliative home care. PREFER: a randomised controlled study. European Journal of Heart Failure 2014;16(10):1142-51.

Markgren R, Brännström M, Lundgren C, Boman K. Impacts of person-centred integrated chronic heart failure and palliative home care on pharmacological heart failure treatment: a substudy of a randomised trial. BMJ Supportive \& Palliative Care 2016;9(1):e10. [DOI: 10.1136/bmjspcare-2015-000894]

Sahlen KG, Boman K, Brännström M. A cost-effectiveness study of person-centred integrated heart failure and palliative home

The effectiveness and cost-effectiveness of hospital-based specialist palliative care for adults with advanced illness and their caregivers 
care: based on a randomised controlled trial. Palliative Medicine 2016;30(3):296-302.

Talabani N, Ängerud KH, Boman K, Brännström M. Patients' experiences of person-centred integrated heart failure care and palliative care at home: an interview study. $B M J$ Supportive \& Palliative Care 2017;0:1-6. [DOI: 10.1136/ bmjspcare-2016-001226. ]

\section{Brumley 2007 \{published data only\}}

* Brumley R, Enguidanos S, Jamison P, Seitz R, Morgenstern N, Saito $S$, et al. Increased satisfaction with care and lower costs: results of a randomised trial of in-home palliative care. Journal of the American Geriatrics Society 2007;55(7):993-1000.

Enguidanos S, Chambers J. In-home palliative care increased patient satisfaction and reduced use and costs of medical services: commentary. Evidence-Based Medicine 2008;13(1):19.

\section{Carson 2016 \{published data only\}}

* Carson SS, Cox CE, Wallenstein S, Hanson LC, Danis M, Tulsky JA, et al. Effect of palliative care-led meetings for families of patients with chronic critical illness: a randomised controlled trial. Journal of the American Medical Association 2016;316(1):51-62.

Nelson JE, Hanson LC, Keller KL, Carson SS, Cox CE, Tulsky JA, et al. The voice of surrogate decision-makers. Family responses to prognostic information in chronic critical illness. American Journal of Respiratory and Critical Care Medicine 2017;196(7):864-72.

\section{Cheung 2010 \{published data only\}}

Cheung W, Aggarwal G, Fugaccia E, Thanakrishnan G, Milliss D, Anderson $\mathrm{R}$, et al. Palliative care teams in the intensive care unit: a randomised, controlled, feasibility study. Critical Care and Resuscitation 2010;12(1):28-35.

\section{Edmonds 2010 \{published data only\}}

* Edmonds P, Hart S, Wei Gao, Vivat B, Burman R, Silber E, et al. Palliative care for people severely affected by multiple sclerosis: evaluation of a novel palliative care service. Multiple Sclerosis 2010;16(5):627-36. [PMID: 20305044]

Higginson IJ, Vivat B, Silber E, Saleem T, Burman R, Hart S, et al. Study protocol: delayed intervention randomised controlled trial within the Medical Research Council (MRC) Framework to assess the effectiveness of a new palliative care service. BMC Palliative Care 2006;5:7.

\section{El-Jawahri 2016 \{published data only\}}

* El-Jawahri A, LeBlanc T, VanDusen H, Traeger L, Greer JA, Pirl WF, et al. Effect of inpatient palliative care on quality of life 2 weeks after hematopoietic stem cell transplantation: a randomised clinical trial. Journal of the American Medical Association 2016;316(20):2094-103.

El-Jawahri A, Traeger L, Greer JA, VanDusen H, Fishman SR, LeBlanc TW, et al. Effect of inpatient palliative care during hematopoietic stem-cell transplant on psychological distress 6 months after transplant: results of a randomised clinical trial. Journal of Clinical Oncology 2017;35(32):3714-21.
VanDusen H, LeBlanc TW, Traeger L, Greer JA, Pirl WF, Vicki A, et al. Inpatient integrated palliative and transplant care to improve family caregiver (FC) outcomes of patients hospitalised for hematopoietic stem cell transplantation (HCT). Journal of Clinical Oncology 2016;34(26):235.

\section{Farquhar 2014 \{published data only\}}

* Farquhar MC, Prevost AT, McCrone P, Brafman-Price B, Bentley A, Higginson IJ, et al. Is a specialist breathlessness service more effective and cost-effective for patients with advanced cancer and their carers than standard care? Findings of a mixed-method randomised controlled trial. BMC Medicine 2014;12(194). [DOI: 10.1186/s12916-014-0194-2]

Farquhar MC, Prevost AT, McCrone P, Higginson IJ, Gray J, Brafman-Kennedy B, et al. Study protocol: phase III singleblinded fast-track pragmatic randomised controlled trial of a complex intervention for breathlessness in advanced disease. Trials 2011;12(1):130. [DOI: 10.1186/1745-6215-12-130]

Javadzadeh S, Chowienczyk S, Booth S, Farquhar M. Comparison of respiratory health-related quality of life in patients with intractable breathlessness due to advanced cancer or advanced COPD. BMJ Supportive \& Palliative Care 2016;6(1):105-8.

\section{Farquhar 2016 \{published data only\}}

* Farquhar MC, Prevost AT, McCrone P, Brafman-Price B, Bentley A, Higginson IJ, et al. The clinical and cost effectiveness of a Breathlessness Intervention Service for patients with advanced non-malignant disease and their informal carers: mixed findings of a mixed method randomised controlled trial. Trials 2016;17(1):185. [DOI: 10.1186/s13063-016-1304-6]

Farquhar MC, Prevost AT, McCrone P, Higginson IJ, Gray J, Brafman-Kennedy B, et al. Study protocol: phase III singleblinded fast-track pragmatic randomised controlled trial of a complex intervention for breathlessness in advanced disease. Trials 2011;12(1):130. [DOI: 10.1186/1745-6215-12-130]

\section{Franciosi 2019 \{published data only\}}

Franciosi V, Maglietta G, Degli Esposti C, Caruso G, Cavanna L, Berte R, et al. Early palliative care and quality of life of advanced cancer patients - a multicenter randomized clinical trial. Annals of Palliative Medicine 2019;8(4):381-9. [PMID: 30943735]

\section{Gade 2008 \{published data only\}}

Gade G, Venohr I, Conner D, McGrady K, Beane J, Richardson $\mathrm{RH}$, et al. Impact of an inpatient palliative care team: a randomised controlled trial. Journal of Palliative Medicine 2008;11(2):180-90.

\section{Groenvold 2017 \{published data only\}}

* Groenvold M, Petersen MA, Damkier A, Neergaard MA, Nielsen JB, Pedersen L, et al. Randomised clinical trial of early specialist palliative care plus standard care versus standard care alone in patients with advanced cancer: the Danish Palliative Care Trial. Palliative Medicine 2017;31(9):814-24.

Johnsen AT, Damkier A, Vejlgaard TB, Lindschou J, Sjøgren P, Gluud $C$, et al. A randomised, multicentre clinical trial of specialised palliative care plus standard treatment versus

The effectiveness and cost-effectiveness of hospital-based specialist palliative care for adults with advanced illness and their caregivers 
standard treatment alone for cancer patients with palliative care needs: the Danish palliative care trial (DanPaCT) protocol. BMC Palliative Care 2013;12(1):37.

Johnsen AT, Petersen MA, Gluud C, Lindschou J, Fayers P, Sjogren $P$, et al. Detailed statistical analysis plan for the Danish Palliative Care Trial (DanPaCT). Trials 2014;15:376.

\section{Grudzen 2016 \{published data only\}}

Grudzen C, Richardson L, Morrison RS. Randomised controlled trial of ED-triggered palliative care in patients with metastatic solid tumours (TH347-C). Journal of Pain and Symptom Management 2015;49(2):352.

* Grudzen CR, Richardson LD, Johnson PN, Hu M, Wang B, Ortiz JM, et al. Emergency department-Initiated palliative care in advanced cancer: a randomised clinical trial. JAMA Oncology 2016;2(5):591-8.

Kandarian B, Morrison RS, Richardson LD, Ortiz J, Grudzen CR. Emergency department-initiated palliative care for advanced cancer patients: protocol for a pilot randomised controlled trial. Trials 2014;15(251). [DOI: 10.1186/1745-6215-15-251]

\section{Higginson 2009 \{published data only\}}

Higginson IJ, Costantini M, Silber E, Burman R, Edmonds P. Evaluation of a new model of short-term palliative care for people severely affected with multiple sclerosis: a randomised fast-track trial to test timing of referral and how long the effect is maintained. Postgraduate Medical Journal 2011;87(1033):769-75.

Higginson IJ, Hart S, Burman R, Silber E, Saleem T, Edmonds P. Randomised controlled trial of a new palliative care service: compliance, recruitment and completeness of follow-up. BMC Palliative Care 2008;7(1):7. [DOI: 10.1186/1472-684X-7-7]

Higginson IJ, Hart S, Silber E, Burman R, Edmonds P. Symptom prevalence and severity in people severely affected by multiple sclerosis. Journal of Palliative Care 2006;22(3):158-65.

* Higginson IJ, McCrone P, Hart SR, Burman R, Silber E, Edmonds PM. Is short-term palliative care cost-effective in multiple sclerosis? A randomised phase II trial. Journal of Pain and Symptom Management 2009;38(6):816-26.

Higginson IJ, Vivat B, Silber E, Saleem T, Burman R, Hart S, et al. Study protocol: delayed intervention randomised controlled trial within the Medical Research Council (MRC) Framework to assess the effectiveness of a new palliative care service. BMC Palliative Care 2006;5(7). [DOI: 10.1186/1472-684X-5-7]

\section{Higginson 2014 \{published data only\}}

Bausewein C, Jolley C, Reilly C, Lobo P, Kelly J, Bellas H, et al. Development, effectiveness and cost-effectiveness of a new out-patient Breathlessness Support Service: study protocol of a phase III fast-track randomised controlled trial. BMC Pulmonary Medicine 2012;12(58):1-10. [DOI: 10.1186/1471-2466-12-58]

Dzingina MD, Reilly CC, Bausewein C, Jolley CJ, Moxham J, $\mathrm{McC}$ rone $\mathrm{P}$, et al. Variations in the cost of formal and informal health care for patients with advanced chronic disease and refractory breathlessness: a across-sectional secondary analysis. Palliative Medicine 2017;31(4):369-77.

* Higginson IJ, Bausewein C, Reilly CC, Gao W, Gysels M, Dzingina $\mathrm{M}$, et al. An integrated palliative and respiratory care service for patients with advanced disease and refractory breathlessness: a randomised controlled trial. Lancet Respiratory Medicine 2014;2(12):979-87.

\section{Hopp 2016 \{published data only\}}

Hopp FP, Zalenski RJ, Waselewsky D, Burn J, Camp J, Welch RD, et al. Results of a hospital-based palliative care intervention for patients with an acute exacerbation of chronic heart failure. Journal of Cardiac Failure 2016;22(12):1033-6.

\section{Janssens 2019 \{published data only\}}

* Janssens JP, Weber C, Herrmann FR, Cantero C, Pessina A, Matis $C$, et al. Can early introduction of palliative care limit intensive care, emergency and hospital admissions in patients with severe chronic obstructive pulmonary disease? A pilot randomized study. Respiration; International Review of Thoracic Diseases 2019;97(5):406-15. [PMID: 30650418]

Veron C, Pautex S, Weber C, Janssens JP, Cedraschi C. Recollection of participating in a trial: a qualitative study of patients with severe and very severe chronic obstructive pulmonary disease. PLOS One 2018;13(9):e0204701.

Weber C, Stirnemann J, Herrmann FR, Pautex S, Janssens JP. Can early introduction of specialised palliative care limit intensive care, emergency and hospital admissions in patients with severe and very severe COPD? A randomised study. BMC Palliative Care 2014;13(1):1-7.

\section{Jingfen 2017 \{published data only\}}

Jingfen RA, Tong ZH, Yanling RE, Yanli LI, Cuimin ZH. Influence of palliative care based on knowledge-belief-action model on the cancer-related fatigue and quality of life for patients with advanced lung cancer. Anti-Tumor Pharmacy 2017;7(1):124-8.

\section{Kane 1984 \{published data only\}}

Kane RL, Klein SJ, Bernstein L, Rothenberg R, Wales J. Hospice role in alleviating the emotional stress of terminal patients and their families. Medical Care 1985;23(3):189-97.

Kane RL, Klein SJ, Bernstein L, Rothenberg R. The role of hospice in reducing the impact of bereavement. Journal of Clinical Epidemiology 1986;39(9):735-42.

* Kane RL, Wales J, Bernstein L, Leibowitz A, Kaplan S. A randomised controlled trial of hospice care. Lancet 1984;323(8382):890-4. [PMID: 6143195]

Wales J, Kane R, Robbins S, Bernstein L, Krasnow R. UCLA hospice evaluation study. Methodology and instrumentation. Medical Care 1983;21(7):734-44.

\section{Lowther 2015 \{published data only\}}

Lowther K, Harding R, Simms V, Ahmed A, Ali Z, Gikaara N, et al. Active ingredients of a person-centred intervention for people on HIV treatment: analysis of mixed methods trial data. BMC Infectious Diseases 2018;18(1):27. 
Lowther K, Higginson IJ, Simms V, Gikaara N, Ahmed A, Ali Z, et al. A randomised controlled trial to assess the effectiveness of a nurse-led palliative care intervention for HIV positive patients on antiretroviral therapy: recruitment, refusal, randomisation and missing data. BMC Research Notes 2014;7(600). [DOI: 10.1186/1756-0500-7-600]

* Lowther K, Selman L, Simms V, Gikaara N, Ahmed A, Ali Z, et al. Nurse-led palliative care for HIV-positive patients taking antiretroviral therapy in Kenya: a randomised controlled trial. Lancet HIV 2015;2(8):e328-34.

Lowther K, Simms V, Selman L, Sherr I, Gwyther L, Kariuki H, et al. Treatment outcomes in palliative care: the TOPCare study. A mixed methods phase III randomised controlled trial to assess the effectiveness of a nurse-led palliative care intervention for HIV positive patients on antiretroviral therapy. BMC Infectious Diseases 2012;12(1):1-10. [DOI: 10.1186/1471-2334-12-288]

\section{Ma 2019 \{published data only\}}

* Ma J, Chi S, Buettner B, Pollard K, Muir M, Kolekar C, et al. Early palliative care consultation in the medical ICU: a cluster randomized crossover trial. Critical Care Medicine 2019;47(12):1707-15. [DOI: 10.1097/CCM.0000000000004016 ]

Ma J, Chi S, Buettner B, Pollard K, Muir M, Kolekar C, et al. Early palliative care consultation in the medical intensive care unit - a clustered randomized crossover trial (TH371B). Journal of Pain and Symptom Management 2019;57(2):396-7.

\section{McCaffrey 2013 \{published data only\}}

McCaffrey N, Agar M, Harlum J, Karnon J, Currow D, Eckermann S. Is home-based palliative care cost-effective? An economic evaluation of the Palliative Care Extended Packages at Home (PEACH) pilot. BMJ Supportive \& Palliative Care 2013;3(4):431-5.

\section{McCorkle 2015 \{published data only\}}

McCorkle R, Jeon S, Ercolano E, Lazenby M, Reid A Davies M, et al. An advanced practice nurse coordinated multidisciplinary intervention for patients with late-stage cancer: a cluster randomised trial. Journal of Palliative Medicine 2015;18(11):962-9.

\section{McWhinney 1994 \{published data only\}}

McWhinney IR, Bass MJ, Donner A. Evaluation of a palliative care service: problems and pitfalls. BMJ 1994;309(6965):1340-2. [PMID: 7532501]

\section{Mendoza-Galindo 2018 (abstract only) \{published data only\}} Mendoza-Galindo L, Arce-Salinas C, Ramirez-Morales R, AllendePerez S, Monreal-Carrillo E, Arzate-Mireles C, et al. Impact of early palliative care in hospitalisation and emergency room visits among breast cancer patients treated at Instituto Nacional De Cancerologia, Mexico City. Supportive Care in Cancer 2018;26(2 Suppl 1):S206-7.

Ramirez-Morales R, Arce-Salinas C, Mendoza-Galindo L, Allende-Perez S, Monreal-Carrillo E, Verastegui-Aviles E, et al. Cost reduction in hospitalization and emergency room visits associated to early palliative care intervention among breast cancer patients. Supportive Care in Cancer 2018;26(2 Suppl 1):S43.

Nottelmann 2018 \{published data only\}

* Nottelmann L, Groenvold M, Petersen MA, Vejlgaard T, Jensen LH. A single-center randomised clinical trial of palliative rehabilitation versus standard care alone in patients with newly diagnosed non-resectable cancer. Journal of Clinical Oncology 2018;36(34 Suppl):75.

Nottelmann L, Groenvold M, Vejlgaard TB, Petersen MA, Jensen $\mathrm{LH}$. A parallel-group randomized clinical trial of individually tailored, multidisciplinary, palliative rehabilitation for patients with newly diagnosed advanced cancer: the PalRehab study protocol. BMC Cancer 2017;17:560.

Nottelmann L, Jensen LH, Vejlgaard TB, Groenvold M. A new model of early, integrated palliative care: palliative rehabilitation for newly diagnosed patients with non-resectable cancer. Supportive Care in Cancer 2019;27(9):3291-300.

O'Riordan 2019 \{published data only\}

O'Riordan D, Rathfon M, Dracup K, Rabow M, Pantilat S, De Marco T. A randomised clinical trial of palliative care for people with heart failure: baseline characteristics (S750). Journal of Pain and Symptom Management 2014;47(2):496.

* O'Riordan D, Rathfon M, Joseph D, Hawgood J, Rabow M, Dracup K, et al. Feasibility of implementing a palliative care intervention for people with heart failure: learnings from a pilot randomised clinical trial. Journal of Palliative Medicine 2019;22(12):1583-8. [DOI: 10.1089/jpm.2018.0633]

\section{Ozcelik 2014 \{published data only\}}

Ozcelik H, Fadiloglu C, Karabulut B, Uyar M. Examining the effect of the case management model on patient results in the palliative care of patients with cancer. American Journal of Hospice \& Palliative Medicine 2014;31(6):655-64.

Rodin 2019 \{published data only\}

Rodin G, Malfitano C, Rydall A, Lo C, Schimmer AD, Marmar C, et al. Emotion and Symptom-focused Engagement (EASE): a randomised pilot trial of an integrated psychosocial and palliative care intervention for individuals with acute leukemia (AL). Journal of Cinical Oncology 2017;35(15_suppl):7041.

* Rodin G, Malfitano C, Rydall A, Schimmer A, Marmar CM, Mah K, et al. Emotion And Symptom-focused Engagement (EASE): a randomised phase II trial of an integrated psychological and palliative care intervention for patients with acute leukemia. Supportive Care in Cancer 2019;28:163-76. [DOI: https://doi.org/10.1007/s00520-019-04723-2]

Rogers 2017 \{published data only\}

Mentz RJ, Tulsky JA, Granger BB, Anstrom KJ, Adams PA, Dodson GC, et al. The palliative care in heart failure trial: rationale and design. American Heart Journal 2014;168(5):645-51.

* Rogers JG, Patel CB, Mentz RJ, Granger BB, Steinhauser KE, Fiuzat $M$, et al. Palliative care in heart failure: the PAL-HF

The effectiveness and cost-effectiveness of hospital-based specialist palliative care for adults with advanced illness and their caregivers 
randomised, controlled clinical trial. Journal of the American College of Cardiology 2017;70(3):331-41.

\section{Sidebottom 2015 \{published data only\}}

Sidebottom AC, Jorgenson A, Richards H, Kirven J, Sillah A. Inpatient palliative care for patients with acute heart failure: outcomes from a randomised trial. Journal of Palliative Medicine 2015;18(2):134-42.

\section{Solari 2018 \{published data only\}}

Giovannetti AM, Borreani C, Bianchi E, Giordano A, Cilia S, Cipollari S, et al. Participant perspectives of a home-based palliative approach for people with severe multiple sclerosis: a qualitative study. PLOS One 2018;13(7):e0200532.

Solari A, Giordano A, Grasso MG, Confalonieri P, Patti F, Lugaresi A, et al. Home-based palliative approach for people with severe multiple sclerosis and their carers: study protocol for a randomized controlled trial. Trials 2015;16:184.

* Solari A, Giordano A, Patti F, Grasso MG, Confalonieri P, Palmisano $L$, et al. Randomized controlled trial of a home-based palliative approach for people with severe multiple sclerosis. Multiple Sclerosis 2018;24(5):663-74.

\section{Tattersall 2014 \{published data only\}}

Tattersall M, Martin A, Devine R, Ryan J, Jansen J, Hastings L, et al. Early contact with palliative care services: a randomised trial of metastatic cancer patients with $<12$ months survival expectation. Journal of Palliative Care \& Medicine 2011;4(170):2. [DOI: 10.4172/2165-7386.1000170]

\section{Temel 2010 \{published data only\}}

Greer JA, Pirl WF, Jackson VA, Muzikansky A, Lennes IT, Heist RS, et al. Effect of early palliative care on chemotherapy use and end-of-life care in patients with metastatic non-small-cell lung cancer. Journal of Clinical Oncology 2012;30(4):394-400.

Greer JA, Tramontano AC, McMahon PM, Pirl WF, Jackson VA, El-Jawahri A, et al. Cost analysis of a randomised trial of early palliative care in patients with metastatic non-small-cell lung cancer. Journal of Palliative Medicine 2016;19(8):842-8.

Jacobsen J, Jackson V, Dahlin C, Greer J, Perez-Cruz P, Billings JA, et al. Components of early outpatient palliative care consultation in patients with metastatic non-small cell lung cancer. Journal of Palliative Medicine 2011;14(4):459-64.

Nipp RD, Greer J, Traeger L, Gallagher ER, Park ER, Jackson VA, et al. Which patients experience improved quality of life (QOL) and mood from early palliative care (PC)? Journal of Clinical Oncology 2014;32(31):16.

Nipp RD, Greer JA, El-Jawahri A, Traeger L, Gallagher ER, Park ER, et al. Age and gender moderate the impact of early palliative care in metastatic non-small cell lung cancer. Oncologist 2016;21(1):119-26.

Pirl WF, Greer JA, Traeger L, Jackson V, Lennes IT, Gallagher ER, et al. Depression and survival in metastatic non-small-cell lung cancer: effects of early palliative care. Journal of Clinical Oncology 2012;30(12):1310-5.
Temel JS, Greer J, Gallagher E, Admane S, Pirl WF, Jackson V, et al. Effect of early palliative care (PC) on quality of life (QOL), aggressive care at the end-of-life (EOL), and survival in stage IV NSCLC patients: results of a phase III randomised trial. Journal of Clinical Oncology 2010;28(15):7509.

Temel JS, Greer JA, Admane S, Gallagher ER, Jackson VA, Lynch TJ, et al. Longitudinal perceptions of prognosis and goals of therapy in patients with metastatic non-small-cell lung cancer: results of a randomised study of early palliative care. Journal of Clinical Oncology 2011;29(17):2319-26.

* Temel JS, Greer JA, Muzikansky A, Gallagher ER, Admane S, Jackson VA, et al. Early palliative care for patients with metastatic non-small-cell lung cancer. New England Journal of Medicine 2010;363(8):733-42.

Yoong J, Park ER, Greer JA, Jackson VA, Gallagher ER, Pirl WF, et al. Early palliative care in advanced lung cancer: a qualitative study. JAMA Internal Medicine 2013;173(4):283-90.

\section{Temel 2017 \{published data only\}}

Temel JS, Greer JA, El-Jawahri A, Pirl WF, Park ER, Jackson VA, et al. Effects of early integrated palliative care in patients with lung and GI cancer: a randomised clinical trial. Journal of Clinical Oncology 2017;35(8):834-41.

Vanbutsele 2018 \{published data only\}

* Vanbutsele G, Pardon K, Van Belle S, Surmont V, De Laat M, Colman R, et al. Effect of early and systematic integration of palliative care in patients with advanced cancer: a randomised controlled trial. Lancet Oncology 2018;19(3):394-404.

Vanbutsele G, Van Belle S, De Laat M, Surmont V, Geboes K, Eecloo K, et al. The systematic early integration of palliative care into multidisciplinary oncology care in the hospital setting (IPAC), a randomised controlled trial: the study protocol. BMC Health Services Research 2015;15(554). [DOI: 10.1186/ s12913-015-1207-3]

\section{Wallen 2012 \{published data only\}}

Slota C, Ulrich CM, Miller-Davis C, Baker K, Wallen GR. Qualitative inquiry: a method for validating patient perceptions of palliative care while enrolled on a cancer clinical trial. BMC Palliative Care 2014;13(1):43. [DOI: 10.1186/1472-684X-13-43]

* Wallen GR, Baker K, Stolar M, Miller-Davis C, Ames N, Yates J, et al. Palliative care outcomes in surgical oncology patients with advanced malignancies: a mixed methods approach. Quality of Life Research 2012;21(3):405-15.

\section{Woo 2019 \{published data only\}}

Woo SM, Song MK, Lee M, Joo J, Kim DH, Kim JH, et al. Effect of early management on pain and depression in patients with pancreatobiliary cancer: a randomized clinical trial. Cancers 2019;11(1):79. [PMID: 30641928]

\section{References to studies excluded from this review}

Bakitas 2017 \{published data only\}

Bakitas M, Dionne-Odom JN, Pamboukian SV, Tallaj J, Kvale E, Swetz KM, et al. Engaging patients and families to create a

The effectiveness and cost-effectiveness of hospital-based specialist palliative care for adults with advanced illness and their caregivers 
feasible clinical trial integrating palliative and heart failure care: results of the ENABLE CHF-PC pilot clinical trial. BMC Palliative Care 2017;16(1):45.

\section{Berglund 2019 \{published data only\}}

Berglund K, Chai E, Moreno J, Reyna MA, Gelfman L. Development of a social work-led primary palliative care model in hospital medicine (FR481C). Journal of Pain and Symptom Management 2019;57(2):436.

\section{Bonsignore 2018 \{published data only\}}

Bonsignore L, Bloom N, Steinhauser K, Nichols R, Allen T, Twaddle $\mathrm{M}$, et al. Evaluating the feasibility and acceptability of a telehealth program in a rural palliative care population: TapCloud for palliative care. Journal of Pain and Symptom Management 2018;56(1):7-14.

\section{Brims 2019 \{published data only\}}

Brims F, Gunatilake S, Lawrie I, Marshall L, Fogg C, Qi C, et al. Early specialist palliative care on quality of life for malignant pleural mesothelioma: a randomised controlled trial. Thorax 2019;74(4):354-61.

\section{Do 2017 \{published data only\}}

Do Carmo TM, Paiva BS, De Oliveira CZ, Nascimento MS, Paiva CE. The feasibility and benefit of a brief psychosocial intervention in addition to early palliative care in patients with advanced cancer to reduce depressive symptoms: a pilot randomised controlled clinical trial. BMC Cancer 2017;17(1):564.

\section{Fischer 2019 \{published data only\}}

Fischer S, Golub M, Plata A, Retrum J, Nielsen E, Lahoff D, et al. Integrating palliative care social workers into sub-acute settings: feasibility of the ALIGN Intervention Trial (S825). Journal of Pain and Symptom Management 2019;57(2):495.

\section{Hanks 2002 \{published data only\}}

Hanks GW, Robbins M, Sharp D, Forbes K, Done K, Peters TJ, et al. The imPaCT study: a randomised controlled trial to evaluate a hospital palliative care team. British Journal of Cancer 2002;87(7):733-9.

\section{Hartman 2019 \{published data only\}}

Hartman A, Ashby M, Buechel V, Williams M, Trantum L, Karlekar M, et al. Abstract TP348: early palliative care consultation in acute stroke. Stroke 2019;50(Suppl 1):ATP348.

\section{Hoek 2017 \{published data only\}}

Hoek PD, Schers HJ, Bronkhorst EM, Vissers KC, Hasselaar Jeroen GJ. The effect of weekly specialist palliative care teleconsultations in patients with advanced cancer - a randomised clinical trial. BMC Medicine 2017;15(1):119.

\section{Jordhoy 2001 \{published data only\}}

Jordhoy MS, Fayers P, Loge JH, Ahlner-Elmqvist M, Kaasa S. Quality of life in palliative cancer care: results from a cluster randomised trial. Journal of Clinical Oncology 2001;19(18):3884-94. [PMID: 11559726]

\section{Kimbell 2018 \{published data only\}}

Kimbell B, Murray SA, Byrne H, Baird A, Hayes PC, MacGilchrist A, et al. Palliative care for people with advanced liver disease: a feasibility trial of a supportive care liver nurse specialist. Palliative Medicine 2018;32(5):919-29.

\section{Maltoni 2016 \{published data only\}}

Maltoni M, Scarpi E, Dall'Agata M, Zagonel V, Berte R, Ferrari D, et al. Systematic versus on-demand early palliative care: results from a multicentre, randomised clinical trial. European Journal of Cancer 2016;65:61-8.

\section{Nordly 2019 \{published data only\}}

Nordly M, Benthien KS, Vadstrup ES, Kurita GP, Von HeymannHoran $A B$, Von Der Maase $H$, et al. Systematic fast-track transition from oncological treatment to dyadic specialised palliative home care: DOMUS - a randomised clinical trial. Palliative Medicine 2019;33(2):135-49.

\section{O'Mahony 2017 \{published data only\}}

O'Mahony S, Johnson TJ, Amer S, McHugh ME, McHenry J, Fosler L, et al. Integration of palliative care advanced practice nurses Into intensive care unit teams. American Journal of Hospice \& Palliative Care 2017;34(4):330-4.

\section{Pantilat 2010 \{published data only\}}

Pantilat SZ, O'Riordan DL, Dibble SL, Landefeld CS. Hospital-based palliative medicine consultation: a randomised controlled trial. Archives of Internal Medicine 2010;170(22):2038-40. [PMID: 21149765]

\section{Rabow 2004 \{published data only\}}

Rabow MW, Dibble SL, Pantilat SZ, McPhee SJ. The comprehensive care team: a controlled trial of outpatient palliative medicine consultation. Archives of Internal Medicine 2004;164(1):83-91.

\section{Scarpi 2019 \{published data only\}}

Scarpi E, Dall'Agata M, Zagonel V, Gamucci T, Berte R, Sansoni E, et al. Systematic vs. on-demand early palliative care in gastric cancer patients: a randomised clinical trial assessing patient and healthcare service outcomes. Supportive Care in Cancer 2019;27(7):2425-34.

\section{Schenker 2018 \{published data only\}}

Schenker Y, Bahary N, Claxton R, Childers J, Chu E, Kavalieratos $D$, et al. A pilot trial of early specialty palliative care for patients with advanced pancreatic cancer: challenges encountered and lessons learned. Journal of Palliative Medicine 2018;21(1):28-36.

\section{Spatuzzi 2017 \{published data only\}}

Spatuzzi R, Giulietti MV, Ricciuti M, Merico F, Meloni C, Fabbietti P, et al. Quality of life and burden in family caregivers of patients with advanced cancer in active treatment settings and hospice care: a comparative study. Death Studies 2017;41(5):276-83.

\section{Sussman 2018 \{published data only\}}

Sussman J, Bainbridge D, Whelan TJ, Brazil K, Parpia S, Wiernikowski J, et al. Evaluation of a specialised oncology

The effectiveness and cost-effectiveness of hospital-based specialist palliative care for adults with advanced illness and their caregivers 
nursing supportive care intervention in newly diagnosed breast and colorectal cancer patients following surgery: a cluster randomised trial. Supportive Care in Cancer 2018;26(5):1533-41.

\section{Ullrich 2017 \{published data only\}}

Ullrich A, Ascherfeld L, Marx G, Bokemeyer C, Bergelt C, Oechsle K. Quality of life, psychological burden, needs, and satisfaction during specialized inpatient palliative care in family caregivers of advanced cancer patients. BMC Palliative Care 2017;16(1):31.

\section{Veronese 2017 \{published data only\}}

Veronese S, Gallo G, Valle A, Cugno C, Chio A, Calvo A, et al. Specialist palliative care improves the quality of life in advanced neurodegenerative disorders: NE-PAL, a pilot randomised controlled study. BMJ Supportive \& Palliative Care 2017;7(2):164-72.

\section{Wong 2016 \{published data only\}}

Wong FK, Ng AY, Lee PH, Lam PT, Ng JS, Ng NH, et al. Effects of a transitional palliative care model on patients with endstage heart failure: a randomised controlled trial. Heart 2016;102(14):1100-8.

\section{Yang 2018 \{published data only\}}

Yang GM, Teo I, Neo SH, Tan D, Cheung YB. Pilot randomised phase II trial of the enhancing quality of life in patients (EQUIP) intervention for patients with advanced lung cancer. American Journal of Hospice \& Palliative Care 2018;35(8):1050-6.

\section{Zimmermann 2014 \{published data only\}}

Zimmermann C, Swami N, Krzyzanowska M, Hannon B, Leighl N, Oza A, et al. Early palliative care for patients with advanced cancer: a cluster-randomised controlled trial. Lancet 2014;383(9930):1721-30. [PMID: 24559581]

\section{References to studies awaiting assessment}

\section{Aljohani 2015 \{published data only\}}

Aljohani A. Early interdisciplinary palliative care for patients with non-small-cell lung cancer. In: 20th Congress of the Asian Pacific Society of Respirology, Kuala Lumpur (http:// www.apsresp.org/congress/apsr2015/ oral-presentations.htm). Vol. 75. 2015

\section{References to ongoing studies}

\section{ACTRN12618001045202 \{published data only\}}

ACTRN12618001045202. Collaborative Supportive Care for Patients with Complex Chronic Disease [Collaborative supportive care for life-limiting chronic conditions: A prospective randomised controlled study comparing supportive care with standard care]. anzctr.org.au/Trial/Registration/ TrialReview.aspx?id=375135\&isReview=true (first received 1 June 2018)

\section{CHICTR1800014482 \{published data only\}}

CHICTR1800014482. Palliative care in end-stage heart failure and application of deep learning [Palliative care in endstage heart failure and application of deep learning]. http:// www.chictr.org.cn/com/25/showprojen.aspx?proj=23243 (first received 16 January 2016).

Courtright 2016 \{published data only\}

Courtright KR, Madden V, Gabler NB, Cooney E, Small DS, Troxel A, et al. Rationale and design of the randomised evaluation of default access to palliative services (REDAPS) trial. Annals of the American Thoracic Society 2016;13(9):1629-39.

DRKS00013922 \{published data only\}

DRKS00013922. Early palliative care for patients with symptomatic heart failure. https://www.drks.de/drks_web/ PdfDownstreamServlet?ID=DRKS00013922\&LOCALE=en (first received 16 March 2018).

\section{Graney 2019 \{published data only\}}

Graney BA, Au DH, Baron AE, Cheng A, Combs SA, Glorioso TJ, et al. Advancing Symptom Alleviation with Palliative Treatment (ADAPT) trial to improve quality of life: a study protocol for a randomized clinical trial. Trials 2019;20(1):355. [DOI: 10.1186/ s13063-019-3417-1]

\section{Hutt 2018 \{published data only\}}

Hutt E, Da Silva A, Bogart E, Le Lay-Diomande S, Pannier D, Delaine-Clisant $S$, et al. Impact of early palliative care on overall survival of patients with metastatic upper gastrointestinal cancers treated with first-line chemotherapy: a randomised phase III trial. BMJ Open 2018;8(1):e015904.

IRCT20160521027993N1 \{published data only\}

IRCT20160521027993N1. Palliative care and self efficacy in elderly with CHF [The effect of palliative care education on self efficacy of elderly with chronic heart failure]. https://en.irct.ir/ trial/30889 (first received 28 June 2018).

\section{IRCT20160914029817N6 \{published data only\}}

IRCT20160914029817N6. Effect of non-drug palliative care on quality of life in patients with chronic obstructive pulmonary disease [Effect of non-drug palliative care on quality of life in patients with chro]. https://en.irct.ir/trial/31684 (first received 15 June 2018).

\section{IRCT20180531039925N1 \{published data only\}}

Vashani HB. The effect of palliative care program on the quality of life of children with leukemia [The effect of palliative care program on the quality of life of children with leukemia]. https://en.irct.ir/trial/31751 (first received 18 August 2018).

\section{Kluger 2019 \{published data only\}}

Kluger BM, Katz M, Galifianakis N, Pantilat SZ, Kutner JS, Sillau S, et al. Does outpatient palliative care improve patientcentered outcomes in Parkinson's disease: rationale, design, and implementation of a pragmatic comparative effectiveness trial. Contemporary Clinical Trials 2019;79:28-36.

\section{Matsumoto 2016 \{published data only\}}

Matsumoto Y. Early specialised palliative care in Japan: a feasibility study. Annals of Oncology 2016;27(Suppl 7):mdw466

The effectiveness and cost-effectiveness of hospital-based specialist palliative care for adults with advanced illness and their caregivers 


\section{NCT01828775 \{published data only\}}

Responsible party: City of Hope Medical Centre. Palliative Care Intervention in Improving Quality of Life, Psychological Distress, and Communication in Patients With Solid Tumors Receiving Treatment [Integration of palliative care for cancer patients on phase I trials]. https://clinicaltrials.gov/ct2/show/NCT01828775 (first received 11 April 2013).

\section{NCT01846520 \{published data only\}}

Responsible party: City of Hope Medical Centre. Family Caregiver Palliative Care Intervention in Supporting Caregivers of Patients With Stage II-IV Gastrointestinal, Gynecologic, Urologic and Lung Cancers [A Randomized Trial of a Family Caregiver Palliative Care Intervention]. https://clinicaltrials.gov/ ct2/show/NCT01846520 (first received 3 May 2013).

\section{NCT01983956 \{published data only\}}

Responsible party: University Hospital Inselspital. A structured early palliative care intervention for patients with advanced cancer - a randomised controlled trial with a nested qualitative study (SENS Trial) (SENS) [A Structured Early Palliative Care Intervention for Patients With Advanced Cancer - a Randomized Controlled Trial With a Nested Qualitative Study (SENS Trial)]. https://clinicaltrials.gov/ct2/show/NCT01983956 (first received 14 November 2013).

\section{NCT02139917 \{published data only\}}

Responsible party: Bonnie, TAM. Effects of a transitional palliative care model on patients with end-stage renal failure (ESRF) [Effects of a Transitional Palliative Care Model on Patients With ESRF]. https://clinicaltrials.gov/ct2/show/ NCT02139917 (first received 16 May 2014).

\section{NCT02308865 \{published data only\}}

Responsible party: University Hospital, Lille. Early Palliative Care in Metastatic Lung Cancer in Northern France (IMPAQ) [Impact of early palliative care on quality of life and survival of patients with non-small-cell metastatic lung cancer in Northern France]. https://clinicaltrials.gov/ct2/show/NCT02308865 (first received 4 December 2014).

\section{NCT02375997 \{published data only\}}

Responsible party: Shen, L. Early Palliative Care in Metastatic Esophageal Squamous Carcinoma (ESCC) and Gastric Cancer [Early palliative care with standard oncology care versus standard oncology care alone in Metastatic Esophageal Squamous Carcinoma (ESCC) and gastric cancer]. https:// clinicaltrials.gov/ct2/show/NCT02375997 (first received 3 March 2015).

\section{NCT02533921 \{published data only\}}

Responsible party: University of Colorado, Denver. Does outpatient palliative care improve patient-centered outcomes in Parkinson's Disease? [Does outpatient palliative care improve patient-centered outcomes in Parkinson's Disease?]. https:// clinicaltrials.gov/ct2/show/NCT02533921 (first received 27 August 2015)

\section{NCT02543541 \{published data only\}}

Responsible party: Case Comprehensive Cancer Centre. A Pilot Study of Structured Palliative Care for Patients Enrolled on
Phase I Clinical Trials [A Pilot Study of Structured Palliative Care for Patients Enrolled on Phase I Clinical Trials]. https:// clinicaltrials.gov/ct2/show/NCT02543541 (first received 7 September 2015).

\section{NCT02631811 \{published data only\}}

Responsible party: Hospices Civils de Lyon. Early Palliative Care in Patient With Acute Leukaemia (Pablo Hemato) [Impact on Quality of Life of an Early Management Supportive Care of Patients With Acute Leukemia in First Relapse.]. https:// clinicaltrials.gov/ct2/history/NCT02631811?V_3=View (first received 10 December 2015).

\section{NCT02712229 \{published data only\}}

Responsible party: Schenker, Y. A Primary Palliative Care Intervention for Patients With Advanced Cancer (CONNECT) [A Cluster Randomized Trial of a Primary Palliative Care Intervention (CONNECT) for Patients With Advanced Cancer]. https://clinicaltrials.gov/ct2/show/NCT02712229 (first received 18 March 2016).

\section{NCT02719938 \{published data only\}}

Responsible party: University of North Carolina, Chapel Hill. Triggered Palliative Care for Advanced Dementia [Triggered Palliative Care for Advanced Dementia]. https:// clinicaltrials.gov/ct2/show/NCT02719938 (first received 25 March 2016).

\section{NCT02786524 \{published data only\}}

Responsible party: Harris, K. Effect of Outpatient Symptom Management on Gynecologic Oncology Patients Receiving Chemotherapy [A Randomized Study to Evaluate the Effect of Outpatient Symptom Management on Symptom Burden in Advanced Stage or Recurrent Gynecologic Oncology Patients Receiving Chemotherapy]. https://clinicaltrials.gov/ct2/show/ NCT02786524 (first received 1 June 2016).

\section{NCT02868112 \{published data only\}}

Responsible party: Nipp, R. Transdisciplinary Intervention Integrating Geriatric And Palliative Care With Oncology Care For Older Adults With Cancer [Pilot study of a transdisciplinary intervention integrating geriatric and palliative care with oncology care for older adults with cancer]. https:// clinicaltrials.gov/ct2/show/NCT02868112 (first received 16 August 2016).

\section{NCT02929966 \{published data only\}}

Responsible party: Nava, S. Palliative Care in Pulmonary Fibrosis (PULFIP) [Effect of Palliative Care in Patients With End Stage Pulmonary Fibrosis: a Randomized Control Study]. https://clinicaltrials.gov/ct2/show/NCT02929966 (first received 11 October 2016).

\section{NCT02975869 \{published data only\}}

Responsible party: El-Jawahri, A. A Collaborative Palliative and Oncology Care Model for Patients With Acute Myeloid Leukemia [Randomized Trial of a Collaborative Palliative and Oncology Care Model for Patients With Acute Myeloid Leukemia]. https:// clinicaltrials.gov/ct2/show/NCT02975869 (first received 29 November 2016)

The effectiveness and cost-effectiveness of hospital-based specialist palliative care for adults with advanced illness and their caregivers 


\section{NCT03022630 \{published data only\}}

Responsible party: Bernard, G. Palliative Care for Non-Malignant Diseases (COMPASS Trial) (COMPASS) [The Creation of Models for Palliative Assessments to Support Severe Illness (COMPASS) Investigation: Testing Early and Ongoing Implementation of Palliative Care for Incurable Non-malignant Diseases]. https:// clinicaltrials.gov/ct2/show/NCT03022630 (first received 22 November 2019).

\section{NCT03088202 \{published data only\}}

Loge, JH. PALLION - PALLiative Care In ONcology (PALLION) [PALLION - PALLiative Care In ONcology - a Cluster-randomized Trial to Improve the Care for Cancer Patients With a Short Life Expectancy]. https://clinicaltrials.gov/ct2/show/NCT03088202 (first received 23 March 2017).

\section{NCT03170466 \{published data only\}}

Responsible party: Kavalieratos, D. Primary Palliative Care in Heart Failure: A Pilot Trial [Primary Palliative Care in Heart Failure: A Pilot Trial]. https://clinicaltrials.gov/ct2/show/ NCT03170466 (first received 31 May 2017).

\section{NCT03181854 \{published data only\}}

Responsible party: Yun, YH. Randomized Controlled Trial of Integrated Early Palliative Care [Randomized Controlled Trial of Integrated Early Palliative Care for Advanced Cancer Patients]. https://clinicaltrials.gov/ct2/show/NCT03181854 (first received 9 June 2017).

\section{NCT03229343 \{published data only\}}

Responsible party: Assistance Publique - Hôpitaux de Paris. Impact of a Systematic Palliative Care on Quality of Life, in Advanced Idiopathic Pulmonary Fibrosis. (PALIF) [Impact of a Systematic Palliative Care on Quality of Life, in Advanced Idiopathic Pulmonary Fibrosis (IPF). A Randomized Multi-center Trial.]. https://clinicaltrials.gov/ct2/show/NCT03229343 (first received 25 July 2017 ).

\section{NCT03310918 \{published data only\}}

Responsible party: El-Jawahri, A. Randomized Trial of a Collaborative Palliative and Leukemia Care Model for Patients With Acute Myeloid Leukemia Receiving Non-Intensive Therapy [A Collaborative Palliative and Leukemia Care Model for Patients With AML Receiving Non-Intensive Therapy]. https:// clinicaltrials.gov/ct2/show/NCT03310918 (first received 16 October 2016).

\section{NCT03456323 \{published data only\}}

Responsible party: Baldwin, M. Post-ICU Palliative Care Intervention (PIPCI) Trial [Post-ICU Palliative Care Consultation Intervention Pilot Trial in Older Survivors of Acute Respiratory Failure]. https://clinicaltrials.gov/ct2/show/NCT03456323 (first received 7 March 2018).

\section{Weber 2014 \{published data only\}}

Weber C, Stirnemann J, Herrmann FR, Pautex S, Janssens JP. Can early introduction of specialised palliative care limit intensive care, emergency and hospital admissions in patients with severe and very severe COPD? A randomised study. BMC Palliative Care 2014;13(1):1-7. [DOI: 10.1186/1472-684X-13-47]

\section{Additional references}

\section{Allen 2008}

Allen RS, Hilgeman MM, Ege MA, Shuster JL Jr, Burgio LD. Legacy activities as interventions approaching the end of life. Journal of Palliative Medicine 2008;11(7):1029-38.

\section{Aoun 2017}

Aoun SM, Rumbold B, Howting D, Bolleter A, Breen LJ. Bereavement support for family caregivers: the gap between guidelines and practice in palliative care. PLOS ONE 2017;12(10):e0184750.

\section{Bajwah 2017}

Bajwah S, Yi D, Grande G, Todd C, Costantini M, Murtagh FE, et al. The effectiveness and cost-effectiveness of inpatient specialist palliative care in acute hospitals for adults with advanced illness and their caregivers. Cochrane Database of Systematic Reviews 2017, Issue 9. Art. No: CD012780. [DOI: 10.1002/14651858.CD012780]

\section{Baker 1981}

Baker TH. A cost analysis of three hospice programs. Los Angeles: Kaiser Permanente Medical Care Program 1981.

\section{Bloom 2016}

Bloom DE, Luca DL. The global demography of aging: facts, explanations, future. In: Piggott J, Woodland A, editors(s). Handbook of the Economics of Population Aging. Oxford: Elsevier, 2016:3-56.

\section{Breen 2014}

Breen LJ, Aoun SM, O'Connor M, Rumbold B. Bridging the gaps in palliative care bereavement support: an international perspective. Death Studies 2014;38(1):54-61.

\section{Broad 2013}

Broad JB, Gott M, Kim H, Boyd M, Chen H, Connolly MJ. Where do people die? An international comparison of the percentage of deaths occurring in hospital and residentia aged care settings in 45 populations, using published and available statistics. International Journal of Public Health 2013;58(2):257-67.

\section{Burge 2012}

Burge F, Lawson B, Mitchell G. How to move to a palliative approach to care for people with multimorbidity. $B M J$ 2012;345:e6324.

\section{C-TAC 2015}

Coalition to Transform Advanced Care (C-TAC). Policy agenda: options to transform advanced care. www.thectac.org/wpcontent/uploads/2015/02/C_TAC-Policy-Agenda.pdf (accessed 31 January 2017).

\section{Cancer Care Ontario 2016}

Cancer Care Ontario. Symptom management guides. cancercare.on.ca/cms/oneaspxportalld=1377\&\%20pageld= \%2058189 (accessed 31 May 2020).

The effectiveness and cost-effectiveness of hospital-based specialist palliative care for adults with advanced illness and their caregivers 


\section{CAPC 2018}

Centre to Advance Palliative Care. Growth of palliative care in U.S hospitals: 2018 snapshot (2000 - 2016). Centre to Advance Palliative Care 2018.

\section{Cohen 1988}

Cohen J. Statistical Power Analysis for the Behavioral Sciences. 2nd edition. New York: Lawrence Erlbaum Associates, 1988.

\section{Cohen 2001}

Cohen SR, Boston P, Mount BM, Porterfield P. Changes in quality of life following admission to palliative care units. Palliative Medicine 2001;15(5):363-71. [PMID: 11591087]

\section{Crown 2013}

Crown. More care, less pathway: a review of the Liverpool Care Pathway. www.gov.uk/government/uploads/system/uploads/ attachment_data/file/212450/Liverpool_Care_Pathway.pdf (accessed 14 May 2019).

\section{Curran 2012}

Curran GM, Bauer M, Mittman B, Pyne JM, Stetler C. Effectiveness-implementation hybrid designs: combining elements of clinical effectiveness and implementation research to enhance public health impact. Medical Care 2012;50(3):217-26.

\section{Dalgaard 2014}

Dalgaard KM, Bergenholtz H, Nielsen ME, Timm H. Early integration of palliative care in hospitals: a systematic review on methods, barriers, and outcome. Palliative \& Supportive Care 2014;12(6):495-513. [PMID: 24621947]

\section{Daveson 2014}

Daveson BA, Harding R, Shipman C, Mason BL, Epiphaniou E, Higginson IJ, et al. The real-world problem of care coordination: a longitudinal qualitative study with patients living with advanced progressive illness and their unpaid caregivers. PLOS ONE 2014;9(5):e95523.

\section{Deeks 2011}

Deeks JJ, Higgins JPT, Altman DG: Cochrane Statistical Methods Group. Chapter 9: Analysing data and undertaking metaanalyses. In: Higgins JP, Green S, editor(s). Cochrane Handbook of Systematic Reviews of Interventions Version 5.1.0 (updated 2011). The Cochrane Collaboration, 2011. Available from handbook.cochrane.org.

\section{Dixon 2015}

Dixon J, King D, Matosevic T, Clark M, Knapp M. Equity in the provision of palliative care in the UK: review of evidence. London School of Economics and Political Science. 2015.

\section{DoH 2008}

Department of Health. End of life care strategy: promoting high quality care for adults at the end of their life. www.gov.uk/ government/uploads/system/uploads/attachment_data/ file/136431/End_of_life_strategy.pdf (accessed 31 January 2017).

\section{Doyle 1998}

Doyle, D. The provision of palliative care. In: Doyle D, Hanks GW, MacDonald N, editors(s). Oxford Textbook of Palliative Medicine. 2nd edition. New York: Oxford University Press, 1998:41-53.

\section{Drummond 1996}

Drummond MF, Jefferson TO. Guidelines for authors and peer reviewers of economic submissions to the BMJ.BMJ 1996;313(7052):275-83.

\section{Etkind 2017}

Etkind SN, Bone AE, Gomes B, Lovell N, Evans CJ, Higginson IJ, et al. How many people will need palliative care in 2040? Past trends, future projections and implications for services. BMC Medicine 2017;15(1):102.

\section{Evans 2014}

Evans CJ, Ho Y, Daveson BA, Hall S, Higginson IJ, Gao W, et al. Place and cause of death in centenarians: a population-based observational study in England, 2001 to 2010. PLOS Medicine 2014;11(6):e1001653.

\section{Evans 2019}

Evans CJ, Ison L, Ellis-Smith C, Nicholson C, Costa A, Oluyase AO, et al. Service delivery models to maximise quality of life for older people at the end of life: a rapid review. Milbank Quarterly 2019;97(1):113-75.

\section{Evers 2005}

Evers S, Goossens M, De Vet H, Van Tulder M, Ament A. Criteria list for assessment of methodological quality of economic evaluations: consensus on health economic criteria. International Journal of Technology Assessment in Health Care 2005;21(2):240-5.

\section{Gaertner 2017}

Gaertner J, Siemens W, Meerpohl JJ, Antes G, Meffert C, Xander C, et al. Effect of specialist palliative care services on quality of life in adults with advanced incurable illness in hospital, hospice, or community settings: systematic review and meta-analysis. BMJ 2017;357:j2925.

\section{Gomes 2012}

Gomes B, Higginson IJ, Calanzani N, Cohen J, Deliens L, Daveson BA, et al. Preferences for place of death if faced with advanced cancer: a population survey in England, Flanders, Germany, Italy, the Netherlands, Portugal and Spain. Annals of Oncology 2012;23(8):2006-15. [PMID: 22345118]

\section{Gomes 2013}

Gomes B, Calanzani N, Curiale V, McCrone P, Higginson IJ. Effectiveness and cost-effectiveness of home palliative care services for adults with advanced illness and their caregivers. Cochrane Database of Systematic Reviews 2013, Issue 6. Art. No: CD007760. [DOI: 10.1002/14651858.CD007760.pub2]

\section{GRADEpro GDT 2015 [Computer program]}

McMaster University (developed by Evidence Prime) GRADEpro GDT. Version accessed 31 January 2017. Hamilton (ON): McMaster University (developed by Evidence Prime), 2015. Available at gradepro.org.

The effectiveness and cost-effectiveness of hospital-based specialist palliative care for adults with advanced illness and their caregivers 


\section{Grande 2017}

Grande GE, Austin L, Ewing G, Leary N, Roberts C. Assessing the impact of a Carer Support Needs Assessment Tool (CSNAT) intervention in palliative home care: a stepped wedge cluster trial. BMJ Supportive \& Palliative Care 2017;7(3):326.

\section{Guyatt 1987}

Guyatt GH, Berman LB, Townsend M, Pugsley SO, Chambers LW. A measure of quality of life for clinical trials in chronic lung disease. Thorax 1987;42(10):773-8.

\section{Guyatt 2011}

Guyatt G, Oxman AD, Akl EA, Kunz R, Vist G, Brozek J, et al. GRADE guidelines: 1 . Introduction - GRADE evidence profiles and summary of findings tables. Journal of Clinical Epidemiology 2011;64(4):383-94. [PMID: 21195583]

\section{Guyatt 2013}

Guyatt G, Oxman AD, Sultan S, Brozek J, Glasziou P, AlonsoCoello P, et al. GRADE guidelines: 11 . Making an overall rating of confidence in effect estimates for a single outcome and for all outcomes. Journal of Clinical Epidemiology 2013;66(2):151-7.

\section{Gysels 2004}

Gysels M, Richardson A, Higginson IJ. Communication training for health professionals who care for patients with cancer: a systematic review of effectiveness. Supportive Care in Cancer 2004;12(10):692-700.

\section{Harding 2012}

Harding R, List S, Epiphaniou E, Jones H. How can informal caregivers in cancer and palliative care be supported? An updated systematic literature review of interventions and their effectiveness. Palliative Medicine 2012;26(1):7-22.

\section{Harris 2013}

Harris I, Murray SA. Can palliative care reduce futile treatment? A systematic review. BMJ Supportive \& Palliative Care 2013;3(4):389-98.

\section{Haun 2017}

Haun MW, Estel S, Rücker G, Friederich HC, Villalobos M, Thomas M, et al. Early palliative care for adults with advanced cancer. Cochrane Database of Systematic Reviews 2017, Issue 6. Art. No: CD011129. [DOI: 10.1002/14651858.CD011129.pub2]

\section{Higgins 2011}

Higgins JPT, Deeks JJ, Altman DG, Cochrane Statistical Methods Group. Chapter 16: Special topics in statistics. In: Higgins JP, Green S, editor(s). Cochrane Handbook for Systematic Reviews of Interventions Version 5.1.0 (updated March 2011). The Cochrane Collaboration, 2011. Available from handbook.cochrane.org.

\section{Higgins 2011a}

Higgins JP, Green S, editor(s). Cochrane Handbook for Systematic Reviews of Interventions Version 5.1.0 (updated March 2011). The Cochrane Collaboration, 2011. Available from handbook.cochrane.org.

\section{Higgins 2011b}

Higgins JPT, Altman DG, Sterne JAC, Cochrane Statistical Methods Group and the Cochrane Bias Methods Group. Chapter 8: Assessing risk of bias in included studies. In: Higgins JP, Green $\mathrm{S}$, editor(s). Cochrane Handbook for Systematic Reviews of Interventions Version 5.1.0 (updated March 2011). The Cochrane Collaboration, 2011. Available from handbook.cochrane.org.

\section{Higginson 2002}

Higginson IJ, Finlay I, Goodwin DM, Cook AM, Hood K, Edwards AG, et al. Do hospital-based palliative teams improve care for patients or families at the end of life? Journal of Pain and Symptom Management 2002;23(2):96-106.

\section{Higginson 2003}

Higginson IJ, Finlay IG, Goodwin DM, Hood K, Edwards AG, Cook $A$, et al. Is there evidence that palliative care teams alter end-of-life experiences of patients and their caregivers? Journal of Pain and Symptom Management 2003;25(2):150-68.

\section{Higginson 2010}

Higginson IJ, Evans CJ. What is the evidence that palliative care teams improve outcomes for cancer patients and their families? Cancer Journal 2010;16(5):423-35.

\section{Hudson 2005}

Hudson PL, Aranda S, Hayman-White K. A psycho-educational intervention for family caregivers of patients receiving palliative care: a randomised controlled trial. Journal of Pain and Symptom Management 2005;30(4):329-41.

\section{Husereau 2013}

Husereau D, Drummond M, Petrou S, Carswell C, Moher D, Greenberg D, et al. Consolidated Health Economic Evaluation Reporting Standards (CHEERS) statement. BMJ 2013;346:f1049.

\section{Imison 2017}

Imison C, Curry N, Holder H, Castle-Clarke S, Nimmons D, Appleby J, et al. Shifting the balance of care: great expectations. Research report. Nuffield Trust 2017.

\section{JLA 2015}

James Lind Alliance. Palliative and end of life care Priority Setting Partnership: Putting patients, carers and clinicians at the heart of palliative and end of life care research (PeolcPSP). January 2015. palliativecarepsp.files.wordpress.com/2015/01/ peolcpsp_final_report.pdf (accessed 31 January 2017).

\section{Kamal 2013}

Kamal AH, Currow DC, Ritchie CS, Bull J, Abernethy AP. Community-based palliative care: the natural evolution for palliative care delivery in the US. Journal of Pain and Symptom Management 2013;46(2):254-64.

\section{Kashihara 2012}

Kashihara D, Carper K. National Health Care Expenses in the U.S. Civilian Noninstitutionalised Population, 2009. Statistical Brief \#355. January 2012. meps.ahrq.gov/data_files/ publications/st355/stat355.pdf (accessed 30 May 2019).

The effectiveness and cost-effectiveness of hospital-based specialist palliative care for adults with advanced illness and their caregivers 


\section{Lafond 2014}

Lafond S, Arora S, Charlesworth A, McKeon A. Into the red? The state of the NHS' finances. An analysis of NHS expenditure between 2010 and 2014. July 2014. www.nuffieldtrust.org.uk/ files/2017-01/into-the-red-nhs-finances-web-final.pdf (accessed 31 January 2017).

\section{Lau 2006}

Lau J, Ioannidis JPA, Terrin N, Schmid CH, Olkin I. The case of the misleading funnel plot. BMJ 2006;333(7568):597-600.

\section{Lee 2016}

Lee Cue H, Cook S, Lee Ji S, Han B. Comparison of two meta-analysis methods: inverse-variance-weighted average and weighted sum of z-scores. Genomics \& Informatics 2016;14(4):173-80.

\section{Liberati 2009}

Liberati A, Altman DG, Tetzlaff J, Mulrow C, Gøtzsche PC, loannidis JPA, et al. The PRISMA statement for reporting systematic reviews and meta-analyses of studies that evaluate health care interventions: explanation and elaboration. PLOS Medicine 2009;6(7):e1000100.

\section{Lichtenthal 2011}

Lichtenthal WG, Nilsson M, Kissane DW, Breitbart W, Kacel E, Jones EC, et al. Underutilization of mental health services among bereaved caregivers with prolonged grief disorder. Psychiatric Services 2011;62(10):1225-9.

\section{Lin 2017}

Lin L, Chu H. Quantifying publication bias in meta-analysis. Biometrics 2018;74(3):785-94. [PMID: 29141096]

\section{Luckett 2014}

Luckett T, Phillips J, Agar M, Virdun C, Green A, Davidson PM. Elements of effective palliative care models: a rapid review. $B M C$ Health Services Research 2014;14:136. [PMID: 24670065]

\section{May 2014}

May P, Normand C, Morrison RS. Economic impact of hospital inpatient palliative care consultation: review of current evidence and directions for future research. Journal of Palliative Medicine 2014;17(9):1054-63.

\section{McCaffree 1976}

McCaffree KM, Harkins EM. Final report for evaluation of nursing home care. Seattle: Battelle Human Affairs Research Centers 1976.

\section{Meier 2011}

Meier DE. Increased access to palliative care and hospice services: opportunities to improve value in health care. Milbank Quarterly 2011;89(3):343-80.

\section{Murtagh 2014}

Murtagh FE, Bausewein C, Verne J, Groeneveld El, Kaloki YE, Higginson IJ. How many people need palliative care? A study developing and comparing methods for population-based estimates. Palliative Medicine 2014;28(1):49-58.

\section{National End of Life Care Programme 2010}

NHS National End of Life Care Programme. Holistic common assessment of supportive and palliative care needs for adults requiring end of life care. www.bl.uk/collection-items/holisticcommon-assessment-of-the-supportive-and-palliative-careneeds-of-adults-requiring-end-of-life-care-1 (accessed 31 May 2020).

\section{NCCN 2016}

National Comprehensive Cancer Network. NCCN guidelines version 1. 2016 palliative care. www.nccn.org/ professionals/ physician_gls/pdf/ palliative.pdf (accessed 31 May 2020) (accessed 31 May 2020).

\section{NCP 2013}

National Consensus Project (NCP) for Quality Palliative Care. Clinical Practice Guidelines for Quality Palliative Care, 3rd edition, 2013. www.hpna.org/multimedia/NCP_Clinical_ Practice_Guidelines_3rd_Edition.pdf (accessed 30 May 2019).

\section{NCPC 2012}

National Council for Palliative Care (NCPC), developed in collaboration with: Association for Palliative Medicine of Great Britain and Ireland, Consultant Nurse in Palliative Care Reference Group, Marie Curie Cancer Care, National Council for Palliative Care, Palliative Care Section of the Royal Society of Medicine. Commissioning Guidance for Specialist Palliative Care: Helping to deliver commissioning objectives. December 2012. www.ncpc.org.uk/sites/default/ files/CommissioningGuidanceforSpecialistPalliativeCare.pdf (accessed 30 May 2019).

\section{NHS England 2016}

NHS England. Specialist level palliative care: information for commissioners. NHS England.

\section{NICE 2012}

National Institute for Health and Care Excellence. NICE process and methods guides. In: The NICE Public Health Guidance Development Process. London: National Institute for Health and Care Excellence, 2012.

\section{Palliative Care Australia 2005}

Palliative Care Australia. A guide to palliative care service development: a population based approach, February 2005. palliativecare.org.au/wp-content/uploads/2015/05/A-guideto-palliative-care-service-development-a-population-basedapproach.pdf (accessed 31 January 2017).

\section{Payne 2010a}

Payne S, Hudson P, Grande G, Oliviere O, Tishelman C, Pleschberger $\mathrm{S}$, et al. White paper on improving support for family carers in palliative care: Part 1: Recommendations from the European association for palliative care (EAPC) task force on family carers. European Journal of Palliative Care 2010;17(5):238-45.

\section{Payne 2010b}

Payne S, Hudson P, Grande G, Oliviere O, Tishelman C, Pleschberger $S$, et al. White Paper on improving support for family carers in palliative care: Part 2: Recommendations

The effectiveness and cost-effectiveness of hospital-based specialist palliative care for adults with advanced illness and their caregivers 
from the European Association for Palliative Care (EAPC) Task Force on Family Carers. European Journal of Palliative Care 2010;17(6):286-90.

\section{PHE 2017}

Public Health England. Cost-effective commissioning of end of life care: understanding the health economics of palliative and end of life care, 2017. www.gov.uk/government/publications/ end-of-life-care-economic-tool (accessed 30 May 2019).

\section{Philips 2004}

Philips Z, Ginnelly L, Sculpher M, Claxton K, Golder S, Riemsma $\mathrm{R}$, et al. Review of guidelines for good practice in decision-analytic modelling in health technology assessment. Health Technology Assessment 2004;8(36):iii-iv, ix-xi, 1-158.

\section{Piggott 2004}

Piggott M, McGee H, Feuer D. Has CONSORT improved the reporting of randomized controlled trials in the palliative care literature? A systematic review. Palliative Medicine 2004;18(1):32-8.

\section{Pinnock 2011}

Pinnock H, Kendall M, Murray SA, Worth A, Levack P, Porter M, et al. Living and dying with severe chronic obstructive pulmonary disease: multi-perspective longitudinal qualitative study. BMJ 2011;342:d142.

\section{Pivodic 2016}

Pivodic L, Pardon K, Morin L, Addington-Hall J, Miccinesi G, Cardenas-Turanzas $\mathrm{M}$, et al. Place of death in the population dying from diseases indicative of palliative care need: a crossnational population-level study in 14 countries. Journal of Epidemiology \& Community Health 2016;70(1):17-24.

\section{Prince 2015}

Prince MJ, Wu F, Guo Y, Gutierrez Robledo LM, O'Donnell M, Sullivan R, et al. The burden of disease in older people and implications for health policy and practice. Lancet 2015;385(9967):549-62.

\section{Quill 2013}

Quill TE, Abernethy AP. Generalist plus specialist palliative care - creating a more sustainable model. New England Journal of Medicine 2013;368(13):1173-5.

\section{RCP 2016}

Royal College of Physicians. End of life care audit dying in hospital: national report for England 2016. www.rcplondon.ac.uk/projects/outputs/end-life-care-auditdying-hospital-national-report-england-2016 (accessed 30 May 2019).

\section{RevMan 2014 [Computer program]}

Nordic Cochrane Centre, The Cochrane Collaboration Review Manager 5 (RevMan 5). Version 5.3. Copenhagen: Nordic Cochrane Centre, The Cochrane Collaboration, 2014.

\section{Rodgers 2009}

Rodgers M, Sowden A, Petticrew M, Arai L, Roberts H, Britten N, et al. Testing methodological guidance on the conduct of narrative synthesis in systematic reviews: effectiveness of interventions to promote smoke alarm ownership and function. Evaluation 2009;15(1):49-73.

\section{Roscoe 2010}

Roscoe LA, Schocken DD. Measuring quality of life at the end of life. In: Preedy VR, Watson RR, editors(s). Handbook of Disease Burdens and Quality of Life Measures. New York: Springer, 2010:2687-703.

\section{Schunemann 2011}

Schünemann HJ, Oxman AD, Vist GE, Higgins JPT, Deeks JJ, Glasziou P, et al, Cochrane Applicability and Recommendations Methods Group. Chapter 12: Interpreting results and drawing conclusions. In: Higgins JP, Green S, editor(s). Cochrane Handbook for Systematic Reviews of Interventions Version 5.1.0 (updated March 2011). The Cochrane Collaboration, 2011. Available from handbook.cochrane.org.

\section{Shepperd 2011}

Shepperd S, Wee B, Straus SE. Hospital at home: home-based end of life care. Cochrane Database of Systematic Reviews 2011, Issue 7. Art. No: CD009231. [DOI: 10.1002/14651858.CD009231.pub2]

\section{Shrier 2008}

Shrier I, Boivin J-F, Platt RW, Steele RJ, Brophy JM, Carnevale F, et al. The interpretation of systematic reviews with metaanalyses: an objective or subjective process? BMC Medical Informatics and Decision Making 2008;8:19.

\section{Sleeman 2019}

Sleeman KE, De Brito M, Etkind S, Nkhoma K, Guo P, Higginson IJ, et al. The escalating global burden of serious health-related suffering: projections to 2060 by world regions, age groups, and health conditions. Lancet Global Health 2019;7(7):E883-92.

\section{Sterne 2001}

Sterne JAC, Egger M, Smith GD. Systematic reviews in health care: Investigating and dealing with publication and other biases in meta-analysis. BMJ 2001;323(7304):101-5.

\section{Sterne 2011}

Sterne JAC, Egger M, Moher D, Cochrane Bias Methods Group. Chapter 10: Addressing reporting biases. In: Higgins JP, Green $\mathrm{S}$, editor(s). Cochrane Handbook for Systematic Reviews of Interventions Version 5.1.0 (updated March 2011). The Cochrane Collaboration, 2011. Available from handbook.cochrane.org.

\section{Van Tulder 2003}

Van Tulder M, Furlan A, Bombardier C, Bouter L, Editorial Board of the Cochrane Collaboration Back Review Group. Updated method guidelines for systematic reviews in the Cochrane Back and Neck Review Group. Spine 2003;28(12):1290-9.

\section{Virdun 2015}

Virdun C, Luckett T, Davidson PM, Phillips J. Dying in the hospital setting: a systematic review of quantitative studies identifying the elements of end-of-life care that patients and

The effectiveness and cost-effectiveness of hospital-based specialist palliative care for adults with advanced illness and their caregivers 
their families rank as being most important. Palliative Medicine 2015;29(9):774-96.

\section{Walsh 2011}

Walsh J, Young JM, Harrison JD, Butow PN, Solomon MJ, Masya L, et al. What is important in cancer care coordination? A qualitative investigation. European Journal of Cancer Care 2011;20(2):220-7.

\section{Ware 1979}

Ware JE Jr, Johnston SA, Davies-Avery A, Brook RH. Santa Monica: Rand Corporation. Vol. 3. 1979 (R-1987/3-HEW).

\section{WHO 2002}

World Health Organization. WHO definition of palliative care. www.who.int/cancer/palliative/definition/en/ (accessed 31 May 2020).

\section{WHOQOL Group 1995}

World Health Organization Quality of Life Group (WHOQOL). The World Health Organization Quality of Life (WHOQOL) Assessment: position paper from the World Health Organization. Social Science and Medicine 1995;41(10):1403-9.

\section{Williams 1995}

Williams A. The role of the Euroqol instrument in QALY calculations. Centre for Health Economics, University of York 1995.

\section{CHARACTERISTICS OF STUDIES}

Characteristics of included studies [ordered by study ID]

\section{WPCA/WHO 2014}

World Palliative Care Alliance (WPCA), World Health Organization (WHO). Global atlas of palliative care at the end of life, January 2014. www.who.int/cancer/publications/palliativecare-atlas/en/ (accessed 31 January 2017).

\section{Zimmermann 2004}

Zimmermann C, Rodin G. The denial of death thesis: sociological critique and implications for palliative care. Palliative Medicine 2004;18(2):121-8.

\section{Zimmermann 2008}

Zimmermann C, Riechelmann R, Krzyzanowska M, Rodin G, Tannock I. Effectiveness of specialized palliative care: a systematic review. Journal of the American Medical Association 2008;299(14):1698-709.

\section{Zimmermann 2012}

Zimmermann C. Acceptance of dying: a discourse analysis of palliative care literature. Social Science and Medicine 2012;75(1):217-24.

\section{Zimmermann 2019}

Zimmermann C, Ryan S, Hannon B, Saltman A, Rodin G, Mak E, et al. Team-based outpatient early palliative care: a complex cancer intervention. BMJ Supportive \& Palliative care 2019. [PMID: 31406013]

* Indicates the major publication for the study

\section{Ahronheim 2000}

\section{Study characteristics}

$\begin{array}{ll}\text { Methods } & \text { Design: RCT } \\ \text { Who led PC intervention: Multidisciplinary team } \\ \text { Core team: Clinical nurse specialist/certified geriatrician }\end{array}$

Participants

Country and regions: USA, New York

Recruitment: Eligible patients admitted to Mount Sinai Hospital over a 3-year period were identified through daily rounds by the palliative care team nurse. Patients were assessed by the palliative care nurse as to appropriateness for inclusion.

Inclusion criteria: Patients were eligible if they had advanced dementia and had been hospitalised for acute illness. Advanced dementia was defined as Functional Assessment Staging Tool (FAST) of stage 6 or greater, with a stable baseline neurological deficit for at least 1 month.

Exclusion criteria: Not described

Number of patients: $\mathrm{N}=99$ (48 intervention and 51 control)

Diseases: Advanced dementia

Patient characteristics: Mean age in years (range): 83.9 (63 - 99) years in intervention group, 85.6 (72 100) years in control group; $77.1 \%$ female in intervention group, $86.3 \%$ female in control group.

The effectiveness and cost-effectiveness of hospital-based specialist palliative care for adults with advanced illness and their caregivers 65 (Review)

Copyright (c) 2020 The Cochrane Collaboration. Published by John Wiley \& Sons, Ltd. 
Ahronheim 2000 (Continued)

Deaths at end of study (N (\%)): $\mathrm{N}=24: 12(25 \%)$ intervention and $12(23.5 \%)$ control

Withdrawals/other dropouts ( $\mathrm{N}(\%))$ : Not reported

Interventions Name: Inpatient palliative care vs usual hospital care

Type: Training in palliative care unclear

Service base: Mount Sinai Hospital, a tertiary care referral centre and teaching hospital for Mount Sinai School of Medicine

Team: Consisted of master's level clinical nurse specialist and one or more attending level, certified geriatricians who held academic appointments in the Departments of Medicine and Geriatrics

Intervention condition: The intervention consisted of palliative care consultation by the team nurse and physician investigator, who then visited the patient and discussed management with available members of the primary healthcare team in the hospital on a daily basis, excluding weekends. The palliative care team also met with family unpaid caregivers or other surrogates when they were available and attempted to arrange meetings after hours. If face-to-face meetings could not be arranged, discussions were held over the phone. During encounters with health professionals or family unpaid caregivers, the palliative care team discussed various care options. The goal of the intervention was to maximise patient comfort with avoidance of painful or invasive treatments, including hospital admission, diagnostic tests, and invasive procedures, unless needed for symptom control. Recommendations regarding palliative care interventions were made to the hospital inpatient team but contact between or after hospitalisations were generally with the family. On readmission, the patient was identified through a computerised system. Consent to continue in the study was obtained from the surrogate by phone, and the inpatient providers were contacted.

Duration: Time of initial randomisation until final discharge or in-hospital death

Control condition: The control group was treated by the primary care team without the input of the palliative care team.

Outcomes
Mortality
Site of discharge
Length of stay
Number of readmissions
Use of nonpalliative procedures
Do-not-resuscitate (DNR) orders and cardiopulmonary resuscitation (CPR)
Systemic antibiotics
Whether a decision was made to forgo life-sustaining treatments
Antibiotics
Intravenous fluids
Blood drawing
Whether a decision was made to adopt an overall palliative care plan

Assessment points: After informed consent, complete history was obtained and physician examination performed. After this baseline evaluation, patients were randomised and outcomes assessed until final discharge or in-hospital death. Date of death for patients who survived the hospitalisation was ascertained by telephone follow-up.

Resource use/costs Number of hospital admissions

The effectiveness and cost-effectiveness of hospital-based specialist palliative care for adults with advanced illness and their caregivers 
Ahronheim 2000 (Continued)

Number of rehospitalisations

Mean length of stay post-randomisation

Time horizon: Start of hospitalisation to final discharge or in-hospital death

Notes

Funding source: This work was supported by grants from The Greenwall Foundation and The Kornfeld Foundation.

Declarations of interest among primary researchers: Apart from funding, no further study author disclosure statements were made.

Power considerations: Not reported

\section{Risk of bias}

\begin{tabular}{lll}
\hline Bias & Authors' judgement & Support for judgement \\
\hline $\begin{array}{l}\text { Random sequence genera- } \\
\text { tion (selection bias) }\end{array}$ & Unclear risk & $\begin{array}{l}\text { Quote from main publication: "patients were randomly assigned to either the } \\
\text { intervention or to the control group". }\end{array}$ \\
& $\begin{array}{l}\text { Comment: there was insufficient information about the random sequence gen- } \\
\text { eration process. }\end{array}$
\end{tabular}

Allocation concealment Unclear risk Comment: allocation concealment was not described.

(selection bias)

$\begin{array}{ll}\begin{array}{l}\text { Blinding of participants } \\ \text { and personnel (perfor- }\end{array} & \text { Low risk } \\ \text { lead to bias in the outcomes. }\end{array}$

mance bias), objective lead to bias in the outcomes.

outcomes

\section{Blinding of outcome as- Low risk} sessment (detection bias), objective outcomes

\begin{abstract}
Quote from main publication: "in order to maintain blindness for the research assistant during data gathering from the chart, consultation did not include written notes on the chart... A research assistant blinded to randomization status gathered information from the charts of patients in both arms of the study; data obtained included demographic characteristics, advance directives, comorbidities and physical findings, interventions, and care plans".
\end{abstract}

Comment: probably done

Incomplete outcome data Unclear risk Comment: number of dropouts were not reported.

(attrition bias)

All outcomes

Selective reporting (re- Unclear risk Comment: protocol was not available.
porting bias)

\begin{tabular}{lll}
\hline Other bias & Low risk & Comment: study appeared to be free of other biases. \\
\hline Size of study & Unclear risk & $\begin{array}{l}\text { Comment: intervention group had } 48 \text { participants and the control } 51 \text { partici- } \\
\text { pants. }\end{array}$ \\
\hline
\end{tabular}

Bajwah 2015

\section{Study characteristics}

\begin{tabular}{l} 
Methods Design: RCT \\
\hline The effectiveness and cost-effectiveness of hospital-based specialist palliative care for adults with advanced illness and their caregivers \\
(Review) \\
Copyright $\odot 2020$ The Cochrane Collaboration. Published by John Wiley \& Sons, Ltd.
\end{tabular}

Copyright ( 2020 The Cochrane Collaboration. Published by John Wiley \& Sons, Ltd. 
Bajwah 2015 (Continued)

\author{
Fast-track phase II RCT \\ Who led PC intervention: Nurse-led intervention
}

Core team: PC specialist nurse. The patient, their carer, hospital-2-home nurse, general practitioner, community matron/district nurse, respiratory nurse, community palliative care nurse (and any other relevant health or social care professional) were invited to attend the case conference.

Participants

Country and regions: UK, London

Recruitment: October 2011 to October 2013. Patients with a clinical diagnosis of advanced idiopathic fibrotic lung disease (IPF by American Thoracic Society/European Respiratory Society criteria or fibrotic nonspecific interstitial pneumonia) were recruited from the inpatient and outpatient settings in a specialist ILD centre (Royal Brompton Hospital, London). Where possible, patient and carer dyads were recruited.

Inclusion criteria: Patients included were considered to have end-stage disease as judged by either high resolution CT or composite physiologic index scores. A subsequent amendment allowed recruitment of patients considered to have end-stage disease clinically who were too unwell to complete pulmonary function tests. To be included, patients and carers had to be $>18$ years old, possess sufficient mental capacity and be able to complete questionnaires in English.

Exclusion criteria: Patients/informal unpaid caregiver

i) Any patient/informal unpaid caregiver unable to give informed consent

ii) Any patient/informal unpaid caregiver less than 18 years of age

iii) Participants who are unable to understand/speak English

iv) Participants who are remaining as an inpatient in the hospital or being transferred to another inpatient facility (e.g. hospice unit, for terminal care)

v) Participants whose prognosis is less than 1 week or judged too unwell by the research team to take part in serial interviews

Number of patients: $\mathrm{N}=53$ (26 intervention and 27 control)

Diseases: Idiopathic pulmonary fibrosis (IPF), nonspecific interstitial pneumonia

Patient characteristics: Mean age in years (SD): 67.1 (10.9) years in intervention group, 70.6 (10.3) years in control group; $23 \%$ female in intervention group, $33 \%$ female in control group

Number of unpaid caregivers: $\mathrm{N}=45$ (19 intervention and 26 control)

unpaid caregivercharacteristics: Mean age in years (SD): 61.3 (14) years in intervention group, 60.3 (13.1) years in control group; $68 \%$ female in intervention group, $77 \%$ female in control group

Deaths at end of study ( $(\%))$ : Total $N=10: 1(3.8 \%)$ intervention and $9(33.3 \%)$ control

Withdrawals/other dropouts $(\mathrm{N}(\%)):$ Total $\mathrm{N}=12: 6(23.1 \%)$ intervention and $6(22.2 \%)$ control

Name:.Case conference intervention (hospital-2-home) delivered alongside best standard care vs standard care

Type: Specialist palliative care. Included a palliative care specialist nurse who delivered the intervention. The nurse had received training on delivery of the intervention from specialist nurses delivering the cancer hospital-2-home intervention

Service base: Hospital

Team: A palliative care specialist nurse delivered the intervention. The patient, their carer, hospital-2-home nurse, general practitioner, community matron/district nurse, respiratory nurse and community palliative care nurse (and any other health or social care professional involved in their care or identified as important by the patient) were invited to attend the case conference.

The effectiveness and cost-effectiveness of hospital-based specialist palliative care for adults with advanced illness and their caregivers 
Intervention condition: The hospital-2-home intervention was delivered alongside best standard care. The fast-track group received the intervention after 1 week, the waiting list group after 4 weeks. The intervention aimed to provide a quality comprehensive palliative care assessment and streamlining of transfer of data between specialist and community settings improving co-ordination of care and communication while codifying responsibility for the patient, carer and health professionals. The Hospital-2-Home intervention included a case conference (multiprofessional and holistic) and a care plan (care individualised to each patient and carer). A palliative care specialist nurse who had received training on the intervention delivered it. Clinical supervision was provided to assist in identifying and advising on strategies to address problems compromising effective management of the palliative care concerns of these patients and carers. During the case conference, current and anticipated palliative care concerns (physical, psychological, social and spiritual concerns) were discussed. An action plan was agreed upon for each concern and a responsible healthcare professional allocated for each item. The individualised care plan was then communicated to the patient and carer and health and social care professionals.

\section{Duration: 8 weeks}

Control condition: All patients received best standard care throughout the study: Patients remained under ILD specialist care for the full duration of the study. Referrals to community health professionals (as deemed necessary by the ILD team) continued throughout the study. These could include referrals to community nursing (such as community matron or district nurses), respiratory services and community palliative care teams.

\section{Primary outcome:}

Palliative Care Outcome Scale (POS) assessed by the patient

\section{Secondary outcome:}

POS assessed by the carer

Breathlessness assessed using the D12 and MRC breathlessness scale

Symptom control assessed using POS

Quality of life of patients assessed using the Kings Brief Interstitial Lung Disease (KBILD) questionnaire and the St Georges Respiratory Questionnaire (SGRQ)

Quality of life of carers assessed using the unpaid caregiver Quality of Life Index

Patient anxiety and depression assessed using the Hospital Anxiety and Depression Scale

Carer anxiety and depression assessed using the Hospital Anxiety and Depression Scale

Carer burden assessed using the Zarit Burden Inventory

Preferred place of care and death

Patient use of other services

Consent and recruitment rates

Percentage of patients in the fast-track group receiving case conferences within 14 days

Assessment points: After consent and baseline interview, patients were randomised to fast-track or waiting list groups. Primary and secondary outcome data were collected by postal questionnaire at baseline in both groups. Subsequent time points were 4 weeks and 8 weeks after receiving the intervention in the fast-track group and just before receiving the intervention and 4 weeks after receiving the intervention in the waiting list group.

Resource use/costs

Patient use of other services

Carer's assessment of patient's use of services

The effectiveness and cost-effectiveness of hospital-based specialist palliative care for adults with advanced illness and their caregivers (Review)

Copyright $\odot 2020$ The Cochrane Collaboration. Published by John Wiley \& Sons, Ltd. 
Bajwah 2015 (Continued)

Notes
Funding source: This study was funded partly by a grant from Marie Curie. The remainder of the funding was from the Royal Marsden and Royal Brompton Palliative Care Research Fund which is funded from charitable sources.

Declarations of interest among primary researchers: No competing interest was declared by the authors.

Power considerations: Fifty-two patients were needed to enable estimation of change in POS between baseline and 4 weeks with accurate precision (assuming a SD of 2, a $95 \% \mathrm{CI}$ for the difference between the fast-track and waiting list groups would be 2.2 units wide, i.e. mean difference \pm 1.1 units).

\section{Risk of bias}

Bias Authors' judgement Support for judgement

Random sequence genera- Low risk tion (selection bias)
Quote from main publication: "after consent and baseline interview, patients were randomised to fast-track or waiting list groups... Treatment allocation (fast-track/waiting list group) was by computer generated random permuted blocks (by the Institute of Cancer Research) with stratification dependent on severity of patient Palliative Care Outcome Scale (POS) at baseline (patients with a POS score $\geq 28$ were classed as severe)".

Comment: probably done

Allocation concealment Low risk
(selection bias)

Quote from main publication: "treatment allocation (fast-track/waiting list group) was by computer generated random permuted blocks (by the Institute of Cancer Research) with stratification dependent on severity of patient Palliative Care Outcome Scale (POS) at baseline (patients with a POS score $\geq 28$ were classed as severe)".

Comment: allocation appeared to be by an independent group.

\begin{tabular}{|c|c|c|}
\hline $\begin{array}{l}\text { Blinding of participants } \\
\text { and personnel (perfor- } \\
\text { mance bias), subjective } \\
\text { outcomes }\end{array}$ & High risk & Comment: no blinding \\
\hline $\begin{array}{l}\text { Blinding of participants } \\
\text { and personnel (perfor- } \\
\text { mance bias), objective } \\
\text { outcomes }\end{array}$ & Low risk & $\begin{array}{l}\text { Information registered for this trial, NCT01450644, reported there was no } \\
\text { masking (open-label). } \\
\text { Comment: participants and personnel were not blinded but this was unlikely } \\
\text { to lead to bias in the objective outcomes. }\end{array}$ \\
\hline $\begin{array}{l}\text { Blinding of outcome as- } \\
\text { sessment (detection bias), } \\
\text { subjective outcomes }\end{array}$ & High risk & Comment: no blinding \\
\hline $\begin{array}{l}\text { Blinding of outcome as- } \\
\text { sessment (detection bias), } \\
\text { objective outcomes }\end{array}$ & Low risk & $\begin{array}{l}\text { Information registered for this trial, NCT01450644, reported there was no } \\
\text { masking (open-label). } \\
\text { Comment: lack of blinding of outcome assessment was unlikely to lead to bias } \\
\text { in objective outcomes. }\end{array}$ \\
\hline $\begin{array}{l}\text { Incomplete outcome data } \\
\text { (attrition bias) } \\
\text { All outcomes }\end{array}$ & High risk & $\begin{array}{l}\mathrm{N}=23(88.5 \%) \text { completers in intervention group vs } \mathrm{N}=21 / 24(77.8 \% / 88.9 \%) \\
\text { completers in control group. In intervention group, loss to follow-up occurred } \\
\text { due to death }(\mathrm{N}=1) \text {, withdrawal }(\mathrm{N}=1) \text { and went on holiday }(\mathrm{N}=1) \text {. In con- } \\
\text { trol group, loss to follow-up occurred due to death }(\mathrm{N}=6) \text {. Patients lost to fol- } \\
\text { low-up were excluded from analysis. }\end{array}$ \\
\hline
\end{tabular}


Bajwah 2015 (Continued)

Selective reporting (re- High risk Comment: some of the outcomes in the protocol were not reported e.g. cost. porting bias) Furthermore, some outcomes reported in the study were not prespecified in the protocol e.g. carer's burden and end-of-life preferences.

Other bias Unclear risk

Comment: there were differences between the intervention and control groups at baseline in patients' age, gender, ethnicity, percentage with comorbidities. However, it was unclear if these differences were significant as no statistical test was carried out. It was also unclear if they were controlled for.

\begin{tabular}{ll}
\hline Size of study $\quad$ Unclear risk & $\begin{array}{l}\text { Comment: intervention group had a total of } 45 \text { participants and the control } 53 \\
\text { participants. }\end{array}$
\end{tabular}

Bakitas 2009

\section{Study characteristics}

Methods

Design: RCT (multisite)

Who led PC intervention: Nurse-led palliative care intervention

Core team: Advanced practice palliative care nurse specialists. Intervention participants and their unpaid caregiver were invited to attend monthly group shared medical appointments (SMAs) led by a certified palliative care physician and nurse practitioner. Palliative care-certified nurse practitioner and physician, psychologists, and other team members, met biweekly to review the advanced practice nurses' audiotaped educational sessions and to provide feedback on difficult patient management issues.

Participants

Country and regions: USA, New Hampshire and Vermont

Recruitment: November 2003 to May 2007. Research assistants (RAs) at the cancer centre and the Veterans Affairs Medical Center (VAMC) attended weekly gastrointestinal (GI), genitourinary (GU), breast, and thoracic cancer management meetings (tumour boards) in which newly diagnosed patients were discussed. The RAs also reviewed clinic schedules to identify potentially eligible patients. The clinician then approached the patients to obtain permission for the RA to provide them with more information about the study. From affiliated outreach clinics, following discussion of the study with clinic staff, the main study site was informed of a potentially interested patient.

Inclusion criteria: Patients identified at the Norris Cotton Cancer Centre's tumour boards with a lifelimiting cancer (prognosis of approximately 1 year) were eligible if they were within 8 to 12 weeks of a new diagnosis of gastrointestinal tract (unresectable stage III or IV), lung (stage IIIB or IV non-small cell or extensive small cell), genitourinary tract (stage IV), or breast (stage IV and visceral crisis, lung or liver metastasis, oestrogen receptor negative [ER-], human epidermal growth factor receptor 2 positive [Her 2 neu + ]) cancer. Patients were asked to select a unpaid caregiver to participate in the study. Patients who did not select a unpaid caregiver were not excluded.

Exclusion criteria: Patients with impaired cognition ( $<17$ on a modified Mini-Mental State Examination), an Axis I psychiatric disorder (schizophrenia, bipolar disorder), or active substance use were excluded.

Number of patients enrolled (survival outcomes sample): $\mathrm{N}=322$ (161 intervention and 161 control)

Number of patients (patient outcomes sample): $N=279$ (145 intervention and 134 control)

Diseases: Gastrointestinal tract (42\%), lung (33\%), genitourinary tract $(13 \%)$ and breast cancer $(11 \%)$

Patient characteristics: Mean age in years (SD): 65.4 (10.3) years in intervention group, 65.2 (11.7) years in control group; $37.9 \%$ female in intervention group, $41.8 \%$ female in control group.

Number of unpaid caregivers enrolled: $\mathrm{N}=220$ (116 intervention and 104 control)

The effectiveness and cost-effectiveness of hospital-based specialist palliative care for adults with advanced illness and their caregivers 
Number of unpaid caregivers ( unpaid caregiveroutcomes sample): $\mathrm{N}=198$ (108 intervention and 90 control)

unpaid caregivercharacteristics ( unpaid caregiveroutcomes sample): Mean age in years (SD): 58 (11.9) years in intervention group, 59.9 (13) years in control group; 76.9\% female in intervention group, $77.8 \%$ female in control group

Deaths at end of study (survival outcomes sample) ( $(\%)): \mathrm{N}=231: 112(69.6 \%)$ intervention and $119(73.9 \%)$ control

Withdrawals/other dropouts from enrollment ( $N(\%)): N=100: 45(28 \%)$ intervention and $55(34.2 \%)$ control

Withdrawals/other dropouts (patient outcome sample) (N (\%)): N = 57: $29(20 \%)$ intervention and 28 (20.9\%) control

Interventions

Name: Advanced practice palliative care nurse specialist-administered palliative care intervention concurrent with anti-cancer treatment for patients with advanced cancer and a unpaid caregiver vs usual care (project ENABLE II).

Early PC: Randomised controlled trial of a palliative care intervention compared with usual care for persons newly diagnosed with advanced cancer (8 to 12 weeks of a new diagnosis)

Type: Specialist palliative care. Included advanced practice palliative care nurse specialists

Service base: Oncology clinics of the National Cancer Institute-designated comprehensive cancer centre and affiliated outreach clinics, and the academically affiliated Veterans Affairs Medical Center (VAMC)

Provider of service: Advanced practice palliative care nurse specialists

Intervention condition: The intervention, based on the chronic care model, used a case management, educational approach to encourage patient activation, self-management, and empowerment. A manualised, telephone-based format was used to improve access to palliative care in a rural population. One of 2 advanced practice nurses with palliative care specialty training conducted 4 initial structured educational and problem-solving sessions and at least monthly telephone follow-up sessions until the participant died or the study ended. A bereavement follow-up call was made to the unpaid caregiver. The nurses began all contacts with an overall assessment by administering the Distress Thermometer, an 11-point rating scale (0-10) of distress. If distress intensity was $>3$, the nurses explored the sources of distress and identified if the participant would like to apply the problem-solving approach to address his or her issues. The education manual, contained the 4 modules of problem-solving, communication and social support, symptom management, advance care planning and unfinished business, and an appendix listing supportive care resources. Following the 4 formal sessions, the advanced practice nurse was available by telephone and also contacted the participant (or unpaid caregiver) at least monthly (until the participant's death) to follow-up on active issues and to assess the need for referral to appropriate care resources. Additionally, intervention participants and their unpaid caregiver were invited to attend monthly group shared medical appointments (SMAs).

Duration: Enrollment until death or study completion

Control condition: Participants assigned to usual care were allowed to use all oncology and supportive services without restrictions including referral to the institutions' interdisciplinary palliative care service. The cancer centre site has a consultative interdisciplinary palliative care team (PCT) comprised of a physician and nurse practitioners which provided care for both inpatient and outpatients. Oncologists could refer patients for assessment by this team while patients were receiving anticancer treatments. Patients and family members were often followed through death and bereavement.

Outcomes

\section{Primary outcomes:}

Patient-reported quality of life measured by the Functional Assessment of Chronic Illness Therapy-Palliative Care (FACIT-Pal)

Symptom intensity measured by a modified Edmonton Symptom Assessment Scale (ESAS)

The effectiveness and cost-effectiveness of hospital-based specialist palliative care for adults with advanced illness and their caregivers 
Bakitas 2009 (Continued)

Resource use

\section{Secondary outcomes:}

Mood measured by the Center for Epidemiological Studies Depression Scale (CES-D)

unpaid caregiver burden measured by the Montgomery Borgatta unpaid caregiver Burden Scale

Perceptions of end-of-life care measured by a revised version of the After Death Bereaved Family Member Interview (ADI)

Survival

\section{Assessment points:}

Baseline assessment was followed by randomisation. Outcomes were assessed at baseline, 1 month, and every 3 months until death or study completion.

Number of days in the intensive care unit (ICU)

Number of emergency department visits

Time horizon: Enrollment until death or study completion

Declarations of interest among primary researchers: No financial disclosure was reported.

Power considerations: The original target sample size of 400 was chosen to provide $80 \%$ power to detect treatment effects of at least 0.35 SDs for scores on the Functional Assessment of Chronic Illness Therapy for Palliative Care, ESAS, and CES-D based on a t-test comparing the treatment groups with respect to the last observed value with a 2-sided value of.01. However, at the planned study completion date, the final total study enrollment was 322 due to slightly slower accrual than anticipated.

\section{Risk of bias}

\section{Bias}

Authors' judgement Support for judgement

Random sequence genera- Low risk tion (selection bias)

Quote from publication: "participants were randomised equally into either the intervention or the usual care group using computer-generated random numbers. There were separate randomisation schemes for the cancer center and the VAMC participants (in order to ensure an equal distribution of patients in intervention and control groups from each of these primary sites). Participants from all other sites randomised according to the cancer center scheme, as large numbers of participants were not anticipated. Randomisation was blocked using random block sizes and was also stratified by diagnosis (lung, breast, and GI and GU cancers) to control for differential effects of treatment regimens and disease course".

Comment: probably done

Allocation concealment Unclear risk (selection bias)

Quote from main publication: "referring clinicians were neither informed nor formally blinded to participant assignment".

Comment: allocation concealment was not adequately described.

Blinding of participants High risk Comment: no blinding

and personnel (perfor-

mance bias), subjective

outcomes

The effectiveness and cost-effectiveness of hospital-based specialist palliative care for adults with advanced illness and their caregivers 
Bakitas 2009 (Continued)

Blinding of participants Low risk Information obtained from the trial registry indicated it was open-label. and personnel (performance bias), objective Comment: participants and personnel were not blinded but this was unlikely outcomes to lead to bias in objective outcomes.

$\begin{array}{ll}\text { Blinding of outcome as- } & \text { High risk } \quad \text { Comment: no blinding } \\ \text { sessment (detection bias), } & \end{array}$

(detection bias)

subjective outcomes

Blinding of outcome as- Low risk

sessment (detection bias),

objective outcomes

Information obtained from the trial registry indicated it was open-label.

Comment: lack of blinding of outcome assessment was unlikely to lead to bias in assessment of objective outcomes.

\section{Incomplete outcome data Unclear risk}

(attrition bias)

All outcomes
$\mathrm{N}=113(77.9 \%)$ in intervention group vs $\mathrm{N}=105(78.4 \%)$ in control group completed $\geq 1$ follow-up. In the intervention group, $29(20 \%)$ patients withdrew and $19(13.1 \%)$ died. In the control group, 28 (20.9\%) withdrew and $28(20.9 \%)$ died. Missing data were included in primary outcome analysis.

Comment: although missing data were included in primary outcome analysis, they were not included in secondary outcome analysis.
Comment: one outcome (problem-solving skills) included on the trial registry was not reported in the publication. Trial Registration no is NCT00253383. Furthermore, quality of care (After Death Bereaved Family Member Interview) was stated as a primary outcome on the trial registry but reported as a secondary outcome in the publication.

\begin{tabular}{lll}
\hline Other bias & Low risk & Comment: study appeared to be free of other biases. \\
\hline Size of study & Low risk & $\begin{array}{l}\text { Comment: each of the groups had above } 200 \text { participants (patients and unpaid } \\
\text { caregivers) in each arm. }\end{array}$ \\
\hline
\end{tabular}

\section{Bakitas 2015}

\section{Study characteristics}

Methods Design: RCT (multisite RCT)

Fast-track RCT

Who led PC intervention: Multidisciplinary team

Core team: Board-certified palliative care clinician/advanced practice palliative care nurse

Participants

Country and regions: USA. Lebanon, New Hampshire and White River Junction, Vermont

Recruitment: October 2010 to March 2013. Research co-ordinators reviewed all outpatient clinicians' schedules and tumour board lists using eligibility criteria to identify potential participants.

Inclusion criteria: English-speaking, age $\geq 18$ years with advanced-stage solid tumour or haematologic malignancy, oncologist-determined prognosis of 6 to 24 months, and able to complete baseline questionnaires; data supplement provides detailed criteria. After providing signed consent, patient participants were asked to select a unpaid caregiver, defined as "someone who knows you well and is involved in your medical care," to participate; however, patients were not excluded if they did not identify a unpaid caregiver. 
Exclusion criteria: Exclusions included impaired cognition (Callahan score $\leq 4$ ), active axis I psychiatric disorder (schizophrenia, bipolar) or substance use disorder, uncorrectable hearing disorder, or unreliable telephone service. There were no formal unpaid caregiver exclusion criteria.

Number of patients: $N=207$ (104 intervention and 103 control)

Diseases: Lung ( $N=88,43 \%)$, breast ( $N=23,11 \%)$, gastrointestinal tract $(N=50,24 \%)$, other solid tu$\operatorname{mour}(N=20,10 \%)$, genitourinary tract $(N=16,8 \%)$ and haematologic malignancy $(N=10,5 \%)$.

Patient characteristics: Mean age in years (SD): 64.03 (10.3) years in intervention group, 64.6 (9.6) years in control group; $46 \%$ female in intervention group, $49 \%$ female in control group

Number of unpaid caregivers: $\mathrm{N}=122$ (61 intervention and 61 control)

unpaid caregivercharacteristics: Mean age in years (SD): 61 (11.6) years in intervention group, 57.9 (11.9) years in control group; $77 \%$ female in intervention group, $80.3 \%$ female in control group

Number of unpaid caregivers who completed after-death questionnaires: $N=44$ (19 intervention and 25 control)

unpaid caregivercharacteristics for those who completed after-death questionnaires: Mean age in years (SD): 62.1 (11.9) years in intervention group, 61.2 (8.6) years in control group; $78.9 \%$ female in intervention group, $88 \%$ female in control group

Deaths at end of study ( $\mathrm{N}(\%)): \mathrm{N}=17: 2(2.9 \%)$ intervention and $14(13.6 \%)$ control

Withdrawals/other dropouts ( $\mathrm{N}(\%)): \mathrm{N}=77: 42(40.4 \%)$ intervention and $35(34 \%)$ control

Interventions
Name: Early palliative care alongside standard oncology care (ENABLE III telehealth concurrent PC model with standard oncology care) vs standard oncology care

Early PC: Early palliative care was defined as initiating palliative care within 30 to 60 days of diagnosis.

Type: Specialist palliative care. Included a board-certified palliative care clinician and advanced practice palliative care nurse specialists.

Service base: National Cancer Institute cancer centre, a Veterans Affairs Medical Centre, and community outreach clinics

Provider of service: Board-certified palliative care clinician and an advanced practice palliative care nurse

Intervention condition: ENABLE includes initial in-person, standardised outpatient palliative care consultation by a board-certified palliative care clinician and six structured weekly telephone coaching sessions by an advanced practice nurse using a manualised curriculum (i.e. Charting Your Course: An Intervention for Patients With Advanced Cancer). Sessions one to three focussed on problem-solving, symptom management, self-care, identification and co-ordination of local resources, communication, decision-making, and advance care planning. Sessions four to six comprised Outlook, a life-review approach that encourages participants to frame advanced illness challenges as personal growth opportunities. After the six Charting Your Course sessions, monthly follow-up calls reinforced prior content and identified new challenges or care co-ordination issues. The principal investigator, reviewed all PC consultation notes, and digitally recorded nurse coach sessions for protocol adherence. She met with the nurse coaches weekly to review and provide feedback on difficult cases. There was also a unpaid caregiver-specific intervention (such as cultivating communication skills with patient and healthcare clinicians).

Duration: Enrollment until death or study completion

Control condition: Usual oncology care, provided to all patients, was directed by a medical oncologist and consisted of anticancer and symptom control treatments and consultation with oncology and supportive care specialists, including a clinical PC team. The latter was provided whenever requested, regardless of group assignment. 
Bakitas 2015 (Continued)

Patient-reported quality of life measured by the Functional Assessment of Chronic Illness Therapy-Palliative Care (FACIT-Pal) and Treatment Outcome Index

unpaid caregiver QoL measured by the CGQOL Scale-Cancer (CQOL-C)

Symptom impact measured by the Quality of life at end-of-life symptom impact subscale

Patient and unpaid caregiver mood measured by the Center for Epidemiological Studies Depression Scale (CES-D)

One year and overall survival

Location of death

unpaid caregiver grief measured by the Prigerson Inventory of Complicated Grief-Short Form (PG13)

unpaid caregiver burden measured by the Montgomery-Borgatta CG Burden (MBCB) Scale

\section{Assessment points:}

Randomisation was done and then assessments were carried out. Subsequent to signed informed consent, research co-ordinators administered questionnaires by telephone at baseline; at 6, 12, 18, and 24 weeks; and every 12 weeks thereafter until death or study completion.

Resource use/costs

Patient-reported hospital and intensive care unit (ICU) days and emergency department (ED) visits

Chemotherapy use in last 14 days

Time horizon: Enrollment until death or study completion

Notes

Funding source: Supported by Grant No. R01NR011871-01 from the National Institute for Nursing Research; by a Cancer and Leukemia Group B Foundation Clinical Scholar Award; by the Foundation for Informed Medical Decision-Making; by Grants No. P30CA023108, UL1 TR001086, and R03NR014915; an NIH/NINR Small Research Grant 1R03NR014915-01 (Zhigang Li); by Norris Cotton Cancer Center pilot funding; by the Dartmouth-Hitchcock Section of Palliative Medicine; by a National Palliative Care Research Center Junior Career Development Award (M.A.B.); by Grant No. 5R25CA047888 from the University of Alabama at Birmingham Cancer Prevention and Control Training Program (J.N.D.-O.); and by Mentored Research Scholar Grant No. MRSG 12-113-01-CPPB in Applied and Clinical Research from the American Cancer Society (K.D.L.)

Declarations of interest among primary researchers: Authors stated that they had no relationship to disclose.

Power considerations: Authors calculated a target sample size of 360 to provide $80 \%$ power to detect a 6-point difference in the FACIT-Pal and 2.5-point difference in the CES-D based on a t-test comparing the 3-month group differences with a two-sided value of.05 using ENABLE II standard deviations of 17 for the FACIT-Pal and seven for the CES-D. However at the planned study completion date, the final enrollment was 207 because of slower than anticipated accrual. On the basis of the final sample size, the 3-month detectable differences were: FACIT-Pal, 7.7 points; CES-D, 3.2 points.

\section{Risk of bias}

\begin{tabular}{lll}
\hline Bias & Authors' judgement & Support for judgement \\
\hline $\begin{array}{l}\text { Random sequence genera- } \\
\text { tion (selection bias) }\end{array}$ & Low risk & $\begin{array}{l}\text { Quote from main publication: "random assignment was on a one-to-one basis } \\
\text { using computer-generated randomly permuted treatment assignments with } \\
\text { randomly assigned block sizes of two and four stratified by disease (six cate- } \\
\text { gories) and enrollment site (four clinics)". } \\
\text { Comment: probably done }\end{array}$ \\
& \\
\hline
\end{tabular}


Bakitas 2015 (Continued)

Allocation concealment $\quad$ Unclear risk $\quad$ Allocation concealment was not mentioned.
(selection bias)

Blinding of participants

High risk

Comment: no blinding

and personnel (perfor-

mance bias), subjective

outcomes

\begin{tabular}{ll}
\hline $\begin{array}{l}\text { Blinding of participants } \\
\text { and personnel (perfor- }\end{array}$ & Low risk \\
$\begin{array}{l}\text { mance bias), objective } \\
\text { outcomes }\end{array}$ & $\begin{array}{l}\text { Comment: participants and personnel were not blinded but this was unlikely } \\
\text { to lead to bias in objective outcomes. }\end{array}$ \\
\hline
\end{tabular}

Blinding of outcome as- Low risk Low risk of bias due to blinding of data collectors
$\begin{aligned} & \text { sessment (detection bias), } \\ & \text { subjective outcomes }\end{aligned}$

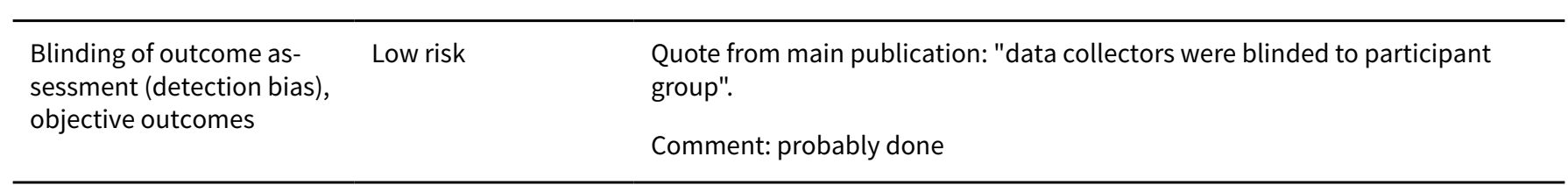

\begin{tabular}{|c|c|c|}
\hline $\begin{array}{l}\text { Incomplete outcome data } \\
\text { (attrition bias) } \\
\text { All outcomes }\end{array}$ & Low risk & $\begin{array}{l}\mathrm{N}=59(56.7 \%) \text { completers in intervention group vs } \mathrm{N}=54(52.4 \%) \text { completers } \\
\text { in control group. In the intervention group, } 12(11.5 \%) \text { patients did not receive } \\
\text { the intervention (did not start intervention }(\mathrm{N}=9) \text { and died before start }(\mathrm{N}= \\
\text { 3)) and } 33(31.7 \%) \text { discontinued intervention for various reasons such as not } \\
\text { interested }(\mathrm{N}=14) \text {, passive withdrawal }(\mathrm{N}=6) \text {, overwhelmed }(\mathrm{N}=6) \text {, moved } \\
\text { care }(\mathrm{N}=3) \text {, too ill }(\mathrm{N}=2) \text {, too well }(\mathrm{N}=1) \text { and no reason }(\mathrm{N}=1) \text {. In the control } \\
\text { group, } 22(21.4 \%) \text { patients did not receive allocated intervention (did not start } \\
\text { intervention }(\mathrm{N}=8) \text { and died before start }(\mathrm{N}=14)) \text { and } 27(26.2 \%) \text { discontinued } \\
\text { intervention for various reasons such as not interested }(\mathrm{N}=11) \text {, passive with- } \\
\text { drawal }(\mathrm{N}=4) \text {, overwhelmed ( } \mathrm{N}=3) \text {, moved care }(\mathrm{N}=3) \text {, too ill (N=1), too well } \\
(\mathrm{N}=5) \text { and no reason }(\mathrm{N}=0) \text {. Missing data were included in analysis. Maximum } \\
\text { likelihood estimates used }\end{array}$ \\
\hline
\end{tabular}

Comment: low risk of bias

\begin{tabular}{|c|c|c|}
\hline $\begin{array}{l}\text { Selective reporting (re- } \\
\text { porting bias) }\end{array}$ & Low risk & All outcomes were reported. \\
\hline \multirow[t]{2}{*}{ Other bias } & Low risk & $\begin{array}{l}\text { Quote from main publication: "the early group had significantly less educa- } \\
\text { tion, higher weekly alcoholic beverage use, and higher clinical trial enroll- } \\
\text { ment... All intention to treat analyses were adjusted for baseline values". }\end{array}$ \\
\hline & & Comment: adjustment was probably done. \\
\hline Size of study & Unclear risk & $\begin{array}{l}\text { Unclear risk of bias because the number of participants in each group was be- } \\
\text { tween } 50 \text { and } 199 .\end{array}$ \\
\hline
\end{tabular}

Bekelman 2018

\section{Study characteristics}

$\begin{array}{ll}\text { Methods } & \text { Design: } \text { RCT } \\ & \text { Single-blind, multisite RCT } \\ & \text { Who led PC intervention: Multidisciplinary team }\end{array}$

The effectiveness and cost-effectiveness of hospital-based specialist palliative care for adults with advanced illness and their caregivers (Review)

Copyright $\odot 2020$ The Cochrane Collaboration. Published by John Wiley \& Sons, Ltd. 
Core team: Nurse/social worker. A collaborative care team comprising the nurse and social worker, a primary care clinician, palliative care physician, and cardiologist provided case review and supervision.

\section{Participants}

\section{Country and regions: USA, Colorado}

Recruitment: Patients with heart failure were identified through the study sites' electronic health records.

Inclusion criteria: Patients with chronic heart failure and reduced health status who were likely to need the additional resources provided by the intervention. The diagnosis was defined using previously validated administrative data supplemented with data on required diuretic dosing (furosemide $\geq$ $80 \mathrm{mg} / \mathrm{d}$ or equivalent), left ventricular ejection fraction of $40 \%$ or less, brain-type natriuretic peptide (BNP) levels of $250 \mathrm{pg} / \mathrm{mL}$ or more, or N-terminal prohormone level of BNP of $1000 \mathrm{pg} / \mathrm{mL}$ or more. During the study screening process, patients who reported reduced heart failure-specific health status (a Kansas City Cardiomyopathy Questionnaire Short Version [KCCQ] score of $\leq 70$ ) or reported at least 1 of the study's target symptoms (fatigue, shortness of breath, pain, and/or depression) were targeted for enrollment. Early in the study, the cut-offs for diuretic dosing and BNP were relaxed, and both reduced heart failure-specific health status and 1 of the target symptoms were required to increase the eligible study population while still enrolling symptomatic patients.

Exclusion criteria: Patients with active substance abuse or serious mental illness were excluded.

Number of patients: $\mathrm{N}=314$ (157 intervention and 157 control)

Diseases: Symptomatic heart failure

Patient characteristics: Mean age in years (SD): 64.5 (10.9) years in intervention group, 66.5 (11.8) years in control group; $18.5 \%$ female in intervention group, $24.2 \%$ female in control group

Deaths at end of study ( $(\%)): \mathrm{N}=8.3(1.9 \%)$ in intervention and $5(3.2 \%)$ in control

Withdrawals/other dropouts $(\mathrm{N}(\%)): \mathrm{N}=32.16(10.2 \%)$ in intervention and $16(10.2 \%)$ in control

Type: The nurse and social worker who provided the CASA intervention were not specialist palliative care clinicians. However, they were trained to provide the intervention and they both worked with the patients' primary care clinicians and were supervised by a study primary care clinician, cardiologist, and palliative care physician.

Service base: The CASA trial was a National Institutes of Health-funded trial in 3 health systems (urban safety net, Veterans Affairs, and academically affiliated health systems).

Team: Core staff included a nurse and social worker. However, a collaborative care team comprising the nurse and social worker, a primary care clinician, palliative care physician, and cardiologist provided case review and supervision.

Intervention condition: The CASA intervention included 3 components. A registered nurse addressed symptoms, a social worker provided structured psychosocial care, and a team (including the nurse and social worker, a primary care clinician, palliative care physician, and cardiologist) reviewed patients' care and provided orders for tests and medications to patients' clinicians for review and signature. The patient and nurse selected an initial symptom on which to focus. The nurse assessed and managed symptoms using structured guidelines developed for the study, including disease-specific, behavioural, and palliative approaches. The nurse was trained in helping communication, motivational interviewing, and the symptom guidelines. Six nurse intervention follow-up assessments by telephone (1-2 per month) were planned using a structured symptom rating scale. The nurse applied motivational interviewing to promote changes in health behaviours. The nurse had access to a PhD-level clinical nurse specialist to discuss difficult issues regarding symptom management. The social worker provided a structured telephone based psychosocial intervention to help patients with heart failure adjust to living with illness and address depression symptoms, if present. The psychosocial intervention was operationalised in a treatment manual and was based on interpersonal and behavioural activation psychotherapies. The following topics were included in approximately 6 counselling sessions: grief and loss, change in role, behavioural activation, and pacing. The social worker also provided support to

The effectiveness and cost-effectiveness of hospital-based specialist palliative care for adults with advanced illness and their caregivers 
patients' informal unpaid caregivers as needed. The social worker received psychosocial intervention training and follow-up supervision. The nurse and the social worker discussed the patients in weekly collaborative care team meetings with a team that included a palliative care physician.

Duration: 6 months

Control condition: Patients in the usual care group received care at the discretion of their clinicians, which could include care from cardiology, palliative care and mental health. Patients were also given an information sheet that outlined self-care for heart failure. Patients who had significant depressive symptoms were notified of this, and their clinicians were also contacted. Referring clinicians then assumed responsibility for depression care.

\section{Outcomes}

\section{Primary outcome}

Patient-reported heart-failure specific health status assessed using the Kansas City Cardiomyopathy Questionnaire (KCCQ)

\section{Secondary outcomes}

Depression measured by the Patient Health Questionnaire (PHQ-9)

Anxiety measured by the Generalized Anxiety Disorder (GAD-7) Questionnaire

Overall symptom distress measured by the General Symptom Distress Scale (GSDS)

Pain measured by the PEG (3 items derived from the Brief Pain Inventory)

Fatigue measured by the Patient Reported Outcomes Measurement Information System (PROMIS) Short Form

Shortness of breath measured by the Memorial Symptom Assessment Scale

unpaid caregiver depression assessed using the Patient Health Questionnaire-8 (PHQ-8)

unpaid caregiver burden assessed using the Zarit Burden Inventory (ZBI)

Number of hospitalisations

Mortality

\section{Assessment points:}

Measures were completed in person, by mail, or by telephone at baseline and at 3, 6, and 12 months by personnel who did not provide the intervention and were unaware of treatment arm assignment and intervention activities.

Resource use/costs Number of hospitalisations

Use of other services

Time horizon: enrollment to one year

Funding source: This work was supported by grant R01-013422 from the National Institute of Nursing Research, National Institutes of Health; grant UL1 TR001082 from the National Institutes of Health/National Center for Advancing Translational Sciences Colorado Clinical and Translational Science Award; and grant CDA 08-022 from the Veterans Affairs Health Services Research and Development Service.

Declarations of interest among primary researchers: None reported

Power considerations: The authors planned a sample size of 312 to detect a change in KCCQ score of 6 to 8 points. They anticipated a $25 \%$ dropout rate owing to death etc. As the SD for the KCCQ has ranged from 15 to 20 in prior studies, with this sample size, they had $86 \%$ power to detect a change of 6 points (assuming an SD of 15) or 8 points (assuming an SD of 20) (2-sided test, $\alpha=.05$ ). 
Bekelman 2018 (Continued)

Risk of bias

\begin{tabular}{lll}
\hline Bias & Authors' judgement & Support for judgement \\
\hline $\begin{array}{ll}\text { Random sequence genera- } \\
\text { tion (selection bias) }\end{array}$ & Low risk & $\begin{array}{l}\text { Quote from main publication: "the randomisation sequence was comput- } \\
\text { er-generated using random block sizes and stratification by study site and was } \\
\text { concealed from study personnel". }\end{array}$ \\
& Comment: probably done
\end{tabular}

\begin{tabular}{|c|c|c|}
\hline \multirow[t]{2}{*}{$\begin{array}{l}\text { Allocation concealment } \\
\text { (selection bias) }\end{array}$} & Low risk & $\begin{array}{l}\text { Quote from main publication: "the randomisation sequence was comput- } \\
\text { er-generated using random block sizes and stratification by study site and was } \\
\text { concealed from study personnel". }\end{array}$ \\
\hline & & Comment: probably done \\
\hline
\end{tabular}

Blinding of participants High risk Comment: no blinding
and personnel (performance bias), subjective outcomes

\begin{tabular}{ll}
\hline $\begin{array}{l}\text { Blinding of participants } \\
\text { and personnel (perfor- }\end{array}$ & Low risk \\
$\begin{array}{l}\text { mance bias), objective } \\
\text { outcomes }\end{array}$ & $\begin{array}{l}\text { Quote from main publication: "because of the nature of the intervention, par- } \\
\text { ticipants could not be blinded". }\end{array}$ \\
& $\begin{array}{l}\text { Comment: participants were not blinded but this was unlikely to lead to bias in } \\
\text { objective outcomes. }\end{array}$
\end{tabular}

Blinding of outcome as- Low risk Low risk of bias due to blinding of outcome assessors
sessment (detection bias),
subjective outcomes

Blinding of outcome as-
sessment (detection bias),

\begin{abstract}
Quote from main publication: "measures were completed in person, by mail, or by telephone at baseline and at 3,6, and 12 months by personnel who did not provide the intervention and were unaware of treatment arm assignment and intervention activities".
\end{abstract}

Comment: participants were not blinded while personnel who sometimes assisted in completing measures were blinded. Given that objective outcomes such as mortality are unlikely to be affected by lack of blinding, a low risk of bias was given.

Incomplete outcome data Low risk (attrition bias)

$\mathrm{N}=124(79 \%)$ completers in intervention group vs $\mathrm{N}=124$ (79\%) completers in control group. In the intervention group, $3(1.9 \%)$ patients died, 6 withdrew and 10 were lost to follow-up. The remaining 14 were not accounted for. In the control group, 5 (3.2\%) patients died, 2 withdrew and 14 were lost to follow-up. The remaining 12 were not accounted for. Missing data were included in analysis. Maximum likelihood estimates used.

Comment: low risk of bias

\begin{tabular}{|c|c|c|}
\hline $\begin{array}{l}\text { Selective reporting (re- } \\
\text { porting bias) }\end{array}$ & High risk & $\begin{array}{l}\text { Some outcomes in the protocol were not reported in the study such as the } \\
\text { FACIT-Sp for spiritual well-being, QUAL-E and EQ-5D-5L for quality of life. In ad- } \\
\text { dition, unpaid caregiver outcomes were not reported. }\end{array}$ \\
\hline
\end{tabular}

Other bias Unclear risk

Quote from main publication: "baseline characteristics were balanced between groups, except those in the intervention group were significantly more likely to have a biventricular pacemaker and to be less short of breath compared with those in the control group".

The effectiveness and cost-effectiveness of hospital-based specialist palliative care for adults with advanced illness and their caregivers 


\begin{tabular}{ll}
\hline Size of study $\quad$ Unclear risk & $\begin{array}{l}\text { Unclear risk of bias because the number of participants in each group was be- } \\
\text { tween } 50 \text { and } 199\end{array}$
\end{tabular}

Brannstrom 2014

\section{Study characteristics}

Design: RCT
Whothods led PC intervention: Multidisciplinary team (intervention was organised in close co-operation
with out-of-hours palliative advanced home care who were fully informed of the identities of the pa-
tients and knew how to respond to calls).
Core team: Physician/nurse. Clinical team comprising specialists in palliative and heart failure care, i.e.
specialised nurses, palliative care nurses, cardiologist, palliative care physician, physiotherapist, and
occupational therapist held regular meetings about the patient twice a month. The team was responsi-
ble for total care.

Participants Country and regions: Sweden

Recruitment: January 2011 to October 2012. Patients with a confirmed diagnosis of CHF and cared for at the Department of Medicine-Geriatrics or primary healthcare centres and who met the criteria of the European Society of Cardiology were asked to participate in the study.

Inclusion criteria: CHF with NYHA functional classes III-IV symptoms and at least one of the following: one hospitalised episode of worsening heart failure that resolved with the injection/infusion of diuretics or the addition of other heart failure treatment in the preceding 6 months and regarded as being 'optimally treated' according to the responsible physician; need for frequent or continual IV support; chronically poor QoL based on a visual analogue scale (VAS) score < 50; signs of cardiac cachexia, defined as an involuntary non-oedematous weight loss $\geq 6 \%$ of total body weight within the preceding 6 12 months; and life expectancy of $<1$ year.

Exclusion criteria: Patients who did not want to participate in the study; had severe communication problems; had severe dementia or other serious diseases in which heart failure was of secondary importance; with other life-threatening illnesses as their primary diagnosis and an expected short survival time; whose primary care centre responsible for their care was located $>30 \mathrm{~km}$ from the hospital; and who were already participating in another clinical trial.

Number of patients: $\mathrm{N}=72$ (36 intervention and 36 control)

Diseases: Chronic heart failure (NYHA class III - IV)

Patient characteristics: mean age in years (SD): 81.9 (7.2) years in intervention group, 76.6 (10.2) years in control group; $27.8 \%$ female in intervention group, $30.6 \%$ female in control group

Deaths at end of study $(\mathrm{N}(\%)): \mathrm{N}=12.8(22.2 \%)$ in intervention and $4(11.1 \%)$ in control

Withdrawals/other dropouts (N (\%)): Not stated

Type: Specialist palliative care. Patients in the intervention group were offered a multidisciplinary approach involving collaboration between specialists in palliative and heart failure care, i.e. specialised nurses, palliative care nurses, cardiologist, palliative care physician, physiotherapist, and occupational therapist.

Service base: County hospital located in Northern Sweden 
Team: Clinical team comprised specialists in palliative and heart failure care, i.e. specialised nurses, palliative care nurses, cardiologist, palliative care physician, physiotherapist, and occupational therapist.

Intervention condition: Patients in the intervention group were offered a multidisciplinary approach involving collaboration between specialists in palliative and heart failure care. The patients were also offered structured, person-centred care (PCC) at home. The patient's narrative was recorded in a structured manner and a mutual care plan was developed that included the goals and strategies for implementation and follow-up. The intervention was carried out as follows: (i) after identifying a patient who fulfilled the inclusion criteria, a responsible physician and nurse were identified for each patient; (ii) the patient received a thorough medical examination by the physician with identification of comorbidities and assessment of physiological, social, and spiritual needs; (iii) meeting with nurses who used a model for person-centred palliative care comprising self-image, self-determination, social relationships, symptom control, synthesis, and surrender, and continued through; (iv) regular meetings about the patients' conditions within the team twice a month; and ( $v$ ) brief discussions took place between the team members at the unit and information was shared by the documentation in medical records and phone calls. The team was responsible for the total care, i.e. including comorbidities.

Duration: 6 months

Control condition: Usual care was provided mainly by general practitioners or doctors and/or the nurse-led heart failure clinic at the Medicine-Geriatrics department.

Outcomes

\section{Outcomes}

Symptom burden assessed using the ESAS

Health-Related Quality of Life assessed using the EQ-5D and the Kansas City Cardiomyopathy Questionnaire

Functional classes

Mortality

Cost

Assessment points: After randomisation, prospective assessments were made at baseline and 1, 3, and 6 months of follow-up using the Edmonton Symptom Assessment Scale (ESAS), Euro Qol (EQ-5D), and Kansas City Cardiomyopathy Questionnaire (KCCQ).

Resource use/costs

Resource utilisation: number of hospitalisations, number of days spent in hospital, number of physician and nurse visits, phone calls and/or drug prescriptions at the outpatient clinics of the hospitals and at the primary healthcare centres.

Time horizon: Study enrollment to study completion at 6 months

Data sources: The data were collected over a total of 6 months between 2011 and 2013. Authors calculated cost based on the time spent for each patient. When services were given as part of our intervention, minutes were documented and used to calculate costs for each staff category. Healthcare services offered as part of the standard care (both primary healthcare and hospital care) were calculated similarly but based on assumptions made on timing. These assumptions were based on recommendations from the county council and according to practice in the area. Costs were calculated by multiplying the allocated time for given services by the average salaries.

Analytical perspective: Provider perspective

Funding source: This work was supported by the Swedish Association of Local Authorities and Regions, the Swedish Heart and Lung Association, and the Ronnbaret Foundation Skelleftea Municipality.

Declarations of interest among primary researchers: None declared

Power considerations: The PREFER model was created to improve the mean symptom burden by at least $25 \%$ compared with the control group. With a power of $80 \%$, significance level of $P<0.05$, and an

The effectiveness and cost-effectiveness of hospital-based specialist palliative care for adults with advanced illness and their caregivers 
Brannstrom 2014 (Continued)

estimated dropout rate of 15\%, 31 patients were needed in each arm (total 62 patients). Due to patients dropping out, mainly due to deaths (12 patients), the number of participants was increased to 36 in each arm.

\section{Risk of bias}

\begin{tabular}{lll}
\hline Bias & Authors' judgement & Support for judgement \\
\hline $\begin{array}{l}\text { Random sequence genera- } \\
\text { tion (selection bias) }\end{array}$ & Low risk & $\begin{array}{l}\text { Quote from main publication: "patients were randomized with envelopes in } \\
\text { blocks of } 20 \text { to the PREFER intervention group }(n=36) \text { or to usual care }(n=36) " . \\
\end{array}$ \\
& & Comment: probably done \\
\hline
\end{tabular}

\begin{tabular}{ll}
\hline $\begin{array}{l}\text { Allocation concealment } \\
\text { (selection bias) }\end{array}$ & Unclear risk
\end{tabular}

\begin{tabular}{ll}
\hline $\begin{array}{l}\text { Blinding of participants } \\
\text { and personnel (perfor- } \\
\text { mance bias), subjective } \\
\text { outcomes }\end{array}$ & High risk \\
\hline $\begin{array}{l}\text { Blinding of participants } \\
\begin{array}{l}\text { and personnel (perfor- } \\
\text { mance bias), objective }\end{array}\end{array}$ & Low risk \\
outcomes & $\begin{array}{l}\text { Quote from main publication: "this was a prospective randomised study with } \\
\text { an open non-blinded design". }\end{array}$ \\
& $\begin{array}{l}\text { Comment: participants and personnel were not blinded but this was unlikely } \\
\text { to lead to bias in objective outcomes such as mortality. }\end{array}$
\end{tabular}

\begin{tabular}{lll}
\hline $\begin{array}{l}\text { Blinding of outcome as- } \\
\text { sessment (detection bias), } \\
\text { subjective outcomes }\end{array}$ & High risk & High risk of bias due to lack of blinding \\
\hline $\begin{array}{l}\text { Blinding of outcome as- } \\
\begin{array}{l}\text { sessment (detection bias), } \\
\text { objective outcomes }\end{array}\end{array}$ & Low risk & $\begin{array}{l}\text { Quote from main publication: "this was a prospective randomised study with } \\
\text { an open non-blinded design". } \\
\text { Comment: lack of blinding of outcome assessment was unlikely to lead to bias } \\
\text { in objective outcomes such as mortality. }\end{array}$ \\
\hline
\end{tabular}

\begin{tabular}{ll}
\hline $\begin{array}{l}\text { Incomplete outcome data } \\
\text { (attrition bias) }\end{array}$ & High risk \\
$\begin{array}{l}\text { All outcomes } \\
\text { in control group. In the intervention group, } 8(22.2 \%) \text { patients died while } 4 \\
(11.1 \%) \text { deaths were recorded in the control group during the study. Missing } \\
\text { data appeared to have been excluded from the analysis. }\end{array}$ \\
Comment: high risk of bias
\end{tabular}

\begin{tabular}{|c|c|c|}
\hline $\begin{array}{l}\text { Selective reporting (re- } \\
\text { porting bias) }\end{array}$ & High risk & $\begin{array}{l}\text { Although primary outcomes and secondary outcomes were specified on the } \\
\text { clinical trials registry NCT01304381, it was not specified in the published pa- } \\
\text { per. Quality of life assessed using the Kansas City Cardiomyopathy Question- } \\
\text { naire (KCCQ), functional class and mortality were outcomes in the published } \\
\text { paper but not mentioned on the clinical trial registry. } \\
\text { Comment: high risk of bias }\end{array}$ \\
\hline Other bias & Low risk & $\begin{array}{l}\text { Quote from main publication: "the two groups were balanced with respect to } \\
\text { baseline characteristics except for mean age". } \\
\text { Comment: given that the authors controlled for age, a decision was made not } \\
\text { to rate down for imbalance bias. Rather, a low risk of bias was rated. }\end{array}$ \\
\hline
\end{tabular}

\begin{tabular}{ll}
\hline Size of study $\quad$ High risk & $\begin{array}{l}\text { High risk of bias because the number of participants in each group was less } \\
\text { than } 50\end{array}$
\end{tabular}

The effectiveness and cost-effectiveness of hospital-based specialist palliative care for adults with advanced illness and their caregivers 
Brumley 2007

\section{Study characteristics}

Design: RCT
Multisite RCT
Who led PC intervention: Multidisciplinary team (physicians conduct home visits and are available
along with nursing services on a 24-hour on-call basis)
Core team: Patient/family/physician/nurse/social worker. Additional team members, including spiri-
tual counsellor or chaplain, bereavement co-ordinator, home health aide, pharmacist, dietitian, volun-
teer, physical therapist, occupational therapist, and speech therapist, join the core care team in service
provision as needed.

\section{Participants Country and regions: Hawaii and Colorado, USA.}

Recruitment: Discharge planners, primary care physicians, and other specialty physicians referred potentially eligible terminally ill patients to the study. For those meeting the initial criteria, the intake clerk contacted the primary care physician to determine the prognosis. Once eligibility was determined, the intake clerk gained informed consent from the patient to participate in the study.

Inclusion criteria: Patients with a primary diagnosis of CHF, COPD, or cancer and a life expectancy of 12 months or less, have visited the emergency department or hospital at least once within the previous year; and scored $70 \%$ or less on the Palliative Performance Scale.

Exclusion criteria: Not explicitly stated

Number of patients: $\mathrm{N}=297$ (145 intervention and 152 control)

Diseases: Cancers $(\mathrm{N}=138,46 \%)$, $\mathrm{COPD}(\mathrm{N}=62,21 \%)$ and $\mathrm{CHF}(\mathrm{N}=97,33 \%)$

Patient characteristics:. Mean age in years (SD): 73.9 (11.1) years in intervention group, 73.7 (13) years in control group; $45 \%$ female in intervention group, $53 \%$ female in control group

Deaths at end of study ( $\mathrm{N}(\%)): \mathrm{N}=8: 8(5.5 \%)$ in intervention and none in control

Withdrawals/other dropouts $(\mathrm{N}(\%)): \mathrm{N}=5: 2(1.4 \%)$ in intervention and $3(2 \%)$ in control

Service base: Two group-model, closed-panel, non-profit health maintenance organisations (HMOs) providing integrated healthcare services in Hawaii and Colorado

Team: The IHPC programme uses an interdisciplinary team approach, with the core team consisting of the patient and family plus a physician, nurse, and social worker with expertise in symptom management and biopsychosocial intervention. Additional team members, including spiritual counsellor or chaplain, bereavement co-ordinator, home health aide, pharmacist, dietitian, volunteer, physical therapist, occupational therapist, and speech therapist, join the core care team in service provision, as needed.

Intervention condition: The IHPC programme is an interdisciplinary home-based healthcare programme. Modelled after hospice programs in that it offers pain management and other comfort care in the patient's home, the IHPC programme also features important modifications. Upon admission, the team assesses the physical, medical, psychosocial, and spiritual needs of the patient and family. All patients received initial assessments from physicians, nurses, and social workers. Additional team members join the core care team in service provision, as needed. The team convenes to develop a care plan in accordance with the wishes of the patient and the family. Frequency of subsequent medical visits is based on the individual needs of the patient. Physicians conduct home visits and are available along with nursing services on a 24-hour on-call basis. Advanced care planning is provided that involves pa-

The effectiveness and cost-effectiveness of hospital-based specialist palliative care for adults with advanced illness and their caregivers 

care.

Duration: Participants enrolled in the IHPC arm received palliative care until death or transfer to a hospice programme.

Control condition: Usual care consisted of standard care to meet the needs of the patients and followed Medicare guidelines for home healthcare criteria. These services included home health services, acute care services, primary care services, and hospice care. Additionally, they received ongoing home care when they met the Medicare-certified criteria for an acute condition.

Outcomes

Resource use/costs

\section{Outcomes}

Patient satisfaction with care assessed using the Reid-Gundlach Satisfaction with Services instrument

Site of death

Service use

Cost of care

Survival

Assessment points: Interviews were conducted via telephone by undergraduate and graduate-level research assistants blinded to group assignment within 48 hours of study enrollment and every 30, 60, 90 , and 120 days to gather demographic information and satisfaction with services.

Resource use: emergency department visits, hospitalisation, enrollment and days in hospice

Time horizon: Medical service use data were collected from the time the patient enrolled in the study until the time of death or the end of the study period.

Data sources: Resource use data for each participant were collected retrospectively from the non-profit HMO mainframe database, from the time the patient enrolled in the study until the time of death or end of study (2002 to 2004). Costs were calculated using actual costs for contracted medical services (services provided by non-HMO contracted facilities in Colorado) and proxy cost estimates for all services provided within the HMO. Costs were in 2002 USD.

Analytical perspective: Not clear

Notes $\quad$ Funding source: This study was funded by the Kaiser Permanente Garfield Memorial Fund.

Declarations of interest among primary researchers: Richard Brumley, Nora Morgenstern, Sherry Saito, and Rae Seitz are employed as physician partners in the Permanente Medical Group of Southern California, Colorado, and Hawaii, respectively. Susan Enguidanos is employed by Partners in Care Foundation and conducted this work through a subcontract with the Garfield Memorial Fund. Paula Jamison and Jorge Gonzalez are employed by Partners in Care Foundation and serve a consultative role through this employment to Kaiser Permanente. Kristine Hillary is employed by Kaiser Permanente. Janet Mcllwaine was employed by Kaiser Permanente.

Power considerations: Based on methods established previously, it was determined that, using a significance criterion of.05, a sample size of 300 would be necessary for a statistical power of 0.80 , using nondirectional (two-tailed) tests to detect whether the intervention had a significant effect on medical care costs.

\section{Risk of bias}

\begin{tabular}{lll}
\hline Bias & Authors' judgement & Support for judgement \\
\hline $\begin{array}{l}\text { Random sequence genera- } \\
\text { tion (selection bias) }\end{array}$ & Low risk & $\begin{array}{l}\text { Quote from main publication: "group assignment was determined by blocked } \\
\text { randomisation using a computer-generated random number chart, stratified } \\
\text { according to study site". }\end{array}$ \\
\hline
\end{tabular}

The effectiveness and cost-effectiveness of hospital-based specialist palliative care for adults with advanced illness and their caregivers 
Brumley 2007 (Continued)

Comment: probably done

\begin{tabular}{lll}
\hline $\begin{array}{l}\text { Allocation concealment } \\
\text { (selection bias) }\end{array}$ & Unclear risk & Allocation concealment was not described in the study. \\
\hline $\begin{array}{l}\text { Blinding of participants } \\
\text { and personnel (perfor- } \\
\text { mance bias), subjective } \\
\text { outcomes }\end{array}$ & High risk & Comment: no blinding \\
\hline $\begin{array}{l}\text { Blinding of participants } \\
\text { and personnel (perfor- } \\
\text { mance bias), objective } \\
\text { outcomes }\end{array}$ & Low risk & Participants and personnel were not blinded \\
\hline
\end{tabular}

Blinding of outcome as- Low risk Low risk of bias because outcome assessors were blinded

sessment (detection bias),

subjective outcomes

\section{Blinding of outcome as- Low risk}

sessment (detection bias),

objective outcomes
Quote from main publication: "undergraduate- and graduate-level research assistants, blinded to group assignments, were recruited and trained to conduct telephone interviews with the patient or, if the patient was unable to participate, the primary unpaid caregiver".

Comment: outcome assessors were blinded.

$\mathrm{N}=145(93.5 \%)$ completers in intervention group vs $\mathrm{N}=152(98.1 \%)$ completers in control group. Only completers were included in analysis. In the intervention group, 10 (6.9\%) people were lost to follow-up [8 (5.5\%) deaths and $2(1.4 \%)$ people withdrew from the study]. In the control group, 3 (2\%) people withdrew.

Comment: low risk because attrition was less than $10 \%$
Incomplete outcome data Low risk

(attrition bias)

All outcomes
Selective reporting (re- Unclear risk Unclear as protocol not available porting bias)
Quote from main publication: "there were no significant differences between study groups in baseline measures other than satisfaction. Satisfaction with services was measured at baseline after study assignment. Those randomised to intervention demonstrated significantly higher satisfaction with services at baseline than those assigned to usual care $(P=0.03)$ ".

Comment: given that the authors did not state that they controlled for difference in satisfaction levels at baseline, an unclear risk of bias rating was given.

$\begin{array}{ll}\text { Size of study Unclear risk } & \begin{array}{l}\text { Unclear risk of bias because the number of participants in each group was be- } \\ \text { tween } 50 \text { and } 199\end{array}\end{array}$

Carson 2016

\section{Study characteristics}

\begin{tabular}{ll}
\hline Methods & Design: RCT \\
Multicentre RCT \\
Who led PC intervention: Multidisciplinary team
\end{tabular}

The effectiveness and cost-effectiveness of hospital-based specialist palliative care for adults with advanced illness and their caregivers 
Core team: Palliative care physician/nurse practitioner. Could include social workers, chaplains, or other disciplines, as needed.

\section{Participants}

Country and regions: USA, Northeastern and Southeastern United States

Recruitment: October 2010 to November 2014. Patients were identified by screening of ICU records and discussion with ICU clinicians.

Inclusion criteria: Patients $\geq 21$ years treated in medical ICUs were eligible if they required at least 7 days of mechanical ventilation uninterrupted for 96 hours or longer and were not expected to be weaned or to die within 72 hours. For the first year of the study, patients were eligible if they required at least 10 days of mechanical ventilation. Family members were eligible if they had the responsibility of healthcare decision-making for the patient.

Exclusion criteria: Patients who were mechanically ventilated at an outside hospital for $>7$ days or who had chronic neuromuscular disease, trauma, or burns. Patients were excluded if a surrogate decision-maker was not available or lacked English proficiency, the primary physician refused to grant permission to investigators to approach the patient or family, or the investigators were the attending physicians. Patients previously admitted to the study ICU or who had a palliative care consultation prior to screening

Number of family surrogate decision-makers: $\mathrm{N}=365$ (184 intervention and 181 control)

Number of patients: $\mathrm{N}=256$ (130 intervention and 126 control)

Diseases: Disease not specified but all patients were adults treated in medical ICUs

Patient characteristics: Mean age in years $(95 \% \mathrm{Cl}): 58$ (55.2 - 60.8) years in intervention group, 57 (54 59.7 ) years in control group; $51 \%$ female in intervention group, $52 \%$ female in control group

Family surrogate decision-makers characteristics: Mean age in years ( $95 \% \mathrm{Cl}): 51$ (48.8 - 52.8) years in intervention group, 51 (48.6 - 52.7) years in control group; $70 \%$ female in intervention group, $72 \%$ female in control group

Deaths among patients at end of study $(\mathrm{N}(\%))$ : Total $\mathrm{N}=8: 8(6.2 \%)$ in intervention and none in control

Withdrawals/other dropouts among family surrogate decision-makers (N (\%)): Total $N=53: 21$ (11.4\%) intervention and $32(17.7 \%)$ control

Interventions

Name: Structured family meetings led by palliative care specialists and provision of an informational brochure (intervention) vs provision of an informational brochure and routine family meetings conducted by ICU teams (control)

Type: Specialist palliative care. Included a palliative care physician.

Service base: 4 medical intensive care units

Team: Comprised of a palliative care physician and nurse practitioner and could include social workers, chaplains, or other disciplines, as needed.

Intervention condition: A validated and widely available brochure describing chronic critical illness was provided to the family surrogate decision-makers. Research co-ordinators then scheduled a minimum of 2 meetings with the support and information team. The protocol provided for scheduling of additional meetings at the request of the family, ICU physician, or support and information team clinicians. Support and information team clinicians conducted pre-meetings with ICU physicians to review each patient's condition, prognosis, and previous discussions of goals of care. The support and information team clinicians also reviewed estimates of 1-year prognosis based on the ProVent 14 score. The support and information team meetings were structured according to a set of objectives and recommended topics. Support and information team clinicians were trained by reviewing the main objectives of the meeting templates that appeared in the original protocol; however, they were allowed some flexibility for adapting the content to the particular needs of each family. After the meetings with family members, the support and information team provided feedback to the ICU clinicians not in attendance.

The effectiveness and cost-effectiveness of hospital-based specialist palliative care for adults with advanced illness and their caregivers 
Duration: The first meeting with the support and information team was conducted after 7 days of mechanical ventilation at the onset of chronic critical illness and when a tracheostomy is often considered. The second meeting was conducted after further treatment was provided for a period approximating the mean duration of mechanical ventilation after tracheostomy for patients who achieved ventilator liberation.

Control condition: The ICU clinicians managed all formal and informal family meetings per their usual practice without input from the palliative care specialists. Family surrogate decision-makers in the control group received the same informational brochure as the intervention group. Clinicians were able to formally consult palliative care clinicians at their discretion, and this was encouraged if they needed assistance with symptom management or for transfer to hospice.

\section{Primary outcome:}

Anxiety and depression of surrogate assessed using the Hospital Anxiety and Depression Scale (HADS)

\section{Secondary outcomes:}

Post-traumatic Stress Disorder (PTSD) of the surrogate assessed using the Impact of Event Scale-Revised (IES-R)

Discussion of patient preferences

Family satisfaction with care assessed using the 24-item Family Satisfaction in the Intensive Care Unit survey

Hospital length of stay for patients

90-day survival of patients

Assessment points: Research co-ordinators interviewed family surrogate decision-makers prior to patient randomisation to collect demographics and prehospitalisation activities of daily living and instrumental activities of daily living. Research co-ordinators blinded to group assignment interviewed surrogate decision-makers immediately after the second support and information team meeting for the intervention group and 10 days after randomisation for the control group, unless the patient had died. All surrogate decision-makers were interviewed again by telephone for follow-up beginning 90 days after randomisation.

\section{Resource use/costs This relates to resource use by patients}

Hospital length of stay

Number of ICU days

Ventilator days

Time horizon: period of hospitalisation Research.

Declarations of interest among primary researchers: One of the authors reported having a consulting agreement with the Research Triangle Institute related to quality of care in long-term acute care hospitals. No other disclosures were reported.

Power considerations: Based on a previous study, it was determined that 150 family members in the intervention group and the control group would provide a sufficient sample to detect a minimal clinically important difference of 1.5 for mean total HADS score with $90 \%$ power and a type I error of $5 \%$.

\section{Risk of bias}


Carson 2016 (Continued)

Random sequence genera- Low risk tion (selection bias)
Quote from main publication: "after enrollment of patients and family members, patients were randomized to the intervention or the control group using a computer-generated, web-based randomization system with blinding of allocation. The randomization was stratified by study site in block sizes varying from 8 to $10 "$.

Comment: probably done
Quote from main publication: "after enrollment of patients and family members, patients were randomized to the intervention or the control group using a computer-generated, web-based randomization system with blinding of allocation".

Comment: probably done

Blinding of participants High risk Comment: no blinding

and personnel (perfor-

High risk

Comment: no blinding

mance bias), subjective

outcomes

\begin{tabular}{ll}
\hline $\begin{array}{l}\text { Blinding of participants } \\
\text { and personnel (perfor- }\end{array}$ & Low risk \\
$\begin{array}{l}\text { mance bias), objective } \\
\text { outcomes }\end{array}$ & $\begin{array}{l}\text { Participants were not blinded. } \\
\text { Comment: participants and personnel were not blinded but this was unlikely } \\
\text { to lead to bias in objective outcomes. }\end{array}$ \\
\hline
\end{tabular}

Blinding of outcome as- Unclear risk sessment (detection bias), subjective outcomes

Study did not state whether outcome assessors were blinded to group allocation when assessing secondary outcomes.

Blinding of outcome as-
sessment (detection bias),
objective outcomes risk

Quote from main publication: "observers were blinded to group allocation for the measurement of the primary outcomes... The research co-ordinator at each study site who had knowledge of group assignments was not involved in collection of the primary outcomes through family interviews. A research assistant at each study site who was blinded to group assignments conducted these interviews".

Comment: lack of blinding of outcome assessment was unlikely to lead to bias in objective outcomes.

Incomplete outcome data High risk
(attrition bias)

$\mathrm{N}=163(88.6 \%)$ completers (surrogates) in intervention group vs $\mathrm{N}=149$

All outcomes

$(82.3 \%)$ completers (surrogates) in control group. In the intervention group, 21

(11.4\%) patients refused to participate and $6(3.3 \%)$ were unavailable. In control group, $15(8.3 \%)$ refused to participate and 17 (9.4\%) were unavailable. Missing data were excluded from the analysis.

Comment: high risk of bias because the study used 'completer' analysis and > $10 \%$ of surrogates did not complete the study

Selective reporting (re- High risk
porting bias)

One of the secondary outcomes specified in the protocol (e.g. physician-surrogate discordance score) was not reported. Furthermore, the original protocol specified 3 co-primary endpoints for anxiety and depression (HADS scores), PTSD (IES-R scores), and discussion of patient preferences, it was decided before enrollment that total HADS score should be the primary outcome, which was consistent with the power analysis.

Comment: high risk of bias

\begin{tabular}{ll}
\hline Other bias $\quad$ Low risk $\quad$ Study appeared to be free of other biases. \\
\hline
\end{tabular}

The effectiveness and cost-effectiveness of hospital-based specialist palliative care for adults with advanced illness and their caregivers 
Carson 2016 (Continued)

$\begin{array}{ll}\text { Size of study } \quad \text { Low risk } & 200\end{array}$

\section{Study characteristics}

$\begin{array}{ll}\text { Methods } & \text { Design: RCT (patient, family and staff) } \\ & \text { Single-centre, unblinded, RCT } \\ \text { Who led PC intervention: Multidisciplinary team } \\ \text { Core team: Physician/registrar/resident/clinical nurse consultant }\end{array}$

Recruitment: May 2006 and October 2008 (29 months): patients with a terminal or pre-terminal condition were eligible if the treating intensivist indicated to the patient or the patient's surrogate that they believed treatment should not be escalated or should be withdrawn. Patients were then enrolled in the study if they met the selection criteria.

Inclusion criteria: Aged 18 years or older; pre-terminal or terminal condition, and the duty intensivist and parent treating teams believed that continuing current treatment or escalating treatment was unlikely to result in a significant improvement in the patient's medical condition; duty intensivist deemed it appropriate that a not-for-resuscitation (NFR) order be written for the patient; patient was deemed unlikely to survive more than 1 week if treatment was either withdrawn or not escalated; patient was expected to stay in the ICU for at least another 48 hours; patient or surrogate was willing to consent to the completion of two questionnaires during the end-of-life process; no reason to believe that the patient or family would object to a palliative care team being involved in the patient's end-of-life care.

Exclusion criteria: Patient unable to give consent or participate in the decision-making process and had no readily available legal surrogate decision-maker to give consent, or was under control of the Guardianship Board; palliative care team not available to see the patient within the next 24 hours; patient unlikely to survive until review by the palliative care team; patient, surrogate or treating medical teams had already specifically requested palliative care involvement in end-of-life care; no independent intensivist was available to approach the patient or family for consent

Number of patients: $\mathrm{N}=20$ (10 intervention and 10 control)

Diseases: Actual diseases were not specified. However, admission codes were stated. The admission code for those not admitted from the operating theatre included cardiovascular $(N=3)$, gastroenterology $(N=1)$, neurology $(N=1)$, respiratory $(N=6)$, sepsis $(N=4)$, trauma $(N=2)$, other $(N=1)$. Two patients were admitted from the operating theatre.

Patient characteristics: Median age in years (IQR): 83 (14) years in intervention group, 74 (20) years in control group; $50 \%$ female in intervention group, $70 \%$ female in control group

Deaths at end of study ( $(\%))$ : Total $N=16: 9(90 \%)$ intervention and $7(70 \%)$ control; differences between intervention and control not statistically significant

Number of families: $\mathrm{N}=9$ ( 5 intervention and 4 control)

Family characteristics: Not provided

Number of staff: 18 teams of nurses ( 9 intervention and 9 control): 17 team of intensivists ( 8 intervention and 9 control)

Staff characteristics: Not provided 
Withdrawals/other drop-outs: No patient was lost to follow-up. However, for one patient, neither questionnaire was given to family or staff as the patient died suddenly soon after the enrollment discussion. For another patient, the authors decided not to administer the second questionnaire to family or staff as the patient died 24 hours after enrollment. One patient was discharged alive to the ward, and the authors decided not to administer the second questionnaire to the same intensivist.

Interventions

Name: Palliative care consultation in addition to usual ICU end-of-life care vs usual ICU care

Type: Training in palliative care unclear

Service base: 14 -bed general ICU in an urban, tertiary hospital

Team: Comprised a physician, registrar, resident and clinical nurse consultant, and undertook ward rounds daily.

Intervention condition: The intervention was a consultation and subsequent management by a palliative care team. The first consultation occurred within 24 hours of randomisation. The intervention was provided in addition to usual ICU care commensurate with the patient's medical condition.

Duration: From enrollment until after the patient had died or been discharged from the ICU

Control condition: The control group received usual ICU care but no palliative care consultation.

Primary outcomes:
ICU and hospital length of stay
Satisfaction with quality of care of families, intensivists, and bed
Secondary outcomes:
ICU and hospital mortality
Number of medical teams caring or consulting for the patient
Individual domain scores of the satisfaction questionnaire

Assessment points: Questionnaires were administered to patients' families, nursing staff and intensivists immediately after randomisation and again after the patient had died or been discharged from the ICU.

Resource use/costs ICU and hospital length of stay

Time horizon: From enrollment until death for hospital length of stay or enrollment to discharge from the ICU for ICU length of stay

\section{Notes}

Funding source: Department of Intensive Care and the Department of Palliative Care, Concord Repatriation General Hospital

Declarations of interest among primary researchers: Apart from funding, no further study author disclosure statements were made.

Power considerations: No power calculation reported

\section{Risk of bias}

Bias Authors' judgement Support for judgement

Random sequence genera- Low risk tion (selection bias)
Quote from main publication: "patients were randomly allocated to an intervention or control group. Allocations were computer-generated by an independent statistician using the biased coin technique, and stored sequentially in sealed envelopes". 


\begin{tabular}{ll}
\hline $\begin{array}{l}\text { Allocation concealment } \\
\text { (selection bias) }\end{array}$ & $\begin{array}{l}\text { Quote from main publication: "allocations were computer-generated by an in- } \\
\text { dependent statistician using the biased coin technique, and stored sequential- } \\
\text { ly in sealed envelopes". }\end{array}$ \\
& Comment: probably done
\end{tabular}

Blinding of participants High risk Comment: no blinding
and personnel (perfor-
mance bias), subjective
outcomes

\begin{tabular}{ll}
\hline $\begin{array}{l}\text { Blinding of participants } \\
\text { and personnel (perfor- }\end{array}$ & Low risk \\
$\begin{array}{l}\text { mance bias), objective } \\
\text { outcomes }\end{array}$ & $\begin{array}{l}\text { Quote from main publication: "the study was a single-centre, unblinded, ran- } \\
\text { domised controlled feasibility trial". }\end{array}$ \\
& $\begin{array}{l}\text { Comment: there was no blinding but this was unlikely to lead to bias in objec- } \\
\text { tive outcomes. }\end{array}$
\end{tabular}

Blinding of outcome as- $\quad$ High risk
$\begin{aligned} & \text { sessment (detection bias), } \\ & \text { subjective outcomes }\end{aligned}$

$\begin{array}{ll}\begin{array}{l}\text { Blinding of outcome as- } \\ \text { sessment (detection bias), }\end{array} & \text { Low risk }\end{array}$

objective outcomes

Comment: there was no blinding but this was unlikely to lead to bias in objective outcomes.

Incomplete outcome data High risk

Quote from publication: "for one patient, neither questionnaire was given to family or staff as the patient died suddenly soon after the enrolment discussion. For another patient, the authors decided not to administer the second questionnaire to family or staff as the patient died 24 hours after enrolment... One patient was discharged alive to the ward 24 hours after the enrolment discussion, and the authors also decided not to administer the second questionnaire to the same intensivist". Analysis was intention-to-treat except for data derived from questionnaires.

Comment: questionnaire administration was varied leading to a high risk of incomplete outcome data.

\begin{tabular}{ll}
\hline $\begin{array}{l}\text { Selective reporting (re- } \\
\begin{array}{l}\text { porting bias) } \\
\text { Low risk }\end{array}\end{array}$ & $\begin{array}{l}\text { The study protocol was available and all of the study's prespecified (primary } \\
\text { and secondary) outcomes that were of interest in the review have been report- } \\
\text { ed in the prespecified way. }\end{array}$
\end{tabular}

Other bias Unclear risk

The authors stated that "patients allocated to standard ICU end-of-life care were younger, were more likely to be female, and had higher APACHE II scores than those allocated to receive a consultation with the palliative care team". However, it was unclear whether these differences were statistically significant. The authors did not state whether they controlled for these differences.

\begin{tabular}{ll}
\hline Size of study $\quad$ High risk & $\begin{array}{l}\text { High risk of bias because the number of participants in each group was less } \\
\text { than } 50\end{array}$
\end{tabular}

Edmonds 2010

\section{Study characteristics}

The effectiveness and cost-effectiveness of hospital-based specialist palliative care for adults with advanced illness and their caregivers (Review)

Copyright ( 2020 The Cochrane Collaboration. Published by John Wiley \& Sons, Ltd. 
Edmonds 2010 (Continued)

Methods

\author{
Design: Fast-track RCT \\ Who led PC intervention: Multidisciplinary team
}

Core team: A part-time consultant in palliative medicine with a special interest in neurological conditions, a part-time clinical nurse specialist and a full time administrator

Participants

Country and regions: UK, Southeast London

Recruitment: June 2004 and July 2005. A consultant in palliative medicine not part of the service initially screened all referrals. Patients were then sent a letter giving information about the trial and inviting them to participate. The interviewer telephoned patients several days after receipt of the letter, and arranged to meet them to explain more about the study, agree consent, ask if the nearest carer/family member could be approached, and complete the baseline interview.

Inclusion criteria: Patients identified by referring clinicians as potentially benefiting from a specialist palliative care assessment. Referrers were advised to use a score of $>8$ on the Expanded Disability Status Scale (EDSS) as a benchmark but were encouraged to refer on the basis of need, rather than disability.

Exclusion criteria: There were no specific exclusion criteria for the study.

Number of patients: $\mathrm{N}=52$ (26 intervention and 26 control)

Diseases: Multiple sclerosis (MS)

Patient characteristics: Mean age in years: 53 years in intervention group, 53 years in control group; $65.4 \%$ female in intervention group, $73.1 \%$ female in control group

Deaths at end of study ( $\mathrm{N}(\%)):$ Total $\mathrm{N}=4: 1(3.8 \%)$ intervention and $3(11.5 \%)$ control

Withdrawals/other dropouts: Total $\mathrm{N}=2$ : none in intervention and $2(7.7 \%)$

Number of unpaid caregivers: Numbers were unclear. Different numbers reported under unpaid caregiver outcomes

Family characteristics: Not provided

Name: Multiprofessional palliative care team assessment and follow-up in addition to usual care vs usual care

Type: Specialist palliative care. The service comprised a part-time consultant in palliative medicine with a special interest in neurological conditions, a part-time clinical nurse specialist and a full time administrator

\title{
Service base: King's College Hospital
}

Team: Comprised a part-time consultant in palliative medicine with a special interest in neurological conditions, a part-time clinical nurse specialist and a full time administrator. The consultant and nurse specialist for the palliative care service had access to a consultant neurologist. Patients thought to benefit from specialist neurological review could be seen in clinic jointly by the neurology and palliative care consultants and other relevant healthcare professionals. The MS palliative care service worked closely with the existing HSPC team, specifically utilising time from the palliative care psychosocial worker.

Intervention condition: At the initial assessment by a member of the multiprofessional team, the following information was collected: demographics, ability to communicate, main symptom issues, current medication, psychological concerns, social issues (including care package and agencies involved), carer concerns and any advanced care planning issues. Following this, an action plan was formulated and communicated to the primary healthcare team and other involved professionals as appropriate. Follow-up telephone calls or visits were arranged depending on clinical need. The clinical team met weekly to discuss the case-load and for the palliative care consultant to input into the management of all the patients. Patients with ongoing specialist palliative care needs were referred onto existing spe- 
cialist community palliative care teams in the area where the patient lived. Intervention was offered in addition to standard best practice services.

Duration: 12 weeks

Control condition: People affected by MS received a variety of services, including nurse services (including nurses specialising in MS), physiotherapy, neurology and rehabilitation services. In addition, district nurses, social services and general practitioners provided support in the community. A few patients received home physiotherapy, occupational therapy and/or attended specialist rehabilitation services or clinics. Inpatient care, including rehabilitation was available as required, as were other specialist services, including continence advice, psychiatry and/or psychology. Charities such as the MS Society provided information on available services and organised support groups.

\section{Outcomes}

\section{Outcomes:}

Patient symptoms and concerns assessed using the Palliative care Outcome Scale (POS) and MS-POS

Quality of life assessed using the physical and psychological subscales of the Multiple Sclerosis Impact Scale (MSIS)

unpaid caregiver burden assessed using the 12-item Zarit Burden Interview

unpaid caregiver mastery assessed using the modified Lawton positivity questionnaire

Assessment points: Assessments were carried out at baseline (before randomisation), then at 6 weeks, 12 weeks and 24-26 weeks for those in the fast-track group. Usual care patients undertook an additional interview 16-18 weeks post-baseline, after they had received the palliative care service.

\begin{tabular}{ll}
\hline Resource use/costs & None reported \\
\hline Notes & Funding source: The MS Society \\
& Declarations of interest among primary researchers: None declared \\
& Power considerations: The authors estimated that a sample of 25 patients in each group would en- \\
able them to detect clinically significant differences of greater than 1.6 on the POS (for individual \\
items), where items had a standard deviation of less than 2 , at $P<0.05$, power $80 \%$. Based on the local \\
patient numbers of people with an EDSS $>8$, they estimated that they would identify 3 to 4 patients per \\
week and be able to recruit and follow-up two of these. Recruitment over a year would therefore give \\
$50-52$ patients.
\end{tabular}

\section{Risk of bias}

Bias Authors' judgement Support for judgement

Random sequence genera- Low risk tion (selection bias)
Quote from main publication: "consenting patients newly referred to the new service were randomised to receive the palliative care service either immediately (fast-track, FI) or after a 12-week wait (standard best practice, SI). Randomisation was conducted by independent statistical colleagues using the minimisation method immediately after baseline interview".

Comment: probably done
Quote from main publication: "consenting patients newly referred to the new service were randomised to receive the palliative care service either immediately (fast-track, FI) or after a 12-week wait (standard best practice, SI). Randomisation was conducted by independent statistical colleagues using the minimisation method immediately after baseline interview". 
Edmonds 2010 (Continued)

Blinding of participants and personnel (performance bias), subjective outcomes
High risk Quote from main publication: "after the baseline interview, details of those patients randomised to fast track were immediately passed to the palliative care service. Those patients randomised to standard best practice were notified and details were kept with the research team until after the third research interview at 12 weeks, when details were passed to the clinical team".

Comment: not done

Blinding of outcome as-
sessment (detection bias), $\quad$ High risk There was no blinding of outcome assessors.

subjective outcomes

Incomplete outcome data Low risk (attrition bias)

All outcomes
We rated a low risk of bias because there were $96.1 \%$ completers in the intervention group and $80.8 \%$ completers in the control group. The authors carried out the analysis with both imputed and non-imputed data in order to test for sensitivity; in the results only the non-imputed data were shown as there was no noticeable difference in the results.

Comment: low risk of bias
Selective reporting (re- High risk Some prespecified outcomes such as use of health and social services were porting bias) not reported.

\begin{tabular}{lll}
\hline Other bias & Low risk & Study appeared to be free of other biases. \\
\hline Size of study & High risk & $\begin{array}{l}\text { High risk of bias because the number of participants in each group was less } \\
\text { than } 50\end{array}$ \\
\hline
\end{tabular}

El-Jawahri 2016

\section{Study characteristics}

\begin{tabular}{ll}
\hline Methods & Design: RCT \\
& Single-centre, unblinded RCT \\
& Who led PC intervention: Multidisciplinary team \\
Core team: Palliative care physician/advanced practice nurses
\end{tabular}

Participants Country and regions: USA, Boston

Recruitment: August 2014 to January 2016. Consecutively eligible patients with planned autologous or allogeneic haematopoietic stem cell transplantation (HCT) were identified during the weekly transplant team meetings. Research staff obtained permission from the treating oncologist to approach eligible patients and their unpaid caregivers within 72 hours of their transplant admission (HCT hospitalisation).

Inclusion criteria: Patients aged $\geq 18$ years who could speak English or complete questionnaires with minimal assistance from an interpreter. Enrolled patients were asked to identify a unpaid caregiver who could be invited to participate in the unpaid caregiver portion of this study. Patients without a unpaid caregiver were still eligible to participate.

Exclusion criteria: Patients with a history of HCT or those with psychiatric or comorbid disease that the oncologist believed would interfere with adherence to study procedures were excluded.

Number of patients: $\mathrm{N}=160$ (81 intervention and 79 control)

Diseases: Adults with haematologic malignancies undergoing autologous/allogeneic HCT

The effectiveness and cost-effectiveness of hospital-based specialist palliative care for adults with advanced illness and their caregivers 
El-Jawahri 2016 (Continued)

Patient characteristics: Mean age in years (SD): 57.2 (12.7) years in intervention group, 56.9 (14.1) years in control group; $59.3 \%$ female in intervention group, $54.4 \%$ female in control group

Deaths at end of study ( $\mathrm{N}(\%)):$ Total $\mathrm{N}=3: 3(3.7 \%)$ in intervention and none in control

Withdrawals/other dropouts $(\mathrm{N}(\%))$ : Total $\mathrm{N}=8: 3(3.7 \%)$ intervention and $5(6.3 \%)$ control

Number of unpaid caregivers: $\mathrm{N}=94$ (49 intervention and 45 control)

unpaid caregivercharacteristics: Mean age in years (SD): 54.4 (14.6) years in intervention group, 54.3 (13.7) years in control group; $66.7 \%$ female in intervention group, $73.3 \%$ female in control group

Interventions

Name: Early palliative care integrated with standard transplant care vs standard transplant care alone

Definition of early PC: Not explicitly defined but palliative care intervention primarily focussed on managing patients' physical and psychological symptoms during hospitalisation for HCT.

Type: Specialist palliative care. The three palliative care clinicians (two nurse practitioners and one board-certified physician) underwent a half-day training focussed on addressing the main topics covered by the intervention.

Service base: Massachusetts General Hospital

Team: Comprised an inpatient palliative care physician and two advanced practice nurses

Intervention condition: Intervention patients met with the inpatient palliative care physician or advanced practice nurse within 72 hours of randomisation. The palliative care clinician followed patients up during their hospitalisation. Patients, unpaid caregivers, and the palliative care clinicians were permitted to initiate additional visits as needed. Participants did not have outpatient palliative care follow-up after discharge. The palliative care intervention primarily focussed on managing patients' physical and psychological symptoms during hospitalisation for HCT and did not include advance care planning, goals-of-care and code status discussions, or end-of-life decision-making. Study investigators created an intervention manual that provided guidelines for addressing symptoms and psychological distress. After each visit, the palliative care clinicians communicated their recommendations in person to the transplant team and documented their recommendations in the medical record.

Duration: Period of hospitalisation

Control condition: Control patients received standard transplant care with the supportive care measures instituted by the transplant team. Patients, unpaid caregivers, and transplant clinicians were permitted to request consultation with palliative care clinicians.

\section{Primary outcome:}

Quality of life assessed using the Functional Assessment of Cancer Therapy - Bone Marrow Transplant (FACT-BMT)

\section{Secondary outcomes:}

Anxiety and depression assessed using the 14-item Hospital Anxiety and Depression Scale (HADS). Depression was also assessed using the Patient Health Questionnaire 9 (PHQ-9)

Fatigue assessed using the 13-item FACT Fatigue subscale

Symptom burden assessed using the Edmonton Symptom Assessment Scale (ESAS)

Post-traumatic Stress Disorder (PTSD) assessed using the 17-item PTSD Checklist-Civilian Version

Distress assessed using the National Comprehensive Cancer Network Distress Thermometer Checklist

Incidence of acute and chronic graft-vs-host disease

Nonrelapse mortality

Overall survival

The effectiveness and cost-effectiveness of hospital-based specialist palliative care for adults with advanced illness and their caregivers 
El-Jawahri 2016 (Continued)

unpaid caregiver quality of life assessed using the unpaid caregiver Oncology QOL questionnaire

unpaid caregiver's mood (depression and anxiety) assessed using the 14-item Hospital Anxiety and Depression Scale (HADS). unpaid caregiver depression was also assessed using the Patient Health Questionnaire 9 (PHQ-9)

Assessment points: Participants completed study questionnaires prior to randomisation and during the second week of hospitalisation for HCT (at patients' blood count nadir; i.e. the period during HCT hospitalisation when patients experience the lowest blood cell counts and highest toxicity and symptom burden: day 5 after stem cell infusion for autologous and day 8 after stem cell infusion for allogeneic HCT, with a 2-day window) and at 3 and 6 months after HCT.

Resource use/costs Number of PC visits

Time horizon: enrollment to discharge from hospital

Notes Funding source: This work was supported by funds from the National Palliative Care Research Foundation and grant K24 CA 181253 from the National Cancer Institute.

Declarations of interest among primary researchers: One of the authors reported receipt of personal fees for consulting or advisory board membership from Bayer, Millennium, Incyte, Seattle Genetics, and Insys. No other disclosures were reported.

Power considerations: A sample size of 160 patients (80 patients in each group) was estimated to be sufficient with $80 \%$ power to detect a 6-point change in QoL (FACT-BMT) from baseline to week 2 using a 2-sample t-test with an $\alpha=0.05$ statistical significance level and a rate of attrition of $15 \%$. All reported $P$ values were 2-sided.

\section{Risk of bias}

\begin{tabular}{lll}
\hline Bias & Authors' judgement & Support for judgement \\
\hline $\begin{array}{ll}\text { Random sequence genera- } \\
\text { tion (selection bias) }\end{array}$ & Low risk & $\begin{array}{l}\text { Quote from main publication: "patients were then randomized to the palliative } \\
\text { care intervention or standard transplant care using a computer-generated 1:1 } \\
\text { randomization stratified by type of HCT (autologous, myeloablative allogene- } \\
\text { ic, or reduced intensity allogeneic HCT)." } \\
\end{array}$ \\
& Comment: probably done
\end{tabular}

Allocation concealment $\quad$ Unclear risk
(selection bias)

Quote from main publication: "patients were then randomized to the palliative (selection bias) care intervention or standard transplant care using a computer-generated 1:1 randomization stratified by type of HCT (autologous, myeloablative allogeneic, or reduced intensity allogeneic HCT)."

Comment: allocation concealment not described

\begin{tabular}{ll}
\hline $\begin{array}{l}\text { Blinding of participants } \\
\text { and personnel (perfor- } \\
\text { mance bias), subjective } \\
\text { outcomes }\end{array}$ & High risk \\
\hline $\begin{array}{l}\text { Blinding of participants } \\
\begin{array}{l}\text { and personnel (perfor- } \\
\text { mance bias), objective }\end{array}\end{array}$ & Low risk \\
$\begin{array}{l}\text { outcomes } \\
\text { Q }\end{array}$ & $\begin{array}{l}\text { Quote from main publication: "non-blinded randomized clinical trial among } \\
160 \text { adults with haematologic malignancies undergoing autologous/allogeneic }\end{array}$ \\
& HCT and their unpaid caregivers". \\
& $\begin{array}{l}\text { Comment: participants and personnel were not blinded but this was unlikely } \\
\text { to lead to bias in objective outcomes. }\end{array}$
\end{tabular}

Blinding of outcome as-
$\begin{aligned} & \text { sessment (detection bias), } \\ & \text { subjective outcomes risk }\end{aligned}$

The effectiveness and cost-effectiveness of hospital-based specialist palliative care for adults with advanced illness and their caregivers 
El-Jawahri 2016 (Continued)

Blinding of outcome as- Low risk Quote from main publication: "non-blinded randomized clinical trial among sessment (detection bias), objective outcomes 160 adults with hematologic malignancies undergoing autologous/allogeneic HCT and their unpaid caregivers".

Comment: there was no blinding but this was unlikely to lead to bias in objective outcomes.

Incomplete outcome data Low risk (attrition bias)

All outcomes
$\mathrm{N}=75(92.6 \%)$ completers in intervention group vs $\mathrm{N}=74$ (93.7\%) completers in control group. Multiple imputation was used for missing observations. 80 $(98.8 \%)$ patients in intervention group and $77(97.5 \%)$ in control group were included in the primary analysis. Furthermore, because of a clerical error, the first 38 study patients did not complete the nausea item, which was therefore omitted from the composite ESAS score analyses (range, 0-90).

\begin{tabular}{lll}
\hline $\begin{array}{l}\text { Selective reporting (re- } \\
\text { porting bias) }\end{array}$ & High risk & $\begin{array}{l}\text { All the study's prespecified outcomes have not been reported and an addition- } \\
\text { al outcome ( unpaid caregiver coping) was added. }\end{array}$ \\
\hline Other bias & Low risk & Study appeared to be free of other biases. \\
\hline Size of study & Unclear risk & $\begin{array}{l}\text { Unclear risk of bias because the number of participants in each group was be- } \\
\text { tween } 50 \text { and } 199\end{array}$ \\
\hline
\end{tabular}

Farquhar 2014

\section{Study characteristics}

Design: RCT
Single-centre Phase III fast-track single-blind mixed-method RCT
Who led PC intervention: Multidisciplinary team
$\begin{aligned} & \text { Core team: Palliative care medical consultant/clinical specialist occupational therapist/clinical spe- } \\ & \text { cialist physiotherapist/administrator }\end{aligned}$

Country and regions: UK, Addenbrooke's catchment area seeing patients from Cambridgeshire, Hertfordshire and Essex and, where practical, further afield

Recruitment: November 2008 to January 2012. Consecutive cancer patients referred to Breathlessness Intervention Service (BIS) (from primary or secondary care) were invited to participate by letter.

Inclusion criteria: Patients were eligible if they met BIS referral criteria (that is, diagnosed appropriately-treated cause of breathlessness, troubled by breathlessness in spite of optimisation of underlying illness, and might benefit from a self-management programme). Recruited patients were asked to identify their informal carers who were also invited to participate. All participating patients and informal carers gave informed consent.

Exclusion criteria: Patients were excluded if they had received BIS previously. Patients who were unwilling to participate in the trial continued to have access to BIS.

Number of patients: $\mathrm{N}=67$ ( 35 intervention and 32 control)

Diseases: Advanced cancer. Lung ( $N=33,49 \%)$, breast $(N=13,19 \%)$ rectal/bowel $(N=4,6 \%)$, prostate $(N=3,4 \%)$, lymphoma $(N=3,4 \%)$, mesothelioma $(N=3,4 \%)$, gastro-oesophageal junction $(N=2,3 \%)$, renal $(N=2,3 \%)$, endometrial $(N=1,2 \%)$, hepatocellular $(N=1,2 \%)$, bladder $(N=1,2 \%)$ and unknown primary $(\mathrm{N}=1,2 \%)$.

Patient characteristics:.Mean age in years (SD): 70 (9.4) years in intervention group, 67 (13.3) years in control group; 59\% female in intervention group, $62 \%$ female in control group

The effectiveness and cost-effectiveness of hospital-based specialist palliative care for adults with advanced illness and their caregivers 
Number of carers: $N=41$ (20 intervention and 21 control)

Carer characteristics: Mean age in years (SD): 65.6 (13.4) years in intervention group, 63.5 (12.2) years in control group; $70 \%$ female in intervention group, $67 \%$ female in control group

Deaths at end of study $(\mathrm{N}(\%)): \mathrm{N}=2: 2(5.7 \%)$ in intervention group and 0 in control group

Withdrawals/other dropouts $(\mathrm{N}(\%)): \mathrm{N}=11: 5(14.3 \%)$ in intervention group and $6(18.8 \%)$ in control group

Name: Breathlessness Intervention Service (BIS) plus standard care vs standard care

Type: Specialist palliative care. Includes a palliative care medical consultant (with dedicated clinical sessions and a research interest in breathlessness), a clinical specialist occupational therapist (lead clinician for the service), a clinical specialist physiotherapist and an administrator.

Service base: Addenbrooke's Hospital. BIS is a secondary care service which provides care in a community setting, predominantly seeing patients in their own homes.

Team: A palliative care medical consultant (with a research interest in breathlessness), a clinical specialist occupational therapist (lead clinician for the service), a clinical specialist physiotherapist and an administrator

Intervention condition: The Breathlessness Intervention Service (BIS) is a multidisciplinary complex intervention combining nonpharmacological and pharmacological interventions to support breathless patients with advanced disease, theoretically underpinned by a palliative care approach. Consultations usually occur in the patient's own home. First stage of intervention is mainly nonpharmacological (selection and application as clinically indicated). Second stage interventions likely to be applied concurrently with first stage interventions include pharmacological review, referral to specialist services or acupuncture.

Duration: Two weeks

Control condition: Standard care was defined as specialist outpatient appointments in secondary care (for example, oncology) which may include specialist nurse input, and primary care services.

\section{Primary outcome:}

Patient distress due to breathlessness measured using a numerical rating scale

\section{Secondary outcomes:}

Disease-specific health-related quality of life assessed using the Chronic Respiratory Questionnaire (CRQ)

Patient anxiety and depression using the Hospital Anxiety and Depression Scale (HADS)

Carer distress due to patient breathlessness measured using a numerical rating scale

Carer anxiety and depression using the Hospital Anxiety and Depression Scale (HADS)

Service use assessed using the Client Services Receipt Inventory (CSRI)

Patients and carers' expectations and experiences of BIS explored using qualitative topic-guided interviews

For health economic analyses: EQ-5D and measure of service use assessed using the Client Services Receipt Inventory (CSRI)

Assessment points: Participating patients and carers completed a baseline interview (t1: week 1 ) before randomisation. These interviews included the quantitative patient- and carer-reported measures and qualitative interviews (carers were interviewed separately where possible). A two-week follow-up interview (t3: week 3) was designed to represent BIS completion for the intervention arm, or end of waiting-list period prior to BIS for controls. A final interview ( $\mathrm{t} 5$ : week 5 ) was conducted four weeks 
Farquhar 2014 (Continued)

from baseline; this represented two weeks after BIS for intervention arm and completion of BIS for controls.

Resource use/costs

Measure of service use assessed using the Client Services Receipt Inventory (CSRI)

Informal care (unpaid hours/week from family/ friends performing specific tasks) was valued at average UK wages (GBP 11.21/hour). Costs of BIS visits were estimated at GBP 91 (based on specialist nurse contacts which averaged the rehabilitation specialists' wages) and phone contacts at one-quarter of this.

Time Horizon: Costs were calculated combining service use data (CSRI) for eight weeks and two weeks prior to baseline and t3, respectively, with UK 2011/2012 unit costs.

Data sources: A generic health status measure (EQ-5D) and measure of service use (Client Services Receipt Inventory (CSRI) were administered for health economic analyses. Costs were calculated combining service use data (CSRI) for eight weeks and two weeks prior to baseline and $t 3$, respectively, with UK 2011/2012 unit costs. Informal care (unpaid hours/week from family/ friends performing specific tasks) was valued at average UK wages (GBP 11.21/hour). Costs of BIS visits were estimated at GBP 91 (based on specialist nurse contacts which averaged the rehabilitation specialists' wages) and phone contacts at one-quarter of this.

Analytical perspective: Not clear

Notes

Funding source: The study was supported by the following funders: NIHR Research for Patient Benefit (for Phase III RCT funding); Macmillan Cancer Support (post-doctoral fellowship); The Gatsby Foundation for the initial funding of BIS; and AT Prevost was supported by the National Institute for Health Research (NIHR) Biomedical Research Centre at Guy's and St Thomas' NHS Foundation Trust and King's College London.

Declarations of interest among primary researchers: The authors declared that Sara Booth (SB) was the founder and one of the clinicians providing BIS but had no role in data collection. The other authors declared that they had no competing interests.

Power considerations: A sample size of 60 randomised patients ( 26 analysed per arm, allowing for dropout) provided $80 \%$ power to detect a 2-point difference in mean distress at two weeks between groups $(S D=2.5$, alpha $=5 \%)$, with increased precision anticipated from adjustment for baseline.

\section{Risk of bias}

\begin{tabular}{lll}
\hline Bias & Authors' judgement & Support for judgement \\
\hline $\begin{array}{l}\text { Random sequence genera- } \\
\text { tion (selection bias) }\end{array}$ & Low risk & $\begin{array}{l}\text { Quote from main publication: "participants were randomised to one of two } \\
\text { groups using randomly permuted blocks of random size two, four and six, gen- } \\
\text { erated by the study statistician using a computer programme and concealed } \\
\text { within sealed opaque envelopes until allocation notification } \\
\text { by the intervention deliverer". } \\
\text { Comment: probably done }\end{array}$ \\
& \\
&
\end{tabular}

\begin{tabular}{ll}
$\begin{array}{l}\text { Allocation concealment } \\
\text { (selection bias) }\end{array}$ & Low risk \\
& $\begin{array}{l}\text { Quote from main publication: "participants were randomised to one of two } \\
\text { groups using randomly permuted blocks of random size two, four and six, gen- } \\
\text { erated by the study statistician using a computer programme and concealed } \\
\text { within sealed opaque envelopes until allocation notification } \\
\text { by the intervention deliverer". } \\
\text { Comment: probably done }\end{array}$ \\
\hline
\end{tabular}

Blinding of participants High risk Comment: no blinding


Farquhar 2014 (Continued)

Blinding of outcome as- Low risk Quote from main publication: "data collection-design facilitated resessment (detection bias), searcher-blinding to group allocation for the collection of primary and key secondary outcomes at the key measurement point, that is, planned unblinding occurred during the two-week follow-up interview (t3) only after collection of this outcome data and prior to qualitative data collection about the intervention".

Comment: researcher who collected data was blinded.

Incomplete outcome data High risk (attrition bias)

All outcomes
$\mathrm{N}=28(75 \%)$ completers in intervention group vs $\mathrm{N}=26(77 \%)$ completers in control group. Missing data were excluded from the analysis: 7 (25\%) patients were missing in the intervention group due to death $(\mathrm{N}=2)$ and deterioration $(\mathrm{N}=5)$ while $6(23 \%)$ patients were missing in the control group due to deterioration of their condition.

Selective reporting (re- High risk
porting bias)

Some outcomes such as patient and carer social functioning, carer assessment of patient's breathlessness, carer distress due to patient's breathlessness, carer quality of life, carer anxiety and depression and unpaid caregiver burden were not reported in the study. The trial registration on ClinicalTrials.gov is NCT00678405.

\begin{tabular}{lll}
\hline Other bias & Low risk & Study appeared to be free of other biases. \\
\hline Size of study & Unclear risk & $\begin{array}{l}\text { Unclear risk of bias because the number of participants in each group was be- } \\
\text { tween } 50 \text { and } 199\end{array}$ \\
\hline
\end{tabular}

Farquhar 2016

\section{Study characteristics}

Methods

\section{Design: RCT}

Single-centre phase III fast-track single-blind mixed method RCT

Who led PC intervention: Multidisciplinary team

Core team: Palliative care medical consultant/clinical specialist occupational therapist/clinical specialist physiotherapist

\section{Participants}

\section{Country and regions: UK}

Recruitment: Consecutive patients with nonmalignant disease who were referred to BIS (from primary or secondary care) were invited to participate in the trial, by letter.

Inclusion criteria: Patients were eligible if they met BIS referral criteria (they had a diagnosed appropriately treated cause of breathlessness, were troubled by breathlessness in spite of optimisation of underlying illness, and might benefit from a self-management programme). Recruited patients were asked to identify their informal carers who were also invited to participate.

Exclusion criteria: Patients were excluded (from the trial only) if they had received BIS previously.

Number of patients: $\mathrm{N}=87$ (44 intervention and 43 control)

Diseases: Advanced nonmalignant disease comprising COPD $(\mathrm{N}=73,84 \%)$ and other nonmalignant disease $(N=14,16 \%)$

Patient characteristics: Mean age in years (SD): 72.3 (10.6) years in intervention group, 72.2 (9.4) years in control group; $36 \%$ female in intervention group, $42 \%$ female in control group 
Number of carers: $\mathrm{N}=57$ (29 intervention and 28 control)

Carer characteristics: Mean age in years (SD): 62.5 (14.82) years in intervention group, 62 (12.02) years in control group; $79 \%$ female in intervention group, $79 \%$ female in control group

Deaths at end of study ( $\mathbf{N}(\%)): \mathrm{N}=2: 1(2.3 \%)$ in intervention and $1(2.3 \%)$ in control

Withdrawals/other dropouts ( $\mathbf{N}(\%)): \mathrm{N}=6: 2(4.5 \%)$ in intervention and $4(9.3 \%)$ in control

Name: Breathlessness Intervention Service (BIS) plus standard care vs standard care

Type: Specialist palliative care. Included a palliative care medical consultant, a clinical specialist occupational therapist and a clinical specialist physiotherapist

Service base: Addenbrooke's Hospital. BIS is a secondary care service which provides care in a community setting, predominantly seeing patients in their own homes.

Team: A palliative care medical consultant, a clinical specialist occupational therapist and a clinical specialist physiotherapist

Intervention condition: The Breathlessness Intervention Service (BIS) is a multidisciplinary complex intervention combining nonpharmacological and pharmacological interventions to support breathless patients with advanced disease, theoretically underpinned by a palliative care approach. Consultations usually occurred in the patient's own home. First stage of intervention was nonpharmacological (selection and application as clinically indicated). Second stage of intervention depended on outcome of first stage interventions and included pharmacological interventions.

Duration: Four weeks

Control condition: Standard care was defined as specialist outpatient appointments in secondary care (for example, oncology) which may include specialist nurse input, and primary care services.

\section{Primary outcome}

Patient distress due to breathlessness measured using a numeric rating scale (NRS)

\section{Secondary outcomes}

Patient quality of life measured by the Chronic Respiratory Questionnaire (CRQ)

Patient anxiety and depression measured by the Hospital Anxiety and Depression Scale (HADS)

Carer-reported outcome measures included an NRS for carer distress due to patient breathlessness

Carer anxiety and depression measured by the Hospital Anxiety and Depression Scale (HADS)

Patient use of other services assessed using the Client Service Receipt Inventory (CSRI)

Patients and carers' expectations and experiences of BIS explored using qualitative topic-guided interviews

For health economic analyses: EQ-5D and the CSRI

Assessment points: Participating patients completed a baseline interview (t1) prior to randomisation. This interview included both the quantitative patient-reported measures and the qualitative topic-guided interviews. Carers were interviewed separately, where possible. Similar mixed method follow-up interviews were conducted with both patients and carers at each subsequent follow-up interview (t2-t5) at fortnightly intervals. Interview two (t2; two weeks after baseline) represented the midway point in either receiving the BIS intervention for the intervention arm or the waiting-list period for the controls; interview three ( $\mathrm{t} 3$ ) was designed to represent BIS completion for the intervention arm, or the end of the waiting-list period prior to commencing BIS for the controls; interview four (t4) represented the midway point in receiving the BIS intervention for the controls (no t4 was conducted with patients and carers on the intervention arm); and the final interview ( $\mathrm{t} 5$; eight weeks from baseline) 
Farquhar 2016 (Continued) was designed to represent four weeks after BIS for the intervention arm, and the completion of BIS for the controls.

Resource use/costs Measure of service use assessed using the Client Services Receipt Inventory (CSRI)

The cost of the intervention was calculated at GBP 91 per contact based on specialist nursing input costs, with phone contacts costed at $25 \%$ of this.

Time Horizon: Costs were calculated by combining service use data (collected for the two months prior to baseline and at four-week follow-up) with UK 2011/12 unit costs.

Data sources: The EQ-5D and Client Service Receipt Inventory (CSRI) were administered for the health economic analyses. Costs were calculated by combining service use data (collected for the two months prior to baseline and at four-week follow-up) with UK 2011/12 unit costs. The cost of the intervention was calculated at $£ 91$ per contact based on specialist nursing input costs, with phone contacts costed at $25 \%$ of this. Costs were combined with the primary outcome and EQ-5D-derived quality-adjusted life years (QALYs), with uncertainty explored using cost-effectiveness planes.

Analytical perspective: Not clear

Funding source: This paper presented independent research commissioned by the NIHR under its Research for Patient Benefit (RfPB) programme (Grant Reference Number PB-PG-0107-11134).

Declarations of interest among primary researchers: MF, ATP, PM, BBP, AB, IJH, CJT, SB had support from NIHR (Research for Patient Benefit grant) for the submitted work; MF had support from Macmillan Cancer Support (Post-Doctoral Fellowship) for the submitted work; IJH had support from Cicely Saunders International; SB had support from Macmillan Cancer Support (funded online learning module connected to BIS), the Gatsby Foundation (funded the pilot BIS and its evaluation), and a capacity building grant from NIHR, and SB was the founder and one of the clinicians providing BIS that may be relevant to the submitted work and started an MRC methods research programme in collaboration with $\mathrm{IJH}$, who has published an evaluation of a breathlessness service.

Power considerations: A sample size of 60 randomised patients ( 26 analysed per arm, with allowance for dropout) provided $80 \%$ power to detect a 2-point difference in mean distress between groups (SD = 2.5 , alpha $=5 \%$ ), with increased precision anticipated from adjustment for baseline.

\section{Risk of bias}

\begin{tabular}{lll}
\hline Bias & Authors' judgement & Support for judgement \\
\hline $\begin{array}{l}\text { Random sequence genera- } \\
\text { tion (selection bias) }\end{array}$ & Low risk & $\begin{array}{l}\text { Quote from main publication: "participants were randomised to one of two } \\
\text { groups using randomly permuted blocks of random size 2, } 4 \text { and } 6, \text { generated } \\
\text { using a computer programme by the study statistician and concealed within } \\
\text { sealed opaque envelopes until allocation notification by the intervention de- } \\
\text { liverer". }\end{array}$ \\
& $\begin{array}{l}\text { Comment: probably done } \\
\text { Allocation concealment } \\
\text { (selection bias) }\end{array}$ & $\begin{array}{l}\text { Quote from main publication: "participants were randomised to one of two } \\
\text { groups using randomly permuted blocks of random size 2, } 4 \text { and 6, generated } \\
\text { using a computer programme by the study statistician and concealed within } \\
\text { sealed opaque envelopes until allocation notification by the intervention de- } \\
\text { liverer". }\end{array}$ \\
& Comment: probably done
\end{tabular}

Blinding of participants High risk Comment: no blinding
and personnel (perfor-
mance bias), subjective
outcomes

The effectiveness and cost-effectiveness of hospital-based specialist palliative care for adults with advanced illness and their caregivers 
Farquhar 2016 (Continued)

Blinding of outcome as- Low risk Quote from main publication: "data collection was designed to facilitate resessment (detection bias), subjective outcomes searcher blinding to group allocation for the collection of primary and key secondary outcomes at $\mathrm{t} 3$. That is, researcher blinding was explained to study participants on recruitment, they were reminded at the start of $t 2$ not to let the researcher know their group allocation, and at the start of $\mathrm{t} 3$ they were asked not to let the researcher know their group allocation until the researcher came to open their group allocation envelope just prior to CSRI completion (after the collection of primary and key secondary outcomes at $\mathrm{t} 3)^{\prime}$."

Comment: researcher who collected data was blinded.

$\begin{array}{ll}\begin{array}{l}\text { Incomplete outcome data } \\ \text { (attrition bias) }\end{array} & \mathrm{N}=41(93.2 \%) \text { completers in intervention group vs } \mathrm{N}=38(88.4 \%) \text { completers } \\ \text { All outcomes } & \text { in control group. Missing data were excluded from the analysis: } 3(6.8 \%) \text { pa- } \\ & \text { tients were missing in the intervention group due to death }(\mathrm{N}=1) \text { and lost to } \\ & \text { study }(\mathrm{N}=2) \text { while } 5(11.6 \%) \text { patients were missing in the control group due to } \\ \text { death }(\mathrm{N}=1) \text { and lost to study }(\mathrm{N}=4) .\end{array}$

\begin{tabular}{lll}
\hline $\begin{array}{l}\text { Selective reporting (re- } \\
\text { porting bias) }\end{array}$ & High risk & $\begin{array}{l}\text { Social functioning was not reported in the study. The trial registration on Clini- } \\
\text { calTrials.gov is NCT00678405. }\end{array}$ \\
\hline Other bias & Low risk & Study appeared to be free of other biases. \\
\hline Size of study & Unclear risk & $\begin{array}{l}\text { Unclear risk of bias because the number of participants in each group was be- } \\
\text { tween } 50 \text { and } 199\end{array}$ \\
\hline
\end{tabular}

Franciosi 2019

\title{
Study characteristics
}

Design: RCT (multicentre RCT)
Who led PC intervention: Multidisciplinary team
$\begin{aligned} & \text { Core team: Double-boarded certified oncologists and palliative care physicians and nurses involved } \\ & \text { full-time in palliative care }\end{aligned}$

Participants Country and regions: Italy, Emilia-Romagna in Northern Italy

Recruitment: November 2014 to March 2016. Follow-up was completed in November 2016. Consecutively enrolled patients were identified by research personnel.

\begin{abstract}
Inclusion criteria: Age $>18$ years; pathologically confirmed NSCLC, pancreatic, gastric or biliary tract cancer diagnosed within the previous 8 weeks; Eastern Cooperative Oncology Group (ECOG) performance status (PS) 0,1 , or 2; metastatic or locally advanced disease (but not susceptible to loco-regional treatments); life expectancy > three months; eligibility for first-line chemotherapy and/or biological therapy; completion of the QoL questionnaire; provision of written informed consent at enrollment
\end{abstract}

Exclusion criteria: Patients already receiving care from the palliative care service or previously treated with chemotherapy and/or biological therapy for advanced disease, as well as patients with NSCLC with EGFR mutation, were excluded.

Number of patients: $\mathrm{N}=281$ (142 intervention and 139 control)

Diseases: Advanced cancer: lung (non-small cell), pancreatic, gastric and biliary

Patient characteristics: Median age in years (IQR): 68.5 (12) years in intervention group, 68 (11) years in control group; $32 \%$ female in intervention group, $38 \%$ female in control group 
Deaths at end of study (N (\%)): $N=97: 48(33.8 \%)$ intervention and $49(35.3 \%)$ control had died by six months.

Withdrawals/other dropouts ( $\mathbf{N}(\%)): \mathrm{N}=7: 4(2.8 \%)$ in intervention group and $3(2.2 \%)$ in control group

Interventions Name: Early palliative care integrated with usual care vs usual care

Early palliative care (EPC): Patients were diagnosed with NSCLC, pancreatic, gastric or biliary tract cancer within the previous 8 weeks.

Type: Double-boarded certified oncologists and palliative care physicians and nurses involved fulltime in palliative care

Service base: Five University and Community Hospital Cancer Centres in Northern Italy

Team: Composed of an oncologist specialised in palliative care (PC) and a nurse involved full-time in PC

Intervention condition: Patients met with the PC team within 2 weeks after enrollment, and at least every 2 weeks thereafter for 24 weeks. After protocol amendment, follow-up visits were scheduled every 3 weeks. Additional visits with the PC team could be scheduled at the discretion of the patient, oncologist, or PC provider. General guidelines for the PC visits were adapted from the protocol of the Temel et al. study. The PC team documented provided care in the patient's medical record. Physical and psychosocial symptoms were assessed using validated instruments, and the necessary interventions enacted according to individual patient and family needs.

Duration: Enrollment to 6 months

Control condition: Patients assigned to standard care received anticancer and symptom control treatments provided by oncologists and nurses without formal PC training. They were offered no formal intervention, but PC referral was not denied, if requested. The patients in the control arm who were referred to the PC team did not cross over to the EPC group or follow the specified PC protocol.

Outcomes

\section{Primary outcomes:}

Quality of life at 12 weeks assessed using the Functional Assessment of Cancer Therapy-General (FACTG)

\section{Secondary outcomes:}

Survival

Use of end-of-life (EoL) care defined as the percentage of deceased patients who used the following in the 30 days preceding death: chemotherapy use, hospital admission and emergency room visit

\section{Assessment points:}

Patients were assessed at baseline, 12 weeks and 24 weeks

Resource use/costs

Percentage of deceased patients who used the following in the 30 days preceding death:

Chemotherapy use

Hospital admission

Emergency room visit

Time horizon: 30 days before death

Notes

Funding source: This work was supported by the Programma di ricerca Regione-Università, Regione Emilia-Romagna, bando "Ricerca per il Governo clinico" 2013. 
Declaration of interest among primary researchers: The authors stated that they had no conflicts of interest to declare.

Power considerations: To detect a significant between-group difference of at least 6.5 points in the change in the FACT-G score between t0 and t1, through a two-tail unpaired Student's t-test with $80 \%$ power and $5 \%$ alpha, the authors estimated a sample size of 186 patients. This quota was increased by $30 \%$ (243 patients) based on the estimated number of deaths within 12 weeks (20\%) and lost to follow-up (10\%) at T1.

\section{Risk of bias}

\begin{tabular}{lll}
\hline Bias & Authors' judgement & Support for judgement \\
\hline $\begin{array}{ll}\text { Random sequence genera- } \\
\text { tion (selection bias) }\end{array}$ & Low risk & $\begin{array}{l}\text { Quote from main publication: "eligible patients were randomised before an- } \\
\text { ticancer treatment to one of the two groups on a 1:1 allocation rate. To take } \\
\text { into account centre heterogeneity, stratified randomisation was performed. }\end{array}$ \\
& $\begin{array}{l}\text { Lists using a permuted block balanced procedure were generated for each par- } \\
\text { ticipating centre with the SAS v8 Statistical Software, and for each list a seed } \\
\text { was defined. Lists were saved and implemented in a web-based e-CRF, to au- } \\
\text { tomatically assign the results of the randomisation, thus ensuring allocation } \\
\text { concealment". } \\
\text { Comment: probably done }\end{array}$
\end{tabular}

$\begin{array}{ll}\begin{array}{l}\text { Allocation concealment } \\ \text { (selection bias) }\end{array} & \text { Quote from main publication: "lists using a permuted block balanced proce- } \\ & \text { dure were generated for each participating centre with the SAS v8 Statistical } \\ & \text { Software, and for each list a seed was defined. Lists were saved and imple- } \\ & \text { mented in a web-based e-CRF, to automatically assign the results of the ran- } \\ \text { domisation, thus ensuring allocation concealment". }\end{array}$

Comment: probably done

\begin{tabular}{|c|c|c|}
\hline $\begin{array}{l}\text { Blinding of participants } \\
\text { and personnel (perfor- } \\
\text { mance bias), subjective } \\
\text { outcomes }\end{array}$ & High risk & $\begin{array}{l}\text { Quote from main publication: "... patients and clinicians could not be blinded } \\
\text { to group assignment". } \\
\text { Comment: not done }\end{array}$ \\
\hline
\end{tabular}

Blinding of participants Low risk and personnel (performance bias), objective outcomes

Quote from main publication: "... patients and clinicians could not be blinded to group assignment".

Comment: participants and personnel were not blinded but this was unlikely to lead to bias in objective outcomes such as survival.
Blinding of outcome as- Low risk sessment (detection bias), subjective outcomes

\begin{abstract}
Quote from main publication: "although complete masking of intervention was not feasible, blinding was ensured for healthcare staff in charge of data collection on QoL, which is very important since outcome measures involve some subjectivity".

Comment: probably done
\end{abstract}

Blinding of outcome as- Low risk sessment (detection bias), objective outcomes

\begin{abstract}
Quote from main publication: "although complete masking of intervention was not feasible, blinding was ensured for healthcare staff in charge of data collection on QoL, which is very important since outcome measures involve some subjectivity".
\end{abstract}

Comment: probably done

\begin{tabular}{ll}
\hline $\begin{array}{l}\text { Incomplete outcome data } \\
\text { (attrition bias) }\end{array}$ & Low risk \\
All outcomes & $\mathrm{N}=90(63.4 \%)$ completers in intervention group vs $\mathrm{N}=87(62.6 \%)$ com- \\
& pleters in control group. Number of deaths and withdrawals were similar \\
& between groups. $48(33.8 \%)$ deaths and $4(2.8 \%)$ withdrawals/dropouts oc- \\
& curred in the intervention group while $49(35.3 \%)$ deaths and $3(2.2 \%)$ with-
\end{tabular}

The effectiveness and cost-effectiveness of hospital-based specialist palliative care for adults with advanced illness and their caregivers (Review)

Copyright (c) 2020 The Cochrane Collaboration. Published by John Wiley \& Sons, Ltd. 
drawals/dropouts occurred in the control group. Imputation method was used for missing observations as described in the FACIT Administration and Scoring Guidelines.

Comment: Given that imputation was done for missing observations, a low risk of bias was rated.

\begin{tabular}{ll}
\hline $\begin{array}{l}\text { Selective reporting (re- } \\
\text { porting bias) }\end{array}$ & Low risk \\
\end{tabular}

Other bias Unclear risk Quote from main publication: "Baseline characteristics were well matched between the two arms. Known prognostic factors, including age, sex, ECOG PS and presence of metastases were also balanced. Only a greater number of patients was observed with gastric cancer in the control arm and with biliary cancer in the intervention arm".

Comment: Given that it was unclear whether this difference was statistically significant, an unclear risk of bias rating was given.

\begin{tabular}{ll}
\hline Size of study $\quad$ Unclear risk & $\begin{array}{l}\text { Unclear risk of bias because the number of participants in each group was be- } \\
\text { tween } 50 \text { and } 199\end{array}$ \\
\hline
\end{tabular}

Gade 2008

\section{Study characteristics}

Methods Design: RCT

Multicentre RCT

Who led PC intervention: Multidisciplinary team (PC team was available Monday through Friday and a palliative care physician was on call after hours)

Core team: Palliative care physician/nurse/hospital social worker/chaplain

Participants

Country and regions: USA, Denver, Portland, and San Francisco

Recruitment: June 2002 and December 2003. Eligible patients were members of the same integrated health plan from three regions: Denver, Colorado, San Francisco, California, and Portland, Oregon. Referrals were received from all medical services and inpatient units.

Inclusion criteria: Eligible patients were $\geq 18$ years, hospitalised with at least one life-limiting diagnosis, and whose attending physician indicated they "would not be surprised if the patient died within 1 year."

Exclusion criteria: Patients were excluded if they had impaired cognitive status and no surrogate or were currently enrolled in hospice or other PC studies.

Number of patients: $\mathrm{N}=517$ (280 intervention and 237 control)

Diseases: Cancer, congestive heart failure (CHF), myocardial infarction (MI), other heart disease, chronic obstructive pulmonary disease (COPD), other pulmonary disease, end-stage renal disease (ESRD), organ failure, stroke, dementia

Patient characteristics: Mean age in years (SD): 73.6 (12.6) years in intervention group, 73.1 (13.2) years in control group; $59 \%$ female in intervention group, $51 \%$ female in control group

Deaths at end of study (N (\%)): No death

Withdrawals/other dropouts $(\mathrm{N}(\%))$ : Total $\mathrm{N}=5: 5(1.8 \%)$ intervention and none in control.

The effectiveness and cost-effectiveness of hospital-based specialist palliative care for adults with advanced illness and their caregivers 
Gade 2008 (Continued)

Interventions
Name: Interdisciplinary inpatient palliative care consultative service (IPCS) vs usual hospital care

Type: Specialist palliative care. Included a palliative care physician

Service base: A hospital in Denver, Portland, and San Francisco

Team: The IPCS teams included a palliative care physician and nurse, hospital social worker and chaplain.

Intervention condition: All teams provided care in accordance with key palliative care components which were adapted from Weismann 1997. The teams assessed patients' needs for symptom management, psychosocial and spiritual support, end-of-life planning, and post-hospital care. The team then met with the patient/family to address symptoms, diagnosis, prognosis, and goals of care. After the patient/family meeting, the team convened briefly to synthesise a palliative care plan and organise follow-up by team members. IPCS provided consultation on intervention patients to the attending, involved subspecialists and staff on all aspects of palliative care, including treatment recommendations. A palliative care physician was on call after hours. The teams collaborated with the attendings and discharge planners in preparing for the patient's discharge. If intervention patients were readmitted to the hospital, they were again followed by IPCS for palliative care needs. Each site was visited early in the study to assess protocol adherence.

Duration: Period of hospitalisation

Control condition: San Francisco and Portland hospitals were part of a managed care organisation's (MCO) delivery system. Denver's community hospital had a contract with the MCO. All hospitals had MCO hospitalist physicians. At two sites hospitalists served as the attending physicians. Portland's hospital used a combination of MCO hospitalists and primary care internists. All hospitals had social workers and chaplains on staff that provided direct patient services to usual care patients.

\section{Primary outcomes:}

Symptom control assessed using the Physical Area scale of the Modified City of Hope Patient Questionnaires (MCOHPQ).

Levels of emotional and spiritual support assessed using items taken from the MCOHPQ Emotional/Relationship Area and Spiritual Area scales.

Patient satisfaction assessed using the MCOHPQ Place of Care Environment scale and the Doctors, Nurses/Other Care Providers Communication scale.

Total health services costs at 6 months post-index hospitalisation.

\section{Secondary measures:}

Survival

Number of advance directives (ADs) at discharge

Hospice utilisation within the 6 months post-index hospitalisation

Assessment points: After a baseline questionnaire was administered, the patient was randomly assigned to IPCS or usual hospital care. Surveys were administered to patients or proxies at study enrollment and within 2 weeks following index hospitalisation discharge to measure symptom control as well as emotional and spiritual support. Patient satisfaction was also measured within 2 weeks of index hospital discharge.

Resource use/costs

Healthcare costs

Intensive care admissions

Hospice utilisation

Time horizon: Enrollment to discharge from hospital 
Data sources: Costs were computed for all health services used within the 6 months following index hospitalisation discharge. Costs of health services were assigned using the predetermined internal MCO rate structure and vendor contracts based on per diem and case rate calculations.

Analytical perspective: Not clear

Notes $\quad$ Funding source: Garfield Memorial Fund

Declarations of interest among primary researchers: Apart from funding, no further study author disclosure statements were made.

Power considerations: Not reported

\section{Risk of bias}

\begin{tabular}{lll}
\hline Bias & Authors' judgement & Support for judgement \\
\hline $\begin{array}{ll}\text { Random sequence genera- } \\
\text { tion (selection bias) }\end{array}$ & Low risk & $\begin{array}{l}\text { Quote from main publication: "after a baseline questionnaire was adminis- } \\
\text { tered the patient was randomly assigned to IPCS or UC using a computer-gen- } \\
\text { erated, randomized assignment list for each site". } \\
\end{array}$ \\
& & \\
& & \\
&
\end{tabular}

\begin{tabular}{ll}
\hline $\begin{array}{l}\text { Allocation concealment } \\
\text { (selection bias) }\end{array}$ & Unclear risk
\end{tabular}

\begin{tabular}{lll}
$\begin{array}{l}\text { Blinding of participants } \\
\text { and personnel (perfor- } \\
\text { mance bias), subjective } \\
\text { outcomes }\end{array}$ & High risk & Comment: no blinding \\
\hline $\begin{array}{l}\text { Blinding of participants } \\
\text { and personnel (perfor- } \\
\text { mance bias), objective }\end{array}$ & Low risk & $\begin{array}{l}\text { Participants and personnel were not blinded but this was unlikely to lead to } \\
\text { bias in objective outcomes. }\end{array}$
\end{tabular}
outcomes

\begin{tabular}{|c|c|c|}
\hline $\begin{array}{l}\text { Blinding of outcome as- } \\
\text { sessment (detection bias), } \\
\text { subjective outcomes }\end{array}$ & Unclear risk & $\begin{array}{l}\text { Unclear risk of bias as there was no mention of blinding of outcome assess- } \\
\text { ment }\end{array}$ \\
\hline
\end{tabular}

\begin{tabular}{|c|c|c|}
\hline $\begin{array}{l}\text { Blinding of outcome as- } \\
\text { sessment (detection bias), } \\
\text { objective outcomes }\end{array}$ & Low risk & $\begin{array}{l}\text { There was no mention of blinding of outcome assessment but this was unlikely } \\
\text { to lead to bias in objective outcomes. }\end{array}$ \\
\hline
\end{tabular}

\begin{tabular}{|c|c|c|}
\hline $\begin{array}{l}\text { Incomplete outcome data } \\
\text { (attrition bias) } \\
\text { All outcomes }\end{array}$ & Low risk & $\begin{array}{l}\mathrm{N}=275(98.2 \%) \text { completers in intervention group vs } \mathrm{N}=237(100 \%) \text { completers } \\
\text { in control group. } 5(1.8 \%) \text { patients in the intervention group withdrew their } \\
\text { consent and were dropped from the study. The } 275(98.2 \%) \text { patients in the in- } \\
\text { tervention and } 237 \text { in control group were included in the analysis. }\end{array}$ \\
\hline
\end{tabular}

Comment: low risk of bias because of the very small number of dropouts

Selective reporting (re- Unclear risk Unclear because the protocol of this study was not available
porting bias)

\begin{tabular}{|c|c|c|}
\hline Other bias & High risk & $\begin{array}{l}\text { Quote from publication: "there were no differences in any baseline measures } \\
\text { between the IPCS and UC groups except for the life-limiting diagnoses of }\end{array}$ \\
\hline
\end{tabular}

Comment: Given that the authors did not control for the highlighted differences, a decision was made to rate down for imbalance bias. 
Gade 2008 (Continued)

Size of study Low risk Low risk of bias because the number of patients in each group exceeded 200

Groenvold 2017

\section{Study characteristics}

Design: RCT
Multicentre RCT
Who led PC intervention: Multidisciplinary team
Core team: Doctors/nurses/psychologists were part of the team in all the 6 specialised palliative care
centres. 5 teams included physiotherapists, 4 social workers, 3 volunteers, 3 chaplains, 1 pharmacist
and 1 had a secretary.

\section{Participants Country and regions: Denmark}

Recruitment: May 2011 to December 2013: consecutive patients who were in oncological treatment or follow-up at five different Departments of Oncology were screened for palliative care needs by research nurses if they had cancer stage IV or cancer in the central nervous system grade III/IV; were $\geq 18$ years; lived in the area of one of the participating SPC centres; had no contact with an SPC during the previous year.

Inclusion criteria: Patients were eligible for the trial if they scored at least $50 \%$ of the score representing maximal symptom or maximally reduced functioning on at least one of the following seven scales: physical function, role function, emotional function, nausea/ vomiting, pain, dyspnoea or lack of appetite; had at least four additional symptoms (defined as a score of at least $33 \%$ of the score corresponding to maximal symptom burden or maximally reduced functioning) as measured by any of the 13 remaining scales (global health status/QoL excluded).

Exclusion criteria: Patients were excluded from the trial if they did not understand Danish well enough to fill in a questionnaire or were considered incapable of complying with the trial protocol.

Number of patients: $\mathrm{N}=297$ (145 intervention and 152 control group)

Diseases: Cancer: lung, digestive system, breast, other

Patient characteristics: Mean/median age not presented. The following was the age distribution in the intervention group: $<50$ (10), 50-59 (27), 60-69 (65), 70-79 (36), $\geq 80$ (7) and control group < 50 (15), $50-59$ (25), 60-69 (58), 70-79 (45), $\geq 80$ (9). \% of females in intervention group was $57 \%$ and $59 \%$ in control group.

Deaths at end of study ( $\mathrm{N}(\%))$ : Total $\mathrm{N}=30: 15(10.3 \%)$ intervention and $15(9.9 \%)$ control

Definition of early PC: Defined as 'usual SPC' initiated at an earlier time than would otherwise have been the case

Type: Training in palliative care unclear

\section{Service base: 6 Danish SPC centres}

Team: Doctors, nurses and psychologists were part of the team in all the 6 specialised palliative care centres. 5 of the teams included physiotherapists, 4 included social workers, 3 had volunteers, 3 had chaplains, 1 had a pharmacist and 1 had a secretary.

Intervention condition: Patients in the intervention group were referred to a SPC team, and the number and frequency of contacts with the SPC team and the treatments and other interventions were de- 
termined by the patient's needs, following the European Association for Palliative Care White Paper, the WHO guidelines and national and local guidelines. The common understanding was that SPC is a complex and multidisciplinary intervention that is adapted to each patient. The interventions were given by the staff normally providing the interventions. Intervention fidelity was not assessed since there was not a specific manual for the intervention.

Duration: The trial period was eight weeks

Control condition: There was very limited description of standard care. Standard care potentially included palliative care provided by the Departments of Oncology, general practitioners (GPs) or home care services.

Primary outcomes:
Change in the patient's primary need (the most severe of the seven EORTC QLQ-C30 scales)
Secondary outcomes:
Change in the seven EORTC QLQ-C30 scales
Survival
Assessment points:
Patients received a questionnaire at baseline and at 3- and 8-week follow-up including the EORTC QLQ-
C3023 and additional instruments.

Resource use/costs Contact with SPC team

Assessment of healthcare service use stated in protocol

Notes $\quad$ Funding source: This work was supported by The Tryg Foundation (7-10-0838A, 7-12-0754) and the
Danish Cancer Society (R16-A695, R114-A7232-14-S3).

Declaration of interest among primary researchers: The author(s) declared no potential conflicts of interest with respect to the research, authorship and/or publication of this article.

Power considerations: Assuming a difference of 7.5 points in the primary outcome, the planned sample size was 300 (alpha: 5\%; beta: 10\%; standard deviation (SD): 20).

\section{Risk of bias}

\begin{tabular}{lll}
\hline Bias & Authors' judgement & Support for judgement \\
\hline $\begin{array}{l}\text { Random sequence genera- } \\
\text { tion (selection bias) }\end{array}$ & Low risk & $\begin{array}{l}\text { Quote from main publication: "central randomisation via telephone was car- } \\
\text { ried out by the Copenhagen Trial Unit (CTU), which was independent of the tri- } \\
\text { al administration office. The allocation sequence was computer-generated 1:1 } \\
\text { with varying block size of } 8 \text { and } 12 \text { per strata and was kept unknown for all in- } \\
\text { vestigators. Randomisation was stratified by 'primary need'". }\end{array}$ \\
& $\begin{array}{l}\text { Comment: probably done } \\
\text { Allocation concealment } \\
\text { (selection bias) }\end{array}$ & $\begin{array}{l}\text { Quote from main publication: "central randomisation via telephone was car- } \\
\text { ried out by the Copenhagen Trial Unit (CTU), which was independent of the tri- } \\
\text { al administration office. The allocation sequence was computer-generated 1:1 } \\
\text { with varying block size of } 8 \text { and } 12 \text { per strata and was kept unknown for all in- } \\
\text { vestigators". }\end{array}$ \\
& Comment: probably done \\
\hline
\end{tabular}

Blinding of participants $\quad$ High risk $\quad$ Comment: patients were not blinded.
and personnel (perfor-

The effectiveness and cost-effectiveness of hospital-based specialist palliative care for adults with advanced illness and their caregivers 
Groenvold 2017 (Continued) mance bias), subjective outcomes

\begin{tabular}{ll}
\hline $\begin{array}{l}\text { Blinding of participants } \\
\text { and personnel (perfor- } \\
\text { mance bias), objective }\end{array}$ & Low risk \\
outcomes & $\begin{array}{l}\text { Patients were not blinded although investigators and those who analysed the } \\
\text { data were blinded. }\end{array}$ \\
& $\begin{array}{l}\text { Comment: a low risk of bias was given because lack of blinding was unlikely to } \\
\text { lead to bias in objective outcomes such as survival. }\end{array}$
\end{tabular}

\begin{tabular}{ll}
\hline $\begin{array}{l}\text { Blinding of outcome as- } \\
\text { sessment (detection bias), } \\
\text { subjective outcomes }\end{array}$ & Low risk
\end{tabular}$\quad \begin{aligned} & \text { Investigators and those who analysed the data were blinded. A low risk of bias } \\
& \text { was given. }\end{aligned}$

\begin{tabular}{ll}
\hline $\begin{array}{l}\text { Blinding of outcome as- } \\
\text { sessment (detection bias), } \\
\text { objective outcomes }\end{array}$ & Low risk
\end{tabular}$\quad \begin{aligned} & \text { Investigators and those who analysed the data were blinded. } \\
& \text { Comment: low risk of bias was given because blinding or a lack of it would not } \\
& \text { lead to bias in the assessment of objective outcomes such as survival. }\end{aligned}$

\begin{tabular}{|c|c|c|}
\hline $\begin{array}{l}\text { Incomplete outcome data } \\
\text { (attrition bias) } \\
\text { All outcomes }\end{array}$ & Low risk & $\begin{array}{l}\text { For whole population, missing outcome data not balanced between interven- } \\
\text { tion } N=32(22 \%) \text { and control groups } N=39(26 \%) \text {. The reasons for missing da- } \\
\text { ta also differed between groups with the exception of number of deaths. } 20 \\
(13 \%) \text { people in the control group did not answer the questionnaire compared } \\
\text { to } 9(6.2 \%) \text { in the intervention group. Higher levels of administrative failure } \\
\text { were recorded in the intervention group }(5.5 \%) \text { compared to the control group } \\
(2 \%) .89 .7 \% \text { of people in intervention and } 90 \% \text { of control were included in the } \\
\text { primary analysis. Multiple imputations were carried out for missing data with } \\
\text { the exception of people who died. }\end{array}$ \\
\hline
\end{tabular}

$\begin{array}{ll}\begin{array}{l}\text { Selective reporting (re- } \\ \text { porting bias) }\end{array} & \text { High risk } \\ & \begin{array}{l}\text { Rated "high risk" because some of the secondary outcomes stated in the pro- } \\ \text { tocol were not reported. Examples included cancer patients' satisfaction with } \\ \text { the healthcare system measured using the FAMCARE-p16, anxiety and depres- } \\ \text { sion measured using the Hospital Anxiety and Depression Scale (HAD scale) } \\ \text { and economic consequences per week from the start of the trial to a minimum } \\ \text { of three months after the end of the intervention. }\end{array}\end{array}$

\begin{tabular}{lll}
\hline Other bias & Low risk & Study appeared to be free of other biases. \\
\hline Size of study & Unclear risk & $\begin{array}{l}\text { Unclear risk of bias because the number of participants in each group was be- } \\
\text { tween } 50 \text { and } 199\end{array}$ \\
\hline
\end{tabular}

Grudzen 2016

\section{Study characteristics}

\begin{tabular}{ll}
\hline Methods & Design: RCT \\
& Single-centre, single-blind, RCT \\
& Who led PC intervention: Multidisciplinary team \\
Core team: Physician/nurse practitioner/social worker/chaplain & \\
Country and regions: USA, New York City \\
Recruitment: June 2011 to April 2014 (34 months). Research assistants screened the electronic med- \\
ical record emergency department (ED) track board for patients with the stated specific advanced can- \\
cer staging criteria 8 to 12 hours daily except Saturdays. Medical oncologists at the institution were \\
able to opt out of participation.
\end{tabular}

The effectiveness and cost-effectiveness of hospital-based specialist palliative care for adults with advanced illness and their caregivers 
Inclusion criteria: Patients eligible for participation were those with a known advanced cancer that met the staging criteria, who were able to speak English or Spanish fluently, and who were being admitted to or observed in the hospital.

Exclusion criteria: Patients who were unable to answer questions because of severe pain or lethargy, those who had been seen by palliative care in the past, or if they had evidence of cognitive impairment based on the 6-item screener. Patients planning to leave the immediate geographic area (i.e. move to another state or country) were also excluded.

Number of patients: $\mathrm{N}=136$ (69 intervention and 67 control)

Diseases: Advanced cancer: breast, colorectal, lung and other

Patient characteristics: Mean age in years (SD): 55.1 (13.1) years in intervention group, 57.8 (14.7) years in control group; $57 \%$ female in intervention group, $55 \%$ female in control group

Deaths at end of study (N (\%)): Total N = 85: 41(59.4\%) intervention and $44(65.7 \%)$ control had died by the one-year mark

\begin{abstract}
Interventions
Name: Emergency department-initiated PC consultation vs usual care

Type: Training in PC unclear

Service base: Mount Sinai Hospital (MSH) ED

Team: Comprised a physician, a nurse practitioner, a social worker, and a chaplain

Intervention condition: Inpatient comprehensive palliative care consultation consisted of 3 components: (1) symptom assessment and treatment, (2) goals of care and advance care plans, and (3) transition planning. The team used validated symptom assessments to make recommendations for symptom management to consulting physicians using National Comprehensive Cancer Network (NCCN) guidelines. The palliative care team met with patients, families, and care teams to identify goals of care, complete advance directives, and communicate difficult news (if requested) using standardised communication protocols. If admitted, the team saw patients daily to monitor implementation and results of treatment recommendations and to assess for new and ongoing symptoms. The team also worked with the patients' social workers and family to facilitate transition management consistent with goals of care. If the team found ongoing palliative care needs that were expected to continue after discharge, they referred patients to the outpatient palliative care clinic.
\end{abstract}

Duration: Enrollment to discharge from hospital

Control condition: Participants assigned to the usual care arm completed the same baseline interviews and follow-up as intervention participants. If requested by the admitting team or oncologist, participants may also have received a palliative care consultation.

\title{
Primary outcomes:
}

Quality of life at 12 weeks assessed using the Functional Assessment of Cancer Therapy-General (FACTG)

\section{Secondary outcomes:}

Survival at one year

Major depressive disorder at 12 weeks assessed using the Patient Health Questionnaire-9 (PHQ-9)

Healthcare utilisation at 180 days (hospital days, hospice use, and ICU admission)

\section{Assessment points:}

12 weeks for quality of life and major depressive disorder, one year for survival and 180 days for healthcare utilisation 
Grudzen 2016 (Continued)

\section{Hospice use}

ICU admission

Time horizon: Enrollment to 180 days

Notes

Funding source: This work was supported by a Mentored Research Scholar Grant from the American Cancer Society and by a Mid-Career Investigator Award in Patient Oriented Research (K24 AG022345) from the National Institute on Aging.

Declaration of interest among primary researchers: Apart from funding, no further study author disclosure statements were made.

Power considerations: Sample size was estimated based on "time to palliative care" in patients with advanced cancer. The baseline mean time to consultation for such patients seen by palliative care was 9 days $(S D=12)$. It was estimated that the intervention would decrease this number by at least $50 \%$, to 4.5 days (estimated $S D=6$ days). Calculations employed two-tailed tests $(\alpha=0.05$, with $\beta=0.80)$. The authors planned to enroll at least 140 patients. Authors expected to have at least $80 \%$ power with $\alpha=$ 0.05 (two-sided) to detect clinically meaningful differences with 70 subjects/group.

\section{Risk of bias}

\begin{tabular}{lll}
\hline Bias & Authors' judgement & Support for judgement \\
\hline $\begin{array}{l}\text { Random sequence genera- } \\
\text { tion (selection bias) }\end{array}$ & Unclear risk & $\begin{array}{l}\text { Quote from main publication: "participants were randomized via prespecified } \\
\text { balanced block randomization in blocks of 50". }\end{array}$ \\
& $\begin{array}{l}\text { Comment: there was insufficient information about the sequence generation } \\
\text { process. }\end{array}$ \\
\hline $\begin{array}{l}\text { Allocation concealment } \\
\text { (selection bias) }\end{array}$ & Low risk & $\begin{array}{l}\text { Quote from main publication: "after the baseline survey was completed, the } \\
\text { research co-ordinator then relayed the participant information to a separate } \\
\text { research staff member (the "randomizer") with no role in study recruitment, } \\
\text { follow-up, or analysis...If the participant was assigned to the intervention } \\
\text { group, the randomizer then paged the palliative care consultation team to re- } \\
\text { lay information about the participant (name, medical record number, ED at- } \\
\text { tending, and oncologist of record) and the reason for consultation. If assigned } \\
\text { to the usual care group, no further action was necessary. The list linking partic- } \\
\text { ipant name and group assignment was stored on a secure network computer } \\
\text { under password protection, and was accessible only to the randomizer". }\end{array}$ \\
& $\begin{array}{l}\text { Comment: probably done } \\
\end{array}$
\end{tabular}

\begin{tabular}{|c|c|c|}
\hline $\begin{array}{l}\text { Blinding of participants } \\
\text { and personnel (perfor- } \\
\text { mance bias), subjective } \\
\text { outcomes }\end{array}$ & High risk & $\begin{array}{l}\text { Quote from main publication: "it was not feasible to blind participants or care } \\
\text { providers to participant assignment". } \\
\text { Comment: high risk of bias due to lack of blinding }\end{array}$ \\
\hline $\begin{array}{l}\text { Blinding of participants } \\
\text { and personnel (perfor- } \\
\text { mance bias), objective } \\
\text { outcomes }\end{array}$ & Low risk & $\begin{array}{l}\text { Quote from main publication: "It was not feasible to blind participants or care } \\
\text { providers to participant assignment". } \\
\text { Comment: there was no blinding of participants and personnel but this was } \\
\text { unlikely to lead to bias for objective outcomes. }\end{array}$ \\
\hline $\begin{array}{l}\text { Blinding of outcome as- } \\
\text { sessment (detection bias), } \\
\text { subjective outcomes }\end{array}$ & Unclear risk & $\begin{array}{l}\text { Information on blinding of outcome assessment was not provided in the study. } \\
\text { Comment: an unclear risk of bias was given because relevant information was } \\
\text { not provided. }\end{array}$ \\
\hline
\end{tabular}


Grudzen 2016 (Continued)

Blinding of outcome as- Low risk Information on blinding of outcome assessment was not provided in the study. sessment (detection bias), objective outcomes However, this was unlikely to lead to bias in the assessment of objective outcomes.

$\begin{array}{ll}\begin{array}{l}\text { Incomplete outcome data Low risk } \\ \text { (attrition bias) }\end{array} & \mathrm{N}=53(77 \%) \text { completers in intervention group vs } \mathrm{N}=43(64 \%) \text { completers in } \\ \text { All outcomes } & \text { control group. However, all patients were included in the analysis. The authors } \\ & \text { stated that "we chose a conservative method of carrying baseline values for- } \\ & \text { ward to account for missing depression and QoL follow-up measures". }\end{array}$

Selective reporting (re- High risk
porting bias)

All of the study's prespecified (primary and secondary) outcomes were not reported in the prespecified way. Furthermore, some outcomes such as depression and survival stated as primary outcomes in the protocol were presented as secondary outcomes in the main publication.

\begin{tabular}{lll}
\hline Other bias & Low risk & Study appeared to be free of other biases. \\
\hline Size of study & Unclear risk & $\begin{array}{l}\text { Unclear risk of bias because the number of participants in each group was be- } \\
\text { tween } 50 \text { and } 199\end{array}$ \\
\hline
\end{tabular}

Higginson 2009

\section{Study characteristics}

Design: RCT (patient and unpaid caregiver)
Fast-track phase II RCT
Who led PC intervention: Multidisciplinary team
Core team: Part-time consultant in palliative medicine/part-time clinical nurse specialist (PC nurse)/
administrator/psychosocial worker

Participants Country and regions: UK, South East London

Recruitment: Length of recruitment was not stated: patients living with multiple sclerosis (MS) and deemed (by clinicians) to have one or more unresolved symptoms, psychosocial concerns, end-of-life issues, progressive illness, or complex needs were referred. Educational seminars informed local health and social care professionals about the Palliative Care Teams (PCTs). Referrals were screened by a consultant in palliative medicine who was independent from delivering the service.

Inclusion criteria: Patients in South East London, living with MS and deemed (by clinicians) to have one or more of unresolved symptoms, psychosocial concerns, end-of-life issues, progressive illness, or complex needs (i.e. palliative care needs). unpaid caregivers were identified through patients.

Exclusion criteria: Referring staff or the screening deemed patients had very urgent needs or were deteriorating rapidly when immediate referral to the service was offered.

Number of patients: $\mathrm{N}=52$ (26 intervention and 26 control)

Diseases: Multiple sclerosis

Patient characteristics: Mean age (SD): 53 (10.5) years in intervention group, 53 (10.4) years in control; $65 \%$ female in intervention group, $73 \%$ female in control.

Deaths at end of study ( $\mathrm{N}(\%))$ : Total $\mathrm{N}=4: 1(3.8 \%)$ intervention and $3(11.5 \%)$ control

Withdrawals/other dropouts $(\mathrm{N}(\%))$ : Total $\mathrm{N}=2$ : none in intervention and $2(7.7 \%)$ control

Name: Multiprofessional palliative care team in addition to usual care vs usual care 
Type: Specialist palliative care. Team included one part-time consultant in palliative medicine and one part-time clinical nurse specialist (PC nurse)

Service base: The multiprofessional PC team was based in a large teaching hospital.

Team: One part-time consultant in palliative medicine, one part-time clinical nurse specialist (PC nurse), one administrator and one psychosocial worker

Intervention condition: Patients in the intervention group received the new palliative care service immediately (fast-track). Patients were visited in their own homes or sometimes outpatient clinics, nursing homes, or hospital. The PCT undertook assessments, suggested ways to improve physical, emotional, social, and other problems, provided specialist welfare benefits advice and bereavement support, and liaised with and acted as a catalyst for local services, both primary and specialist teams. After initial assessment, treatment was recommended.

Duration: The intervention was for three months.

Control condition: Control group patients received usual care for 12 weeks, after which they were offered the palliative care service. Community and hospital services (including neurologists, MS nurses, rehabilitation, neurological, and social services) were offered as usual.

Outcomes

Cost

Palliative care needs and concerns assessed using the Palliative care Outcome Scale (POS-8)

Pain item of the POS-8

unpaid caregiver burden assessed using the Zarit Carer Burden Inventory (ZBI)

Assessment points: Baseline (before randomisation) and at 6, 12, 18 (only control group - after they received PCT), and 24 weeks

Resource use/costs

Health, social and voluntary services (district/practice nurse, MS nurse, palliative care nurse, other nurse, general practice, specialist (home), specialist (hospital), specialist (ward), specialist (other), occupational therapist/physiotherapist, dietician/chiropodist/dentist, speech therapist, social services, informal unpaid caregiver, day centre, inpatient care, respite care)

Time horizon: From enrollment to death or study end. Data regarding the use of health and social services in the previous three months were collected at each interview using a standard schedule.

Costs: Mean service costs (health, social and voluntary services) in the three months prior to baseline assessment and 12-week follow-up interviews

Currency: 2005 GBP

Data sources: Research assistants (not blinded to group allocation) collected resource use data in the last 3 months from patients in face-to-face interview using an adapted version of the Client Service Receipt Inventory; data were collected at baseline (before randomisation) and at 6 and 12 weeks, at 18 weeks (the latter only for the control group, after receiving the service) and 24 weeks. Service costs were calculated by combining resource use data with nationally applicable unit costs. Informal care costs were estimated by assuming that in the absence of a unpaid caregiver, the help would need to be provided by a home care worker; the unit costs of the latter were, therefore, used as a "shadow price". Costs were in 2005 GBP.

Analytical perspective: Costs were assessed using a broad perspective including costs to health, social and voluntary services, and informal unpaid caregivers.

Declarations of interest among primary researchers: Apart from funding, no further study author disclosure statements were made. 
Power considerations: Study estimated that a sample of more than 25 patients in each arm would enable it to detect differences of $>2$ on the POS- 8 at $P<0.05$, power $80 \%$ (with SD of 2.25 ) at 12 weeks.

Cost-effectiveness was assessed only when both cost and outcome data were present.

\section{Risk of bias}

Bias Authors' judgement Support for judgement

Random sequence genera- Low risk tion (selection bias)
Quote from main publication: "the researcher e-mailed relevant data to independent statisticians who conducted the randomization using the minimization method to give an equal balance of gender, age, date of diagnosis, and according to whether patients could or could not communicate".

Comment: probably done
Quote from publication: "the researcher e-mailed relevant data to independent statisticians who conducted the randomization using the minimization method...the statistician informed researchers who then informed patients of their allocation".

Comment: probably done
Blinding of participants and personnel (performance bias), subjective outcomes

Blinding of outcome assessment (detection bias), subjective outcomes
High risk

Quote from publication: "we were unable to blind the interviewers or participants from group allocation".

Comment: high risk of bias due to lack of blinding
High risk

Quote from publication: "research assistants (not blinded to group allocation) collected resource use data in the last 3 months from patients in face-to-face interview using an adapted version of the Client Service Receipt Inventory".

Comment: high risk of bias due to lack of blinding

$\mathrm{N}=25(96.2 \%)$ completers in intervention group vs $\mathrm{N}=21(80.8 \%)$ completers in control group. There was one death in the intervention group and three in the control group. One patient was withdrawn from the control group due to protocol violation and another due to severe illness. Although, the authors separately explored missing data and tested imputations (last-value-carried-forward, next-value-carried-backward, and mean value) in a sensitivity analysis, the number of patients analysed for the POS- 8 and POS pain (outcome measures) differed from the number of patients randomly assigned to the intervention and control groups.

\begin{tabular}{lll}
\hline $\begin{array}{l}\text { Selective reporting (re- } \\
\text { porting bias) }\end{array}$ & Low risk & The protocol was available and all stated outcomes were reported. \\
\hline Other bias & Low risk & Study appeared to be free of other biases. \\
\hline Size of study & High risk & $\begin{array}{l}\text { High risk of bias because the number of participants in each group was less } \\
\text { than } 50\end{array}$ \\
\hline
\end{tabular}

\section{Higginson 2014}

\section{Study characteristics}

Methods Design: RCT

Fast-track phase III RCT

The effectiveness and cost-effectiveness of hospital-based specialist palliative care for adults with advanced illness and their caregivers 
Higginson 2014 (Continued)

Who led PC intervention: Multidisciplinary team

Core team: Palliative care physician/respiratory medicine physician/physiotherapist/occupational therapist

Participants

Country and regions: South London

Recruitment: October 2010 and September 2012. The authors screened for potential patients across three large teaching hospitals and via general practitioners.

Inclusion criteria: Patients had to meet all criteria: refractory breathlessness on exertion or rest (MRC dyspnoea scale score $\geq 2$ ), despite optimum treatment of the underlying disease, as deemed by the identifying clinician; advanced disease such as cancer, chronic obstructive pulmonary disease (COPD), chronic heart failure, interstitial lung disease, and motor neuron disease; willing to engage with shortterm home physiotherapy and occupational therapy; and able to provide informed consent

Exclusion criteria: Breathlessness of unknown cause; a primary diagnosis of chronic hyperventilation syndrome; completely house- (or hospital- or nursing home-) bound, despite offer of free transport to clinic; or within 2 weeks of treatment for an acute exacerbation. Such patients were reapproached after 2 weeks.

Number of patients: $\mathrm{N}=105$ (53 intervention and 52 control)

Diseases: Cancer, chronic obstructive pulmonary disease (COPD), heart failure, interstitial lung disease and other.

Patient characteristics: Mean age (SD): 66 (11) years in intervention group, 68 (11) years in control; $47 \%$ female in intervention group, $37 \%$ female in control.

Deaths at end of study ( $(\%))$ : Total $N=4: 1(1.9 \%)$ intervention and $3(5.8 \%)$ control

Withdrawals/other dropouts $(\mathrm{N}(\%))$ : Total $\mathrm{N}=19: 10(18.9 \%)$ in intervention and $9(17.3 \%)$ control refractory breathlessness (breathlessness support service) vs usual care.

Early PC: Early palliative care was integrated with respiratory services for patients with advanced disease and refractory breathlessness. The authors were explicit about early palliative care.

Type: Specialist palliative care. Team included a palliative care physician.

Service base: Hospital

Team: Palliative care physician, respiratory medicine physician, physiotherapist, occupational therapist

Intervention condition: The breathlessness support service (BSS) was a multiprofessional integrated service that combined respiratory, physiotherapy, occupational therapy, and palliative care assessment and management. It brought together assessment and treatment of physical, emotional, psychological, and spiritual concerns, through one point of access. The service comprised a first outpatient clinic appointment with respiratory medicine and palliative care clinicians assessing present treatment and concerns. The patient (and family, if present) was given a breathlessness pack including information, management, and pacing guidance, a hand-held fan or water spray, and a poem and helped to agree a crisis plan. A home assessment was done 2-3 weeks after the clinic visit to assess the need for walking and home aids and adaptations, reinforcement of self-management, and further guidance on pacing and exercises, including a DVD, when appropriate. A final clinic appointment with a palliative care specialist was arranged to agree further actions and a discharge plan.

Duration: The intervention was for six weeks.

Control condition: Control group patients continued with optimum management as provided by their usual services in accordance with relevant UK guidance to ensure best practice. After the 6-week (primary endpoint) research interview, these patients were offered BSS. 
Higginson 2014 (Continued)

Outcomes

\section{Primary outcome}

Breathlessness mastery at 6 weeks determined according to one domain of the quality of life measure, the Chronic Respiratory Disease Questionnaire.

\section{Secondary outcomes}

Severity of breathlessness on average, at worst, at rest and on exertion in the previous 24 hours

Activity (assessed by London Chest Activity of Daily Living questionnaire)

Other domains of the Chronic Respiratory Disease Questionnaire (breathlessness, fatigue, and emotional function)

Quality of life assessed using the EQ-5D

Palliative needs assessed using the Palliative care Outcome Scale (POS)

Depression and anxiety assessed using Hospital Anxiety and Depression Scale (HADS)

Spirometry

Patient survival

Assessment points: Research data were collected in face-to-face interviews with patients, usually in their own homes, at baseline and 6 weeks follow-up (the primary endpoint).

\section{Resource use/costs Hospital inpatient days in previous 3 months}

Time horizon: Data regarding hospital inpatient days in the previous three months was collected using a standard schedule.

Costs: Cost of formal care in the previous 3 months

Currency: $2011-12$ unit costs GBP

Data sources: Data regarding hospital inpatient days in the previous three months at baseline was collected using a standard schedule. We calculated costs by combining Client Service Receipt Inventory data with UK 2011-12 unit costs.

Analytical perspective: Not clear

Notes

Funding source: Mainly by a National Institute for Health Research (NIHR) grant from Research for Patient Benefit (PBPG-0808-17311)

Declarations of interest among primary researchers: The authors declared no competing interests.

Power considerations: On the basis of the primary outcome, the Chronic Respiratory Disease Questionnaire mastery domain, the authors estimated that more than 34 patients per group would detect a mean difference of 0.70 (SD 1), a value of less than 0.05 at power $80 \%$. To allow for a conservative estimated attrition of $40 \%$, the authors planned to recruit at least 110 patients.

\section{Risk of bias}

\section{Bias}

Authors' judgement Support for judgement

Random sequence genera- Low risk tion (selection bias) 
Comment: probably done

\begin{tabular}{|c|c|c|}
\hline $\begin{array}{l}\text { Allocation concealment } \\
\text { (selection bias) }\end{array}$ & Low risk & $\begin{array}{l}\text { Quote from main publication: "randomisation was computer generated cen- } \\
\text { trally by the independent Clinical Trials Unit in a 1:1 ratio, by minimisation to } \\
\text { balance four potential confounders: cancer versus non-cancer, breathlessness } \\
\text { severity, presence of an informal unpaid caregiver, and ethnicity". }\end{array}$ \\
\hline
\end{tabular}

\begin{tabular}{|c|c|c|}
\hline $\begin{array}{l}\text { Blinding of participants } \\
\text { and personnel (perfor- } \\
\text { mance bias), subjective } \\
\text { outcomes }\end{array}$ & High risk & $\begin{array}{l}\text { Quote from main publication: "patients were aware of treatment allocation, } \\
\text { and were asked not to disclose this information to interviewers or research } \\
\text { nurses". }\end{array}$ \\
\hline
\end{tabular}

Comment: high risk of bias due to lack of blinding

\begin{tabular}{|c|c|c|}
\hline $\begin{array}{l}\text { Blinding of participants } \\
\text { and personnel (perfor- } \\
\text { mance bias), objective } \\
\text { outcomes }\end{array}$ & Low risk & $\begin{array}{l}\text { This study was a single-blind RCT in which the research interviewers/nurses } \\
\text { were blinded. Quote from main publication: "Patients were aware of treatment } \\
\text { allocation, and were asked not to disclose this information to interviewers or } \\
\text { research nurses". }\end{array}$ \\
\hline
\end{tabular}

Comment: patients were not blinded but this was unlikely to lead to bias in objective outcomes such as survival.

Blinding of outcome as- $\quad$ Unclear risk
sessment (detection bias),

Quote from main publication: "research nurses/interviewers were masked to sessment (detection bias) search nurse could have seen breathlessness support service equipment (e.g. hand-held fan and information sheets) in patients' home, which could have biased their interviews.

Comment: unclear risk because there was the possibility that interviews could have been biased

Blinding of outcome as- $\quad$ Low risk
sessment (detection bias),
objective outcomes
Quote from main publication: "Research nurses/interviewers were masked to treatment allocation". However, the authors also acknowledged that the re- search nurse could have seen breathlessness support service equipment (e.g. hand-held fan and information sheets) in patients' home, which could have bi- ased their interviews.
Comment: although there was the possibility that the interviews by research nurses/interviewers could have been biased, this was unlikely to lead to bias in the assessment of objective outcomes.

Incomplete outcome data High risk
(attrition bias)
All outcomes

$\mathrm{N}=42(79.2 \%)$ completers in intervention group vs $\mathrm{N}=40$ (76.9\%) completers in control group. Missing data were excluded from the analysis: 11 (20.8\%) patients were missing in the intervention group due to death $(N=1)$, withdrawal $(\mathrm{N}=6)$, unable to contact $(\mathrm{N}=4)$ and $12(23.1 \%)$ patients were missing in control group due to death $(\mathrm{N}=3)$, withdrawal $(\mathrm{N}=7)$, and unable to contact $(\mathrm{N}=$ 2).

\begin{tabular}{lll}
\hline $\begin{array}{l}\text { Selective reporting (re- } \\
\text { porting bias) }\end{array}$ & High risk & $\begin{array}{l}\text { There were protocol deviations. Economic evaluation was not carried out as } \\
\text { specified in the protocol. }\end{array}$ \\
\hline Other bias & Low risk & Study appeared to be free of other biases. \\
\hline Size of study & Unclear risk & $\begin{array}{l}\text { Unclear risk of bias because the number of participants in each group was be- } \\
\text { tween } 50 \text { and } 199 \text { participants }\end{array}$ \\
\hline
\end{tabular}


Hopp 2016

\section{Study characteristics}

Design: RCT (multicentre RCT)
Who led PC intervention: Multidisciplinary team
Core team: Physician/advanced nurse practitioner. Other professionals (chaplains and social workers)
participated as requested.

Participants Country and regions: USA

Recruitment: September 2006 to June 2008. Patients were screened with the use of computer-generated lists of diagnoses, and charts were reviewed for eligibility criteria.

Inclusion criteria: Participants had an admission diagnosis of acute heart failure (HF) (ICD-9 codes and subcodes associated with 425 and 428), a 1-year mortality risk of $\geq 33 \%$ based on Enhanced Feedback for Effective Cardiac Treatment (EFFECT) score, and/or New York Heart Association (NYHA) functional class III or IV.

Exclusion criteria: Cognitive impairment, a life-limiting non-HF illness, acute severe psychiatric illness other than major depression, symptoms too severe for study consent, anticipated cardiac transplantation, or an attending physician who rejected the need for palliative care consultation (PCC).

Number of patients: $\mathrm{N}=85$ (43 intervention and 42 control)

Diseases: Acute heart failure

Patient characteristics: Mean age (SD): 67 (11) years in intervention group, 68 (13) years in control; $39.5 \%$ female in intervention group, $57.1 \%$ female in control.

Deaths at end of study ( $\mathrm{N}(\%))$ : Total $\mathrm{N}=19: 11$ (\% not presented because denominator was unclear) intervention and 8 ( $\%$ not presented because denominator was unclear) control

Withdrawals/other dropouts ( $(\%)):$ Not stated

Interventions

Name: Hospital-based palliative care consultation vs usual care

Type: Training in palliative care unclear

Service base: 3 hospitals in a predominantly African-American urban community

Team: The Palliative Care Consultation (PCC) team included a physician and advanced nurse practitioner. Other professionals (chaplains and social workers) participated as requested.

Intervention condition: The PCC team included a physician and advanced nurse practitioner. Other professionals (chaplains and social workers) participated as requested. Clinical interviews assessed for uncontrolled distressing symptoms, goals of care, advance care planning, code status, and desired post-treatment residential setting. All PCC patients had at least 1 palliative care consultation, with the opportunity for additional meetings, as desired.

Duration: 3 to 6 months after randomisation

Control condition: Not described

Outcomes Primary outcome:

The primary outcome assessed after 3-6 months was a dichotomous (election vs nonelection) measure of comfort-oriented care, which included

(1) outpatient hospice,

(2) inpatient hospice,

(3) a "do not resuscitate" (DNR) order during the index or a subsequent hospitalisation, or 
(4) a DNR order at home or at a nursing home, as assessed by means of telephone interviews and medical records.

Secondary outcomes were not stated.

\section{Assssement point:}

After written informed consents were obtained, baseline questionnaires were completed and randomisation to palliative care consultation (PCC) and control groups conducted. 3-6 months after randomisation

\begin{tabular}{|c|c|}
\hline Resource use/costs & None reported \\
\hline \multirow[t]{3}{*}{ Notes } & $\begin{array}{l}\text { Funding source: This work was supported by the Blue Cross/Blue Shield Foundation of Michigan (grant } \\
\text { no. 1074-II PIRAP, Rob Zalenski, Principal Investigator) }\end{array}$ \\
\hline & Declarations of interest among primary researchers: No disclosures were made by the authors. \\
\hline & $\begin{array}{l}\text { Power considerations: The study was powered to detect a moderate to large effect of } \geq 20 \% \text { absolute } \\
\text { group differences in palliative outcomes ( } 24 \% \text { PCC vs } 4 \% \text { control). A sample size of } 88 \text { had } 80 \% \text { power } \\
\text { to detect the prespecified } 20 \% \text { proportion difference with a significance, or alpha, of } 0.05(P=.05) \text {. }\end{array}$ \\
\hline
\end{tabular}

\section{Risk of bias}

Bias Authors' judgement Support for judgement

Random sequence genera- Unclear risk tion (selection bias)
Quote from main publication: "after written informed consents were obtained, baseline questionnaires were completed and randomisation to PCC and control groups conducted".

Comment: there was insufficient information about the random sequence generation process.

Allocation concealment $\quad$ Unclear risk $\quad$ Allocation concealment was not described.
(selection bias)

Blinding of participants Low risk
and personnel (perfor-

Low risk mance bias), objective outcomes

Quote from main publication: "this was a prospective open-label behavioral intervention pilot trial of PCC versus usual care in patients with advanced HF who were hospitalised for an acute HF exacerbation at 1 of 3 hospitals in a predominantly African American urban community".

Comment: there was no blinding but this was unlikely to lead to bias in objective outcomes.

\section{Blinding of outcome as- Low risk} sessment (detection bias), objective outcomes

Quote from main publication: "this was a prospective open-label behavioral intervention pilot trial of PCC versus usual care in patients with advanced HF who were hospitalised for an acute HF exacerbation at 1 of 3 hospitals in a predominantly African American urban community".

Comment: there was no blinding but this was unlikely to lead to bias in the assessment of objective outcomes.

Incomplete outcome data High risk
(attrition bias) All outcomes
It was reported that 19 (23.8\%) of 80 patients died 3-6 months after randomisation with no difference by group ( 11 intervention and 8 control, $P=0.47$ ). The percentage of attrition for intervention and control group could not be calculated due to lack of clarity on the denominator.

Comment: given that attrition was $>10 \%$, judgment of a high risk of bias was made. 
Hopp 2016 (Continued)

Selective reporting (re- Unclear risk $\quad$ Furthermore, patient satisfaction and quality of life were reported at baseporting bias)

line but not at follow-up. No information was given regarding how the authors measured those outcomes. It was unclear if these were part of the outcomes in this study as the protocol was not available.

\begin{tabular}{lll}
\hline Other bias & Low risk & Study appeared to be free of other biases. \\
\hline Size of study & High risk & $\begin{array}{l}\text { High risk of bias because the number of participants in each group was less } \\
\text { than } 50\end{array}$ \\
\hline
\end{tabular}

\section{Janssens 2019}

\section{Study characteristics}

Design: RCT (single-centre RCT)
Who led PC intervention: Multidisciplinary team
Core team: Nurses with experience in palliative care and a palliative care physician

Participants Country and regions: Switzerland, Geneva

Recruitment: September 2013 to July 2016. Patients were recruited among subjects followed by Geneva University Hospitals on long-term oxygen therapy (LTOT) and/or home non-invasive ventilation (NIV), and subjects hospitalised for acute exacerbation of COPD (AECOPD) in our general internal medicine and geriatric wards.

Inclusion criteria: COPD defined according to GOLD (Global Initiative for Obstructive Lung Diseases) criteria (FEV1/FVC $<70 \%$ ) stage III or IV (FEV1 < 50\% predicted) and LTOT and/or home NIV and/or one or more hospital admissions in the previous year for an acute exacerbation.

Exclusion criteria: Moderate or severe cognitive impairment (Mini Mental State Examination [MMSE] score $<23$ ) and cancer.

Number of patients: $\mathrm{N}=49$ (26 intervention and 23 control)

Diseases: COPD

Patient characteristics: Mean age (SD): 70.8 (8.4) years in intervention group, 71.3 (8.1) years in control; $46.2 \%$ female in intervention group, $60.9 \%$ female in control

Deaths at end of study (N (\%)): Total N = 8: $4(15.4 \%)$ intervention and $4(17.4 \%)$ control

Withdrawals/other dropouts (N (\%)): Not stated

Interventions Name: Early palliative care (EPC) at home with usual care vs usual care

EPC: The authors reported that they chose patients with LTOT and/or NIV and/or hospitalised within the preceding year. Patients hospitalised for AECOPD are at high risk of subsequent readmission or post-discharge mortality; those under LTOT and/or home NIV also have a limited survival, justifying the chosen criteria.

Type: Specialist palliative care. Included nurses with experience in palliative care and a palliative care physician

Service base: Geneva University Hospitals

Team: Included nurses with experience in palliative care and a palliative care physician who supervised the PC interventions and defined medical strategies whenever symptom scores were abnormal (ESAS items $>4)$.

The effectiveness and cost-effectiveness of hospital-based specialist palliative care for adults with advanced illness and their caregivers 
Intervention condition: EPC group patients met the community ambulatory palliative care team as soon as possible after inclusion and monthly for 12 months. Home visits by nurses from the palliative care consultation lasted approximately $90 \mathrm{~min}$ and focussed on symptom assessment and management using the Edmonton Symptom Assessment Scale (ESAS) (If intensity of pain, dyspnoea, mood, anxiety, and appetite were $>4 / 10$ and the patient agreed, a consultation with a palliative care physician (or other specialist) was suggested), nutrition, understanding of illness and coping, anticipation and decision-making, support of relatives, social-spiritual needs, co-ordination between different health providers and alternative approaches such as relaxation. All cases were discussed with a physician specialised in palliative care, whom the patient could consult whenever appropriate. The EPC group also received standard care throughout the study period.

Duration: 12 months

Control condition: Control group patients had no contact with the palliative care team. For all patients under LTOT and/or home NIV, specialised nurses provided regular home visits to check on all aspects related to respiratory support. Treatments prescribed by a primary care physician or pulmonologist were not modified. Because the palliative care team was distinct from those providing standard care, the risk of 'contamination' of the control group by the early palliative care intervention was minimal.

Outcomes

\section{Primary outcomes}

Admissions to emergency wards

Hospitalisations

Admissions to ICUs

\section{Secondary outcomes}

Symptoms assessed using the Edmonton Symptom Assessment Scale (ESAS)

Health-related quality of life (HRQOL) assessed using the SF-36

Mood disturbances assessed using the Hospital Anxiety and Depression Scale (HADS-A and HADS-D)

Advanced care planning (ACP)

Survival

Appreciation of intervention

\section{Assssement points:}

After written informed consents were obtained and completion of baseline assessments, patients were randomised to EPC and control groups. Every month, data related to primary outcomes were recorded through hospital files, contact with the patient and his/her GP. Data related to the secondary outcomes were collected every three months.

$\begin{array}{ll}\text { Resource use/costs } & \begin{array}{l}\text { Admissions to emergency wards } \\ \text { Hospitalisations }\end{array} \\ \text { Admissions to ICU } \\ \text { Use of antibiotics }\end{array}$

Notes

Funding source: Swiss National Foundation for Research, within a programme focussing on palliative care. The authors also received additional funding from the Lancardis Foundation and the Pulmonary League of Geneva, both non-profit organisations devoted to supporting healthcare and research.

Declarations of interest among primary researchers: The authors declared that they had no conflict of interest to report regarding the study. 
Power considerations: Regarding the primary outcome, the authors calculated that a sample size of 80 patients per group would be adequate with an $80 \%$ power to detect a difference in hospital admissions of $20 \%$ between both groups at an a significance level (2-sided test) of 0.05 (obtained from study protocol).

\section{Risk of bias}

Bias Authors' judgement Support for judgement

Random sequence genera- Low risk tion (selection bias)

Quote from main publication: "patients were randomly assigned to the early palliative care group (EPC) or the standard care group (SC) in a 1:1 ratio without stratification and with randomised block sizes ranging from 4 to 6 , using sealed envelopes prepared with a computer program by... a co-investigator not involved in the inclusion of the patients".

Comment: probably done

Allocation concealment Low risk
(selection bias)

Allocation concealment Low risk

Quote from main publication: "patients were randomly assigned to the early palliative care group (EPC) or the standard care group (SC) in a 1:1 ratio without stratification and with randomised block sizes ranging from 4 to 6 , using sealed envelopes prepared with a computer program by... a co-investigator not involved in the inclusion of the patients".

Comment: probably done

$\begin{array}{ll}\begin{array}{l}\text { Blinding of participants } \\ \text { and personnel (perfor- }\end{array} & \text { High risk }\end{array} \begin{aligned} & \text { Information obtained from the trial registry indicated it was open-label. } \\ & \begin{array}{l}\text { mance bias), subjective } \\ \text { outcomes }\end{array}\end{aligned}$

\begin{tabular}{ll}
\hline $\begin{array}{l}\text { Blinding of participants } \\
\text { and personnel (perfor- }\end{array}$ & Low risk \\
$\begin{array}{l}\text { mance bias), objective } \\
\text { outcomes }\end{array}$ & $\begin{array}{l}\text { Information obtained from the trial registry indicated it was open-label. } \\
\text { to lead to bias in the objective outcomes assessed. }\end{array}$
\end{tabular}

Blinding of outcome as- High risk
Blinding of outcome as- High risk sessment (detection bias), subjective outcomes
Comment: Outcome assessors were not blinded. Consequently, a high risk of bias was rated. Information obtained from the trial registry indicated it was open-label.

\begin{tabular}{ll}
\hline $\begin{array}{l}\text { Blinding of outcome as- } \\
\text { sessment (detection bias), } \\
\text { objective outcomes }\end{array}$ & Low risk
\end{tabular}$\quad \begin{aligned} & \text { Information obtained from the trial registry indicated it was open-label. } \\
& \text { Comment: Outcome assessors were not blinded but this was unlikely to lead } \\
& \text { to bias in the objective outcomes assessed. }\end{aligned}$

\begin{tabular}{ll}
\hline $\begin{array}{l}\text { Incomplete outcome data } \\
\text { (attrition bias) }\end{array}$ & It appeared there were $\mathrm{N}=22(84.6 \%)$ completers in intervention group vs $\mathrm{N}=$ \\
All outcomes & $19(82.6 \%)$ completers in control group. There were $4(15.4 \%)$ deaths in the in- \\
& tervention group and $4(17.4 \%)$ deaths in the control group. Given that missing \\
data were included in the analysis, a low risk of bias rating was given.
\end{tabular}

\begin{tabular}{lll}
\hline $\begin{array}{l}\text { Selective reporting (re- } \\
\text { porting bias) }\end{array}$ & High risk & $\begin{array}{l}\text { Some outcomes such as survival and advanced care planning were not stated } \\
\text { a priori in the clinical trial registry. }\end{array}$ \\
\hline Other bias & Low risk & Study appeared to be free of other biases. \\
\hline Size of study & High risk & $\begin{array}{l}\text { High risk of bias because the number of participants in each group was less } \\
\text { than } 50\end{array}$ \\
\hline
\end{tabular}

The effectiveness and cost-effectiveness of hospital-based specialist palliative care for adults with advanced illness and their caregivers 
Jingfen 2017

\section{Study characteristics}

\begin{tabular}{ll}
\hline Methods & Design: RCT (information provided for sections that were translated) \\
Who led PC intervention: Multidisciplinary team \\
Core team: Physician/senior nurse/junior nurse \\
\hline
\end{tabular}

Participants

Country and regions: China (foreign language study)

Recruitment: March 2015 to August 2016

Inclusion criteria: Patients who are:

1. Clinically diagnosed to have late-stage lung cancer (stages III and IV)

2. Expected life expectancy $>6$ months

3. Radiation therapy post-operation

4. Informed consent to participate

Exclusion criteria: The exclusion criteria are:

1. Inability to adhere to experiment, disagreeable participants

2. Disabilities that can affect visual-auditory understanding, speech

3. Participated in other experiments within recent one month

4. Major mental illnesses and cognitive deficiencies

5. Infectious diseases or immune disorders

Number of patients: $\mathrm{N}=106$ (53 intervention and 53 control)

Diseases: Advanced lung cancer

Patient characteristics: Mean age (SD): 64.25 (10.41) years in intervention group, 63.34 (10.22) years in control; $42 \%$ female in intervention group, $53 \%$ female in control

Deaths at end of study ( $\mathrm{N}(\%))$ : Unclear as not stated in paper

Withdrawals/other dropouts ( $\mathrm{N}(\%))$ : Unclear as not stated in paper

Interventions

Name: Palliative care based on the knowledge-belief-action model vs control (routine nursing intervention)

Type: Specialist palliative care. Clinical team was trained by expert in model of knowledge-belief-action palliative care

\section{Service base: Hospital}

Team: Clinical team comprised main attending physician, senior nurse and junior nurse who were equipped with relevant educational qualifications, good communication and co-ordinating skills.

Intervention condition: Intervention included three stages:

1. First stage of model (hospitalisation 1 to 3 days) promoted health knowledge, including family support

2. Second stage of model (hospitalisation 4 to 6 days) established healthy beliefs/attitudes

3. Third stage of model (hospitalisation 7 days to discharge) addressed behaviour

All patients in the study group were given a 3-month nursing intervention.

Duration: Three months

Control condition: Control patients received a routine nursing intervention.

Outcomes Outcomes

Cancer-related fatigue assessed using the Piper Fatigue Scale

The effectiveness and cost-effectiveness of hospital-based specialist palliative care for adults with advanced illness and their caregivers 126 (Review)

Copyright $\odot 2020$ The Cochrane Collaboration. Published by John Wiley \& Sons, Ltd. 
Jingfen 2017 (Continued)

Quality of life assessed using the Chinese version of the EORTC QLQ-C30

Nursing satisfaction assessed using hospital self-made survey questionnaire

\begin{tabular}{ll}
\hline Resource use/costs & Not reported \\
\hline Notes & $\begin{array}{l}\text { Funding source: Funded by Chengde Science and Technology Bureau, Science and Technology Re- } \\
\text { search and Development Plan }\end{array}$
\end{tabular}

Power considerations: Authors did not include a power calculation.

\section{Risk of bias}

\begin{tabular}{|c|c|c|}
\hline Bias & Authors' judgement & Support for judgement \\
\hline \multirow[t]{2}{*}{$\begin{array}{l}\text { Random sequence genera- } \\
\text { tion (selection bias) }\end{array}$} & Low risk & $\begin{array}{l}\text { Quote from main publication: "106 patients with advanced lung cancer in our } \\
\text { hospital were selected and divided into the study group and control group ac- } \\
\text { cording to the random number table method". }\end{array}$ \\
\hline & & Comment: probably done \\
\hline $\begin{array}{l}\text { Allocation concealment } \\
\text { (selection bias) }\end{array}$ & Unclear risk & Information not provided in the study \\
\hline $\begin{array}{l}\text { Blinding of participants } \\
\text { and personnel (perfor- } \\
\text { mance bias), subjective } \\
\text { outcomes }\end{array}$ & Unclear risk & Information not provided in the study \\
\hline $\begin{array}{l}\text { Blinding of outcome as- } \\
\text { sessment (detection bias), } \\
\text { subjective outcomes }\end{array}$ & Unclear risk & Information not provided in the study \\
\hline $\begin{array}{l}\text { Blinding of outcome as- } \\
\text { sessment (detection bias), } \\
\text { objective outcomes }\end{array}$ & Unclear risk & Information not provided in the study \\
\hline $\begin{array}{l}\text { Incomplete outcome data } \\
\text { (attrition bias) } \\
\text { All outcomes }\end{array}$ & Unclear risk & Information not provided in the study \\
\hline $\begin{array}{l}\text { Selective reporting (re- } \\
\text { porting bias) }\end{array}$ & Unclear risk & Unclear as protocol not available \\
\hline Other bias & Low risk & Study appeared to be free of other biases. \\
\hline Size of study & Unclear risk & $\begin{array}{l}\text { Unclear risk of bias because the number of participants in each group was be- } \\
\text { tween } 50 \text { and } 199 \text { participants }\end{array}$ \\
\hline
\end{tabular}

Kane 1984

\section{Study characteristics}

Methods Design: RCT

Multicentre RCT

The effectiveness and cost-effectiveness of hospital-based specialist palliative care for adults with advanced illness and their caregivers 
Kane 1984 (Continued)

Who led PC intervention: Multidisciplinary team

Core team: Physicians/nurses/social worker/chaplain/volunteers

Participants Country and regions: USA, West Los Angeles

Recruitment: Recruitment period was not stated. For each hospital admission, the primary physician of each patient on the roster was consulted to ascertain whether the patient's condition (cancer) was terminal. When the physician believed that the patient had a terminal prognosis of two weeks to six months and the patient had been informed of this prognosis, the patient was invited to participate in the study.

Inclusion criteria: These criteria included 1) diagnosis of cancer, 2) terminal prognosis (life expectancy of up to 6 months for the inpatients and 1 year for outpatients), 3) residence within a 30-minute driving range of the Wadsworth Hospital if an outpatient, and 4) sufficient responsiveness to the environment to be judged capable of providing informed consent for study participation and the potential of benefiting from the hospice programme

Exclusion criteria: Not stated

Number of patients: $\mathrm{N}=247$ (137 intervention and 110 control)

Diseases: Lung $(\mathrm{N}=89,36 \%)$, prostate $(\mathrm{N}=26,10.5 \%)$, ear, nose and throat $(\mathrm{N}=25,10.1 \%)$, brain $(\mathrm{N}=$ $18,7.3 \%)$, other $(N=89,36 \%)$

Patient characteristics: Mean age (SD): 63.3 years in intervention group, 64 years in control; $2.2 \%$ female in intervention group, $2.8 \%$ female in control

Number of survivors of patients: $\mathrm{N}=96$ (56 intervention and 40 control)

Survivor characteristics: Mean age (SD): 56 (11) years in intervention group, 58 (13) years in control; $87 \%$ female in intervention group, $77 \%$ female in control

Deaths at end of study (N (\%)): Total N = 230: 128 (93.4\%) intervention and $102(92.7 \%)$ in control group

Withdrawals/other dropouts $(\mathrm{N}(\%))$ : Total $\mathrm{N}=10$. Numbers in intervention and control group not given

Interventions Name: Hospice provided both in a special inpatient unit and at home vs usual care

Type: Specialist palliative care as the intervention was a hospice programme

Service base: The hospice programme studied was based at the Veterans Administration (VA) Medical Center, West Los Angeles, Wadsworth Division, a university-affiliated teaching hospital

Team: The hospice included an 11-bed inpatient unit staffed by 2 physicians, 19 nurses, a social worker, a chaplain, and about 30 volunteers; a homecare programme serving about 25 patients at any given time; and a consultation service for patients awaiting admission to the hospice inpatient unit or needing emergency hospital care when no hospice inpatient beds are available.

Intervention condition: Hospice patients were referred to the hospice programme, which conducted its own assessment and developed a treatment plan.

Duration: Enrollment to death

Control condition: Control patients continued under their current care.

Outcomes

\section{Outcomes}

Pain was measured using the McGill Pain Scale.

The symptom scale was adapted from the California Pain Assessment Profile.

Depression was measured using the Centre for Epidemiologic Studies-Depression Scale (CES-D).

The effectiveness and cost-effectiveness of hospital-based specialist palliative care for adults with advanced illness and their caregivers (Review)

Copyright (C) 2020 The Cochrane Collaboration. Published by John Wiley \& Sons, Ltd. 
Kane 1984 (Continued)

Anxiety was measured using a section of the General Well-Being Measure used in the Rand Health Insurance Study.

Satisfaction with care: interpersonal care measured using the interpersonal care scale adapted from the Ware scale, question on the degree of satisfaction with involvement in care adapted from the National Cancer Institute's Hospice Study and physical environment satisfaction scale adapted from McCaffree and Harkins

Mortality

Assessment point: Randomisation was followed by baseline interviews. Both hospice and control patients and their familial unpaid caregivers were interviewed according to a fixed schedule until the patient's death or until a pre-established number of interviews of each type had been conducted. The intervals between interviews varied with different questionnaires.

Resource use/costs

Total inpatient days: general medical, hospice, ICU and intermediate care

Nursing home days

Number of days at home

Radiation treatments

Chemotherapy treatments

Use of surgical procedures: major and minor surgical procedures

Use of diagnostic procedures

Time horizon: Both hospice and control patients and their family unpaid caregivers were interviewed according to a fixed schedule until the patient's death or until a pre-established number of interviews of each type had been conducted.

Data sources: The number of hospital days the patient spent in the hospice ward, the intermediate care ward, a general medical ward, or an intensive care unit was obtained from the charts of each patient who died. For patients who had inpatient stays in other hospitals, the authors obtained the total number of hospital days, but were often unable to obtain the breakdown of days between intensive care unit and other wards. In the cost study, the authors obtained utilisation data from completed inpatient charts. A total of 144 patients had complete data for all cost elements, but bed-day calculations were made on a larger sample of 230 patients. Completed inpatient charts were abstracted to obtain records of the patient's use of drugs, laboratory, radiology, surgical, and diagnostic procedures, chemotherapy, radiation therapy, nuclear medicine, and consultation services. Each of these was assigned a price. The number of times each drug was administered was summed and multiplied by the unit price of the drug to obtain the total drug costs for a given patient over all hospital stays. The authors were able to obtain prices for drugs, major surgical procedures, pathology, and chemotherapy, as well as the cost of a bed in a general medical or intensive-care unit. However, the VA operated two classes of beds for which there was no ready equivalent at UCLA. Wadsworth VA operated an intermediate-care ward for patients not requiring treatment as intensive as that given in a general medical bed. The authors used the VA cost accounting estimates that an intermediate-care bed costs $70 \%$ as much as a general medical bed. For hospice per diem costs, the authors used two alternate assumptions-(1) equal to intermediate care; and (2) equal to a general medical bed.

Analytical perspective: Not clear

Notes

Funding source: This work was supported by grants from the California chapter of the American Cancer Society and the Henry J. Kaiser Family Foundation and by direct assistance from the VA Medical Center, Wadsworth.

Declaration of interest among primary researchers: Not stated

Power considerations: No sample size calculation

\section{Risk of bias}

The effectiveness and cost-effectiveness of hospital-based specialist palliative care for adults with advanced illness and their caregivers 
Kane 1984 (Continued)

\section{Bias Authors' judgement Support for judgement}

Random sequence genera- Unclear risk tion (selection bias)

Quote from main publication: "after informed consent was received from patients and their FCGs, patients were randomly assigned to receive hospice or conventional care; the sampling proportion was deliberately weighted to favour hospice care".

Comment: random sequence generation was unclear.

Allocation concealment Unclear risk It was not clear whether allocation was concealed.
(selection bias)

\begin{tabular}{|c|c|c|}
\hline $\begin{array}{l}\text { Blinding of participants } \\
\text { and personnel (perfor- } \\
\text { mance bias), subjective } \\
\text { outcomes }\end{array}$ & High risk & $\begin{array}{l}\text { Participants and personnel were not blinded. } \\
\text { Comment: high risk of bias due to lack of blinding }\end{array}$ \\
\hline
\end{tabular}
outcomes

$\begin{array}{ll}\begin{array}{l}\text { Blinding of participants } \\ \text { and personnel (perfor- }\end{array} & \text { Low risk } \\ \begin{array}{l}\text { mance bias), objective } \\ \text { outcomes }\end{array} & \begin{array}{l}\text { Comment: There was no blinding but this was unlikely to lead to bias in objec- } \\ \text { tive outcomes. }\end{array}\end{array}$

\begin{tabular}{|c|c|c|}
\hline $\begin{array}{l}\text { Blinding of outcome as- } \\
\text { sessment (detection bias), } \\
\text { subjective outcomes }\end{array}$ & Unclear risk & $\begin{array}{l}\text { Blinding of outcome assessment was unclear. } \\
\text { Comment: an unclear risk of bias was given because blinding of outcome as- } \\
\text { sessment was unclear. }\end{array}$ \\
\hline
\end{tabular}

\begin{tabular}{ll}
\hline $\begin{array}{l}\text { Blinding of outcome as- } \\
\text { sessment (detection bias), } \\
\text { objective outcomes }\end{array}$ & Low risk
\end{tabular}$\quad \begin{aligned} & \text { Clinding of outcome assessment was unclear. } \\
& \end{aligned}$

\begin{tabular}{ll}
$\begin{array}{l}\text { Incomplete outcome data } \\
\text { (attrition bias) }\end{array}$ & High risk \\
All outcomes & $\mathrm{N}=9(7 \%)$ completers in intervention group vs $\mathrm{N}=9(8.2 \%)$ completers in con- \\
& trol group. No information was provided on how missing data was handled. \\
& Comment: high risk of bias \\
\hline
\end{tabular}

\begin{tabular}{lll}
\hline $\begin{array}{l}\text { Selective reporting (re- } \\
\text { porting bias) }\end{array}$ & Unclear risk & Unclear as protocol not available \\
\hline Other bias & Low risk & Study appeared to be free of other biases. \\
\hline Size of study & Unclear risk & $\begin{array}{l}\text { Unclear risk of bias because the number of participants in each group was be- } \\
\text { tween } 50 \text { and } 199\end{array}$ \\
\hline
\end{tabular}

Lowther 2015

\section{Study characteristics}

\begin{tabular}{ll}
\hline Methods & Design: RCT \\
& Who led PC intervention: Nurse-led intervention \\
& $\begin{array}{l}\text { Core team: Nurses. The intervention nurses had a weekly clinical support session with their clinical } \\
\text { palliative care mentor to review complex cases. }\end{array}$ \\
\hline Participants & Country and regions: Mombasa, Kenya
\end{tabular}

The effectiveness and cost-effectiveness of hospital-based specialist palliative care for adults with advanced illness and their caregivers 
Recruitment: Period of recruitment not stated. The authors screened patients waiting onsite for routine clinic appointments. Each day, they drew a random number to select the first waiting patient to screen, then all consecutive waiting patients were subsequently screened.

Inclusion criteria: Authors enrolled adults taking antiretroviral therapy (ART) for at least 1 month, with a pain or symptom score of 3-5 (the score ranges from 0 [best] to 5 [worst]) and reporting that their pain or symptoms had lasted more than 2 weeks.

Exclusion criteria: Authors excluded patients who had pain or symptoms for fewer than 2 weeks, were receiving ART for prevention of mother-to-child transmission, or did not speak English or Swahili.

Number of patients: $\mathrm{N}=120$ (60 intervention and 60 control)

Diseases: People with HIV on ART

Patient characteristics: Mean age (SD): 38.3 (8.2) years in intervention group, 40.5 (9.2) years in control; $80 \%$ female in intervention group, $82 \%$ female in control

Deaths at end of study ( $\mathrm{N}(\%))$ : Total $\mathrm{N}=3: 3(5 \%)$ intervention and none in control group

Withdrawals/other dropouts ( $\mathrm{N}(\%)):$ Total $\mathrm{N}=3: 3(5 \%)$ intervention and none in control group

Interventions

Name: Nurse-led palliative care intervention vs usual care

Type: Specialist palliative care. Nurses received 2 weeks of full-time palliative care training delivered by Kenyan experts from the Kenyan Hospice and Palliative Care Association.

Service base: Outpatient HIV clinics in a community hospital

Team: Two experienced nurses employed by the HIV clinic

Intervention condition: These nurses used a standardised multidimensional assessment and care planning instrument for all patients allocated to the intervention group to provide holistic patient-centred care. The instrument addressed physical, psychological, social, and spiritual well-being and patients' understanding of their illness and adherence to ART. The instrument also included space to plan and review care against prioritised needs. The intervention nurses had a weekly clinical support session with their clinical palliative care mentor to review complex cases. Patients in the intervention group met the trained nurse immediately after allocation, then at 2 weeks, 4 weeks, and for three subsequent monthly appointments, with a total of six appointments over 4 months.

Duration: 5 months

Control condition: Patients allocated to the control group received usual care from the HIV clinic, consisting of monthly clinical assessments once ART was established, with investigations and treatment for any relevant symptoms or problems. Nurses with no exposure to palliative care provided this service, because no palliative care was available beyond the hospice. Patients in the control group received usual monthly appointments (i.e. five appointments during the study).

\section{Primary outcomes:}

Pain assessed using the pain item of the African Palliative care Outcome Scale (APOS)

\section{Secondary outcomes}

Psychological morbidity assessed using the General Household Questionnaire-12 (GHQ-12)

Palliative care-related problems and concerns assessed using the APOS

Adherence to ART

Quality of life assessed using the Medical Outcomes Study-HIV (MOS-HIV) scale

Assessment points: Baseline data was collected before randomisation; quantitative data was collected once a month for 5 months. 
Lowther 2015 (Continued)

Resource use/costs

Notes
None reported in main publication although it was stated that the Client Services Receipt Inventory was used to record the components of care received by patients.

Funding source: Diana Princess of Wales Memorial Fund

Declaration of interest among primary researchers: The authors declared that they had no competing interests.

Power considerations: The authors calculated that they would need to enroll 60 participants in each group, allowing for $6 \%$ attrition, to detect a 1 point change in the pain score on APOS with $5 \%$ precision and $80 \%$ power.

\section{Risk of bias}

Bias Authors' judgement Support for judgement

Random sequence genera- Unclear risk tion (selection bias)
Quote from publication: "participants were randomly assigned (1:1) by block randomisation (block size 40) to either intervention or control".

Comment: insufficient information about sequence generation process

Allocation concealment Unclear risk
(selection bias)

Quote from publication: "participants selected a folded slip of paper from a box offered by the researcher, which was unfolded by the researcher and the assignment (to control or intervention) recorded".

Comment: "unclear risk" was rated because although the slip of paper was folded, they were not included in sealed envelopes which may have reduced the possibility of foreseeing assignments.

\begin{tabular}{|c|c|c|}
\hline $\begin{array}{l}\text { Blinding of participants } \\
\text { and personnel (perfor- } \\
\text { mance bias), subjective } \\
\text { outcomes }\end{array}$ & High risk & $\begin{array}{l}\text { Quote from publication: "participants and investigators were not masked to } \\
\text { allocation". } \\
\text { Comment: high risk due to lack of blinding }\end{array}$ \\
\hline $\begin{array}{l}\text { Blinding of outcome as- } \\
\text { sessment (detection bias), } \\
\text { subjective outcomes }\end{array}$ & High risk & $\begin{array}{l}\text { Quote from publication: "Participants and investigators were not masked to } \\
\text { allocation". } \\
\text { Comment: high risk of bias due to lack of blinding }\end{array}$ \\
\hline $\begin{array}{l}\text { Incomplete outcome data } \\
\text { (attrition bias) } \\
\text { All outcomes }\end{array}$ & Unclear risk & $\begin{array}{l}\mathrm{N}=54(90 \%) \text { completers in intervention group vs } \mathrm{N}=60(100 \%) \text { completers in } \\
\text { control group. } 6 \text { patients in intervention group were excluded from the analy- } \\
\text { sis because they died (3), migrated (2) or withdrew (1) due to time constraints. }\end{array}$ \\
\hline $\begin{array}{l}\text { Selective reporting (re- } \\
\text { porting bias) }\end{array}$ & High risk & Data collected using the Client Services Receipt Inventory was not reported. \\
\hline Other bias & Unclear risk & Study appeared to be free of other biases. \\
\hline Size of study & Unclear risk & $\begin{array}{l}\text { Unclear risk because the number of participants in each group was between } 50 \\
\text { and } 199\end{array}$ \\
\hline
\end{tabular}

Ma 2019

\section{Study characteristics}

Methods Design: Cluster-randomised crossover trial

The effectiveness and cost-effectiveness of hospital-based specialist palliative care for adults with advanced illness and their caregivers 
Ma 2019 (Continued)

Who led PC intervention: Multidisciplinary team

Core team: A physician board-certified in palliative care, nurse practitioners, a palliative care clinical fellow, a social worker, and a chaplain.

\section{Participants}

\section{Country and regions: USA}

Recruitment: August 2017 to May 2018. A member of the research team who was independent of the ICU and palliative care teams screened the electronic medical records of MICU admissions within the previous 24 hours. Up to the first two consecutively screened patients meeting eligibility criteria were enrolled per MICU each weekday in both the intervention and control arms.

Inclusion criteria: Patients who met any of the following criteria: admitted from long-term skilled nursing facility or acute care facility, or admitted from home with activities of daily living dependencies requiring skilled nursing; end-stage neurologic condition; advanced or metastatic cancer; arrest with neurologic compromise; multiple organ system failure; end-stage organ disease; shock; acute respiratory failure and prolonged length of stay or ICU readmission.

Exclusion criteria: History of any stem cell transplant; solid organ transplant within $1 \mathrm{yr}$ of transplant, or actively undergoing workup for solid organ transplant; non-English speaking patients for whom an interpreter was unavailable; patients without capacity to participate in palliative care $(P C)$ discussions with no identifiable surrogate; patients who had received a PC consultation earlier during the same hospitalisation; patients already determined to be do-not-resuscitate/do-not-intubate (DNR/DNI).

Number of patients: $\mathrm{N}=199$ (97 intervention and 102 control)

Diseases: Neurologic, CHF, coronary artery or peripheral vascular disease, end-stage renal disease, solid organ transplant, cancer, HIV, COPD, cirrhosis

Patient characteristics: Mean age (SD): 66 (14) years in intervention group, 62 (12) years in control; $51 \%$ female in intervention group, $45 \%$ female in control

Deaths at end of study (N (\%)): Total N = 71: $34(35.1 \%)$ intervention and $37(36.3 \%)$ in control group

Withdrawals/other dropouts (N (\%)): Not reported

Early PC: Early palliative care consultation within 48 hours of medical intensive care unit (MICU) admission

Type: Specialist palliative care. Included a physician board-certified in palliative care and a palliative care clinical fellow

Service base: Barnes-Jewish hospital

Team: A physician board-certified in palliative care, nurse practitioners, a palliative care clinical fellow, a social worker, and a chaplain

Intervention condition: Intervention arm patients received a palliative care consultation within 48 hours of MICU admission. A palliative care consultation included: chart review of the patient's hospitalisation, meeting with the patient and available healthcare proxies, identification of physical and emotional needs of the patient and family, discussion with the primary team on how best to meet those needs, and communication between all parties with respect to goals, values, and treatment decisions. Formal meetings including the palliative care team, primary team, and the patient or healthcare proxies were encouraged but not mandatory. A care plan for each consultation was discussed by the entire palliative care team at rounds, with additional team members participating as appropriate. The palliative care team continued to follow the patient until discharge from the hospital.

Duration: Hospitalisation to discharge

Control condition: The control arm received standard of care: palliative care could be consulted at the discretion of the MICU clinicians. 
Ma 2019 (Continued)

Outcomes

\section{Primary outcomes:}

Proportion of patients who transitioned to DNR/DNI resuscitation preference before hospital discharge

\section{Secondary outcomes}

MICU length of stay

Hospital length of stay

Discharge to hospice

Duration of mechanical ventilation

Duration of vasopressors

Tracheostomy

Cardiopulmonary resuscitation (CPR)

Mortality

Post-discharge emergency department visits

Hospital readmissions.

Assessment points: During hospitalisation and also post-discharge

$\begin{array}{ll}\text { Resource use/costs } & \text { MICU length of stay } \\ \text { Hospital length of stay } & \text { Duration of mechanical ventilation } \\ \text { Duration of vasopressors } & \text { Tracheostomy } \\ \text { Cardiopulmonary resuscitation (CPR) } & \text { Post-discharge emergency department visits } \\ \text { Hospital readmissions } \\ \text { Cost }\end{array}$

Time Horizon: During hospitalisation and also post-discharge

Funding source: The study was supported by The Foundation for Barnes-Jewish Hospital and Washington University Institute of Clinical and Translational Sciences (ICTS).

Declaration of interest among primary researchers: Drs. Chi, Buettner, Al-Hammadi, and Kollef received support for article research from the NIH. Dr. Buettner's institution received funding from ICTS Just In Time award. Drs. Buettner's and Dans's institutions received funding from Barnes-Jewish Hospital Foundation. Dr. Chen disclosed work for hire. Dr. Kollef's effort was supported by the Barnes-Jewish Hospital Foundation. Dr. Dans' institution also received funding from ICTS at Washington University School of Medicine and Barnes Jewish Hospital Foundation, and she received funding from National Comprehensive Cancer Network (NCCN). The remaining authors disclosed that they do not have any potential conflicts of interest.

Power considerations: Based on preliminary data from the first 60 days of the study, a generalised estimating equation model was used to determine that 96 patients would be required per arm to detect a threefold increase (54\% vs $18 \%$ ) in the primary outcome with $80 \%$ power and a type 1 error of $5 \%$.

The effectiveness and cost-effectiveness of hospital-based specialist palliative care for adults with advanced illness and their caregivers 
Ma 2019 (Continued)

Risk of bias

\begin{tabular}{lll}
\hline Bias & Authors' judgement & Support for judgement \\
\hline $\begin{array}{l}\text { Random sequence genera- } \\
\text { tion (selection bias) }\end{array}$ & Unclear risk & $\begin{array}{l}\text { Quote from main publication: "the two medical ICUs (MICUs) of Barnes-Jewish } \\
\text { Hospital (1,250 beds) comprised of } 16 \text { and } 18 \text { beds respectively were randomly } \\
\text { assigned to intervention or usual care. A washout period of } 6 \text { weeks occurred } \\
\text { halfway through the study during which enrollment was halted and new ad- } \\
\text { missions received usual care, followed by crossover of the MICUs to interven- } \\
\text { tion or usual care". } \\
\text { Comment: random sequence generation was unclear. }\end{array}$ \\
\end{tabular}

Allocation concealment Low risk

(selection bias)

Quote from main publication: "the two medical ICUs (MICUs) of Barnes-Jewish Hospital (1,250 beds) comprised of 16 and 18 beds respectively were randomly assigned to intervention or usual care. A washout period of 6 weeks occurred halfway through the study during which enrollment was halted and new admissions received usual care, followed by crossover of the MICUs to intervention or usual care... The Washington University School of Medicine Human Studies Committee approved this investigation and waived the need for informed consent".

Comment: given that the need for informed consent was waived, it appeared treatment assignment was concealed from participants thereby reducing selection bias.

$\begin{array}{ll}\begin{array}{l}\text { Blinding of participants } \\ \text { and personnel (perfor- }\end{array} & \text { Low risk }\end{array}$
mance bias), objective outcomes

Blinding of outcome as- Low risk sessment (detection bias), objective outcomes
Quote from main publication: "two trained research study team members independently collected process and outcome data for each patient's hospitalisation, and a third team member reconciled any discrepancies. Physicians and study team members were not blinded because of inherent difficulties with blinding of the palliative care intervention."

Comment: lack of blinding was unlikely to lead to bias in objective outcomes.

\begin{tabular}{lll}
\hline $\begin{array}{l}\text { Incomplete outcome data } \\
\text { (attrition bias) } \\
\text { All outcomes }\end{array}$ & High risk & $\begin{array}{l}\mathrm{N}=63(64.9 \%) \text { completers in intervention group vs N=65 (63.7\%) completers } \\
\text { in control group. Some patients were not included in the analysis of the out- } \\
\text { comes and it was unclear how the authors dealt with missing data. }\end{array}$ \\
\hline $\begin{array}{l}\text { Selective reporting (re- } \\
\text { porting bias) }\end{array}$ & High risk & $\begin{array}{l}\text { One of the secondary outcomes (antibiotic usage and duration) specified in } \\
\text { the trial registry was not reported. }\end{array}$ \\
\hline Other bias & Low risk & Study appeared to be free of other biases. \\
\hline Size of study & Unclear risk & $\begin{array}{l}\text { Unclear risk because the number of participants in each group was between 50 } \\
\text { and } 199\end{array}$ \\
\hline
\end{tabular}

McCaffrey 2013

\section{Study characteristics}

Methods Design: RCT

The effectiveness and cost-effectiveness of hospital-based specialist palliative care for adults with advanced illness and their caregivers 
McCaffrey 2013 (Continued)

Pilot phase II RCT

Who led PC intervention: Nurse-led intervention (services provided up to $24 \mathrm{~h} /$ day for up to 5 days)

Core team: Nursing services (utilising agency nursing staff). It also co-ordinates allied health input (occupational therapy, physiotherapy, pastoral care) if equipment or other services are needed.

Participants

Country and regions: Australia, Southwest Sydney, New South Wales

Recruitment: Recruitment sites were an inpatient unit and the community.

Inclusion criteria: Patients were eligible if they had complex or unstable symptom management and high care needs.

Exclusion criteria: Not stated

Number of patients: $\mathrm{N}=31$ (23 intervention and 8 control)

Diseases: Predominantly cancer $(\mathrm{N}=25,80.7 \%)$, non-cancer $(\mathrm{N}=3,9.7 \%)$ and not reported $(\mathrm{N}=3$, 9.7\%)

Patient characteristics: Mean age (SD): 62.8 (14.2) in intervention group, 66 (20.8) in control group; $39.1 \%$ female in intervention group, $50 \%$ female in control group

Deaths at end of study ( $\mathrm{N}(\%)): \mathrm{N}=68 \%$; mean $(95 \% \mathrm{Cl}): 69.6 \%(52.2 \%, 87 \%)$ intervention and $62.5 \%$ $(25 \%, 100 \%)$ control

Withdrawals/other dropouts (N (\%)): None

Name: Home-based palliative care vs usual care

Type: Training in palliative care unclear

Service base: Hospital

Team: Includes nursing services (utilising agency nursing staff). It also co-ordinated allied health input (occupational therapy, physiotherapy, pastoral care) if equipment or other services are needed.

Intervention condition: PEACH was an individualised care package determined by local protocols for community and inpatients. Services were rapidly mobilised, essential equipment was secured, allied health was co-ordinated and higher intensity nursing was provided (up to $24 \mathrm{~h}$ /day for up to 5 days) compared with usual care.

Duration: 28 days

Control condition: Usual care encompassed conventional discharge planning with existing community services including specialist palliative care, access to an after-hours number, and equipment from loan pools.

\section{Primary outcome:}

Number of days out of institutional care (determined from medical records of admissions to hospital and patient/ unpaid caregiver report of date of admission to residential care)

\section{Secondary outcomes:}

Place of death

Days at home

Assessment points: 28 days

Resource use/costs Number of days at home


McCaffrey 2013 (Continued)

PEACH intervention costs (staff administration, travel and direct patient contact time, overheads and consumables)

Cost of specialist palliative care service use

Cost of acute hospital and palliative care unit inpatient lengths of stay and outpatient visits

Time horizon: from study enrollment to 28 days

Data sources: Patient-level data were collected prospectively, including: days at home; place of death; PEACH intervention costs (staff administration, travel and direct patient contact time, overheads and consumables); specialist palliative care service use; acute hospital and palliative care unit inpatient lengths of stay and outpatient visits. Resource use was costed according to the Australian Manual of Resource Items and their Associated Costs, and inpatient stays as recommended by the Australian Medical Services Advisory Committee guidelines. Specialist palliative care services and PEACH costs were estimated using hourly rates of local salaries (plus $30 \%$ on-costs), agency staff costs and equipment hire. PEACH administrative costs were included. Outpatient visits were costed using the National Hospital Cost Data Collection.

Analytical perspective: Healthcare provider perspective

Notes

Funding source: This study was funded by the Australian Government Department of Health and Ageing under the National Palliative Care Program, Palliative Care for People at Home. NM was also funded through the National Palliative Care Program and Flinders University.

Declarations of interest among primary researchers: The authors declared no competing interests.

Power considerations: None reported

\section{Risk of bias}

Bias Authors' judgement Support for judgement

Random sequence genera- Unclear risk tion (selection bias)
Quote from main publication: "participants were randomised to receive $\mathrm{PEACH}$ or usual care in a 3:1 ratio, increasing experience with $\mathrm{PEACH}$ and aiding recruitment and ethics approval".

Comment: there was insufficient information about the random sequence generation process.

\begin{tabular}{lll}
\hline $\begin{array}{l}\text { Allocation concealment } \\
\text { (selection bias) }\end{array}$ & Unclear risk & Allocation concealment was not described. \\
\hline $\begin{array}{l}\text { Blinding of participants } \\
\begin{array}{l}\text { and personnel (perfor- } \\
\text { mance bias), objective }\end{array}\end{array}$ & Low risk & Quote from trial registry: "masking not used" \\
outcomes & $\begin{array}{l}\text { Comment: masking was not carried out but this was unlikely to lead to bias in } \\
\text { objective outcomes. }\end{array}$
\end{tabular}

$\begin{array}{ll}\begin{array}{l}\text { Blinding of outcome as- } \\ \text { sessment (detection bias), } \\ \text { objective outcomes }\end{array} & \text { Low risk }\end{array} \quad \begin{aligned} & \text { Quote from trial registry: "masking not used" } \\ & \text { Comment: masking was not carried out but this was unlikely to lead to bias in } \\ & \text { assessment of objective outcomes. }\end{aligned}$

\begin{tabular}{|c|c|c|}
\hline $\begin{array}{l}\text { Incomplete outcome data } \\
\text { (attrition bias) } \\
\text { All outcomes }\end{array}$ & Low risk & $\begin{array}{l}\mathrm{N}=7(30.4 \%) \text { completers in intervention group vs } \mathrm{N}=3(37.5 \%) \text { completers in } \\
\text { control group. In the intervention group, } 16(69.6 \%) \text { patients died during the } \\
\text { 28-day follow-up while } 5(62.5 \%) \text { died in control group. Complete data were } \\
\text { available and used in cost-effectiveness analysis. }\end{array}$ \\
\hline
\end{tabular}

Selective reporting (re- High risk porting bias)
Some secondary outcomes in the protocol such as change in symptom score, functional status, unpaid caregiver QoL and modified family inventory of 
McCaffrey 2013 (Continued)

needs score were not reported in the main publication. Study was retrospectively registered.

Other bias Unclear risk

There were differences in age, gender, marital status, recruitment site and the percentage of patients with different diagnosis between the intervention and control group at baseline. The authors did not state if these differences were statistically significant and whether they controlled for them.

$\begin{array}{ll}\text { Size of study } \quad \text { High risk } & \begin{array}{l}\text { High risk of bias because the number of participants in each group was less } \\ \text { than } 50\end{array}\end{array}$

McCorkle 2015

\section{Study characteristics}

Design: Cluster-RCT
Who led PC intervention: Multidisciplinary team
Core team: Advanced practice nurse (APNs)/physician assistants (PAs)/medical social workers (MSWs)/
nurse co-ordinators/medical oncologists/surgeons/radiation oncologists worked as a palliative care
unit with the study APN overseeing the co-ordination and implementation of the intervention by differ-
ent members of the team.

Participants Country and regions: USA, Connecticut

Recruitment: August 2010 and December 2012. Eligible patients were identified at weekly tumour boards. Patients' oncologist asked patients if they were interested in the study. Research staff met with interested patients to explain the study, obtain consent, and administer baseline questionnaires.

Inclusion criteria: Eligible patients qualified based on (1) a late-stage cancer diagnosis within 100 days; (2) post-biopsy or surgery with additional treatment recommended; (3) at least one self-reported chronic condition; and (4) age $\geq 21$ years.

Exclusion criteria: Not stated

Number of patients: $\mathrm{N}=146$ (66 intervention and 80 control)

Diseases: Gynaecologic (29), lung (37), gastrointestinal (53), and head and neck cancers (27)

Patient characteristics: Mean age (range): 34 (51.5\%) were < 65 years and 32 (48.5\%) were 65 years and older in intervention group, $57(71.3 \%)$ were $<65$ years and $23(28.7 \%)$ were 65 years and older in control group; $71.2 \%$ female in intervention group, $43.7 \%$ female in control group.

Deaths at end of study $(\mathrm{N}(\%)): \mathrm{N}=10: 7(10.6 \%)$ intervention and $3(3.75 \%)$ control

Withdrawals/other dropouts ( $(\%)): N=44: 23(34.8 \%)$ intervention and $21(26.3 \%)$ control

Name: An advanced practice nurse (APN) co-ordinated multidisciplinary intervention vs enhanced usual care (usual multidisciplinary care plus a copy of the symptom management toolkit with instructions on its use).

Early PC: Eligible patients were those with a late-stage cancer diagnosis within 100 days.

Type: Specialist palliative care. Advanced practice nurses (APNs), physician assistants (PAs), and medical social workers (MSWs) in the two disease-specific clinics participated in three one-hour, one-onone training sessions with the study APN co-ordinator. Training included review of evidence-based symptom protocols, documentation requirements, guidelines on handling adverse events and communication strategies to enhance patient problem-solving, decision-making, and self-efficacy. 
Service base: Four disease-specific multidisciplinary clinics (gynaecologic, lung, gastrointestinal, and head and neck clinics) at Smilow Cancer Hospital ( $\mathrm{SCH}$ ) at Yale-New Haven, Connecticut

Team: Members of each disease-specific multidisciplinary team (APNs, PAs, MSWs, nurse co-ordinators, medical oncologists, surgeons, and radiation oncologists) worked as a palliative care unit with the study APN overseeing the co-ordination and implementation of the intervention by different members of the team.

Intervention condition: The essential components of the intervention included monitoring patients' status, providing symptom management, executing complex care procedures, teaching patients and family unpaid caregivers, clarifying the illness experience, co-ordinating care, responding to the family, enhancing QoL, and collaborating with other providers. In addition, goals of care were discussed. The study APN trained the clinic staff (APNs, PAs, MSWs) in the lung and gynaecological clinics prior to the recruitment of patients. Members of each disease-specific multidisciplinary team worked together as a palliative care unit, each member taking on different functions to ensure all components of the intervention were addressed. The clinic APN oversaw the co-ordination and implementation of the intervention by different members of the team. Intervention fidelity was assessed and monitored by the study APN co-ordinator.

Duration: 10 weeks

Control condition: Patients in this group did not receive the APN-co-ordinated intervention but continued to receive routine oncology care delivered by multidisciplinary members of the head and neck and gastrointestinal disease-specific clinics. Both groups received a copy of the Symptom Management Toolkit, a resource manual describing 28 common symptoms and problems associated with cancer treatment and were instructed on its use.

\section{Outcomes}

\section{Primary outcome}

Symptom distress assessed using the Symptom Distress Scale (SDS)

Health distress assessed using a four-item scale developed by the Stanford Patient Education Research Center

Depression assessed using Personal Health Questionnaire (PHQ-9)

Functional status assessed using the Enforced Social Dependency Scale (ESDS)

Self-reported health assessed using the first item of the Short Form - 12 (SF-12)

\section{Secondary outcomes}

Quality of life assessed using the Functional Assessment of Cancer Therapy-General (FACT-G)

Anxiety assessed using the Hospital Anxiety and Depression Scale (HADS)

Uncertainty assessed using the Mishel Uncertainty in Illness Scale - Community Form (MUIS-C)

Self-efficacy assessed using the Self-Efficacy for Managing Chronic Disease Scale (SEMCD-6)

Assessment points: Standardised scales were used to collect five primary patient outcomes at all three data collection points (baseline - after randomisation, one and three months). The four secondary outcomes were collected at one and three months post-baseline.

\begin{tabular}{ll}
\hline Resource use/costs & None reported \\
\hline Notes & Funding source: Funded by NIH/NINR grant R01NR011872 \\
& Declarations of interest among primary researchers: Apart from funding, no further study author \\
& disclosure statements were made.
\end{tabular}

disclosure statements were made.

Power considerations: Not reported 
McCorkle 2015 (Continued)

Risk of bias

\begin{tabular}{|c|c|c|}
\hline Bias & Authors' judgement & Support for judgement \\
\hline $\begin{array}{l}\text { Random sequence genera- } \\
\text { tion (selection bias) }\end{array}$ & Low risk & $\begin{array}{l}\text { Quote from main publication: "a cluster randomisation procedure was used } \\
\text { to randomise four disease-specific clinics at Smilow Cancer Hospital (SCH) at } \\
\text { Yale-New Haven into two groups: an intervention group (an APN-co-ordinated } \\
\text { multidisciplinary intervention) and an enhanced usual care group (usual multi- } \\
\text { disciplinary care plus a copy of the symptom management toolkit with instruc- } \\
\text { tions on its use). Due to the ongoing interactions of the team members to dis- } \\
\text { cuss and share patients' treatment plans and management strategies in the } \\
\text { disease-specific clinics, it was important to randomise the clinics and not the } \\
\text { patients... Randomisation was done using the ranuni function in conjunction } \\
\text { with the rank procedure in statistical software SAS (SAS version } 9.2 \text { for Win- } \\
\text { dows; SAS Institute Inc., Cary, NC)". }\end{array}$ \\
\hline & & Comment: probably done \\
\hline
\end{tabular}

Allocation concealment $\quad$ Unclear risk
(selection bias)

Comment: unclear

\begin{tabular}{|c|c|c|}
\hline $\begin{array}{l}\text { Blinding of participants } \\
\text { and personnel (perfor- } \\
\text { mance bias), subjective }\end{array}$ & High risk & $\begin{array}{l}\text { Participants and personnel were not blinded. } \\
\text { Comment: high risk of bias due to lack of blinding }\end{array}$ \\
\hline
\end{tabular}

outcomes

\begin{tabular}{|c|c|c|}
\hline $\begin{array}{l}\text { Blinding of outcome as- } \\
\text { sessment (detection bias), } \\
\text { subjective outcomes }\end{array}$ & Unclear risk & $\begin{array}{l}\text { Unclear because no mention was made of this } \\
\text { Comment: unclear risk of bias because blinding of outcome assessment was } \\
\text { unclear }\end{array}$ \\
\hline
\end{tabular}

Incomplete outcome data High risk N =36 (54.5\%) completers in intervention group vs N=56 (70\%) completers in
(attrition bias)

All outcomes control group. Missing data were not balanced in numbers across intervention and control group. There were high proportions of loss-to-follow-up and these were excluded from the analysis. In the intervention group, patients were lostto-follow-up because 22 (33.3\%) were treated at other facilities, 7 (10.6\%) died and $1(1.5 \%)$ withdrew. In the control group, patients were lost-to-follow-up because $20(25 \%)$ were treated at other facilities, $3(3.8 \%)$ died and $1(1.3 \%)$ withdrew.

$\begin{array}{ll}\text { Selective reporting (re- } \quad \text { High risk } & \text { Uncertainty assessed using the Mishel Uncertainty in Illness Scale - Commu- } \\ \text { porting bias) } & \text { nity Form (MUIS-C) was specified as the primary outcome on the clinical trial } \\ & \text { registry, clinicaltrials.gov (NCTO1272024). However, this was reported as a sec- } \\ & \text { ondary outcome in the main publication. Other outcomes that were not speci- } \\ & \text { fied on the trials registry were also reported. }\end{array}$

\begin{tabular}{|c|c|c|}
\hline \multirow[t]{2}{*}{ Other bias } & Unclear risk & $\begin{array}{l}\text { The authors reported that "patient characteristics in both groups were simi- } \\
\text { lar, except patients in the intervention group were older, more likely to be fe- } \\
\text { male, had more chronic conditions, and were diagnosed with later-stage can- } \\
\text { cers". The authors did not state whether they controlled for these variables in } \\
\text { the analysis. }\end{array}$ \\
\hline & & Comment: unclear risk of bias \\
\hline Size of study & Unclear risk & $\begin{array}{l}\text { Unclear risk of bias because the number of participants in each group was be- } \\
\text { tween } 50 \text { and } 199\end{array}$ \\
\hline
\end{tabular}




\section{Study characteristics}

\begin{tabular}{ll}
\hline Methods & Design: RCT (patient and unpaid caregiver) \\
Fast-track RCT \\
$\begin{array}{l}\text { Who led PC intervention: Multidisciplinary team (one of the team nurses, with physician back-up, was } \\
\text { available } 24 \text { hours a day) }\end{array}$
\end{tabular}
available 24 hours a day)

Core team: Palliative care nurses (working one week on, one off)/physician/part-time social worker

\section{Participants}

Country and regions: Canada, London, Ontario, metropolitan area

Recruitment: (date and length not stated). From family physicians and home care $(\mathrm{HC})$ nurses (with "strenuous efforts to attract referrals, including an information sheet for family doctors and presentations to medical meetings" resulting in short lived increases)

Inclusion criteria: Aged $\geq 18$ years; being cared for at home by an eligible unpaid caregiver; having symptomatic cancer which had metastasised or spread to surrounding tissues; and expected to survive for two months.

Exclusion criteria: Not explicitly stated

Number of patients (randomised): 146

Diseases: Cancer (146)

Number of unpaid caregivers who completed questionnaires: 74

Patient and unpaid caregivercharacteristics: Not stated

Deaths at end of study: $\mathrm{N}=36$ (numbers in the intervention and control groups not stated)

Withdrawals/other dropouts: 14 patients (10\%) failed to complete the one-month questionnaire and in 3 patients, the reasons for dropout were not stated.

Type: Specialist palliative care. The palliative care home support team consisted of two experienced palliative care nurses (working one week on, one off), one physician, and a part-time social worker.

Service base: The palliative care home support team was based on a 14-bed palliative care unit.

Team: Two experienced palliative care nurses, one physician, and a part-time social worker

Intervention condition: The team was planned to be a consulting and support service for family physicians and home care nurses. Within three days of referral by a family doctor or nurse, one of the team nurses carried out a full assessment in the home. The nurse's assessment and recommendations were discussed with the team doctor, then sent to the family doctor with copies to the visiting nurse and home care case manager. A consultation by the team doctor was available on request. All new and active cases were discussed at the weekly team meeting. The involvement of the team after the initial assessment depended on the wishes of the patient and family and on negotiation with the family physician and home care nurse. One of the team nurses, with physician back-up, was available 24 hours a day, and patients were given a number to call if their home care nurse or family doctor could not be reached.

\section{Duration: Not stated}

Control condition: Control patients'waiting list' group waited four weeks for assessment by the team. Emergency consultation by the team physician was available for patients in the waiting list group if requested by the family physician. 
McWhinney 1994 (Continued)

Outcomes

\section{Primary outcomes}

Pain assessed using the McGill Pain Questionnaire

Nausea assessed using the Melzack Nausea Questionnaire

\section{Secondary outcomes}

Patient's quality of life (scale not stated)

unpaid caregiver's health (scale not stated)

Assessment points: After randomisation, a research assistant visited the home to provide more details, obtain written consent and explain the study questionnaires and then collect them after three days. Data collection was repeated at one and two months, one month being the main comparison point.

\section{Risk of bias}

\begin{tabular}{lll}
\hline Bias & Authors' judgement & Support for judgement \\
\hline $\begin{array}{ll}\text { Random sequence genera- } \\
\text { tion (selection bias) }\end{array}$ & Low risk & $\begin{array}{l}\text { Quote from main publication: "the project coordinator assessed eligibility and } \\
\text { conducted randomisation using a computer generated table of random num- } \\
\text { bers". } \\
\text { Comment: probably done }\end{array}$ \\
& &
\end{tabular}

\begin{tabular}{ll}
\hline $\begin{array}{l}\text { Allocation concealment } \\
\text { (selection bias) }\end{array}$ & Low risk \\
& $\begin{array}{l}\text { Quote from main publication: "the project coordinator assessed eligibility and } \\
\text { conducted randomisation using a computer generated table of random num- } \\
\text { bers". } \\
\text { Comment: probably done }\end{array}$ \\
\hline
\end{tabular}

\begin{tabular}{|c|c|c|}
\hline $\begin{array}{l}\text { Blinding of participants } \\
\text { and personnel (perfor- } \\
\text { mance bias), subjective }\end{array}$ & High risk & $\begin{array}{l}\text { Patients and unpaid caregivers were not blinded. } \\
\text { Comment: high risk of bias due to lack of blinding }\end{array}$ \\
\hline
\end{tabular}

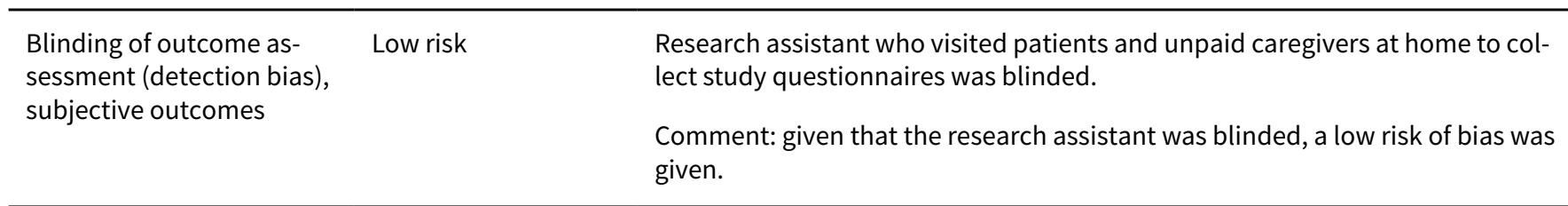

Incomplete outcome data High risk (attrition bias)

All outcomes
Of the 146 patients who were randomised in this study, 53 (36.3\%) were lost to follow-up before one month. $36(24.7 \%)$ died, 14 (9.6\%) failed to complete the one month questionnaires and $3(2.1 \%)$ were unaccounted for by the authors. There were high proportions of loss-to-follow-up.

The effectiveness and cost-effectiveness of hospital-based specialist palliative care for adults with advanced illness and their caregivers (Review) 
McWhinney 1994 (Continued)
Selective reporting (re-
Unclear risk
Unclear as there was no published protocol porting bias)

\begin{tabular}{lll}
\hline Other bias & Unclear risk & $\begin{array}{l}\text { An unclear risk of bias was rated because the sample characteristics at base- } \\
\text { line was not reported. }\end{array}$ \\
\hline Size of study & Unclear risk & $\begin{array}{l}\text { Unclear risk of bias because the number of participants in each group was not } \\
\text { stated. A total of } 146 \text { patients and } 74 \text { unpaid caregivers took part in the trial. }\end{array}$
\end{tabular}

Mendoza-Galindo 2018 (abstract only)

\title{
Study characteristics
}

\begin{tabular}{ll}
\hline Methods & Design: RCT \\
Who led PC intervention: Unclear \\
Core team: Professionals in palliative team were not described. \\
\hline
\end{tabular}

\section{Participants}

\section{Country and regions: Mexico}

Recruitment: Not stated

Inclusion criteria: Newly diagnosed or relapsed metastatic breast cancer patients were included.

Exclusion criteria: Not stated

Number of patients: $\mathrm{N}=53$ (33 intervention and 20 control).

Diseases: Breast cancer

Patient characteristics: Not stated

Deaths at end of study (N (\%)): Not stated

Withdrawals/other dropouts (N (\%)): Not stated

Interventions

Name: Early palliative care vs standard care

Early PC: Not described

Type: Training unclear

Service base: Hospital, Instituto Nacional de Cancerologia in Mexico

Team: Palliative team. However, professionals in palliative team were not described.

Intervention condition: Intervention was provided by a palliative team, which included psychological, nutritional and symptom support.

Duration: Not clear

Control condition: Standard care was given by the attending physician.

\section{Outcomes}

\author{
Outcomes \\ Number of emergency room consultations \\ Number of hospitalisation \\ Hospitalisation length
}


Mendoza-Galindo 2018 (abstract only) (Continued)

Assessment points: Not stated

\begin{tabular}{ll} 
Resource use/costs & $\begin{array}{l}\text { Cost of emergency room consultations } \\
\text { Cost of hospitalisation days } \\
\text { Time Horizon: Unclear }\end{array}$ \\
\hline Notes & $\begin{array}{l}\text { Funding source: Not stated } \\
\text { Declarations of interest among primary researchers: Not stated } \\
\text { Power considerations: Not stated }\end{array}$
\end{tabular}

\section{Risk of bias}

\begin{tabular}{lll}
\hline Bias & Authors' judgement & Support for judgement \\
\hline $\begin{array}{l}\text { Random sequence genera- } \\
\text { tion (selection bias) }\end{array}$ & Unclear risk & $\begin{array}{l}\text { Quote from abstract: "patients were randomized to standard care given by the } \\
\text { attending physician (control arm) or intervention by palliative team". } \\
\text { Comment: unclear risk of bias due to insufficient information in the abstract }\end{array}$ \\
\hline $\begin{array}{l}\text { Allocation concealment } \\
\text { (selection bias) }\end{array}$ & Unclear risk & Comment: unclear risk of bias due to insufficient information in the abstract \\
\hline $\begin{array}{l}\text { Blinding of participants } \\
\text { and personnel (perfor- } \\
\text { mance bias), subjective } \\
\text { outcomes }\end{array}$ & Unclear risk & Comment: unclear risk of bias due to insufficient information in the abstract \\
\hline
\end{tabular}

Blinding of participants Unclear risk Comment: unclear risk of bias due to insufficient information in the abstract
and personnel (perfor-
mance bias), objective
outcomes

Blinding of outcome as- Unclear risk Comment: unclear risk of bias due to insufficient information in the abstract
sessment (detection bias),
subjective outcomes

Blinding of outcome as-
sessment (detection bias),
objective outcomes

\begin{tabular}{lll}
\hline $\begin{array}{l}\text { Incomplete outcome data } \\
\text { (attrition bias) } \\
\text { All outcomes }\end{array}$ & Unclear risk & Comment: unclear risk of bias due to insufficient information in the abstract \\
\hline $\begin{array}{l}\text { Selective reporting (re- } \\
\text { porting bias) }\end{array}$ & Unclear risk & Comment: unclear risk of bias due to insufficient information in the abstract \\
\hline Other bias & Unclear risk & Comment: unclear risk of bias due to insufficient information in the abstract \\
\hline Size of study & High risk & $\begin{array}{l}\text { High risk of bias because the number of participants in each group was less } \\
\text { than } 50\end{array}$ \\
\hline
\end{tabular}


Nottelmann 2018

\section{Study characteristics}

Design: RCT
Who led PC intervention: Multidisciplinary team
Core team: The specialised palliative care team comprised physicians, nurses, physiotherapists, psy-
chologists, a part-time social worker, dietician, occupational therapist, and chaplain.

Participants

Country and regions: Denmark, Vejle

Recruitment: Eligible patients were informed about the project by a doctor or nurse in the outpatient clinic.

Inclusion criteria: First-time non-resectable cancer diagnosed less than 8 weeks before enrollment. Patients with prostatic cancer were eligible, if referred to systemic oncologic treatment for the first time less than 8 weeks before enrollment (e.g. due to failure of anti-hormone treatment). Patients eligible for systemic oncologic treatment at Vejle Hospital and accepted treatment. Patients aged $\geq 18$ years of age with the ability to read and understand Danish. Patients who were able to provide written and oral informed consent.

Exclusion criteria: Other contact with a specialised palliative care unit within 1 year of enrollment and inability to comply with the protocol due to cognitive or other impairment

Number of patients: $\mathrm{N}=281$ (132 intervention and 149 control).

Diseases: Cancer

Patient characteristics: Mean age (SD): 66 (9) years in intervention group. \% female: $42 \%$ female in intervention group. Data not provided for control group.

Deaths at end of study ( $(\%)): \mathrm{N}=4(3 \%)$ in the intervention group. Data not provided for control group

Withdrawals/other dropouts $(\mathrm{N}(\%)): \mathrm{N}=6(4.5 \%)$ in the intervention group. Data not provided for control group

Interventions

Name: Palliative rehabilitation alongside standard oncology treatment vs standard treatment alone

Early PC: Patients were diagnosed with non-resectable solid cancer within the last 8 weeks

Type: Specialist palliative care. Physician and nurse were specialised in palliative care. The specialised palliative care (SPC) team had 15 years of experience in treating patients with life-threatening illnesses and their unpaid caregivers, predominately as home-based specialised palliative care and in the late phases of the disease. The SPC team received a 2-day visit from researchers and palliative rehabilitation clinicians from the British and Irish Health Systems.

Service base: Department of Oncology, Vejle Hospital

Team: Specialised palliative care team consisting of physicians, nurses, physiotherapists, psychologists, a part-time social worker, dietician, occupational therapist and chaplain.

Intervention condition: The intervention consisted of a 'basic offer' and tailored elements. The basic offer was two mandatory consultations and the option of contacting a palliative rehabilitation team directly during the participation period of 12 weeks, if needed. In addition, patients and family unpaid caregivers could be offered participation in a 12-week patient/ unpaid caregiver school combined with individually tailored physical exercise in groups, individual consultations with members of the palliative rehabilitation team, or both. Except for the chaplain, all SPC team members offered individual consultations to patients and family unpaid caregivers in the palliative rehabilitation clinic or over the telephone. The SPC team assembled for weekly multidisciplinary conferences discussing each patient at least once.

Duration: 12 weeks 
Control condition: The control group received standard care at the Department of Oncology. In addition to anticancer treatment, all patients had access to a number of paramedical services available through referral. These services were not open for unpaid caregivers.

Primary outcomes
Symptom/problem prioritised on an adapted form of the EORTC QLQ-C30 by patients
Secondary outcomes
Quality of life assessed using the EORTC QLQ-C30
Survival
Health service utilisation including number and length of hospital admissions and treatments, visits to
outpatient clinics, emergency rooms, and general practitioners

Assessment points: At baseline (before randomisation), 6 weeks and 12 weeks

Resource use/costs Health service utilisation including number and length of hospital admissions and treatments, visits to outpatient clinics, emergency rooms, and general practitioners

Time horizon: From enrollment until three months after final data collection

\section{Notes}

Funding source: This study was supported by the Danish Cancer Society, the Research Council of Lillebaelt Hospital, the Andreas and Grethe Gullev Hansen Foundation and the Hede Nielsen Family Foundation.

Declarations of interest among primary researchers: The authors declared that they had no conflict of interest.

Power considerations: Data from other studies using EORTC QLQ-C30 suggested a SD of less than 25 for a difference between the repeated measurements, and a group difference of 10 for clinical relevance. With a risk of type I error of 0.05 and type II error of $0.10,133$ patients were required in each arm. In order to allow for a dropout rate of approximately $10 \%$, each arm needed 150 patients (total $=300$ patients).

\section{Risk of bias}

\begin{tabular}{lll}
\hline Bias & Authors' judgement & Support for judgement \\
\hline $\begin{array}{l}\text { Random sequence genera- } \\
\text { tion (selection bias) }\end{array}$ & Low risk & $\begin{array}{l}\text { Quote from study protocol: "randomisation is subsequently performed by the } \\
\text { clinical trial unit using a randomisation list from randomiser.org. Patients are } \\
\text { randomised } 1: 1 \text { to the intervention or control group with no further stratifica- } \\
\text { tion used during randomisation". } \\
\text { Comment: probably done }\end{array}$ \\
& & \\
&
\end{tabular}

\begin{tabular}{ll}
\hline $\begin{array}{l}\text { Allocation concealment } \\
\text { (selection bias) }\end{array}$ & Low risk \\
& $\begin{array}{l}\text { Quote from study protocol: "the randomisation list is blinded from anyone in- } \\
\text { volved in informing potential study participants". }\end{array}$
\end{tabular}

Comment: probably done

\begin{tabular}{|c|c|c|}
\hline $\begin{array}{l}\text { Blinding of participants } \\
\text { and personnel (perfor- } \\
\text { mance bias), subjective } \\
\text { outcomes }\end{array}$ & High risk & $\begin{array}{l}\text { Quote from study protocol: "blinding of study participants and health profes- } \\
\text { sionals is not possible". } \\
\text { Comment: not done }\end{array}$ \\
\hline
\end{tabular}

\begin{tabular}{|c|c|c|}
\hline $\begin{array}{l}\text { Blinding of participants } \\
\text { and personnel (perfor- } \\
\text { mance bias), objective } \\
\text { outcomes }\end{array}$ & Low risk & $\begin{array}{l}\text { Quote from study protocol: "Blinding of study participants and health profes- } \\
\text { sionals is not possible". }\end{array}$ \\
\hline
\end{tabular}

The effectiveness and cost-effectiveness of hospital-based specialist palliative care for adults with advanced illness and their caregivers 


Blinding of outcome as-
sessment (detection bias),
subjective outcomes

\begin{tabular}{|c|c|c|}
\hline $\begin{array}{l}\text { Blinding of outcome as- } \\
\text { sessment (detection bias), } \\
\text { objective outcomes }\end{array}$ & Low risk & $\begin{array}{l}\text { Lack of blinding was unlikely to lead to bias in assessment of objective out- } \\
\text { comes. }\end{array}$ \\
\hline $\begin{array}{l}\text { Incomplete outcome data } \\
\text { (attrition bias) } \\
\text { All outcomes }\end{array}$ & Unclear risk & $\begin{array}{l}\text { Data on attrition was only presented for the intervention group. In the in- } \\
\text { tervention group, there were } 4(3 \%) \text { deaths and } 5(4.5 \%) \text { withdrawal of con- } \\
\text { sent/dropouts. }\end{array}$ \\
\hline $\begin{array}{l}\text { Selective reporting (re- } \\
\text { porting bias) }\end{array}$ & Unclear risk & Comment: Unclear risk of bias due to insufficient information in the abstract \\
\hline Other bias & Unclear risk & Comment: Unclear risk of bias due to insufficient information in the abstract \\
\hline Size of study & Unclear risk & $\begin{array}{l}\text { Unclear risk of bias because the number of participants in each group was be- } \\
\text { tween } 50 \text { and } 199 .\end{array}$ \\
\hline
\end{tabular}

O'Riordan 2019

\section{Study characteristics}

\begin{tabular}{ll}
\hline Methods & Design: RCT \\
Who led PC intervention: Multidisciplinary team \\
Core team: Nurse practitioner/physician/social worker/chaplain \\
\hline
\end{tabular}

Participants

\section{Country and regions: USA}

Recruitment: Study recruited inpatients with heart failure (HF) being actively managed during the current admission or had been within the past 6 months. Eligible patients were screened by conducting a review of new inpatient admissions to the medicine and cardiology services. Eligibility was confirmed through review of the electronic health record (EHR). The research co-ordinator contacted the patient's attending physician prior to approaching the patient to obtain informed consent.

Inclusion criteria: Inclusion criteria included:

- HF primary diagnosis or symptomatic/active HF in current hospitalisation or within prior 6 months

- NYHA Class II - IV

$\cdot \geq 18$ years of age

- English speaking

- Able to give informed consent and pass Short Portable Mental Status Questionnaire (SPMSQ)

- Medicine, cardiology and HF service

- No previous palliative care or hospice care

Exclusion criteria: Exclusion criteria included:

- Pulmonary hypertension 
O'Riordan 2019 (Continued)

\author{
- Right heart failure \\ - Left ventricular device (LVAD) \\ during index admission \\ -Pre/post heart, liver, lung transplant \\ - Homeless or live outside bay area \\ - Active illicit drug use
}

- Percutaneous coronary intervention ( $\mathrm{PCI})$, coronary arterial bypass graft (CABG) or valve replacement

Number of patients enrolled: $N=39$ (22 intervention and $17 / 18$ control)

Number of patients who participated in study: $N=30$ (16 intervention and 14 control)

Diseases: Heart failure

Patient characteristics: Mean age (SD): 71 (18) years in intervention group, 59 (19) years in control; $69 \%$ female in intervention group, $28 \%$ female in control group

Deaths at end of study ( $(\%)): N=2: 1(6.3 \%)$ in intervention and $1(7.1 \%)$ in control group

Withdrawals/other dropouts ( $(\%)): N=8: 5(31.3 \%)$ in intervention and $3(21.4 \%)$ in control group

Interventions

Name: Interdisciplinary palliative care intervention (Symptom Management Service - HF [SMS-HF]) alongside standard cardiology care vs standard care

Type: Training unclear

Service base: Large, urban, academic medical centre in the US

Team: Inpatient palliative care team consisting of a nurse practitioner, physician, social worker and chaplain

Intervention condition: Patients received a six-month palliative care intervention provided by the interdisciplinary SMS-HF inpatient palliative care team. The SMS-HF team provided direct care to the patient including prescribing medications for symptoms, discussing advance care planning and completing appropriate documentation, and providing psychosocial and spiritual support and services. The patients first contact the SMS-HF team during hospitalisation. They received a one-week, in-person follow-up assessment, and five monthly consultations, of which at least two were in person, with the remainder conducted via telephone and including all members of the SMS-HF team. Additional contacts with the SMS-HF team were scheduled as needed. Patients in the SMS-HF group who were re-admitted to the same hospital were followed by the inpatient palliative care team. Standard electronic health record (EHR) templates were used to document in-person and telephone care and communicate recommendations to the cardiology team.

\title{
Duration: 6 months
}

Control condition: The patients randomised to usual care received guideline-driven HF treatment. Authors assessed all symptoms and quality of life (QoL) at enrollment and symptoms, QoL, satisfaction, advance care planning documentation, and resource utilisation at follow-up three and six months later.

\section{Outcomes}

Quality of life assessed using the Minnesota Living with HF Questionnaire (MLHFQ) and the Functional Assessment of Chronic Illness Therapy with the palliative care subscale (FACIT-PAL)

Pain assessed using the Brief Pain Inventory (BPI).

Anxiety and depression assessed using the Hospital Anxiety and Depression Scale (HADS)

Symptoms assessed using the Edmonton Symptom Assessment Scale (ESAS)

The effectiveness and cost-effectiveness of hospital-based specialist palliative care for adults with advanced illness and their caregivers 
O'Riordan 2019 (Continued)

Fatigue assessed using the Brief Fatigue Inventory (BFI)

Dyspnoea assessed using the BORG Scale

Patient satisfaction assessed using an unvalidated scale

Assessment points: Patients were randomised and completed the survey at 3 time points (baseline, three and six-month follow-up). Patients completed the baseline survey in the hospital. The follow-up survey was mailed to patients at three and six months for both usual care and intervention groups.

\begin{tabular}{|c|c|}
\hline Resource use/costs & $\begin{array}{l}\text { Number of readmissions to hospital } \\
\text { Number of hospital visits } \\
\text { Time horizon: Study enrollment to six months }\end{array}$ \\
\hline Notes & $\begin{array}{l}\text { Funding source: The National Palliative Care Research Center and the Alafi Family Foundation } \\
\text { Declarations of interest among primary researchers: Not stated } \\
\text { Power considerations: Based on previous research from the HF clinic, the authors projected it would } \\
\text { be feasible to obtain complete baseline and follow-up data from } 64 \text { patients over a } 24 \text {-month peri- } \\
\text { od. Using the SD for the MLHFQ (SD }=25.4) \text { and the BPI (SD }=1.99) \text { and using paired t-tests to estimate } \\
\text { the minimum detectable difference, the authors calculated that with } 80 \% \text { power and } P=0.05 \text {, a sam- } \\
\text { ple size of } 32 \text { patients in each group would enable them to observe a minimum detectable difference } \\
\text { on the BPI of } 1.4 \text { points and on the MLHFQ of } 16.4 \text { points, while for pain the detectable difference was } \\
\text { smaller than the clinically meaningful value ( } 2 \text {-points). }\end{array}$ \\
\hline
\end{tabular}

\section{Risk of bias}

Bias Authors' judgement Support for judgement

Random sequence genera- Unclear risk tion (selection bias)
Quote from main publication: "patients were randomised within blocks of six to either SMS-HF or usual care to reduce potential bias and confounding. A member of the research team with no contact with the study patients conducted a random assignment procedure to prevent any bias in the allocation to groups".

Comment: it was unclear how the random sequence was generated.

\begin{tabular}{ll}
\hline $\begin{array}{l}\text { Allocation concealment } \\
\text { (selection bias) }\end{array}$ & $\begin{array}{l}\text { Quote from main publication: "a member of the research team with no contact } \\
\text { with the study patients conducted a random assignment procedure to prevent } \\
\text { any bias in the allocation to groups". }\end{array}$ \\
& Comment: allocation concealment was unclear.
\end{tabular}

\begin{tabular}{|c|c|c|}
\hline $\begin{array}{l}\text { Blinding of participants } \\
\text { and personnel (perfor- } \\
\text { mance bias), subjective }\end{array}$ & High risk & $\begin{array}{l}\text { Participants and personnel were not blinded. } \\
\text { Comment: high risk of bias due to lack of blinding }\end{array}$ \\
\hline
\end{tabular}
Quote from main publication: "a member of the research team with no contact
with the study patients conducted a random assignment procedure to prevent
Comment: allocation concealment was unclear.

\begin{tabular}{|c|c|c|}
\hline $\begin{array}{l}\text { Blinding of outcome as- } \\
\text { sessment (detection bias), } \\
\text { subjective outcomes }\end{array}$ & Unclear risk & $\begin{array}{l}\text { Unclear because it was not mentioned } \\
\text { Comment: unclear risk of bias because blinding of outcome assessment was } \\
\text { not mentioned }\end{array}$ \\
\hline
\end{tabular}

\begin{tabular}{|c|c|c|}
\hline $\begin{array}{l}\text { Incomplete outcome data } \\
\text { (attrition bias) } \\
\text { All outcomes }\end{array}$ & High risk & $\begin{array}{l}\mathrm{N}=16(72.7 \%) \text { completers in intervention group vs } \mathrm{N}=14(77.8 \%) \text { completers } \\
\text { in control group. In the intervention group, one }(6.3 \%) \text { death occurred, } 2 \\
(12.5 \%) \text { patients dropped out and } 3(18.8 \%) \text { patients were determined not el- } \\
\text { igible immediately after enrollment. In control group, one }(7.1 \%) \text { death oc- }\end{array}$ \\
\hline
\end{tabular}

The effectiveness and cost-effectiveness of hospital-based specialist palliative care for adults with advanced illness and their caregivers (Review)

Copyright ( 2020 The Cochrane Collaboration. Published by John Wiley \& Sons, Ltd. 

the analysis.

\begin{tabular}{ll}
\hline $\begin{array}{l}\text { Selective reporting (re- } \\
\text { porting bias) }\end{array}$ & High risk \\
& $\begin{array}{l}\text { Information published on the clinical trials registry NCT01461681 indicate that } \\
\text { the primary outcome for the study was depression assessed using the Center } \\
\text { for Epidemiologic Studies Depression Scale (CES-D). However, in the published } \\
\text { study, depression was not specified as the primary outcome. Other additional } \\
\text { outcomes such as quality of life, pain and symptoms were also assessed. }\end{array}$
\end{tabular}

\begin{tabular}{ll}
\hline Other bias & High risk \\
& $\begin{array}{l}\text { Quote from main publication: "The intervention group had significantly more } \\
(P=0.03) \text { women }(69 \%, n=11) \text { than the usual care group }(28 \%, n=4) " .\end{array}$ \\
& $\begin{array}{l}\text { Comment: study did not control for difference between intervention and con- } \\
\text { trol group. High risk was rated due to imbalance bias. }\end{array}$
\end{tabular}

\begin{tabular}{ll}
\hline Size of study $\quad$ High risk & $\begin{array}{l}\text { High risk of bias because the number of participants in each group was less } \\
\text { than } 50\end{array}$
\end{tabular}

\section{Study characteristics}

\begin{tabular}{ll}
\hline Methods & Design: RCT \\
Who led PC intervention: Multidisciplinary team \\
Core team: Medical oncologist/case manager nurse/clinical nurse/algologist/psychiatrist/physical \\
therapy expert/social services expert/liaison consultant nurse with a doctorate in psychiatry
\end{tabular}

\section{Participants Country and regions: Turkey, Izmir}

Recruitment: September 2009 to September 2011. The authors used the criteria of the National Comprehensive Cancer Network clinical practice guide for palliative care for the selection of patients. They selected inpatients who had an acute need for palliative care.

Inclusion criteria: Older than 18 years, fully conscious, co-operative and oriented, no sight or hearing problems, capable of verbal communication, patient with advanced stage cancer, life expectancy of between 6 and 12 months, a performance level of 50 or less on the Karnofsky Performance Scale (KPS), patient with cancer having 1 or more uncontrollable symptoms, and patient with cancer receiving palliative care.

\section{Exclusion criteria: Not described}

Number of patients: $\mathrm{N}=44$ (22 intervention and 22 control)

Diseases: Cancers: gastrointestinal, genitourinary, breast, sarcoma, lung and unknown primary tumour

Patient characteristics: Mean age (SD): 52.59 (13.31) in intervention group, 53.63 (12.31) in control group; $81.8 \%$ female in intervention group, $68.2 \%$ female in control group

Deaths at end of study (N (\%)): No death

Withdrawals/other dropouts (N (\%)): No dropout

Type: Training in palliative care unclear

Service base: Tulay Aktas Oncology Hospital, Medical Oncology Clinic, Ege University 
Team: Included a medical oncologist, a case manager nurse, and a clinical nurse, an algologist, a psychiatrist, a physical therapy expert, a social services expert, and a liaison consultant nurse with a doctorate in psychiatry.

Intervention condition: Palliative care was provided by a multidisciplinary team, based on the case management model. Initially, the patients met with the medical oncologist and the registered nurse (RN) when they were accepted into the palliative care programme. The case management nurse, along with the clinical nurse, followed up the patient and family from admission to discharge. The intervention group received immediate consultation and follow-up by the palliative care team based on a philosophy of multidisciplinary care. After a comprehensive symptom diagnosis, effective symptom management, psychosocial stress management, social support, care and training support, and family counselling services were organised. Symptom follow-up and monitoring were made by case manager nurse with Edmonton Symptom Assessment System (ESAS). Reconsultation and treatment arrangement were made for uncontrolled symptoms of the patients. The care and training requirements of the patient and his family were provided by the case manager nurse and service nurses. Family counselling was provided by psychiatry nurse. Patients were provided with personal care, and a training book was used to train both patients and their families. Individualised patient/family education was given by the case manager nurse. The educational book "Palliative Care for Cancer Patients and Their Families" was also given to the usual care group, but individualised education was given only to the case management group.

Duration: The period of hospitalisation: day of admission to hospital until the day of discharge

Control condition: When usual care patients were admitted to the Oncology Department, an oncologist obtained a medical history, examined the patient, and ordered various tests. Treatment plans were then made, and orders were given to the ward nurses. The nurses provided treatment to the patients according to the doctor's orders and implemented usual nursing care. The educational book "Palliative Care for Cancer Patients and Their Families" was also given to the usual care group.

\section{Outcomes Outcomes:}

Level of symptoms assessed using the Edmonton Symptom Assessment System (ESAS)

Quality of life assessed using the European Organization for Research and Treatment (EORTC) Quality of Life Questionnaire C30 (QLQ-C30)

Patient and family satisfaction assessed using Patient and Family Satisfaction Forms that was created by the researcher based on the FAMCARE questionnaire

Resource use/costs Length of stay in hospital

Direct costs in USD

Time horizon: Period of hospitalisation

Data sources: The Patient Expenditure Record Form was created by listing direct health expenditure, which consisted of all expenses incurred while in hospital. Items such as the following appeared in the form among the direct expenses: medicines used from the start of the patient's stay in hospital, medical equipment, laboratory and diagnosis tests, consultations, professional care, and hospital stay expenses (including those of companions). On the patient's discharge from hospital, costs were recorded on the form by obtaining the expenses list from the clinic secretary. Total costs for each patient were calculated in US dollars from the expenses lists.

Analytical perspective: Not clear

Funding source: The authors received no financial support for the research, authorship, and/or publication of this article.

Declarations of interest among primary researchers: The authors declared no potential conflicts of interest with respect to the research, authorship, and/or publication of this article.

Power considerations: While the research power that is necessary to show the difference in palliative care service from routine clinic care was $90 \%$, minimum sample width that was necessary to find a

The effectiveness and cost-effectiveness of hospital-based specialist palliative care for adults with advanced illness and their caregivers 
Ozcelik 2014 (Continued)

meaningful difference between the 2 averages in the working group was determined as 18 . However, the authors ended the study with 22 experimental and 22 working group patients.

\section{Risk of bias}

Bias Authors' judgement Support for judgement

Random sequence genera- Unclear risk tion (selection bias)
Quote from main publication: "in this study, patients with advanced stage cancer receiving palliative care who met the specified criteria and agreed to take part in the research were divided randomly according to age, sex, and education level into 2 groups a control group and an experimental group".

Comment: unclear as no mention of how the random sequence was generated

\begin{tabular}{ll}
\hline $\begin{array}{l}\text { Allocation concealment } \\
\text { (selection bias) }\end{array}$ & Unclear risk
\end{tabular}

\begin{tabular}{lll}
\hline $\begin{array}{l}\text { Blinding of participants } \\
\text { and personnel (perfor- } \\
\text { mance bias), subjective } \\
\text { outcomes }\end{array}$ & High risk & $\begin{array}{l}\text { Participants and personnel were not blinded. } \\
\text { Comment: high risk of bias due to lack of blinding }\end{array}$ \\
\hline $\begin{array}{l}\text { Blinding of outcome as- } \\
\text { sessment (detection bias), } \\
\text { subjective outcomes }\end{array}$ & Unclear risk & $\begin{array}{l}\text { Unclear as blinding of outcome assessment was not stated } \\
\text { Comment: unclear risk of bias because blinding of outcome assessment was } \\
\text { not mentioned }\end{array}$
\end{tabular}

Incomplete outcome data Low risk There was no loss to follow-up reported.

(attrition bias)

All outcomes

\begin{tabular}{lll}
\hline $\begin{array}{l}\text { Selective reporting (re- } \\
\text { porting bias) }\end{array}$ & Unclear risk & Unclear as the protocol of this study was not available \\
\hline Other bias & Unclear risk & $\begin{array}{l}\text { There were differences between the intervention and control group in gender, } \\
\text { marital status, educational level, disease duration, tumour type and Karnosfky } \\
\text { performance score. However, no testing for statistically significant differences } \\
\text { was carried out and it was unclear if differences were controlled for. }\end{array}$ \\
\hline Size of study & High risk & $\begin{array}{l}\text { High risk of bias because the number of participants in each group was less } \\
\text { than } 50\end{array}$ \\
\hline
\end{tabular}

Rodin 2019

\section{Study characteristics}

\begin{tabular}{ll}
\hline Methods & Design: RCT \\
& Who led PC intervention: Multidisciplinary team \\
& $\begin{array}{l}\text { Core team: EASE-psy was delivered by a mental health clinician while the core team in EASE-phys was } \\
\text { a palliative care physician and nurse. Other multidisciplinary team members were involved as needed. }\end{array}$ \\
\hline Participants & $\begin{array}{l}\text { Country and regions: Canada, Toronto } \\
\text { Recruitment: March } 2015 \text { to November } 2016 . \text { Eligible patients were identified through clinical records } \\
\text { Centre (PM), Toronto, Canada. }\end{array}$ \\
\hline
\end{tabular}

The effectiveness and cost-effectiveness of hospital-based specialist palliative care for adults with advanced illness and their caregivers 
Inclusion criteria: Newly diagnosed or recently relapsed with acute myeloid leukaemia (AML) or acute lymphocytic leukaemia (ALL) within 1 month of inpatient admission to PM; receiving (or expected to receive) induction chemotherapy with curative intent; > 18 years old; fluent in English; and no cognitive impairment

Exclusion criteria: Cognitive screening test score below cutoff (i.e. Short Orientation-Memory-ConcentrationTest (SOMC) score <20); and already receiving psychological/psychiatric counselling or palliative care services at PM at the time of recruitment

Number of patients: $\mathrm{N}=42$ (22 intervention and 20 control)

Diseases: Acute leukaemia (AL) (all included patients were newly diagnosed with acute leukaemia)

Patient characteristics: Mean age (SD): 51.59 (16.66) in intervention group and 54.25 (15.19) in control group; $36.4 \%$ female in intervention group, $40 \%$ female in control group

Deaths at end of study (N (\%)): None

Withdrawals/other dropouts $(\mathrm{N}(\%)): \mathrm{N}=7: 6(27.3 \%)$ intervention and $1(5 \%)$ control

Name: Emotion And Symptom-focused Engagement (EASE) plus usual care vs usual care alone

Early PC: Eligible patients were newly diagnosed or recently relapsed with acute myeloid leukaemia (AML) or acute lymphocytic leukaemia (ALL) within 1 month of inpatient admission to PM (all recruited patients were newly diagnosed with acute leukaemia).

Type: Specialist palliative care. The core team in EASE-phys was a palliative care physician and nurse. Other multidisciplinary team members were involved as needed.

Service base: Princess Margaret Cancer Centre (PM), University Health Network (UHN), Toronto, Canada

Team: EASE-psy was delivered by a mental health clinician while the core team in EASE-phys was a palliative care physician and nurse. Other multidisciplinary team members were involved, as needed.

Intervention condition: EASE integrated a novel psychotherapeutic intervention (EASE-psy) with systematic screening of physical symptoms and triggered referral for early palliative care (EASE-phys) to target traumatic stress and physical symptoms. EASE-psy included 8-12 psychotherapeutic sessions, approximately 30-60 min each, delivered over 8 weeks by a trained mental health clinician. It was based on principles of supportive psychotherapy and trauma-focussed CBT applied to patients with life-threatening or advanced disease. The first 8 sessions occurred during hospitalisation, adjusted depending on the patient's ability to participate. The remaining 4 sessions occurred weekly or bi-weekly after discharge, in co-ordination with outpatient clinic visits. EASE-phys consisted of systematic screening of physical symptoms with the ESAS-AL, with triggered referral to early palliative care. The ESASAL was administered 2 to 3 times weekly during the inpatient stay and weekly after discharge. When there was a score $\geq 4$ (moderate to severe) on any physical symptom, a palliative care referral was triggered and ESAS-AL screening for that participant was taken over by the EASE-phys team until all symptom scores were $<4$. At that point, the research team resumed ESAS-AL administration, with re-referral, if needed. The EASE-phys team applied symptom management guidelines used routinely in palliative care at PM. As long as symptom scores were $\geq 4$, follow-up from the EASE-phys team occurred 3 times weekly for inpatients in-person and weekly for outpatients in-person or by telephone.

Duration: 12 weeks

Control condition: Patients receiving induction chemotherapy for AL were admitted to one of three dedicated AL inpatient wards at PM. Care was provided by a multidisciplinary team including physicians, nurses, and allied health personnel dedicated to the treatment of AL. Participants in the control group received no formal trial intervention, but any referral to psychosocial or palliative care services was not delayed or denied. At PM, all newly diagnosed patients with AL are referred to a social worker. However, these social workers did not routinely provide structured psychotherapy. At the end of the trial, participants in the control group were offered EASE on compassionate grounds, without further completion of questionnaires or symptom screening. 
Rodin 2019 (Continued)

Outcomes

\section{Primary outcome}

Severity of traumatic stress symptoms measured by the 30-item Stanford Acute Stress Reaction Questionnaire (SASRQ).

\section{Secondary outcomes}

Physical symptom burden measured by the Memorial Symptom Assessment Scale (MSAS)

Pain assessed using the Brief Pain Inventory (BPI)

Quality of life (QoL) measured using the Functional Assessment of Chronic Illness Therapy-Spiritual Well-Being Scale (FACIT-Sp)

Depression assessed by the Beck Depression Inventory-II (BDI-II)

Patient satisfaction with care measured using the 16-item Family Satisfaction with Care - Patient Version (FAMCARE-P16)

Attachment security assessed with the Brief Experiences in Close Relationships Scale (ECR-M16)

Emotional support assessed with Clinical Evaluation Questionnaire (CEQ)

Assessment points: Assessments were conducted at baseline (before randomisation) and at 4, 8, and 12 weeks.
Referral to palliative care, social work and psychiatry

Time horizon: 12 weeks

Funding source: This trial was funded by the Canadian Cancer Society Research Institute (CCSRI grant no. 702603; GR and CZ, Co-Principal Investigators). This research was also supported in part by the Princess Margaret Cancer Centre and Princess Margaret Cancer Foundation, University Health Network, Toronto, Ontario, Canada, and the Ontario Ministry of Health and Long-term Care.

Declarations of interest among primary researchers: One of the authors reported personal fees and grants outside this submitted work from Novartis, Takeda Pharmaceuticals, Otsuka Pharmaceutical, Jazz Pharmaceuticals, Medivir, and Abbvie. The remaining authors declared no conflicts of interest.

Power considerations: The main outcome criteria for this phase II trial were related to feasibility. Therefore, the trial was powered to detect only medium to large effects. The authors planned to recruit 50 participants, in order to detect a medium effect size of 0.56 (Cohen's d) between groups at the primary endpoint on the SASRQ, assuming a correlation between repeated measurements of 0.72 on the SASRQ observed in their earlier longitudinal study of patients with AL.

\section{Risk of bias}

\begin{tabular}{lll}
\hline Bias & Authors' judgement & Support for judgement \\
\hline $\begin{array}{l}\text { Random sequence genera- } \\
\text { tion (selection bias) }\end{array}$ & Low risk & $\begin{array}{l}\text { Quote from main publication: "upon providing written informed consent and } \\
\text { completing baseline measures, participants were allocated by permuted block } \\
\text { randomisation (variable block size) either to EASE plus usual care or to usu- } \\
\text { al care alone, with stratification by age }(\leq 60 \text { vs. }>60) \text { and type of AL (AML or } \\
\text { ALL)... The PM Department of Biostatistics, which is independent of the trial } \\
\text { team, developed the randomisation procedures, managed the logbook, and } \\
\text { provided the computer-generated randomisation allocation to research staff } \\
\text { after patients' completion of the baseline assessment." } \\
\text { Comment: probably done }\end{array}$ \\
&
\end{tabular}

\begin{tabular}{|c|c|c|}
\hline $\begin{array}{l}\text { Allocation concealment } \\
\text { (selection bias) }\end{array}$ & Low risk & $\begin{array}{l}\text { Quote from main publication: "the PM Department of Biostatistics, which is in- } \\
\text { dependent of the trial team, developed the randomisation procedures, man- }\end{array}$ \\
\hline
\end{tabular}


aged the logbook, and provided the computer-generated randomisation allocation to research staff after patients' completion of the baseline assessment."

Comment: probably done

Blinding of participants and personnel (performance bias), subjective outcomes
High risk

Quote from main publication: "we report here results of an unblinded phase II randomised controlled trial (RCT) for patients with newly diagnosed AL..."

Comment: not done

\section{Blinding of participants Low risk and personnel (perfor- \\ Quote from main publication: "we report here results of an unblinded phase II randomised controlled trial (RCT) for patients with newly diagnosed AL..." mance bias), objective outcomes Comment: there was no blinding but this was unlikely to lead to bias in objec- tive outcomes.}

Blinding of outcome as-
sessment (detection bias),
subjective outcomes

Quote from main publication: "we report here results of an unblinded phase II randomised controlled trial (RCT) for patients with newly diagnosed AL..."

subjective outcomes

\section{Comment: not done}

Blinding of outcome as-
sessment (detection bias),

Quote from main publication: "we report here results of an unblinded phase II sessment (detection bias) randomised controlled trial (RCT) for patients with newly diagnosed AL..."

objective outcomes

Comment: there was no blinding but this was unlikely to lead to bias in objective outcomes.

Incomplete outcome data Low risk
(attrition bias)
All outcomes

$\mathrm{N}=16(72.7 \%)$ completers in intervention group vs $\mathrm{N}=19(95 \%)$ completers in control group. There were no deaths during the trial. Missing data were included in the analysis using maximum likelihood estimates.

Comment: low risk of bias

\begin{tabular}{lll}
\hline $\begin{array}{l}\text { Selective reporting (re- } \\
\text { porting bias) }\end{array}$ & High risk & $\begin{array}{l}\text { Some prespecified outcomes such as attachment security and emotional sup- } \\
\text { port by clinical services were not reported. }\end{array}$ \\
\hline Other bias & Low risk & Study appeared to be free of other biases. \\
\hline Size of study & High risk & $\begin{array}{l}\text { High risk of bias because the number of participants in each group was less } \\
\text { than } 50\end{array}$ \\
\hline
\end{tabular}

Rogers 2017

\section{Study characteristics}

\begin{tabular}{ll}
\hline Methods & Design: RCT \\
Who led PC intervention: Multidisciplinary team \\
Core team: Palliative care nurse practitioner/hospice and palliative medicine board-certified physi- \\
cian. The intervention was performed in collaboration with each patient's clinical cardiology team.
\end{tabular}

The effectiveness and cost-effectiveness of hospital-based specialist palliative care for adults with advanced illness and their caregivers 
Inclusion criteria: Age > 18 y; hospitalisation for acute heart failure (HF) (either systolic HF or HF with preserved ejection fraction) or within 2 weeks of discharge of a hospitalisation for acute HF; dyspnoea at rest or minimal exertion plus $\geq 1$ sign of volume overload; previous HF hospitalisation within the past $1 \mathrm{yr}$; ESCAPE risk score $\geq 4$ indicating $>50 \%$ predicted 6 -month mortality; anticipated discharge from hospital with anticipated ability to return to outpatient follow-up appointments; or subjects aged > 18 $\mathrm{y}$, hospitalised with acute HF with signs/symptoms of volume overload who do not meet all other eligibility criteria may also be considered for enrollment if they can be categorised into one of the following high-risk groups:

1. Support with chronic inotropes without plans for cardiac transplant or cardiac assist device and anticipated discharge from hospital

2. Multiple hospitalisations for $\mathrm{HF}$ in the past 12 months (minimum 3 ) and anticipated discharge from hospital

3. No prior hospitalisation for HF in the past 12 months but with an ESCAPE score $>4$ and anticipated discharge from hospital

Exclusion criteria: Failure to meet severity of illness criteria in the Evaluation Study of Congestive Heart Failure and Pulmonary Artery Catheterisation Effectiveness risk score. Other reasons for exclusion were acute coronary syndrome within 30 days; cardiac resynchronisation therapy within the past 3 months or current plan to implant; active myocarditis, constrictive pericarditis; severe stenotic valvular disease amendable to surgical intervention; anticipated heart transplant or ventricular assist device within 6 months; renal replacement therapy; non-cardiac terminal illness; women who are pregnant or planning to become pregnant; inability to comply with study protocol.

Number of patients: $\mathrm{N}=150$ (75 intervention and 75 control)

Diseases: Advanced heart failure

Patient characteristics: Mean age (SD): 71.9 (12.4) years in intervention group, 69.8 (13.4) years in control group. $44 \%$ females in intervention and $50.7 \%$ females in control group

Deaths at end of study (\%)): $29 \%$ of patients died but numbers in intervention and control group not provided

Withdrawals/other dropouts (N (\%)): N = 96: 47 (62.7\%) intervention and 49 (65.3\%) control usual care

Type: Specialist palliative care. Included a certified palliative care nurse practitioner and a hospice and palliative medicine board-certified physician

Service base: Duke University Hospital

Team: A certified palliative care nurse practitioner and a hospice and palliative medicine board-certified physician

Intervention condition: The study team assessed and managed the multiple domains of quality of life for patients with advanced HF, including physical symptoms, psychosocial and spiritual concerns, and advance care planning. A certified palliative care nurse practitioner co-ordinated these aspects of the patient's care in collaboration with a hospice and palliative medicine board-certified physician. The intervention was performed in collaboration with each patient's clinical cardiology team and focussed on shared goal-setting to combine HF symptom amelioration with palliative care goals. After hospital discharge, the PAL-HF nurse practitioner actively participated in the ongoing management of the patients. Patients were screened for depression and anxiety with the HADS. Patients who screened positive were considered for referral to a mental health provider as well as for possible use of symptomatic medical therapies, stress management resources and psychotherapy. Spiritual concerns were assessed by the study nurse practitioner, and these details were shared with the intervention team. Goals of care were iteratively assessed by the intervention nurse practitioner. After the 6-month intervention period was completed, the nurse practitioner continued to contact the patients in the intervention arm every 3 months to provide ongoing support and clinical care. 
Rogers 2017 (Continued)

\section{Duration: 6 months}

Control condition: Usual care patients were managed by a cardiologist-directed team with HF expertise. Inpatient care was focussed on symptom relief and use of evidence-based therapies as detailed in current guidelines. Inpatient palliative care consultation was not denied to usual care patients. After discharge, these patients received outpatient follow-up with their general practitioners as well as an $\mathrm{HF}$ cardiologist or nurse practitioner with care focussed on guidelines-based medication titration and serial monitoring of end-organ function.

Outcomes

Primary outcomes:

Quality of life assessed by two different questionnaires, the Kansas City Cardiomyopathy Questionnaire (KCCQ) and Functional Assessment of Chronic Illness Therapy - Palliative Care (FACIT-Pal) scale

\section{Secondary outcomes:}

Depression and anxiety assessed using the Hospital Anxiety and Depression Scale (HADS)

Spiritual well-being assessed using the Functional Assessment of Chronic Illness Therapy - Spiritual Well-Being (FACIT-Sp) scale

Hospitalisations

Mortality

Assessment points: After trial enrollment, subjects underwent reassessment of their clinical status and primary outcome questionnaires at weeks $2,6,12$, and 24 . Secondary outcomes were assessed at weeks 2, 12 and 24 .

Resource use/costs Number of hospital encounter records

Number of clinic encounter records

Number of primary care contacts

Number of cardiology contacts

Number of telephone contacts

Number of rehabilitation clinic contacts

Number of emergency department/urgent care contacts

Number of hospitalisations

Time horizon: From enrollment until death or end of the study National Institute of General Medical Sciences.

Declarations of interest among primary researchers: Dr. Johnson received research support from projects funded by the National Institute on Aging. Dr. Krishnamoorthy has worked on projects funded by research grants to the Duke Clinical Research Institute from the NIH, Novartis, Daiichi-Sankyo, and Eli Lilly; and has received support to attend educational conferences from HeartWare, Thoratec, and Medtronic. Dr. Mark has received consulting fees from Medtronic; and has received research funding from Eli Lilly, Bristol-Myers Squibb, Pfizer, AstraZeneca, Merck and Company, Oxygen Theraputics, and Gilead. Dr. Tulsky has received research funding from PCORI. All other authors have reported that they have no relationships relevant to the contents of this paper to disclose. P.K. Shah served as Guest Editor-in-Chief for this paper. Barry H. Greenberg served as Guest Editor for this paper.

Power considerations: The KCCQ overall summary and FACIT-PAL scores were selected as the co-primary endpoints. Assuming a common SD of 12 points for the KCCQ overall summary score, the planned sample size of 200 subjects ( 100 per arm) was projected to provide $80 \%$ power to detect a difference of 4.8 points. As noted earlier, a 5-point change in this score is the smallest change that is clinically signifi-

The effectiveness and cost-effectiveness of hospital-based specialist palliative care for adults with advanced illness and their caregivers 
Rogers 2017 (Continued)

cant at the individual patient level. For the FACIT-PAL co-primary endpoint, the sample size of 200 subjects was projected to provide $80 \%$ power to detect a difference of 10 points assuming a SD of 25 . Sample size calculations were based on a 2-sample Student t-test with a type I error rate of 0.05 . The data and safety monitoring board, in consultation with the sponsoring agency, recommended a sample size reduction to 150 subjects, based upon enrollment rates, a mortality rate that was lower than predicted, and observed outcomes differences at that intermediate time point.

\section{Risk of bias}

\begin{tabular}{lll}
\hline Bias & Authors' judgement & Support for judgement \\
\hline $\begin{array}{ll}\text { Random sequence genera- } \\
\text { tion (selection bias) }\end{array}$ & Unclear risk & $\begin{array}{l}\text { Quote from main publication: "enrolled patients were randomised in a 1:1 al- } \\
\text { location to usual care (UC) alone or UC plus palliative care intervention (UC + } \\
\text { PAL) using a complete randomisation scheme". }\end{array}$ \\
& & Comment: unclear as random sequence generation was not well described
\end{tabular}

\begin{tabular}{ll}
\hline $\begin{array}{l}\text { Allocation concealment } \\
\text { (selection bias) }\end{array}$ Unclear risk & Allocation concealment was not described.
\end{tabular}

(selection bias)

\begin{tabular}{|c|c|c|}
\hline $\begin{array}{l}\text { Blinding of participants } \\
\text { and personnel (perfor- } \\
\text { mance bias), subjective } \\
\text { outcomes }\end{array}$ & High risk & $\begin{array}{l}\text { Quote from main publication: "the trial was unblinded because blinding of the } \\
\text { intervention was not feasible". } \\
\text { Comment: high risk of bias due to lack of blinding }\end{array}$ \\
\hline
\end{tabular}

Blinding of participants Low risk and personnel (perfor-

Quote from main publication: "the trial was unblinded because blinding of the mance bias), objective outcomes intervention was not feasible".

Comment: there was no blinding but this was unlikely to lead to bias in objective outcomes such as mortality.

$\begin{array}{lll}\text { Blinding of outcome as- } & \text { High risk } & \begin{array}{l}\text { Quote from main publication: "the trial was unblinded because blinding of the } \\ \text { sessment (detection bias), }\end{array}\end{array}$
subjective outcomes

Comment: high risk of bias due to lack of blinding

$\begin{array}{ll}\begin{array}{l}\text { Blinding of outcome as- } \\ \text { sessment (detection bias), } \\ \text { objective outcomes }\end{array} & \text { Low risk }\end{array} \quad \begin{aligned} & \begin{array}{l}\text { Quote from main publication: "the trial was unblinded because blinding of the } \\ \text { intervention was not feasible". }\end{array} \\ & \begin{array}{l}\text { Comment: there was no blinding but this was unlikely to lead to bias in the as- } \\ \text { sessment of objective outcomes such as mortality. }\end{array}\end{aligned}$

Incomplete outcome data High risk
(attrition bias)

$\mathrm{N}=28(37.3 \%)$ completers in intervention group vs $\mathrm{N}=26(34.7 \%)$ completers All outcomes in control group. At the point of primary analysis ( 6 months), $N=41(54.7 \%)$ completers in intervention group vs $\mathrm{N}=40(53.3 \%)$ in control group. At the end of the study, $40(53.3 \%)$ patients died and $7(9.3 \%)$ withdrew from the intervention group while in the control group $38(50.7 \%)$ died and $11(14.7 \%)$ withdrew. Missing data were not included in the analysis. Overall, there was high attrition across intervention and control groups.

Comment: high risk of bias

\begin{tabular}{lll}
\hline $\begin{array}{l}\text { Selective reporting (re- } \\
\text { porting bias) }\end{array}$ & High risk & $\begin{array}{l}\text { Some outcomes specified in the protocol such as unpaid caregiver satisfac- } \\
\text { tion, quality of care from the family member's perspective and cost were not } \\
\text { reported in the published study. }\end{array}$ \\
\hline Other bias & Low risk & Study appeared to be free from other biases. \\
\hline Size of study & Unclear risk & $\begin{array}{l}\text { Unclear risk of bias because the number of participants in each group was be- } \\
\text { tween } 50 \text { and } 199\end{array}$
\end{tabular}

The effectiveness and cost-effectiveness of hospital-based specialist palliative care for adults with advanced illness and their caregivers 


Design: RCT
Who led PC intervention: Multidisciplinary team
Core team: Physicians board-certified in hospice and palliative medicine/clinical nurse specialists
board-certified in advanced practice palliative care nursing/social worker/chaplain

Participants

Country and regions: USA, Minneapolis, Minnesota

Recruitment: April 2012 to February 2013. Potentially eligible patients were identified using reports from the electronic health record (EHR). Eligibility was verified by reviewing patient records and talking with a floor nurse if needed. Patients determined to be eligible were visited by the research nurse who explained the study and enrolled those interested in participating.

Inclusion criteria: Patients were considered eligible for the study if they were adult inpatients with a diagnosis of acute HF.

Exclusion criteria: Patients in the intensive care unit (ICU), on a ventilator, undergoing evaluation for a heart transplant or a left ventricular assist device (LVAD), post-transplant or post-LVAD, determined to be actively dying, or if they had cognitive impairments such that informed consent and data collection would not be possible or if they spoke limited English. Patients who had already had a palliative care order request by their attending physician during the hospital stay were ineligible.

Number of patients: $\mathrm{N}=232$ (116 intervention and 116 control)

Diseases: Acute heart failure

Patient characteristics: Mean age (SD): 76 (11.9) years in intervention group, 70.9 (13.6) years in control group. $52.6 \%$ females in intervention and $42.2 \%$ females in control group

Deaths at end of study ( $(\%)): \mathrm{N}=19: 14(12.1 \%)$ intervention and $5(4.3 \%)$ in control group

Withdrawals/other dropouts ( $\mathrm{N}(\%)): \mathrm{N}=93: 49(42.2 \%)$ intervention and $44(37.9 \%)$ control

Type: Specialist palliative care. Included physicians who were board-certified in hospice and palliative medicine and nurse specialists board-certified in advance practice palliative care nursing

Service base: Abbott Northwestern Hospital (ANW), 629-bed tertiary-care facility in Minneapolis, Minnesota

Team: The ANW inpatient palliative care team at the time of the study included four physicians boardcertified in hospice and palliative medicine, two clinical nurse specialists board-certified in advanced practice palliative care nursing, a social worker, and a chaplain.

Intervention condition: After patients were randomised to the intervention group, an order for palliative care was entered, and triaged by the palliative care team with a goal of conducting the palliative care consult within 24 hours of the order. Providers did an initial consult and then determined whether further appointments were necessary. The study intervention differed from the standard palliative care process in two ways. First, baseline study measures of symptom burden, depression, and QoL were available to the providers to review at the time of the consultation. Second, the study paid only for the initial palliative care consultation and any subsequent visits were billed to the patient's insurance as standard care. Actions of palliative care providers during visits generally included assessment of symptom burdens; emotional, spiritual, and psychosocial aspects of care; co-ordination of care orders; recommendations for change in current or future treatment; referrals; and future care planning assessment and discussions. All HF patients received a referral to the ACP process through their discharge orders. 
Sidebottom 2015 (Continued)

Duration: Period of hospitalisation

Control condition: This was not described.

\begin{tabular}{ll}
\hline Outcomes & Primary outcome \\
Symptom burden assessed usi & Depressive symptoms assessed \\
& Quality of life assessed using the \\
Secondary outcomes & Advanced care planning (ACP) \\
Inpatient 30-day readmission \\
Hospice use \\
Mortality
\end{tabular}

Assessment points: After enrollment and baseline data collection, patients were randomised to the study group and notified of their groups. Outcomes were assessed at the time of enrollment, and also at 1 and 3 months. ACP completion, hospice admission and mortality were assessed within 6 months of the study hospitalisation.

Resource use/costs 30-day inpatient readmission

Hospice use

Time horizon: Readmission to hospital within 30 days was assessed as well as hospice use within 6 months.
Funding source: This study was funded by the Abbott Northwestern Hospital Foundation.

Declarations of interest among primary researchers: The authors stated that no competing financial interests existed.

Power considerations: Power calculations were done to identify a sample size using mean baseline values of summary scores from 26 pilot study patients for each of the three study data collection instruments. Calculations assumed an alpha of 0.05 and $80 \%$ power. Results indicated a sample size of 500 ( 250 per group) would be sufficient to detect an effect size (Cohen's d) of 0.25 , which equates to minimum detectable mean differences between intervention and control groups of 1.5 points in the PHQ- 9 , 6.4 points in the MLHF Questionnaire, and 3.3 points in the ESAS.

\section{Risk of bias}

Bias Authors' judgement Support for judgement

Random sequence genera- Unclear risk tion (selection bias)
Quote from main publication: "patients hospitalised with acute HF were randomised to receive a PC consult with follow-up as determined by provider or standard care".

Comment: there was no description of the random sequence generation process.

Allocation concealment $\quad$ Unclear risk $\quad$ Allocation concealment was not described.
(selection bias)

Blinding of participants

High risk

Quote from main publication: "after enrollment and baseline data collection, and personnel (perforpatients were immediately randomised to the study group and notified of

The effectiveness and cost-effectiveness of hospital-based specialist palliative care for adults with advanced illness and their caregivers 
Sidebottom 2015 (Continued) mance bias), subjective outcomes whether they were in the intervention or control group so they would know whether to expect a visit from a palliative care provider".

Comment: high risk due to lack of blinding
Blinding of participants Low risk and personnel (performance bias), objective outcomes
Quote from main publication: "After enrollment and baseline data collection, patients were immediately randomised to the study group and notified of whether they were in the intervention or control group so they would know whether to expect a visit from a palliative care provider".

Comment: participants and personnel were not blinded but this was unlikely to lead to bias in objective outcomes.

Blinding of outcome as- Unclear risk Unclear risk of bias because blinding of outcome assessment was unclear
sessment (detection bias),
subjective outcomes

$\begin{array}{ll}\begin{array}{l}\text { Blinding of outcome as- } \\ \text { sessment (detection bias), } \\ \text { objective outcomes }\end{array} & \text { It was unclear whether there was blinding of outcome assessment. } \\ & \begin{array}{l}\text { Comment: Although blinding of outcome assessment was unclear, this was un- } \\ \text { likely to lead to bias in assessment of objective outcomes. }\end{array}\end{array}$

Incomplete outcome data High risk
(attrition bias)

\begin{abstract}
$\mathrm{N}=65(56 \%)$ completed all surveys in intervention group vs $\mathrm{N}=78(67.2 \%)$ completed all surveys in control group. In the intervention group, $14(12.1 \%)$ patients died, 20 (17.2\%) withdrew/dropped out for other reasons, $15(12.9 \%)$ and $14(12.1 \%)$ surveys were not completed for unknown reasons at 1-month and 3-month data collection, respectively. In the control group, 5 (4.3\%) patients died, 5 (4.3\%) withdrew, $21(18.1 \%)$ and $18(15.5 \%)$ surveys were not completed for unknown reasons at 1-month and 3-month data collection, respectively. Missing data were excluded from the analysis. Overall, there was high attrition (> 10\%) across intervention and control groups.
\end{abstract}

Comment: high risk of bias

\begin{tabular}{lll}
\hline $\begin{array}{l}\text { Selective reporting (re- } \\
\text { porting bias) }\end{array}$ & Unclear risk & Unclear as the protocol was not available \\
\hline Other bias & Low risk & $\begin{array}{l}\text { There was a statistically significant difference in age between intervention and } \\
\text { control groups at baseline. However, the authors adjusted for age, gender and } \\
\text { marital status. }\end{array}$ \\
& $\begin{array}{l}\text { Comment: Given that the authors controlled for the highlighted difference, a } \\
\text { decision was made not to rate down for imbalance bias. Rather a low of bias } \\
\text { was rated. }\end{array}$ \\
\hline Size of study & Unclear risk & $\begin{array}{l}\text { Unclear risk of bias because the number of participants in each group was be- } \\
\text { tween } 50 \text { and } 199\end{array}$ \\
\hline
\end{tabular}

Solari 2018

\section{Study characteristics}

$\begin{array}{ll}\text { Methods } & \text { Design: RCT (multicentre RCT) } \\ \text { Who led PC intervention: Multidisciplinary team } \\ \text { Core team: Physician (neurologist or psychiatrist), a nurse (case manager and team leader), a psychol- } \\ \text { ogist, and a social worker }\end{array}$

The effectiveness and cost-effectiveness of hospital-based specialist palliative care for adults with advanced illness and their caregivers 
Solari 2018 (Continued)

Participants
Country and regions: Italy: Milan, Rome and Catania

Recruitment: January 2015 to November 2015. Patients were recruited from three Italian centres.

Inclusion criteria: Participants were non-institutionalised adults (age $\geq 18$ years) with severe multiple sclerosis (MS) and their primary carers. Other patient inclusion criteria were primary or secondary progressive MS. Expanded Disability Status Scale (EDSS) score $\geq 8.0$, complex symptoms, and $\geq 2$ unmet care needs. The carer was his or her next of kin and was designated by the patient except for patients with severe cognitive compromise.

Exclusion criteria: Hospitalised/institutionalised patients, patients already receiving palliative care and dyads living out of study area

Number of adult-carer dyads: $\mathrm{N}=78$ (52 intervention and 26 control)

Diseases: Severe multiple sclerosis

Patient characteristics: Mean age (SD): 60.5 (9.7) years in intervention group, 56.8 (9.5) years in control group. $62 \%$ females in intervention and $46 \%$ females in control group

Carer characteristics: Mean age (SD): 60.1 (13.9) years in intervention group, 60.8 (11.1) years in control group. $62 \%$ females in intervention and $61 \%$ females in control group

Deaths at end of study ( $\mathrm{N}(\%)): \mathrm{N}=4: 4(7.7 \%)$ intervention and $0(0 \%)$ in control group

Patient withdrawals/other dropouts $(\mathrm{N}(\%)): \mathrm{N}=5: 3(5.8 \%)$ intervention and $2(7.7 \%)$ control

Carer withdrawals/other dropouts ( $(\%)): N=4: 2(3.8 \%)$ intervention and $2(7.7 \%)$ control

Name: Home-based palliative approach (HPA) combined with usual care vs usual care

Type: Specialist palliative care. Nurses of the Milan and Rome centres had degrees and worked fulltime in palliative care; the Catania nurse attended a week-long individual training course. Prior to study start, all team members were trained in the HPA intervention.

Service base: Three Italian centres

Team: Each centre had a HPA team consisting of a physician (neurologist or psychiatrist), a nurse (case manager and team leader), a psychologist, and a social worker.

Intervention condition: After a comprehensive assessment of the dyad needs based on direct observation and on visit 1 information, the HPA team defined the contents of the intervention, involving the dyad and the patient caring physician. Subsequently, the team verified programme implementation and reviewed it as necessary. The team was not on call for dyads. In the event of emergencies, dyads contacted the patient caring physician or emergency medical services. All team activities were recorded in the PeNSAMI patient study record, which was kept at the patient's home and available to all health professionals/ unpaid caregivers. Three and six months after trial initiation, the HPA team met again to share experiences, fine-tune the protocol, and discuss difficult cases.

Duration: Six months

Control condition: Usual care consisted of the health and social services provided by the Italian $\mathrm{Na}$ tional Health Service in the study area. Dyads assigned to usual care received the three examiner visits (visits 1-3) and the monthly telephone interviews, but not the HPA team visits (except visit 0). At the end of the study, dyads who received usual care were offered the HPA.

\section{Outcomes Primary outcome}

Health-related quality of life assessed using the Schedule for the Evaluation of Individual Quality of Life - Direct Weighting (SEIQoL-DW)

Symptom burden assessed using the Palliative Care Outcome Scale-Symptoms-MS, POS-S-MS

\section{Secondary outcomes}

The effectiveness and cost-effectiveness of hospital-based specialist palliative care for adults with advanced illness and their caregivers (Review)

Copyright (c) 2020 The Cochrane Collaboration. Published by John Wiley \& Sons, Ltd. 
Solari 2018 (Continued)

Quality of life assessed using the European Quality of life Five Dimensions (EQ-5D-3L)

Anxiety and depression assessed using the Hospital Anxiety and Depression Scale (HADS)

Functional independence assessed using the Functional Independence Measure (FIM)

Carer quality of life assessed using the Short Form 36 (SF-36) and the EQ-5D-3L

Carer depression and anxiety assessed using the Hospital Anxiety and Depression Scale (HADS)

Carer burden assessed using the Zarit Burden Interview (ZBI)

Adverse events

Assessment points: After enrollment, patients were randomised (2: 1) to receive HPA or usual care. Outcomes were assessed at baseline, and after three and six months.

Resource use/costs Direct and indirect tangible costs assessed by the MS Foundation Costs Questionnaire (MSCQ)

Time horizon: Six telephone interviews are performed on a monthly basis by a trained interviewer who administered the full MSCQ (at three and six months) and pertinent MSCQ sections (at one, two, four and five months).

Notes $\quad$ Funding source: The Fondazione Italiana Sclerosi Multipla (FISM)

Declarations of interest among primary researchers: A.S. has been a board member of Biogen Idec, Merck Serono and Novartis and has received speaker honoraria from Genzyme, Merck Serono, and Excemed. F.P. received honoraria for speaking activities from Bayer Schering, Biogen Idec, Merck Serono, Novartis, and Sanofi Aventis. He has served as advisory board member of Bayer Schering, Biogen Idec, Merck Serono, and Novartis. M.G.G. has received research funding from Merck Serono and consulting and speaking fees from Biogen Idec. P.C. has been a board member of Biogen Idec, received travel grants from Sanofi Aventis, Biogen Dompe, and Merck Serono. P.Z. and M.A.B. are board members of the Fondazione Italiana Sclerosi Multipla (charitable organisation). All other authors declared that they had no competing interests.

Power considerations: The authors set a power of $80 \%$ for the SEIQOL-DW and $85 \%$ for the POS-SMS. For the POS-S-MS, the authors calculated that a sample size of 62 patients would yield a power of $85 \%$ to detect a mean score change of -0.4 (SD, 0.5$)$ in the HPA group compared to a change of 0.2 (SD, 0.8 ; null hypothesis) in the usual care group, at an a level of 0.05 . Assuming $20 \%$ dropout, 49 patients were required in the HPA group and 25 patients in the usual care group (total sample size 74). For the SEIQOL-DW, the authors calculated that a sample size of 32 patients would yield a power of $80 \%$ to detect a mean score change of 12.1 (SD, 12.8) in the HPA group compared to a change of -7.4 (SD, 19.3) in the usual care group, at an a level of 0.05 . Assuming $20 \%$ dropout, 25 patients were required in the HPA group and 13 in the usual care group (total sample size 38 ).

\section{Risk of bias}

Bias Authors' judgement Support for judgement

Random sequence genera- Low risk tion (selection bias)
Quote from main publication: "dyads were randomly assigned (2:1) to receive HPA or usual care. Allocation to treatment groups was done using a third-party, web-based computerised randomisation procedure with stratified minimisation for expanded disability status scale (EDSS) score, presence of severe cognitive compromise (clinical judgment), and centre".

Comment: probably done
Quote from main publication: "allocation to treatment groups was done using a third-party, web-based computerised randomisation procedure with stratified minimisation for expanded disability status scale (EDSS) score, presence of severe cognitive compromise (clinical judgment), and centre". 


$\begin{array}{lll}\begin{array}{l}\text { Blinding of participants } \\ \text { and personnel (perfor- }\end{array} & \text { High risk } & \text { Participants and personnel were not blinded, only the outcome assessors (ex- } \\ \text { aminers) were. }\end{array}$
mance bias), subjective outcomes

$\begin{array}{ll}\begin{array}{l}\text { Blinding of participants } \\ \text { and personnel (perfor- }\end{array} & \text { Low risk }\end{array}$
mance bias), objective outcomes

Comment: participants and personnel were not blinded (only the outcome assessors 'examiners' were blinded) but this was unlikely to lead to bias in objective outcomes such as serious adverse events.

Blinding of outcome as- $\quad$ Low risk
sessment (detection bias),
subjective outcomes
subjective outcomes
Quote from main publication: "in this randomised, examiner-blind, controlled study, we recruited patients from three Italian centers".

Comment: probably done

\begin{tabular}{lll}
\hline $\begin{array}{l}\text { Blinding of outcome as- } \\
\begin{array}{l}\text { sessment (detection bias), } \\
\text { objective outcomes }\end{array}\end{array}$ & Low risk & $\begin{array}{l}\text { Quote from main publication: "in this randomised, examiner-blind, controlled } \\
\text { study, we recruited patients from three Italian centers". } \\
\text { Comment: probably done }\end{array}$ \\
\hline $\begin{array}{l}\text { Incomplete outcome data } \\
\text { (attrition bias) }\end{array}$ & Low risk & $\mathrm{N}=50$ dyads completers in intervention group vs $\mathrm{N}=26$ dyads completers in \\
All outcomes & control group. In the intervention group, $4(7.7 \%)$ intervention patients died \\
and $3(5.8 \%)$ withdrew/dropped out for other reason. $2(3.8 \%)$ carers in the in- \\
tervention group also withdrew. In the control group, no patients died and 2 \\
$(7.7 \%)$ withdrew consent. $2(7.7 \%)$ carers in the control group also withdrew \\
consent.
\end{tabular}

Comment: low risk of bias because attrition was less than $10 \%$

\begin{tabular}{lll}
\hline $\begin{array}{l}\text { Selective reporting (re- } \\
\text { porting bias) }\end{array}$ & High risk & Some outcomes such as EQ-5D, Core-POS and MSCQ were not reported. \\
\hline Other bias & Unclear risk & $\begin{array}{l}\text { There appeared to be differences between the intervention and control groups } \\
\text { in gender, age and occupation. However, no testing for statistically significant } \\
\text { differences was carried out. }\end{array}$ \\
\hline Size of study & Unclear risk & $\begin{array}{l}\text { Unclear risk of bias because the number of participants in each group was be- } \\
\text { tween } 50 \text { and } 199\end{array}$ \\
\hline
\end{tabular}

Tattersall 2014

\section{Study characteristics}

\begin{tabular}{ll}
\hline Methods & Design: RCT \\
& Who led PC intervention: Nurse-led intervention \\
& Core team: Palliative care nurse consultant. Other members of the team were not described. \\
\hline Participants & $\begin{array}{l}\text { Country and regions: Australia, Department of Medical Oncology, Royal Prince Alfred Hospital (RPAH) } \\
\text { Camperdown, New South Wales }\end{array}$
\end{tabular}

The effectiveness and cost-effectiveness of hospital-based specialist palliative care for adults with advanced illness and their caregivers (Review)

Copyright (c) 2020 The Cochrane Collaboration. Published by John Wiley \& Sons, Ltd. 
Recruitment: April 2003 to January 2005: ambulatory patients with newly detected incurable metastatic cancer attending a medical oncology clinic with a life expectancy of less than 12 months were invited to take part.

Inclusion criteria: Ambulatory patients with newly detected incurable metastatic cancer attending a medical oncology clinic with a life expectancy of less than 12 months

Exclusion criteria: Previous contact with palliative care

Number of patients: $\mathrm{N}=120$ (60 intervention and 60 control)

Diseases: Gastrointestinal cancer (44), lung cancer (23), gynaecological cancer (19), breast cancer (17), prostate cancer (2) and other primary sites (15)

Patient characteristics: Mean age (SD): 63 (11.2) years in intervention group, 64 (11.1) in control group; $47 \%$ female in intervention group, $57 \%$ female in control group

Deaths at end of study (N (\%)): Total N = 70: 39 (65\%) intervention and $31(51.7 \%)$ in control group.

Withdrawals/other dropouts ( $\mathrm{N}(\%))$ : Unclear

Name: Early contact with palliative care services plus standard oncologic care vs standard oncologic care

Early PC (EPC): Early contact with a palliative care nurse consultant with ongoing oncologist care

Type: Specialist palliative care. Included a palliative care nurse consultant

Service base: Medical oncology clinic

Team: Palliative care nurse consultant who served as a link to palliative care services in the hospital and community

Intervention condition: Patients met with a palliative care nurse consultant (PC nurse), a member of the hospital palliative care team. She outlined available palliative care services including advice about symptom control, and she offered to arrange review by a palliative care physician, and provided contact details for the palliative care service. The PC nurse offered to telephone the patient monthly to check on their well-being, or, if the patient preferred, provided her contact details.

Duration: Intervention continued during the lifespan of the patient.

Control condition: Standard oncologic care was given in line with the oncologist's recommendation. Control patients were referred to the palliative care service when recommended by the oncologist.

\section{Primary outcome}

Quality of life assessed using the McGill Quality of Life (MQLL) questionnaire

Symptom severity assessed using the Rotterdam Symptom Checklist (RSC)

Feeling supported assessed using the Supportive Care Needs - Short Form Questionnaire (SCNS - Short)

\section{Secondary outcomes}

End-of-life experiences

Number of lines of chemotherapy

Place of death

Survival

Assessment points: Baseline (after randomisation); the following baseline study questionnaires (MQoL and RSC) were completed monthly whilst the SCNS was to be completed every 4 months until death. 
Tattersall 2014 (Continued)

Resource use/costs Contact with palliative care services: palliative care nurse and physician

Time horizon: From study enrollment to death

Notes

Funding source: Supported by an NHMRC strategic palliative care research grant no: 219141

Declarations of interest among primary researchers: Apart from funding, no further study author disclosure statements were made.

Power considerations: A sample size of 150 patients was sought to provide over $80 \%$ power to detect an effect size of 0.50 (SD) at the two-sided $5 \%$ level of significance based on a two-sample t-test.

\section{Risk of bias}

\begin{tabular}{lll}
\hline Bias & Authors' judgement & Support for judgement \\
\hline $\begin{array}{ll}\text { Random sequence genera- } \\
\text { tion (selection bias) }\end{array}$ & Low risk & $\begin{array}{l}\text { Quote from main publication: "for allocation of the participants, a comput- } \\
\text { er-generated list of random numbers was used". } \\
\end{array}$ \\
& & Comment: probably done
\end{tabular}

\begin{tabular}{ll}
\hline $\begin{array}{l}\text { Allocation concealment } \\
\text { (selection bias) }\end{array}$ & Low risk \\
& $\begin{array}{l}\text { Quote from main publication: "allocation was concealed using sequentially } \\
\text { numbered, opaque sealed envelopes". }\end{array}$ \\
& Comment: probably done
\end{tabular}

\begin{tabular}{|c|c|c|}
\hline $\begin{array}{l}\text { Blinding of participants } \\
\text { and personnel (perfor- } \\
\text { mance bias), subjective }\end{array}$ & High risk & $\begin{array}{l}\text { Quote from trial registry: "masking not used" } \\
\text { Comment: high risk of bias due to lack of blinding }\end{array}$ \\
\hline
\end{tabular}

\section{outcomes}

\begin{tabular}{ll}
\hline $\begin{array}{l}\text { Blinding of participants } \\
\text { and personnel (perfor- }\end{array}$ & Quote from trial registry: "masking not used" \\
$\begin{array}{l}\text { mance bias), objective } \\
\text { outcomes }\end{array}$ & $\begin{array}{l}\text { Comment: although masking was not carried out, this was unlikely to lead to } \\
\text { bias in objective outcomes such as place of death. }\end{array}$
\end{tabular}

\begin{tabular}{lll}
\hline $\begin{array}{l}\text { Blinding of outcome as- } \\
\begin{array}{l}\text { sessment (detection bias), } \\
\text { subjective outcomes }\end{array}\end{array}$ & High risk & $\begin{array}{l}\text { Quote from trial registry: "masking not used" } \\
\text { Comment: high risk of bias due to lack of blinding }\end{array}$ \\
\hline $\begin{array}{l}\text { Blinding of outcome as- } \\
\begin{array}{l}\text { sessment (detection bias), } \\
\text { objective outcomes }\end{array}\end{array}$ & Low risk & Quote from trial registry: "masking not used" \\
& $\begin{array}{l}\text { Comment: although masking was not carried out, this was unlikely to lead to } \\
\text { bias in assessment of objective outcomes such as place of death. }\end{array}$
\end{tabular}

\begin{tabular}{ll}
\hline $\begin{array}{l}\text { Incomplete outcome data } \\
\text { (attrition bias) }\end{array}$ & High risk \\
All outcomes & $\mathrm{N}=11(18.3 \%)$ completers in intervention group vs $\mathrm{N}=18$ (30\%) completers \\
& $\begin{array}{l}\text { in control group. } 10(16.7 \%) \text { patients in the intervention group and } 11(18.3 \%) \\
\text { in control were alive but did not complete the questionnaire battery at } 12\end{array}$ \\
months. The reasons for non-completion were not stated. Overall, there was \\
high attrition across intervention and control groups. \\
Comment: high risk of bias
\end{tabular}

\begin{tabular}{|c|c|c|}
\hline $\begin{array}{l}\text { Selective reporting (re- } \\
\text { porting bias) }\end{array}$ & Low risk & $\begin{array}{l}\text { Study appeared to be free of reporting bias as all the outcomes listed in the tri- } \\
\text { al registry were reported. }\end{array}$ \\
\hline Other bias & Low risk & $\begin{array}{l}\text { The authors reported that there were differences between the groups in the } \\
\text { time since initial cancer diagnosis (mean of } 29 \text { versus } 34 \text { months in the early } \\
\text { referral and standard care groups, respectively), and the oncologists' estimate } \\
\text { of likely survival (e.g. } 11 \text { versus } 20 \text { patients with estimates of }>12 \text { months like- }\end{array}$ \\
\hline
\end{tabular}


Comment: given that the authors controlled for the highlighted differences, a decision was made not to rate down for imbalance bias. Rather a low risk of bias was rated.

\begin{tabular}{ll}
\hline Size of study Unclear risk & $\begin{array}{l}\text { Unclear risk of bias because the number of participants in each group was be- } \\
\text { tween } 50 \text { and } 199\end{array}$
\end{tabular}

Temel 2010

\section{Study characteristics}

\begin{tabular}{ll}
\hline Methods & Design: RCT \\
& Who led PC intervention: Multidisciplinary team \\
Core team: Board-certified palliative care physicians/advanced practice nurses
\end{tabular}

Participants Country and regions: USA, Massachusetts

Recruitment: June 2006 to July 2009. Patients who presented to the outpatient thoracic oncology clinic were invited by their medical oncologists to enroll in the study. Physicians were encouraged, but not required, to offer participation to all eligible patients; no additional screening or recruitment measures were used.

Inclusion criteria: Patients were eligible to participate if they had pathologically confirmed metastatic non-small-cell lung cancer diagnosed within the previous 8 weeks and an Eastern Cooperative Oncology Group (ECOG) performance status of 0,1 , or 2 and were able to read and respond to questions in English.

Exclusion criteria: Patients who were already receiving care from the palliative care service were not eligible..

Number of patients: $\mathrm{N}=151$ (77 intervention and 74 control)

Diseases: Metastatic non-small-cell lung cancer

Patient characteristics: Mean age (SD): 64.98 (9.73) years in intervention group, 64.87 (9.41) years in control; 42 (55\%) female in intervention group, 36 (49)\% female in control

Deaths at end of study $(\mathrm{N}(\%)): \mathrm{N}=27: 10(13 \%)$ intervention and $17(23 \%)$ control

Withdrawals/other dropouts ( $\mathrm{N}(\%)): \mathrm{N}=17: 7(9.1 \%)$ intervention and $10(13.5 \%)$ control

Early PC (EPC): Patients were eligible to participate if they had pathologically confirmed metastatic non-small-cell lung cancer diagnosed within the previous 8 weeks.

Type: Specialist palliative care. Included board-certified palliative care physicians and advanced-practice nurses

Service base: Massachusetts General Hospital (MGH), Boston

Team: Board-certified palliative care physicians and advanced practice nurses

Intervention condition: Patients met with a member of the palliative care team, which consisted of board-certified palliative care physicians and advanced-practice nurses, within 3 weeks after enrollment and at least monthly in the outpatient setting until death. Additional visits with the palliative care 
Temel 2010 (Continued)

service were scheduled at the discretion of the patient, oncologist, or palliative care provider. General guidelines for the palliative care visits in the ambulatory setting were adapted from the National Consensus Project for Quality Palliative Care. Using a template in the electronic medical record, palliative care clinicians documented the care they provided according to these guidelines with particular attention to assessing physical and psychosocial symptoms, establishing goals of care, assisting with decision-making regarding treatment, and co-ordinating care on the basis of the individual needs of the patient. All the participants continued to receive routine oncologic care throughout the study period.

Duration: Intervention group patients met with a member of the palliative care team within 3 weeks after enrollment and at least monthly thereafter in the outpatient setting until death.

Control condition: Patients who were randomly assigned to standard care were not scheduled to meet with the palliative care service unless a meeting was requested by the patient, the family, or the oncologist.

\section{Outcomes Primary outcome}

Change in quality of life (QoL) from baseline to week 12 assessed using the Functional Assessment of Cancer Therapy-Lung (FACT-L) scale

\section{Secondary outcomes}

Mood assessed using the Hospital Anxiety and Depression Scale (HADS) and Patient Health Questionnaire-9 (PHQ-9).

Survival

Location of death

Cost analysis

Assessment points: Participants completed baseline questionnaires before randomisation. Follow-up assessments of quality of life and mood were performed at 12 weeks. Participants who had no scheduled clinic visits within this period received the questionnaires by mail.

Resource use/costs

Number of palliative care visits

Use of health services and end-of-life care including anticancer therapy, medication prescriptions, referral to hospice, hospital admissions, emergency department visits

Time horizon: Study enrollment until death or date of censoring (Dec 1, 2009)

Data sources: Data were collected from the electronic medical record on the use of health services and end-of-life care, including anticancer therapy, medication prescriptions, referral to hospice, hospital admissions, emergency department visits, and the date and location of death.

Analytical perspective: Not clear

Funding source: Funded by an American Society of Clinical Oncology Career Development Award and philanthropic gifts from the Joanne Hill Monahan Cancer Fund and Golf Fights Cancer.

Declarations of interest among primary researchers: Dr. Temel reported receiving payment for developing continuing medical education (CME) programs from Informedical; and Dr. Lynch served on the board of Infinity Pharmaceuticals, receiving consulting fees from Roche, Boehringer Ingelheim, Merck, AstraZeneca, Bristol-Myers Squibb, and Sanofi-Aventis, royalties from Partners HealthCare, and payment for developing CME programs from Informedical. No other potential conflict of interest relevant to this article was reported.

Power considerations: The authors estimated that with 120 patients, the study would have $80 \%$ power to detect a significant between-group difference in the change in the TOI score from baseline to 12 weeks, with a medium effect size of $0.5 \mathrm{SD}$. The protocol was amended to allow for the enrollment of an additional 30 participants in order to compensate for the loss of any patients to follow-up.

The effectiveness and cost-effectiveness of hospital-based specialist palliative care for adults with advanced illness and their caregivers 
Temel 2010 (Continued)

Risk of bias

\begin{tabular}{lll}
\hline Bias & Authors' judgement & Support for judgement \\
\hline $\begin{array}{l}\text { Random sequence genera- } \\
\text { tion (selection bias) }\end{array}$ & Unclear risk & $\begin{array}{l}\text { Quote from main publication: "eligible patients were enrolled within } 8 \text { weeks } \\
\text { after diagnosis and were randomly assigned to one of the two groups in a 1:1 } \\
\text { ratio without stratification". }\end{array}$ \\
& $\begin{array}{l}\text { Comment: there was insufficient information about the random sequence gen- } \\
\text { eration process. }\end{array}$
\end{tabular}

\begin{tabular}{|c|c|c|}
\hline $\begin{array}{l}\text { Allocation concealment } \\
\text { (selection bias) }\end{array}$ & Unclear risk & Allocation concealment was not described. \\
\hline $\begin{array}{l}\text { Blinding of participants } \\
\text { and personnel (perfor- } \\
\text { mance bias), subjective } \\
\text { outcomes }\end{array}$ & High risk & $\begin{array}{l}\text { Quote from main publication: "We enrolled ambulatory patients with newly di- } \\
\text { agnosed metastatic non-small-cell lung cancer in a nonblinded, randomised, } \\
\text { controlled trial of early palliative care integrated with standard oncologic care, } \\
\text { as compared with standard oncologic care alone". }\end{array}$ \\
\hline & & Comment: high risk of bias due to lack of blinding \\
\hline
\end{tabular}

\begin{tabular}{|c|c|c|}
\hline $\begin{array}{l}\text { Blinding of participants } \\
\text { and personnel (perfor- } \\
\text { mance bias), objective } \\
\text { outcomes }\end{array}$ & Low risk & $\begin{array}{l}\text { Quote from main publication: "We enrolled ambulatory patients with newly di- } \\
\text { agnosed metastatic non-small-cell lung cancer in a nonblinded, randomised, } \\
\text { controlled trial of early palliative care integrated with standard oncologic care, } \\
\text { as compared with standard oncologic care alone". }\end{array}$ \\
\hline
\end{tabular}

outcomes

Comment: there was no blinding but this was unlikely to lead to bias in objective outcomes such as survival.

$\begin{array}{ll}\begin{array}{l}\text { Blinding of outcome as- } \\ \text { sessment (detection bias), } \\ \text { subjective outcomes }\end{array} & \text { High risk }\end{array} \quad \begin{aligned} & \text { Quote from main publication: "We enrolled ambulatory patients with newly di- } \\ & \text { agnosed metastatic non-small-cell lung cancer in a nonblinded, randomised, } \\ & \text { controlled trial of early palliative care integrated with standard oncologic care, } \\ & \text { as compared with standard oncologic care alone". }\end{aligned}$

Comment: high risk of bias due to lack of blinding

Blinding of outcome as- Low risk sessment (detection bias), objective outcomes

Quote from main publication: "We enrolled ambulatory patients with newly diagnosed metastatic non-small-cell lung cancer in a nonblinded, randomised, controlled trial of early palliative care integrated with standard oncologic care, as compared with standard oncologic care alone".

Comment: there was no blinding but this was unlikely to lead to bias in assessment of objective outcomes such as survival.

Incomplete outcome data Low risk (attrition bias)

All outcomes
$\mathrm{N}=60(78 \%)$ completers in intervention group vs $\mathrm{N}=47(64 \%)$ completers in control group. $17(22 \%)$ patients in the intervention group did not complete 12-week follow-up assessment for a number of reasons: died $(n=10)$, transferred care $(n=1)$, form mailed but not returned $(n=1)$, and refused, hospitalised or too ill $(n=5) .27(36 \%)$ in control group did not complete 12-week follow-up assessment for a number of reasons: died $(n=17)$, withdrew $(n=1)$, form mailed but not returned $(n=3)$, and refused, hospitalised or too ill ( $n=$ 6). For intention-to-treat analyses, a conservative method of carrying baseline values forward to account for all missing patient-reported outcome data, including data that were missing owing to death was used.

Comment: low risk of bias was rated because the study used baseline observation-carried-forward to deal with missing patient-reported outcome data.

\begin{tabular}{|c|c|c|}
\hline $\begin{array}{l}\text { Selective reporting (re- } \\
\text { porting bias) }\end{array}$ & High risk & $\begin{array}{l}\text { One of the outcomes, family unpaid caregiver satisfaction, has not been re- } \\
\text { ported in any of the publications for this study. }\end{array}$ \\
\hline
\end{tabular}


Temel 2010 (Continued)

\begin{tabular}{lll} 
Other bias & Low risk & Study appeared to be free of other biases. \\
\hline Size of study & Unclear risk & $\begin{array}{l}\text { Unclear risk of bias because the number of participants in each group was be- } \\
\text { tween } 50 \text { and } 199\end{array}$
\end{tabular}

Temel 2017

\section{Study characteristics}

\begin{tabular}{ll}
\hline Methods & Design: RCT \\
& Who led PC intervention: Multidisciplinary team \\
Core team: Physicians/advanced practice nurses
\end{tabular}

\section{Participants Country and regions: USA, Massachusetts}

Recruitment: May 2011 to July 2015. Study staff screened consecutive patients who presented to the oncology clinics and notified clinicians via email when patients were eligible to participate. Oncology clinicians invited their patients to enroll in the study.

Inclusion criteria: Patients were eligible to participate if they were within 8 weeks of a diagnosis of incurable lung (NSCLC, small-cell, or mesothelioma) or noncolorectal GI (pancreatic, oesophageal, gastric, or hepatobiliary) cancer. Patients were also required to receive their care at Massachusetts General Hospital (MGH), be $\geq 18$ years of age, have no history of therapy for metastatic disease, have an Eastern Cooperative Oncology Group performance status of 0 to 2, and be able to read and respond to questions in English or complete questionnaires with minimal assistance.

Exclusion criteria: Patients who were already receiving palliative care services, needed immediate referral for palliative care or hospice, or who had significant psychiatric or other comorbid disease prohibiting participation

Number of patients: $\mathrm{N}=350$ (175 intervention and 175 control)

Diseases: Lung (non-small-cell, small-cell, neuroendocrine, mesothelioma, epidermal growth factor receptor (EGFR) mutation, anaplastic lymphoma kinase (ALK) translocation) and noncolorectal cancer (pancreatic, oesophageal/GE junction, gastric, hepatobiliary)

Patient characteristics: Mean age (SD): 65.64 (11.26) years in intervention group, 64.03 (10.46) years in control; $48 \%$ female in intervention group, $44 \%$ female in control

Deaths at end of study (N (\%)): $N=59: 27(15.4 \%)$ intervention and $32(18.3 \%)$ control

Withdrawals/other dropouts (N (\%)): N = 49: 30 (17.1\%) intervention and 19 (10.9\%) control

Early PC (EPC): Patients were eligible to participate if they were within 8 weeks of a diagnosis of incurable lung (NSCLC, small-cell, or mesothelioma) or noncolorectal GI (pancreatic, oesophageal, gastric, or hepatobiliary) cancer.

Type: Specialist palliative care. Included palliative care clinicians

Service base: Massachusetts General Hospital (MGH), Boston

Team: Physicians and advanced practice nurses

Intervention condition: Patients assigned to early palliative care met with a member of the outpatient palliative care team within 4 weeks of enrollment and at least once per month until death. Consisting of physicians and advanced practice nurses, the MGH outpatient team practices per guidelines of the 
Temel 2017 (Continued)

National Consensus Project for Quality Palliative Care. Palliative care clinicians contacted patients via telephone when an in-person visit was not possible. The patient, oncologist, or palliative care clinician could schedule additional palliative care visits at their discretion. Finally, for patients who were admitted to MGH, the inpatient palliative care team observed them throughout their hospitalisation.

Duration: Intervention continued at least once per month until the patient's death.

Control condition: Patients assigned to usual oncology care were able to meet with a palliative care clinician only upon request by the oncologist, patient, or family. All patients, regardless of group assignment, continued to receive routine oncology care throughout the study period.

Primary outcome
Change in quality of life (QoL) from baseline to week 12 assessed using the Functional Assessment of
Cancer Therapy - General (FACT-G) scale
Secondary outcomes
Change in QoL from baseline to week 24 assessed using the Functional Assessment of Cancer Therapy
General (FACT-G) scale
Depression assessed using the Patient Health Questionnaire-9 (PHQ-9)
Anxiety and depression assessed using the Hospital Anxiety and Depression Scale (HADS)
Differences in end-of-life communication assessed using the Prognosis and Treatment Perceptions
Questionnaire

Assessment points: Patients completed a demographic questionnaire and baseline self-report measures after providing written informed consent and before random assignment. Follow-up assessments occurred at 12 weeks and 24 weeks.

Resource use/costs

Number of palliative care visits

Time horizon: Study enrollment to 24 weeks
Funding source: Supported by the National Institutes of Health Grant No. NCT01401907 and National Institute of Nursing Research Grant No. R01- NR012735

Declarations of interest among primary researchers: Four of the authors provided disclosure information.

Power considerations: The primary outcome was change in FACT-G score from baseline to 12 weeks; a 4- to 5-point change in FACT-G score is considered clinically meaningful. The authors estimated that with 280 patients, the study would have $80 \%$ power to detect a 4-point difference in the change in FACT-G scores from baseline to 12 weeks between study groups (with $P<0.05$ ). Given the rate of missing data observed in a previous study by the authors, the sample size was increased to 350 patients.

\section{Risk of bias}

\begin{tabular}{lll}
\hline Bias & Authors' judgement & Support for judgement \\
\hline $\begin{array}{l}\text { Random sequence genera- } \\
\text { tion (selection bias) }\end{array}$ & Low risk & $\begin{array}{l}\text { Quote from main publication: "the Office of Data Quality randomly assigned } \\
\text { patients in a 1:1 fashion to receive early integrated PC and oncology care ver- } \\
\text { sus usual oncology care, stratified by cancer type, using a computer-generated } \\
\text { number sequence, which was concealed until after group assignment". }\end{array}$ \\
& \begin{tabular}{l} 
Comment: probably done \\
\hline $\begin{array}{l}\text { Allocation concealment } \\
\text { (selection bias) }\end{array}$ \\
Low risk
\end{tabular} & $\begin{array}{l}\text { Quote from main publication: "the Office of Data Quality randomly assigned } \\
\text { patients in a 1:1 fashion to receive early integrated PC and oncology care ver- } \\
\text { sus usual oncology care, stratified by cancer type, using a computer-generated } \\
\text { number sequence, which was concealed until after group assignment". }\end{array}$ \\
\hline
\end{tabular}

The effectiveness and cost-effectiveness of hospital-based specialist palliative care for adults with advanced illness and their caregivers 
Blinding of participants and personnel (performance bias), subjective outcomes
High risk

Quote from main publication: "we enrolled patients with newly diagnosed incurable cancers from Massachusetts General Hospital (MGH) in a nonblinded, randomized trial of early palliative integrated with oncology care compared with usual oncology care".

Comment: high risk of bias due to lack of blinding
Blinding of outcome as- $\quad$ High risk
sessment (detection bias),

subjective outcomes
Quote from main publication: "we enrolled patients with newly diagnosed incurable cancers from Massachusetts General Hospital (MGH) in a nonblinded, randomized trial of early PC integrated with oncology care compared with usual oncology care".

Comment: high risk of bias due to lack of blinding

\section{Incomplete outcome data High risk} (attrition bias)

All outcomes
$\mathrm{N}=118(67.4 \%)$ completers in intervention group vs $\mathrm{N}=124(70.9 \%)$ completers in control group. $30(17.1 \%)$ patients in the intervention group did not complete 24-week follow-up assessment for a number of reasons: hospitalised/hospice $(n=8)$, transferred care $(n=9)$, withdrew consent $(n=7)$, mailed and not returned $(n=1)$ and refused $(n=5) .19(10.9 \%)$ in control group did not complete 24-week follow-up assessment for a number of reasons: hospitalised/hospice $(n=7)$, transferred care $(n=3)$, withdrew consent $(n=1)$, mailed and not returned $(n=2)$, refused $(n=5)$ and clinic staff missed patient $(n=1)$. Missing data were excluded from the analysis.

Comment: high risk of bias
Quote from main publication: "we also compared patient-reported outcomes between the two groups by using a terminal decline joint modeling approach, which models the trend in outcomes backward from death, rather than prospectively from enrollment. We did not prespecify the use of this approach in our protocol as it was first published in 2013, after initiation of this study".

Comment: post hoc analysis

\begin{tabular}{ll}
\hline Other bias & $\begin{array}{l}\text { Quote from main publication: "to examine QoL and mood we used indepen- } \\
\text { dent-samples t-tests and analysis of covariance (ANCOVA) models that con- } \\
\text { trolled for baseline criterion scores and potential confounders such as age and } \\
\text { comorbidity, which were imbalanced between groups and associated with } \\
\text { outcomes of interest". The authors therefore controlled for baseline criterion } \\
\text { scores and potential confounders. }\end{array}$ \\
& $\begin{array}{l}\text { Comment: Given that the authors controlled for the highlighted differences, } \\
\text { a decision was made not to rate down for imbalance bias. Rather a low risk of } \\
\text { bias was rated. }\end{array}$ \\
\hline Size of study & Unclear risk of bias because the number of participants in each group was be- \\
& tween 50 and 199
\end{tabular}

Vanbutsele 2018

\section{Study characteristics}

\begin{tabular}{ll}
\hline Methods & Design: RCT \\
Who led PC intervention: Nurse-led intervention
\end{tabular}

The effectiveness and cost-effectiveness of hospital-based specialist palliative care for adults with advanced illness and their caregivers 
Recruitment: April 2013 to February 2016. Study recruited patients with advanced cancer from the Medical Oncology, Thoracic Oncology, and Digestive Oncology departments of Ghent University Hospital in Flanders, Belgium. Patients were identified for recruitment by a trained clinical research assistant and the treating oncologists. Both outpatients and inpatients were considered for inclusion. Oncologists described the study to patients and all participants provided written informed consent.

Inclusion criteria: Eligible patients were $\geq 18$ years, and had an advanced cancer diagnosis due to a solid tumour, a European Cooperative Oncology Group performance status of 0 - 2, an estimated life expectancy of 12 months, were within the first 12 weeks of a new primary tumour or had a diagnosis of progression, and were able to read and respond to questions in Dutch. Patients recruited from a hospital other than Ghent University Hospital had to be within the first 12 weeks of a disease progression event or still on first-line treatment.

Exclusion criteria: Patients with haematological malignancies because they have a less predictable disease trajectory than those presenting with solid tumours. Patients who had received one or more palliative care consultations at any time, or one palliative care consultation in the 6 months before diagnosis or disease progression. Patients deemed cognitively impaired at the discretion of the oncologist and psychologist

Number of patients: $\mathrm{N}=186$ (92 intervention and 94 control)

Diseases: Gastrointestinal [pancreas $(N=25)$, biliary tract $(N=11)$, oesophagus $(N=6)$, gastro-oesophageal $(N=7)$, gastric $(N=7)$, colorectal $(N=15)]$, lung $(N=51)$, head and neck $(N=19)$, breast $(N=$ 14), melanoma $(N=15)$, genitourinary [prostate $(N=6)$, bladder $(N=4)$, kidney $(N=6)$ ]

Patient characteristics: Median age (IQR): 64.5 (57.3 - 71) years in intervention group, 65 (57 - 71) years in control; $36 \%$ female in intervention group, $27 \%$ female in control

Deaths at end of study ( $\mathrm{N}(\%)): \mathrm{N}=47: 22(23.4 \%)$ intervention and $25(27.2 \%)$ control

Withdrawals/other dropouts (N (\%)): $\mathrm{N}=106: 44(47.8 \%)$ intervention and 62 (66\%) control

Interventions
Name: Systematic early integration of palliative care in oncological care vs usual oncology care

Early PC (EPC): Patients were within the first 12 weeks of a new primary tumour or had a diagnosis of progression. Patients recruited from a hospital other than Ghent University Hospital had to be within the first 12 weeks of a disease progression event or still on first-line treatment.

Type: Specialist palliative care. Included specialist palliative care nurse and palliative care physician. The palliative care physician involved in this study has a specialty degree in anaesthesiology and was a trained professional in specialised palliative care.

Service base: Medical Oncology, Thoracic Oncology, and Digestive Oncology departments of Ghent University Hospital in Flanders, Belgium

Team: Palliative care physicians and palliative care nurses

Intervention condition: Patients had a first consultation with a specialised palliative care nurse within 3 weeks of enrollment. Hospital consultations between patients and palliative care nurses were organised monthly until the patient's death, and coincided with planned oncological staff meetings. The palliative care physician visited patients after referral from the palliative care nurse. The early palliative care intervention used a previously published study (Temel 2010) as a guide, with the intervention consisting of four major components:

(1) training sessions for palliative care nurses and physician about cancer treatments, use of the intervention documents and the administration of the Edmonton Symptom Assessment Scale (ESAS)

(2) semi-structured, monthly palliative care consultations by palliative care nurses allowed for individualised patient care. These consultations focussed on illness understanding and perception, symptom 
Vanbutsele 2018 (Continued)

burden, psychological coping, spiritual coping, and medical decision-making. If needed, patients were referred to other health-care professionals.

(3) Monthly symptom assessments using ESAS by palliative care nurses

(4) Integration of palliative care into oncological care through participation of palliative care nurses in the weekly multidisciplinary oncology meetings and their reporting in the electronic patient file.

Duration: Hospital consultations between patients and palliative care nurses were organised monthly until the patient's death.

Control condition: Standard oncology care was provided by a multidisciplinary team, including oncologists, other medical specialists, psychologists, social workers, dieticians, and specialist nurses. In routine clinical practice, the palliative care team is only involved on demand, often late in the disease trajectory, and their services are not systematically offered to all patients from oncology departments. All patients could have further consultations with the specialist nurse, dietician, and psychologist on demand.

\section{Primary outcomes}

Quality of life assessed using the European Organisation for Research and Treatment of Cancer Quality of Life Questionnaire Core 30 items (EORTC QLQ-C30)

\section{Secondary outcomes}

Quality of life assessed using the McGill Quality of Life (MQOL) Questionnaire

Patient's mood assessed using the Hospital Anxiety and Depression Scale (HADS)

Depression assessed using the Patient Health Questionnaire-9 (PHQ-9)

Understanding of illness assessed using the questionnaire used by Temel and colleagues

European Organisation for Research and Treatment of Cancer Quality-of-Life Questionnaire Core 30 items (EORTC QLQ-C30 functioning and symptoms scales)

McGill Quality of Life (MQOL) Questionnaire functioning scales

Overall survival

\section{Assessment points:}

Enrolled patients completed follow-up questionnaires administered by the data manager at 12,18 , and 24 weeks, and 6 weekly thereafter until death. Patients who were not willing or able to complete the questionnaires at the hospital received them by mail.

Resource use/costs

Number of consultations with the palliative care team

Frequency of contact with a psychologist, dietician, social worker, or a specialist nurse

Time horizon: from study enrollment to 24 weeks

Notes

Funding source: Research Foundation Flanders, Flemish Cancer Society (Kom Op Tegen Kanker).

Declarations of interest among primary researchers: The authors declared no competing interests.

Power considerations: With 59 patients in each group, the study had a power of $80 \%$ to detect a 12 point difference in the global health status/quality of life scale EORTC QLQ-C30 score from baseline to 12 weeks between both study groups assuming a SD of 23.0. Based on a previous study, the authors expected an overall dropout rate of $35 \%$, accounting for the planned inclusion of 182 patients.

\section{Risk of bias}

The effectiveness and cost-effectiveness of hospital-based specialist palliative care for adults with advanced illness and their caregivers 
Vanbutsele 2018 (Continued)

\section{Bias Authors' judgement Support for judgement}

Random sequence genera- Low risk tion (selection bias)
Quote from main publication: "patients were randomly assigned (1:1) to either systematic early integration of palliative care in oncological care or to usual oncological care. We generated the randomisation list using the permuted block method (block size of 4), stratified according to treating department. Computer generated sequences were created by a statistician using the PLAN procedure in SAS (version 9.4)".

Comment: probably done
Quote from main publication: "the allocation sequence was only available to an independent administrative assistant and was unknown to the investigators. The research assistant enrolled the patients and obtained patient study numbers and the corresponding allocation from the administrative assistant".

Comment: probably done

\begin{tabular}{ll}
$\begin{array}{l}\text { Blinding of participants } \\
\text { and personnel (perfor- } \\
\text { mance bias), subjective }\end{array}$ & High risk \\
outcomes & $\begin{array}{l}\text { Quote from main publication: "in this non-blinded, randomized, controlled tri- } \\
\text { al..." }\end{array}$ \\
\hline
\end{tabular}

$\begin{array}{ll}\begin{array}{l}\text { Blinding of participants } \\ \text { and personnel (perfor- } \\ \text { mance bias), objective }\end{array} & \text { Low risk } \\ \begin{array}{l}\text { outcomes } \\ \text { al..." }\end{array} & \begin{array}{l}\text { Comment: participants and personnel were not blinded but this was unlikely } \\ \text { to lead to bias in objective outcomes such as survival. }\end{array}\end{array}$

\begin{tabular}{|c|c|c|}
\hline $\begin{array}{l}\text { Blinding of outcome as- } \\
\text { sessment (detection bias), } \\
\text { subjective outcomes }\end{array}$ & High risk & $\begin{array}{l}\text { Quote from main publication: "masking those individuals giving the interven- } \\
\text { tion, those assessing the outcomes and analysing the data was not possible". } \\
\text { Comment: high risk of bias due to lack of blinding }\end{array}$ \\
\hline
\end{tabular}

Blinding of outcome as- Low risk Quote from main publication: "masking those individuals giving the interven-
sessment (detection bias), $\quad$ tion, those assessing the outcomes and analysing the data was not possible". objective outcomes Comment: outcome assessors were not blinded but this was unlikely to lead to bias in the assessment of objective outcomes such as survival.

Incomplete outcome data Low risk
(attrition bias)

$\mathrm{N}=51(55.4 \%)$ completers in intervention group vs $\mathrm{N}=45$ (47.9\%) completers All outcomes in control group. Multiple imputation was carried out for missing data and they were included in the analysis.

Comment: given the fact that multiple imputations were carried out, a low risk of bias was rated.

\section{Selective reporting (re- Unclear risk} porting bias)

\begin{abstract}
Quote from main publication: "Additional protocol-specified secondary endpoints not reported here include patient illness trajectory and end-of-life care; unpaid caregiver's mood, understanding of the patient's illness, satisfaction with care, and impact on quality of life; and impact on advance-care planning and end-of-life decision-making, as reported by the patient's physician". However, the authors stated that "Data regarding whether physicians discussed advance-care planning and end-of-life care with their patients, patient quality of life near death, and unpaid caregiver-reported outcomes will be reported elsewhere".
\end{abstract}

Comment: the above highlighted secondary endpoints were not reported but will be reported subsequently. 
Vanbutsele 2018 (Continued)

\begin{tabular}{|c|c|c|}
\hline Other bias & Low risk & Study appeared to be free of other biases. \\
\hline Size of study & Unclear risk & $\begin{array}{l}\text { Unclear risk of bias because the number of participants in each group was be- } \\
\text { tween } 50 \text { and } 199\end{array}$ \\
\hline
\end{tabular}

Wallen 2012

\section{Study characteristics}

Design: RCT
Who led PC intervention: Multidisciplinary team
Core team: Full-time attending physicians/nurse practitioners/nurse thanatologist/physician fellow in
Hospice and Palliative medicine. The extended team included spiritual ministry, social work, recreation
therapy, counselling, nutrition, acupuncture, acupressure, massage, reiki, rehabilitation medicine.

Participants

Country and regions: USA, Bethesda, Maryland

Recruitment: Patients with advanced malignancies who were undergoing surgical procedures in National Cancer Institute ( $\mathrm{NCl})$ Surgery Branch clinical trials were recruited.

Inclusion criteria: Not described

Exclusion criteria: Not described

Number of patients: $\mathrm{N}=152$ (76 intervention and 76 control)

Diseases: Advanced cancer

Patient characteristics: Mean age (SD): 52.43 (10.42) years in intervention group, 52.38 (3.01) in control group. Gender distribution in the intervention and control group was not described.

Deaths at end of study (N (\%)): Number of deaths was not provided.

Withdrawals/other dropouts (including deaths) ( $(\%)): \mathrm{N}=102: 54(71.1 \%)$ intervention and 48 $(63.2 \%)$ control

Interventions

Name: Hospital-based early palliative care vs standard oncology care

Early PC (EPC): Early palliative care was begun postoperatively. The philosophy of the palliative care team was to provide comfort care for symptom burden earlier in the disease process to improve quality of life.

Type: Specialist palliative care. Included professionals with training in palliative care

Service base: National Institutes of Health Clinical Centre, Bethesda

Team: Two full-time attending physicians, three nurse practitioners, a nurse thanatologist, and one physician fellow in Hospice and Palliative medicine. The extended team included spiritual ministry, social work, recreation therapy, counselling, nutrition, acupuncture, acupressure, massage, reiki, rehabilitation medicine.

Intervention condition: The hospital-based pain and palliative care service (PPCS) was a consult team available to all patients who were actively participating in research studies throughout the National Institutes of Health $(\mathrm{NIH})$ Clinical Centre. The patients were seen as inpatients as well as in an outpatient clinic setting. Each consult included a full assessment of pain and other symptoms, what treatments had been implemented, and what were the most bothersome and disruptive to the patient. The consult also covered emotional and spiritual distress. The patient was usually offered varied modalities of treatment, pharmacologic as well as complementary therapies. 
Wallen 2012 (Continued)

Duration: The intervention was provided until 12 months: interviews were conducted pre-surgically and at follow-up visits up to 1 year.

Control condition: Standard pain and symptom management provided to the control group included individual consultations such as nutrition, social work, spiritual ministry, recreation therapy, occupational therapy, physical therapy, and/or clinical psychiatry. If standard pain and symptom management were insufficient to meet the needs of a patient, study participants were permitted to cross over to the treatment arm of the study at the clinical discretion of the attending physician.

Primary outcomes
Pain intensity and unpleasantness assessed using the Gracely Pain Scale
Symptom burden assessed using the Symptom Distress Scale
Secondary outcomes
Mood assessed using the Centre for Epidemiologic Studies - Depression Scale (CES-D)
Social support
Satisfaction with pain and symptom management
Assessment points: After randomisation, face-to-face interviews were conducted prior to surgery,
within the first 24 hrs postoperatively, and during follow-up staging visits at 4-6 weeks, 3, 6, 9, and 12
months. Pain and symptom outcomes were assessed at all time intervals. Secondary outcomes were
assessed less frequently with mood assessed at baseline, 6 and 12 months.

Resource use/costs None reported

Notes $\quad$ Funding source: Not reported

Declarations of interest among primary researchers: Not reported

Power considerations: Not reported

\section{Risk of bias}

Bias Authors' judgement Support for judgement

Random sequence genera- Unclear risk tion (selection bias)

Quote from main publication: "once written consent was obtained, patients were randomized to the standard care (control) or early palliative care (treatment) group".

Comment: unclear as random sequence generation was not well described

\begin{tabular}{|c|c|c|}
\hline $\begin{array}{l}\text { Allocation concealment } \\
\text { (selection bias) }\end{array}$ & Unclear risk & Allocation concealment was not described. \\
\hline $\begin{array}{l}\text { Blinding of participants } \\
\text { and personnel (perfor- } \\
\text { mance bias), subjective } \\
\text { outcomes }\end{array}$ & High risk & $\begin{array}{l}\text { Participants and personnel were not blinded. } \\
\text { Comment: high risk of bias due to lack of blinding }\end{array}$ \\
\hline $\begin{array}{l}\text { Blinding of outcome as- } \\
\text { sessment (detection bias), } \\
\text { subjective outcomes }\end{array}$ & Unclear risk & $\begin{array}{l}\text { Unclear risk of bias was given because blinding of outcome assessment was } \\
\text { not mentioned. }\end{array}$ \\
\hline $\begin{array}{l}\text { Incomplete outcome data } \\
\text { (attrition bias) } \\
\text { All outcomes }\end{array}$ & High risk & $\begin{array}{l}\mathrm{N}=22(28.9 \%) \text { completers in intervention group vs } \mathrm{N}=28(36.8 \%) \text { completers } \\
\text { in control group. In the intervention group } 54 \text { patients dropped out due to } \\
\text { death or disease progression while } 48 \text { patients in the control group dropped } \\
\text { out for these reasons. }\end{array}$ \\
\hline
\end{tabular}

The effectiveness and cost-effectiveness of hospital-based specialist palliative care for adults with advanced illness and their caregivers 
Wallen 2012 (Continued)

Comment: given the high levels of attrition, a high risk of bias was rated.

Selective reporting (re- High risk porting bias)
Data for mood measured using the Centre for Epidemiologic Studies Depression Scale (CES-D) was not presented at baseline and also during follow-up. Also, the protocol included the palliative care outcome scale (POS) as one of the outcome measures but this was not presented in the published study.

\begin{tabular}{lll}
\hline Other bias & Low risk & Study appeared to be free of other biases. \\
\hline Size of study & Unclear risk & $\begin{array}{l}\text { Unclear risk of bias because the number of participants in each group was be- } \\
\text { tween } 50 \text { and } 199\end{array}$ \\
\hline
\end{tabular}

Woo 2019

\section{Study characteristics}

\begin{tabular}{ll}
\hline Methods & Design: RCT \\
& Who led PC intervention: Multidisciplinary team \\
Core team: Nurses and physicians
\end{tabular}

Participants Country and regions: South Korea

Recruitment: Patients who presented to the outpatient cancer clinic between April 2012 and May 2016 were invited by their physicians to enroll in the study; all the physicians in the clinic agreed to approach, recruit, and obtain consent from their patients.

Inclusion criteria: Patients were eligible to participate if they had pathologically confirmed locally advanced or metastatic pancreatic or biliary tract cancer diagnosed within the previous 8 weeks, a Karnofsky performance rating scale $\geq 50 \%$ and cancer-related pain (BPI worst pain score $>3$ ) and/or depression (CES-D > 16).

\section{Exclusion criteria: Not stated}

Number of patients: $\mathrm{N}=288$ (144 intervention and 144 control)

Diseases: Pancreatic or biliary tract cancer

Patient characteristics: Median age (range): 66 (40 - 86) years in intervention group, 67 (42 - 89 ) in control group. 56\% female in intervention group, $55 \%$ female in control

Deaths at end of study (N (\%)): N = 207: 100 (69.4\%) intervention and 107 (74.3\%) control

Withdrawals/other dropouts (N (\%)): N = 48: 25 (17.4\%) intervention and $23(16 \%)$ control

Early PC (EPC): Patients were eligible to participate if they had pathologically confirmed locally advanced or metastatic pancreatic cancer or biliary tract cancer diagnosed within the previous 8 weeks.

Type: Training in palliative care unclear

\section{Service base: National Cancer Centre, Korea}

Team: Nurses and two physician experts in pancreatic cancer or biliary tract cancer

Intervention condition: EPC included: (1) Nursing assessment of pain and depression, (2) pain control based on National Comprehensive Cancer Network (NCCN) guidelines, (3) depression control by psychoeducation and/or consultation with a psychiatric specialist, and (4) patient education. Nursing assessment of pain included a brief evaluation of each patient's mood state with the CES-D. Patients 
Woo 2019 (Continued)

with CES-D scores $>25$ were referred to psychiatric specialists. The interventions was delivered by telephone or during regularly scheduled outpatient care. Follow-up intervention visits or telephone coaching were scheduled daily until BPI worst pain score was $\leq 3$. In addition, telephone calls were triggered when patients reported inadequate symptom improvement, nonadherence to medication, adverse effects, or suicidal ideation, or when patients requested to be contacted.

Duration: Participants were contacted at baseline and at 1, 3, 6, 9, and 12 months.

Control condition: The control group received no formal intervention but were informed of their depressive and pain symptoms. Their screening results were provided to their physician. Usual oncology care (UOC) was directed by an attending physician and consisted of anticancer and symptom control treatments and consultation with psychiatric and pain care specialists. The latter were provided whenever requested, regardless of group assignment.

Outcomes

\section{Primary outcomes}

Pain assessed using the Brief Pain Inventory

Depression assessed using the Centre for Epidemiological Studies - Depression Scale (CES-D)

\section{Secondary outcomes}

Quality of life assessed using the European Organisation for Research and Treatment of Cancer Core Quality of Life questionnaire (EORTC QLQ-C30) Korean version

Sleep disturbance assessed using the Insomnia Severity Index (ISI)

Satisfaction with pain control assessed on a 5 -point scale ( 1 = very good; $5=$ very poor)

Patient and investigator's global assessment

Clinical global impression score assessed using the Clinical Global Impression-Improvement (CGI-I scale)

Survival

Assessment points: Participants were contacted at baseline and at 1, 3, 6, 9 and 12 months.

Resource use/costs None reported

Notes

Funding source: The study was supported by grants from the National Cancer Center, Korea.

Declarations of interest among primary researchers: The authors indicated that they had no potential conflicts of interest.

Power considerations: The target sample size, calculated for a power of $80 \%$ and a corrected twosided alpha of 0.025 and allowing a dropout rate of $18-20 \%$, was 144 per group.

\section{Risk of bias}

Bias Authors' judgement Support for judgement

Random sequence genera- Unclear risk tion (selection bias)

Quote from publication: "patients...were randomised 1:1 within 8 weeks after diagnosis to receive either EPC or usual oncology care. Patients were stratified based on tumor type (PC or BTC) and symptom type (pain only, depression only, and pain and depression)".

Comment: there was insufficient information about the random sequence generation process.

Allocation concealment Unclear risk Allocation concealment was not described.


Woo 2019 (Continued)

Blinding of participants and personnel (performance bias), subjective outcomes
High risk Quote from publication: "lack of blinding is always an issue in clinical research regarding early palliative care for patients with advanced cancer. This bias could not be avoided due to the lack of patient blinding in this prospective, randomised controlled trial".

Comments: participants and personnel were not blinded.
Blinding of participants Low risk and personnel (performance bias), objective outcomes
Quote from publication: "lack of blinding is always an issue in clinical research regarding early palliative care for patients with advanced cancer. This bias could not be avoided due to the lack of patient blinding in this prospective, randomised controlled trial".

Comments: participants and personnel were not blinded but this was unlikely to lead to bias in objective outcomes such as survival.

\begin{tabular}{|c|c|c|}
\hline $\begin{array}{l}\text { Blinding of outcome as- } \\
\text { sessment (detection bias), } \\
\text { subjective outcomes }\end{array}$ & Unclear risk & It was not stated whether outcome assessors were blinded. \\
\hline $\begin{array}{l}\text { Blinding of outcome as- } \\
\text { sessment (detection bias), } \\
\text { objective outcomes }\end{array}$ & Low risk & $\begin{array}{l}\text { It was not stated whether outcome assessors were blinded but this was unlike- } \\
\text { ly to lead to bias in objective outcomes such as survival. }\end{array}$ \\
\hline $\begin{array}{l}\text { Incomplete outcome data } \\
\text { (attrition bias) } \\
\text { All outcomes }\end{array}$ & Low risk & $\begin{array}{l}\mathrm{N}=19(13.2 \%) \text { completers in intervention group vs } \mathrm{N}=14(9.7 \%) \text { completers } \\
\text { in control group. In the intervention group } 125 \text { patients }(86.8 \%) \text { dropped out } \\
\text { due to death and other reasons while } 130 \text { patients }(90.3 \%) \text { in the control group } \\
\text { dropped out for these reasons. } \\
\text { Comment: given that there was imputation of missing values, a low risk of bias } \\
\text { was rated. }\end{array}$ \\
\hline $\begin{array}{l}\text { Selective reporting (re- } \\
\text { porting bias) }\end{array}$ & Unclear risk & Study protocol was not available. \\
\hline Other bias & Low risk & Study appeared to be free of other biases. \\
\hline Size of study & Unclear risk & $\begin{array}{l}\text { Unclear risk of bias because the number of participants in each group was be- } \\
\text { tween } 50 \text { and } 199\end{array}$ \\
\hline
\end{tabular}

ACP: advanced care planning

$\mathrm{AL}$ : acute leukemia

BIS: Breathlessness Intervention Service

BPI: Brief Pain Inventory

CHF: chronic heart failure

$\mathrm{Cl}$ : Confidence interval

COPD: chronic obstructive pulmonary disease

CT: Computerised Tomography

DVD: Digital Versatile Disc

D12: Dyspnoea 12 breathlessness scale

EGFR: epidermal growth factor receptor

EPC: Early Palliative Care

GI: Gastrointestinal

GP: General Practitioner

GU: Genitourinary

h: hours

HSPC: Hospital Specialist Palliative Care

ICD: International Classification of Disease

ICU: intensive care unit

IHPC: In Home Palliative Care

The effectiveness and cost-effectiveness of hospital-based specialist palliative care for adults with advanced illness and their caregivers 
ILD: Interstitial Lung Disease

km: kilometers

$\mathrm{mg} / \mathrm{d}$ : milligram/day

MICU: medical intensive care unit

MRC: Modified Research Council breathlessness scale

NIHR: National Institute for Health Research

NSCLC: non-small cell lung cancer

NYHA: New York Heart Association

$\mathrm{N} / \mathrm{n}$ : Number

PC: palliative care

$\mathrm{PCT}$ : palliative care team

$\mathrm{pg} / \mathrm{mL}$ : picogram/milliliter

QoL: quality of life

$\mathrm{RCT}$ : randomised controlled trial

SD: Standard deviation

SPC: specialist palliative care

UCLA: University of California, Los Angeles

v: versus

WHO: World Health Organization

y / yrs: years

Characteristics of excluded studies [ordered by study ID]

\begin{tabular}{|c|c|}
\hline Study & Reason for exclusion \\
\hline Bakitas 2017 & This study was not a randomised controlled trial. \\
\hline Berglund 2019 & Intervention was not provided by a multidisciplinary team. \\
\hline Bonsignore 2018 & This study was not a randomised controlled trial. \\
\hline Brims 2019 & The control group included palliative care as part of routine usual care. \\
\hline Do 2017 & $\begin{array}{l}\text { Study compared a psychosocial intervention combined with early palliative care vs early pallia- } \\
\text { tive care vs standard cancer treatment. The control group included palliative care as part of routine } \\
\text { usual care. }\end{array}$ \\
\hline Fischer 2019 & Intervention was not provided by a multidisciplinary team. \\
\hline Hanks 2002 & $\begin{array}{l}\text { This study compared the effectiveness of a multidisciplinary specialist palliative care team (PCT) } \\
\text { ('full-PCT') with limited telephone advice ('telephone-PCT', the control group). The control group } \\
\text { included palliative care as part of routine usual care. }\end{array}$ \\
\hline Hartman 2019 & $\begin{array}{l}\text { This study was not a randomised controlled trial as it used an alternate day assignment to allocate } \\
\text { participants to the intervention and control groups. }\end{array}$ \\
\hline Hoek 2017 & $\begin{array}{l}\text { This study compared weekly teleconsultations from a hospital-based specialist palliative care con- } \\
\text { sultation team (SPCT) with palliative home care provided by GPs (supported by the SPCT) accord- } \\
\text { ing to the standard referral procedures. The control group included palliative care as part of rou- } \\
\text { tine usual care. }\end{array}$ \\
\hline Jordhoy 2001 & This study did not conceal its allocation sequence. It was a cluster-RCT. \\
\hline Kimbell 2018 & This study was not a randomised controlled trial. \\
\hline Maltoni 2016 & The intervention was not provided by a multidisciplinary team. \\
\hline
\end{tabular}




\begin{tabular}{|c|c|}
\hline Study & Reason for exclusion \\
\hline Nordly 2019 & $\begin{array}{l}\text { Study included both hospital-based and hospice-based specialist palliative care and did not sepa- } \\
\text { rate their results. }\end{array}$ \\
\hline O'Mahony 2017 & This study was not a randomised controlled trial. \\
\hline Pantilat 2010 & $\begin{array}{l}\text { This study was not a randomised controlled trial as it used an alternate day assignment to allocate } \\
\text { participants to the intervention and control groups. }\end{array}$ \\
\hline Rabow 2004 & This study did not conceal its allocation sequence. It was a cluster-RCT. \\
\hline Scarpi 2019 & The intervention was not provided by a multidisciplinary team. \\
\hline Schenker 2018 & The intervention was not provided by a multidisciplinary team. \\
\hline Spatuzzi 2017 & This study was not a randomised controlled trial. \\
\hline Sussman 2018 & The intervention was not provided by a multidisciplinary team. \\
\hline Ullrich 2017 & This study was not a randomised controlled trial. \\
\hline Veronese 2017 & The intervention was not hospital-based specialist palliative care. It was home palliative care. \\
\hline Wong 2016 & The control group included palliative care as part of routine usual care. \\
\hline Yang 2018 & The intervention was not provided by a multidisciplinary team. \\
\hline Zimmermann 2014 & This study did not conceal its allocation sequence. It was a cluster-RCT. \\
\hline
\end{tabular}

GP: General Practitioners

PCT: Palliative Care Team

RCT: Randomised Controlled Trial

SPCT: Specialist Palliative Care Consultation Team

vs: versus

Characteristics of studies awaiting classification [ordered by study ID]

Aljohani 2015

\begin{tabular}{ll}
\hline Methods & RCT \\
\hline Participants & Patients with newly diagnosed non-small-cell lung cancer \\
\hline Interventions & Early palliative care integrated with standard oncological care \\
\hline Outcomes & $\begin{array}{l}\text { Primary outcome measured: quality of life at } 12 \text { weeks assessed using the Edmonton Symptom As- } \\
\text { sessment Scale (ESAS) }\end{array}$ \\
\hline Notes & $\begin{array}{l}\text { Only the abstract was published. We found no preregistration entry for this study and the author } \\
\text { could not be contacted. Consequently, there was insufficient information as to the nature of pallia- } \\
\text { tive care team and setting. }\end{array}$
\end{tabular}

RCT: Randomised Controlled Trial

The effectiveness and cost-effectiveness of hospital-based specialist palliative care for adults with advanced illness and their caregivers 
Characteristics of ongoing studies [ordered by study ID]

\section{ACTRN12618001045202}

Study name
Collaborative supportive care for life-limiting chronic conditions: a prospective randomised controlled study comparing supportive care with standard care

\begin{tabular}{|c|c|}
\hline Methods & $\mathrm{RCT}$ \\
\hline \multirow[t]{6}{*}{ Participants } & Country: Australia \\
\hline & Recruitment: Not stated \\
\hline & Number of patients: Unknown as study is ongoing \\
\hline & Diseases: Adults with life-limiting chronic conditions \\
\hline & Patient characteristics: Patients aged 18 years and over \\
\hline & $\begin{array}{l}\text { Deaths at end of study: Unknown as study is ongoing } \\
\text { Withdrawals/other dropouts: Unknown as study is ongoing }\end{array}$ \\
\hline \multirow[t]{5}{*}{ Interventions } & Type: Specialist palliative care \\
\hline & Service base: Hospital \\
\hline & $\begin{array}{l}\text { Team: Palliative care, renal medicine, general medicine, cardiac failure medicine, social work, ad- } \\
\text { vance care planning and complex care nurse practitioners }\end{array}$ \\
\hline & $\begin{array}{l}\text { Description: Multidisciplinary supportive care clinic management and ongoing follow-up as clini- } \\
\text { cally required. Face-to-face consultations with patients and their carers with a palliative care physi- } \\
\text { cian and/or general medicine physician, nurse practitioner, and social work. Medical issues will be } \\
\text { reviewed, symptoms managed, and care planning issues reviewed. The clinic review will be on an } \\
\text { as-required basis after the initial review. These may be up to weekly if needed. All participants are } \\
\text { reviewed until death. Patients' family physicians will be provided with a summary of the consulta- } \\
\text { tion and a care plan to each patient. Each clinic will also have a multidisciplinary meeting to dis- } \\
\text { cuss each patient's needs. }\end{array}$ \\
\hline & Duration: Unclear \\
\hline \multirow[t]{12}{*}{ Outcomes } & Primary outcomes: \\
\hline & Health professional-rated score for symptom severity \\
\hline & Quality of life assessed using the McGills Quality of Life Questionnaire \\
\hline & Symptom assessment assessed using the palliative care 'symptom assessment scale' \\
\hline & Secondary outcomes: \\
\hline & Advance care plans \\
\hline & Emergency room attendances \\
\hline & General practitioner episodes of contact \\
\hline & Hospital admissions \\
\hline & Number of community service referrals \\
\hline & Number of episodes of contact with other healthcare professionals \\
\hline & Number of patient/carer episodes of contact (composite score) \\
\hline
\end{tabular}

The effectiveness and cost-effectiveness of hospital-based specialist palliative care for adults with advanced illness and their caregivers 
ACTRN12618001045202 (Continued)

Starting date

July 2018

\section{Contact information}

Notes

\section{CHICTR1800014482}

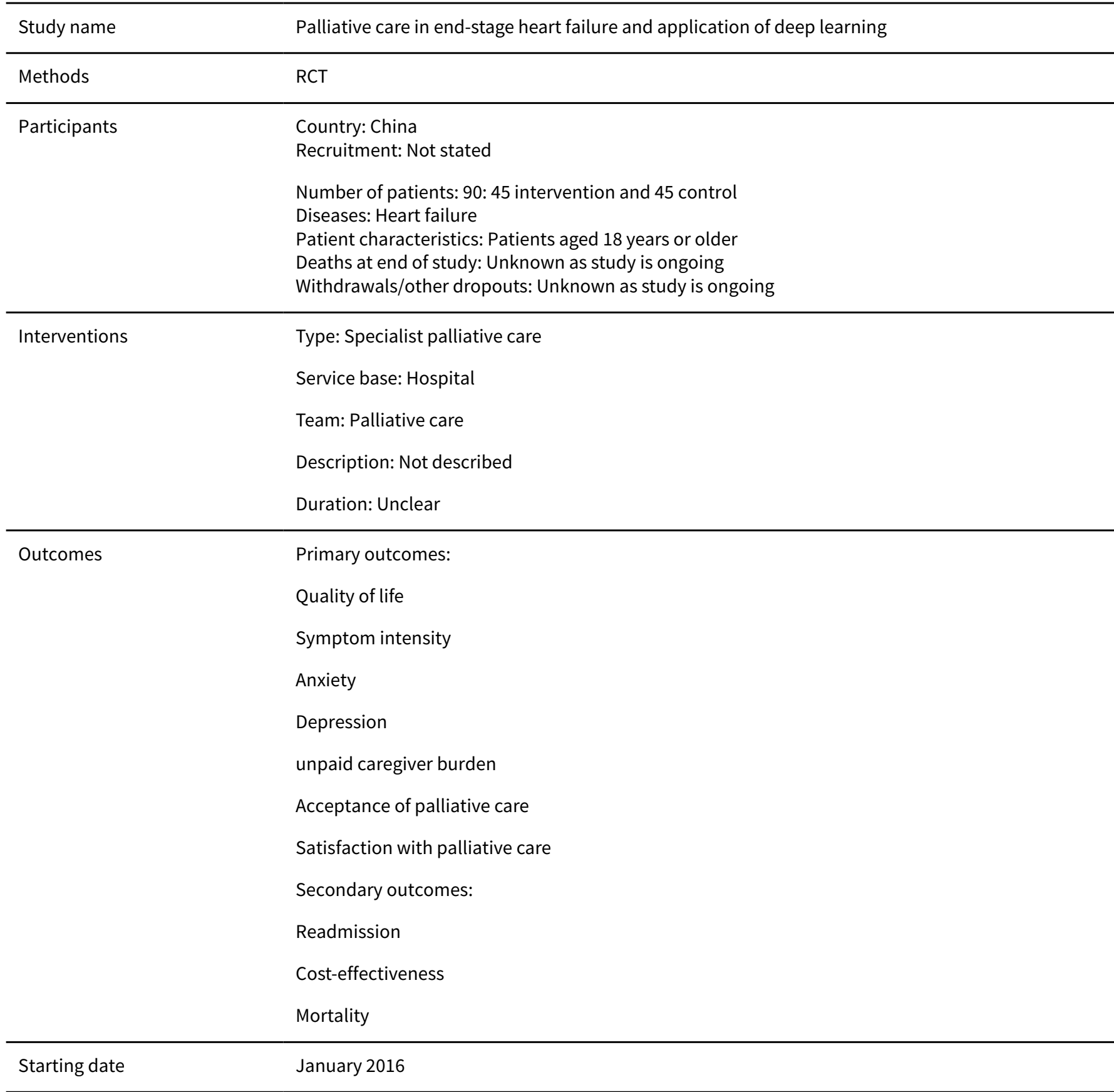

Contact information

The effectiveness and cost-effectiveness of hospital-based specialist palliative care for adults with advanced illness and their caregivers 
CHICTR1800014482 (Continued)

Notes

Courtright 2016

Study name The randomised evaluation of default access to palliative services (REDAPS) trial

Methods Multicentre pragmatic, stepped-wedge cluster-RCT

Participants

\section{Country: USA}

Recruitment: An electronic health record (EHR)-based algorithm to identify eligible patients was constructed based on ICD-9 and -10th Revisions, Clinical Modification codes (67 - 69) that are present on admission. In addition, nurses complete a 5 -item electronic checklist during intake to denote the disease-specific eligibility criteria. The eligibility criteria were chosen to include patients (1) with sufficiently complex needs that they are likely to benefit from specialty palliative care, (2) who could be identified relatively easily from the electronic health record, and (3) who differ from the populations most commonly included in prior or ongoing studies of palliative care.

Number of patients: Unknown as study is ongoing

Diseases: Advanced chronic obstructive pulmonary disease, dementia or end-stage renal disease Patient characteristics: Patients aged 65 years or older

Deaths at end of study: Unknown as study is ongoing

Withdrawals/other dropouts: Unknown as study is ongoing

Interventions

Type: Specialist palliative care

Service base: Hospital

Team: Palliative care team

Description: REDAPS is a pragmatic, stepped-wedge cluster-randomised trial in which hospitals adopt the intervention of electronically triggered, default palliative care consultation orders for eligible patients at randomly assigned times (wedges). During the intervention phase of the trial at each hospital, a palliative care consult order is entered by default for all eligible patients. It is initially an inactive "standing order" that is generated with a future start date. Physicians caring for patients who trigger the default order are notified and instructed on how to cancel it if they wish. If it is not cancelled within 24 hours, the standing order becomes active. In keeping with the principles of pragmatic trials testing real-world effectiveness of interventions, palliative care teams retain discretion regarding prioritisation of patients, provision of services, and documentation practices within a standardised palliative care consultation form.

Duration: All hospitals contribute at least 3.5 months of control data before adopting the intervention. Similarly, the end of the 31-month study period includes a 3.5-month period during which all hospitals are using the intervention. This design enables comparisons of outcomes before and after implementation within hospitals as well as comparisons among hospitals at given time points.

Outcomes

Primary outcome:

Composite measure of in-hospital mortality and length of stay.

Secondary outcomes:

Palliative care process measures

Documentation of goals of care

Documentation of family meetings

Documentation of durable power of attorney, surrogate, or proxy

Documentation of pain assessment

Palliative care team visits per patient

Use of bowel regimen for patients on opioids

The effectiveness and cost-effectiveness of hospital-based specialist palliative care for adults with advanced illness and their caregivers (Review)

Copyright $\odot 2020$ The Cochrane Collaboration. Published by John Wiley \& Sons, Ltd. 
Courtright 2016 (Continued)

\author{
Clinical outcomes: \\ Pain scores (excluding patients with dementia) \\ Dyspnoea \\ Code status (most recent at time of death or discharge) \\ Hospital mortality \\ Intensive Care Unit (ICU) mortality \\ Transfer to ICU after randomisation \\ Cardiopulmonary resuscitation after randomisation \\ Days of mechanical ventilation \\ Hospital discharge disposition \\ 30-d hospital readmissions \\ Economic outcomes \\ Direct cost per hospitalisation \\ Direct cost per day
}

Starting date March 2016

Contact information

Notes

DRKS00013922

\begin{tabular}{ll} 
Study name & Early palliative care for patients with symptomatic heart failure \\
\hline Methods & RCT \\
\hline Participants & Country: Germany \\
Recruitment: Not stated \\
Number of patients: 200 \\
Diseases: Heart failure \\
Patient characteristics: Patients aged 18 years or older \\
Deaths at end of study: Unknown as study is ongoing \\
Withdrawals/other dropouts: Unknown as study is ongoing
\end{tabular}

Interventions

Type: Specialist palliative care

Service base: Unclear

Team: Palliative care team

Description: Early Integration of Palliative Care (EIPC, proactive) with monthly visits with monitoring of symptoms and treatment, psychological support in addition to treatment of control group

Duration: Unclear

\begin{tabular}{ll}
\hline Outcomes & Primary outcome: \\
& Health-related quality of life assessed at 12 months and measured by the Functional Assessment \\
of Chronic Illness Therapy; Palliative Care scale (FACIT-PAL) and the Kansas City Cardiomyopathy \\
Questionnaire (KCCQ) \\
Secondary outcomes: \\
Depression and anxiety score (HADS-D) every 3 months
\end{tabular}

The effectiveness and cost-effectiveness of hospital-based specialist palliative care for adults with advanced illness and their caregivers 
Spiritual well-being (measured via the FACIT-Spiritual Well-Being scale (FACIT-Sp)) at baseline and end of study

Number of hospital readmissions

Total number of hospital days due to heart failure (end of study)

Overall survival (end of study)

Medical resource and cost assessment (end of study)

\section{Starting date}

\section{Contact information}

Notes

Graney 2019

Study name

Advancing symptom alleviation with palliative treatment (ADAPT) trial to improve quality of life: a study protocol for a randomized clinical trial

Methods Multisite RCT

\section{Participants}

\section{Country: USA}

Recruitment: Eligible subjects at both study sites will be identified electronically using validated combinations of diagnostic codes. Study personnel will screen individual medical records to confirm eligibility. After confirmation, patients' primary care providers will be contacted to confirm the study team can contact their patients and to explain the study. With primary care provider approval, veterans will be mailed letters describing the study and providing contact information for study staff if they are interested in participating. If an eligible veteran does not contact the study team, they are contacted by telephone.

Number of patients: 300

Diseases: Chronic heart failure (CHF), interstitial lung disease (ILD) and COPD

Patient characteristics: Study to include veterans with CHF, ILD and COPD

Deaths at end of study: Unknown as study is ongoing

Withdrawals/other dropouts: Unknown as study is ongoing

Team: Palliative care team comprising a registered nurse (RN) and Master's level social worker (MSW) who integrate into a larger collaborative care team ('Team') that includes a representative primary care provider (PCP) and palliative care specialist. Specialist support with a cardiologist or pulmonologist is available for the Team for additional management recommendations, if needed.

Description: The intervention is a multidisciplinary, team-based approach to addressing symptoms and psychosocial needs of participants. The team-based approach is based on the evidence-based collaborative care model of healthcare delivery.

Duration: 6 months

\begin{tabular}{ll}
\hline Outcomes & Primary outcome: \\
& $\begin{array}{l}\text { Patient-reported quality of life assessed using the Functional Assessment for Chronic illness Thera- } \\
\text { py-General (FACT-G) questionnaire }\end{array}$ \\
\hline
\end{tabular}




\section{Secondary outcomes:}

Symptom experience measured using the General Symptom Distress Scale (GSDS)

Depression assessed using the Patient Health Questionnaire-8 (PHQ-8)

Health-related quality of life assessed using the Quality of Life at the End of Life (QUAL-E) questionnaire, Kansas City Cardiomyopathy Questionnaire (KCCQ-12); the Clinical COPD Questionnaire (CCQ); or the King's Brief Interstitial Lung Disease (K-BILD)

Hospitalisations

Advanced care planning communication and documentation

\section{Hutt 2018}

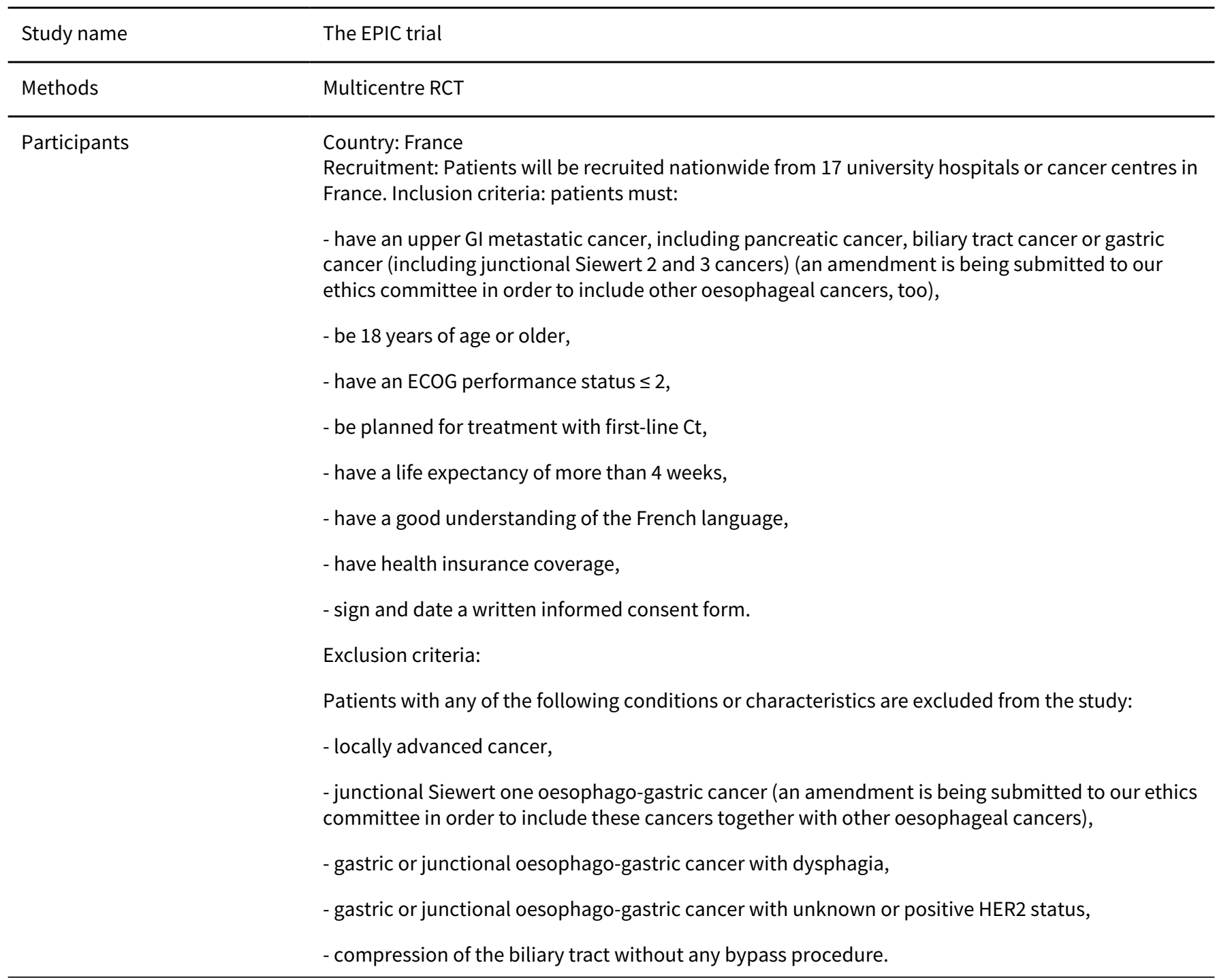

The effectiveness and cost-effectiveness of hospital-based specialist palliative care for adults with advanced illness and their caregivers 
Hutt 2018 (Continued)

Number of patients: Sample size of 480 patients proposed

Diseases: Metastatic upper $\mathrm{Gl}$ cancers

Patient characteristics: Unknown as study is ongoing

Deaths at end of study: Unknown as study is ongoing

Withdrawals/other dropouts: Unknown as study is ongoing

Interventions

Type: Early palliative care

Service base: Hospital

Team: Palliative care specialists

Intervention: Medical oncologists will be in charge of the patient for chemotherapy (Ct) administration and for supportive care, in accordance with professional practices. PC specialists will be in charge of PC/EPC visits. In the experimental arm (Ct + EPC), five PC visits are scheduled. The first visit (V1) will be scheduled within the first 3 weeks after randomisation. The remaining four visits will be scheduled every month. The content of each of the five PC visits will be described by the PC physician and documented in the database following a specific checklist developed by PC physicians.

Duration: 24 weeks

Control: In order to match with standard practice in France, participants allocated to the standard arm (Ct alone) are not scheduled to meet with the PC service, but a PC visit can be performed anytime if requested by the patient, the family or the oncologist.

Overall survival (as intent-to treat analysis)

Secondary outcomes:

Overall survival (per protocol analysis)

One-year survival rate (intent-to treat and per protocol analyses)

Quality of Life assessed with the QLQ-C30 questionnaire at baseline, and after inclusion, every 8 weeks until patient withdrawal from the study

Depression and anxiety assessed with the HADS scale (Hospital Anxiety and Depression Scale) at baseline, and after inclusion, every 8 weeks during 24 weeks

Time Until Definitive Deterioration (TUDD) for quality of life scores was defined as the time from randomisation to the first observation of a definitive deterioration of QLQ-C30 score or death

Presence or lack of advanced directives

Actual description of the PC package

Number of patients receiving chemotherapy in their last 30 days of life

Starting date August 2016

Contact information

\section{Notes}

\section{IRCT20160521027993N1}

Study name The effect of palliative care education on self-efficacy of elderly with chronic heart failure


IRCT20160521027993N1 (Continued)

\begin{tabular}{ll} 
Methods & RCT \\
\hline Participants & Country: Iran \\
Recruitment: Not stated. & Number of patients: 48 \\
Diseases: Chronic heart failure \\
Patient characteristics: Unknown as study is ongoing \\
Deaths at end of study: Unknown as study is ongoing \\
Withdrawals/other dropouts: Unknown as study is ongoing
\end{tabular}

Interventions

Type: Unclear

Service base: Unclear as not stated

Team: Unclear

Intervention: Participants will be trained for 6 weeks according to the WHO protocol, in groups of 6 to 8 people, once a week. The educational content included physical problems management and strategies for promoting physical activity, self-care (diet, sleep pattern, proper use of drugs and no smoking) and psychological and spiritual care and social communication.

Duration: 6 weeks

Control: Routine care without any intervention

\begin{tabular}{ll}
\hline Outcomes & Primary outcome: \\
& Self-efficacy assessed using Sullivan's cardiac self-efficacy questionnaire
\end{tabular}

Starting date June 2018

Contact information

Notes

\section{IRCT20160914029817N6}

Study name Effect of non-drug palliative care on quality of life in patients with chronic obstructive pulmonary
disease

\begin{tabular}{ll}
\hline Methods & RCT \\
\hline Participants & Country: Iran \\
& Recruitment: Not stated \\
& Number of patients: 60 \\
& Diseases: COPD \\
& Patient characteristics: Unknown as study is ongoing \\
& Deaths at end of study: Unknown as study is ongoing \\
& Withdrawals/other dropouts: Unknown as study is ongoing \\
\hline
\end{tabular}

Interventions

Type: Unclear

Service base: Unclear as not stated

Team: Unclear 
IRCT20160914029817N6 (Continued)

Intervention: COPD patients will receive palliative care using the training and implementation of the protocol from the time of admission. Training sessions will include: first day - giving pamphlets and training on smoking cessation methods and diet, day two - teaching effective breathing techniques, day three - lung physiotherapy, day four - cold steaming.

Duration: Not stated

Control: Receives routine nursing care.

\begin{tabular}{ll}
\hline Outcomes & $\begin{array}{l}\text { Primary outcome } \\
\text { Quality of life assessed using the SF-12 }\end{array}$ \\
\hline Starting date & June 2018 \\
\hline Contact information & \\
\hline Notes & \\
\hline
\end{tabular}

\section{IRCT20180531039925N1}

\begin{tabular}{ll}
\hline Study name & The effect of palliative care program on the quality of life of chil \\
\hline Methods & Country: Iran \\
Recruitment: Not stated. & \\
& Number of patients: 60 \\
Diseases: Leukaemia \\
Patient characteristics: Unknown as study is ongoing \\
Deaths at end of study: Unknown as study is ongoing \\
Withdrawals/other dropouts: Unknown as study is ongoing
\end{tabular}

Type: Unclear
Service base: Unclear as not stated
Team: Unclear
Intervention: In the intervention group, palliative care will be performed for 4 weeks.
Duration: Not stated
Control: No intervention

Interventions Primary outcome:

Quality of life

Outcomes June 2018

\section{Starting date}

Contact information

\section{Notes}


Kluger 2019

Study name

Does outpatient palliative care improve patient-centered outcomes in Parkinson's disease: rationale, design, and implementation of a pragmatic comparative effectiveness trial

Methods
Recruitment: Included: 1) Referrals from colleagues within academic practices; 2) Referrals from
community physicians notified about the study through personal connections, solicitations
through email and letters, and continuing medical education (CME) events; 3) Community talks giv-
en to patients by investigators or patient/ unpaid caregiver advisors sponsored by support groups
or local PD organisations; 4) Advertisements through local PD organisations (e.g. newsletters); and
5) Postings on clinical research websites
Number of patients: 210
Diseases: Parkinson's disease
Patient characteristics: Unknown as study is ongoing
Deaths at end of study: Unknown as study is ongoing
Withdrawals/other dropouts: Unknown as study is ongoing

Participants

Type: Unclear

Service base: Unclear as not stated

Team: Neurologist with PC experience and informal training with a board-certified palliative medicine specialist

Intervention: Team-based outpatient PC

Duration: 12 months

Control: Usual care

\begin{tabular}{ll}
\hline Interventions & Primary outcomes: \\
& Quality of life \\
& unpaid caregiver distress \\
& Secondary outcomes: \\
& Symptom burden \\
& Spiritual well-being \\
& Mood \\
\hline Outcomes & October 2015 \\
\hline Starting date & \\
\hline Contact information & \\
\hline Notes & \\
\hline
\end{tabular}

\section{Matsumoto 2016}

\begin{tabular}{ll}
\hline Study name & Early specialised palliative care in Japan \\
\hline Methods & RCT \\
\hline Participants & Country: Japan
\end{tabular}

The effectiveness and cost-effectiveness of hospital-based specialist palliative care for adults with advanced illness and their caregivers 
Inclusion criteria: (1) Histologically or cytologically proven lung cancer (2) Stage IV non-small cell lung cancer or extensive-disease small cell lung cancer (3) Negative or unknown of EGFR gene mutation (4) Negative or unknown of ALK fusion gene (5) Undergoing first-line chemotherapy at institutions participating in this study (6) Not performed anticancer therapy (chemotherapy, radical surgery, definitive radiotherapy) (7) Initial administration of the first-line chemotherapy in inpatient hospital setting (8) Age $>=20$ years (9) Written informed consent for participation in this study

Exclusion criteria: (1) Already consulted outpatient palliative medicine clinic or outpatient psycho-oncology clinic before entry to this study, and scheduled to receive the medical care subsequently (2) Already received intervention of specialty palliative care service before diagnosis (3) With severe cognitive impairment (4) Unable to read and respond to questions in Japanese (5) Participated in other intervention studies which prohibits participation in this study (6) Judged to be inappropriate for participation to this study

Number of patients: Unknown as study is ongoing

Diseases: Advanced lung cancer

Patient characteristics: Unknown as study is ongoing

Deaths at end of study: Unknown as study is ongoing

Withdrawals/other dropouts: Unknown as study is ongoing

Service base: Unclear as not stated

Team: Information obtained from contacting the author indicates that the nurse-led, screening-triggered early specialist palliative care intervention involves other healthcare professionals such as doctors or psychologists

Intervention: Nurse-led screening-triggered early specialised palliative care (ESPC) intervention

Duration: Not stated

Control: Usual care

Change in the score on the Functional Assessment of Cancer Therapy-Lung Trial Outcome Index (FACT-L TOI) from baseline to 3 months

Secondary outcomes:

\section{FACT-L score}

FACT-L Lung Cancer Subscale (LCS) score

Proportion of patients with depression assessed by Patient Health Questionnaire 9 (PHQ-9)

PHQ-9 score

Proportion of patients with anxiety assessed by generalised anxiety disorder 7-item (GAD-7)

GAD-7 score

EuroQol-5 Dimension (EQ-5D) score

Proportion of responses to each item in the questionnaire about illness understanding

Proportion of responses to each item in the questionnaire about medical services received

One-year survival rate

Overall survival 
Visiting time with patients taken for each profession belonging to specialised palliative care service to meet with patients

Place of death as a ratio outcome

Days from the last chemotherapy to death

Days of hospital stay in general wards during one month before death

Days of hospice usage during one month before death

Proportion of hospice usage during one month before death

Proportion of cardiopulmonary resuscitation

\begin{tabular}{ll}
\hline Starting date & January 2017 \\
\hline Contact information & $\begin{array}{l}\text { Only the abstract was published. The author was contacted and provided a link to the trial registra- } \\
\text { tion details, https://upload.umin.ac.jp/cgi-open-bin/ctr/ctr_view.cgi?recptno=R000029335 }\end{array}$ \\
\hline Notes &
\end{tabular}

\section{NCT01828775}

Study name Integration of palliative care for cancer patients on phase I Trials

Methods RCT

\section{Participants}

\section{Country: USA}

Recruitment: Inclusion Criteria:

- Patients diagnosed with solid tumours who are eligible for participation in phase I clinical trials of investigational cancer therapies

- Patients who have signed an informed consent for participation in phase I clinical trials

- Able to read or understand English - this is included because the intervention and study materials (including outcome measures) are only in English

- Ability to read and/or understand the study protocol requirements, and provide written informed consent

\section{Exclusion Criteria:}

- Patients diagnosed with haematologic (as a population distinct from solid tumours and different trials) cancers

Number of patients: Sample size of 480 participants proposed

Disease: Patients diagnosed with solid tumours

Patient characteristics: Unknown as still ongoing

Deaths at end of study: Unknown as still ongoing

Withdrawals/other dropouts: Unknown as still ongoing 
Intervention: Patients receive part I of the Palliative Care Intervention ( $\mathrm{PCl}$ ) comprising quantitative surveys, comprehensive palliative care assessment by the Research Nurses, and goals of care discussions beginning prior to administration of the first dose of phase I treatment. Patients then receive part II of the $\mathrm{PCl}$ comprising recommendations from the interdisciplinary team, patient educational sessions, and supportive care referrals following the first dose of phase I treatment and which is completed within one month of the first treatment.

Duration: Unclear

Usual care: Delayed PCI

Change in overall QoL scores, assessed by the Functional Assessment of Cancer Therapy-General (FACT-G) and Functional Assessment of Chronic Illness Therapy-Spirituality (FACIT-Sp)

Change in psychological distress, assessed using the Psychological Distress Thermometer

Satisfaction with communication, measured by the Family Satisfaction with Advanced Cancer Care (FAMCARE)

Patients' symptom intensity and symptom interference with daily activities

Total numbers of supportive care referrals (social work, dietitian, chaplaincy, psychologist/psychiatrist)

Total number of unscheduled outpatient encounters and inpatient admissions

Total number of hospice referrals

Retention on the phase I trial

Patient satisfaction with the $\mathrm{PCI}$

Secondary outcomes:

Change in overall QoL scores, assessed by FACT-G and FACIT-Sp

Change in psychological distress, assessed using the Psychological Distress Thermometer

Satisfaction with communication, measured by FAMCARE

Patients' symptom intensity and symptom interference with daily activities

Total numbers of supportive care referrals (social work, dietitian, chaplaincy, psychologist/psychiatrist)

Total number of unscheduled outpatient encounters and inpatient admissions

Total number of hospice referrals

Retention on the phase I trial

Patient satisfaction with the $\mathrm{PCl}$

Starting date September 2014

Contact information

\section{Notes}


NCT01846520

Study name A randomized trial of a family unpaid caregiver palliative care intervention

Methods Country: USA

Participants

Recruitment: Inclusion Criteria:

- Primary family unpaid caregivers of cancer patients with gastrointestinal (colorectal, pancreatic, gastric), gynaecologic, urinary or lung cancers who are entering the City of Hope for treatment or follow-up

- Primary family unpaid caregivers of cancer patients who are diagnosed with stage II-IV disease

- Primary family unpaid caregivers of cancer patients with $>6$ months prognosis

- Living within a 50 mile radius of the City of Hope

Number of family unpaid caregivers: Sample size of 200 participants proposed

Diseases: Patients with stage II-IV gastrointestinal, gynaecologic, urologic and lung cancers

Patient characteristics: Unknown as still ongoing

Deaths at end of study: Unknown as still ongoing

Withdrawals/other dropouts: Unknown as still ongoing

Service base: Unclear

Team: Advanced practice nurse

Intervention: Participants receive $\mathrm{FCPCl}$ with an advanced practice nurse (APN), comprising 4 home education sessions once weekly followed by 4 telephone support sessions for 30 minutes once monthly and 24-hour telephone support available for 6 months.

Duration: 6 months

Control: Usual care

Outcomes Primary outcomes:

Effects of Family unpaid caregiver Palliative Care Intervention (FCPCI) on unpaid caregiver burden

Effects of $\mathrm{FCPCl}$ on caregiving skills preparedness

Effects of $\mathrm{FCPCl}$ on Quality of Life (QoL)

Effects of $\mathrm{FCPCl}$ on psychological distress

Secondary outcomes:

unpaid caregiver's self-care behaviour

unpaid caregivers' resource use

Identification of subgroups of family unpaid caregivers who benefit most from the FCPCI in relation to sociodemographic, health status, and patient characteristics

Family unpaid caregivers' satisfaction with the FCPCI

unpaid caregiver out-of-pocket costs 
NCT01846520 (Continued)

Notes

Study name A structured early palliative care intervention for patients with advanced cancer - a randomized controlled trial with a nested qualitative study (SENS Trial)

\begin{tabular}{|c|c|}
\hline Methods & $\mathrm{RCT}$ \\
\hline Participants & $\begin{array}{l}\text { Country: Switzerland } \\
\text { Recruitment: Inclusion Criteria: } \\
\text { - Diagnosed within the last } 16 \text { weeks } \\
\text { - Metastatic or locally advanced, not amenable to curative treatment, non-small cell lung cancer } \\
\text { (NSCLC), or } \\
\text { - Metastatic or locally advanced, not amenable to curative treatment, colorectal cancer, or } \\
\text { - Metastatic or locally advanced, not amenable to curative treatment, prostate cancer, or } \\
\text { - Metastatic or locally advanced, not amenable to curative treatment, breast cancer with visceral } \\
\text { and/or brain metastasis, or } \\
\text { - Metastatic or locally advanced, not amenable to curative treatment, bladder/ urothelium cancer, } \\
\text { or } \\
\text { - Metastatic or locally advanced, not amenable to curative treatment, pancreatic cancer } \\
\text { - Diagnosis is histologically confirmed } \\
\text { - Eastern Cooperative Oncology Group (ECOG) Performance Status of } 0,1 \text { or } 2 \\
\text { - At least } 18 \text { years of age at the time of enrollment } \\
\text { - Signed informed consent with understanding of the study procedures and the investigational na- } \\
\text { ture of the study } \\
\text { Exclusion Criteria: } \\
\text { - Presence of delirium or dementia or other reason for lack of ability to give informed consent } \\
\text { - Inability to communicate adequately in German } \\
\text { - Patient's lack of accountability, inability to appreciate the nature, meaning and consequences of } \\
\text { the study and to formulate his/her own wishes correspondingly } \\
\text { - Patients already receiving care from an inpatient palliative care service } \\
\text { Dumber of patients: Sample size of } 150 \text { patients proposed } \\
\text { Withdrawals/other dropouts: Unknown as still ongoing } \\
\text { Diseases Advanced cancer }\end{array}$ \\
\hline
\end{tabular}

Interventions

Type: Early Palliative Care Intervention

Service base: Unclear

Team: Palliative care physicians and nurses

Intervention: The structured approach intervention with the SENS model is based on the biopsychosocial-spiritual model of care and the WHO definitions of palliative care as well as the NCCN Practice Guidelines for Palliative Care. It supports the assessment of areas and complexity of concerns from the patient perspective, determines the priority and structures the support needed. The intervention is performed by palliative care physicians and nurses collaboratively. It is utilised as baseline assessment and afterwards integrated in each routine oncology care outpatient and inpa- 
NCT01983956 (Continued)

tient visit. Depending on the goals, it may be applied between routine visits. In addition, patients will receive usual oncology care throughout the study period.

Duration: Unclear

Control: Patients in the usual care group will receive routine oncology care throughout the study. This incorporates a routine assessment according to the standard SAKK - protocol which assesses overall symptoms. Patients are not seen by nurses during a routine visit to the outpatient clinic unless they need a blood withdrawal or any intravenous or subcutaneous treatment. Only nursing staff of the palliative care unit are familiar with using the SENS-assessment instrument. Participants assigned to usual care may meet with the palliative care service on request according to established practice.

\section{Outcomes}

Primary outcomes:

Distress over six months as measured with the National Comprehensive Cancer Network (NCCN) Distress thermometer

Secondary outcomes:

Quality of life as measured by Functional Assessment of Cancer Therapy - General (FACT-G)

Palliative Outcome Scale (POS)

Overall survival

Location of death

Healthcare utilisation

Starting date December 2013

Contact information

Notes

\section{NCT02139917}

\begin{tabular}{ll}
\hline Study name & Effects of a transitional palliative care model on patients with end-stage renal failure (ESRF) \\
\hline Methods & RCT \\
\hline Participants & Country: Hong Kong \\
Recruitment: & Inclusion Criteria: \\
- Patient with chronic kidney disease and diabetic mellitus with creatinine $\geq 350$ milli mole (uM) or \\
those without diabetic mellitus with creatinine $\geq 450$ milli mole (uM) who refused renal replace- \\
ment therapy (RRT); \\
- Patient not suitable for long-term renal replacement therapy (RRT) after assessment by renal \\
team (e.g. multiple comorbidities, poor functional status and social support) \\
- Identified as ESRF patient eligible for palliative care without prior renal replacement therapy \\
- Living within the hospital service area \\
- Ability to be contacted by phone \\
Exclusion Criteria:
\end{tabular}

The effectiveness and cost-effectiveness of hospital-based specialist palliative care for adults with advanced illness and their caregivers 
NCT02139917 (Continued)

- Discharged to nursing home or other institution

- Inability to communicate

- Cognitive impairment, mini-mental stage examination $(\mathrm{MMSE})<20$

- Diagnosed with severe psychiatric disorders such as schizophrenia and bipolar disorder

Number of patients: 176 participants proposed

Diseases: end-stage renal failure (ESRF)

Patient characteristics: Unknown as still ongoing Deaths at end of study: Unknown as still ongoing Withdrawals/other dropouts: Unknown as still ongoing

Type: Transitional Palliative Care Model
Service base: Unclear
Team: Unclear
Intervention: Transitional palliative care includes:-
• telephone follow-up for early identification of signs and symptoms
- home visit for spiritual support
Duration: Unclear
Control: Customary care receives care:-
- hospital based medical follow-up
- general nursing assessment and advice

Outcomes Primary outcomes:

Healthcare utilisation composite - The dates of rehospitalisations, length of stay, and number of other hospital services used, including clinics and emergency room visits, will be extracted from the hospital administrative systems.

Secondary outcomes:

Perceived outcomes composite (quality of life, unpaid caregiver burden, satisfaction with care). Quality of life will be measured by an ESRF-specific quality of life measure. The unpaid caregiver burden will be measured by the Zarit unpaid caregiver Burden Scale (ZCBS).

Satisfaction with care will be measured by the 15 -item questionnaire

Starting date August 2014

Contact information

\section{Notes}

\section{NCT02308865}

$\begin{array}{ll}\text { Study name } & \begin{array}{l}\text { Impact of early palliative care on quality of life and survival of patients with non-small-cell } \\ \text { metastatic lung cancer in Northern France }\end{array}\end{array}$

Methods RCT

Participants Country: Northern France

The effectiveness and cost-effectiveness of hospital-based specialist palliative care for adults with advanced illness and their caregivers 
Recruitment: Inclusion Criteria:

- Being diagnosed with non-small cell lung cancer

- Proven histologically

- Metastatic proven imaging (MRI, CT Scanner, PET scan) Stage IV (any T, any N, M1)

- Prior to secondary chemotherapy treatment.

- Age $>18$ years

- $\mathrm{PS} \leq 2$

- Patient able to understand the nature, purpose and methodology of the study

- Signed informed consent

Exclusion Criteria:

- Age $<18$ years

- Patient already supported by palliative care

- Patient with an activating EGFR mutation or EML4-ALK rearrangement or ROS1 gene translocation

- Patient under trusteeship/guardianship

Number of patients: Sample size of 144 patients proposed

Diseases: Metastatic lung cancer

Patient characteristics: Unknown as still ongoing

Deaths at end of study: Unknown as still ongoing

Withdrawals/other dropouts: Unknown as still ongoing

Interventions

Type: Early palliative care

Service base: Unclear

Team: Multidisciplinary palliative care monthly consultations with a doctor, a nurse, a psychologist and possibility of a physical therapist and a chaplain

Intervention: Multidisciplinary palliative care monthly consultations with a doctor, a nurse, a psychologist and possibility of a physical therapist and a chaplain in addition to standard onco-pneumologic care

Duration: Unclear

Control: Patient supported by the Oncology Respiratory service for the treatment of their disease by chemotherapy and for the treatment of complications

Outcomes

Primary outcome:

Quality of life measured at 12 weeks by the TOI score

Secondary outcomes:

Survival

Events - Presence of any of the following: chemotherapy, use of resuscitation or no treatment-limiting decision 14 days before death

Quality of life measured by the score TOI at 12 weeks and by the Echelle SCNS - SF34 scale, FACT-L, PHQ-9 and HADS questionnaires at 12 and 21 weeks 


\begin{tabular}{|c|c|}
\hline Methods & $\mathrm{RCT}$ \\
\hline Participants & 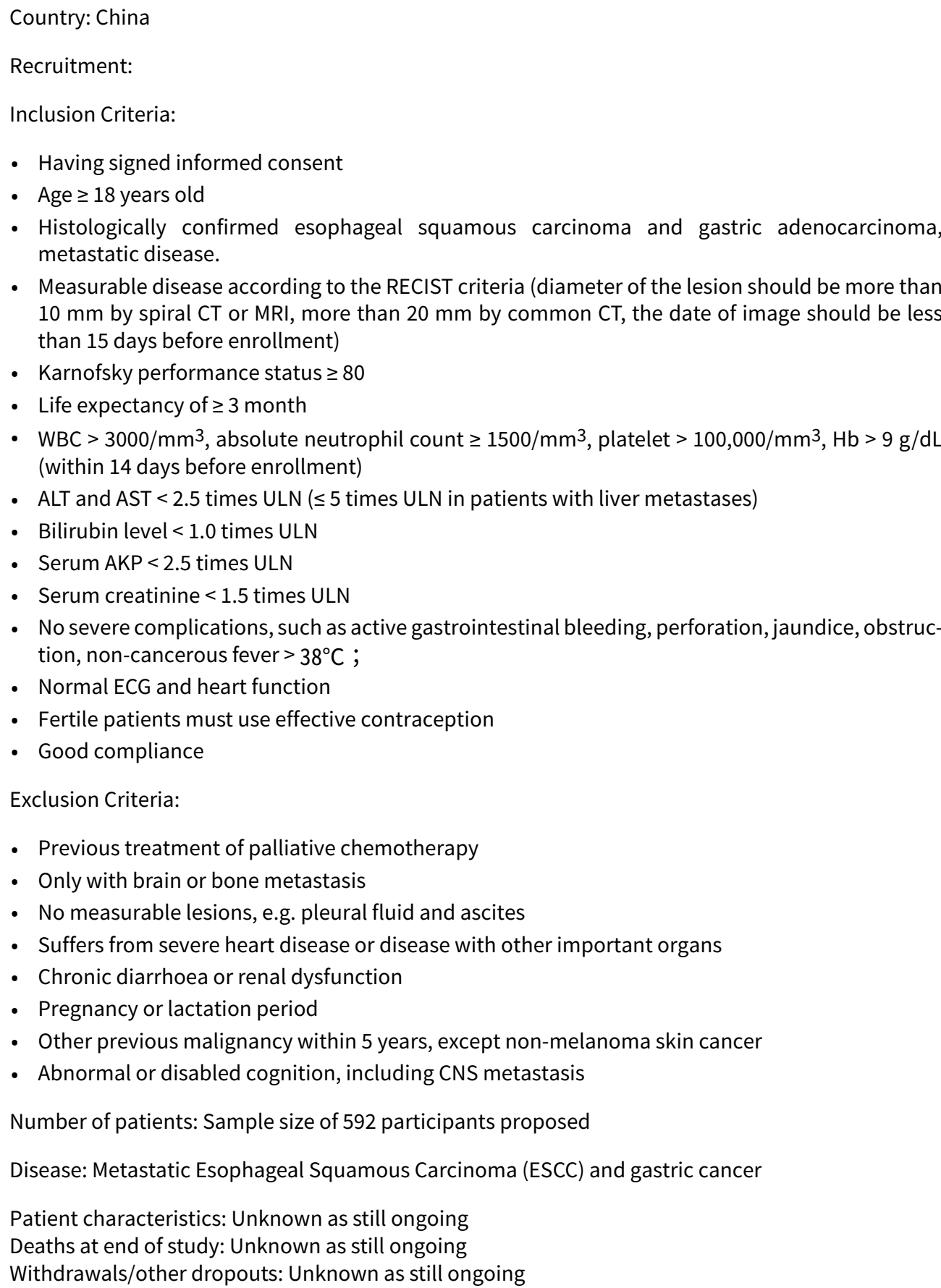 \\
\hline Interventions & $\begin{array}{l}\text { Type: Early palliative care } \\
\text { Service base: Unclear } \\
\text { Team: Unclear }\end{array}$ \\
\hline
\end{tabular}

The effectiveness and cost-effectiveness of hospital-based specialist palliative care for adults with advanced illness and their caregivers 
NCT02375997 (Continued)

Intervention: Standard oncology care plus palliative care

Duration: Unclear

Control: Standard oncology care

Outcomes Primary outcomes:

Overall survival. Time from randomisation to death

Secondary outcomes:

Quality of life scores measured on the quality of life form

Overall response rate: complete response rate plus partial response rate

Adverse events: number of participants with adverse events as a measure of safety and tolerability

Starting date October 2014

Contact information

Notes

NCT02533921

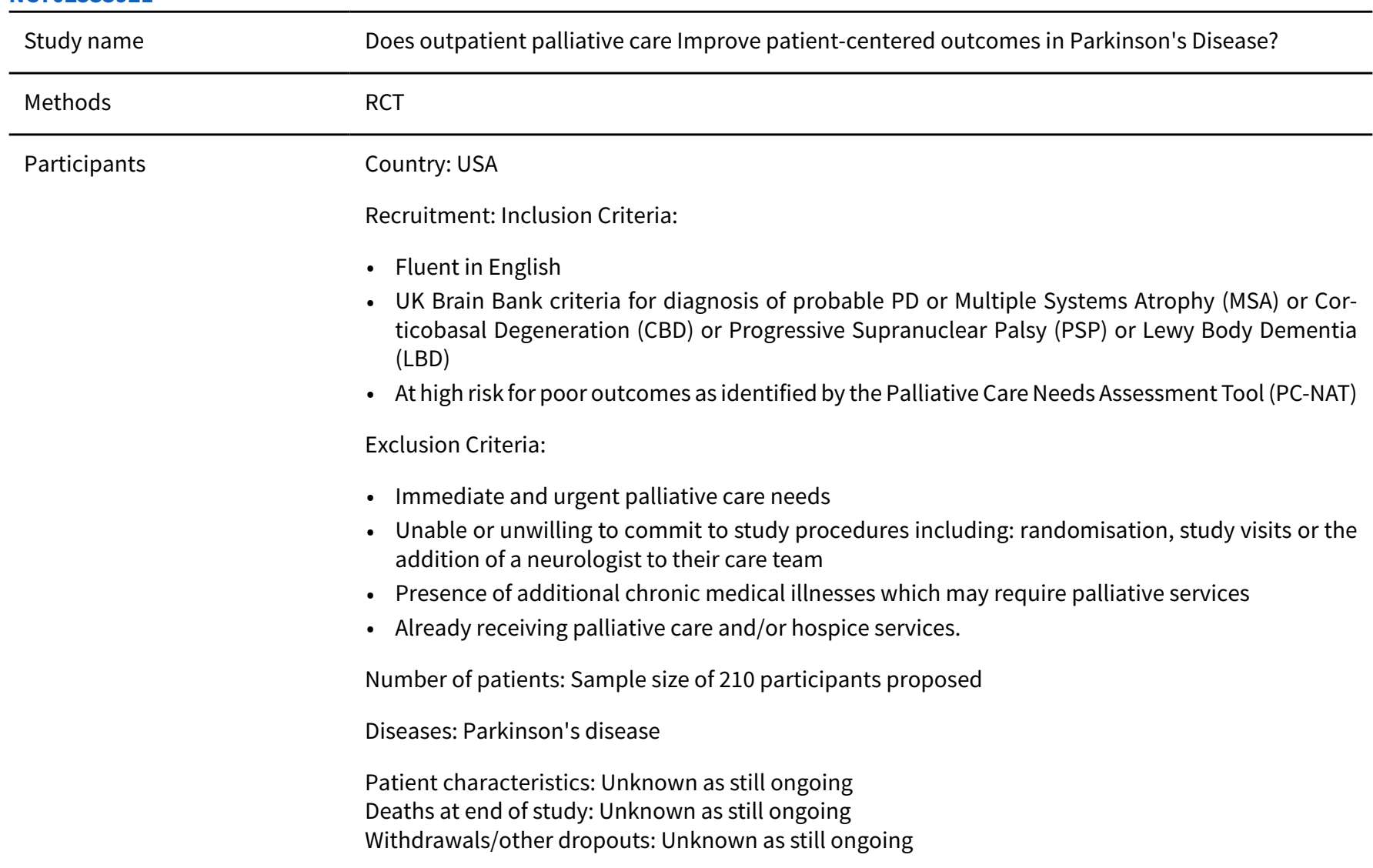

Interventions

Type: Outpatient palliative care

Service base: Hospital

The effectiveness and cost-effectiveness of hospital-based specialist palliative care for adults with advanced illness and their caregivers 
NCT02533921 (Continued)

Team: Interdisciplinary outpatient palliative care team

Intervention: Usual care augmented by an outpatient interdisciplinary palliative care team

Duration: 6 months

Control: Usual care as in including both a Primary Care Physician (PCP) and neurologist

Outcomes Primary outcomes:

Changes in the subjects quality of life (QoL). The QOL-AD (Quality of Life in Alzheimer's Disease) survey will be used to measure the differences in the quality of life between groups.

Changes in unpaid caregiver distress. The Zarit unpaid caregiver Burden Interview Form (ZBI) will be used to measure differences in unpaid caregiver Distress between groups.

Secondary outcomes:

Changes in patient and unpaid caregiver mood. The Hospital Anxiety and Depression Scale (HADS) will be used to quantify changes in anxiety and depression.

Starting date October 2015

Contact information

Notes

\section{NCT02543541}

Study name A pilot study of structured palliative care for patients enrolled on phase I clinical trials

Methods RCT

\section{Participants}

\section{Country: USA}

Recruitment: Patient Inclusion Criteria:

- Patients must be enrolled in a phase 1 clinical trial and be within 2 weeks of starting the experimental therapy or intervention.

- Patients are eligible to enroll on this study with or without the enrollment of their unpaid caregiver.

\section{Patient Exclusion Criteria:}

- Patients diagnosed with a haematologic malignancy

unpaid caregiver Inclusion Criteria:

- Any unpaid caregiver is considered eligible for this study; the unpaid caregiver is the person identified by the patient as the one who provides the most regular physical and/or emotional support.

- unpaid caregivers must be willing to complete surveys at baseline and on a monthly basis.

unpaid caregiver Exclusion Criteria:

- unpaid caregivers who are solely professional, paid unpaid caregivers

Number of participants: Sample size of 132 participants proposed

Diseases: Cancer

Patient characteristics: Unknown as still ongoing

The effectiveness and cost-effectiveness of hospital-based specialist palliative care for adults with advanced illness and their caregivers 
NCT02543541 (Continued)

Deaths at end of study: Unknown as still ongoing

Withdrawals/other dropouts: Unknown as still ongoing

Interventions

Type: Structured palliative care

Service base: Hospital

Team: A team of trained specialists which include doctors, nurses, social workers and spiritual care providers. The team can make referrals to other specialists such as psychologists or nutritionist, if needed.

Intervention: Supportive care for the patient and unpaid caregiver will be provided by the outpatient palliative care team which includes clinicians with specialised palliative care training, social workers, spiritual care specialists and mental health clinical nurse specialist.

Duration: Unclear

Control: Supportive care for the patient and unpaid caregiver will be provided by the treating oncologist.

Average total MSAS score: assessment of patient burden

Average total FACT-G score: measure of patient quality of life

Reason for study discontinuation (patient-reported outcome): measure of central tendency for why patients discontinued the phase I study. Patient-reported outcomes for the reason for study discontinuation will be qualitatively assessed

Duration on study: measure of central tendency of the duration patients were on the phase I study. Duration on study will serve as the primary objective for sample size determination.

Adverse events: measure of central tendency of adverse events

Secondary outcomes:

Mean number of hours of palliative care services: mean number of hours utilised by patients and unpaid caregivers who received structured palliative care

Type of palliative care services: measure of central tendency for the type of palliative care services utilised by patients and unpaid caregivers who received structured palliative care

\section{Other Outcome Measures:}

Frequency of adverse events of patients receiving structured and usual supportive care Average days on study

Change in FACT-G score: change in patient symptom burden when compared to baseline between patients who received structured palliative care and those who received standard supportive care Change in MSAS score: change in quality of life when compared to baseline between patients who received structured palliative care and those who received standard supportive care Change in CRA score: change in unpaid caregiver burden when compared to baseline between those who received structured palliative care and those who received standard supportive care Change in QOLLTI-F score: change in unpaid caregiver quality of life as assessed through change in QOLLTI-F score when compared to baseline between those who received structured palliative care and those who received standard supportive care 
NCT02631811

Study name

Impact on quality of life of an early management supportive care of patients with acute leukemia in first relapse

\begin{tabular}{ll}
\hline Methods & RCT \\
\hline Participants & Recruitment: Inclusion Criteria: \\
- $>18$ years old \\
- Acute lymphoblastic or myeloblastic leukaemia at first relapse and diagnosed within 8 weeks be- \\
- Pore inclusion \\
- Patients in whom a curative strategy (transplant) is not considered \\
- Informed signed consent \\
Exclusion Criteria: \\
- Unable to answer the questionnaire \\
- Psychiatric disorders other than depression \\
- Persons under guardianship \\
Number of participants: Sample size of 80 participants proposed \\
Diseases: Acute leukaemia \\
Patient characteristics: Unknown as still ongoing \\
Deaths at end of study: Unknown as still ongoing \\
Withdrawals/other dropouts: Unknown as still ongoing \\
\hline
\end{tabular}

Interventions

Type: Early palliative care

Service base: Hospital

Team: A multidisciplinary palliative specialist team of physician, nurse and psychologist.

Intervention: Patients will be seen by palliative care team at least once a month until the 12th week, more if needed. The symptom and suffering will be assessed by a multidisciplinary palliative specialist team of physician, nurse and psychologist. Physical, psychological, social and existential suffering will be addressed.

Duration: 12 weeks

Control: Patients will be supported by the support care team if asked by the oncologist.

Outcomes Primary outcome:

Measure of quality of life: quality of life measured by FACT-Leu questionnaire (compared between the 2 groups)

Secondary outcomes:

Measure of symptoms intensity: symptom intensity measured by ESAS questionnaire (compared between the 2 groups)

Measure of depression: score of depression measured by HADS questionnaire (compared between the 2 groups)

Measure of anxiety: score of anxiety measured by HADS questionnaire (compared between the 2 groups) 
NCT02631811 (Continued)

Measure of the quality of the end-of-life: within the last month of life, several parameters will be studied to evaluate the quality of the end-of-life like number of admissions in emergency unit.

Overall survival

Starting date November 2015

Contact information

Notes

NCT02712229

$\begin{array}{ll}\text { Study name } & \begin{array}{l}\text { A cluster randomized trial of a primary palliative care intervention (CONNECT) for patients with ad } \\ \text { vanced cancer }\end{array}\end{array}$
vanced cancer

\begin{tabular}{ll}
\hline Methods & RCT \\
\hline Participants & Country: USA \\
Recruitment: Participants will be
\end{tabular}

- (1) patients with advanced cancer receiving care at a participating clinic;

- (2) their unpaid caregivers;

- (3) their oncology staff nurses, oncologists, and practice managers.

Patient eligibility criteria. Inclusion criteria:

(1) adults ( $\geq 21$ years old);

(2) the oncologist "would not be surprised if the patient died in the next year";

(3) Eastern Cooperative Oncology Group performance status (ECOG PS) of $\leq 2$;

(4) planning to receive ongoing care from a participating oncologist and willing to be seen at least monthly.

\section{Exclusion criteria:}

(1) unable to read and respond to questions in English;

(2) cognitive impairment or inability to consent to treatment, as determined by the patient's oncologist;

(3) unable to complete baseline interview;

(4) ECOG PS of 3 (capable of limited self-care; confined to bed or chair $>50 \%$ of waking hours) or 4 (cannot carry on any self-care; totally confined to bed or chair);

(5) haematologic malignancy.

unpaid caregiver eligibility criteria. Inclusion criteria:

(1) adults ( $\geq 21$ years old);

(2) family member or friend of an eligible patient.

Exclusion criteria:

(1) unable to read and respond to questions in English; 
(2) unable to complete the baseline interview. Patients will be asked to select as unpaid caregiver the person who is most likely to accompany them to visits or help with their care should they need it.

Clinician eligibility criteria:

Oncology staff nurses who undergo training to deploy CONNECT, oncologists, and practice managers at participating sites will be eligible to participate.

Number of participants: Sample size of 672 patients and their unpaid caregivers proposed Diseases: Advanced cancer

Patient characteristics: Unknown as still ongoing

Deaths at end of study: Unknown as still ongoing

Withdrawals/other dropouts: Unknown as still ongoing

Interventions

Type: A primary palliative care intervention (CONNECT)

Service base: Hospital

Team: Care management by oncology nurses

Intervention: At clinics randomised to the CONNECT intervention, oncology nurses will be selected by a nurse advisory panel to receive standardised primary palliative care training. A multi-step deployment strategy will be employed to orient oncologists and implement CONNECT processes. CONNECT nurses will administer CONNECT to enrolled patients and unpaid caregivers. An intervention fidelity monitoring and maintenance plan will be implemented to ensure high quality and consistent delivery of the intervention.

Duration: Unclear

Control: At clinics randomised to usual care, enrolled patients and unpaid caregivers will continue to receive supportive oncology care according to usual practice.

Outcomes

Primary outcomes:

Quality of Life - patient. The investigators will compare change in the 3-month FACIT-Pal scores between enrolled patients at intervention clinics and enrolled patients at usual care clinics.

Secondary outcomes:

Symptom burden - patient. The investigators will compare change in the 3-month Edmonton Symptom Assessment Scale (ESAS) scores between enrolled patients at intervention clinics and enrolled patients at usual care clinics.

Depression and anxiety symptoms - patient. The investigators will compare change in the 3-month Hospital Anxiety and Depression Scale (HADS) scores between enrolled patients at intervention clinics and enrolled patients at usual care clinics.

Depression and anxiety symptoms - unpaid caregiver. The investigators will compare change in the 3-month Hospital Anxiety and Depression Scale (HADS) scores between enrolled unpaid caregivers at intervention clinics and enrolled unpaid caregivers at usual care clinics.

unpaid caregiver burden - unpaid caregiver. The investigators will compare change in the 3-month Zarit Burden Interview-Short scores between enrolled unpaid caregivers at intervention clinics and enrolled unpaid caregivers at usual care clinics.

Healthcare utilisation. To inform future dissemination efforts and aid in understanding optimal financing models, the investigators will calculate implementation costs of the intervention and determine the effects of CONNECT on healthcare utilisation, including hospitalisations, chemotherapy use, and hospice use.

Survival - patients. The investigators will calculate survival time from date of enrollment using the Kaplan-Meier method.

The effectiveness and cost-effectiveness of hospital-based specialist palliative care for adults with advanced illness and their caregivers 
Patient-oncologist therapeutic relationship. The investigators will compare change in the 3-month Human Connection Scale scores between enrolled patients at intervention clinics and enrolled patients at usual care clinics.

Hope - patients and unpaid caregivers. The investigators will compare change in the 3-month Herth Hope Index scores between enrolled patients and unpaid caregivers at intervention clinics and enrolled patients and unpaid caregivers at usual care clinics.

Self-efficacy - patients and unpaid caregivers. The investigators will compare 3-month scores on the Cancer Behavior Inventory-Brief (patients) and the unpaid caregiver Inventory ( unpaid caregivers) between enrolled patients and unpaid caregivers at intervention clinics and enrolled patients and unpaid caregivers at usual care clinics.

Satisfaction - unpaid caregivers. The investigators will compare change in the 3-month FAMCARE-2 scores between enrolled unpaid caregivers at intervention clinics and enrolled unpaid caregivers at usual care clinics.

Distress - patients. The investigators will compare change in the 3-month scores on the Distress Thermometer between enrolled patients at intervention clinics and enrolled patients at usual care clinics.

Illness understanding, care preferences, advance care planning - patients. The investigators will compare change in patient illness understanding, care preferences, and advance care planning between enrolled patients at intervention clinics and enrolled patients at usual care clinics.

Burnout - clinicians. The investigators will compare burnout (Maslach Burnout Inventory) between clinicians at intervention clinics and usual care clinics.

Satisfaction with CONNECT and recommendations for improvement. The investigators will assess satisfaction with CONNECT and recommendations for improvement among clinicians at intervention clinics annually.

Starting date July 2016

Contact information

Notes

NCT02719938

Study name Triggered palliative care for advanced dementia

Methods RCT

Participants

Country: USA

Recruitment: Inclusion criteria

- Diagnosis of dementia from Alzheimer's or other underlying cause

- Global Deterioration Scale (GDS) Stage 5, 6 or 7

- Acute illness hospitalisation

Exclusion Criteria:

- No English-speaking family decision-maker

- Primary physician expects study to be too stressful for family unpaid caregiver

Number of participants: Sample size of 120 participants proposed

Diseases: Advanced dementia

Patient characteristics: Unknown as still ongoing

Deaths at end of study: Unknown as still ongoing

Withdrawals/other dropouts: Unknown as still ongoing

The effectiveness and cost-effectiveness of hospital-based specialist palliative care for adults with advanced illness and their caregivers 
NCT02719938 (Continued)

Interventions
Type: Triggered palliative care

Service base: Hospital

Team: Interdisciplinary Palliative Care consultation during hospitalisation with post-discharge collaborative care by a Palliative Care Nurse Practitioner and outpatient primary care physician

Intervention: Specialty interdisciplinary Palliative Care consultation during hospitalisation with post-discharge collaborative care by a Palliative Care Nurse Practitioner and outpatient primary care physician. Clinical care will be augmented by evidence-based educational materials for dementia unpaid caregivers.

Duration: Unclear

Control: Usual care

Outcomes

Hospital transfers: includes emergency department visits and hospital admissions during measured interval

Secondary outcomes:

Comfort: comfort at the End of Life in Dementia (CAD-EOLD) instrument, consisting of 14 Likert-scaled items measuring comfort in the final phase of life with dementia. Scores range from 14-42, with higher scores indicating greater comfort.

unpaid caregiver Strain: Family Distress in Advanced Dementia instrument, a 21-item questionnaire designed to detect strain in family unpaid caregivers in dementia. unpaid caregivers are asked a series of items about emotional distress, preparedness, and relations with healthcare providers scored 1-5.

Access to hospice or palliative care: percent with access to specialty palliative care healthcare provider or hospice within 30 days post-discharge

POLST (Physician Orders for Life Sustaining Treatment): percent with POLST form completed and signed

Palliative care domains: number of palliative care domains addressed in treatment plan, using the Palliative Care Domain score which is scored 0 (not addressed) or 1 (addressed) for each of 10 possible domains of a palliative care treatment plan - prognosis, overall goals of care, physical symptoms, psychiatric symptoms, spiritual needs, and 5 treatment preferences: resuscitation, artificial feeding, intravenous fluids, antibiotics, and hospitalisation. Scores are summed for a total possible score of $0-10$, with higher scores indicating greater attention to palliative care needs in the treatment plan.

Burdensome treatment: number of burdensome treatments per patient, defined as a count per patient of use of the following treatments: feeding tube, central intravenous line, surgical procedure, intensive care transfer, ventilator use, cardiopulmonary resuscitation use at any time during the time frame of measurement 
NCT02786524

Study name
A randomized study to evaluate the effect of outpatient symptom management on symptom burden in advanced stage or recurrent gynecologic oncology patients receiving chemotherapy

\begin{tabular}{|c|c|}
\hline Methods & $\mathrm{RCT}$ \\
\hline Participants & $\begin{array}{l}\text { Country: USA } \\
\text { Recruitment: Inclusion criteria } \\
\text { - Female } \\
\text { - } 18 \text { years of age or older } \\
\text { - Diagnosis of Stage III, IV, or recurrent gynaecologic malignancy (uterine, ovarian, cervical, vulvar, } \\
\text { vaginal, fallopian tube, primary peritoneal) } \\
\text { - Receiving active intravenous, intraperitoneal, or oral chemotherapy } \\
\text { - Patient at University of Michigan Gynecologic Oncology Clinic } \\
\text { Exclusion Criteria: } \\
\text { - Male } \\
\text { - Less than } 18 \text { years of age } \\
\text { - Patients without a diagnosis of a gynaecologic malignancy } \\
\text { - Patients not receiving intravenous, intraperitoneal or oral chemotherapy at the time of enroll- } \\
\text { ment } \\
\text { - Patients receiving radiation therapy with chemo-sensitization. } \\
\text { Number of participants: Sample size of } 180 \text { participants proposed } \\
\text { Diseases: Gynaecologic malignancies } \\
\text { Patient characteristics: Unknown as still ongoing } \\
\text { Deaths at end of study: Unknown as still ongoing } \\
\text { Withdrawals/other dropouts: Unknown as still ongoing }\end{array}$ \\
\hline
\end{tabular}

Interventions

Type: Specialised symptom management and supportive care intervention

Service base: A specialised symptom management and supportive care clinic

Team: Symptom management providers

Intervention: Patients randomised to this arm are referred to a specialised symptom management and supportive care clinic and seen within two weeks. Patients will be seen in follow-up as recommended by the symptom management providers, and at each visit they will complete the ESAS-r and NCCN distress thermometer and return their responses either in person or by mail to the study team in a pre-addressed postage-paid envelope.

Duration: Unclear

Control: Patients randomised to this arm receive standard symptom management care by their primary gynaecologic oncologist and complete the NCCN distress thermometer and ESAS-r at each visit, every 3-4 weeks.

Outcomes

Primary outcomes:

Change in patient-reported symptom burden as determined by the Edmonton Symptom Assessment System: To determine if referral to a symptom management clinic improves the symptom burden quantified by the patients' Edmonton Symptom Assessment System (ESAS) scores

Change in patient-reported symptom burden as determined by the National Comprehensive Cancer Network Distress Screening Tool: To determine if referral to a symptom management clinic improves the symptom burden quantified by the patients' National Comprehensive Cancer Network (NCCN) Distress Screening Tool scores

Secondary outcomes:

The effectiveness and cost-effectiveness of hospital-based specialist palliative care for adults with advanced illness and their caregivers 
Baseline symptom burden and palliative care needs: To determine the baseline symptom burden and palliative care needs upon enrollment of patients with advanced stage or recurrent gynaecologic malignancies receiving chemotherapy

Change in patient-reported distress: To measure changes in patient-reported distress using the NCCN Distress Thermometer

Change in symptom burden: To evaluate changes in patients' symptom burden with the ESAS-r to evaluate the ongoing sustained effect of the intervention

Change in patient adherence to symptom management programme: To analyse patients in the symptom management arms with a 3-item questionnaire to determine what factors are associated with adherence to a symptom management programme

Barriers to symptom management and supportive care: To analyse barriers that prevented patients from attending the Symptom Management and Supportive Care Clinic, as identified in a 3-item questionnaire

Overall survival rate: Overall survival will be compared between the two study arms.

Starting date $\quad$ February 2016

Contact information

Notes

NCT02868112

Study name Pilot study of a transdisciplinary intervention integrating geriatric and palliative care with oncology care for older adults with cancer

\begin{tabular}{ll}
\hline Methods & RCT \\
\hline Participants & Reuntry: USA \\
- Age 65 or older & - Diagnosed with incurable (defined as metastatic or receiving chemotherapy with palliative intent) \\
& esophageal, gastric, pancreas, hepatobiliary, colorectal, or lung cancer within the prior 8 weeks \\
(including patients with prior diagnosis of cancer who developed incurable disease) & Verbal fluency in English \\
- Planning to receive care at Massachusetts General Hospital (MGH) \\
Exclusion Criteria: \\
- Unwilling or unable to participate in the study \\
- Significant psychiatric, cognitive or other comorbid disease which the treating clinician believes \\
prohibits informed consent or participation in the study \\
- No medical problems for geriatric clinician to address (e.g. comorbidities, polypharmacy, etc.) \\
Number of participants: Sample size of 62 participants proposed \\
Diseases: Incurable cancer \\
Patient characteristics: Unknown as still ongoing \\
Deaths at end of study: Unknown as still ongoing \\
Withdrawals/other dropouts: Unknown as still ongoing
\end{tabular}

Interventions

Type: Transdisciplinary Intervention Integrating Geriatric And Palliative Care With Oncology Care

Service base: Unclear

The effectiveness and cost-effectiveness of hospital-based specialist palliative care for adults with advanced illness and their caregivers 211 (Review)

Copyright $\odot 2020$ The Cochrane Collaboration. Published by John Wiley \& Sons, Ltd. 
Intervention: Patients randomised to the transdisciplinary intervention will undergo evaluation with a board-certified geriatric clinician, who will tailor their care based on the results of a brief geriatric screening tool, completed prior to their visit. A two-visit transdisciplinary intervention will be tested with the first visit occurring within four weeks of enrollment and the second visit four weeks after the initial visit.

\section{Duration: Unclear}

Control: Participants receiving usual oncology care will not meet routinely with geriatric clinicians, though they may receive a geriatrics consult at their request or at the discretion of their treating team.

\section{Outcomes}

\section{Primary outcome:}

Perceptions of the necessary components of a transdisciplinary intervention integrating geriatrics and palliative care with oncology care for older patients with incurable GI and lung cancers. Investigators will use qualitative assessment methods to characterise the sample and explore participant perceptions of the supportive care needs of older patients with cancer.

Secondary outcomes:

Rates of study enrollment (proportion of eligible patients who enroll in the study; to demonstrate feasibility)

Rates of study completion (the proportion of participants who complete both study visits; to demonstrate feasibility)

Mean change in quality of life (measured with the Functional Assessment of Cancer Therapy (FACT) - General) scores between treatment groups from baseline to week 12. Investigators will compare mean change in QoL scores between treatment groups from baseline to week 12.

Mean change in symptom scores (measured continuously with the Edmonton Symptom Assessment System-revised (ESAS-r)) between treatment groups from baseline to week 12. Investigators will compare mean change in symptom scores between treatment groups from baseline to week 12.

Mean change in depression scores (measured continuously with the Geriatric Depression Scale (GDS)) between treatment groups from baseline to week 12. Investigators will compare mean change in depression scores between treatment groups from baseline to week 12 .

Rates of post-intervention moderate/severe symptoms (measured as presence or absence of moderate/severe symptoms using the Edmonton Symptom Assessment System-revised (ESAS-r)) between treatment groups. Investigators will compare rates of post-intervention moderate/severe symptoms (defined as ESAS scores $\geq 4$ ) between treatment groups.

Rates of post-intervention depression symptoms (measured as presence or absence of depression symptoms using the Geriatric Depression Scale (GDS)) between treatment groups. Investigators will compare rates of post-intervention depression symptoms (defined as GDS scores > 5) between treatment groups.

Mean change in activities of daily living (measured continuously using a subscale of the Medical Outcomes Study (MOS) Physical Health) between treatment groups from baseline to week 12. Investigators will compare mean change in activities of daily living between treatment groups from baseline to week 12.

Mean change in instrumental activities of daily living (subscale of the Multidimensional Functional Assessment Questionnaire from the Older American Resources and Services (OARS)) between treatment groups from baseline to week 12 . Investigators will compare mean change in instrumental activities of daily living between treatment groups from baseline to week 12 . 
Mean change in illness perceptions (measured continuously using the Brief Illness Perceptions Questionnaire (BIPQ)) between treatment groups from baseline to week 12. Investigators will compare mean change in illness perceptions between treatment groups from baseline to week 12 .

Mean change in self-efficacy (measured continuously using the Perceived Efficacy in Patient-Physician Interactions (PEPPI)) between treatment groups from baseline to week 12. Investigators will compare mean change in self-efficacy between treatment groups from baseline to week 12 .

Rates of post-intervention deficits in activities of daily living (measured as presence or absence of deficits in activities of daily living using a subscale of the Medical Outcomes Study (MOS) Physical Health) between treatment groups. Investigators will compare rates of post-intervention deficits in activities of daily living between treatment groups.

Rates of post-intervention deficits in instrumental activities of daily living (subscale of the Multidimensional Functional Assessment Questionnaire from the Older American Resources and Services (OARS)) between treatment groups. Investigators will compare rates of post-intervention deficits in instrumental activities of daily living between treatment groups.

Rates of hospice enrollment prior to death (measured as rates of hospice enrollment prior to death) between treatment groups. Investigators will compare rates of hospice enrollment prior to death between treatment groups.

Acceptability of the Transdisciplinary Intervention to older patients with incurable GI and lung cancers. Investigators will evaluate acceptability using exit assessment data. Participants will rate their satisfaction with the structure, timing and content of the intervention, using Likert-type scale responses.

Starting date October 2016

Contact information

Notes

NCT02929966

Study name Effect of palliative care in patients with end stage pulmonary fibrosis: a randomized control study

\begin{tabular}{ll}
\hline Methods & RCT \\
\hline
\end{tabular}

Participants

Country: Italy

Recruitment: Inclusion Criteria:

- diagnosis of idiophathic pulmonary fibrosis

- resting partial arterial oxygen pressure $(\mathrm{PaO} 2)<60 \mathrm{mmHg}$

- decline in Forced Vital Capacity (FVC) $>10 \%$ in the last 6 months

- stage 3 according to the GAP index

Exclusion Criteria:

- active treatment with antifibrotic drug

- concomitant cancer

Number of participants: Sample size of 50 participants proposed

Diseases: Idiopathic pulmonary fibrosis

Patient characteristics: Unknown as still ongoing

Deaths at end of study: Unknown as still ongoing

Withdrawals/other dropouts: Unknown as still ongoing 
NCT02929966 (Continued)

Service base: Unclear

Team: Unclear

Intervention: The patients will receive the usual respiratory care that included both the 'classical' treatments with antifibrotic drugs and oxygen therapy PLUS a palliative care programme that includes psychological support, spiritual care and respiratory therapist support.

Duration: Unclear

Control: The patients will receive the usual respiratory care that included both the 'classical' treatments with antifibrotic drugs and oxygen therapy

\begin{tabular}{ll}
\hline Outcomes & Primary outcome: \\
& Quality of life assessed using the Maugeri Respiratory Questionnaire reduced form \\
& Depression assessed using the Centre for Epidemiologic Studies Depression score (CES-D) \\
& Dyspnoea score assessed using the Borg scale \\
& Secondary outcomes: \\
& Survival \\
\hline Starting date & July 2016 \\
\hline Contact information & \\
\hline Notes & \\
\hline
\end{tabular}

\section{NCT02975869}

$\begin{array}{ll}\text { Study name } & \begin{array}{l}\text { Randomized trial of a collaborative palliative and oncology care model for patients with acute } \\ \text { myeloid leukemia (AML) }\end{array}\end{array}$

\begin{tabular}{ll}
\hline Methods & RCT \\
\hline Participants & Recruitment: Inclusion Criteria: \\
- Hospitalised patients with high-risk AML, defined as: \\
- Newly diagnosed patients with AML $\geq 60$ years of age \\
- Newly diagnosed AML with antecedent haematologic disorder \\
- Newly diagnosed therapy-related AML \\
- Relapsed AML \\
Exclusion Criteria: \\
- Patients already receiving palliative care \\
- Major psychiatric illness or comorbid conditions prohibiting compliance with study procedures \\
- A diagnosis of acute promyelocytic leukaemia (APML) \\
Number of patients: Sample size of 160 patients proposed \\
Diseases: Acute myeloid leukaemia \\
Patient characteristics: Unknown as still ongoing \\
Deaths at end of study: Unknown as still ongoing
\end{tabular}

The effectiveness and cost-effectiveness of hospital-based specialist palliative care for adults with advanced illness and their caregivers 


Type: Collaborative palliative care and oncology care
Service base: Hospital
Team: Palliative care and oncology clinicians
Intervention: Collaborative care from palliative care and leukaemia will be given
Duration: Unclear
Control: Standard leukaemia care

\section{Outcomes} Primary outcome:

Change in patients' FACT-Leukemia score from baseline to week-2 between study arms Secondary outcomes:

Change in FACT-Leukemia scores from baseline to months 1,3 , and 6 , and longitudinally between the study arms

Patients' depressive symptoms (as per HADS), and major depressive syndrome (as per PHQ-9) at baseline, week 2, months 1,3 , and 6 , and longitudinally between the two arms

Change In patients' fatigue scores (as per FACT-fatigue) from baseline to week 2, baseline to months 1,3 , and 6 , and longitudinally between study arms

Symptom burden (as per ESAS) at baseline, week 2, months 1, 3, and 6, and longitudinally between study arms

Patient-reported PTSD (as per PCL) at week 2, months 1, 3, and 6, and longitudinally between study arms

Patient-report of discussion of end-of-life (EoL) care preferences between study arms (time frame: 6 months)

Rates of documentation of EoL care preferences (i.e. code status yes, documented vs. no) In the electronic health record between the two study arms within 30 days of death

Rates of chemotherapy administration within 3, 7, 14, and 30 days of death between the two study arms

Rates of hospitalisations within 3, 7, 14, and 30 days of death between the study arms

Rates of hospice utilisation and length-of-stay in hospice at the EoL between the study arms

Starting date September 2016

Contact information

Notes

\section{NCT03022630}

Study name

The Creation Of Models for Palliative Assessments to Support Severe illlness (COMPASS) investigation: Testing early and ongoing implementation of palliative care for incurable non-malignant diseases 
NCT03022630 (Continued)

Methods RCT

Participants

Country: USA

Recruitment: Inclusion Criteria:

- Inpatient at Vanderbilt University Medical Center with advanced cirrhotic liver disease, whose treating hepatologist indicates a 'No' response to the question, "Would you be surprised if this patient died within 1 year?"

Exclusion Criteria:

- Age $<18$ years

- Receipt of liver transplant at the time of potential enrollment

- Inability to give written informed consent (patient or surrogate decision-maker)

- Inability to respond to questions in English

- Treating hepatologist denies permission to enroll

- Receiving hepatology care at non-Vanderbilt sites (to ensure appropriate follow-up)

Number of participants: Sample size of 63 participants proposed

Diseases: Liver disease

Patient characteristics: Unknown as still ongoing

Deaths at end of study: Unknown as still ongoing

Withdrawals/other dropouts: Unknown as still ongoing

Interventions

Type: Integrated comprehensive palliative care services

Service base: Hospital

Team: A palliative care physician or nurse practitioner

Intervention: Participants who are randomly assigned to the intervention arm will receive informational materials, comprehensive inpatient palliative care consultations with a palliative care physician or nurse practitioner, in addition to standard hepatic care. If the patient is discharged, follow-up consults will be provided by a palliative care nurse via telephone contact. Telephone contacts will occur on a flexible schedule (e.g. weekly, bi-weekly, monthly) based on the needs and wishes of the patient, at a minimum frequency of once a month. If a need for further care is identified from a telephone contact, appropriate follow-up (referral, appointment, clinical communication, etc.) will occur per standard procedures. If the participant is readmitted, the consultation schedule will restart.

Standard care will be provided as per the hospital guideline.

Duration: 1 year

Control: Participants randomised to the usual care arm will not be scheduled to meet with the palliative care service unless a consult is requested by the patient, the family, or treating physician. These consultations would include the same palliative care services as the intervention arm, excluding the informational patient materials and telephone consultations.

Outcomes

Primary outcome:

Time to first hospital readmission within 1-year post randomisation. Assessment of the impact of palliative care services on time to first hospital readmission

Secondary outcomes:

Days alive out of hospital: from randomisation to 6 months post-randomisation compared across arms

Total days in hospital: from randomisation to 1 year post-randomisation compared across arms

Total days in ICU: from randomisation to 1 year post-randomisation compared across arms

The effectiveness and cost-effectiveness of hospital-based specialist palliative care for adults with advanced illness and their caregivers 
Number of hospital readmissions: from randomisation to 1 year post-randomisation compared across arms

Median length of hospital stay per admission: from randomisation to 1 year post-randomisation compared across arms

Hospice Referral: number of transfers to hospice within 1 year post-randomisation compared across arms

Time to hospice placement: number of days from hospice referral to time to hospice placement

Chronic Liver Disease Questionnaire (CLDQ). Change in liver disease-related quality of life: the CLDQ is a 29-item questionnaire measuring 6 domains. Item scores range from 1 to 7 with higher scores indicating better quality of life.

EQ-5D-5L. Change in generic health status: the EQ-5D-5L is a 5-item questionnaire with responses ranging from absence of symptom to extreme experience of the symptom.

PROMIS Emotional Distress - Anxiety - Short Form 4a. Change in mood (anxiety): the PROMIS Emotional Distress - Anxiety - Short Form 4a contains 4 items that measure anxiety on a 5-point Likert scale with higher scores indicating increased symptomatology.

PROMIS Emotional Distress - Depression - Short Form 4a. Change in mood (depression): the PROMIS Emotional Distress - Depression - Short Form 4a contains 4 items that measure depression on a 5-point Likert scale with higher scores indicating increased symptomatology.

Satisfaction with care (Quality of End-of-Life Care: Questionnaire for Patient). Change in patient satisfaction with care: the Quality of End-of-Life Care: Questionnaire for Patient is an 11-item questionnaire, with items scored on a 10-point Likert type scale with higher scores indicating better quality of care.

Kingston unpaid caregiver Stress Scale: will be used to measure unpaid caregiver stress (lay unpaid caregivers, not institutional staff) and was designed to monitor change in an individuals stress over time. Ten items are grouped into three categories: caregiving, family, and financial issues.

Satisfaction with care (Quality of End-of-Life Care: Questionnaire for Patient adapted for unpaid caregiver); measures change in unpaid caregiver satisfaction with care with wording modified to apply to unpaid caregivers. The questionnaire is an 11-item questionnaire, with items scored on a 10-point Likert type scale with higher scores indicating better quality of care.

Liver Transplant Status: number of deferred, listed, and declined listing for liver transplant compared across arms

MELD Score: change in MELD score compared across arms

Completed liver transplants: number of patients with completed liver transplants compared across arms

Physical symptoms: number of documented physical symptoms (ascites, variceal bleeding, encephalopathy, etc.) compared across arms

Presence of advance directives: percentage of patients with documented advance care directives compared across arms

Survival: survival rate compared across arms

Provider satisfaction: the 'ICU Provider Satisfaction Survey with the Palliative Care Program:Veterans Affairs of Ann Arbor' instrument is available online and has been modified for the current study by removing the 'ICU' reference and revising 'pain' to symptoms more relevant to the current population. 
NCT03022630 (Continued)

Contact information

Notes

NCT03088202

Study name

PALLION - PALLiative Care In ONcology - a cluster-randomized trial to improve the care for cancer patients with a short life expectancy

\begin{tabular}{|c|c|}
\hline Methods & Multicentre cluster-RCT \\
\hline Participants & $\begin{array}{l}\text { Country: Norway } \\
\text { Recruitment: Inclusion Criteria: } \\
\text { - A verified metastatic or locally advanced cancer of the upper GI tract, lower GI tract, pancreas, } \\
\text { liver, breast, bladder, prostate, kidney, cholangiocarcinoma, or malignant melanoma } \\
\text { - Defined as a palliative care patient, with expected life expectancy of }<12 \text { months } \\
\text { - Scheduled to start what is perceived as last-line chemotherapy (definition: tumour-directed med- } \\
\text { ical therapy). Observe: different time points for inclusion and line of treatment apply for the spe- } \\
\text { cific diagnoses } \\
\text { - Age > } 18 \text { years } \\
\text { - Fluency in written and oral Norwegian } \\
\text { - Physically and cognitively able to provide written informed consent, based on clinical judgement } \\
\text { - Scheduled to receive all oncological and specialised palliative treatment at the participating hos- } \\
\text { pital } \\
\text { - World Health Organization (WHO) performance status } 0-2 \\
\text { Exclusion Criteria: } \\
\text { - Any serious psychiatric diagnosis (e.g. psychotic, bipolar disorder), substance abuse or cognitive } \\
\text { impairment as judged by standard clinical criteria (disturbed consciousness, disorientation to } \\
\text { time/place and attention deficits) at the discretion of the attending physician that precludes com- } \\
\text { pletion of PROs } \\
\text { - A cancer diagnosis other than the ones above } \\
\text { - Multiple malignancies } \\
\text { - Serious substance abuse } \\
\text { - Already included in a palliative care programme } \\
\text { Number of participants: Sample size of } 600 \text { participants proposed } \\
\text { Diseases: Advanced cancer } \\
\text { Patient characteristics: Unknown as still ongoing } \\
\text { Deaths at end of study: Unknown as still ongoing } \\
\text { Withdrawals/other dropouts: Unknown as still ongoing }\end{array}$ \\
\hline
\end{tabular}

Interventions

Type: Educational programme, standardised care pathways and early palliative care

Service base: Hospital

Team: Unclear

Intervention: Comprises

- Educational programme: E-learning lectures, group exercises, skills training

- Standardised care pathways: Pathways for systematic follow-up

- Early palliative care: Compulsory referral to palliative care at inclusion

Duration: Unclear

The effectiveness and cost-effectiveness of hospital-based specialist palliative care for adults with advanced illness and their caregivers 
NCT03088202 (Continued)

Control: Usual care

Outcomes Primary outcomes:

Use of chemotherapy: number of patients who receive chemotherapy during their last 3 months of life

Use of chemotherapy: number of patients who receive chemotherapy during their last month of life

Secondary outcomes:

Use of artificial nutrition: number of patients who receive artificial nutrition during their last month of life

Use of concomitant medication: number of patients who receive concomitant medication during their last month of life

Starting date March 2017

Contact information

Notes

NCT03170466

\begin{tabular}{ll}
\hline Study name & Primary palliative care in heart failure: a pilot trial \\
\hline Rethods & RCT \\
\hline Participants & Recruitment: Inclusion Criteria: \\
- New York Heart Association Class III or IV heart failure & - or more hospitalisations in the past year due to heart failure \\
& Exclusion Criteria: \\
- Less than 40 years old \\
- Currently awaiting a transplant \\
- Received palliative care within the past 12 months \\
- Pregnant or intends to be within the next 12 months \\
- No regular phone access \\
- Not fluent in English \\
- Failed the Callahan 6-item Screener \\
- Does not intent to regularly attend clinic for the next 12 months \\
Number of participants: Sample size of 30 participants proposed \\
Diseases: Advanced heart failure \\
Patient characteristics: Unknown as still ongoing \\
Deaths at end of study: Unknown as still ongoing \\
Withdrawals/other dropouts: Unknown as still ongoing
\end{tabular}

Interventions

Type: Primary palliative care plus usual care

Service base: Hospital

Team: Cardiology nurses with training in palliative care. The nurse will act as a liaison to communicate concerns to the patient's cardiologist and primary care physician

The effectiveness and cost-effectiveness of hospital-based specialist palliative care for adults with advanced illness and their caregivers 
Intervention: The intervention will be delivered through four primary mechanisms. First, an existing HF nurse will deliver the intervention to patients during regularly scheduled visits. Second, telephone calls will reinforce topics. Third, patients will regularly report symptoms through the MyUPMC patient portal. Fourth, the nurse will act as a liaison to communicate concerns to the patient's cardiologist and primary care physician, as well as facilitating other resources (e.g. home health). In addition, follow-up assessments will be completed via phone or email at least 2 weeks post-intervention delivery. unpaid caregivers will complete surveys during the first in-person visit and then during the follow-up assessments.

\section{Duration: Unclear}

Control: Patients randomised to the control arm of this study will continue to receive the standard of high-quality HF care provided to all patients. Control patients may still receive palliative care outside of the study.

\begin{tabular}{ll}
\hline Outcomes & Primary outcome: \\
& Feasibility of enrolling 30 patients via attempting to enroll 30 patients \\
& Secondary outcomes: \\
& $\begin{array}{l}\text { Intervention acceptability through interviews with patients, unpaid caregivers, and healthcare } \\
\text { providers. }\end{array}$ \\
& $\begin{array}{l}\text { Intervention fidelity via the Intervention Fidelity Monitoring Report. This will be by assessing inter- } \\
\text { vention fidelity by audio-recording the interventions and having independent reviewers listen to } \\
\text { them and complete the Intervention Fidelity Monitoring Report. }\end{array}$ \\
\hline Starting date & October 2017 \\
\hline Contact information & \\
\hline Notes & \\
\hline
\end{tabular}

\section{NCT03181854}

\begin{tabular}{|c|c|}
\hline Study name & Randomized controlled trial of integrated early palliative care for advanced cancer patients \\
\hline Methods & $\mathrm{RCT}$ \\
\hline Participants & $\begin{array}{l}\text { Country: South Korea } \\
\text { Recruitment: Inclusion Criteria: } \\
\text { - Subject } 20 \text { years and older. } \\
\text { - Subject who has an advanced cancer diagnosis (histologically or cytologically confirmed) due to } \\
\text { a solid tumour } \\
\text { - Subject whose ECOG performance status is between } 0 \text { to } 2 \\
\text { - Subject with an estimated life expectancy of } 12 \text { months and less (assessed by the treating oncol- } \\
\text { ogist) } \\
\text { - Subject who volunteers } \\
\text { Exclusion Criteria: } \\
\text { - Inability to speak, understand or write Korean } \\
\text { - Medical conditions that would limit adherence to participation of the clinical trial (as confirmed } \\
\text { by their referring physician; e.g. dyspnoea) } \\
\text { - Suspension of all cancer treatment } \\
\text { - Palliative care consultation at any time or in palliative care }\end{array}$ \\
\hline
\end{tabular}

The effectiveness and cost-effectiveness of hospital-based specialist palliative care for adults with advanced illness and their caregivers 
NCT03181854 (Continued)

Number of participants: sample size of 144 participants proposed

Diseases: advanced cancer

Patient characteristics: Unknown as still ongoing

Deaths at end of study: Unknown as still ongoing

Withdrawals/other dropouts: Unknown as still ongoing

Interventions

Type: Integrated early palliative care

Service base: Unclear

Team: Palliative care team (PCT). PCT doctor mentioned but unclear if other health and care professionals will be involved

Intervention: Consultation with PCT doctor every 3 weeks. Telephone coaching once a week for 3 months and once in 2 weeks for another 3 months

Duration: 6 months

Control: Usual palliative care can be provided if desired.

Change in level of EORTC QLQ-C15-PAL. A questionnaire developed to assess the quality of life of palliative cancer care patients

Secondary outcomes:

Change in level of MQOL: a questionnaire that measures psychological, existential well-being, and support

Change in level of EQ-5D of EuroQoL: a questionnaire that measures mobility, self-care, daily activity, pain/discomfort, and anxiety/depression

Change in level of PHQ-9: 9-question instrument given to patients in a primary care setting to screen for the presence and severity of depression

Change in level of understanding the illness: 2 questions to assess how patients understand the prognosis of their illness

Change in level of crisis overcoming capability (SAT-SF). A questionnaire about goal of life, current crisis/goal, positivity, preparation and practice

Change in advance care preference: questions about advance directive and treatment preference in case of terminal condition

Medical cost and utilisation of CAM: overall medical cost savings (cost-effectiveness) and use of complementary and alternative medicine

1-year survival

Changes of CQOL: a questionnaire that measures quality of life and burden for family unpaid caregivers

Change in level of PHQ-9 of family unpaid caregivers: 9-question instrument given to unpaid caregivers in a primary care setting to screen for the presence and severity of depression

Change in level of understanding the illness by family unpaid caregivers: 2 questions to assess how family unpaid caregivers understand the prognosis of patients' illness

Change in level of crisis overcoming capability (SAT-SF) of family unpaid caregivers: a questionnaire about goal of life, current crisis/goal, positivity, preparation and practice of family unpaid caregivers

Change in advance care preference of family unpaid caregivers: questions about family unpaid caregivers' preference on advance directive and treatment in case of terminal condition

The effectiveness and cost-effectiveness of hospital-based specialist palliative care for adults with advanced illness and their caregivers 
NCT03181854 (Continued)

Change in Quality Care questionnaire

Starting date

September 2017

Contact information

Notes

NCT03229343

Study name

Impact of a systematic palliative care on quality of life in advanced idiopathic pulmonary fibrosis (IPF). A randomized multi-centre trial

\begin{tabular}{|c|c|}
\hline Methods & $\mathrm{RCT}$ \\
\hline Participants & $\begin{array}{l}\text { Country: France } \\
\text { Recruitment: Inclusion Criteria: } \\
\text { - Age > } 40 \text { years } \\
\text { - Patient with confirmed diagnosis of IPF according to the American Thoracic Society (ATS)/Euro- } \\
\text { pean Respiratory Society (ERS)/Japanese Respiratory Society (JRS)/Latin American Thoracic As- } \\
\text { sociation (ALAT) criteria. The patient may be included regardless of the date of diagnosis } \\
\text { - Advanced IPF with forced vital capacity (FVC) }<50 \% " \text { of predicted value and/or diffusing capacity } \\
\text { for carbon monoxide ((DLCO) < } 30 \% \text { of predicted value or inability to achieve the functional res- } \\
\text { piratory investigations (EFR) due to respiratory severity. EFR dated less than } 3 \text { months } \\
\text { - Absence of argument for acute or subacute exacerbation in the last } 6 \text { months } \\
\text { - Patient who can be followed in ambulatory consultation/outpatient consultation } \\
\text { - Informed consent signed (signed by the patient or in the presence of a third party for patients who } \\
\text { are poorly fluent in French) } \\
\text { - Affiliation to the social security system } \\
\text { Exclusion Criteria: } \\
\text { - Patient unable to respond to quality of life questionnaires } \\
\text { - Inability (physical or mental) to give a written informed consent } \\
\text { - Acute exacerbation of fibrosis in the previous } 6 \text { months } \\
\text { - Patient eligible for a pulmonary transplant } \\
\text { - Participation in other therapeutic trial } \\
\text { - Patient cannot be followed in ambulatory consultation } \\
\text { - Patient under trustee } \\
\text { Number of participants: Sample size of } 120 \text { participants proposed } \\
\text { Diseases: Advanced idiopathic pulmonary fibrosis } \\
\text { Patient characteristics: Unknown as still ongoing } \\
\text { Deaths at end of study: Unknown as still ongoing } \\
\text { Withdrawals/other dropouts: Unknown as still ongoing }\end{array}$ \\
\hline
\end{tabular}

Interventions

Type: Systematic palliative care

Service base: Unclear

Team: A palliative intervention staff and a chest physician team

Intervention: Supportive care jointly with pneumological consultation, monthly, starting at month 0 and continuing up to month 6

Duration: 6 months

The effectiveness and cost-effectiveness of hospital-based specialist palliative care for adults with advanced illness and their caregivers 
NCT03229343 (Continued)

Control: Pneumological consultation performed at months 0,3 and 6

Outcomes

Primary outcome:

The benefit of a systematic, formalised and joint intervention of palliative intervention staff and a chest physician team on quality of life, evaluated after 6 months by the Short Form (36) Health Survey

Secondary outcomes:

The benefit of the systematic, formalised and joint intervention of a supportive care and a pneumologist team on mood and depression evaluated by the Hospital Anxiety and Depression questionnaire

The benefit of the systematic, formalised and joint intervention of a supportive care and a pneumologist team on understanding of diagnosis and therapeutic objectives and frequency of drafting of advance directives will be evaluated by the illness understanding questionnaire.

The benefit of the systematic, formalised and joint intervention of a supportive care and a pneumologist team on respiratory symptoms (dyspnoea) will be evaluated by St George's respiratory questionnaire (SGRQ) and Transition Dyspnea Index (TDI).

The benefit of the systematic, formalised and joint intervention of a supportive care and a pneumologist team on the course of care, the use of palliative care stays and the duration of hospital stays (number and duration of hospitalisations)

The benefit of the systematic, formalized and joint intervention of a supportive care and a pneumologist team on the overall survival (survival follow-up visit at month 12).

The benefit of the systematic, formalised and joint intervention of a supportive care and a pneumologist team on the overall survival measured between inclusion and date of death or last news

Carrying out a medico-economic study evaluating the incremental cost-utility and cost-effectiveness ratio (overall survival criterion) evaluated by the medico-economic questionnaire: EuroQol five dimensions questionnaire (EQ-5D)

Starting date

December 2017

Contact information

Notes

\section{NCT03310918}

Study name

Randomized trial of a collaborative palliative and leukemia care model for patients with acute myeloid leukemia receiving non-Intensive therapy

\begin{tabular}{ll}
\hline Methods & RCT \\
\hline Participants & $\begin{array}{l}\text { Country: USA } \\
\text { Recruitment: Inclusion Criteria: }\end{array}$ \\
- Patients with AML receiving non-intensive therapy including hypomethylating agents, sin- \\
gle-agent chemotherapy, targeted therapy agents, or single or combination non-intensive agents \\
offered on a clinical trial, including the following populations: newly diagnosed AML, relapsed \\
AML, primary refractory AML \\
- The ability to provide informed consent \\
- The ability to comprehend English or complete questionnaires with minimal assistance of an in- \\
terpreter
\end{tabular}

The effectiveness and cost-effectiveness of hospital-based specialist palliative care for adults with advanced illness and their caregivers 
NCT03310918 (Continued)

\section{Exclusion Criteria:}

- Patients not receiving care at MGH

- Patients receiving intensive chemotherapy (requiring 4-6 week hospitalisation)

- Patients receiving supportive care alone

- Major psychiatric illness or comorbid conditions prohibiting compliance with study procedures

- Patients already receiving palliative care

Number of patients: Sample size of 320 patients proposed

Diseases: Acute myeloid leukaemia

Patient characteristics: Unknown as still ongoing

Deaths at end of study: Unknown as still ongoing

Withdrawals/other dropouts: Unknown as still ongoing

Interventions

Type: Collaborative palliative care and oncology care

Service base: Hospital

Team: Physicians and advanced practice nurses trained in the care of patients facing serious illness Intervention: Collaborative palliative and oncology care comprises the following:

- 1st palliative care visit within 96 hours of randomisation in the outpatient or hospital

- In outpatient setting: at least once weekly for the first 30 days and then at least twice per month; thereafter palliative care clinic visits or contact via telephone

- During hospital admissions to MGH: at least twice weekly palliative care visits

Standard care will be provided as per the hospital guideline.

Duration: 2 years

Control: Standard care will be provided as per the hospital guideline and may involve palliative care consults only upon request.

Outcomes

Primary outcomes:

Time from documentation of end-of-life care preferences to death

Secondary outcomes:

Rates of documentation of end-of-life care preferences at least one week prior to death

Patient report of discussing end-of-life care preferences based on an item from the perception of treatment and prognosis questionnaire

Comparison of rate of hospitalisation between the study arms

Rate of hospice utilisation and length-of-stay in hospice

Change in quality of life using the FACT-Leuk

Quality of end-of-life care using the FAMCARE

Symptom burden using the ESAS

Mood using the HADS

Other outcome measures:

Chemotherapy administration

Rates of death in hospital

The effectiveness and cost-effectiveness of hospital-based specialist palliative care for adults with advanced illness and their caregivers 
NCT03310918 (Continued)

unpaid caregiver-reported discussion of end-of-life care preferences as measured by the perception of treatment and prognosis questionnaire

Starting date October 2017

Contact information

Notes

NCT03456323

$\begin{array}{ll}\text { Study name } & \begin{array}{l}\text { Post-ICU palliative care consultation intervention pilot trial in older survivors of acute respiratory } \\ \text { failure }\end{array}\end{array}$
failure

\begin{tabular}{ll}
\hline Methods & RCT \\
\hline Participants & Country: USA \\
& Recruitment: Inclusion criteria:
\end{tabular}

- Age $\geq 50$ years

- Acute respiratory failure requiring invasive mechanical ventilation, non-invasive mechanical ventilation (continuous or bi-level positive pressure), or high flow nasal cannula for greater than 24 hours in a Columbia University Medical Center medical or surgical ICU

Exclusion Criteria:

- Hospital discharge directly from ICU

- Already received palliative care consultation during the hospitalisation

- Planned discharge to hospice or home hospice

- Respiratory failure due to neurologic diagnosis (intracranial haemorrhage, stroke, or coma)

- Pre-existing neurologic disease or stroke with motor deficits. Older adults with motor diseases (e.g. Parkinson's disease) will be excluded from frailty measurements because they could present with frailty characteristics from a single disease

- Psychiatric history of bipolar disorder, schizoaffective disorder, or schizophrenia

- Current alcoholism or drug abuse

- Not English or Spanish speaking. Many surveys are not validated in other languages besides English or Spanish.

- No healthcare proxy or surrogate also consenting to participate

- Expected to be discharged to a location > 20 miles from Columbia University Medical Center

- Status post-heart, -lung, or -liver transplantation. These patients are not representative of the larger population of older adult survivors of acute respiratory failure.

Number of participants: Sample size of 80 participants proposed

Diseases: Acute respiratory failure

Patient characteristics: Unknown as results have not been published

Deaths at end of study: Unknown as results have not been published

Withdrawals/other dropouts: Unknown as results have not been published

Type: Palliative care consultation

Service base: Hospital

Team: Palliative care consultation team will be led by one of the board-certified palliative care physicians.

Intervention: The palliative care consultation team will be led by one of the board-certified palliative care physicians at Columbia University Medical Center. Over one or more visits, the palliative

The effectiveness and cost-effectiveness of hospital-based specialist palliative care for adults with advanced illness and their caregivers 
care consultation team will first review intervention participants' medical records and baseline Edmonton Symptom Assessment System (ESAS) scores. They will also directly assess participants' physical and psychological symptoms. They will provide supportive counselling, make treatment recommendations for burdensome symptoms to the primary team of physicians, and will address goals of care. They will document these activities in structured electronic medical record consultation notes.

\section{Duration: Unclear}

Control: Patient-surrogate pairs randomised to usual care will not have a palliative care consultation intervention offered, and will receive care by their primary physicians. However, if a palliative care consultation is requested after randomisation to usual care by the primary team of physicians and/or the patient/surrogate, it will be provided.

Annual enrollment rate: the number of patients who enroll compared to the number of patients who enroll and decline enrolling over 1 year

Adherence to the palliative care consultation intervention: proportion of patient-surrogate pairs randomised to a palliative care consultation who actually agree to have the palliative care consultation prior to hospital discharge

Crossover from usual care to post-ICU palliative care: proportion of patient-surrogate pairs randomised to usual care who end up receiving a post-ICU palliative care consultation prior to hospital discharge

Fidelity of the palliative care intervention: documentation in electronic medical record consultation notes of (a) burdensome symptoms, (b) supportive counselling, (c) symptom treatment recommendations, and (d) addressing goals-of-care

Secondary outcomes:

Change in Edmonton Symptom Assessment System (ESAS) scores: changes in patients' ESAS symptoms (best (0) to worse (10)) from randomisation to hospital discharge, and from hospital discharge to 1-month follow-up. ESAS symptoms are: pain, fatigue, drowsiness, nausea, lack of appetite, depression, anxiety, shortness of breath, and well-being.

Change in Hospital Anxiety and Depression Scores (HADS) for surrogates: changes in surrogates' HADS scores (best (0) to worst (42)) from randomisation to hospital discharge, and from hospital discharge to 1-month follow-up

Other outcome measures:

Number of patients with moderate-to-severe fatigue (ESAS fatigue scores greater than or equal to 4) who are recommended for and receive methylphenidate treatment New limitation of life-sustaining therapy: number of patients who elect DNR after randomisation Enrollment in hospice: number of patients who enroll in inpatient hospice or home hospice Acute-care readmissions: number of patients who are re-admitted to an acute care hospital within 1 month and 3 months

Starting date March 2018

Contact information 
Weber 2014

Study name
Early palliative care for patients with severe and very severe chronic obstructive pulmonary disease (COPD)

\section{Methods}

Participants

\section{RCT}

\section{Country: Geneva, Switzerland}

Recruitment: Potential participants will receive information from the research nurse and will be advised as to what is involved if participating in the trial. The study nurse will arrange an appointment for the baseline assessment with the interested patient. This first appointment will take place in the patient's home or at the hospital, according to the patient's choice. At this appointment, written informed consent for participation in the study will be obtained from participants. The inclusion criteria are: COPD defined according to GOLD criteria (FEV1/FVC $<70 \%$ ) stage III or IV (FEV1 < $50 \%$ predicted)

- and/or long-term treatment with either domiciliary oxygen or home mechanical ventilation

- and/or one or more hospital admissions in the previous year for an acute exacerbation

The exclusion criteria are:

- Patients in their last days of life (patient bedbound and/or semi comatose and/or takes only fluid and/or no longer able to take oral drugs)

- Patients with cognitive impairment: Mini Mental status Examination $\leq 23$ at the day of inclusion of patients

- Patients with active cancer

Number of patients: Sample size of 90 patients/group proposed

Diseases: Chronic Obstructive Pulmonary Disease

Patient characteristics: Unknown as results have not been published

Deaths at end of study: Unknown as results have not been published

Withdrawals/other dropouts: Unknown as results have not been published

Interventions

Type: Early specialist palliative care

Service base: Hospital

Team: Psychiatrist, pulmonologist, nurse and specialised palliative care physician

Intervention: Patients assigned to the early palliative care group will meet a nurse attached to the community ambulatory palliative care unit of the HUG (USPC) within three weeks of inclusion and at least monthly thereafter during one year after inclusion. This nurse has a long experience in palliative care. The first consultation will take place at the patient's home, unless the patient prefers to have the consultation at the pulmonary division. It will take approximately $1.5 \mathrm{~h}$ to assess and discuss the different items. The subsequent monthly consultations will last approximately $30 \mathrm{~min}-$ utes according to the needs of the patients. A consultation with a specialised palliative care physician or another specialist (psychiatrist, pulmonologist) will be organised if intensity of pain, dyspnoea, mood, anxiety and appetite disturbances are $>4 / 10$ on visual analogic scales (VAS) and both the patient and his/her physician agree. The individualised palliative care assessment and treatment plan will be forwarded in writing to the physician in charge of the patient within 24 hours of the consultation. At the end of each consultation, the items discussed during the consultations will be collected. Participants who refuse to attend one or more palliative care interventions will be contacted by phone by the palliative care nurse to understand their reasons. If the patient, agrees a new appointment will be fixed.

Duration: One year

Control: Patients randomised to the control group, i.e. who receive standard care alone, will not meet with the palliative support team unless the patient himself, his family or his treating physician request a meeting. These patients will remain in the control group, as this is already part of present practice. Patients who present acute exacerbations will be treated by the participant's GP 
Weber 2014 (Continued)

or pulmonologist or by the emergency division according to usual practices and recommendations of our institution.

Primary outcomes:
Hospital, ICU and emergency admissions will be collected in both groups from the medical records
once a month. Length of stay in the different divisions, type of divisions (i.e. internal medicine, re-
habilitation) and reasons for admissions will be collected.
Secondary outcomes:
Mood and anxiety of patients will be measured with the self-assessment instrument: the Hospital
Anxiety and Depression Scale (HADS).
Quality of life will be measured with the SF-36 short form: a self-assessment scale validated in this
population, and the COPD Assessment Test (CAT): a short, simple instrument for quantifying the
symptom burden of COPD in routine practice.
Use of antibiotics during the last three months will be recorded.
Completion of advance directives, documented preferences for resuscitation, or nomination of sur-
rogate decision-maker will also be recorded.

Starting date

September 2013

Contact information

Notes

ALK: anaplastic lymphoma kinase

ALT:Alanine Aminotransferase

AKP: Alkaline Phosphatase

AML:Acute Myeloid Leukemia

AST: aspartate aminotransferase

${ }^{\circ} \mathrm{C}$ : centigrade

CAM: Complementary and Alternative Medicine

COPD: chronic obstructive pulmonary disorder

CQOL: unpaid caregiver Quality of Life

CRA: Caregiver Reseaction Assessment

$\mathrm{CT} / \mathrm{Ct}$ : Computerised Tomography

d: day/s

ECG: echocardiogram

ECOG: Eastern Cooperative Oncology Group

EGFR: Epidermal Growth Factor Receptor

EML4-ALK: echinoderm microtubule associated protein-like 4- anaplastic lymphoma kinase

EoL: End of Life

EORTC: European Organization for Research and Treatment of Cancer

EPC: early palliative care

EQ-5D: EuroQol-5 Dimension

ESAS-r: Edmonton Symptom Assessment System Revised

FACT-G: Functional Assessment of Cancer Therapy-General

FACT-L: Functional Assessment of Cancer Therapy-Lung (FACT-L)

FACT-Leu: Functional Assessment of Cancer Therapy - Leukemia

FACIT-Pal: Functional Assessment of Chronic Illness Therapy - Palliative Care

FAMCARE: Family Care

FEV1/FVC: Forced Expiratory Volume in the first one second/Forced Vital

g/dL: grams/decilitre

GAP: Gender Age Physiology

GI: gastrointestinal

GOLD: Global Initiative for Chronic Obstructive Lung Disease

h: hour/s

The effectiveness and cost-effectiveness of hospital-based specialist palliative care for adults with advanced illness and their caregivers 
Hb: Haemoglobin

HER2: human epidermal growth factor receptor 2

HF: heart failure

ICD: International Classification of Diseases

ICU: intensive care unit

M: Metastasis

MELD: Model for End-Stage Liver Disease

$\mathrm{mm}$ : millimetre

$\mathrm{mmHg}$ : millimetre of mercury

MRI: Magnetic Resonance Imaging

MSAS: Memorial Symptom Assessment Scale

MQOL: McGill Quality of Life Questionnaire

$\mathrm{N}$ : Number / Node

NCCN: National Comprehensive Cancer Network

PC: palliative care

PCL: PTSD Checklist

PD: Parkinson's Disease

PET: Positron Emission Tomography

PHQ-9: Patient Health Questionnaire-9

PRO: patient reported outcomes

PROMIS: Patient-Reported Outcomes Measurement Information System

PS: Performance Status

QLQ-C15-PAL: Quality of Life Questionnaire Core 15 Palliative Care

QoL: quality of life

QOLLTI-F: Quality of Life in Life-Threatening Illness -Family carer version

$\mathrm{RCT}$ : randomised controlled trial

RECIST: Response Evaluation Criteria in Solid Tumours

ROS1: Receptor Tyrosine Kinase

SAT-SF: Crisis Overcoming Capability

SCNS: Supportive Care Needs Survey

SF-12: Short Form Survey 12

$\mathrm{T}$ : Tumour

ULN: Upper Limit of Normal

WBC: White Blood Cells

WHO: World Health Organization

\section{DATA AND ANALYSES}

\section{Comparison 1. Patient health-related quality of life}

\begin{tabular}{|c|c|c|c|c|}
\hline Outcome or subgroup title & No. of studies & $\begin{array}{l}\text { No. of partici- } \\
\text { pants }\end{array}$ & Statistical method & Effect size \\
\hline $\begin{array}{l}1.1 \mathrm{HSPC} \text { versus usual care on patient } \\
\text { HRQoL: adjusted endpoint values }\end{array}$ & 10 & 1344 & $\begin{array}{l}\text { Std. Mean Difference (IV, } \\
\text { Random, } 95 \% \mathrm{CI} \text { ) }\end{array}$ & $0.26[0.15,0.37]$ \\
\hline $\begin{array}{l}\text { 1.2 HSPC versus usual care on patient } \\
\text { HRQoL: adjusted endpoint values (exclud- } \\
\text { ing McCorkle 2015) }\end{array}$ & 9 & 1280 & $\begin{array}{l}\text { Std. Mean Difference (IV, } \\
\text { Random, } 95 \% \mathrm{CI} \text { ) }\end{array}$ & $0.29[0.18,0.40]$ \\
\hline $\begin{array}{l}\text { 1.3 HSPC versus usual care on patient } \\
\text { HRQoL: unadjusted endpoint values }\end{array}$ & 9 & 1201 & $\begin{array}{l}\text { Std. Mean Difference (IV, } \\
\text { Random, } 95 \% \mathrm{CI} \text { ) }\end{array}$ & $0.41[0.11,0.70]$ \\
\hline $\begin{array}{l}\text { 1.4 HSPC versus usual care on patient } \\
\text { HRQoL: unadjusted endpoint values (ex- } \\
\text { cluding McCorkle 2015) }\end{array}$ & 8 & 1137 & $\begin{array}{l}\text { Std. Mean Difference (IV, } \\
\text { Random, } 95 \% \mathrm{CI} \text { ) }\end{array}$ & $0.46[0.13,0.78]$ \\
\hline
\end{tabular}

The effectiveness and cost-effectiveness of hospital-based specialist palliative care for adults with advanced illness and their caregivers 


\begin{tabular}{lllll}
\hline Outcome or subgroup title & No. of studies & $\begin{array}{l}\text { No. of partici- } \\
\text { pants }\end{array}$ & Statistical method & Effect size \\
\hline $\begin{array}{l}\text { 1.5 HSPC versus usual care on patient } \\
\text { HRQoL: unadjusted change values }\end{array}$ & 9 & 1278 & $\begin{array}{l}\text { Std. Mean Difference (IV, } \\
\text { Random, 95\% CI) }\end{array}$ & $0.67[0.16,1.18]$ \\
\hline
\end{tabular}

\section{Analysis 1.1. Comparison 1: Patient health-related quality of life, Outcome 1: HSPC versus usual care on patient HRQOL: adjusted endpoint values}

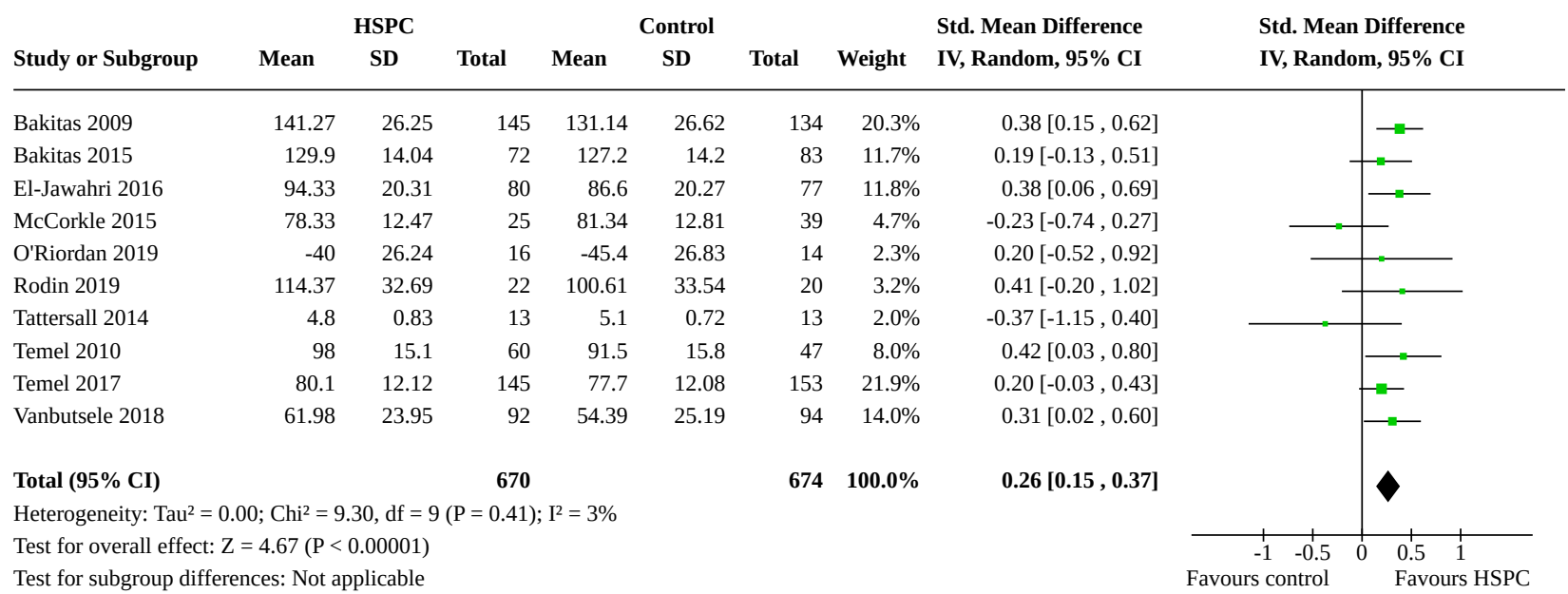

Analysis 1.2. Comparison 1: Patient health-related quality of life, Outcome 2: HSPC versus usual care on patient HRQoL: adjusted endpoint values (excluding McCorkle 2015)

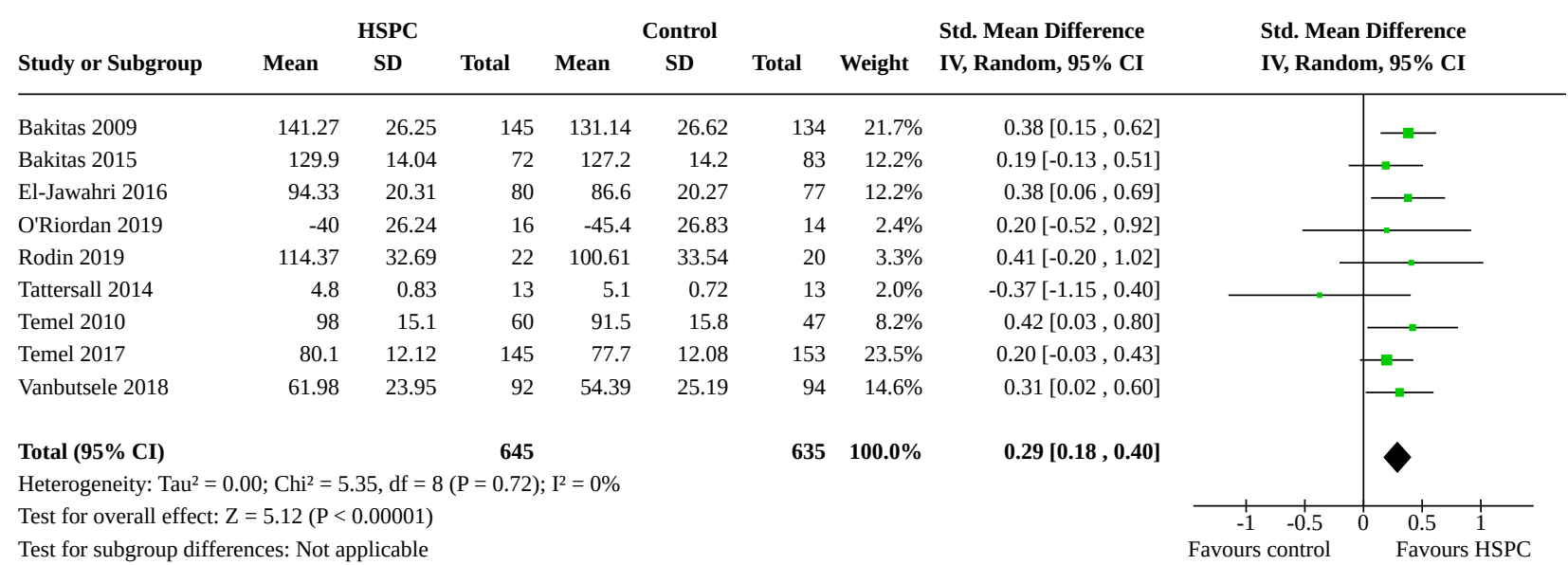


Analysis 1.3. Comparison 1: Patient health-related quality of life, Outcome 3: HSPC versus usual care on patient HRQoL: unadjusted endpoint values

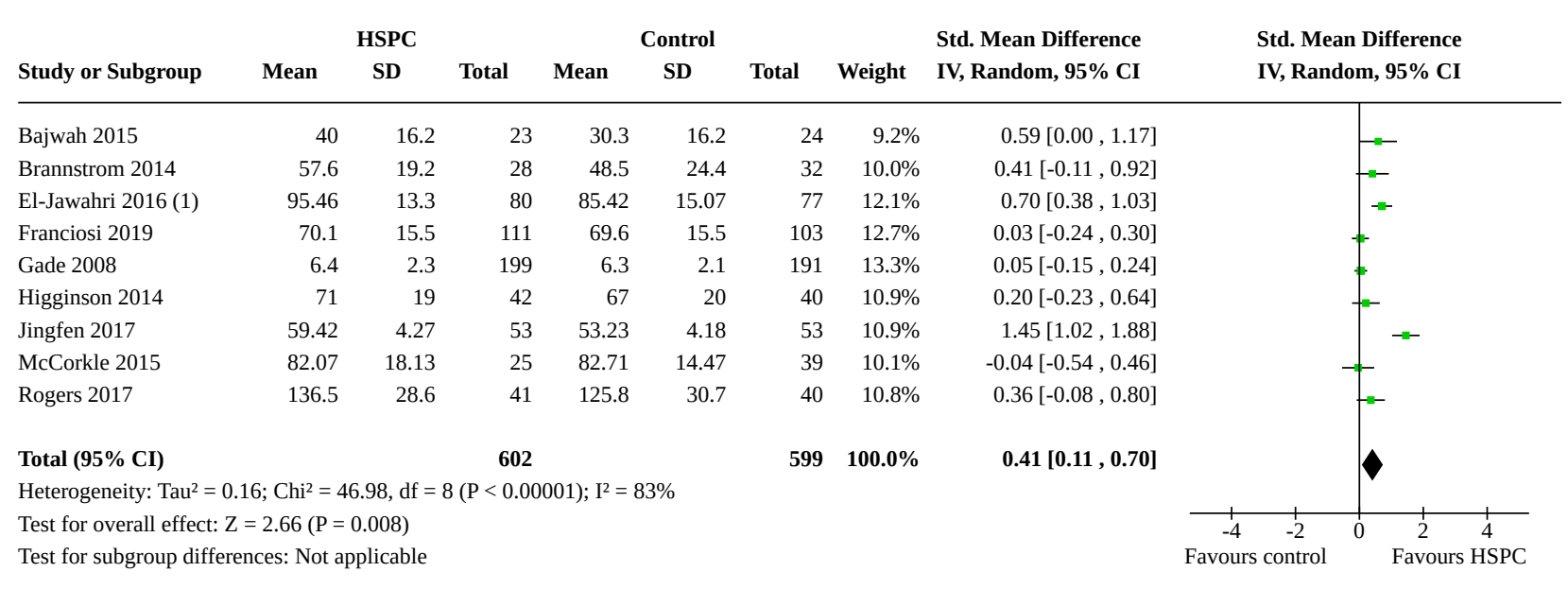

Footnotes

(1) $95 \%$ CI were estimated from graph

Analysis 1.4. Comparison 1: Patient health-related quality of life, Outcome 4: HSPC versus usual care on patient HRQoL: unadjusted endpoint values (excluding McCorkle 2015)

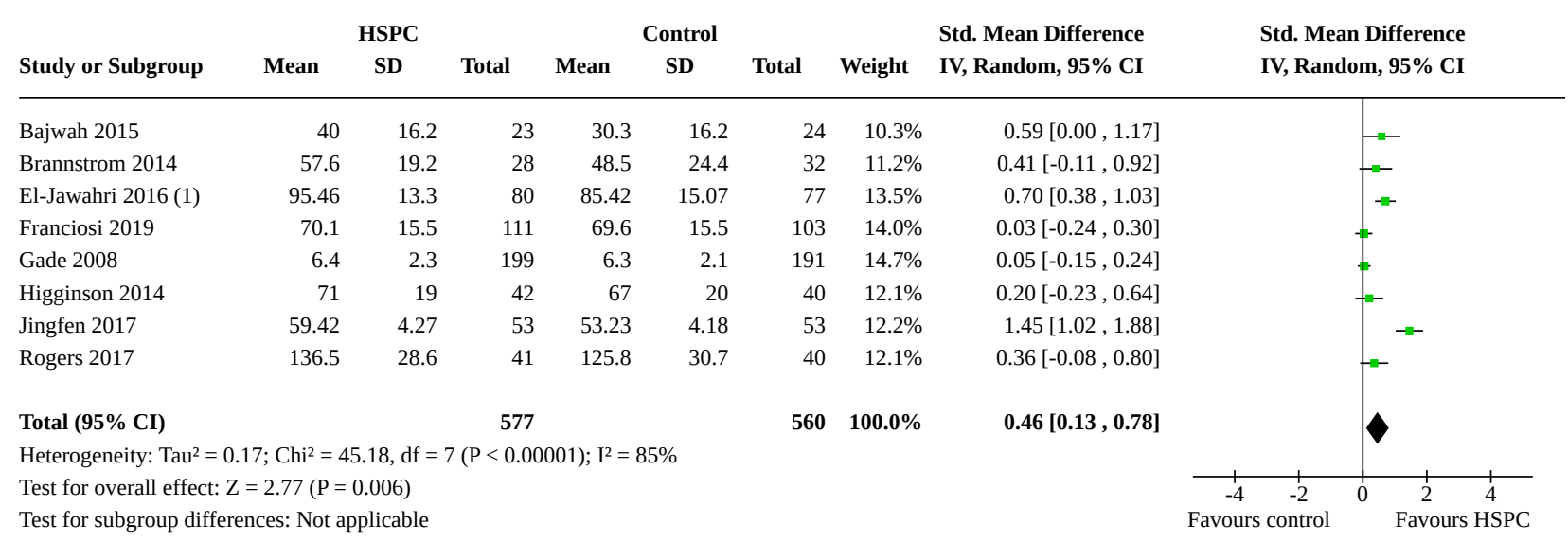

Footnotes

(1) $95 \%$ CI were estimated from graph 
Analysis 1.5. Comparison 1: Patient health-related quality of life, Outcome 5: HSPC versus usual care on patient HRQoL: unadjusted change values

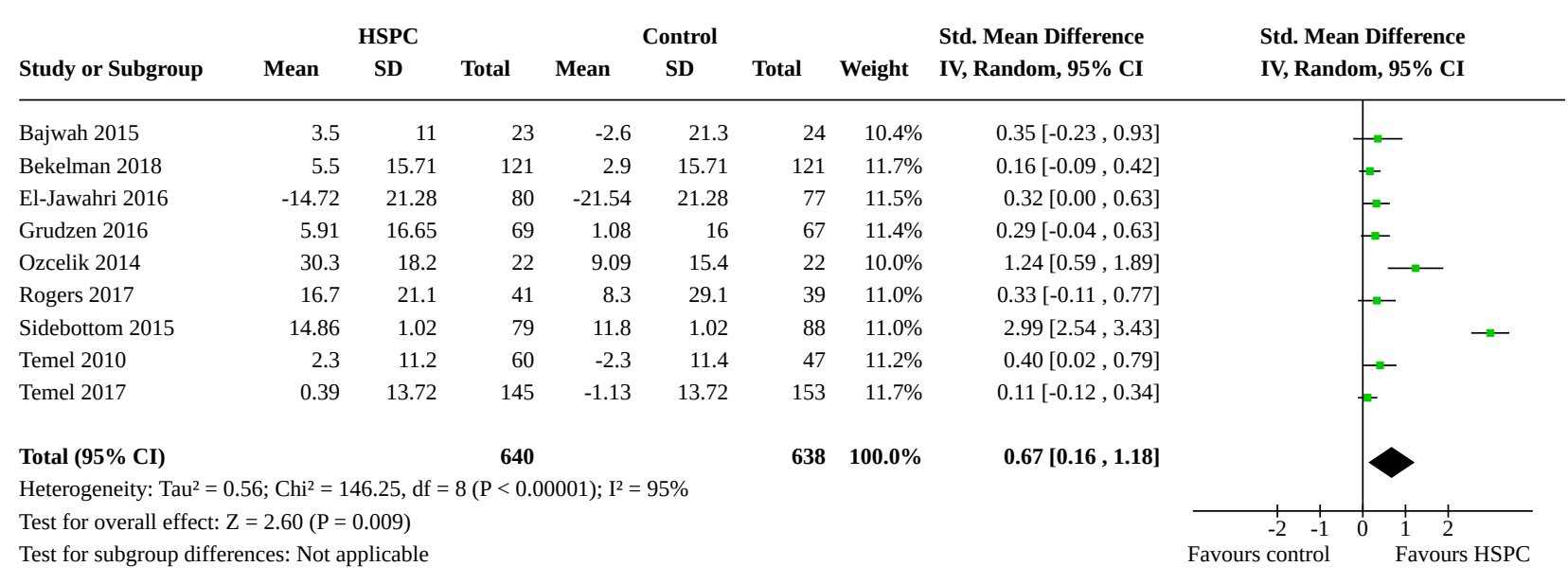

\section{Comparison 2. Patient symptom burden}

\begin{tabular}{|c|c|c|c|c|}
\hline Outcome or subgroup title & No. of studies & $\begin{array}{l}\text { No. of partici- } \\
\text { pants }\end{array}$ & Statistical method & Effect size \\
\hline $\begin{array}{l}2.1 \mathrm{HSPC} \text { versus usual care on patient } \\
\text { symptom burden: adjusted endpoint val- } \\
\text { ues }\end{array}$ & 6 & 761 & $\begin{array}{l}\text { Std. Mean Difference (IV, } \\
\text { Random, } 95 \% \mathrm{Cl} \text { ) }\end{array}$ & $\begin{array}{l}-0.26[-0.41 \\
-0.12]\end{array}$ \\
\hline $\begin{array}{l}2.2 \mathrm{HSPC} \text { versus usual care on patient } \\
\text { symptom burden: unadjusted endpoint } \\
\text { values }\end{array}$ & 6 & 833 & $\begin{array}{l}\text { Std. Mean Difference (IV, } \\
\text { Random, } 95 \% \mathrm{CI} \text { ) }\end{array}$ & $-0.17[-0.54,0.20]$ \\
\hline $\begin{array}{l}2.3 \mathrm{HSPC} \text { versus usual care on patient } \\
\text { symptom burden: unadjusted endpoint } \\
\text { values (excluding McCorkle 2015) }\end{array}$ & 5 & 769 & $\begin{array}{l}\text { Std. Mean Difference (IV, } \\
\text { Random, } 95 \% \mathrm{CI} \text { ) }\end{array}$ & $-0.19[-0.62,0.24]$ \\
\hline $\begin{array}{l}2.4 \mathrm{HSPC} \text { versus usual care on patient } \\
\text { symptom burden: adjusted change values }\end{array}$ & 4 & 353 & $\begin{array}{l}\text { Std. Mean Difference (IV, } \\
\text { Random, } 95 \% \mathrm{Cl} \text { ) }\end{array}$ & $-1.31[-3.27,0.64]$ \\
\hline $\begin{array}{l}\text { 2.5 HSPC versus usual care on patient } \\
\text { symptom burden: adjusted change values } \\
\text { (excluding McCorkle 2015) }\end{array}$ & 3 & 289 & $\begin{array}{l}\text { Std. Mean Difference (IV, } \\
\text { Random, } 95 \% \mathrm{CI} \text { ) }\end{array}$ & $-1.79[-4.29,0.70]$ \\
\hline $\begin{array}{l}\text { 2.6 HSPC versus usual care on patient } \\
\text { symptom burden: unadjusted change val- } \\
\text { ues }\end{array}$ & 6 & 641 & $\begin{array}{l}\text { Std. Mean Difference (IV, } \\
\text { Random, } 95 \% \mathrm{CI} \text { ) }\end{array}$ & $-0.44[-0.94,0.06]$ \\
\hline
\end{tabular}


Analysis 2.1. Comparison 2: Patient symptom burden, Outcome 1: HSPC versus usual care on patient symptom burden: adjusted endpoint values

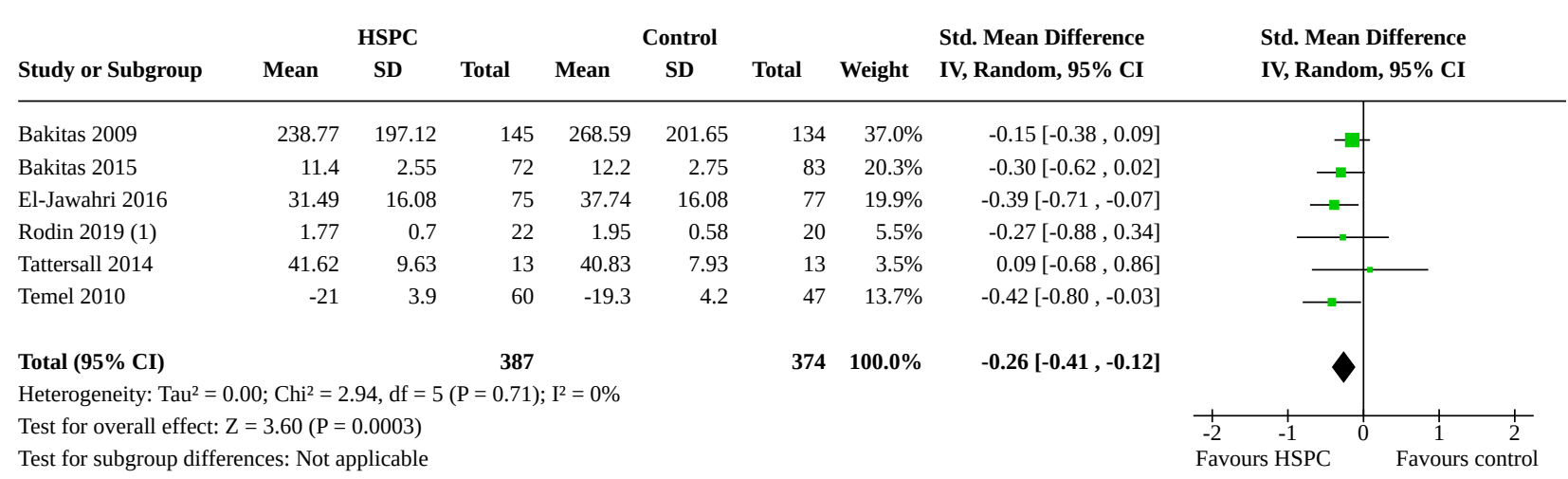

Footnotes

(1) Data from the severity subscale of the Memorial Symptom Assessment Scale (MSAS) was used in meta-analysis

Analysis 2.2. Comparison 2: Patient symptom burden, Outcome 2: HSPC versus usual care on patient symptom burden: unadjusted endpoint values

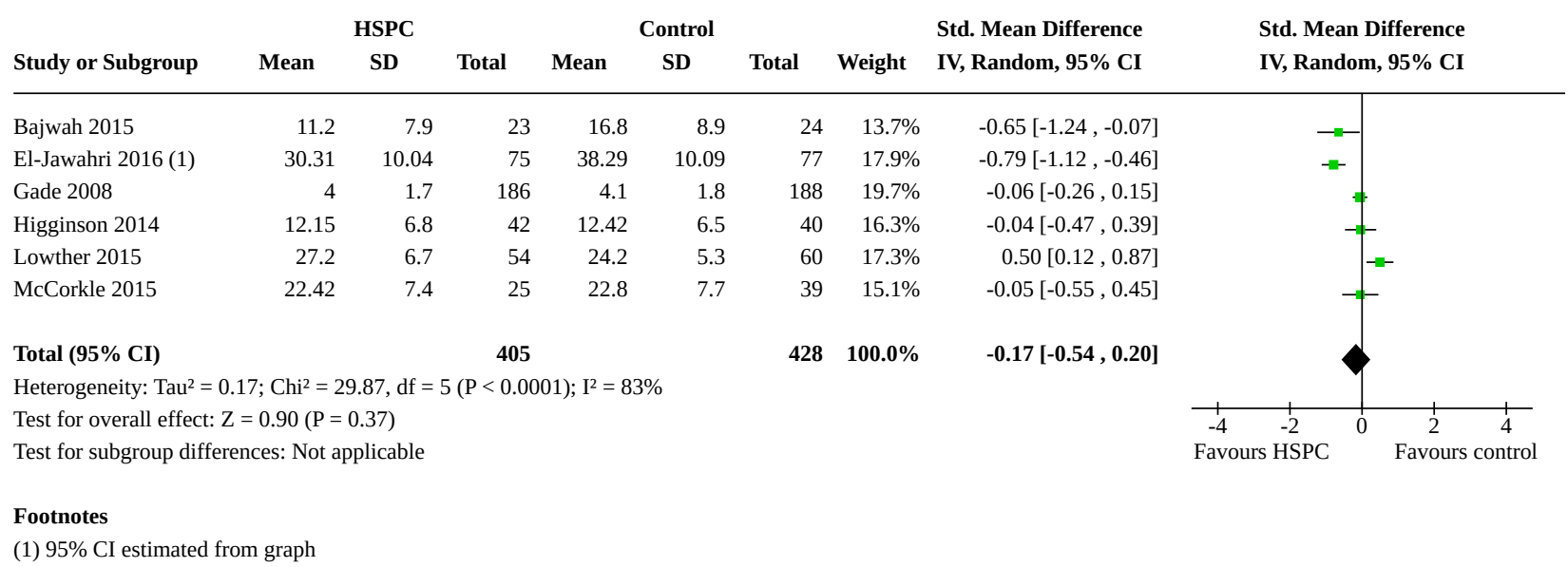

Analysis 2.3. Comparison 2: Patient symptom burden, Outcome 3: HSPC versus usual care on patient symptom burden: unadjusted endpoint values (excluding McCorkle 2015)

\begin{tabular}{|c|c|c|c|c|c|c|c|c|c|c|}
\hline \multirow[b]{2}{*}{ Study or Subgroup } & \multirow[b]{2}{*}{ Mean } & \multicolumn{2}{|l|}{ HSPC } & \multicolumn{3}{|c|}{ Control } & \multirow[b]{2}{*}{ Weight } & \multirow{2}{*}{$\begin{array}{l}\text { Std. Mean Difference } \\
\text { IV, Random, 95\% CI }\end{array}$} & \multirow{2}{*}{\multicolumn{2}{|c|}{$\begin{array}{l}\text { Std. Mean Difference } \\
\text { IV, Random, 95\% CI }\end{array}$}} \\
\hline & & SD & Total & Mean & SD & Total & & & & \\
\hline Bajwah 2015 & 11.2 & 7.9 & 23 & 16.8 & 8.9 & 24 & $16.6 \%$ & $-0.65[-1.24,-0.07]$ & $\longrightarrow$ & \\
\hline El-Jawahri 2016 (1) & 30.31 & 10.04 & 75 & 38.29 & 10.09 & 77 & $21.0 \%$ & $-0.79[-1.12,-0.46]$ & - & \\
\hline Gade 2008 & 4 & 1.7 & 186 & 4.1 & 1.8 & 188 & $22.8 \%$ & $-0.06[-0.26,0.15]$ & & \\
\hline Higginson 2014 & 12.15 & 6.8 & 42 & 12.42 & 6.5 & 40 & $19.3 \%$ & $-0.04[-0.47,0.39]$ & & \\
\hline Lowther 2015 & 27.2 & 6.7 & 54 & 24.2 & 5.3 & 60 & $20.3 \%$ & $0.50[0.12,0.87]$ & & —— \\
\hline Total (95\% CI) & & & 380 & & & 389 & $100.0 \%$ & $-0.19[-0.62,0.24]$ & & \\
\hline \multicolumn{11}{|c|}{ Heterogeneity: Tau $^{2}=0.20 ;$ Chi $^{2}=29.73, \mathrm{df}=4(\mathrm{P}<0.00001) ; \mathrm{I}^{2}=87 \%$} \\
\hline \multicolumn{9}{|c|}{ Test for overall effect: $\mathrm{Z}=0.88(\mathrm{P}=0.38)$} & $\begin{array}{ccc}1 & 1 & + \\
-1 & -0.5 & 0\end{array}$ & $\begin{array}{ll}1 \\
0.5 & 1\end{array}$ \\
\hline \multicolumn{9}{|c|}{ Test for subgroup differences: Not applicable } & Favours HSPC & Favours control \\
\hline
\end{tabular}

The effectiveness and cost-effectiveness of hospital-based specialist palliative care for adults with advanced illness and their caregivers 


\section{Analysis 2.4. Comparison 2: Patient symptom burden, Outcome 4: HSPC versus usual care on patient symptom burden: adjusted change values}

\begin{tabular}{|c|c|c|c|c|c|c|c|c|c|}
\hline Study or Subgroup & Mean & $\begin{array}{l}\text { HSPC } \\
\text { SD }\end{array}$ & Total & Mean & Sontrol & Total & Weight & $\begin{array}{l}\text { Std. Mean Difference } \\
\text { IV, Random, 95\% CI }\end{array}$ & $\begin{array}{l}\text { Std. Mean Difference } \\
\text { IV, Random, 95\% CI }\end{array}$ \\
\hline Edmonds 2010 & -1 & 2.7 & 25 & 1.1 & 2.8 & 21 & $24.9 \%$ & $-0.75[-1.35,-0.15]$ & - \\
\hline McCorkle 2015 & -0.24 & 4.76 & 25 & -0.89 & 4.92 & 39 & $25.1 \%$ & $0.13[-0.37,0.63]$ & - \\
\hline Sidebottom 2015 & -11 & 0.99 & 79 & -6.7 & 0.99 & 88 & $25.0 \%$ & $-4.32[-4.88,-3.76]$ & - \\
\hline Solari 2018 & -2.3 & 6.69 & 50 & -0.3 & 5.69 & 26 & $25.1 \%$ & $-0.31[-0.79,0.17]$ & \\
\hline Total (95\% CI) & & & 179 & & & 174 & $100.0 \%$ & $-1.31[-3.27,0.64]$ & \\
\hline \multicolumn{10}{|c|}{ Heterogeneity: $\mathrm{Tau}^{2}=3.90 ; \mathrm{Chi}^{2}=162.38, \mathrm{df}=3(\mathrm{P}<0.00001) ; \mathrm{I}^{2}=98 \%$} \\
\hline \multicolumn{9}{|c|}{ Test for overall effect: $\mathrm{Z}=1.31(\mathrm{P}=0.19)$} & -4 \\
\hline \multicolumn{9}{|c|}{ Test for subgroup differences: Not applicable } & Favours HSPC \\
\hline
\end{tabular}

Analysis 2.5. Comparison 2: Patient symptom burden, Outcome 5: HSPC versus usual care on patient symptom burden: adjusted change values (excluding McCorkle 2015)

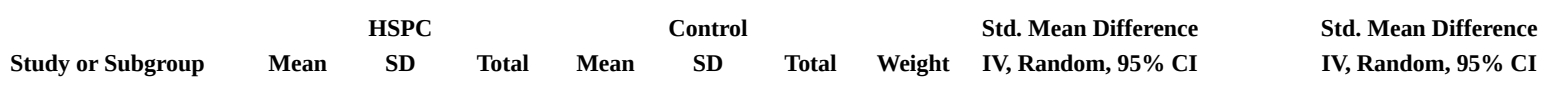

\begin{tabular}{|c|c|c|c|c|c|c|c|c|c|c|}
\hline Edmonds 2010 & -1 & 2.7 & 25 & 1.1 & 2.8 & 21 & $33.2 \%$ & $-0.75[-1.35,-0.15]$ & - & \\
\hline Sidebottom 2015 & -11 & 0.99 & 79 & -6.7 & 0.99 & 88 & $33.3 \%$ & $-4.32[-4.88,-3.76]$ & \multirow[t]{2}{*}{-} & \\
\hline Solari 2018 & -2.3 & 6.69 & 50 & -0.3 & 5.69 & 26 & $33.5 \%$ & $-0.31[-0.79,0.17]$ & & \\
\hline Total (95\% CI) & & & \multicolumn{3}{|l|}{154} & 135 & $100.0 \%$ & $-1.79[-4.29,0.70]$ & & \\
\hline \multicolumn{9}{|c|}{ Heterogeneity: $\mathrm{Tau}^{2}=4.80 ; \mathrm{Chi}^{2}=126.75, \mathrm{df}=2(\mathrm{P}<0.00001) ; \mathrm{I}^{2}=98 \%$} & & \\
\hline \multicolumn{6}{|c|}{ Test for overall effect: $\mathrm{Z}=1.41(\mathrm{P}=0.16)$} & & & & $\begin{array}{ll}1 & 1 \\
-4 & -2\end{array}$ & 2 \\
\hline \multicolumn{6}{|c|}{ Test for subgroup differences: Not applicable } & & & & \multicolumn{2}{|c|}{ Favours HSPC Favours control } \\
\hline
\end{tabular}

Analysis 2.6. Comparison 2: Patient symptom burden, Outcome 6: HSPC versus usual care on patient symptom burden: unadjusted change values

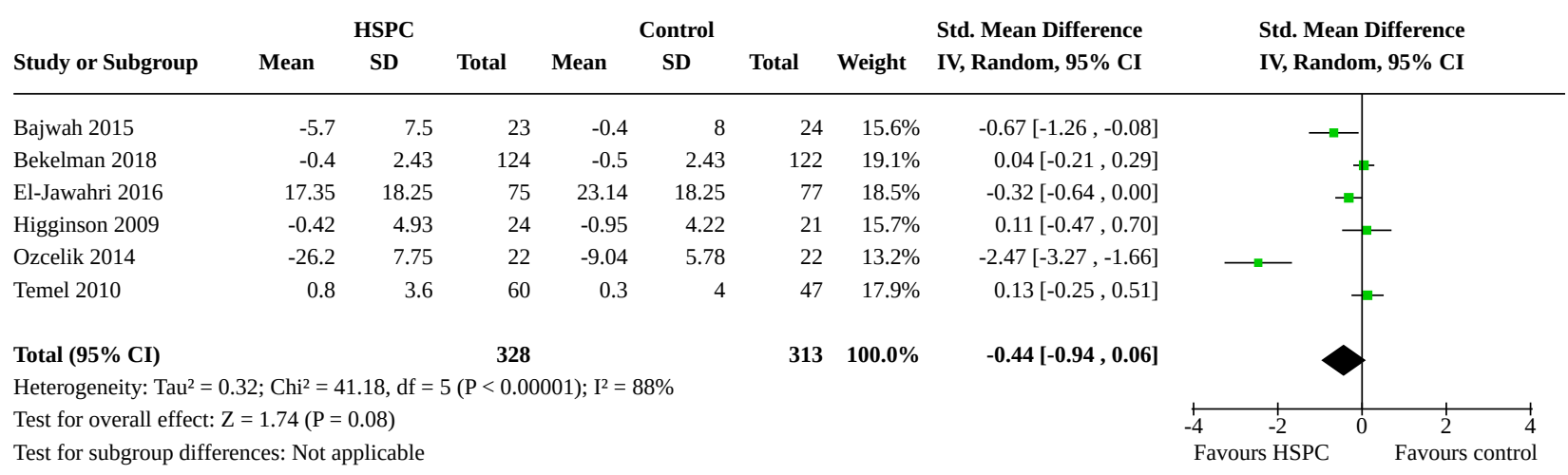

Comparison 3. Patient satisfaction with care

\begin{tabular}{|c|c|c|c|c|}
\hline Outcome or subgroup title & No. of studies & $\begin{array}{l}\text { No. of partici- } \\
\text { pants }\end{array}$ & Statistical method & Effect size \\
\hline $\begin{array}{l}\text { 3.1 HSPC versus usual care on patient satis- } \\
\text { faction with care: adjusted endpoint values }\end{array}$ & 2 & 337 & $\begin{array}{l}\text { Std. Mean Difference (IV, } \\
\text { Random, } 95 \% \mathrm{CI} \text { ) }\end{array}$ & $0.36[0.14,0.57]$ \\
\hline
\end{tabular}

The effectiveness and cost-effectiveness of hospital-based specialist palliative care for adults with advanced illness and their caregivers 234 (Review)

Copyright ( 2020 The Cochrane Collaboration. Published by John Wiley \& Sons, Ltd. 
Analysis 3.1. Comparison 3: Patient satisfaction with care, Outcome 1: HSPC versus usual care on patient satisfaction with care: adjusted endpoint values

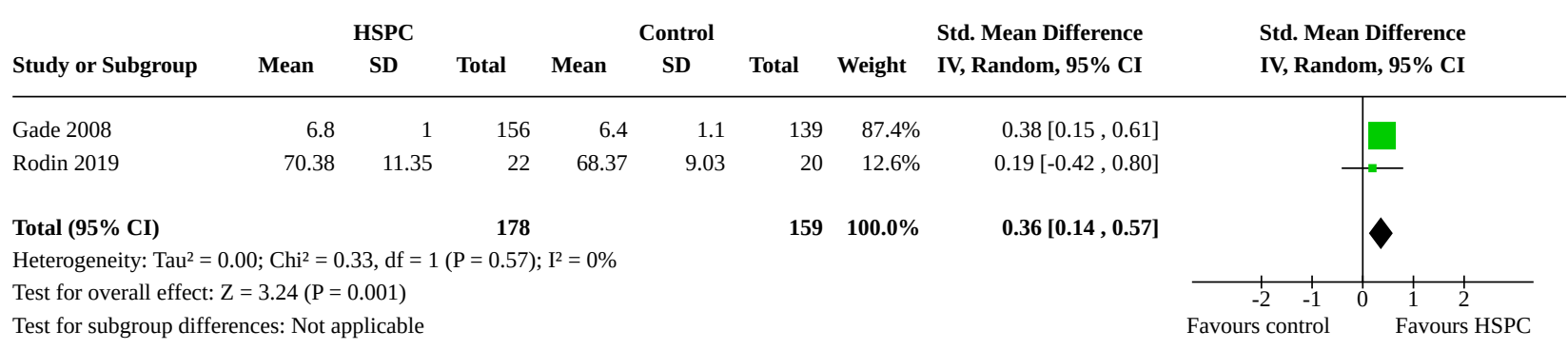

\section{Comparison 4. Achieving patient preferred place of death}

\begin{tabular}{lllll}
\hline Outcome or subgroup title & No. of studies & $\begin{array}{l}\text { No. of partici- } \\
\text { pants }\end{array}$ & Statistical method & Effect size \\
\hline $\begin{array}{l}\text { 4.1 HSPC versus usual care on home } \\
\text { deaths }\end{array}$ & 7 & 861 & $\begin{array}{l}\text { Odds Ratio (M-H, Random, } \\
95 \% \mathrm{Cl})\end{array}$ & $1.63[1.23,2.16]$ \\
\hline
\end{tabular}

\section{Analysis 4.1. Comparison 4: Achieving patient preferred place of death, Outcome 1: HSPC versus usual care on home deaths}

\begin{tabular}{|c|c|c|c|c|c|c|c|c|c|}
\hline \multirow[b]{2}{*}{ Study or Subgroup } & \multicolumn{2}{|c|}{ HSPC } & \multicolumn{2}{|c|}{ Control } & \multicolumn{2}{|r|}{ Odds Ratio } & \multirow{2}{*}{\multicolumn{2}{|c|}{$\begin{array}{c}\text { Odds Ratio } \\
\text { M-H, Random, 95\% CI }\end{array}$}} & \\
\hline & Events & Total & Events & Total & Weight & M-H, Random, 95\% CI & & & \\
\hline Bajwah 2015 & 5 & 8 & 5 & 13 & $2.4 \%$ & $2.67[0.43,16.39]$ & & & \\
\hline Bakitas 2009 & 67 & 112 & 62 & 119 & $29.4 \%$ & $1.37[0.81,2.31]$ & & 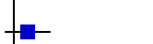 & \\
\hline Bakitas 2015 & 27 & 50 & 28 & 59 & $14.0 \%$ & $1.30[0.61,2.77]$ & & $=$ & \\
\hline Brumley 2007 & 81 & 118 & 54 & 113 & $27.8 \%$ & $2.39[1.40,4.09]$ & & $\rightarrow$ & \\
\hline McCaffrey 2013 & 13 & 23 & 6 & 8 & $2.5 \%$ & $0.43[0.07,2.62]$ & & & \\
\hline Tattersall 2014 & 13 & 58 & 8 & 53 & $8.4 \%$ & $1.63[0.61,4.30]$ & & & \\
\hline Temel 2010 & 40 & 61 & 36 & 66 & $15.5 \%$ & $1.59[0.78,3.25]$ & & - & \\
\hline Total (95\% CI) & & 430 & & 431 & $100.0 \%$ & $1.63[1.23,2.16]$ & & & \\
\hline Total events: & 246 & & 199 & & & & & & \\
\hline \multicolumn{5}{|c|}{ Heterogeneity: $\mathrm{Tau}^{2}=0.00 ; \mathrm{Chi}^{2}=5.11, \mathrm{df}=6(\mathrm{P}=0.53) ; \mathrm{I}^{2}=0 \%$} & & 0.01 & $\frac{1}{1}$ & 10 & 100 \\
\hline \multicolumn{5}{|c|}{ Test for overall effect: $\mathrm{Z}=3.38(\mathrm{P}=0.0007)$} & & Fav & urs control & Favours H & SPC \\
\hline
\end{tabular}

\section{Comparison 5. Pain}

\begin{tabular}{llll}
\hline Outcome or subgroup title & No. of studies & $\begin{array}{l}\text { No. of partici- } \\
\text { pants }\end{array}$ & Statistical method \\
\hline $\begin{array}{l}5.1 \mathrm{HSPC} \text { versus usual care on pain: ad- } \\
\text { justed endpoint values }\end{array}$ & 4 & 525 & $\begin{array}{l}\text { Std. Mean Difference (IV, } \\
\text { Random, 95\% CI) }\end{array}$ \\
\hline $\begin{array}{l}5.2 \mathrm{HSPC} \text { versus usual care on pain: ad- } \\
\text { justed change values }\end{array}$ & 2 & 218 & $\begin{array}{l}\text { Std. Mean Difference (IV, } \\
\text { Random, } 95 \% \mathrm{CI})\end{array}$ \\
\hline \hline
\end{tabular}

The effectiveness and cost-effectiveness of hospital-based specialist palliative care for adults with advanced illness and their caregivers 


\begin{tabular}{lllll}
\hline Outcome or subgroup title & No. of studies & $\begin{array}{l}\text { No. of partici- } \\
\text { pants }\end{array}$ & Statistical method & Effect size \\
\hline $\begin{array}{l}5.3 \mathrm{HSPC} \text { versus usual care on pain: un- } \\
\text { adjusted change values }\end{array}$ & 2 & 291 & $\begin{array}{l}\text { Std. Mean Difference (IV, } \\
\text { Random, 95\% CI) }\end{array}$ & $-0.93[-3.05,1.19]$ \\
\hline
\end{tabular}

Analysis 5.1. Comparison 5: Pain, Outcome 1: HSPC versus usual care on pain: adjusted endpoint values

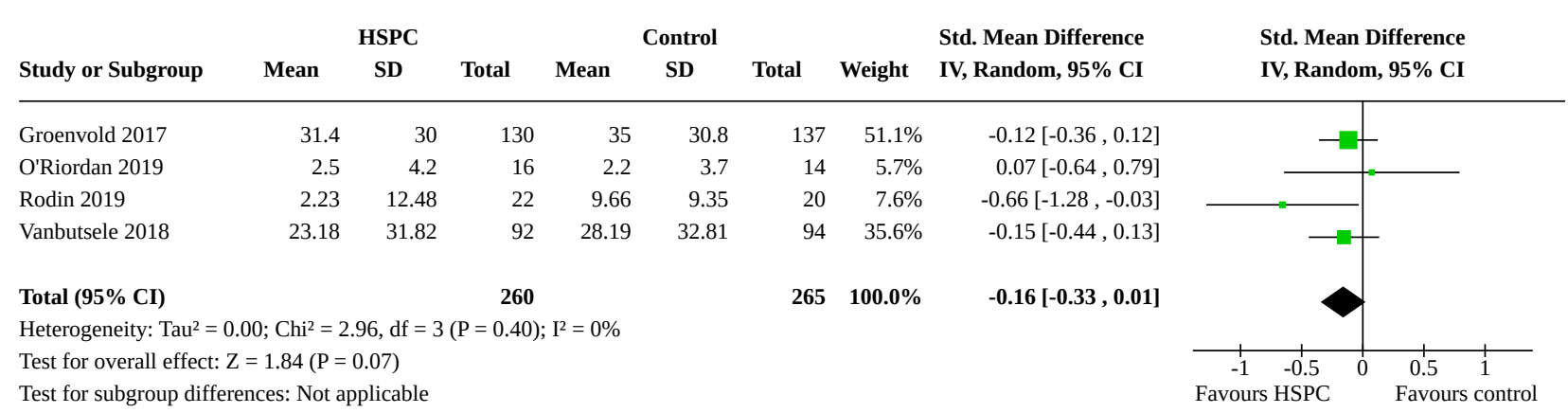

\section{Analysis 5.2. Comparison 5: Pain, Outcome 2: HSPC versus usual care on pain: adjusted change values}

\begin{tabular}{|c|c|c|c|c|c|c|c|c|c|}
\hline Study or Subgroup & Mean & $\begin{array}{l}\text { HSPC } \\
\text { SD }\end{array}$ & Total & Mean & $\begin{array}{l}\text { ontrol } \\
\text { SD }\end{array}$ & Total & Weight & $\begin{array}{l}\text { Std. Mean Difference } \\
\text { IV, Random, } 95 \% \text { CI }\end{array}$ & $\begin{array}{l}\text { Std. Mean Difference } \\
\text { IV, Random, 95\% CI }\end{array}$ \\
\hline Higginson 2009 & -0.46 & 1.29 & 26 & 0.3 & 1.11 & 25 & $22.9 \%$ & $-0.62[-1.18,-0.06]$ & $\multimap-$ \\
\hline Sidebottom 2015 & -0.93 & 1.02 & 79 & -0.49 & 1.02 & 88 & $77.1 \%$ & $-0.43[-0.74,-0.12]$ & \\
\hline Total $(95 \%$ CI) & & & 105 & & & 113 & $100.0 \%$ & $-0.47[-0.74,-0.20]$ & \\
\hline \multicolumn{10}{|c|}{ Heterogeneity: Tau $^{2}=0.00 ; \mathrm{Chi}^{2}=0.34, \mathrm{df}=1(\mathrm{P}=0.56) ; \mathrm{I}^{2}=0 \%$} \\
\hline \multicolumn{9}{|c|}{ Test for overall effect: $\mathrm{Z}=3.44(\mathrm{P}=0.0006)$} & $\begin{array}{lll}+ & + & + \\
-2 & -1 & 0\end{array}$ \\
\hline \multicolumn{9}{|c|}{ Test for subgroup differences: Not applicable } & rs HSPC \\
\hline
\end{tabular}

\section{Analysis 5.3. Comparison 5: Pain, Outcome 3: HSPC versus usual care on pain: unadjusted change values}

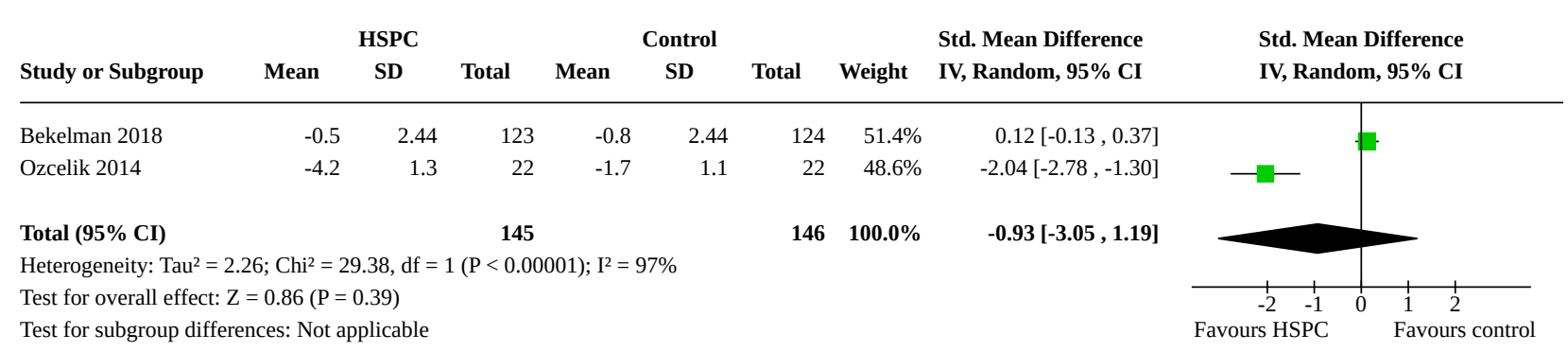

\section{Comparison 6. Patient anxiety}

\begin{tabular}{lllll}
\hline Outcome or subgroup title & No. of studies & $\begin{array}{l}\text { No. of partici- } \\
\text { pants }\end{array}$ & Statistical method & Effect size \\
\hline $\begin{array}{l}\text { 6.1 HSPC versus usual care on patient } \\
\text { anxiety: adjusted endpoint values }\end{array}$ & 5 & 384 & $\begin{array}{l}\text { Mean Difference (IV, Ran- } \\
\text { dom, 95\% Cl) }\end{array}$ & $-0.63[-2.22,0.96]$ \\
\hline
\end{tabular}

The effectiveness and cost-effectiveness of hospital-based specialist palliative care for adults with advanced illness and their caregivers 236 (Review)

Copyright (c) 2020 The Cochrane Collaboration. Published by John Wiley \& Sons, Ltd. 


\begin{tabular}{|c|c|c|c|c|}
\hline Outcome or subgroup title & No. of studies & $\begin{array}{l}\text { No. of partici- } \\
\text { pants }\end{array}$ & Statistical method & Effect size \\
\hline $\begin{array}{l}\text { 6.2 HSPC versus usual care on patient } \\
\text { anxiety: adjusted endpoint values (ex- } \\
\text { cluding McCorkle 2015) }\end{array}$ & 4 & 320 & $\begin{array}{l}\text { Mean Difference (IV, Ran- } \\
\text { dom, } 95 \% \mathrm{CI} \text { ) }\end{array}$ & $\begin{array}{l}-1.60[-2.56 \\
-0.65]\end{array}$ \\
\hline $\begin{array}{l}\text { 6.3 HSPC versus usual care on patient } \\
\text { anxiety: unadjusted endpoint values }\end{array}$ & 4 & 273 & $\begin{array}{l}\text { Mean Difference (IV, Ran- } \\
\text { dom, } 95 \% \mathrm{CI} \text { ) }\end{array}$ & $-0.90[-2.52,0.71]$ \\
\hline $\begin{array}{l}\text { 6.4 HSPC versus usual care on patient } \\
\text { anxiety: unadjusted endpoint values (ex- } \\
\text { cluding McCorkle 2015) }\end{array}$ & 3 & 209 & $\begin{array}{l}\text { Mean Difference (IV, Ran- } \\
\text { dom, } 95 \% \mathrm{CI} \text { ) }\end{array}$ & $-1.48[-3.52,0.56]$ \\
\hline $\begin{array}{l}\text { 6.5 HSPC versus usual care on patient } \\
\text { anxiety: unadjusted change values }\end{array}$ & 4 & 496 & $\begin{array}{l}\text { Std. Mean Difference (IV, } \\
\text { Random, } 95 \% \mathrm{CI} \text { ) }\end{array}$ & $\begin{array}{l}-0.62[-1.02 \\
-0.21]\end{array}$ \\
\hline $\begin{array}{l}\text { 6.6 HSPC versus usual care on patient } \\
\text { anxiety in different populations: adjusted } \\
\text { endpoint values }\end{array}$ & 5 & 384 & $\begin{array}{l}\text { Mean Difference (IV, Ran- } \\
\text { dom, } 95 \% \mathrm{CI} \text { ) }\end{array}$ & $-0.63[-2.22,0.96]$ \\
\hline 6.6.1 Cancer populations & 3 & 275 & $\begin{array}{l}\text { Mean Difference (IV, Ran- } \\
\text { dom, } 95 \% \mathrm{CI} \text { ) }\end{array}$ & $-0.65[-3.03,1.74]$ \\
\hline 6.6.2 Non-cancer populations & 2 & 109 & $\begin{array}{l}\text { Mean Difference (IV, Ran- } \\
\text { dom, } 95 \% \mathrm{CI} \text { ) }\end{array}$ & $-0.82[-2.45,0.80]$ \\
\hline $\begin{array}{l}\text { 6.7 HSPC versus usual care on patient } \\
\text { anxiety in different populations: adjust- } \\
\text { ed endpoint values (excluding McCorkle } \\
\text { 2015) }\end{array}$ & 4 & 320 & $\begin{array}{l}\text { Mean Difference (IV, Ran- } \\
\text { dom, } 95 \% \mathrm{CI} \text { ) }\end{array}$ & $\begin{array}{l}-1.60[-2.56 \\
-0.65]\end{array}$ \\
\hline 6.7.1 Cancer populations & 2 & 211 & $\begin{array}{l}\text { Mean Difference (IV, Ran- } \\
\text { dom, } 95 \% \mathrm{CI} \text { ) }\end{array}$ & $\begin{array}{l}-1.91[-3.12 \\
-0.70]\end{array}$ \\
\hline 6.7.2 Non-cancer populations & 2 & 109 & $\begin{array}{l}\text { Mean Difference (IV, Ran- } \\
\text { dom, } 95 \% \mathrm{CI} \text { ) }\end{array}$ & $-0.82[-2.45,0.80]$ \\
\hline $\begin{array}{l}\text { 6.8 EPC vs LPC on patient anxiety: adjust- } \\
\text { ed endpoint values }\end{array}$ & 5 & 384 & $\begin{array}{l}\text { Mean Difference (IV, Ran- } \\
\text { dom, } 95 \% \mathrm{CI} \text { ) }\end{array}$ & $-0.63[-2.22,0.96]$ \\
\hline 6.8.1 Early palliative care (EPC) & 2 & 221 & $\begin{array}{l}\text { Mean Difference (IV, Ran- } \\
\text { dom, } 95 \% \mathrm{CI} \text { ) }\end{array}$ & $-0.57[-3.94,2.79]$ \\
\hline 6.8.2 Late palliative care (LPC) & 3 & 163 & $\begin{array}{l}\text { Mean Difference (IV, Ran- } \\
\text { dom, } 95 \% \mathrm{CI} \text { ) }\end{array}$ & $-0.81[-2.14,0.52]$ \\
\hline $\begin{array}{l}6.9 \text { Effect of MDT-led services on patient } \\
\text { anxiety: adjusted endpoint values }\end{array}$ & 5 & 384 & $\begin{array}{l}\text { Mean Difference (IV, Ran- } \\
\text { dom, } 95 \% \mathrm{CI} \text { ) }\end{array}$ & $-0.63[-2.22,0.96]$ \\
\hline 6.9.1 MDT-led services & 5 & 384 & $\begin{array}{l}\text { Mean Difference (IV, Ran- } \\
\text { dom, } 95 \% \mathrm{CI} \text { ) }\end{array}$ & $-0.63[-2.22,0.96]$ \\
\hline $\begin{array}{l}\text { 6.10 Effect of MDT-led services on patient } \\
\text { anxiety: adjusted endpoint values (ex- } \\
\text { cluding McCorkle 2015) }\end{array}$ & 4 & 320 & $\begin{array}{l}\text { Mean Difference (IV, Ran- } \\
\text { dom, } 95 \% \mathrm{CI} \text { ) }\end{array}$ & $\begin{array}{l}-1.60[-2.56 \\
-0.65]\end{array}$ \\
\hline
\end{tabular}




\begin{tabular}{|c|c|c|c|c|}
\hline Outcome or subgroup title & No. of studies & $\begin{array}{l}\text { No. of partici- } \\
\text { pants }\end{array}$ & Statistical method & Effect size \\
\hline 6.10.1 MDT-led services & 4 & 320 & $\begin{array}{l}\text { Mean Difference (IV, Ran- } \\
\text { dom, } 95 \% \mathrm{CI} \text { ) }\end{array}$ & $\begin{array}{l}-1.60[-2.56 \\
-0.65]\end{array}$ \\
\hline $\begin{array}{l}\text { 6.11 HSPC versus usual care on patient } \\
\text { anxiety in different countries: adjusted } \\
\text { endpoint values }\end{array}$ & 5 & 384 & $\begin{array}{l}\text { Mean Difference (IV, Ran- } \\
\text { dom, } 95 \% \mathrm{CI} \text { ) }\end{array}$ & $-0.63[-2.22,0.96]$ \\
\hline 6.11.1 Studies from USA & 3 & 251 & $\begin{array}{l}\text { Mean Difference (IV, Ran- } \\
\text { dom, } 95 \% \mathrm{CI} \text { ) }\end{array}$ & $-0.32[-3.04,2.39]$ \\
\hline 6.11.2 Studies from UK & 2 & 133 & $\begin{array}{l}\text { Mean Difference (IV, Ran- } \\
\text { dom, } 95 \% \mathrm{CI} \text { ) }\end{array}$ & $-1.02[-2.45,0.42]$ \\
\hline $\begin{array}{l}\text { 6.12 HSPC versus usual care on patient } \\
\text { anxiety in different countries: adjusted } \\
\text { endpoint values (excluding McCorkle } \\
\text { 2015) }\end{array}$ & 4 & 320 & $\begin{array}{l}\text { Mean Difference (IV, Ran- } \\
\text { dom, } 95 \% \mathrm{CI} \text { ) }\end{array}$ & $\begin{array}{l}-1.60[-2.56 \\
-0.65]\end{array}$ \\
\hline 6.12.1 Studies from USA & 2 & 187 & $\begin{array}{l}\text { Mean Difference (IV, Ran- } \\
\text { dom, } 95 \% \mathrm{CI} \text { ) }\end{array}$ & $-1.45[-3.90,1.00]$ \\
\hline 6.12.2 Studies from UK & 2 & 133 & $\begin{array}{l}\text { Mean Difference (IV, Ran- } \\
\text { dom, } 95 \% \mathrm{CI} \text { ) }\end{array}$ & $-1.02[-2.45,0.42]$ \\
\hline
\end{tabular}

\section{Analysis 6.1. Comparison 6: Patient anxiety, Outcome 1: HSPC versus usual care on patient anxiety: adjusted endpoint values}

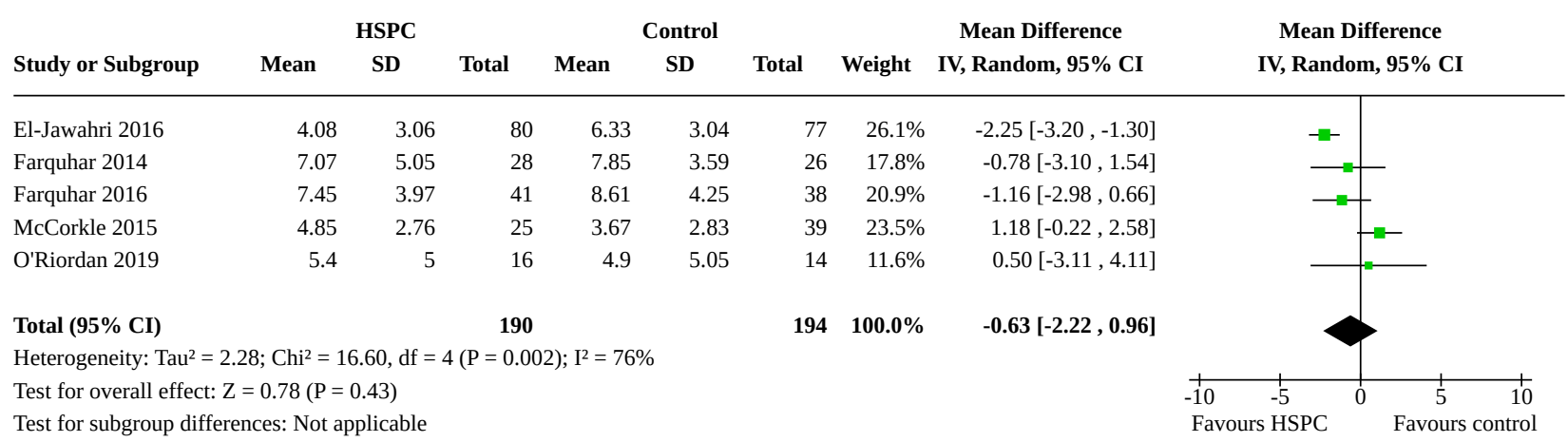


Analysis 6.2. Comparison 6: Patient anxiety, Outcome 2: HSPC versus usual care on patient anxiety: adjusted endpoint values (excluding McCorkle 2015)

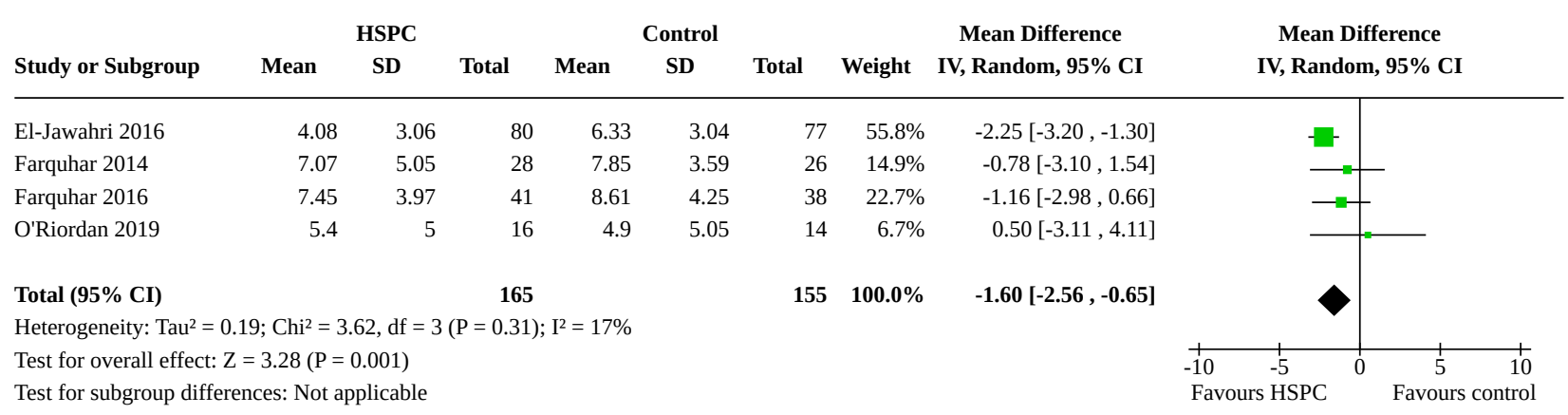

Analysis 6.3. Comparison 6: Patient anxiety, Outcome 3: HSPC versus usual care on patient anxiety: unadjusted endpoint values

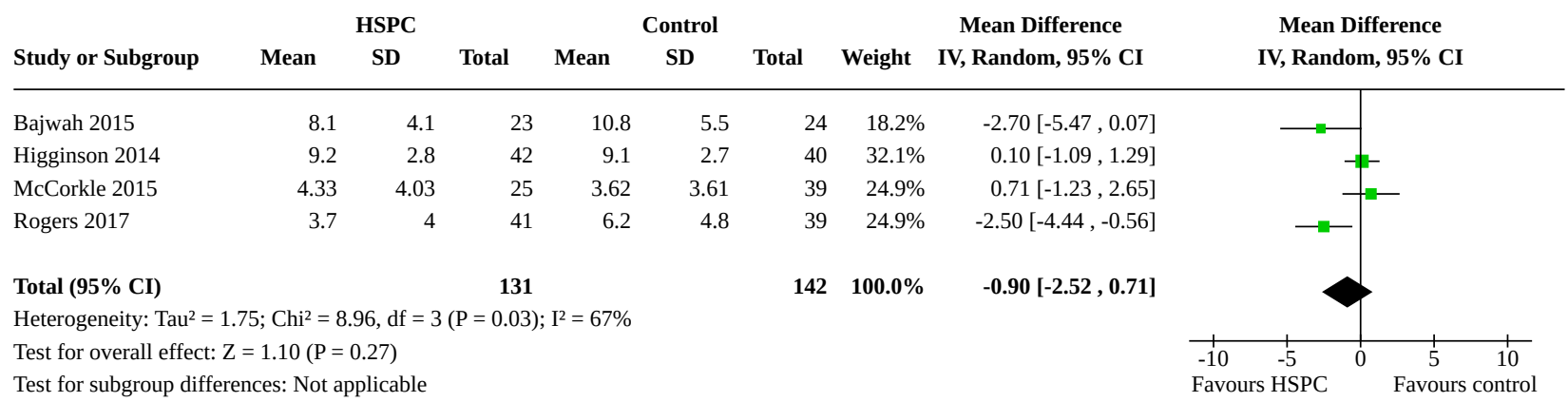

Analysis 6.4. Comparison 6: Patient anxiety, Outcome 4: HSPC versus usual care on patient anxiety: unadjusted endpoint values (excluding McCorkle 2015)

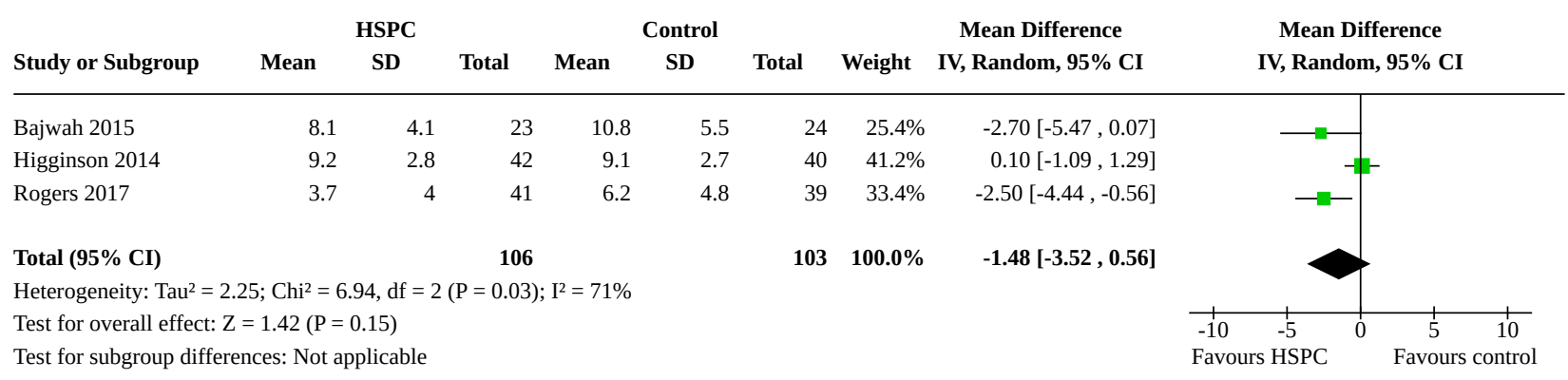




\section{Analysis 6.5. Comparison 6: Patient anxiety, Outcome 5: HSPC} versus usual care on patient anxiety: unadjusted change values

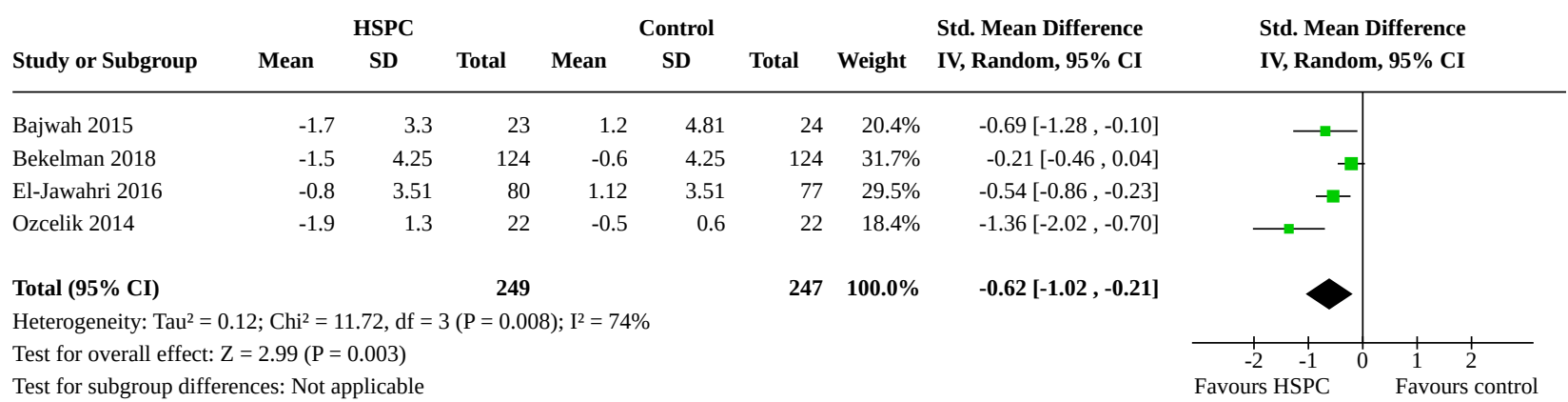

\section{Analysis 6.6. Comparison 6: Patient anxiety, Outcome 6: HSPC versus usual care on patient anxiety in different populations: adjusted endpoint values}

\begin{tabular}{|c|c|c|c|c|c|c|c|c|c|}
\hline Study or Subgroup & Mean & $\begin{array}{l}\text { HSPC } \\
\text { SD }\end{array}$ & Total & Mean & $\begin{array}{l}\text { ontrol } \\
\text { SD }\end{array}$ & Total & Weight & $\begin{array}{c}\text { Mean Difference } \\
\text { IV, Random, 95\% CI }\end{array}$ & $\begin{array}{c}\text { Mean Difference } \\
\text { IV, Random, 95\% CI }\end{array}$ \\
\hline \multicolumn{10}{|c|}{ 6.6.1 Cancer populations } \\
\hline El-Jawahri 2016 & 4.08 & 3.06 & 80 & 6.33 & 3.04 & 77 & $26.1 \%$ & $-2.25[-3.20,-1.30]$ & - \\
\hline Farquhar 2014 & 7.07 & 5.05 & 28 & 7.85 & 3.59 & 26 & $17.8 \%$ & $-0.78[-3.10,1.54]$ & \\
\hline McCorkle 2015 & 4.85 & 2.76 & 25 & 3.67 & 2.83 & 39 & $23.5 \%$ & $1.18[-0.22,2.58]$ & \\
\hline Subtotal (95\% CI) & & & 133 & & & 142 & $67.5 \%$ & $-0.65[-3.03,1.74]$ & \\
\hline \multicolumn{10}{|c|}{ Heterogeneity: Tau $^{2}=3.76 ; \mathrm{Chi}^{2}=15.84, \mathrm{df}=2(\mathrm{P}=0.0004) ; \mathrm{I}^{2}=87 \%$} \\
\hline \multicolumn{10}{|c|}{ Test for overall effect: $\mathrm{Z}=0.53(\mathrm{P}=0.60)$} \\
\hline \multicolumn{10}{|c|}{ 6.6.2 Non-cancer populations } \\
\hline Farquhar 2016 & 7.45 & 3.97 & 41 & 8.61 & 4.25 & 38 & $20.9 \%$ & $-1.16[-2.98,0.66]$ & - \\
\hline O'Riordan 2019 & 5.4 & 5 & 16 & 4.9 & 5.05 & 14 & $11.6 \%$ & $0.50[-3.11,4.11]$ & \\
\hline Subtotal (95\% CI) & & & 57 & & & 52 & $32.5 \%$ & $-0.82[-2.45,0.80]$ & \\
\hline \multicolumn{10}{|c|}{ Heterogeneity: $\mathrm{Tau}^{2}=0.00 ; \mathrm{Chi}^{2}=0.65, \mathrm{df}=1(\mathrm{P}=0.42) ; \mathrm{I}^{2}=0 \%$} \\
\hline \multicolumn{10}{|c|}{ Test for overall effect: $\mathrm{Z}=0.99(\mathrm{P}=0.32)$} \\
\hline Total $(95 \%$ CI) & & & 190 & & & 194 & $100.0 \%$ & $-0.63[-2.22,0.96]$ & \\
\hline \multicolumn{10}{|c|}{ Heterogeneity: $\mathrm{Tau}^{2}=2.28 ; \mathrm{Chi}^{2}=16.60, \mathrm{df}=4(\mathrm{P}=0.002) ; \mathrm{I}^{2}=76 \%$} \\
\hline \multicolumn{9}{|c|}{ Test for overall effect: $\mathrm{Z}=0.78(\mathrm{P}=0.43)$} & $\begin{array}{lll}1 & 1 & 1 \\
-4 & -2 & 0\end{array}$ \\
\hline \multicolumn{9}{|c|}{ Test for subgroup differences: $\mathrm{Chi}^{2}=0.01, \mathrm{df}=1(\mathrm{P}=0.90), \mathrm{I}^{2}=0 \%$} & Irs HSPC \\
\hline
\end{tabular}


Analysis 6.7. Comparison 6: Patient anxiety, Outcome 7: HSPC versus usual care on patient anxiety in different populations: adjusted endpoint values (excluding McCorkle 2015)

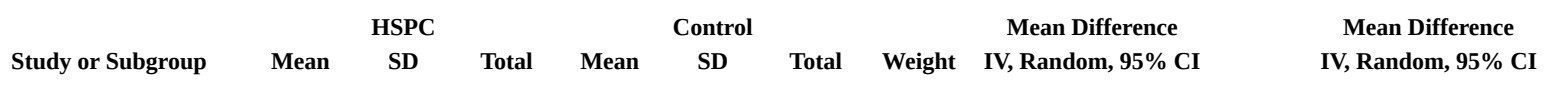

\subsubsection{Cancer populations}

$\begin{array}{lllll}4.08 & 3.06 & 80 & 6.33 & 3.04 \\ 7.07 & 5.05 & 28 & 7.85 & 3.59\end{array}$

$77 \quad 55.8 \%$

$26 \quad 14.9 \%$

$-2.25[-3.20,-1.30]$

Farquhar 2014

$7.07 \quad 5.05$

$\begin{array}{lc}\text { Subtotal }(\mathbf{9 5 \%} \text { CI) } & \mathbf{1 0 8} \\ \text { Heterogeneity: } \mathrm{Tau}^{2}=0.26 ; \mathrm{Chi}^{2}=1.31, \mathrm{df}=1(\mathrm{P}=0.25) ; \mathrm{I}^{2}=24 \%\end{array}$

$103 \quad 70.7 \%$

$-0.78[-3.10,1.54]$

Test for overall effect: $\mathrm{Z}=3.09(\mathrm{P}=0.002)$

6.7.2 Non-cancer populations

\begin{tabular}{|c|c|c|c|c|c|c|c|c|}
\hline Farquhar 2016 & 7.45 & 3.97 & 41 & 8.61 & 4.25 & 38 & $22.7 \%$ & $-1.16[-2.98,0.66]$ \\
\hline O'Riordan 2019 & 5.4 & 5 & 16 & 4.9 & 5.05 & 14 & $6.7 \%$ & $0.50[-3.11,4.11]$ \\
\hline Subtotal $(95 \%$ CI) & & & 57 & & & 52 & $29.3 \%$ & $-0.82[-2.45,0.80]$ \\
\hline
\end{tabular}

Heterogeneity: $\mathrm{Tau}^{2}=0.00 ; \mathrm{Chi}^{2}=0.65, \mathrm{df}=1(\mathrm{P}=0.42) ; \mathrm{I}^{2}=0 \%$

Test for overall effect: $\mathrm{Z}=0.99(\mathrm{P}=0.32)$

Total (95\% CI) 165

Heterogeneity: $\mathrm{Tau}^{2}=0.19 ; \mathrm{Chi}^{2}=3.62, \mathrm{df}=3(\mathrm{P}=0.31) ; \mathrm{I}^{2}=17 \%$

Test for overall effect: $\mathrm{Z}=3.28(\mathrm{P}=0.001)$

Test for subgroup differences: $\mathrm{Chi}^{2}=1.11, \mathrm{df}=1(\mathrm{P}=0.29), \mathrm{I}^{2}=10.0 \%$

$155 \quad 100.0 \% \quad-1.60[-2.56,-0.65]$

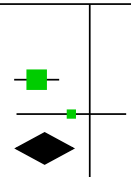

Analysis 6.8. Comparison 6: Patient anxiety, Outcome 8: EPC vs LPC on patient anxiety: adjusted endpoint values

\begin{tabular}{|c|c|c|c|c|c|c|c|c|c|}
\hline & & ISPC & & & ontrol & & & Mean Difference & Mean Difference \\
\hline Study or Subgroup & Mean & SD & Total & Mean & SD & Total & Weight & IV, Random, 95\% CI & IV, Random, 95\% CI \\
\hline
\end{tabular}

6.8.1 Early palliative care (EPC)

Subtotal (95\% CI)

$4.85 \quad 2.76$

25
105

$3.67 \quad 2.83$

$77 \quad 26.1 \%$

$-2.25[-3.20,-1.30]$

$3923.5 \%$

$1.18[-0.22,2.58]$

$-0.57[-3.94,2.79]$

Test for overall effect: $\mathrm{Z}=0.34(\mathrm{P}=0.74)$

6.8.2 Late palliative care (LPC)

$\begin{array}{lrrrrrrrr}\text { Farquhar 2014 } & 7.07 & 5.05 & 28 & 7.85 & 3.59 & 26 & 17.8 \% & -0.78[-3.10,1.54] \\ \text { Farquhar 2016 } & 7.45 & 3.97 & 41 & 8.61 & 4.25 & 38 & 20.9 \% & -1.16[-2.98,0.66] \\ \text { O'Riordan 2019 } & 5.4 & 5 & 16 & 4.9 & 5.05 & 14 & 11.6 \% & 0.50[-3.11,4.11] \\ \text { Subtotal (95\% CI) } & & & \mathbf{8 5} & & & \mathbf{7 8} & \mathbf{5 0 . 3 \%} & \mathbf{- 0 . 8 1}[-\mathbf{2 . 1 4}, \mathbf{0 . 5 2}]\end{array}$

Subtotal (95\% CI)

85

$78 \quad \mathbf{5 0 . 3} \%$

$-0.81[-2.14,0.52]$

Test for overall effect: $\mathrm{Z}=1.19(\mathrm{P}=0.23)$

Total (95\% CI)

190

$194 \quad 100.0 \%$

$-0.63[-2.22,0.96]$

Heterogeneity: Tau $^{2}=2.28 ; \mathrm{Chi}^{2}=16.60, \mathrm{df}=4(\mathrm{P}=0.002) ; \mathrm{I}^{2}=76 \%$

Test for overall effect: $\mathrm{Z}=0.78(\mathrm{P}=0.43)$

Test for subgroup differences: $\mathrm{Chi}^{2}=0.02, \mathrm{df}=1(\mathrm{P}=0.90), \mathrm{I}^{2}=0 \%$

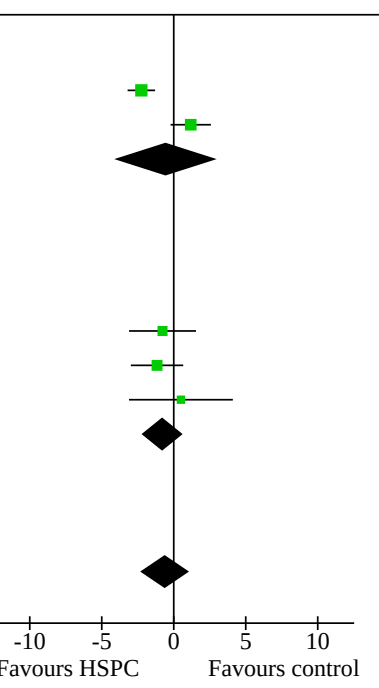

The effectiveness and cost-effectiveness of hospital-based specialist palliative care for adults with advanced illness and their caregivers 
Analysis 6.9. Comparison 6: Patient anxiety, Outcome 9: Effect of MDT-led services on patient anxiety: adjusted endpoint values

\begin{tabular}{|c|c|c|c|c|c|c|c|c|c|}
\hline & & HSPC & & & ontro & & & Mean Difference & Mean Difference \\
\hline Study or Subgroup & Mean & SD & Total & Mean & SD & Total & Weight & IV, Random, 95\% CI & IV, Random, 95\% CI \\
\hline
\end{tabular}

\subsubsection{MDT-led services}

El-Jawahri 2016

Farquhar 2014

$4.08 \quad 3.06$

Farquhar 2016

McCorkle 2015

O'Riordan 2019

$\begin{array}{ll}7.07 & 5.05\end{array}$

$\begin{array}{ll}7.45 & 3.97\end{array}$

$4.85 \quad 2.76$

5.4

5

Subtotal (95\% CI)

Heterogeneity: $\mathrm{Tau}^{2}=2.28 ; \mathrm{Chi}^{2}=16.60, \mathrm{df}=4(\mathrm{P}=0.002) ; \mathrm{I}^{2}=76 \%$

Test for overall effect: $\mathrm{Z}=0.78(\mathrm{P}=0.43)$

Total (95\% CI) 190

Heterogeneity: $\mathrm{Tau}^{2}=2.28 ; \mathrm{Chi}^{2}=16.60, \mathrm{df}=4(\mathrm{P}=0.002) ; \mathrm{I}^{2}=76 \%$

Test for overall effect: $\mathrm{Z}=0.78(\mathrm{P}=0.43)$

Test for subgroup differences: Not applicable

$\begin{array}{rrrr} & & & \\ 3.04 & 77 & 26.1 \% & -2.25[-3.20,-1.30] \\ 3.59 & 26 & 17.8 \% & -0.78[-3.10,1.54] \\ 4.25 & 38 & 20.9 \% & -1.16[-2.98,0.66] \\ 2.83 & 39 & 23.5 \% & 1.18[-0.22,2.58] \\ 5.05 & 14 & 11.6 \% & 0.50[-3.11,4.11] \\ & \mathbf{1 9 4} & \mathbf{1 0 0 . 0 \%} & \mathbf{- 0 . 6 3}[-\mathbf{2 . 2 2} \mathbf{0 . 9 6}]\end{array}$

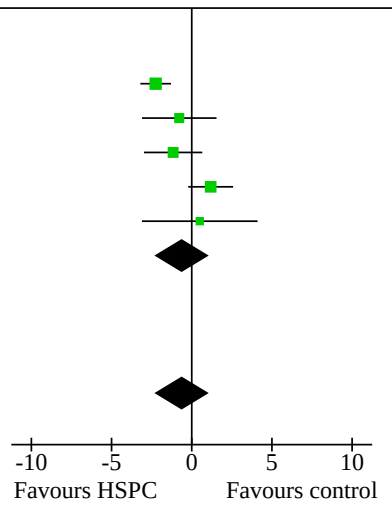

Analysis 6.10. Comparison 6: Patient anxiety, Outcome 10: Effect of MDT-led services on patient anxiety: adjusted endpoint values (excluding McCorkle 2015)

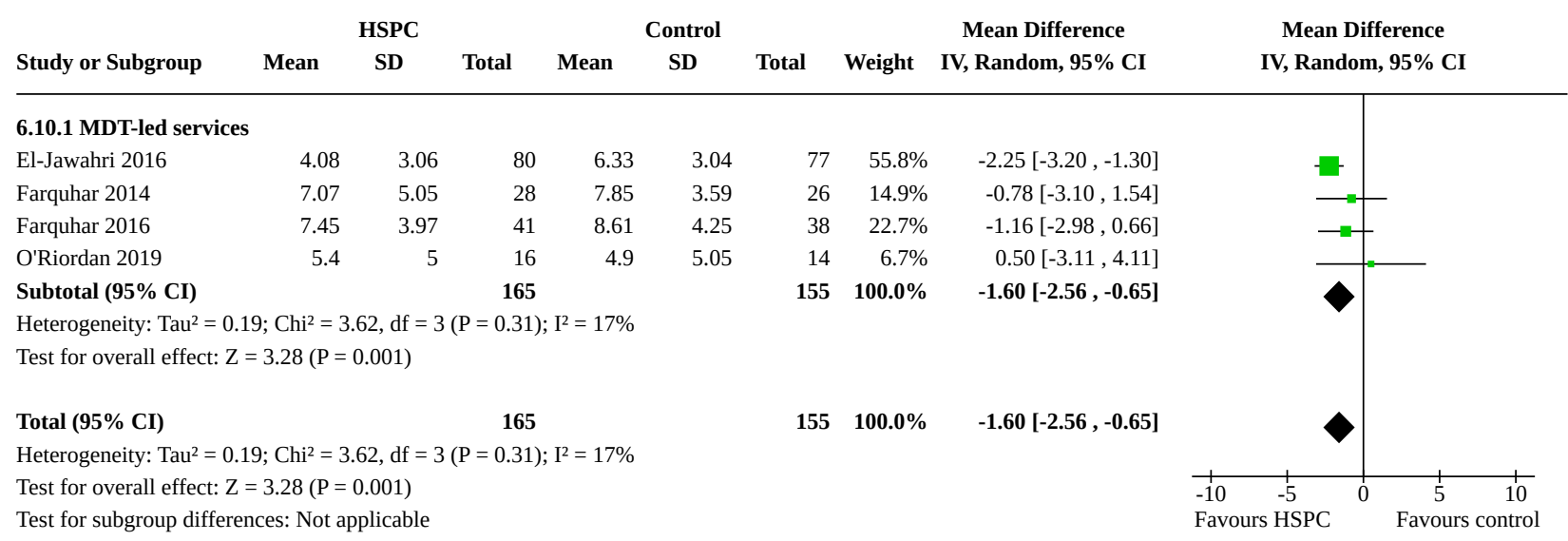


Analysis 6.11. Comparison 6: Patient anxiety, Outcome 11: HSPC versus usual care on patient anxiety in different countries: adjusted endpoint values

\begin{tabular}{|c|c|c|c|c|c|c|c|c|c|}
\hline & & ISPC & & & ontr & & & Mean Difference & Mean Difference \\
\hline Study or Subgroup & Mean & SD & Total & Mean & SD & Total & Weight & IV, Random, 95\% CI & IV, Random, 95\% CI \\
\hline
\end{tabular}

6.11.1 Studies from USA

El-Jawahi 2016

McCorkle 2015

$\begin{array}{ll}4.08 & 3.06\end{array}$

$\begin{array}{lll}80 & 6.33 & 3.04\end{array}$

$77 \quad 26.1 \%$

$-2.25[-3.20,-1.30]$

O'Riordan 2019

$4.85 \quad 2.76$

25

$3.67 \quad 2.83$

$39 \quad 23.5 \%$

Subtotal (95\% CI)

121

$\begin{array}{ll}4.9 & 5.05\end{array}$

$14 \quad 11.6 \%$

$1.18[-0.22,2.58]$

$0.50[-3.11,4.11]$

Heterogeneity: $\mathrm{Tau}^{2}=4.66 ; \mathrm{Chi}^{2}=16.53, \mathrm{df}=2(\mathrm{P}=0.0003) ; \mathrm{I}^{2}=88 \%$

$13061.3 \%$

$-0.32[-3.04,2.39]$

Test for overall effect: $\mathrm{Z}=0.23(\mathrm{P}=0.81)$

6.11.2 Studies from UK

$\begin{array}{lllllllll}\text { Farquhar } 2014 & 7.07 & 5.05 & 28 & 7.85 & 3.59 & 26 & 17.8 \% & -0.78[-3.10,1.54] \\ \text { Farquhar 2016 } & 7.45 & 3.97 & 41 & 8.61 & 4.25 & 38 & 20.9 \% & -1.16[-2.98,0.66]\end{array}$

Subtotal (95\% CI)

69
$0.80) ; \mathrm{I}^{2}=0 \%$

$64 \quad 38.7 \%$

$-1.02[-2.45,0.42]$

Heterogeneity: $\mathrm{Tau}^{2}=0.00 ; \mathrm{Chi}^{2}=0.06, \mathrm{df}=1(\mathrm{P}=0.80) ; \mathrm{I}^{2}=0 \%$
Test for overall effect: $\mathrm{Z}=1.39(\mathrm{P}=0.16)$

Total (95\% CI)

190

Heterogeneity: $\mathrm{Tau}^{2}=2.28 ; \mathrm{Chi}^{2}=16.60, \mathrm{df}=4(\mathrm{P}=0.002) ; \mathrm{I}^{2}=76 \%$

$194 \quad 100.0 \%$

$-0.63[-2.22,0.96]$

Test for overall effect: $\mathrm{Z}=0.78(\mathrm{P}=0.43)$

Test for subgroup differences: $\mathrm{Chi}^{2}=0.20, \mathrm{df}=1(\mathrm{P}=0.66), \mathrm{I}^{2}=0 \%$

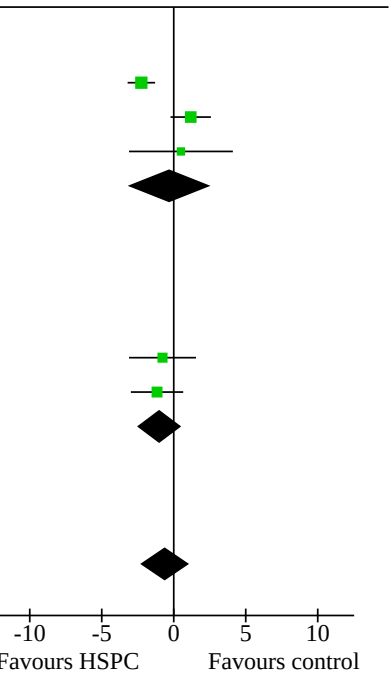

Analysis 6.12. Comparison 6: Patient anxiety, Outcome 12: HSPC versus usual care on patient anxiety in different countries: adjusted endpoint values (excluding McCorkle 2015)

\begin{tabular}{|c|c|c|c|c|c|c|c|c|c|}
\hline & & HSPC & & & ontro & & & Mean Difference & Mean Difference \\
\hline Study or Subgroup & Mean & SD & Total & Mean & SD & Total & Weight & IV, Random, 95\% CI & IV, Random, 95\% CI \\
\hline
\end{tabular}

6.12.1 Studies from USA

El-Jawahri 2016

O'Riordan 2019

$\begin{array}{lllll}4.08 & 3.06 & 80 & 6.33 & 3.04\end{array}$

$77 \quad 55.8 \%$

$-2.25[-3.20,-1.30]$

Subtotal (95\% CI)

$\begin{array}{rrr}5.4 & 5 & 16\end{array}$

4.9

5.05

$14 \quad 6.7 \%$

$0.50[-3.11,4.11]$

Heterogeneity: $\mathrm{Tau}^{2}=1.97 ; \mathrm{Chi}^{2}=2.09, \mathrm{df}=1(\mathrm{P}=0.15) ; \mathrm{I}^{2}=52 \%$

Test for overall effect: $\mathrm{Z}=1.16(\mathrm{P}=0.25)$

6.12.2 Studies from UK

$\begin{array}{lllllllll}\text { Farquhar } 2014 & 7.07 & 5.05 & 28 & 7.85 & 3.59 & 26 & 14.9 \% & -0.78[-3.10,1.54] \\ \text { Farquhar 2016 } & 7.45 & 3.97 & 41 & 8.61 & 4.25 & 38 & 22.7 \% & -1.16[-2.98,0.66] \\ \text { Subtotal (95\% CI) } & & & \mathbf{6 9} & & & \mathbf{6 4} & \mathbf{3 7 . 6 \%} & \mathbf{- 1 . 0 2}[-\mathbf{2 . 4 5}, \mathbf{0 . 4 2}]\end{array}$

Heterogeneity: $\mathrm{Tau}^{2}=0.00 ; \mathrm{Chi}^{2}=0.06, \mathrm{df}=1(\mathrm{P}=0.80) ; \mathrm{I}^{2}=0 \%$

Test for overall effect: $\mathrm{Z}=1.39(\mathrm{P}=0.16)$

Total (95\% CI) 165

Heterogeneity: $\mathrm{Tau}^{2}=0.19 ; \mathrm{Chi}^{2}=3.62, \mathrm{df}=3(\mathrm{P}=0.31) ; \mathrm{I}^{2}=17 \%$

Test for overall effect: $\mathrm{Z}=3.28(\mathrm{P}=0.001)$

Test for subgroup differences: $\mathrm{Chi}^{2}=0.09, \mathrm{df}=1(\mathrm{P}=0.77), \mathrm{I}^{2}=0 \%$

$155 \quad 100.0 \% \quad-1.60[-2.56,-0.65]$

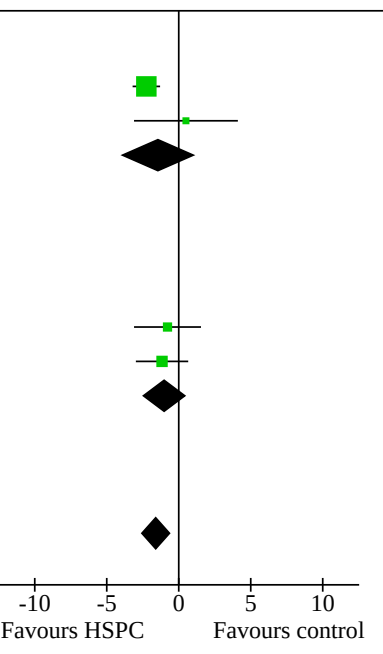

Comparison 7. Unpaid caregiver anxiety

\begin{tabular}{lllll}
\hline Outcome or subgroup title & No. of studies & $\begin{array}{l}\text { No. of partici- } \\
\text { pants }\end{array}$ & Statistical method & Effect size \\
\hline $\begin{array}{l}7.1 \text { HSPC versus usual care on unpaid care- } \\
\text { giver anxiety: unadjusted endpoint values }\end{array}$ & 2 & 351 & $\begin{array}{l}\text { Mean Difference (IV, } \\
\text { Random, 95\% CI) }\end{array}$ & $-0.71[-4.27,2.85]$ \\
\hline
\end{tabular}

The effectiveness and cost-effectiveness of hospital-based specialist palliative care for adults with advanced illness and their caregivers 
Analysis 7.1. Comparison 7: Unpaid caregiver anxiety, Outcome 1: HSPC versus usual care on unpaid caregiver anxiety: unadjusted endpoint values

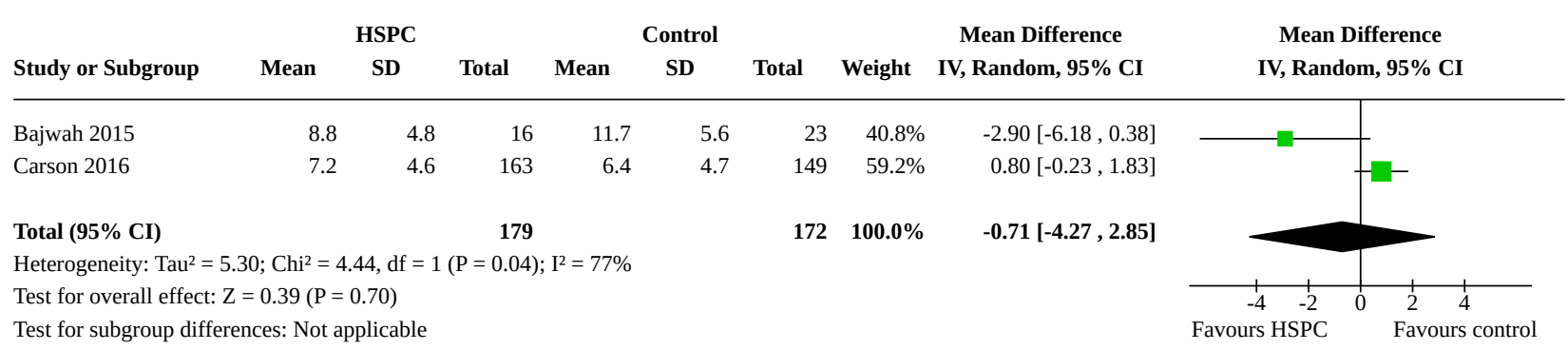

Comparison 8. Patient depression

\begin{tabular}{lllll}
\hline Outcome or subgroup title & No. of studies & $\begin{array}{l}\text { No. of partici- } \\
\text { pants }\end{array}$ & Statistical method & Effect size \\
\hline $\begin{array}{l}\text { 8.1 HSPC versus usual care on patient de- } \\
\text { pression: adjusted endpoint values }\end{array}$ & 8 & 1096 & $\begin{array}{l}\text { Std. Mean Difference (IV, } \\
\text { Random, 95\% Cl) }\end{array}$ & $\begin{array}{l}-0.22[-0.34, \\
-0.10]\end{array}$ \\
\hline $\begin{array}{l}\text { 8.2 HSPC versus usual care on patient de- } \\
\text { pression: unadjusted endpoint values }\end{array}$ & 5 & 350 & $\begin{array}{l}\text { Std. Mean Difference (IV, } \\
\text { Random, 95\% Cl) }\end{array}$ & -0.25 [-0.55, 0.04] \\
\hline $\begin{array}{l}\text { 8.3 HSPC versus usual care on patient de- } \\
\text { pression: unadjusted endpoint values (ex- } \\
\text { cluding McCorkle 2015) }\end{array}$ & 4 & Std. Mean Difference (IV, & -0.34 [-0.65, \\
\hline $\begin{array}{l}\text { 8.4 HSPC versus usual care on patient de- } \\
\text { pression: adjusted change values }\end{array}$ & 2 & Random, 95\% CI) & $-0.03]$ \\
\hline $\begin{array}{l}\text { 8.5 HSPC versus usual care on patient de- } \\
\text { pression: unadjusted change values }\end{array}$ & 4 & 286 & $\begin{array}{l}\text { Mean Difference (IV, Ran- } \\
\text { dom, 95\% Cl) }\end{array}$ & -0.32 [-1.10, 0.45] \\
\hline $\begin{array}{l}\text { 8.6 HSPC versus usual care on patient de- } \\
\text { pression as a binary outcome }\end{array}$ & 3 & $\begin{array}{l}\text { Std. Mean Difference (IV, } \\
\text { Random, 95\% Cl) }\end{array}$ & -0.38 [-0.58, \\
\hline
\end{tabular}


Analysis 8.1. Comparison 8: Patient depression, Outcome 1: HSPC versus usual care on patient depression: adjusted endpoint values

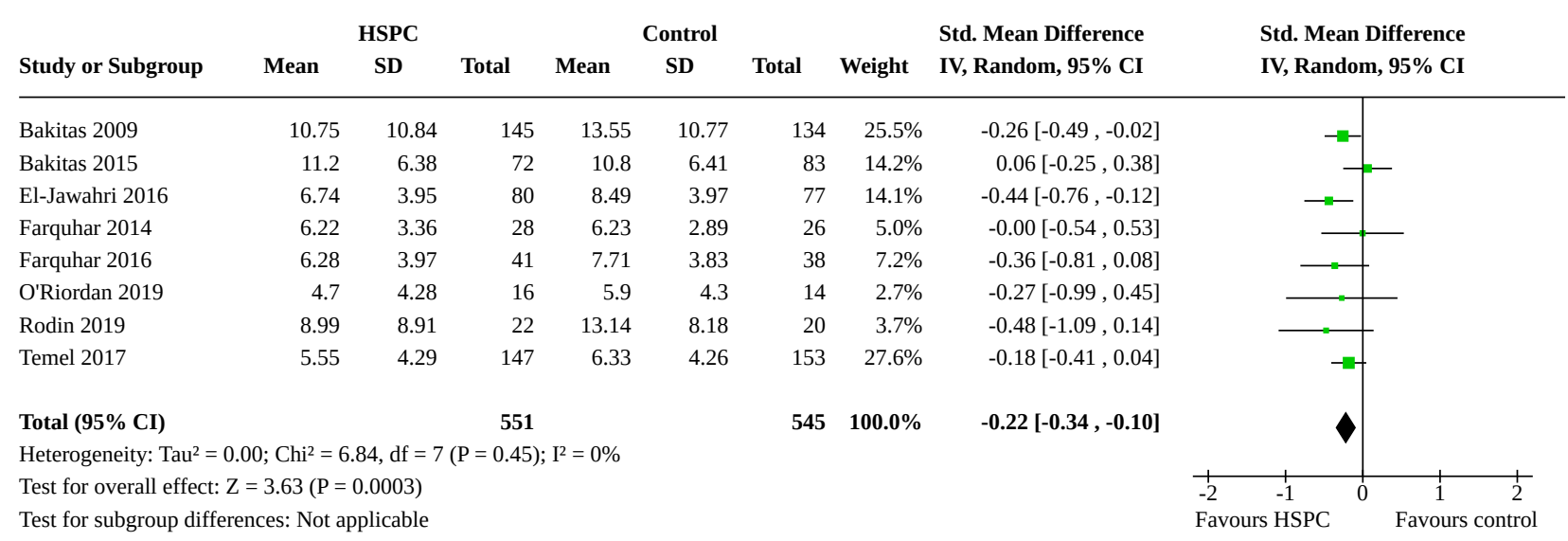

Analysis 8.2. Comparison 8: Patient depression, Outcome 2: HSPC versus usual care on patient depression: unadjusted endpoint values

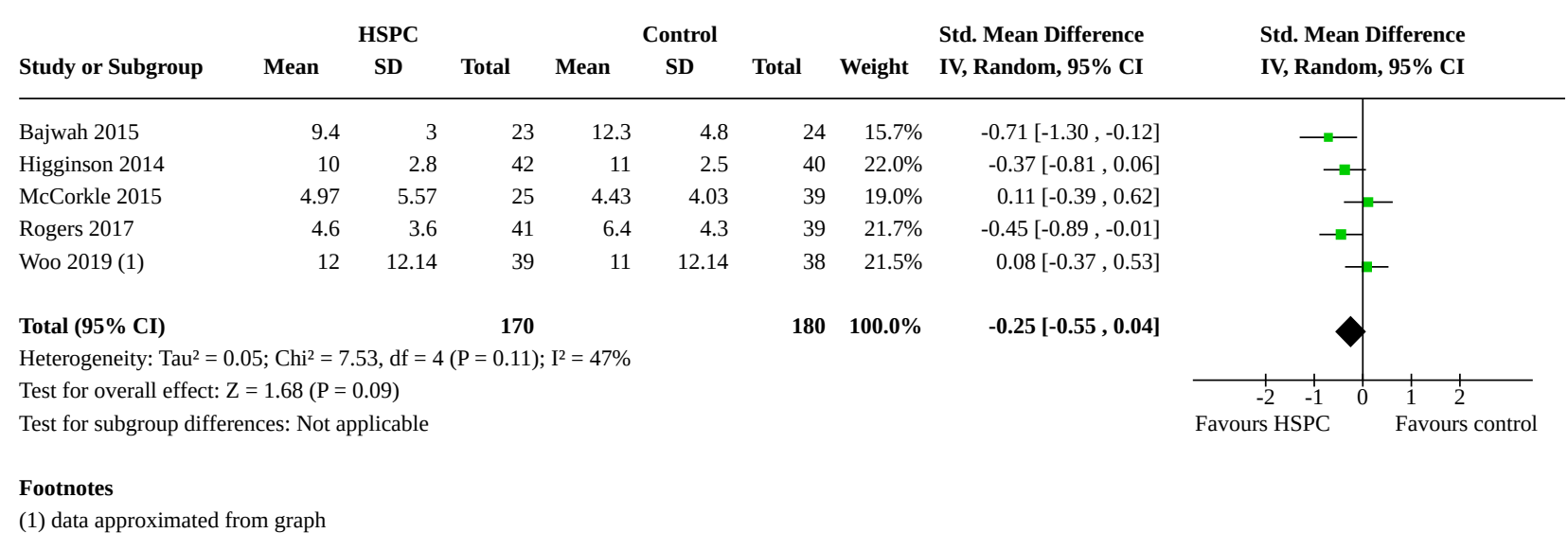

Analysis 8.3. Comparison 8: Patient depression, Outcome 3: HSPC versus usual care on patient depression: unadjusted endpoint values (excluding McCorkle 2015)

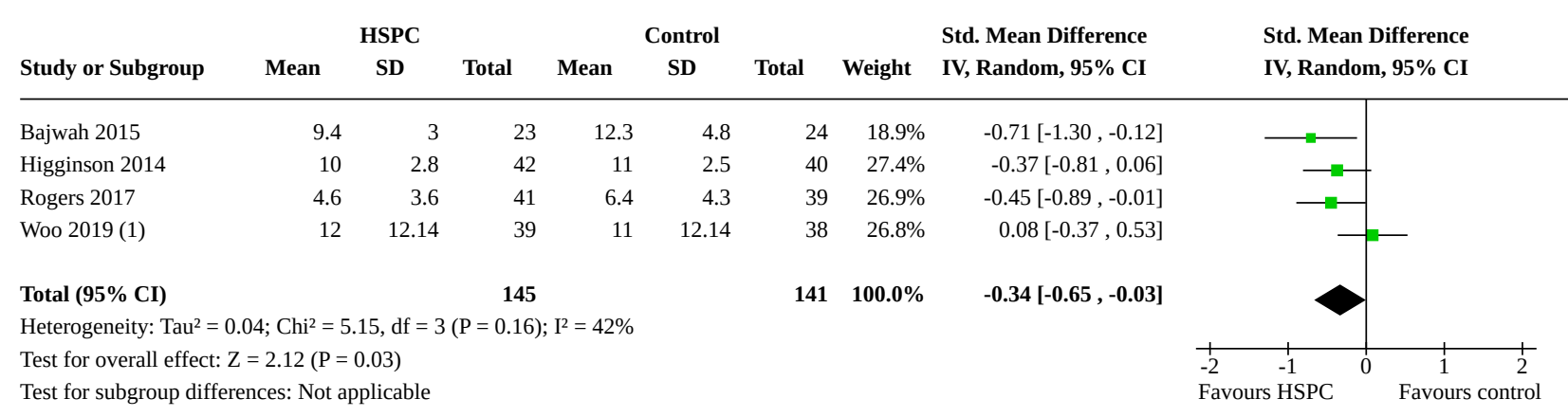

Footnotes

(1) data approximated from graph 
Analysis 8.4. Comparison 8: Patient depression, Outcome 4: HSPC versus usual care on patient depression: adjusted change values

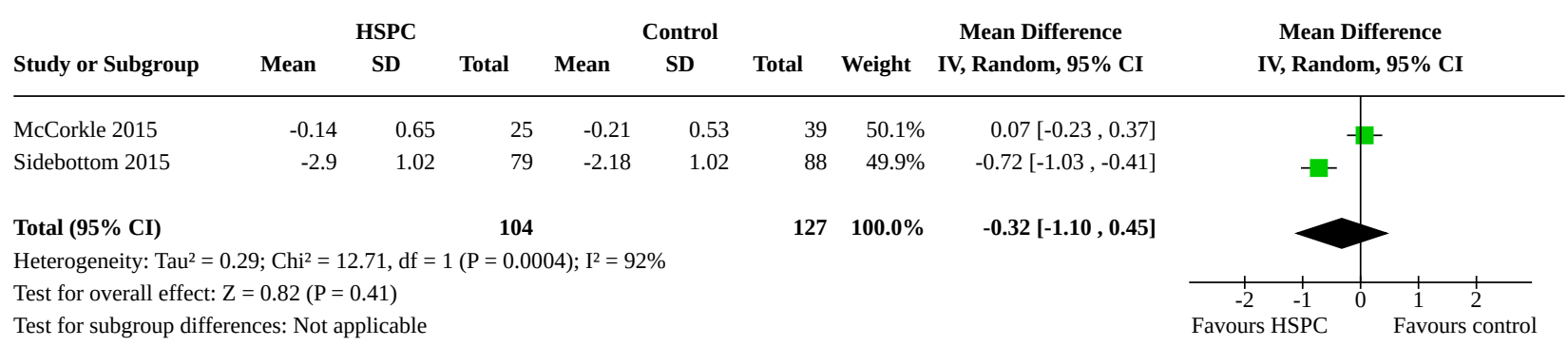

\section{Analysis 8.5. Comparison 8: Patient depression, Outcome 5: HSPC} versus usual care on patient depression: unadjusted change values

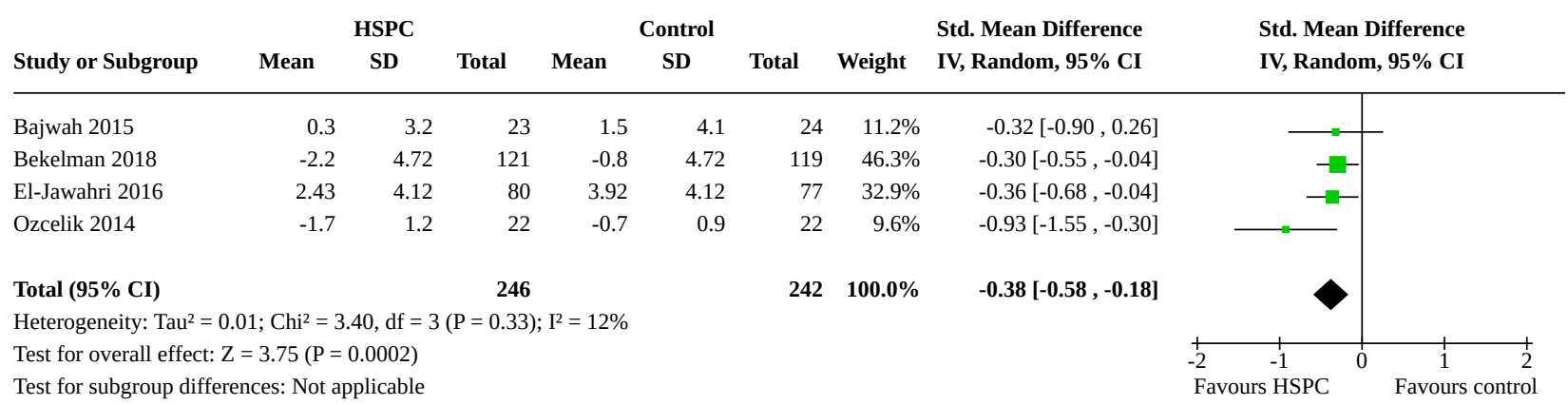

\section{Analysis 8.6. Comparison 8: Patient depression, Outcome 6: HSPC versus usual care on patient depression as a binary outcome}

\begin{tabular}{lccccccc} 
& \multicolumn{2}{c}{ HSPC } & \multicolumn{2}{c}{ Control } & \multicolumn{2}{c}{ Odds Ratio } & \multicolumn{2}{c}{ Odds Ratio } \\
Study or Subgroup & Events & Total & Events & Total & Weight & M-H, Random, 95\% CI & M-H, Random, 95\% CI
\end{tabular}

\begin{tabular}{|c|c|c|c|c|c|c|}
\hline El-Jawahri 2016 & 9 & 57 & 18 & 47 & $28.5 \%$ & $0.30[0.12,0.76]$ \\
\hline Temel 2010 & 24 & 80 & 46 & 77 & $43.9 \%$ & $0.29[0.15,0.56]$ \\
\hline Woo 2019 & 12 & 39 & 14 & 38 & $27.5 \%$ & $0.76[0.30,1.96]$ \\
\hline Total (95\% CI) & & 176 & & 162 & $100.0 \%$ & $0.38[0.21,0.68]$ \\
\hline Total events: & 45 & & 78 & & & \\
\hline
\end{tabular}

Heterogeneity: $\mathrm{Tau}^{2}=0.09 ; \mathrm{Chi}^{2}=2.96, \mathrm{df}=2(\mathrm{P}=0.23) ; \mathrm{I}^{2}=32 \%$

Test for overall effect: $\mathrm{Z}=3.24(\mathrm{P}=0.001)$

Test for subgroup differences: Not applicable

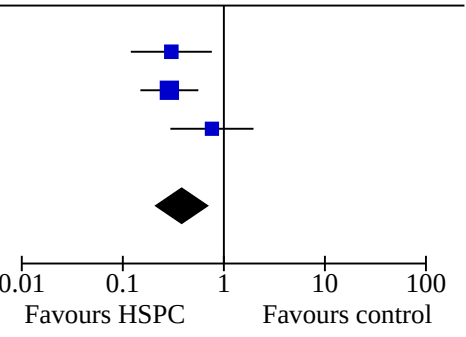

\section{Comparison 9. Unpaid caregiver depression}

\begin{tabular}{lllll}
\hline Outcome or subgroup title & No. of studies & $\begin{array}{l}\text { No. of partici- } \\
\text { pants }\end{array}$ & Statistical method & Effect size \\
\hline $\begin{array}{l}\text { 9.1 HSPC versus usual care on unpaid care- } \\
\text { giver depression: adjusted endpoint values }\end{array}$ & 2 & 413 & $\begin{array}{l}\text { Std. Mean Difference (IV, } \\
\text { Random, 95\% Cl) }\end{array}$ & $-0.02[-0.21,0.18]$ \\
\hline
\end{tabular}

The effectiveness and cost-effectiveness of hospital-based specialist palliative care for adults with advanced illness and their caregivers 


\begin{tabular}{lllll}
\hline Outcome or subgroup title & No. of studies & $\begin{array}{l}\text { No. of partici- } \\
\text { pants }\end{array}$ & Statistical method & Effect size \\
\hline $\begin{array}{l}\text { 9.2 HSPC versus usual care on unpaid care- } \\
\text { giver depression: unadjusted endpoint val- } \\
\text { ues }\end{array}$ & 3 & 420 & $\begin{array}{l}\text { Std. Mean Difference (IV, } \\
\text { Random, 95\% Cl) }\end{array}$ & $-0.29[-0.70,0.12]$ \\
\hline
\end{tabular}

Analysis 9.1. Comparison 9: Unpaid caregiver depression, Outcome 1: HSPC versus usual care on unpaid caregiver depression: adjusted endpoint values

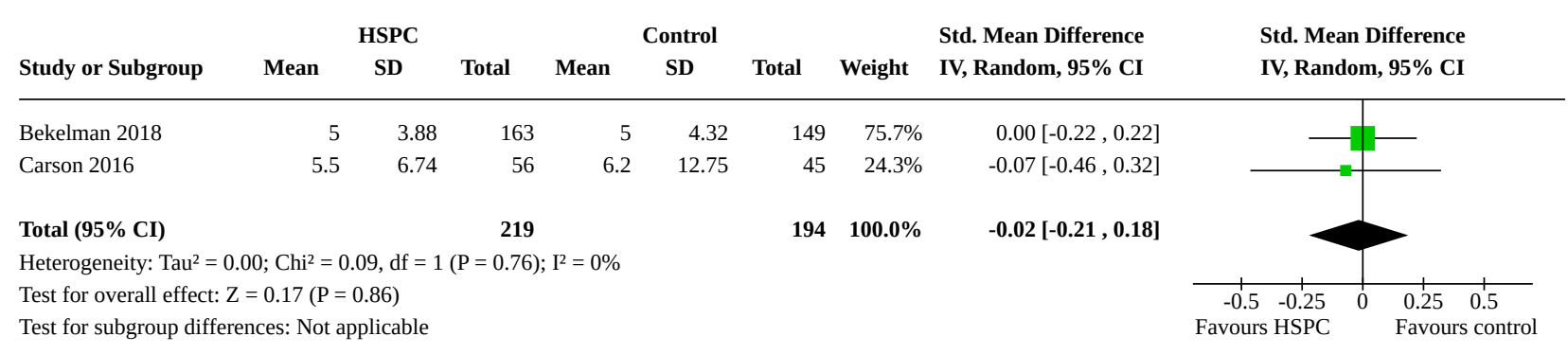

Analysis 9.2. Comparison 9: Unpaid caregiver depression, Outcome 2: HSPC versus usual care on unpaid caregiver depression: unadjusted endpoint values

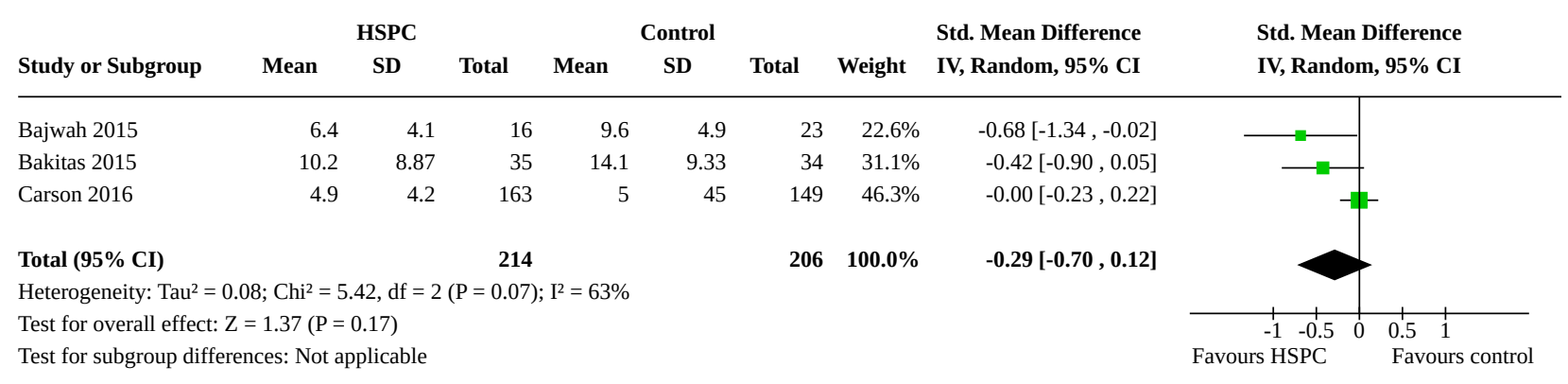

\section{Comparison 10. Unpaid caregiver quality of life}

\begin{tabular}{lllll}
\hline Outcome or subgroup title & No. of studies & $\begin{array}{l}\text { No. of partici- } \\
\text { pants }\end{array}$ & Statistical method & Effect size \\
\hline $\begin{array}{l}\text { 10.1 HSPC versus usual care on unpaid care- } \\
\text { giver quality of life: unadjusted endpoint val- } \\
\text { ues }\end{array}$ & 2 & 105 & $\begin{array}{l}\text { Mean Difference (IV, } \\
\text { Random, 95\% CI) }\end{array}$ & $6.11[0.42,11.81]$ \\
\hline
\end{tabular}


Analysis 10.1. Comparison 10: Unpaid caregiver quality of life, Outcome 1: HSPC versus usual care on unpaid caregiver quality of life: unadjusted endpoint values

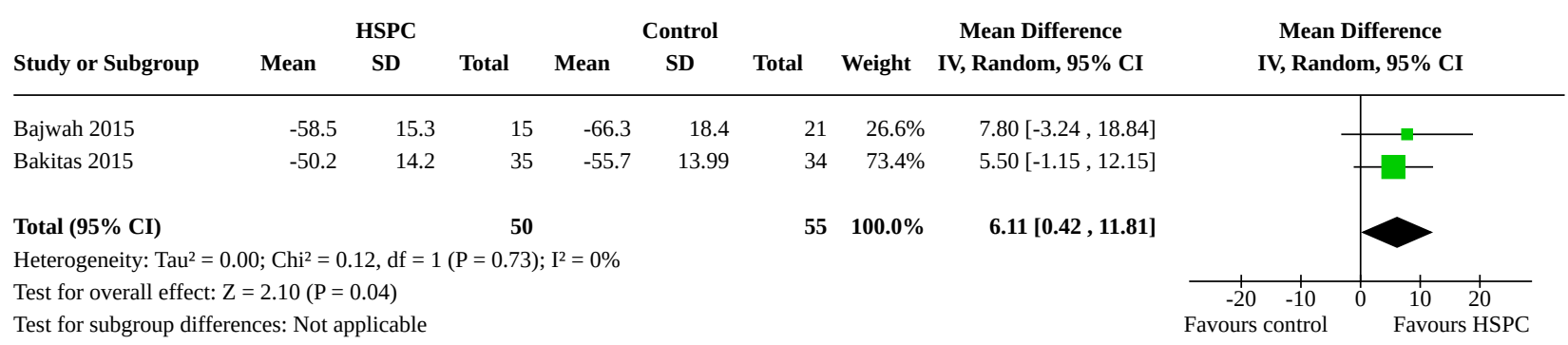

\section{Comparison 11. Unpaid caregiver burden}

\begin{tabular}{lllll}
\hline Outcome or subgroup title & No. of studies & $\begin{array}{l}\text { No. of partici- } \\
\text { pants }\end{array}$ & Statistical method & Effect size \\
\hline $\begin{array}{l}11.1 \text { HSPC versus usual care on unpaid care- } \\
\text { giver burden: adjusted change values }\end{array}$ & 3 & 128 & $\begin{array}{l}\text { Mean Difference (IV, } \\
\text { Random, 95\% Cl) }\end{array}$ & $\begin{array}{l}-3.88[-5.95, \\
-1.80]\end{array}$ \\
\hline
\end{tabular}

\section{Analysis 11.1. Comparison 11: Unpaid caregiver burden, Outcome 1: HSPC versus usual care on unpaid caregiver burden: adjusted change values}

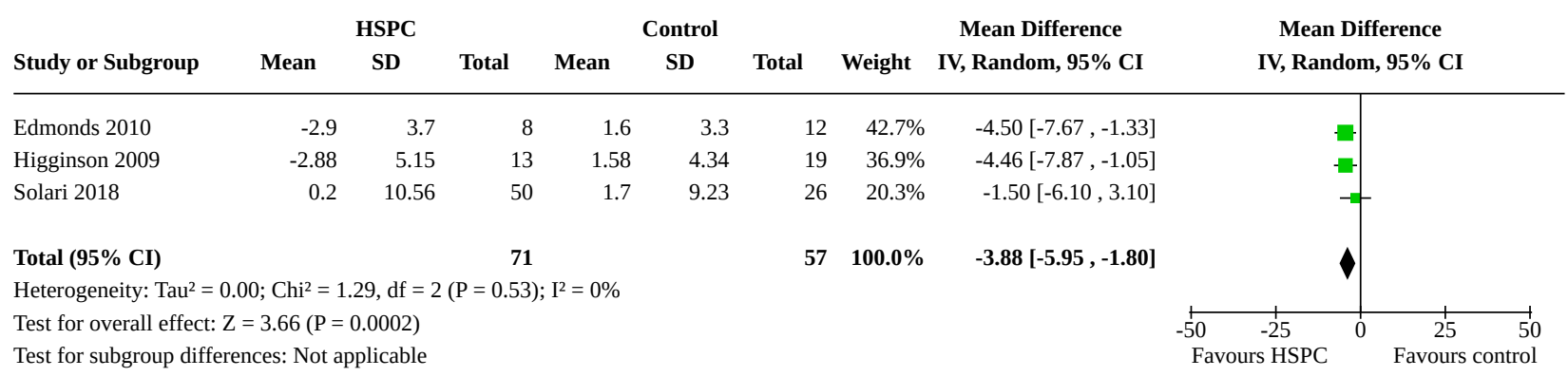

\section{Comparison 12. Patient breathlessness}

\begin{tabular}{lllll}
\hline Outcome or subgroup title & No. of studies & $\begin{array}{l}\text { No. of partici- } \\
\text { pants }\end{array}$ & Statistical method & Effect size \\
\hline $\begin{array}{l}12.1 \text { HSPC versus usual care on patient } \\
\text { breathlessness: adjusted endpoint values }\end{array}$ & 5 & 616 & $\begin{array}{l}\text { Std. Mean Difference (IV, } \\
\text { Random, 95\% Cl) }\end{array}$ & $-0.04[-0.19,0.12]$ \\
\hline $\begin{array}{l}12.2 \text { HSPC versus usual care on patient } \\
\text { breathlessness: unadjusted endpoint val- } \\
\text { ues }\end{array}$ & 2 & 128 & $\begin{array}{l}\text { Std. Mean Difference (IV, } \\
\text { Random, 95\% Cl) }\end{array}$ & $-0.35[-0.70$, \\
\hline $\begin{array}{l}12.3 \text { HSPC versus usual care on patient } \\
\text { breathlessness: unadjusted change values }\end{array}$ & 2 & 292 & Std. Mean Difference (IV, & -0.007 [-1.55, 0.61] \\
\hline
\end{tabular}

The effectiveness and cost-effectiveness of hospital-based specialist palliative care for adults with advanced illness and their caregivers 
Analysis 12.1. Comparison 12: Patient breathlessness, Outcome 1: HSPC versus usual care on patient breathlessness: adjusted endpoint values

\begin{tabular}{|c|c|c|c|c|c|c|c|c|c|}
\hline \multirow{2}{*}{ Study or Subgroup } & \multicolumn{3}{|c|}{ HSPC } & \multicolumn{3}{|c|}{ Control } & \multirow{2}{*}{ Weight } & \multirow{2}{*}{$\begin{array}{l}\text { Std. Mean Difference } \\
\text { IV, Random, } 95 \% \text { CI }\end{array}$} & \multirow{2}{*}{$\begin{array}{l}\text { Std. Mean Difference } \\
\text { IV, Random, 95\% CI }\end{array}$} \\
\hline & Mean & SD & Iotal & Mean & SD & Iotal & & & \\
\hline Farquhar 2014 & 3.43 & 2.95 & 28 & 4.42 & 3.01 & 26 & $8.7 \%$ & $-0.33[-0.87,0.21]$ & - \\
\hline Farquhar 2016 & 4.02 & 2.49 & 41 & 4.05 & 2.57 & 38 & $12.8 \%$ & $-0.01[-0.45,0.43]$ & 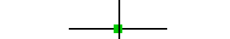 \\
\hline Groenvold 2017 & 36.5 & 32.5 & 130 & 38.7 & 31 & 137 & $43.4 \%$ & $-0.07[-0.31,0.17]$ & 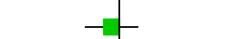 \\
\hline O'Riordan 2019 & 1.9 & 2.64 & 16 & 2.4 & 2.58 & 14 & $4.8 \%$ & $-0.19[-0.91,0.53]$ & \\
\hline Vanbutsele 2018 & 30.86 & 32.88 & 92 & 27.24 & 34.66 & 94 & $30.2 \%$ & $0.11[-0.18,0.39]$ & - \\
\hline Total (95\% CI) & & & 307 & & & 309 & $100.0 \%$ & $-0.04[-0.19,0.12]$ & \\
\hline \multicolumn{10}{|c|}{ Heterogeneity: $\mathrm{Tau}^{2}=0.00 ; \mathrm{Chi}^{2}=2.33, \mathrm{df}=4(\mathrm{P}=0.68) ; \mathrm{I}^{2}=0 \%$} \\
\hline \multicolumn{9}{|c|}{ Test for overall effect: $\mathrm{Z}=0.45(\mathrm{P}=0.65)$} & $\begin{array}{rrr}+1 & 1 & 1 \\
-1 & -0.5 & 0\end{array}$ \\
\hline \multicolumn{9}{|c|}{ Test for subgroup differences: Not applicable } & Irs HSPC Favours \\
\hline
\end{tabular}

Analysis 12.2. Comparison 12: Patient breathlessness, Outcome 2: HSPC versus usual care on patient breathlessness: unadjusted endpoint values

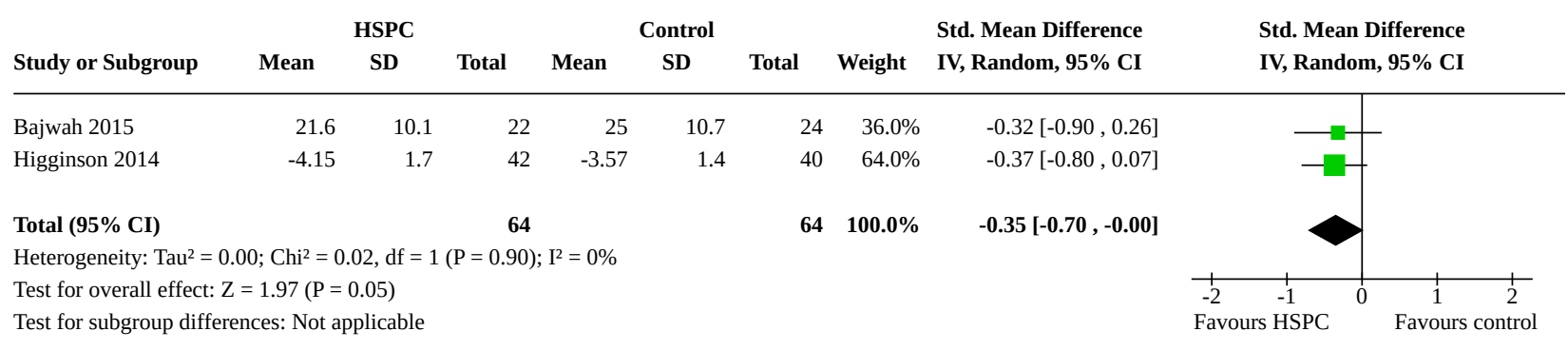

Analysis 12.3. Comparison 12: Patient breathlessness, Outcome 3: HSPC versus usual care on patient breathlessness: unadjusted change values

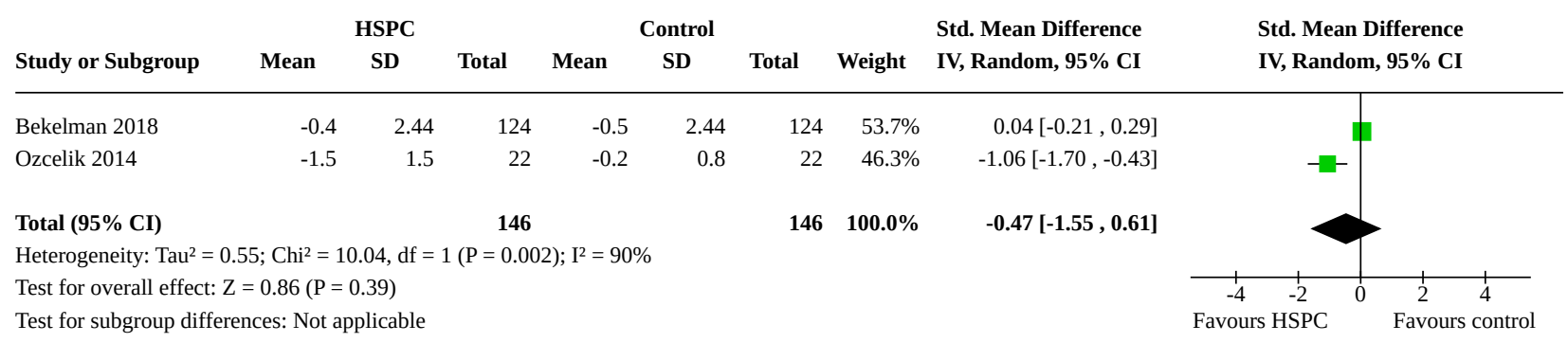

\section{ADDITIONAL TABLES}

Table 1. Taxonomy of the components of hospital-based specialist palliative care in studies that either included certified experts in palliative care or those described as palliative care clinicians

\begin{tabular}{|c|c|c|c|c|c|}
\hline Author & $\begin{array}{l}\text { Symptom con- } \\
\text { trol (e.g. as- } \\
\text { sess symp- } \\
\text { toms, pre- } \\
\text { scribing of } \\
\text { medications) }\end{array}$ & $\begin{array}{l}\text { Deci- } \\
\text { sion-making } \\
\text { (e.g. enquire } \\
\text { about goals } \\
\text { of care) }\end{array}$ & $\begin{array}{l}\text { Future plan- } \\
\text { ning (e.g. ad- } \\
\text { vance care } \\
\text { planning) }\end{array}$ & $\begin{array}{l}\text { Coping and } \\
\text { support (e.g. } \\
\text { emotional } \\
\text { and practical } \\
\text { support) }\end{array}$ & $\begin{array}{l}\text { Care co-ordi- } \\
\text { nation (e.g. } \\
\text { helping with } \\
\text { co-ordinating } \\
\text { care) }\end{array}$ \\
\hline
\end{tabular}

The effectiveness and cost-effectiveness of hospital-based specialist palliative care for adults with advanced illness and their caregivers 249 
Table 1. Taxonomy of the components of hospital-based specialist palliative care in studies that either included certified experts in palliative care or those described as palliative care clinicians (Continued)

\begin{tabular}{|c|c|c|c|c|c|}
\hline Bajwah 2015 & Yes & Yes & Yes & Yes & Yes \\
\hline Bakitas 2009 & Yes & Yes & Yes & Yes & Yes \\
\hline Bakitas 2015 & Yes & Yes & Yes & Yes & Yes \\
\hline Bekelman 2018 & Yes & Yes & No & Yes & Yes \\
\hline Brannstrom 2014 & Yes & Yes & No & Yes & Yes \\
\hline Brumley 2007 & Yes & Yes & Yes & Yes & Yes \\
\hline Carson 2016 & No & Yes & No & Yes & No \\
\hline Edmonds 2010 & Yes & Yes & Yes & Yes & Yes \\
\hline El-Jawahri 2016 & Yes & No & No & Yes & No \\
\hline Farquhar 2014 & Yes & Yes & Yes & Yes & No \\
\hline Farquhar 2016 & Yes & Yes & Yes & Yes & No \\
\hline Franciosi 2019 & Yes & Yes & No & Yes & Yes \\
\hline Gade 2008 & Yes & Yes & Yes & Yes & No \\
\hline Higginson 2009 & Yes & No & Yes & Yes & Yes \\
\hline Higginson 2014 & Yes & Yes & Yes & Yes & Yes \\
\hline Janssens 2019 & Yes & Yes & Yes & Yes & Yes \\
\hline Kane 1984 & Yes & No & Yes & Yes & No \\
\hline Lowther 2015 & Yes & Yes & Yes & Yes & No \\
\hline Ma 2019 & Yes & Yes & No & Yes & Yes \\
\hline McCorkle 2015 & Yes & Yes & No & Yes & Yes \\
\hline McWhinney 1994 & Unclear & Unclear & Unclear & Yes & Unclear \\
\hline Nottelmann 2018 & Yes & Yes & Yes & Yes & Yes \\
\hline Rodin 2019 & Yes & No & No & Yes & No \\
\hline Rogers 2017 & Yes & Yes & Yes & Yes & Yes \\
\hline Sidebottom 2015 & Yes & Yes & Yes & Yes & Yes \\
\hline Solari 2018 & Unclear & Unclear & Unclear & Yes & Unclear \\
\hline Tattersall 2014 & Yes & No & No & Yes & No \\
\hline Temel 2010 & Yes & Yes & No & Yes & Yes \\
\hline
\end{tabular}

The effectiveness and cost-effectiveness of hospital-based specialist palliative care for adults with advanced illness and their caregivers 
Table 1. Taxonomy of the components of hospital-based specialist palliative care in studies that either included certified experts in palliative care or those described as palliative care clinicians (Continued)

\begin{tabular}{llllll} 
Temel 2017 & Yes & Yes & No & Yes & No \\
\hline Vanbutsele 2018 & Yes & Yes & Yes & No & Yes \\
\hline Wallen 2012 & Yes & No & No \\
\hline
\end{tabular}

Table 2. Taxonomy of the components of hospital-based specialist palliative care in studies that were unclear about training in palliative care

\begin{tabular}{|c|c|c|c|c|c|}
\hline Author & $\begin{array}{l}\text { Symptom control } \\
\text { (e.g. assess symp- } \\
\text { toms, prescribing } \\
\text { of medications) }\end{array}$ & $\begin{array}{l}\text { Decision-mak- } \\
\text { ing (e.g. en- } \\
\text { quire about } \\
\text { goals of care) }\end{array}$ & $\begin{array}{l}\text { Future plan- } \\
\text { ning (e.g. ad- } \\
\text { vance care } \\
\text { planning) }\end{array}$ & $\begin{array}{l}\text { Coping and sup- } \\
\text { port (e.g. emo- } \\
\text { tional and practi- } \\
\text { cal support) }\end{array}$ & $\begin{array}{l}\text { Care co-ordi- } \\
\text { nation (e.g. } \\
\text { helping with } \\
\text { co-ordinating } \\
\text { care) }\end{array}$ \\
\hline Ahronheim 2000 & Yes & No & Yes & Yes & No \\
\hline Cheung 2010 & Unclear & Unclear & Unclear & Unclear & Unclear \\
\hline Groenvold 2017 & Unclear & Unclear & Unclear & Unclear & Unclear \\
\hline Grudzen 2016 & Yes & Yes & Yes & Yes & No \\
\hline Hopp 2016 & Yes & Yes & Yes & Yes & No \\
\hline Jingfen 2017 & Yes & Yes & No & Yes & No \\
\hline McCaffrey 2013 & Unclear & Unclear & Unclear & Unclear & Yes \\
\hline $\begin{array}{l}\text { Mendoza-Galindo } 2018 \text { (ab- } \\
\text { stract only) }\end{array}$ & Yes & No & No & Yes & No \\
\hline O'Riordan 2019 & Yes & No & Yes & Yes & No \\
\hline Ozcelik 2014 & Yes & No & Yes & Yes & No \\
\hline Woo 2019 & Yes & No & No & Yes & No \\
\hline
\end{tabular}

Table 3. Health-related quality of life scales and dimensions covered

\begin{tabular}{lll}
\hline $\begin{array}{l}\text { Studies, primary end- } \\
\text { point (PEP), disease } \\
\text { group }\end{array}$ & Scales used & Dimensions covered in scales \\
\hline Bajwah 2015 & $\begin{array}{l}\text { KBILD (used in meta- } \\
\text { analysis) }\end{array}$ & $\begin{array}{l}\text { KBILD is a 15-item questionnaire consisting of three domains (breathlessness } \\
\text { and activities, chest symptoms and psychological) - secondary outcome }\end{array}$ \\
PEP: 4 weeks & SGRQ & $\begin{array}{l}\text { SGRQ is a 50-item instrument designed to measure impact on overall health, } \\
\text { daily life, and perceived well-being in patients with obstructive airways dis- } \\
\text { ease. Part 1 has a symptoms component (frequency and severity) with a 1, 3 } \\
\text { or 12 month recall (several scales); Part 2 has an activities component looking } \\
\text { at activities that cause or are limited by breathlessness and an impact compo- }\end{array}$ \\
\hline $\begin{array}{l}\text { Advanced fibrotic lung } \\
\text { disease }\end{array}$ & &
\end{tabular}

The effectiveness and cost-effectiveness of hospital-based specialist palliative care for adults with advanced illness and their caregivers 
Table 3. Health-related quality of life scales and dimensions covered (Continued)

nent looking at social functioning, psychological disturbances resulting from airways disease and referring to current state as the recall (dichotomous (true) false) except last question (4-point Likert scale) - secondary outcome

\begin{tabular}{|c|c|c|}
\hline $\begin{array}{l}\text { Bakitas } 2009 \\
\text { PEP: } 13 \text { months }\end{array}$ & FACIT-Pal & $\begin{array}{l}\text { Measures physical, emotional, social, and functional well-being in addition to } \\
\text { concerns relevant to persons with life-threatening illness (e.g. feeling peaceful, } \\
\text { reconciling with others) - primary outcome }\end{array}$ \\
\hline
\end{tabular}

Cancer

\begin{tabular}{|c|c|c|}
\hline $\begin{array}{l}\text { Bakitas } 2015 \\
\text { PEP: } 3 \text { months } \\
\text { Cancer }\end{array}$ & $\begin{array}{l}\text { FACIT-Pal (used in meta- } \\
\text { analysis) } \\
\text { Treatment Outcome In- } \\
\text { dex }\end{array}$ & $\begin{array}{l}\text { Measures physical, emotional, social, and functional well-being and additional } \\
\text { concern subscales - study did not specify whether primary or secondary out- } \\
\text { come } \\
\text { TOI, composed of FACIT-Pal physical, functional, and additional concern sub- } \\
\text { scales }\end{array}$ \\
\hline $\begin{array}{l}\text { Bekelman } 2018 \\
\text { PEP: } 6 \text { months } \\
\text { Heart failure }\end{array}$ & KCCQ & $\begin{array}{l}K C C Q \text { is a valid, reliable measure of heart failure-specific health status that is } \\
\text { responsive to change. No further details provided in the study }\end{array}$ \\
\hline $\begin{array}{l}\text { Brannstrom } 2014 \\
\text { PEP: } 6 \text { months } \\
\text { Heart failure }\end{array}$ & $\begin{array}{l}\text { EQ-5D (used in meta- } \\
\text { analysis) } \\
\text { KCCQ }\end{array}$ & $\begin{array}{l}\text { A generic, single index that defines health in the five dimensions of mobility, } \\
\text { self-care, usual activities, pain/discomfort, and anxiety/depression - did not } \\
\text { specify primary or secondary outcomes } \\
\text { Full data not shown in study }\end{array}$ \\
\hline
\end{tabular}

\begin{tabular}{|c|c|c|}
\hline Edmonds 2010 & MSIS & $\begin{array}{l}\text { Multiple Sclerosis Impact Scale (MSIS) is a } 29 \text {-item measure of disease impact. } \\
\text { It has two subscales: physical and psychological subscales. }\end{array}$ \\
\hline
\end{tabular}

Multiple sclerosis

\begin{tabular}{|c|c|c|}
\hline $\begin{array}{l}\text { El-Jawahri } 2016 \\
\text { PEP: } 2 \text { weeks }\end{array}$ & FACT-BMT & $\begin{array}{l}\text { The } 47 \text {-item Functional Assessment of Cancer Therapy-Bone Marrow Trans- } \\
\text { plant which includes subscales assessing physical, functional, emotional, so- } \\
\text { cial well-being, and bone marrow transplant-specific concerns during the past } \\
\text { week, was used to assess patients' QoL - primary outcome }\end{array}$ \\
\hline
\end{tabular}

\begin{tabular}{|c|c|c|}
\hline $\begin{array}{l}\text { Franciosi } 2019 \\
\text { PEP: } 12 \text { weeks } \\
\text { Cancer }\end{array}$ & FACT-G & $\begin{array}{l}\text { Functional Assessment of Cancer Therapy-General (FACT-G) scale. It is a 27- } \\
\text { item internationally validated questionnaire divided into four primary HRQoL } \\
\text { domains: physical well-being, social/family well-being, emotional well-being, } \\
\text { and functional well-being. The total FACT-G score is the sum of the } 4 \text { subscale } \\
\text { scores. }\end{array}$ \\
\hline $\begin{array}{l}\text { Gade } 2008 \\
\text { PEP: at hospital dis- } \\
\text { charge } \\
\text { Mixed diseases com- } \\
\text { prising cancer and non- } \\
\text { cancer }\end{array}$ & MCOHPQ & $\begin{array}{l}\text { MCOHPQ Physical Area scale, emotional/relationship area and spiritual area } \\
\text { scales and MCOHPQ place of care environment scale. Physical Area scale ad- } \\
\text { dresses pain, fatigue, sleep changes, nausea, constipation, diarrhoea, dry } \\
\text { mouth, change in appetite, and shortness of breath. Emotional support items } \\
\text { included: anxiety, burden to family, support they received, isolation, opportu- } \\
\text { nity to discuss illness and possible death, and treatment wishes/goals. Spiritu- } \\
\text { al support included: the importance of participation in spiritual or religious ex- } \\
\text { periences from the Spiritual Area scale, and two items developed by the inves- } \\
\text { tigators: ability to find meaning in one's life, and support given by religion or } \\
\text { spiritual belief. }\end{array}$ \\
\hline
\end{tabular}

MCOHPQ Place of Care Environment scale addressed experiences receiving pain management and symptom relief, psychological and social support, discharge planning, and end-of-life planning - primary outcome. 
Table 3. Health-related quality of life scales and dimensions covered (Continued)
Grudzen 2016
FACT-G
Functional Assessment of Cancer Therapy-General Measure (not specified in
PEP: 12 weeks study) - primary outcome

Cancer

\begin{tabular}{|c|c|c|}
\hline $\begin{array}{l}\text { Higginson } 2014 \\
\text { PEP: } 6 \text { weeks } \\
\text { Mixed diseases com- } \\
\text { prising cancer and non- } \\
\text { cancer }\end{array}$ & $\begin{array}{l}\text { CRQ HROL (presented } \\
\text { in meta-analysis) } \\
\text { EQ-5D }\end{array}$ & $\begin{array}{l}\text { Measures breathlessness mastery, breathlessness, fatigue, and emotional } \\
\text { function - secondary outcome } \\
\text { A generic, single index that defines health in the five dimensions of mobility, } \\
\text { self-care, usual activities, pain/discomfort, and anxiety/depression }\end{array}$ \\
\hline $\begin{array}{l}\text { Janssens } 2019 \\
\text { PEP: } 12 \text { months } \\
\text { COPD }\end{array}$ & SF-36 & $\begin{array}{l}\text { A generalised self-assessment scale assessing different dimensions including } \\
\text { vitality, mental health, general health, physical functioning, role physical, role } \\
\text { emotional, bodily pain, social functioning and health transition }\end{array}$ \\
\hline $\begin{array}{l}\text { Jingfen } 2017 \\
\text { PEP: } 3 \text { months } \\
\text { Cancer }\end{array}$ & $\begin{array}{l}\text { EORTC QLQ-C30-Chi- } \\
\text { nese version }\end{array}$ & Not specified as primary or secondary outcome \\
\hline $\begin{array}{l}\text { McCorkle } 2015 \\
\text { PEP: not stated but } 3 \\
\text { months used in meta- } \\
\text { analysis } \\
\text { Cancer }\end{array}$ & $\begin{array}{l}\text { FACT-G (presented in } \\
\text { meta-analysis) } \\
\text { SF-12 (not used in } \\
\text { meta-analysis because } \\
\text { only its first item was } \\
\text { used) }\end{array}$ & $\begin{array}{l}\text { No information provided in study on dimensions covered by FACT-G - sec- } \\
\text { ondary outcome }\end{array}$ \\
\hline $\begin{array}{l}\text { Nottelmann } 2018 \\
\text { PEP: } 12 \text { weeks } \\
\text { Cancer }\end{array}$ & EORTC QLQ-C30 & $\begin{array}{l}\text { The EORTC QLQ-C30 consists of } 30 \text { items in } 15 \text { scales. In the present study ad- } \\
\text { ditional items measuring role functioning, cognitive functioning, social func- } \\
\text { tioning, dyspnoea, pain, fatigue, insomnia, appetite loss, nausea/vomiting } \\
\text { and constipation were added to the questionnaire to expand these scales to at } \\
\text { least four items in each scale. }\end{array}$ \\
\hline $\begin{array}{l}\text { O'Riordan } 2019 \\
\text { PEP: not stated but ap- } \\
\text { peared to be } 6 \text { months. } \\
6 \text { months was used in } \\
\text { meta-analysis } \\
\text { Heart failure }\end{array}$ & MLHF questionnaire & $\begin{array}{l}\text { MLHF questionnaire measures heart failure-specific health-related quality of } \\
\text { life. No further information provided }\end{array}$ \\
\hline $\begin{array}{l}\text { Ozcelik } 2014 \\
\text { PEP: on discharge } \\
\text { Cancer }\end{array}$ & EORTC QLQ-C30 & $\begin{array}{l}\text { The scale consists of the } 2 \text { subscales 'functional' and 'symptom'. The function- } \\
\text { al section is divided into } 6 \text { subsections: physical, role, cognitive, emotional, } \\
\text { social, and global quality of life. The symptom section includes the following } \\
\text { symptoms: fatigue, nausea and vomiting, pain, dyspnoea, sleep disorders, loss } \\
\text { of appetite, constipation, diarrhoea, and financial impact - primary outcome }\end{array}$ \\
\hline $\begin{array}{l}\text { Rodin } 2019 \\
\text { PEP: } 12 \text { weeks } \\
\text { Cancer }\end{array}$ & FACIT-Sp & $\begin{array}{l}\text { The scale covers physical, social/family, emotional, functional, and spiritual } \\
\text { well-being. }\end{array}$ \\
\hline
\end{tabular}

The effectiveness and cost-effectiveness of hospital-based specialist palliative care for adults with advanced illness and their caregivers 
Table 3. Health-related quality of life scales and dimensions covered (Continued)

$\begin{array}{lll}\text { Rogers } 2017 & \text { FACIT-Pal (presented in } & \begin{array}{l}\text { Assesses quality of life in several domains, including physical well-being, so- } \\ \text { cial/family well-being, emotional well-being, functional well-being, and pallia- } \\ \text { tive care - primary outcome }\end{array} \\ \text { PEP: } 6 \text { months } & \text { KCCQ } & \begin{array}{l}\text { The overall summary score is derived from the physical function, symptom, so- } \\ \text { cial function, and quality-of-life domains. }\end{array}\end{array}$

\begin{tabular}{|c|c|c|}
\hline $\begin{array}{l}\text { Sidebottom } 2015 \\
\text { PEP: not stated but } \\
\text { data presented at } 3 \\
\text { months used in meta- }\end{array}$ & MLHF questionnaire & $\begin{array}{l}\text { The MLHF Questionnaire was created to be representative of the ways HF and } \\
\text { treatments can affect key physical, emotional, social, and mental dimensions } \\
\text { of QoL. It assess how much a person's HF has affected many aspects of their } \\
\text { life during the prior month - primary outcome }\end{array}$ \\
\hline
\end{tabular}

analysis

Heart failure

\begin{tabular}{ll}
\hline Solari 2018 & $\begin{array}{l}\text { SEIQOL-DW question- } \\
\text { naire }\end{array}$ \\
PEP: 6 months & $\begin{array}{l}\text { Schedule for the Evaluation of Individual Quality of Life-Direct Weighting } \\
\text { (SEIQoL-DW). The SEIQoL-DW is administered in an interview in which respon- } \\
\text { dents nominate the five areas of life that are most important in determining } \\
\text { their QoL, and rate the satisfaction/functioning and weight/importance in } \\
\text { each of these areas. The SEIQoL-DW index can range from } 0 \text { to } 100 \text { (best). }\end{array}$ \\
\hline
\end{tabular}

\begin{tabular}{|c|c|c|}
\hline Tattersall 2014 & $\begin{array}{l}\text { McGill QoL Question- } \\
\text { naire }\end{array}$ & $\begin{array}{l}\text { Physical symptoms, psychological symptoms, outlook on life, and meaningful } \\
\text { existence - primary outcome }\end{array}$ \\
\hline PEP: one year & & \\
\hline Cancer & & \\
\hline
\end{tabular}

\begin{tabular}{lll}
\hline Temel 2010 & $\begin{array}{l}\text { FACT-L (presented in } \\
\text { meta-analysis) }\end{array}$ & $\begin{array}{l}\text { Assesses multiple dimensions of the quality of life (physical, functional, emo- } \\
\text { tional, and social well-being) during the previous week. In addition, the lung } \\
\text { cancer subscale (LCS) of the FACT-L scale evaluates seven symptoms specific } \\
\text { to lung cancer - primary outcome }\end{array}$ \\
PEP: 12 weeks & LCS &
\end{tabular}

\begin{tabular}{|c|c|c|}
\hline $\begin{array}{l}\text { Temel } 2017 \\
\text { PEP: } 12 \text { weeks } \\
\text { Cancer }\end{array}$ & FACT-G & $\begin{array}{l}\text { Assesses four dimensions of QoL (physical, functional, emotional, and social } \\
\text { well-being) - primary outcome }\end{array}$ \\
\hline $\begin{array}{l}\text { Vanbutsele } 2018 \\
\text { PEP: } 12 \text { weeks }\end{array}$ & $\begin{array}{l}\text { EORTC QLQ-C30 (pre- } \\
\text { sented in meta-analy- } \\
\text { sis) }\end{array}$ & $\begin{array}{l}\text { Global health status/quality of life scale of the European Organisation for Re- } \\
\text { search and Treatment of Cancer Quality-of-Life Questionnaire Core } 30 \text { items } \\
\text { (EORTC QLQ-C30; version 3) }\end{array}$ \\
\hline Cancer & $\begin{array}{l}\text { McGill QoL question- } \\
\text { naire }\end{array}$ & $\begin{array}{l}\text { Single item scale and overall summary score of the McGill Quality of Life ques- } \\
\text { tionnaire (MQOL). The MQoL incorporates a single item scale of global quali- } \\
\text { ty of life and four subscales, measuring four relevant domains of quality of life } \\
\text { (i.e. physical, psychological, existential/spiritual, and social). }\end{array}$ \\
\hline $\begin{array}{l}\text { Woo } 2019 \\
\text { PEP: } 4 \text { weeks }\end{array}$ & $\begin{array}{l}\text { EORTC QLQ-C30 (Kore- } \\
\text { an version) }\end{array}$ & $\begin{array}{l}\text { EORTC QLQ-C30 (Korean version) assesses multiple dimensions of QoL (physi- } \\
\text { cal, functional, emotional and social well-being) during the previous week. }\end{array}$ \\
\hline Cancer & & \\
\hline
\end{tabular}

COPD:

CRQ HROL:

EORTC QLQ-C30:

EQ-5D:

FACIT-Pal: Functional Assessment of Chronic Illness Therapy for Palliative Care

The effectiveness and cost-effectiveness of hospital-based specialist palliative care for adults with advanced illness and their caregivers 
FACIT-Sp: Functional Assessment of Chronic Illness Therapy-Spiritual Well-Being (FACIT-Sp)

FACT-BMT: Functional Assessment of cancer Therapy - Bone Marrow Transplant

FACT-G: Functional Assessment of Cancer Therapy-General Measure/Functional Assessment of Chronic Illness Therapy-General Measure FACT-L:

HF:

HRQOL:

KBILD: Kings Brief Interstitial Lung Disease

KCCQ: Kansas City Cardiomyopathy Questionnaire

LCS:

MCOHPQ: Modified City of Hope Patient Questionnaires

MLHF: Minnesota Living with Heart Failure Questionnaire

MQoL:

MSIS:

PEP:

QoL:

QUAL-E: Quality of Life at the End of Life (QUAL-E)

SEIQOL-DW:

SF-12:

SF-36:

SGRQ: St Georges Respiratory Questionnaire

TOI:

Table 4. Studies that reported on mortality/survival

\begin{tabular}{|c|c|c|}
\hline Author & Results for Mortality/Survival & $P$ value \\
\hline \multirow[t]{3}{*}{ Ahronheim 2000} & Number of deaths in the sample & 0.96 \\
\hline & Intervention: 12 (25\%) & \\
\hline & Control: $12(25 \%)$ & \\
\hline \multirow[t]{3}{*}{ Bajwah 2015} & Number of deaths in the sample & Not stated \\
\hline & Intervention: $8(32 \%)$ & \\
\hline & Control: 13 (54\%) & \\
\hline \multirow[t]{6}{*}{ Bakitas 2009} & Number of deaths in the sample & Cox proportional hazards model esti- \\
\hline & Intervention: 112 (69.6\%) & risk of death (HR, 0.67 (95\% Cl: 0.496 \\
\hline & Control: 119 (73.9\%) & $\begin{array}{l}\text { to } 0.906) \mathrm{P}=.009 \text { ) in the HSPC group } \\
\text { during the first year of the study and a }\end{array}$ \\
\hline & Survival time (median, $95 \% \mathrm{Cl}$ ) & $\begin{array}{l}\text { greater relative risk after one year, (HR, } \\
1.56(95 \% \mathrm{Cl}: 0.908 \text { to } 2.655)) \text {. }\end{array}$ \\
\hline & Intervention: 14 months (10.6 to 18.4 ) & $\mathrm{P}$ for survival time $=0.14$ \\
\hline & Control: 8.5 months ( 7 to 11.1 ) & \\
\hline \multirow[t]{6}{*}{ Bakitas 2015} & $\begin{array}{l}\text { Number of deaths (authors stated that there were } 109 \text { deaths } \\
(52.7 \%)\end{array}$ & \multirow{6}{*}{$\begin{array}{l}\text { Kaplan-Meier curves illustrated a } 15 \% \\
\text { difference in survival at } 1 \text { year (HSPC, } \\
63 \% \text { vs control, } 48 \% \text {; } P=0.038) \text {. Howev- } \\
\text { er, for the overall log-rank test, } P=0.18 \text {, } \\
\text { suggesting a convergence in overall sur- } \\
\text { vival after } 12 \text { months. }\end{array}$} \\
\hline & Intervention: numbers not provided & \\
\hline & Control: numbers not provided & \\
\hline & Survival time (median) & \\
\hline & Intervention: 18.3 months & \\
\hline & Control: 11.8 months & \\
\hline
\end{tabular}

The effectiveness and cost-effectiveness of hospital-based specialist palliative care for adults with advanced illness and their caregivers 
Table 4. Studies that reported on mortality/survival (Continued)

\begin{tabular}{ll} 
Bekelman 2018 & Number of deaths in the sample \\
& Intervention: $10(6.4 \%)$ \\
& Control: $13(8.3 \%)$ \\
\hline Brannstrom 2014 & Number of deaths in the sample \\
& Intervention: $8(22 \%)$ \\
Control: $4(11.1 \%)$
\end{tabular}

\begin{tabular}{lll}
\hline Brumley 2007 & $\begin{array}{l}\text { Number of deaths (authors highlighted 75\% deaths among } \\
\text { participants) }\end{array}$ & $\mathrm{P}=0.03$ \\
Intervention: numbers not provided & $\begin{array}{l}\text { However, results of the Kaplan-Meier } \\
\text { survival analysis did not show differ- } \\
\text { ences in survival time between study } \\
\text { groups }(\mathrm{P}=0.08) .\end{array}$ \\
Control: numbers not provided & \\
Survival time (mean (SD)) & \\
Intervention: 196 days (SD:164) & \\
Control: 242 days (SD:200) & \\
\hline
\end{tabular}

\begin{tabular}{lll}
\hline Carson 2016 & Survival time (median, IQR) & P for survival time $=0.51$ \\
& Intervention: 19 days (12 to 37$)$ & 90-day survival (HR, $0.95(95 \% \mathrm{Cl}: 0.65$ \\
& to 1.38$), \mathrm{P}=0.96)$. Post hoc adjustment \\
Control: 23 days (12 to 39) & for baseline activities of daily living and \\
& study site did not alter the outcome \\
& $(\mathrm{HR}, 1.01$ (95\% Cl; 0.69 to 1.47$), \mathrm{P}=0.96)$
\end{tabular}

\begin{tabular}{lll}
\hline Cheung 2010 & Number of deaths in the sample & $\mathrm{P}=0.58$ \\
& Intervention: $7(70 \%)$ \\
& Control: $9(90 \%)$ \\
\hline Edmonds 2010 & Number of deaths in the sample & P value not stated \\
Intervention: $1(70 \%)$ & \\
Control: $3(11.5 \%)$
\end{tabular}

\begin{tabular}{lll}
\hline El-Jawahri 2016 & Number of deaths in the sample & P value not stated \\
& Intervention: $3(3.7 \%)$ & \\
Control: 0 & P value not stated \\
\hline Farquhar 2014 & Number of deaths in the sample & \\
Intervention: $2(5.7 \%)$ & P value not stated \\
\hline Carquhar 2016 & Number of deaths in the sample 0 \\
Intervention: $1(2.3 \%)$ & \\
Control: $1(2.3 \%)$
\end{tabular}

The effectiveness and cost-effectiveness of hospital-based specialist palliative care for adults with advanced illness and their caregivers 
Table 4. Studies that reported on mortality/survival (Continued)

$\begin{array}{lll}\text { Franciosi } 2019 & \text { Pumber of deaths in the sample } & \\ & \text { Intervention: } 52(37.4 \%) & \\ \text { Control: } 30(36.6 \%) & \text { P (for difference in number of deaths) }= \\ \text { Gade } 2008 & \text { Number of deaths in the sample } & 0.08 \\ & \text { Intervention: } 173(63 \%) & \text { P (for difference in survival time) }=0.08 \\ & \text { Control: } 132(56 \%) & \\ & \text { Survival time (median, IQR) } \\ & \text { Intervention: } 30 \text { days (6 to } 104) & \\ \text { Control: } 36 \text { days }(13 \text { to } 106) & \end{array}$

\begin{tabular}{ll}
\hline Groenvold 2017 & $\begin{array}{l}\text { Number of deaths in the sample } \\
\text { Intervention: } 25(27 \%)\end{array}$ \\
Control: $22(23 \%)$ & $\begin{array}{l}\mathrm{P}(\text { for difference in survival time })=0.16, \\
\text { but in the adjusted analysis } \mathrm{P}=0.39\end{array}$ \\
& Survival time (median) \\
Intervention: 323 days & Control: 364 days
\end{tabular}

$\begin{array}{lll}\text { Grudzen } 2016 & \text { Number of deaths in the sample } & \begin{array}{l}\text { The P value for difference in median sur- } \\ \text { Intervention: } 41(59.4 \%)\end{array}\end{array}$

Control: 44 (65.7\%)

Survival time (median, 95\% Cl)

Intervention: 289 days (128 to 453 )

Control: 132 days ( 80 to 302 )

\begin{tabular}{ll}
\hline Higginson 2009 & Number of deaths in the sample \\
& Intervention: $1(3.8 \%)$ \\
Control: $3(11.5 \%)$
\end{tabular}

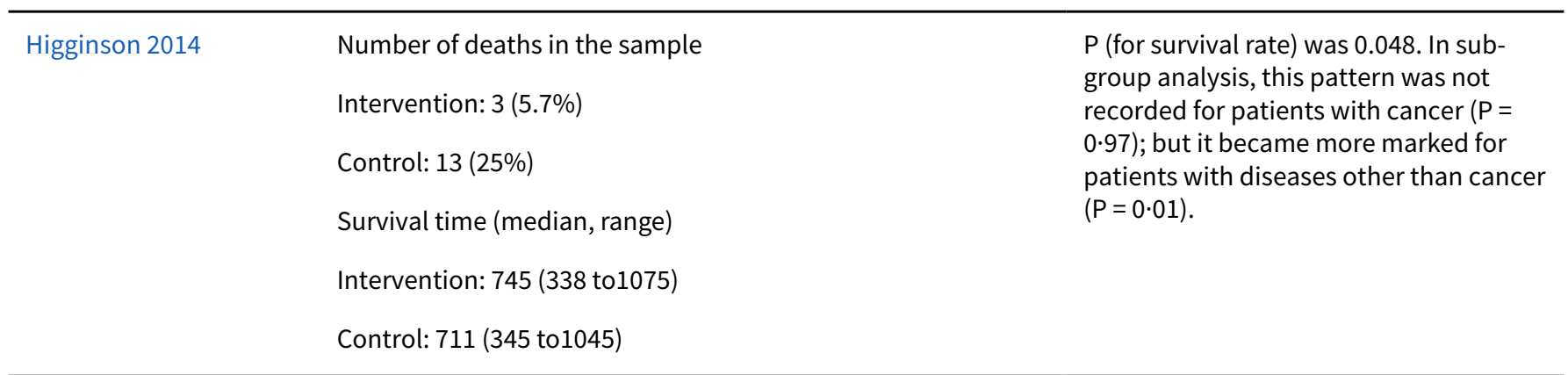

Hopp $2016 \quad$ Number of deaths in the sample (denominator unclear) $\quad P=0.47$
Intervention: 11

The effectiveness and cost-effectiveness of hospital-based specialist palliative care for adults with advanced illness and their caregivers 
Table 4. Studies that reported on mortality/survival (Continued)

Control: 8

\begin{tabular}{|c|c|c|}
\hline Janssens 2019 & $\begin{array}{l}\text { Number of deaths in the sample } \\
\text { Intervention: } 4(15.4 \%) \\
\text { Control: } 4(17.4 \%) \\
\text { Survival time (unclear if mean or median reported) } \\
\text { Intervention: } 454 \text { days ( } 95 \% \mathrm{Cl}: 382 \text { to } 525) \\
\text { Control: } 425 \text { days ( } 95 \% \mathrm{Cl}: 339 \text { to } 509)\end{array}$ & $\begin{array}{l}\text { Survival did not differ between groups } \\
\text { (log-rank test, } P=0.913 \text { ). }\end{array}$ \\
\hline Kane 1984 & $\begin{array}{l}\text { One-third of the sample died within } 45 \text { days after enrollment, } \\
\text { the second third within } 120 \text { days but numbers were not pro- } \\
\text { vided for the intervention and control groups }\end{array}$ & $\begin{array}{l}\text { Authors reported no difference in the } \\
\text { survival patterns of HSPC and control } \\
\text { patients }\end{array}$ \\
\hline Lowther 2015 & $\begin{array}{l}\text { Number of deaths in the sample } \\
\text { Intervention: } 3(5 \%) \\
\text { Control: } 0\end{array}$ & $P$ value not stated \\
\hline Ma 2019 & $\begin{array}{l}\text { Number of deaths in the sample } \\
\text { Intervention: } 34(35.1 \%) \\
\text { Control: } 37(36.3 \%)\end{array}$ & $P=0.87$ \\
\hline
\end{tabular}

\begin{tabular}{lll}
\hline McCaffrey 2013 & Number of deaths in the sample & $\begin{array}{l}\text { Increment (95\% Cl) reported as } 7 \text { (-45.1 } \\
\text { Intervention: } 16(69.6 \%)\end{array}$ \\
Control: $5(62.5 \%)$ & P value not stated \\
\hline McCorkle 2015 & Number of deaths in the sample & \\
& Control: $3(3.8 \%)$
\end{tabular}

\begin{tabular}{ll}
\hline McWhinney 1994 & Authors reported that 36 (24.7\%) patients died before one \\
month but did not provide numbers in the intervention and \\
control group.
\end{tabular}

\begin{tabular}{ll}
\hline O'Riordan 2019 & Number of deaths in the sample P value not stated \\
Intervention: $1(4.5 \%)$ & \\
Control: $1(5.6 \%)$
\end{tabular}

\begin{tabular}{ll}
\hline Rogers 2017 & Number of deaths in the sample P value not stated \\
& Intervention: $23(30.7 \%)$ \\
Control: $20(26.7 \%)$
\end{tabular}

\begin{tabular}{lll}
\hline Sidebottom 2015 & $\begin{array}{l}\text { Number of deaths in the sample } \\
\text { Intervention: } 14(12.1 \%)\end{array}$ & $\begin{array}{l}\text { Results of the survival analysis found } \\
\text { no association between study group as- } \\
\text { signment and death within } 6 \text { months }\end{array}$ \\
Control: $5(4.3 \%)$ &
\end{tabular}

The effectiveness and cost-effectiveness of hospital-based specialist palliative care for adults with advanced illness and their caregivers 
Table 4. Studies that reported on mortality/survival (Continued)

after adjustment for age, gender, and marital status.

\begin{tabular}{ll}
\hline Solari 2018 & Number of deaths in the sample P value not stated \\
Intervention: $3(3 \%)$ & \\
Control: 0
\end{tabular}

\begin{tabular}{lll}
\hline Tattersall 2014 & Number of deaths in the sample & $\mathrm{P}(\log$ rank $)=0.014$. \\
& Intervention: $39(65 \%)$ & The estimated $\mathrm{HR}$ was $1.6(95 \% \mathrm{Cl}: 1.1$ \\
& to $2.3 ; \mathrm{P}=0.015)$. This estimate changed \\
Control: $31(51.7 \%)$ & to $1.5(95 \% \mathrm{Cl} 0.99$ to $2.2 ; \mathrm{P}=0.06)$ when \\
Survival time (median, $95 \% \mathrm{Cl})$ & adjusted for the oncologist's baseline \\
& estimate of likely survival, diagnosis, \\
Intervention: 7 months $(5.2$ to 9.8$)$ & months since diagnosis, and gender.
\end{tabular}

Control: 11.7 months (9.8 to 18.8 )

\begin{tabular}{|c|c|c|}
\hline \multirow[t]{6}{*}{ Temel 2010} & $\begin{array}{l}\text { Number of deaths (authors stated } 105 \text { participants }(70 \%) \text { had } \\
\text { died by the time of analysis) }\end{array}$ & Log-rank $\mathrm{P}=0.02$ \\
\hline & Intervention: numbers not provided & $\begin{array}{l}\text { After adjustment for age, sex, and base- } \\
\text { line Eastern Cooperative Oncology }\end{array}$ \\
\hline & Control: numbers not provided & $\begin{array}{l}\text { Group performance status, the group } \\
\text { assignment remained a predictor of sur- }\end{array}$ \\
\hline & Survival time (median, 95\% Cl) & $\begin{array}{l}\text { vival (HR for death in the standard care } \\
\text { group, } 1.70 ; 95 \% \mathrm{Cl}, 1.14 \text { to } 2.54 ; \mathrm{P}=\end{array}$ \\
\hline & Intervention: 11.6 (6.4 to 16.9 ) months & $0.01)$ \\
\hline & Control: 8.9 (6.3 to 11.4$)$ months & \\
\hline \multirow[t]{3}{*}{ Temel 2017} & Number of deaths in the sample & $P$ value not stated \\
\hline & Intervention: 33 (18.9\%) & \\
\hline & Control: 41 (23.4\%) & \\
\hline \multirow[t]{6}{*}{ Vanbutsele 2018} & $\begin{array}{l}\text { Number of deaths (authors stated that } 121(65 \%) \text { of partici- } \\
\text { pants had died by the end of the study) }\end{array}$ & $P=0.97$ \\
\hline & Intervention: numbers not provided & \\
\hline & Control: numbers not provided & \\
\hline & Survival time (median, $95 \% \mathrm{Cl}$ ) & \\
\hline & Intervention: 312 days (190 to 434 ) & \\
\hline & Control: 343 days ( 253 to 433 ) & \\
\hline
\end{tabular}

$\mathrm{Cl}:$

HR:

HSPC:

IQR:

vs: 
Table 5. Studies that reported on adverse events in patients and/or caregivers

\begin{tabular}{|c|c|c|}
\hline Studies & Participants & Adverse effects in patients/caregivers \\
\hline Bajwah 2015 & Patients and caregivers & $\begin{array}{l}\text { Authors reported no worsening of any outcome after receiving the interven- } \\
\text { tion. }\end{array}$ \\
\hline Bekelman 2018 & Patients & There were no harmful adverse events attributed to the intervention. \\
\hline Groenvold 2017 & Patients & Authors did not observe any harmful effect of the intervention. \\
\hline Higginson 2014 & $\begin{array}{l}\text { Patients (and caregivers } \\
\text { if present) }\end{array}$ & Authors did not observe any harmful effect of the intervention. \\
\hline Lowther 2015 & Patients & Authors did not observe any harmful effect of the intervention. \\
\hline Rodin 2019 & Patients & Authors reported no adverse events during the study. \\
\hline Solari 2018 & Patients and caregivers & $\begin{array}{l}\text { Authors reported } 15 \text { serious adverse events in } 13 \text { patients in the HSPC group } \\
\text { and } 7 \text { in } 7 \text { patients in the control group. Serious adverse events reported in- } \\
\text { cluded aspiration pneumonia, generalised anxiety, breathing difficulty, urine } \\
\text { retention/infection, anarthria, contact dermatitis, dysphagia, vomiting, blad- } \\
\text { der catheter malfunctioning, fever, arrhythmia, necrotising fasciitis, traumatic } \\
\text { wound, macrohaematuria, constipation, abdominalgia and bronchitis. Three } \\
\text { patients in the HSPC group died but this was considered to be unrelated to the } \\
\text { intervention. }\end{array}$ \\
\hline
\end{tabular}

Tattersall $2014 \quad$ Patients Authors reported that more patients in the HSPC group had poorer appetite compared to the control group $(P=0.04)$.

HSPC:

Table 6. Emergency department (ED) use

\begin{tabular}{llll}
\hline Study & Time horizon & Significance and direction & Details \\
\hline Bakitas 2009 & During study period & Wilcoxon rank sum test & Intervention: 0.86 visits \\
& $\mathrm{P}=0.53$ & Control: 0.63 visits \\
& & $\begin{array}{l}\text { Note: } \text { not clear if the figures were means } \\
\text { or medians }\end{array}$
\end{tabular}

Total use covering period before and after enrollment

Poisson generalised linear model

$P=0.32$ for baseline (total sample of 207)

$P=0.21$ for total use in 109 decedents
Intervention for baseline sample (days, $95 \% \mathrm{Cl}): 0.16(0.1$ to 0.25$)$

Control for baseline sample:

$0.21(0.15$ to 0.31

Intervention (total use in 50 decedents):

0.14 (0.09 to 0.2$)$

Control (total use in 59 decedents):

$0.19(0.14$ to 0.26$)$

Brumley $2007 \quad$ During study period Reduced ED use in intervention group Intervention: 20\% had ED visits 
Table 6. Emergency department (ED) use (Continued)

linear regression adjusted for survival, age and severity of illness showed intervention reduced ED visits by $0.35(P=$ 0.02)

\begin{tabular}{|c|c|c|c|}
\hline \multirow[t]{2}{*}{ Janssens 2019} & $\begin{array}{l}\text { Admissions to the } \\
\text { emergency ward } \\
\text { in the year before } \\
\text { study enrollment }\end{array}$ & $\begin{array}{l}\text { There was no difference in admissions } \\
\text { to the emergency ward in the interven- } \\
\text { tion group compared to the control group } \\
\text { (Incidence rate ratio } 1.27,95 \% \mathrm{Cl}: 0.72 \text { to } \\
2.26, \mathrm{P}=0.384 \text { ). }\end{array}$ & $\begin{array}{l}\text { Number of admissions to emergency } \\
\text { ward } \\
\text { Intervention: } 33 \\
\text { Control: } 23\end{array}$ \\
\hline & During study period & $\begin{array}{l}\text { Admission to the emergency ward was } \\
\text { twice as often in the intervention group } \\
\text { compared to the control group (incidence } \\
\text { rate ratio } 2.05,95 \% \mathrm{Cl}: 1.11 \text { to } 3.94, \mathrm{P}= \\
0.014 \text { ). However, after the Benjamini and } \\
\text { Hochberg correction for multiple testing, } \\
\text { this difference was not significant. }\end{array}$ & $\begin{array}{l}\text { Number of admissions to emergency } \\
\text { ward } \\
\text { Intervention: } 37 \\
\text { Control: } 16\end{array}$ \\
\hline Ma 2019 & $\begin{array}{l}\text { During study period } \\
\text { and post-discharge }\end{array}$ & $\begin{array}{l}\text { Patients in the intervention group had } \\
\text { fewer ED visits compared to usual care ( } P \\
=0.0067)\end{array}$ & $\begin{array}{l}\text { \% of ED visits: } \\
\text { Intervention: } 1.3 \% \\
\text { Control: } 12.5 \% \\
\text { P: } 0.0067\end{array}$ \\
\hline $\begin{array}{l}\text { Mendoza-Galindo } \\
2018 \text { (abstract only) }\end{array}$ & Unclear & $P=0.074$ & $\begin{array}{l}\text { Intervention: } 39 \\
\text { Control: } 50\end{array}$ \\
\hline Rogers 2017 & During study period & $P$ value not stated & $\begin{array}{l}\text { Frequency of interactions occurring be- } \\
\text { tween patients and providers } \\
\text { Emergency department/urgent care: } \\
\text { Intervention, mean (SD): } 0.4(0.12) \\
\text { Control, mean (SD): } 0.5(0.11)\end{array}$ \\
\hline Temel 2010 & During study period & $P$ value not stated & $\begin{array}{l}\text { Any emergency department visit from en- } \\
\text { rollment to death: } \\
\text { Intervention: } 53.1 \% \\
\text { Control: } 57.1 \%\end{array}$ \\
\hline & & $P$ value not stated & $\begin{array}{l}\text { Any emergency department visit within } \\
30 \text { days of death: } \\
\text { Intervention: } 22.4 \% \\
\text { Control: } 30.4 \%\end{array}$ \\
\hline
\end{tabular}

$\mathrm{Cl}$ : confidence intervals

ED:

SD: standard deviation

The effectiveness and cost-effectiveness of hospital-based specialist palliative care for adults with advanced illness and their caregivers 
Table 7. Intensive care unit (ICU) use

\begin{tabular}{|c|c|c|c|}
\hline Study & Time horizon & Significance and direction & Details \\
\hline Bakitas 2009 & During study period & $\begin{array}{l}\text { Wilcoxon rank sum test } \\
P>0.99\end{array}$ & $\begin{array}{l}\text { Intervention: } 0.06 \text { days } \\
\text { Control: } 0.06 \text { days } \\
\text { Note: not clear if the figures were means or } \\
\text { medians }\end{array}$ \\
\hline Bakitas 2015 & $\begin{array}{l}\text { Total use covering } \\
\text { period before and af- } \\
\text { ter enrollment }\end{array}$ & $\begin{array}{l}\text { Poisson generalised linear model } \\
P=0.10 \text { for baseline (total sample of } \\
207 \text { ) } \\
P=0.49 \text { for total use in } 109 \text { decedents }\end{array}$ & $\begin{array}{l}\text { Intervention for baseline sample (days, } 95 \% \\
\mathrm{Cl}): 0.52 \text { ( } 0.28 \text { to } 0.95) \\
\text { Control for baseline sample: } \\
0.22 \text { ( } 0.1 \text { to } 0.5) \\
\text { Intervention (total use in } 50 \text { decedents): } \\
0.1 \text { ( } 0.04 \text { to } 0.24) \\
\text { Control (total use in } 59 \text { decedents): } \\
0.15 \text { ( } 0.07 \text { to } 0.3 \text { ) }\end{array}$ \\
\hline Carson 2016 & $\begin{array}{l}\text { Interviewed surro- } \\
\text { gate decision-mak- } \\
\text { ers immediately } \\
\text { after the second sup- } \\
\text { port and information } \\
\text { team meeting } \\
\text { for the intervention } \\
\text { group and } 10 \text { days af- } \\
\text { ter randomisation for } \\
\text { the control group, } \\
\text { unless the patient } \\
\text { had died. All surro- } \\
\text { gate } \\
\text { decision-makers } \\
\text { were interviewed } \\
\text { again by telephone } \\
\text { for } \\
\text { follow-up beginning } \\
90 \text { days after ran- } \\
\text { domisation. }\end{array}$ & $\begin{array}{l}\text { Differences between groups for oth- } \\
\text { er patient outcomes were analysed } \\
\text { based on } t \text { tests, nonparametric tests, } \\
\text { X2 tests (including the Fisher exact } \\
\text { test), or log-rank tests as appropriate. } \\
\text { For total ICU days, } \mathrm{P}=0.51 \\
\mathrm{P} \text { value for after randomisation, } \mathrm{P}= \\
0.72\end{array}$ & $\begin{array}{l}\text { ICU days } \\
\text { Total: } \\
\text { Intervention, median (IQR): } 19 \text { (15 to } 26 \text { ) } \\
\text { Control, median (IQR): } 20 \text { (15 to } 30) \\
\text { After randomisation: } \\
\text { Intervention, median (IQR): } 9 \text { (6 to } 15) \\
\text { Control, median (IQR): } 10 \text { (5 to } 17)\end{array}$ \\
\hline Cheung 2010 & $\begin{array}{l}\text { Enrollment to ICU } \\
\text { discharge }\end{array}$ & $\begin{array}{l}\text { Fisher's exact test and the Mann- } \\
\text { Whitney test } \\
P=0.97\end{array}$ & $\begin{array}{l}\text { Intervention: median (IQR) ICU length of } \\
\text { stay: } 3 \text { (7) days } \\
\text { Control: median (IQR) ICU length of stay: } 5 \\
\text { (8) days }\end{array}$ \\
\hline Grudzen 2016 & During study period & $\begin{array}{l}\text { Index-admission } \\
\text { Fisher exact test } P>0.99 \\
\text { Up to } 180 \text { days } \\
\text { Fisher exact test } P>0.99\end{array}$ & $\begin{array}{l}\text { Hospital days at } 180 \text { days } \\
\text { Index-admission: } \\
\text { Since only } 1 \text { participant had more than } 1 \text { ICU } \\
\text { admission, the authors treated the ICU ad- } \\
\text { mission as a binary outcome. During the in- }\end{array}$ \\
\hline
\end{tabular}

The effectiveness and cost-effectiveness of hospital-based specialist palliative care for adults with advanced illness and their caregivers 
Table 7. Intensive care unit (ICU) use (Continued)

dex-admission, there was no difference between the 2 groups. (Fisher exact test $P>$ 0.99)

Up to 180 days:

There was no difference between the 2 groups (Fisher exact test, $\mathrm{P}>0.99$ ).

$\begin{array}{ll}\text { Gade } 2008 \text { months post-index } & \mathrm{P}=0.04 \\ \text { hospitalisation } & \text { Continuous measures for interven- } \\ & \text { tion and usual care patients were } \\ & \text { compared using t tests for normally } \\ & \text { distributed measures and Wilcoxon } \\ & \text { two-sample tests for measures with } \\ & \text { skewed distributions. }\end{array}$

ICU admissions, median n:

Intervention: 12

Control: 21

\begin{tabular}{|c|c|c|c|}
\hline \multirow[t]{2}{*}{ Janssens 2019} & $\begin{array}{l}\text { Admissions to ICU for } \\
\text { respiratory failure in } \\
\text { the year before study } \\
\text { enrollment }\end{array}$ & $\begin{array}{l}\text { There was no difference in ICU admis- } \\
\text { sions for respiratory failure in the in- } \\
\text { tervention group compared to the } \\
\text { control group (Incidence rate ratio } \\
0.88,95 \% \mathrm{Cl}: 0.26 \text { to } 2.96, \mathrm{P}=0.82 \text { ). }\end{array}$ & $\begin{array}{l}\text { Number of ICU admissions for respiratory } \\
\text { failure in the year before inclusion: } \\
\text { Intervention: } 7 \\
\text { Control: } 7\end{array}$ \\
\hline & During study period & $\begin{array}{l}\text { There was no difference in ICU admis- } \\
\text { sions for respiratory failure in the in- } \\
\text { tervention group compared to the } \\
\text { control group (Incidence rate ratio } \\
4.42,95 \% \mathrm{Cl}: 0.49 \text { to } 20.92, \mathrm{P}=0.16 \text { ). }\end{array}$ & $\begin{array}{l}\text { Number of ICU admissions for respiratory } \\
\text { failure during the study period: } \\
\text { Intervention: } 5 \\
\text { Control: } 1\end{array}$ \\
\hline Kane 1984 & During study period & $P$ value not stated & $\begin{array}{l}\text { Mean number of ICU days per patient: } \\
\text { Intervention, mean per patient: } 0.2 \\
\text { Control, mean per patient: } 0.3\end{array}$ \\
\hline Ma 2019 & During study period & $\begin{array}{l}\text { No difference in ICU duration be- } \\
\text { tween intervention and control group } \\
(P=0.38)\end{array}$ & $\begin{array}{l}\text { ICU duration in days, median (IQR): } \\
\text { Intervention: } 5(3-8) \\
\text { Control: } 5.5(3-10) \\
\text { P: } 0.38\end{array}$ \\
\hline
\end{tabular}

$\mathrm{Cl}$ : confidence intervals

ICU:

IQR: interquartile range

Table 8. Resource use in intensive care unit (ICU)

\begin{tabular}{|c|c|c|c|}
\hline Study & Time horizon & $\begin{array}{l}\text { Significance and direc- } \\
\text { tion }\end{array}$ & Details \\
\hline Carson 2016 & $\begin{array}{l}\text { Interviewed surro- } \\
\text { gate decision-mak- } \\
\text { ers immediately after } \\
\text { the second support } \\
\text { and information team } \\
\text { meeting for the inter- }\end{array}$ & $\begin{array}{l}\text { Differences between } \\
\text { groups for other patient } \\
\text { outcomes were analysed } \\
\text { based on } t \text { tests, nonpara- } \\
\text { metric tests, } x 2 \text { tests (in- } \\
\text { cluding the Fisher exact }\end{array}$ & $\begin{array}{l}\text { Limitations of ICU treatment } \\
\text { Mechanical ventilation: } \\
\text { Intervention, median (IQR): } 40 \text { (31) }\end{array}$ \\
\hline
\end{tabular}

The effectiveness and cost-effectiveness of hospital-based specialist palliative care for adults with advanced illness and their caregivers 
Table 8. Resource use in intensive care unit (ICU) (Continued) vention group and 10 days after randomisation for the control group, unless the patient had died. All surrogate decision-makers were interviewed again by telephone for follow-up beginning 90 days after randomisation. test), or log-rank tests as appropriate.

Mechanical ventilation, $P$ $=0.41$

Dialysis, $P=0.64$

Nutrition, $P=0.60$

Vasopressors, $\mathrm{P}=0.86$
Control, median (IQR): 33 (26)

Dialysis:

Intervention, median (IQR): 13 (10)

Control, median (IQR): 15 (12)

Nutrition:

Intervention, median (IQR): 18 (14)

Control, median (IQR): 21 (17)

Vasopressors:

Intervention, median (IQR): 18 (14)

Control, median (IQR): 19 (15)

$\%$ of patients using mechanical ventilation:

The following were lower in the intervention group compared to the control group: tracheostomy ( $P$ $=0.035)$ and days on mechanical ventilation $(\mathrm{P}=$ 0.042).

Intervention: $53.6 \%$

Control: $56.9 \%$

$P: 0.64$
Haemodialysis:

Intervention: $15.5 \%$

Control: $23.5 \%$

$P: 0.15$

Vasopressors:

Intervention: $48.5 \%$

Control: $50 \%$

P: 0.83

Tracheostomy:

Intervention: $1 \%$

Control: $7.8 \%$

P: 0.035

Cardiopulmonary resuscitation:

Intervention: 5.2\%

Control: $6.9 \%$

$P: 0.61$

Number of days on mechanical ventilation, median (IQR):

Intervention: $4(3-7)$

Control: $6(3-13)$ 
Table 8. Resource use in intensive care unit (ICU)

(Continued)

$$
\begin{aligned}
& \text { P: } 0.042 \\
& \text { Number of days on vasopressors, median (IQR): } \\
& \text { Intervention: } 3(1-6) \\
& \text { Control: } 3(2-6) \\
& \text { P: } 0.91
\end{aligned}
$$

ICU:

IQR: Interquartile Range

Table 9. Hospital admission

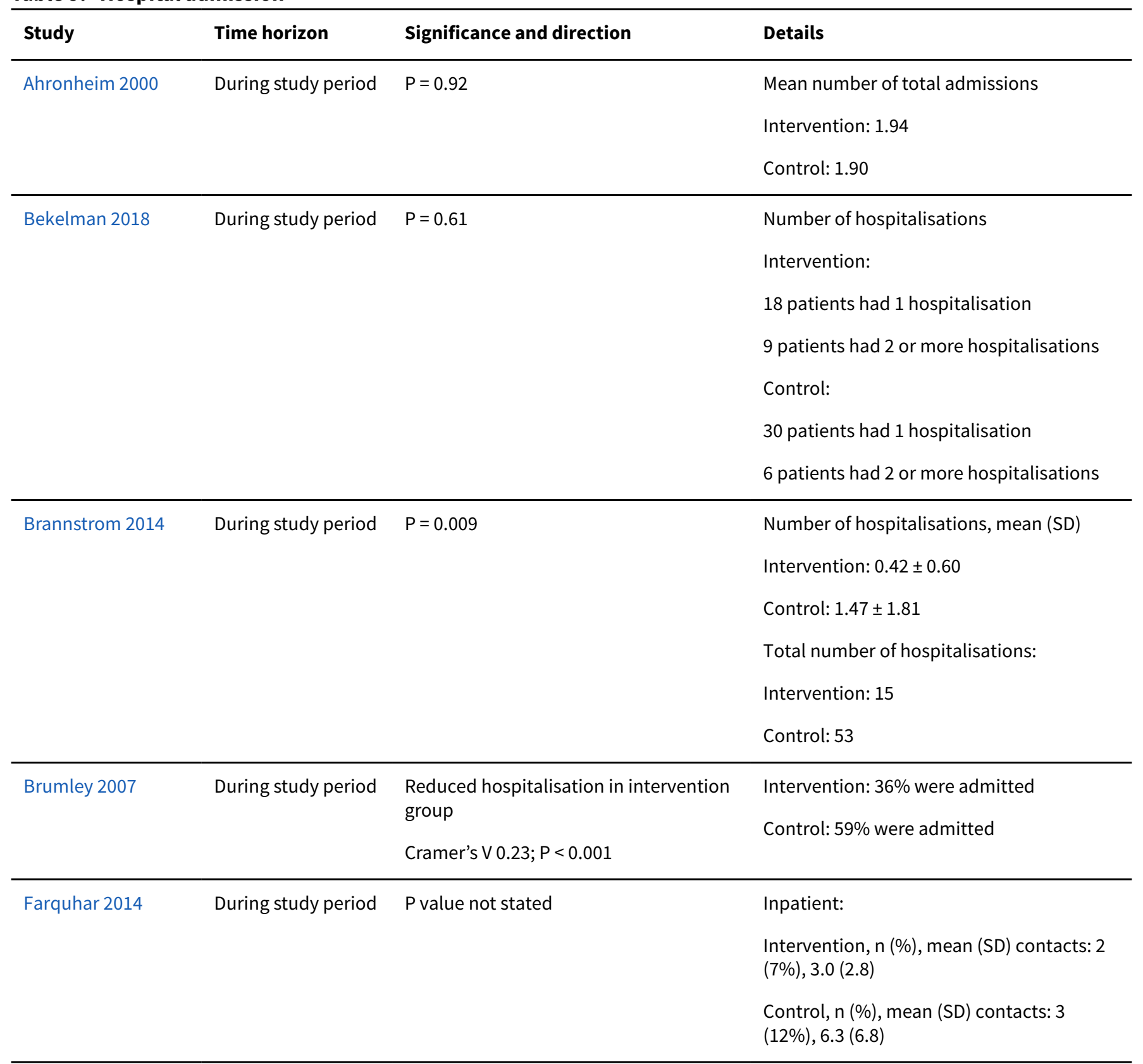

The effectiveness and cost-effectiveness of hospital-based specialist palliative care for adults with advanced illness and their caregivers 
Table 9. Hospital admission (Continued)

Farquhar $2016 \quad$ During study period $P$ value not stated
Intervention, $\mathrm{n}(\%)$, mean (SD) contacts: 6
$(15 \%), 11.5(8.3)$
Control, $\mathrm{n}(\%)$, mean (SD) contacts: $4 \quad(11 \%), 6.0(3.4)$

\begin{tabular}{|c|c|c|c|}
\hline \multirow[t]{4}{*}{ Janssens 2019} & $\begin{array}{l}\text { Hospital admis- } \\
\text { sions for respirato- } \\
\text { ry failure in the year } \\
\text { before study enroll- } \\
\text { ment }\end{array}$ & $\begin{array}{l}\text { There was no difference in hospital ad- } \\
\text { missions for respiratory failure in the in- } \\
\text { tervention group compared to the con- } \\
\text { trol group (incidence rate ratio } 1.18 \text {, } \\
95 \% \mathrm{Cl}: 0.61 \text { to } 2.31, \mathrm{P}=0.60 \text { ). }\end{array}$ & $\begin{array}{l}\text { Number of hospital admissions for respira- } \\
\text { tory failure in the year before inclusion: } \\
\text { Intervention: } 24 \\
\text { Control: } 18\end{array}$ \\
\hline & During study period & $\begin{array}{l}\text { Hospital admission for respiratory fail- } \\
\text { ure was almost twice as often in the in- } \\
\text { tervention group compared to the con- } \\
\text { trol group (incidence rate ratio } 1.87 \text {, } \\
95 \% \mathrm{Cl} \text { : } 1.04 \text { to } 3.48, \mathrm{P}=0.026 \text { ). Howev- } \\
\text { er, after the Benjamini and Hochberg } \\
\text { correction for multiple testing, this dif- } \\
\text { ference was not significant. }\end{array}$ & $\begin{array}{l}\text { Number of hospital admissions for respira- } \\
\text { tory failure during study period: } \\
\text { Intervention: } 38 \\
\text { Control: } 18\end{array}$ \\
\hline & $\begin{array}{l}\text { Hospital admis- } \\
\text { sions for respirato- } \\
\text { ry failure in the year } \\
\text { before study enroll- } \\
\text { ment }\end{array}$ & $\begin{array}{l}\text { There was no difference in hospital ad- } \\
\text { missions for respiratory failure in the in- } \\
\text { tervention group compared to the con- } \\
\text { trol group (incidence rate ratio } 1.18 \text {, } \\
95 \% \mathrm{Cl}: 0.36 \text { to } 4.12, \mathrm{P}=0.77 \text { ). }\end{array}$ & $\begin{array}{l}\text { Other hospitalisations in the year before } \\
\text { inclusion: } \\
\text { Intervention: } 8 \\
\text { Control: } 6\end{array}$ \\
\hline & During study period & $\begin{array}{l}\text { There was no difference in hospital ad- } \\
\text { missions for respiratory failure in the in- } \\
\text { tervention group compared to the con- } \\
\text { trol group (incidence rate ratio } 1.01 \text {, } \\
95 \% \mathrm{Cl}: 0.32 \text { to } 3.28, \mathrm{P}=0.99 \text { ). }\end{array}$ & $\begin{array}{l}\text { Other hospitalisations during study period: } \\
\text { Intervention: } 8 \\
\text { Control: } 7\end{array}$ \\
\hline Ma 2019 & $\begin{array}{l}\text { During study period } \\
\text { and post-discharge }\end{array}$ & $\begin{array}{l}\text { Patients in the intervention group had } \\
\text { fewer hospital readmissions compared } \\
\text { to usual care }(P=0.024)\end{array}$ & $\begin{array}{l}\text { \% of hospital readmissions: } \\
\text { Intervention: } 17.3 \% \\
\text { Control: } 33.3 \% \\
\text { P: } 0.024\end{array}$ \\
\hline $\begin{array}{l}\text { Mendoza-Galindo } \\
2018 \text { (abstract only) }\end{array}$ & Unclear & $\begin{array}{l}\text { There was no difference in number of } \\
\text { hospitalisations. P value not given }\end{array}$ & $\begin{array}{l}\text { Intervention: } 48 \% \\
\text { Control: } 51 \%\end{array}$ \\
\hline Rogers 2017 & During study period & $\begin{array}{l}\text { During the 6-month follow-up, } 30 \% \text { of } \\
\text { patients were hospitalised for HF. No } \\
\text { differences were seen between the } 2 \\
\text { treatment groups in this clinical end- } \\
\text { points through the 6-month follow-up } \\
\text { point. For hospitalisation for non-heart } \\
\text { failure/cardiovascular and hospitalisa- } \\
\text { tion for non-cardiovascular, P value was } \\
\text { not stated }\end{array}$ & $\begin{array}{l}\text { Hospitalisation for HF: } \\
\text { Intervention: } 30.7 \% \\
\text { Control: } 29.3 \% \\
\text { Hospitalisation for non-heart failure/car- } \\
\text { diovascular: } \\
\text { Intervention: } 16 \% \\
\text { Control: } 13 \% \\
\text { Hospitalisation for non-cardiovascular: }\end{array}$ \\
\hline
\end{tabular}

The effectiveness and cost-effectiveness of hospital-based specialist palliative care for adults with advanced illness and their caregivers 266 (Review)

Copyright (C) 2020 The Cochrane Collaboration. Published by John Wiley \& Sons, Ltd. 
Table 9. Hospital admission (Continued)

Intervention: $10.7 \%$

Control: $24 \%$

\begin{tabular}{llll}
\hline Sidebottom 2015 & $\begin{array}{l}\text { Inpatient readmis- } \\
\text { sion for any cause } \\
\text { within 30 days }\end{array}$ & $\begin{array}{l}\text { Survival analysis using proportional } \\
\text { hazards regression }\end{array}$ & $\begin{array}{l}\text { There was no association between study } \\
\text { group assignment and 30-day inpatient } \\
\text { readmission (adjusting for age, gender, } \\
\text { and marital status). }\end{array}$ \\
\hline Temel 2010 & During study period & P value not stated & Any admission from enrollment to death: \\
& & Intervention: $73.5 \%$ \\
& P value not stated & Control: $76.8 \%$ \\
& & Any admission within 30 days of death: \\
& & Intervention: $36.7 \%$ \\
& Control: $53.6 \%$
\end{tabular}

$\mathrm{Cl}:$

$\mathrm{HF}$ :

$\mathrm{n}$ : number

SD: standard deviation

Table 10. Length of hospital admissions

\begin{tabular}{|c|c|c|c|}
\hline Study & Time horizon & Significance and direction & Details \\
\hline Ahronheim 2000 & During study period & $\begin{array}{l}\text { Student's t-test was used } \\
P=0.46\end{array}$ & $\begin{array}{l}\text { Intervention (mean (range)): } 8.8 \text { (1 - 93) } \\
\text { Control (mean (range)): } 9.7(1-63)\end{array}$ \\
\hline Bakitas 2009 & During the study & $\begin{array}{l}\text { Wilcoxon rank sum test } \\
P=0.14\end{array}$ & $\begin{array}{l}\text { Number of hospital days (unclear if mean or me- } \\
\text { dian reported) } \\
\text { Intervention: } 6.6 \text { days } \\
\text { Control: } 6.5 \text { days }\end{array}$ \\
\hline Bakitas 2015 & $\begin{array}{l}\text { Total use covering } \\
\text { period before and } \\
\text { after enrollment }\end{array}$ & $\begin{array}{l}\text { Poisson generalised linear model } \\
P=0.03 \text { for baseline (total sample } \\
\text { of } 207 \text { ) } \\
P=0.26 \text { for total use in } 109 \text { dece- } \\
\text { dents }\end{array}$ & $\begin{array}{l}\text { Intervention for baseline sample (days, } 95 \% \mathrm{Cl} \text { ): } \\
0.69 \text { ( } 0.4 \text { to } 1.18) \\
\text { Control for baseline sample: } \\
1.39 \text { ( } 0.97 \text { to } 1.97) \\
\text { Intervention (total use in } 50 \text { decedents): } \\
0.95 \text { ( } 0.61 \text { to } 1.46) \\
\text { Control (total use in } 59 \text { decedents): } \\
1.3 \text { ( } 0.91 \text { to } 1.86)\end{array}$ \\
\hline Brannstrom 2014 & $\begin{array}{l}\text { During the study } \\
\text { period }\end{array}$ & $\begin{array}{l}\text { P value for total hospital days }= \\
0.011 . \\
\text { The number of days spent in hos- } \\
\text { pital was also significantly lower in }\end{array}$ & $\begin{array}{l}\text { Total hospital days, mean (SD) } \\
\text { Intervention: } 2.9 \text { (8.3) } \\
\text { Control: } 8.5 \text { (12.4) }\end{array}$ \\
\hline
\end{tabular}

The effectiveness and cost-effectiveness of hospital-based specialist palliative care for adults with advanced illness and their caregivers 
Table 10. Length of hospital admissions (Continued)

the intervention group at the Departments of Medicine-Geriatrics (100, range $1-45$ vs. 242 , range 2-46 days) and Surgery (0 vs. 56 , range 2-21 days). Days in other departments did not differ significantly.
Days in Department of Medicine-Geriatrics:

Intervention: 100 (range 1 - 45)

Control: 242 (range 2 - 46)

Days in Department of Surgery:

Intervention: 0

Control: 56

Days in other departments:

Intervention: 3 (range 1 - 2)

Control: $7(1-6)$

Brumley $2007 \quad$ During the study Fewer hospital days in interven- No descriptive data provided
tion group. Linear regression adjusted for survival, age and severity of illness showed intervention reduced hospital days by $4.36(\mathrm{P}<$ 0.001)

\begin{tabular}{|c|c|c|c|}
\hline Carson 2016 & $\begin{array}{l}\text { Interviewed surro- } \\
\text { gate decision-mak- } \\
\text { ers immediately af- } \\
\text { ter the second sup- } \\
\text { port and informa- } \\
\text { tion team meeting } \\
\text { for the intervention } \\
\text { group and } 10 \text { days } \\
\text { after randomisa- } \\
\text { tion for the control } \\
\text { group, unless the } \\
\text { patient had died. } \\
\text { All surrogate deci- } \\
\text { sion-makers were } \\
\text { interviewed again } \\
\text { by telephone for } \\
\text { follow-up begin- } \\
\text { ning } 90 \text { days after } \\
\text { randomisation. }\end{array}$ & $\begin{array}{l}\text { Differences in the number of hos- } \\
\text { pital days were analysed using } \\
\text { nonparametric methods. } \\
\text { P value for total hospital days, } P= \\
0.78 \\
P \text { value for deceased patients, } P= \\
0.60 \\
P \text { value for after randomisation, } P \\
=0.51\end{array}$ & $\begin{array}{l}\text { Hospital days } \\
\text { Total hospital days: } \\
\text { Intervention, median (IQR): } 35 \text { (23 to 52) } \\
\text { Control, median (IQR): } 36 \text { ( } 23 \text { to } 54) \\
\text { For deceased patients: } \\
\text { Intervention ( } 49 \text { deaths), median (IQR): } 25 \text { (18 to } \\
\text { 36) } \\
\text { Control (51 deaths), median (IQR): } 24 \text { (14 to 39) } \\
\text { After randomisation: } \\
\text { Intervention, median (IQR): } 19 \text { (12 to } 37 \text { ) } \\
\text { Control, median (IQR): } 23 \text { (12 to } 39 \text { ) }\end{array}$ \\
\hline Cheung 2010 & During study period & $\begin{array}{l}\text { Fisher's exact test and the Mann- } \\
\text { Whitney test } \\
P=0.44\end{array}$ & $\begin{array}{l}\text { Intervention: median (IQR) hospital length of } \\
\text { stay: } 5 \text { (8) days } \\
\text { Control: median (IQR) hospital length of stay: } 11 \\
\text { (27) days }\end{array}$ \\
\hline El-Jawahri 2016 & During study period & $P$ value not stated & $\begin{array}{l}\text { Duration of HCT hospitalisation, median (range): } \\
\text { Intervention: } 20(12-102) \text { days } \\
\text { Control: } 21(13-40) \text { days }\end{array}$ \\
\hline Gade 2008 & $\begin{array}{l}6 \text { months post-in- } \\
\text { dex hospitalisation }\end{array}$ & $\begin{array}{l}P \text { value for admission to study en- } \\
\text { rollment (days), } P=0.36 \\
P \text { value for study enrollment to } \\
\text { discharge or death in the hospital }\end{array}$ & $\begin{array}{l}\text { Admission to study enrollment (days), median } \\
\text { (IQR): } \\
\text { Intervention: } 3(2,7)\end{array}$ \\
\hline
\end{tabular}

The effectiveness and cost-effectiveness of hospital-based specialist palliative care for adults with advanced illness and their caregivers 268 (Review)

Copyright $\odot 2020$ The Cochrane Collaboration. Published by John Wiley \& Sons, Ltd. 
Table 10. Length of hospital admissions (Continued)

P-value for index hospital length of stay (days), $P=0.57$

Continuous measures for intervention and usual care patients were compared using t tests for normally distributed measures and Wilcoxon two-sample tests for measures with skewed distributions.
Study enrollment to discharge or death in the hospital (days), median (IQR):

Intervention: $3(1,6)$

Control: $2(1,5)$

Index hospital length of stay (days), median (IQR):

Intervention: $7(4,12)$

Control: $7(4,12)$

\begin{tabular}{|c|c|c|c|}
\hline \multirow[t]{7}{*}{ Grudzen 2016} & \multirow[t]{7}{*}{ During study period } & Index-admission & Hospital days at 180 days \\
\hline & & Wilcoxon test & Index-admission: \\
\hline & & $P=0.67$ & The authors found no difference in hospital days \\
\hline & & Up to 180 days & during the index-admission (Wilcoxon test $P=$ \\
\hline & & Wilcoxon test $P=0.14$ & $0.67)$ \\
\hline & & & Up to 180 days: \\
\hline & & & $\begin{array}{l}\text { The intervention group had slightly more hospi- } \\
\text { tal days at } 180 \text { days than the usual care group } \\
\text { (Wilcoxon test } P=0.14 \text { ). }\end{array}$ \\
\hline \multirow[t]{2}{*}{ Higginson 2009} & \multirow[t]{2}{*}{$\begin{array}{l}12 \text { weeks following } \\
\text { enrollment }\end{array}$} & $\begin{array}{l}\text { Authors stated increased institu- } \\
\text { tional days in control group but P } \\
\text { value was not stated. }\end{array}$ & $\begin{array}{l}\text { Intervention: } 4 / 26(17 \%) \text { were institutionalised } \\
\text { for mean } 19.0 \text { days (SD 21.6) }\end{array}$ \\
\hline & & $\begin{array}{l}\text { "The control care patients were } \\
\text { more likely to be (...) admitted to } \\
\text { or seen in hospital". }\end{array}$ & $\begin{array}{l}\text { Control: } 6 / 28 \text { ( } 29 \%) \text { were institutionalised for } \\
\text { mean } 30.7 \text { days (SD 32.1) }\end{array}$ \\
\hline \multirow[t]{2}{*}{ Higginson 2014} & \multirow[t]{2}{*}{$\begin{array}{l}\text { Three months be- } \\
\text { fore baseline inter- } \\
\text { view }\end{array}$} & $P$ value not stated & $\begin{array}{l}\text { Hospital inpatient days } \\
\text { Intervention, mean (SD): } 4.5 \text { (6.8) }\end{array}$ \\
\hline & & & Control, mean (SD): 4.6 (7.6) \\
\hline \multirow[t]{9}{*}{ Kane 1984} & \multirow[t]{9}{*}{ During study period } & $\begin{array}{l}P \text { value for general medical inpa- } \\
\text { tient days, } P<0.05\end{array}$ & Total inpatient days: \\
\hline & & & Intervention, mean per patient: 51 \\
\hline & & $\begin{array}{l}P \text { value for intermediate care inpa- } \\
\text { tient days } P<0.05\end{array}$ & Control, mean per patient: 47.5 \\
\hline & & & General medical: \\
\hline & & & Intervention, mean per patient: 13.2 \\
\hline & & & Control, mean per patient: 20.7 \\
\hline & & & Intermediate care: \\
\hline & & & Intervention, mean per patient: 8.3 \\
\hline & & & Control, mean per patient: 26.5 \\
\hline Ma 2019 & During study period & $\begin{array}{l}\text { No difference in hospital duration } \\
\text { between intervention and control } \\
\text { group }(P=0.43)\end{array}$ & $\begin{array}{l}\text { Hospital duration in days, median (IQR) } \\
\text { Intervention: } 10(6-15)\end{array}$ \\
\hline
\end{tabular}

The effectiveness and cost-effectiveness of hospital-based specialist palliative care for adults with advanced illness and their caregivers 
Table 10. Length of hospital admissions (Continued)

Control: $11(6-19)$

P: 0.43

\begin{tabular}{|c|c|c|c|}
\hline $\begin{array}{l}\text { Mendoza-Galindo } \\
2018 \text { (abstract only) }\end{array}$ & Unclear & $P=0.808$ & $\begin{array}{l}\text { Intervention: } 78 \text { days } \\
\text { Control: } 90 \text { days }\end{array}$ \\
\hline Ozcelik 2014 & During study period & $P=0.07$ & $\begin{array}{l}\text { Intervention, mean (SD): } 9.4 \text { (6.27) days } \\
\text { Control, mean (SD): } 13.9 \text { (11.5) days }\end{array}$ \\
\hline Temel 2010 & During study period & $P$ value not stated & $\begin{array}{l}\text { Median inpatient days (range) from enrollment } \\
\text { to death: } \\
\text { Intervention: } 5(0-50) \\
\text { Control: } 7(0-45)\end{array}$ \\
\hline
\end{tabular}

IQR: interquartile range

SD: standard deviation

Table 11. Palliative care visits during hospitalisation

\begin{tabular}{|c|c|c|c|}
\hline Study & Time horizon & $\begin{array}{l}\text { Significance and } \\
\text { direction }\end{array}$ & Details \\
\hline \multirow[t]{2}{*}{ El-Jawahri 2016} & During study period & $P$ value not stated & Palliative care visits, median (range): \\
\hline & & & $\begin{array}{l}\text { All intervention patients had at least } 2 \text { palliative care visits dur- } \\
\text { ing the first } 2 \text { weeks of their hospitalisation (median number } \\
\text { of visits, } 4 \text {; range, } 2-7) \text {. Intervention participants had at least } 4 \\
\text { palliative care visits during their entire hospitalisation (median } \\
\text { number of visits, } 8 \text {; range, } 4-40) \text {. Two control patients received } \\
\text { a palliative care consultation. A total of } 41.8 \%(146 / 349) \text { of pal- } \\
\text { liative care visits occurred while a family member was present. }\end{array}$ \\
\hline \multirow[t]{3}{*}{ Tattersall 2014} & During study period & $P=0.37$ & Palliative care contact during the last acute hospital admission: \\
\hline & & & Intervention: 42 patients (86\%) \\
\hline & & & Control: 29 patients $(78 \%)$ \\
\hline
\end{tabular}

Table 12. Outpatient clinic visits

\begin{tabular}{|c|c|c|c|}
\hline Study & Time horizon & $\begin{array}{l}\text { Significance and } \\
\text { direction }\end{array}$ & Details \\
\hline \multirow[t]{5}{*}{ Brannstrom 2014} & \multirow[t]{5}{*}{ During study period } & \multirow[t]{2}{*}{$\begin{array}{l}P \text { value for physi- } \\
\text { cian visit, } P=0.000\end{array}$} & Hospital outpatient clinic \\
\hline & & & Physician visit, $\mathrm{n}$, median (range): \\
\hline & & \multirow{2}{*}{$\begin{array}{l}P \text { value for physi- } \\
\text { cian, phone calls } \\
\text { and prescriptions, } P \\
=0.012\end{array}$} & Intervention: 27, $1(4-30)$ \\
\hline & & & Control: 133, $3(2-11)$ \\
\hline & & $\begin{array}{l}P \text { value for nurse } \\
\text { visits, } P=0.003\end{array}$ & Physician, phone calls and prescriptions, $\mathrm{n}$, median (range): \\
\hline
\end{tabular}

The effectiveness and cost-effectiveness of hospital-based specialist palliative care for adults with advanced illness and their caregivers 
Table 12. Outpatient clinic visits (Continued)

$\begin{array}{ll}\text { P value for nurse } & \text { Intervention: } 42,3(0-8) \\ \text { visits, phone calls } & \\ \text { and prescriptions } P & \text { Control: } 86,3(0-10)\end{array}$

$=0.003$
Nurse visits, $n$, median (range):

Intervention: 4, $1(0-4)$

Control: 60, 2 (0 -27)

Nurse, phone calls and prescriptions, $\mathrm{n}$, median (range):

Intervention: 8, $1(0-4)$

Control: 44, $2(0-8)$

Groenvold $2017 \quad$ During study period $\quad$ P values not stated

Contact with the HSPC team, (numbers):

Intervention: 138 patients had at least one face-to-face contact

Control: 13 patients had at least one face-to-face contact

\begin{tabular}{llll}
\hline Higginson 2009 & $\begin{array}{l}12 \text { weeks following } \\
\text { enrollment }\end{array}$ & $\begin{array}{l}\text { Hospital specialist } \\
\text { visits differences } \\
\text { and P value not } \\
\text { stated }\end{array}$ & $\begin{array}{l}\text { Intervention: } 8 \text { patients (35\%) received; mean 1.0 contacts (SD } \\
0.0)\end{array}$ \\
& & Control: 16 patients (76\%) received; mean 1.3 contacts (SD 0.7)
\end{tabular}

\begin{tabular}{lll}
\hline Rogers $2017 \quad \begin{array}{l}\text { During study period } \\
\text { P value not stated }\end{array}$ & $\begin{array}{l}\text { Frequency of interactions occurring between patients and } \\
\text { providers }\end{array}$ \\
& $\begin{array}{l}\text { Total number of clinic encounter records: } \\
\text { Intervention, mean (SD): } 21.9(1.99)\end{array}$ \\
& Control, mean (SD): $20.8(1.92)$ \\
& Cardiology: \\
& Intervention, mean (SD): $2.3(0.55)$ \\
& Control, mean (SD): $3.2(1.0)$ \\
& Rehabilitation clinic: \\
Intervention, mean (SD): $1.4(0.68)$ & Control, mean (SD): $0.9(0.48)$
\end{tabular}

Tattersall $2014 \quad$ During study period P values not stated Contact with palliative care physician consultant:

Intervention: 51 patients (85\%)

Control: 8 patients $(13.3 \%)$

Contact with palliative care physician in the last month of life:

Intervention: 16 patients (26.7\%)

Control: 6 patients (10\%)

Temel $2010 \quad$ During study period P values not stated PC visits:

The effectiveness and cost-effectiveness of hospital-based specialist palliative care for adults with advanced illness and their caregivers 
Table 12. Outpatient clinic visits (Continued)

All the patients assigned to early palliative care, except for one patient who died within 2 weeks after enrollment, had at least one visit with the palliative care service by the 12th week. The average number of visits in the palliative care group was 4 (range, 0 to 8$)$. Ten patients who received standard care (14\%) had a palliative care consultation in the first 12 weeks of the study, primarily to address the management of symptoms, with seven patients having one visit and three having two visits.

Mean number of palliative care visits:

Intervention, mean (range): 6.54 ( 0 to 14 )

Control, mean (range): 0.89 ( 0 to 7 )

Number of palliative care visits split on lung and GI cancer:

The authors stated that "we explored characteristics between patients with lung and $\mathrm{GI}$ cancer and found no differences in baseline measures or in the number of PC visits among those patients who received intervention. However, the GI cancer cohort had a higher proportion of male patients and a greater number of hospitalisations $(P=0.038)$ from baseline to week 24 compared with the lung cancer cohort".

\section{During study period}

$P$ value not stated for some of the comparisons.

However, the authors reported a difference between intervention and control groups for number of consultations with a psychologist $(P=0.02)$
Number of consultations from the palliative care team nurse at 18 weeks:

Intervention, median (IQR): $3(1-4) .82$ patients (89\%) had at least one consultation

Control, median (IQR): 17 patients (18\%) had at least one consultation

PC physician at 18 weeks:

Intervention: 25 patients (27\%)

Control: 1 patient (1\%)

Nurses at 24 weeks:

Intervention, median (IQR): $3(2-5) .55$ patients (60\%) had at least 3 consultations

Control, median (IQR): 12 patients (13\%) had at least 3 consultations

PC physician at 24 weeks:

Intervention: 32 patients (35\%) had at least one consultation

Control: $1(1 \%)$ had one consultation

Number of consultations with a psychologist:

18 weeks:

Intervention: 34 patients (37\%) had at least one consultation

Control: 21 patients (22\%) had at least one consultation

24 weeks: 
Table 12. Outpatient clinic visits (Continued)

No difference was found between intervention and control groups.

Number of consultations with other professionals:

There were no differences between study groups in the number of consultations with a social care nurse $(P=0.87)$, dietician $(P=$ $0.32)$, or specialist nurse $(P=0.28)$ between 18 weeks and baseline; or between 24 weeks and baseline with social care nurse $(P=0.07)$, dietician $(P=0.95)$, or specialist nurse $(P=0.99)$.

Woo $2019 \quad$ During study period $\begin{aligned} & \text { Forwards from en- } \\ & \text { rollment }\end{aligned}$

The proportions that consulted a psychiatrist ( $12 \%$ vs $12 \%$ ) were similar in the intervention and control groups.

HSPC: hospital-based specialist palliative care

IQR: Interquartile range

PC: palliative care

SD: standard deviation

Table 13. Community care

\begin{tabular}{llll}
\hline Study & Time horizon & Significance and direction & Details \\
\hline Bakitas 2015 & $\begin{array}{l}\text { Total use covering } \\
\text { period before and } \\
\text { after enrollment }\end{array}$ & Poisson generalised linear model & Hospice use: \\
& $\mathrm{P}=0.62$ & Intervention, rate $95 \% \mathrm{Cl}: 0.68(0.55$ to 0.84$)$ \\
& & Control, rate $95 \% \mathrm{Cl:} 0.63$ (0.51 to 0.78) \\
\hline
\end{tabular}

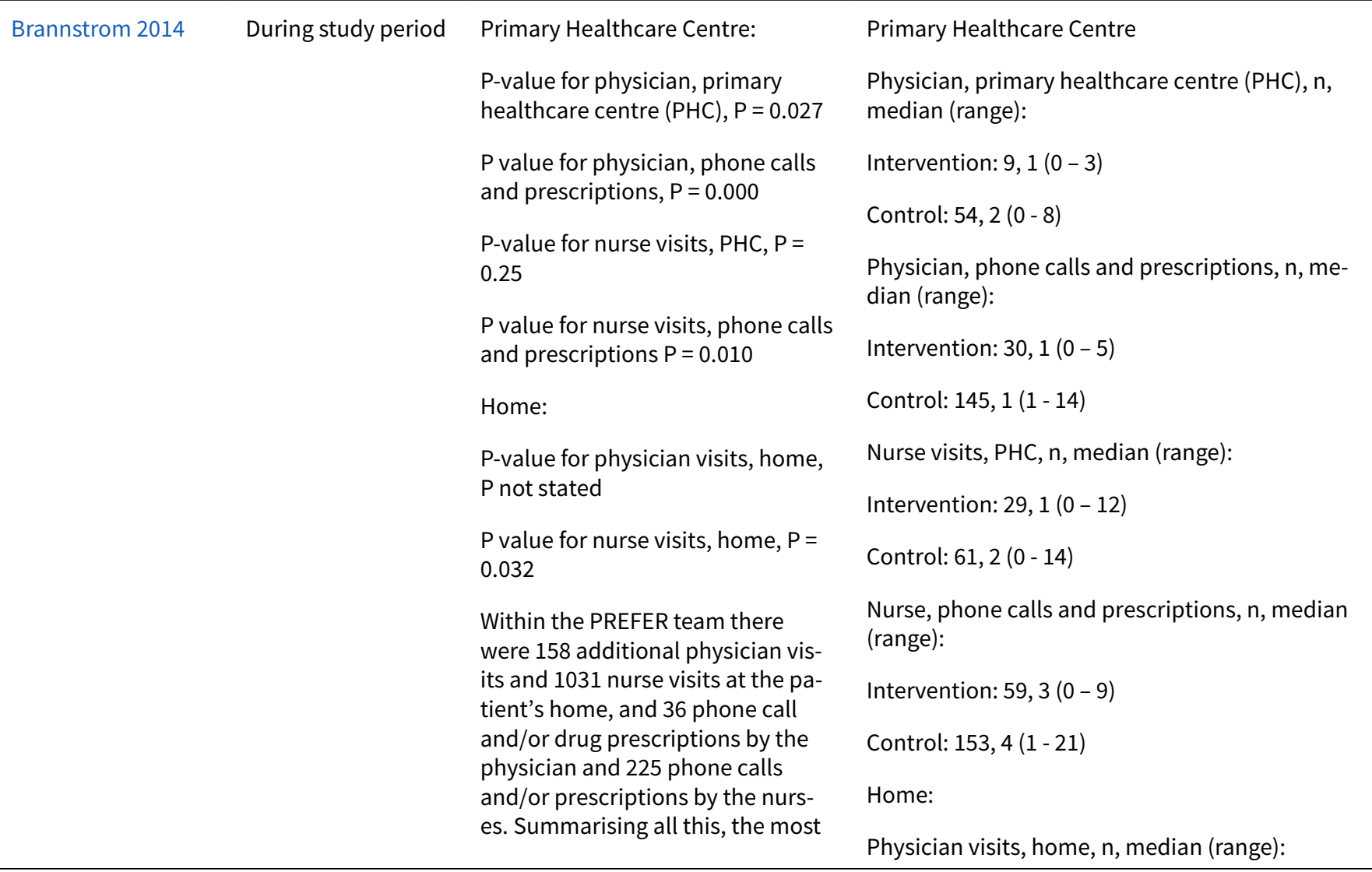

The effectiveness and cost-effectiveness of hospital-based specialist palliative care for adults with advanced illness and their caregivers 
Table 13. Community care (Continued)

striking difference was found between nurse visits in the PREFER group and the usual care group (1075 vs. 230; $P=0.000$ ). On the other hand, phone calls and prescriptions by doctors were more common in the usual care group (108 vs. 231), while physician's visits were somewhat similar (194 vs. 201).
Intervention: 0, $0(0-0)$

Control: 14, $2(1$ - 5)

Nurse visits, home, $\mathrm{n}$, median (range):

Intervention: 11, $2(1-3)$

Control: 109, 5 (1 - 23)

Days in hospice care ( 1 of 2 sites only):

descriptive data not provided

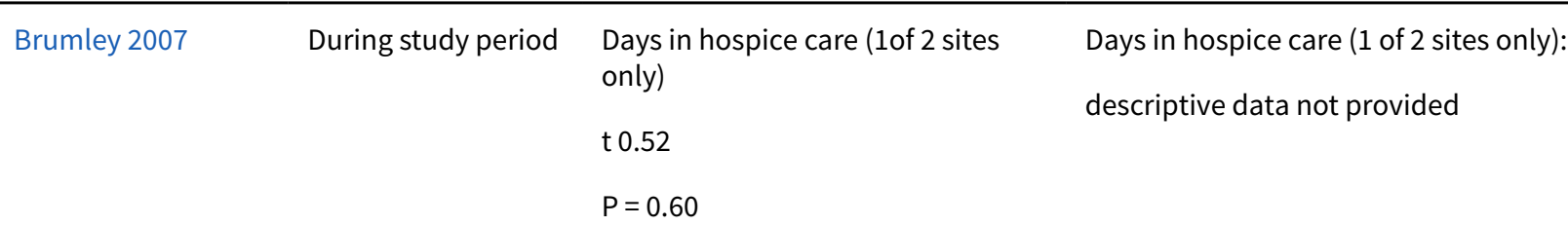

Farquhar $2014 \quad$ During study period $\quad \mathrm{P}$ values not stated

Breathlessness intervention service:

Intervention, $\mathrm{n}(\%)$, mean (SD) contacts: 27 (96\%), $1.9(2.0)$

Control, n (\%), mean (SD) contacts: 2 (8\%), 1.5 (0.7)

$P$ values not stated

GP:

Intervention, $\mathrm{n}(\%)$, mean (SD) contacts: 10 (36\%), $1.2(0.6)$

Control, n (\%), mean (SD) contacts: 13 (50\%), 1.3 (0.5)

Farquhar $2016 \quad$ During study period $\quad \mathrm{P}$ values not stated

Breathlessness intervention service:

Intervention, $\mathrm{n}(\%)$, mean (SD) contacts: 39 (95\%), $2.1(1.0)$

Control, $\mathrm{n}(\%)$, mean (SD) contacts: 2 (5\%), 1.5 (0.7)

Intervention, $\mathrm{n}(\%)$, mean (SD) contacts: 25 (61\%), $1.8(1.2)$

Control, n (\%), mean (SD) contacts: 24 (63\%), 1.6 (0.7)

\begin{tabular}{|c|c|}
\hline 6 months post-in- & $P=0.09$ \\
\hline & $\begin{array}{l}\text { Continuous measures for inter- } \\
\text { vention and control patients were } \\
\text { compared using t tests for nor- } \\
\text { mally distributed measures and } \\
\text { Wilcoxon two-sample tests for } \\
\text { measures with skewed distribu- } \\
\text { tions }\end{array}$ \\
\hline
\end{tabular}

6 months post-in- $\quad P=0.09$

Continuous measures for intervention and control patients were compared using t tests for norWilcoxon two-sample tests for tions
Study enrollment to hospice admission (days), median (IQR):

Intervention: $2(0,23)$

Control: $3(0,37)$

Hospice length of stay (days), median (IQR) 
Table 13. Community care (Continued)

Continuous measures for intervention and control patients were compared using t tests for normally distributed measures and Wilcoxon two-sample tests for measures with skewed distributions.
Intervention: $24(7,94)$

Control: $12(4,48)$

\section{$P=0.5$}

Categorical measures were tested using 2 tests or Fisher's exact test.
Patients admitted to hospice, $\mathrm{n}(\%)$ :

Intervention: 103 (37.1\%)

Control: $96(40.7 \%)$

Grudzen $2016 \quad$ During study period Fisher's exact test $P=0.85 \quad$ Hospice use at 180 days:

Chi ${ }^{2}$ test $\mathrm{P}=0.93 \quad$ Intervention: $28 \%$

Control: $25 \%$

\begin{tabular}{|c|c|c|c|}
\hline \multirow[t]{20}{*}{ Higginson 2009} & 12 weeks following & General practice: & General practice: \\
\hline & & $\begin{array}{l}\text { Authors stated less GP contact in } \\
\text { intervention group but } P \text { values } \\
\text { not stated }\end{array}$ & $\begin{array}{l}\text { Intervention: } 8(35 \%) \text { received; M } 3.8 \text { contacts } \\
\text { (SD 0.5) }\end{array}$ \\
\hline & & & Control: 11 (52\%) received; M 3.4 contacts (SD \\
\hline & & District/practice nurse: & $1.2)$ \\
\hline & & $P$ values not stated & $\begin{array}{l}\text { "Control care patients were more likely to be in } \\
\text { contact with general practitioners" }\end{array}$ \\
\hline & & MS nurse: & \\
\hline & & Authors stated there were no dif- & District/practice nurse: \\
\hline & & ferences (P values not stated) & $\begin{array}{l}\text { Intervention: } 20 \text { (87\%) received; M } 12.3 \text { contacts } \\
\text { (SD 19.7) }\end{array}$ \\
\hline & & Social services: & \\
\hline & & $P$ values not stated & $\begin{array}{l}\text { Control: } 13 \text { (62\%) received; M } 31.9 \text { contacts (SD } \\
50.7)\end{array}$ \\
\hline & & Specialist home visit: & MS nurse: \\
\hline & & $P$ values not stated & $\begin{array}{l}\text { Intervention: } 11(48 \%) \text { received; M } 1.8 \text { contacts } \\
\text { (SD 1.8) }\end{array}$ \\
\hline & & & $\begin{array}{l}\text { Control: } 7 \text { (33\%) received; M } 1.1 \text { contacts (SD } \\
0.2)\end{array}$ \\
\hline & & & $\begin{array}{l}\text { "Receipt of MS nurses was similar in the two } \\
\text { groups". }\end{array}$ \\
\hline & & & Social services: \\
\hline & & & $\begin{array}{l}\text { Intervention: } 10(43 \%) \text { received; M } 6.4 \text { contacts } \\
\text { (SD 7.7) }\end{array}$ \\
\hline & & & $\begin{array}{l}\text { Control: } 8(38 \%) \text { received; M } 4.1 \text { contacts (SD } \\
2.4)\end{array}$ \\
\hline & & & Specialist home visit: \\
\hline & & & $\begin{array}{l}\text { Intervention: } 5 \text { (22\%) received; M } 5.2 \text { contacts } \\
\text { (SD 4.5) }\end{array}$ \\
\hline & & & Control: 0 received \\
\hline
\end{tabular}

The effectiveness and cost-effectiveness of hospital-based specialist palliative care for adults with advanced illness and their caregivers 275 (Review)

Copyright $\odot 2020$ The Cochrane Collaboration. Published by John Wiley \& Sons, Ltd. 
Table 13. Community care (Continued)

Note: authors stated that specialist home visits were most likely to be from the intervention home palliative care team.

\begin{tabular}{|c|c|c|c|}
\hline Kane 1984 & During study period & $P$ value not stated & $\begin{array}{l}\text { Days at home: } \\
\text { Intervention, mean per patient: } 44.8 \\
\text { Control, mean per patient: } 37.9\end{array}$ \\
\hline McCaffrey 2013 & During study period & $\begin{array}{l}\text { No difference as increment, mean } \\
(95 \% \mathrm{Cl})=1(-6.8,8.6)\end{array}$ & $\begin{array}{l}\text { Days at home: } \\
\text { Intervention, mean }(95 \% \mathrm{Cl}): 13.1(8.5,17.7) \\
\text { Control, mean }(95 \% \mathrm{Cl}): 12.1(5.9,18.4)\end{array}$ \\
\hline Rogers 2017 & During study period & $P$ values not stated & $\begin{array}{l}\text { Frequency of interactions occurring between pa- } \\
\text { tients and providers } \\
\text { Primary care: } \\
\text { Intervention, mean (SD): } 4.4(0.93) \\
\text { Control, mean (SD): } 5.2(0.82)\end{array}$ \\
\hline Sidebottom 2015 & $\begin{array}{l}\text { Hospice use within } \\
6 \text { months of study } \\
\text { hospitalisation }\end{array}$ & $\begin{array}{l}\text { Survival analysis using proportion- } \\
\text { al hazards regression } \\
\mathrm{P}=0.36\end{array}$ & $\begin{array}{l}\text { There was no significant association between } \\
\text { study group assignment and hospice use within } \\
6 \text { months (adjusting for age, gender, and marital } \\
\text { status). }\end{array}$ \\
\hline Temel 2010 & During study period & $P=0.09$ & $\begin{array}{l}\text { Median duration of hospice care: } \\
\text { Intervention: } 11 \text { days } \\
\text { Control: } 4 \text { days }\end{array}$ \\
\hline
\end{tabular}

$\mathrm{Cl}:$

GP: General Practitioner

M: mean

MS: Multiple Sclerosis

$\mathrm{n}$ : number

PHC:

PREFER:

SD: standard deviation

Table 14. Informal care

\begin{tabular}{llll}
\hline Study & Time horizon & $\begin{array}{l}\text { Significance and } \\
\text { direction }\end{array}$ & Details \\
\hline Farquhar 2014 & During study period & P value not stated & Breathlessness intervention service: \\
& & Intervention, $n$ (\%), mean (SD) contacts: $22(79 \%), 20.3(20.8)$ \\
& & Control, $n$ (\%), mean (SD) contacts: 25 (96\%), 23.4 (25.2) \\
\hline
\end{tabular}

Higginson 2009 weeks following P value not stated Care by informal caregiver:
enrollment

Intervention: 15/23 (65\%) received; Mean 152.5 contacts (SD 53.7)

The effectiveness and cost-effectiveness of hospital-based specialist palliative care for adults with advanced illness and their caregivers 
Table 14. Informal care (Continued)

$\mathrm{n}$ : number

SD: standard deviation

Table 15. Medications and other resources

\begin{tabular}{|c|c|c|c|}
\hline Study & Time horizon & $\begin{array}{l}\text { Significance and } \\
\text { direction }\end{array}$ & Details \\
\hline \multirow[t]{21}{*}{ Ahronheim 2000} & During study period & Pearson chi ${ }^{2}$ test & New feeding tube \\
\hline & & $P=0.79$ & Intervention: 22 (45.8\%) \\
\hline & & & Control: 22 (43.1\%) \\
\hline & & Pearson chi 2 test & Total feeding tube \\
\hline & & $P=0.66$ & Intervention: 34 (70.8\%) \\
\hline & & & Control: 34 (66.7\%) \\
\hline & & Pearson chi 2 test & Mechanical ventilation \\
\hline & & $P=0.44$ & Intervention: 2 (4.2\%) \\
\hline & & & Control: 4 (7.8\%) \\
\hline & & $\begin{array}{l}\text { Not calculated be- } \\
\text { cause expected fre- } \\
\text { quencies }<5 \text { in at } \\
\text { least } 2 \text { cells }\end{array}$ & $\begin{array}{l}\text { Tracheostomy } \\
\text { Intervention: } 0 \\
\text { Control: } 1\end{array}$ \\
\hline & & $\begin{array}{l}\text { Not calculated be- } \\
\text { cause expected fre- } \\
\text { quencies }<5 \text { in at } \\
\text { least } 2 \text { cells }\end{array}$ & $\begin{array}{l}\text { CPR } \\
\text { Intervention: } 0 \\
\text { Control: } 3(5.9 \%)\end{array}$ \\
\hline & & Pearson $\mathrm{chi}^{2}$ test & Systemic antibiotics (unclear if mean or median presented) \\
\hline & & $P=0.16$ & Intervention: 73 (79.3) \\
\hline & & & Control: 69 (70.4) \\
\hline & & & Interventions during 190 admissions \\
\hline & & Pearson chi² test & $\begin{array}{l}\text { IV for entire admission (unclear if mean or median present- } \\
\text { ed) }\end{array}$ \\
\hline & & $r-0.0<0$ & Intervention: $61(66)$ \\
\hline & & & Control: 79 (81) \\
\hline & & Pearson chi ${ }^{2}$ test & $\begin{array}{l}\text { Indwelling urinary catheter (unclear if mean or median pre- } \\
\text { sented) }\end{array}$ \\
\hline & & $r-0.00$ & Intervention: 41 (44.6) \\
\hline & & & Control: 51 (52) \\
\hline
\end{tabular}


Table 15. Medications and other resources (Continued)

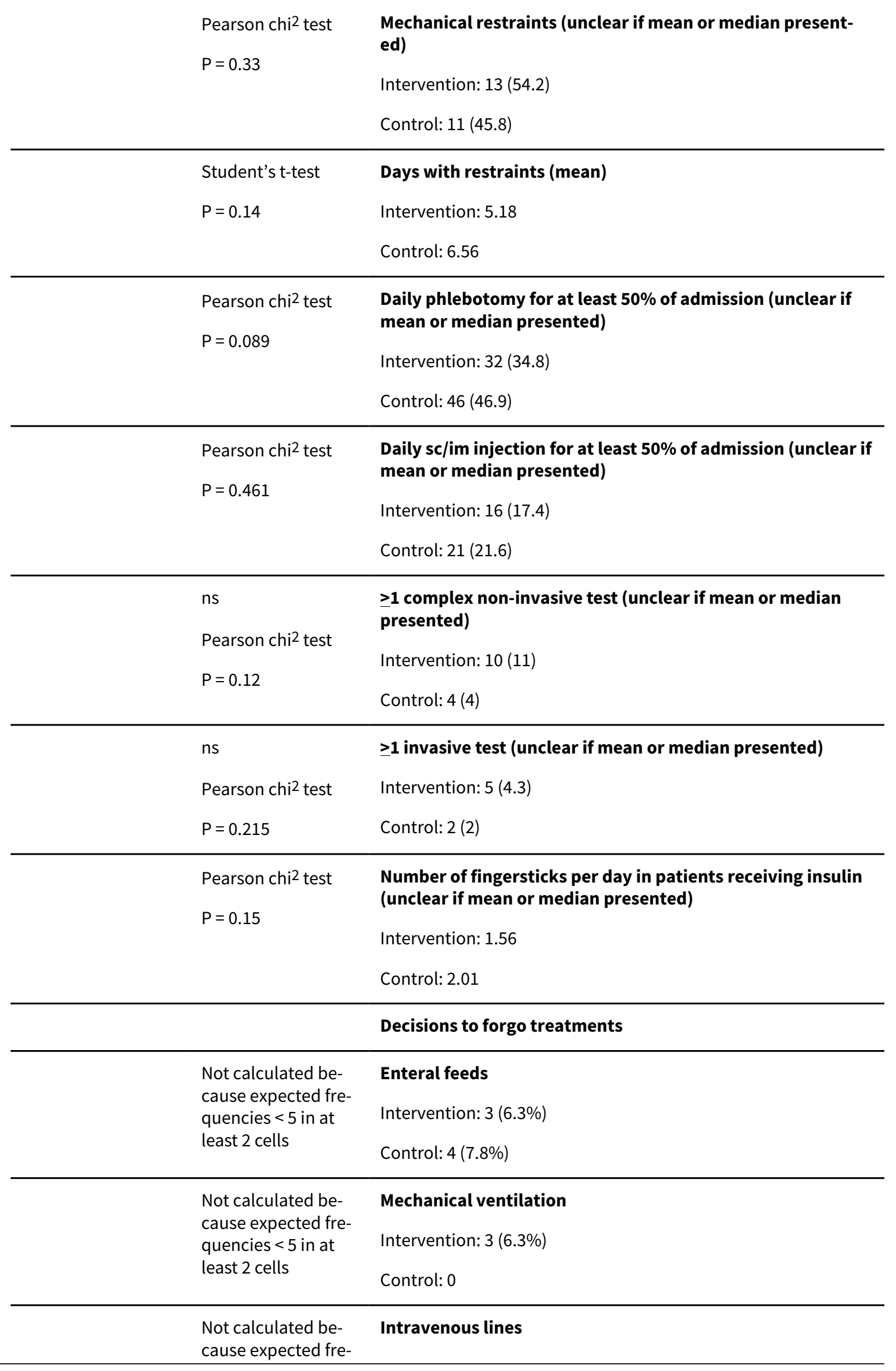

The effectiveness and cost-effectiveness of hospital-based specialist palliative care for adults with advanced illness and their caregivers 
Table 15. Medications and other resources (Continued)

quencies $<5$ in at Intervention: $5(10.4 \%)$

least 2 cells

Control: $1(2 \%)$

\begin{tabular}{lll}
\hline $\begin{array}{l}\text { Not calculated be- } \\
\text { cause expected fre- } \\
\text { quencies }<5 \text { in at } \\
\text { least } 2 \text { cells }\end{array}$ & $\begin{array}{l}\text { Blood draws } \\
\text { Intervention: } 4(8.3 \%)\end{array}$ & Control: 0 \\
\hline $\begin{array}{l}\text { Not calculated be- } \\
\text { cause expected fre- } \\
\text { quencies }<5 \text { in at } \\
\text { least } 2 \text { cells }\end{array}$ & Antibiotics \\
& Intervention: $3(6.3 \%)$ \\
\hline $\begin{array}{ll}\text { Pearson chi2 test } \\
\mathrm{P}=0.65\end{array}$ & CPR in-hospital (unclear if mean or median presented) \\
& Intervention: 62 (67.4) \\
& Control: 63 (64.3)
\end{tabular}

Pearson chi2 $^{2}$ test

CPR nonhospital (unclear if mean or median presented)

$P=0.10$

Intervention: 47 (51.1)

Control: 38 (38.8)

\begin{tabular}{|c|c|c|c|}
\hline Bakitas 2009 & During study period & $\begin{array}{l}P=0.34 \\
\begin{array}{l}\text { Referral to hospice } \\
\text { care }\end{array} \\
\text { Fisher exact test } P= \\
0.75\end{array}$ & $\begin{array}{l}\text { Referral to palliative care } \\
\text { Intervention: } 34 / 145(23.4 \%) \\
\text { Control: } 39 / 134(29.1 \%) \\
\text { Referral to hospice care } \\
\text { Intervention: } 6 / 161(3.7 \%) \\
\text { Control: } 4 / 161(2.5 \%)\end{array}$ \\
\hline Bakitas 2015 & $\begin{array}{l}\text { Total use covering } \\
\text { period before and } \\
\text { after enrollment }\end{array}$ & $\begin{array}{l}\text { Poisson generalised } \\
\text { linear model } \\
P=0.54\end{array}$ & $\begin{array}{l}\text { Chemotherapy in last } 2 \text { weeks of life } \\
\text { Intervention, rate }(95 \% \mathrm{Cl}): 0.08 \text { (0.03 to } 0.2) \\
\text { Control, rate }(95 \% \mathrm{Cl}): 0.05 \text { (0.02 to } 0.15)\end{array}$ \\
\hline Brumley 2007 & During study period & $\begin{array}{l}\text { Referral to hospice } \\
\text { care } \\
\text { (1of } 2 \text { sites only) } \\
\text { Chi2 } \mathrm{P}=0.15 \\
\text { Days in hospice } \\
\text { care (1 of } 2 \text { sites on- } \\
\text { ly) } \\
\text { t } 0.52 \\
P=0.60\end{array}$ & $\begin{array}{l}\text { Referral to hospice care } \\
\text { (1 of } 2 \text { sites only) } \\
\text { Intervention: } 25 \% \\
\text { Control: } 36 \% \\
\text { Days in hospice care (1 of } 2 \text { sites only) } \\
\text { descriptive data not provided }\end{array}$ \\
\hline Carson 2016 & $\begin{array}{l}\text { Interviewed surro- } \\
\text { gate decision-mak- } \\
\text { ers immediately af- }\end{array}$ & $\begin{array}{l}\text { Total ventilator } \\
\text { days, } P=0.59\end{array}$ & $\begin{array}{l}\text { Ventilator days } \\
\text { Total }\end{array}$ \\
\hline
\end{tabular}

The effectiveness and cost-effectiveness of hospital-based specialist palliative care for adults with advanced illness and their caregivers 
Table 15. Medications and other resources (Continued)

ter the second support and informa-

After randomisation, $\mathrm{P}=0.42$

tion team meeting

for the intervention

group and 10 days

after randomisation

for

the control group, unless the patient had died. All surrogate decision-makers were interviewed again by telephone for follow-up beginning 90 days after randomisation.
Intervention, median (IQR): 19 (15 to 31)

Control, median (IQR): 21 (14 to 35)

After randomisation

Intervention, median (IQR): 10 (5 to 20)

Control, median (IQR): 12 (5 to 27 )

Interviewed surro- $P=0.62$
gate decision-mak-
ers immediately
after the second
support and infor-
mation team meet-
ing
for the intervention
group and 10 days
after randomisation
for
the control group,
unless the patient
had died. All surro-
gate
decision-makers
were interviewed
again by telephone
for
follow-up begin-
ning 90 days after
randomisation.

\author{
Hospital discharge disposition (81 patients discharged from \\ the hospital in intervention group and 75 in control group). \\ Home \\ Intervention, median (IQR): 15 (19) \\ Control, median (IQR): 18 (24)
}

Home with paid assistance:

Intervention, median (IQR): 10 (12)

Control, median (IQR): 7 (9)

Hospice

Intervention, median (IQR): 3 (4)

Control, median (IQR): 4 (5)

Acute rehabilitation facility

Intervention, median (IQR): 22 (27)

Control, median (IQR): 15 (20)

Long-term acute care hospital

Intervention, median (IQR): 12 (15)

Control, median (IQR): 12 (16)

Other acute care facility

Intervention, median (IQR): 0

Control, median (IQR): 1 (1)

Skilled nursing facility

Intervention, median (IQR): 19 (23)

Control, median (IQR): 16 (21)

Other 
Table 15. Medications and other resources (Continued)

Intervention, median (IQR): 0

Control, median (IQR): 2 (3)

\begin{tabular}{|c|c|c|c|}
\hline \multirow[t]{3}{*}{ Farquhar 2014} & During study period & $P$ value not stated & $\begin{array}{l}\text { Other hospital care } \\
\text { Intervention, n (\%), mean (SD) contacts: } 15(54 \%), 1.5(0.8) \\
\text { Control, n (\%), mean (SD) contacts: } 14(54 \%), 1.4(0.6)\end{array}$ \\
\hline & & $P$ value not stated & $\begin{array}{l}\text { Nurse } \\
\text { Intervention, n (\%), mean (SD) contacts: } 11(39 \%), 3.0 \text { (3.8) } \\
\text { Control, n (\%), mean (SD) contacts: } 12(46 \%), 1.8(1.6)\end{array}$ \\
\hline & & $P$ value not stated & $\begin{array}{l}\text { Other health professionals } \\
\text { Intervention, } \mathrm{n}(\%) \text {, mean (SD) contacts: } 5(18 \%), 1.2(0.4) \\
\text { Control, } \mathrm{n}(\%) \text {, mean (SD) contacts: } 3(12 \%), 1.0(0.0)\end{array}$ \\
\hline
\end{tabular}

\section{Social care}

Intervention, n (\%), mean (SD) contacts: 4 (14\%), 4.3 (6.5)

Control, n (\%), mean (SD) contacts: 3 (12\%), 15.7 (22.9)

\begin{tabular}{|c|c|c|c|}
\hline \multirow[t]{4}{*}{ Farquhar 2016} & During study period & $P$ value not stated & $\begin{array}{l}\text { Other hospital services } \\
\text { Intervention, } \mathrm{n}(\%) \text {, mean (SD) contacts: } 20 \text { (49\%), } 1.7(1.0) \\
\text { Control, n (\%), mean (SD) contacts: } 19 \text { (50\%), } 2.5(3.5)\end{array}$ \\
\hline & & $P$ value not stated & $\begin{array}{l}\text { Nurse } \\
\text { Intervention, n (\%), mean (SD) contacts: } 21 \text { (51\%), } 2.7 \text { (3.3) } \\
\text { Control, n (\%), mean (SD) contacts: } 16(42 \%), 2.5(2.5)\end{array}$ \\
\hline & & $P$ value not stated & $\begin{array}{l}\text { Other health services } \\
\text { Intervention, } \mathrm{n}(\%) \text {, mean (SD) contacts: } 14(34 \%), 1.5(1.1) \\
\text { Control, n (\%), mean (SD) contacts: } 4 \text { (11\%), } 1.0(0.0)\end{array}$ \\
\hline & & $P$ value not stated & $\begin{array}{l}\text { Social and other care } \\
\text { Intervention, } \mathrm{n}(\%) \text {, mean (SD) contacts: } 8(20 \%), 5.4(4.6) \\
\text { Control, n (\%), mean (SD) contacts: } 9 \text { (24\%), } 11.3(22.8)\end{array}$ \\
\hline
\end{tabular}

Groenvold $2017 \quad$ During study period P value not stated Telephone contact with the HSPC team, $\mathbf{n}$

Intervention: 116 patients had at least one telephone contact

Control: 9 patients had at least one telephone contact

\begin{tabular}{ll}
\hline Higginson 2009 & $\begin{array}{l}12 \text { weeks after en- P value not stated } \\
\text { rollment }\end{array}$
\end{tabular}

\section{Palliative care nurse}

Intervention: 9 (39\%) received; M 3.0 (SD 1.5)

Control: 0 received 
Table 15. Medications and other resources (Continued)

\section{Other nurse}

Intervention: 7 (30\%) received; M 40.0 (SD 63.8)

Control: 7 (33\%) received; M 95.0 (SD 79.6)

Specialist (ward)

Intervention: 5 (22\%) received; M 1.0 (SD 0.0)

Control: 7 (33\%) received; M 9.6 (SD 12.1)

Specialist (other)

Intervention: 4 (17\%) received; M 1.1 (SD 0.3)

Control: 5 (24\%) received; M 1.0 (SD 0.0)

Occupational therapist/physiotherapist

Intervention: 16 (70\%) received; M 10.6 (SD 9.9)

Control: 14 (67\%) received; M 22.5 (SD 47.7)

\section{Dietitian/chiropodist}

Intervention: 12 (52\%) received; M 3.5 (SD 2.5)

Control: 13 (62\%) received; M 2.6 (SD 1.3)

\section{Day centre}

Intervention: 5 (22\%) received;M 20.2(SD 21.0)

Control: 5 (24\%) received; M 20.4 (SD 15.9)

\section{Respite care}

Intervention: 2 (9\%) received; M 9.5 (SD 0.7)

Control: 5 (24\%) received; M 10.0 (SD 5.9)

Janssens $2019 \quad$ During study period $P=0.819$

\section{Use of antibiotics}

The use of antibiotics (for exacerbations not leading to hospital admission) did not differ between groups during the observation period.

\begin{tabular}{ll}
\hline Kane $1984 \quad$ During study period & $\begin{array}{l}\text { Major surgical pro- } \\
\text { cedures } \mathrm{P}<0.05\end{array}$
\end{tabular}

\section{Major surgical procedures}

Intervention, mean per patient: 0.09

Control, mean per patient: 0.01

\section{Minor surgical procedures}

Intervention, mean per patient: 0.42

Control, mean per patient: 0.30

$\begin{array}{ll}\begin{array}{l}\text { Over } 80 \% \text { of both } \\ \text { hospice and con- }\end{array} & \text { Radiation treatments } \\ \text { trol patients had } & \text { Intervention, mean per patient: } 7.4 \\ \text { no radiation treat- } & \\ \text { ments. However, } & \text { Control, mean per patient: } 7.7 \\ \text { those few who did } & \end{array}$

The effectiveness and cost-effectiveness of hospital-based specialist palliative care for adults with advanced illness and their caregivers 
Table 15. Medications and other resources (Continued)

had as many as 48

treatments, hence

the large number.

$P=0.03$

Chemotherapy treatments

Intervention, mean per patient: 1.3

Control, mean per patient: 0.49

\section{Markgren}

2016 (linked to

Brannstrom 2014)
During study period

Only the change in patients receiving full target doses of the ACEIs/angiotensin receptor blockers, BBs and MRAs were higher $(P=0.0009)$ in the intervention arm than in the control arm.

\section{Prescribed medication use}

In the intervention arm, the percentages of angiotensin converting enzyme inhibitors (ACEIs) and mineralocorticoid receptor antagonists (MRAs) increased at the end of the study from baseline, while loop diuretics decreased. Beta-receptor blockers (BBs) decreased somewhat in both groups. The number of patients treated with MRAs differed the most between groups, and increased from $10(28 \%)$ to $15(48 \%)$ in the PREFER arm compared with $13(35 \%)$ vs $13(39 \%)$ in the control group. The change in patients receiving full target doses (+8 vs. +1$)$ of the ACEls/angiotensin receptor blockers, BBs and MRAs were higher $(P=0.0009)$ in the intervention arm than in the control arm.

Diuretics, $P=0.2$

\section{Guideline-driven HF therapies}

Spironolac-

CRT device

tone/eplerenone, $\mathrm{P}$

$=0.9$

Intervention: $20 \%$

Beta-blockers, $\mathrm{P}=$

Control: $35.7 \%$

0.4

\section{ACE1/ARB}

Intervention: 60\%

Control: $35.7 \%$

\section{Diuretics}

Intervention: $86.7 \%$

Control: $64.3 \%$

\section{Spironolactone/eplerenone}

Intervention: $26.7 \%$

Control: $28.6 \%$

Beta-blockers

Intervention: $66.7 \%$

Control: $50 \%$

Medications for other conditions

Cholesterol-lowering medication

Intervention: $73.3 \%$

The effectiveness and cost-effectiveness of hospital-based specialist palliative care for adults with advanced illness and their caregivers 
Table 15. Medications and other resources (Continued)

Control: $50 \%$

\section{Anti-anginal}

Intervention: $20 \%$

Control: $14.3 \%$

Diabetes medication

Intervention: $13.3 \%$

Control: $14.3 \%$

\section{Antidepressants}

Intervention: $20 \%$

Control: $28.6 \%$

\section{Pain medication (NSAIDS and opioids)}

Intervention: 53.3\%

Control: $21.4 \%$

Anxiety medication

Intervention: 0

Control: $7.1 \%$

\section{Constipation}

Intervention: $26.7 \%$

Control: $28.6 \%$

\section{Referral to palliative care}

Intervention: 22 (100\%)

Control: $1(5 \%)$

\section{Referral to social work}

Intervention: 22 (100\%)

Control: 20 (100\%)

Referral to psychiatry

Intervention: 1 (4.5\%)

Control: $1(5 \%)$

Rogers $2017 \quad$ During study period $\quad \mathrm{P}$ value not stated

Frequency of interactions occurring between patients and providers

\section{Total number of hospital encounter records}

Intervention, mean (SD): 2.5 (0.45)

Control, mean (SD): 2.4 (0.35)

\section{Telephone contact}


Table 15. Medications and other resources (Continued)

Intervention, mean (SD): 12.6 (1.2)

Control, mean (SD): 10.6 (0.88)

Temel 2010 During study period $P=0.05$

\section{Aggressive end-of-life care among 105 decedents}

(chemotherapy within 14 days before death, no hospice care, or admission to hospice 3 days or less before death)

Intervention: 54\%

Control: $33 \%$

\section{Chemotherapy within 30 days of death}

Intervention: $32.5 \%$

Control: $42 \%$

ACEI:

ARB:

BB:

CPR: Cardiopulmonary Resuscitation

CRT:

$\mathrm{HF}: I Q R$ : interquartile range

M: mean

MRA:

$\mathrm{n}:$ number

ns:

NSAID:

PREFER:

sc/im: subcutaneous/Intramuscuslar

SD: standard deviation

Table 16. Studies with qualitative components

\begin{tabular}{|c|c|c|c|c|}
\hline Studies & $\begin{array}{l}\text { Participants in- } \\
\text { terviewed }\end{array}$ & $\begin{array}{l}\text { Qualitative ap- } \\
\text { proach }\end{array}$ & Findings of the qualitative study & $\begin{array}{l}\text { Findings of the quantitative } \\
\text { component }\end{array}$ \\
\hline $\begin{array}{l}\text { Bajwah } 2015 \text { (pa- } \\
\text { tients with inter- } \\
\text { stitial lung dis- } \\
\text { ease (ILD)) }\end{array}$ & $\begin{array}{l}5 \text { patients } \\
5 \text { carers } \\
1 \text { ILD consultant } \\
1 \text { ILD CNS } \\
1 \text { community } \\
\text { matron } \\
1 \text { community }\end{array}$ & $\begin{array}{l}\text { Semi-struc- } \\
\text { tured interviews } \\
\text { analysed us- } \\
\text { ing a constant } \\
\text { comparison ap- } \\
\text { proach with- } \\
\text { in framework } \\
\text { analysis }\end{array}$ & $\begin{array}{l}\text { Findings: } \\
\text { Patients and carers interviewed } \\
\text { valued the case conference as } \\
\text { they felt that it "laid everything } \\
\text { on the table" and importantly ad- } \\
\text { dressed concerns and anxieties } \\
\text { that had been playing on patients' } \\
\text { and carers' minds. The qualitative } \\
\text { work also identified lack of early } \\
\text { referral to palliative care by com- }\end{array}$ & $\begin{array}{l}\text { Primary outcome: } \\
\text { Symptom burden } \\
\text { Mean (SD) POS scores at } 4 \text { weeks } \\
\text { were }-5.7(7.5) \text { fast-track vs }-0.4 \\
\text { (8.0) control, (mean change dif- } \\
\text { ference between the two arms } \\
\text { was }-5.3(95 \% \mathrm{Cl}-9.8 \text { to }-0.7) \text { inde- } \\
\text { pendent t test } \mathrm{P}=0.02) \text {; effect size } \\
(95 \% \mathrm{Cl})-0.7(-1.2 \text { to }-0.1) \text {. }\end{array}$ \\
\hline
\end{tabular}

palliative care munity health professionals, denurse spite requests from patients and carers, and some gatekeeping by hospital health professionals.

\section{Secondary outcomes:}

$1 \mathrm{GP}$

Themes from patients:

The secondary outcomes of quality of life, anxiety and depression were superior in the fast-track arm, and none were worse.

Support in the community

Crisis management 
Table 16. Studies with qualitative components (Continued)

Palliative care, psychological support

Advance care planning

Themes from health profession-

als:

GPs - collaboration of care and ef-

ficiency

Community palliative care clinical nurse specialist - individual care plans and practical problems addressed

ILD consultant - symptom control

ILD CNS - empowering health professionals

\begin{tabular}{|c|c|c|}
\hline $\begin{array}{l}\text { Bakitas } 2013 \\
\text { (linked to Bakitas } \\
\text { 2009) }\end{array}$ & $\begin{array}{l}35 \text { oncology clin- } \\
\text { icians compris- } \\
\text { ing } 21 \text { physicians } \\
\text { and } 14 \text { nurse } \\
\text { practitioner }\end{array}$ & $\begin{array}{l}\text { Semi-struc- } \\
\text { tured interviews } \\
\text { analysed using } \\
\text { thematic analy- } \\
\text { sis }\end{array}$ \\
\hline
\end{tabular}

\section{Findings:}

Oncologists believed that integrating palliative care at the time of an advanced cancer diagnosis enhanced patient care and complemented their practice. Five themes comprised oncologists' views on the complementary role of palliative care: (1) "refer early and often," (2) referral challenges: "palliative" equals "hospice"; "Heme patients are different," (3) palliative care as consultants or co-managers, (4) palliative care "shares the load," and (5) ENABLE II facilitated palliative care integration. Self-assessment of their practice with advanced cancer patients comprised four themes: (1) treating the whole patient, (2) focussing on quality versus quantity of life, (3) "some patients just want to fight," and (4) helping with transitions; timing is everything.

\section{Primary outcomes:}

\section{Quality of life:}

The estimated treatment effects (intervention minus usual care) for all participants were a mean (SE) of 4.6 (2) for quality of life $(P=$ 0.02)

\section{Symptom intensity}

The estimated treatment effects (intervention minus usual care) for all participants were a mean (SE) of -27.8 (15) for symptom intensity $(P=0.06)$

Resource use:

Intensity of service did not differ between the 2 groups.

\section{Secondary outcomes:}

The estimated treatment effects (intervention minus usual care) for all participants were a mean (SE) of -1.8 (0.81) for depressed $\operatorname{mood}(P=0.02)$.

\section{Maloney 2013} (linked to Bakitas 2009)

53 patients $(28$ females included)

\section{(ENABLE II)}

(cancer patients)

\section{Semi-struc- \\ Findings:} tured interviews analysed using thematic analysis
Participants' perceptions of intervention benefits were represented by four themes: enhanced problem-solving skills, better coping, feeling empowered, and feeling supported or reassured.

Three themes related to trial participation: helping future patients and contributing to science, gain-

\section{Primary outcomes:}

Quality of life:

The estimated treatment effects (intervention minus usual care) for all participants were a mean (SE) of 4.6 (2) for quality of life ( $P=$ 0.02)

\section{Symptom intensity}

The effectiveness and cost-effectiveness of hospital-based specialist palliative care for adults with advanced illness and their caregivers 
Table 16. Studies with qualitative components (Continued)

ing insight through completion of questionnaires, and trial/intervention aspects to improve. Participants did not describe participation as burdensome but rather described some inconveniences or disappointments such as nonattendance of meetings by other participants and disappointment at not being randomised to the intervention group.

The estimated treatment effects (intervention minus usual care) for all participants were a mean (SE) of -27.8 (15) for symptom intensity $(\mathrm{P}=0.06)$

Intensity of service did not differ between the 2 groups.

\section{Secondary outcomes:}

The estimated treatment effects (intervention minus usual care) for all participants were a mean (SE) of -1.8 (0.81) for depressed $\operatorname{mood}(P=0.02)$.

\section{Outcomes:} tured interviews analysed using content analysis

\section{Findings:}

Two themes and a total of five categories were identified. The first theme was feeling secure and safe through receiving care at home with the categories: having access to readily available care at home, being followed up continuously and having trust in the team members' ability to help. The second theme was being acknowledged as both a person and a patient, with the following two categories: being met as a person, participating in decisions about one's care and receiving help for symptoms of both HF and comorbidities. The team also offered relatives support, which patients appreciated.

Quality of life:

Between-group analysis revealed that patients receiving HSPC had improved HRQoL compared with controls $(57.6 \pm 19.2$ vs. $48.5 \pm$ 24.4 , age-adjusted $\mathrm{P}=0.05$ ). Within-group analysis revealed a $26 \%$ improvement in the

HSPC group for HRQoL $(P=0.046)$ compared with $3 \%(P=0.82)$ in the control group.

Quality of life improved by $24 \%$ ( $P$ $=0.047$ ).

Symptom burden:

Total symptom burden improved by $18 \%(P=0.035)$

Resource use:

Fifteen rehospitalisations (103 days) occurred in the HSPC group, compared with 53 (305 days) in the control group.

\section{Primary outcome:}

tured interviews analysed using framework analysis

\section{Findings:}

Breathlessness intervention service (BIS) reduced fear and worry, and increased confidence in managing breathlessness. Patients and carers consistently identified specific and repeatable aspects of the BIS model and interventions that helped. The multidisciplinary staff expertise was repeatedly noted. How interventions were delivered was important with a suggestion that the intervention sas delivered through the provi
BIS reduced patient distress due to breathlessness (primary outcome: $-1.29 ; 95 \% \mathrm{Cl}-2.57$ to $-0.005 ; P=0.049$ ) significantly more than the control group; $94 \%$ of respondents reported a positive impact $(51 / 53)$

\section{Secondary outcomes:}

Mean CRQ mastery scores improved only negligibly in the intervention arm and remained stable 
Table 16. Studies with qualitative components (Continued)

sion of knowledge, with specialist expertise, which increased patients' and carers' confidence. BIS legitimised breathlessness and increased knowledge whilst making patients and carers feel 'not alone'. for controls. No differences were found between trial arms on other CRQ domains (dyspnoea, fatigue or emotional function). Mean anxiety scores (HADS) remained fairly stable (both arms). Mean depression scores decreased slightly in the intervention arm, increasing slightly for controls. There was little change in other patient or carer outcomes.

BIS had a $66 \%$ likelihood of better outcomes in terms of reduced distress due to breathlessness at lower health/social care costs than standard care $(81 \%$ with informal care costs included).

\begin{tabular}{lll}
\hline Farquhar 2016 & 20 patients (and & Semi-struc- \\
(Non-cancer & associated car- & tured interviews \\
(mostly COPD) & ers) & $\begin{array}{l}\text { analysed us- } \\
\text { ing framework } \\
\end{array}$ \\
& & analysis
\end{tabular}

\section{Findings:}

Patients with non-malignant conditions and their carers described a range of impacts including reduced fear, anxiety, worry, and feelings of panic, as well as feeling more confident about breathlessness. They valued the multidisciplinary staff expertise (their knowledge and understanding of life with breathlessness), the characteristics of the BIS staff (their approachability and attentiveness) and their reassuring and positive approach, and the time BIS gave them to talk about breathlessness with an expert. They reported that being seen at home was especially helpful. The findings suggest that it was not only the provision of these interventions that was important, but also that how they were delivered was key to their impact: delivery of interventions through the provision of knowledge (why and how interventions work or specific guidance on how and when to use a particular intervention) increased patients' and carers' confidence.

\section{Primary outcome:}

There was no difference between groups in the primary outcome ("distress due to breathlessness"), when compared to standard care, of -0.24 (95\% Cl: $-1.30,0.82)$.

\section{Secondary outcomes:}

Mean CRQ mastery scores improved slightly on both arms with greater improvement in the intervention arm. No differences were found between trial arms on other CRQ domains (dyspnoea, fatigue or emotional function). Mean patient anxiety scores decreased slightly for the intervention arm and increased slightly for the control arm and mean depression scores decreased slightly in the intervention arm and remained stable for controls; no between-group difference was found. Mean anxiety scores for carers achieved a greater, 1.65point, reduction in the intervention arm compared with a 0.15 point reduction for controls, adjusted difference of -1.22 (95\% $\mathrm{Cl}:-2.84$ to 0.40$), \mathrm{P}=0.14$. There was little change in other patient or carer secondary outcomes.

Carers of patients randomised to the intervention arm achieved a greater, 1.03-point, reduction in their distress due to their patient's breathlessness compared with a 0.2-point increase for controls, adjusted difference of -0.42 (95\% Cl: -1.86 to 1.02 ), $\mathrm{P}=0.56$. 
Table 16. Studies with qualitative components (Continued)

BIS resulted in extra mean costs of GBP799, reducing to GBP100 when outliers were excluded.

\begin{tabular}{|c|c|c|c|}
\hline $\begin{array}{l}\text { Hopp } 2016 \text { (pa- } \\
\text { tients with heart } \\
\text { failure) }\end{array}$ & 85 patients & $\begin{array}{l}\text { Unclear although } \\
\text { the authors stat- } \\
\text { ed that clinical } \\
\text { records were } \\
\text { qualitatively re- } \\
\text { viewed }\end{array}$ & $\begin{array}{l}\text { Findings: } \\
\text { Patients expressed concerns } \\
\text { about hospital palliative care as it } \\
\text { might prevent them from receiv- } \\
\text { ing more aggressive treatment. } \\
\text { Most patients did not engage with } \\
\text { advanced care options. }\end{array}$ \\
\hline
\end{tabular}

\begin{tabular}{lll}
\hline $\begin{array}{l}\text { Veron } 2018 \\
\text { (linked to } \\
\text { Janssens 2019) }\end{array}$ & $\begin{array}{l}18 \text { patients } \\
(44.4 \% \text { females })\end{array}$ & $\begin{array}{l}\text { Semi-struc- } \\
\text { tured interviews } \\
\text { analysed using } \\
\text { thematic content } \\
\text { analysis }\end{array}$ \\
\hline
\end{tabular}

\section{Findings:}

Patients described poor recollection of the RCT and difficulties understanding the palliative care intervention. No major differences were observed between patients who received the specialised intervention and those who did not. Content analysis emphasised that although they experienced disabling symptoms, participants tended to attribute their limitations to problems other than COPD and some declared that they were not sick. Patients reported restrictions due to oxygen therapy, and the burden of becoming dependent on it. This dependence resulted in intense anxiety, leading participants to focus on the present only. A strong feeling of perceived helplessness emerged from the patients' interviews.

\section{Primary outcome:}

There was no difference between groups in the primary outcome (election vs non-election of measure of comfort-oriented care) (difference $9.3 \%, 95 \% \mathrm{Cl}-11.8 \%$ to $30 \% ; P=0.12$ ).

\section{Primary outcomes:}

Patients in the HSPC group were hospitalised for respiratory failure (incidence rate ratio (IRR) 1.87, $95 \% \mathrm{Cl} 1.04$ to $3.48, \mathrm{P}=0.026$ ) and admitted to the emergency ward (IRR 2.05, 95\% Cl 1.11 to $3.94, \mathrm{P}=$ 0.014 ) twice as often during follow-up than the control group. However, after the Benjamini and Hochberg correction for multiple testing, none of these differences was significant. Furthermore, median values were identical in both groups (hospitalisation: median (IQR): 0.0 ( 1 to 2 ) vs. 1.5 ( 1 to 4 ), P $=0.219$; admissions to emergency wards: $1.0(0 ; 3)$ vs. $1.0(0 ; 4), P=$ 0.484).

\section{Secondary outcomes:}

There was no difference in HRQoL assessed using the SF-36 between the HSPC and control group. There was no difference in anxiety and depression measured by the HADS-anxiety and HADSdepression between the intervention and control group. At inclusion, 3 patients in each group had completed their advanced care planning (ACP) directives ( $P$ $=1.00)$. At the end of the study, 9 patients (35\%) of the intervention group versus $3(13 \%)$ of the control group had completed ACP directives $(P=0.194)$. There was therefore a difference in the number of patients who wrote their ACP directives in favour of the intervention group $(P=0.023)$. Survival did not differ between the groups $(P=0.913) .8$ deaths occurred, 4 in each group. In the intervention group, survival was 454 days (1.24 years; $95 \%$ Cl: 382 
Table 16. Studies with qualitative components (Continued)

to 525 vs. 425 days ( 1.16 years; $95 \% \mathrm{Cl}: 339$ to 509 ) in the control group; $\mathrm{P}=0.592$.
Lowther 2018

(linked to

Lowther 2015)

(HIV patients)
20 patients (predominantly females (85\%))

from the intervention group
Semi-structured interviews analysed using thematic content analysis

\section{Findings:}

Patients reported that having time to talk, appropriate pain medication and effective health education was of therapeutic value for their psychological well-being. Integration of mixed method findings suggested that positive effect in quantitative measures of mental health and well-being were attributable to the active in gredients of: appropriate medication, effective health education and counselling, and having time to talk in clinical encounters. Mechanisms of action included symptom relief, improved understanding of illness and treatment, and support focussed on articulated concerns.

Participants whose quality of life remained static or deteriorated reported concurrent intractable physical or social problems which prevented them from fulfilling their social roles and led to financial difficulties. This in turn led to stress, which was a barrier to positive psychological well-being.
Giovannetti 2018

(linked to Solari

2018) (multiple sclerosis)

\section{2 patients, 15} caregivers, 8 physicians and nine members of HSPC team
Semi-structured interviews analysed using framework method

\section{Findings:}

Three themes emerged from the interviews: 'expectations,' 'met and unmet needs', and 'barriers'. Participants described benefits from the intervention such as improved control of symptoms and reduced sense of isolation of the patient-caregiver dyads. $\mathrm{Pa}$ tient-caregiver dyads valued the expertise of the HSPC team. Limitations identified that included factors related to experimental design (difficulty of dyads in identifying examiner and team roles, additional burden for caregivers); team issues (insufficient team building/supervision, competing priorities); limitations of the intervention itself (insufficient length, lack of rehabilitation input); and external factors (resource limitations, under-responsive services/professionals). The referring

\section{Primary outcome:}

In the control group, median pain score on the pain item of the APOS (range: 0 to 5; 0 indicates worst pain) improved from 1.0 (IQR 0.0 to 2.0) at baseline to 5.0 (3.0 to 5.0) at 4 months; in the HSPC group, it improved from 1.0 (0.0 to 2.0) at baseline to 4.5 (3.0 to 5.0) at 4 months. There was no between-group difference (coefficient $-0.01,95 \% \mathrm{Cl}-0.36$ to $0.34, \mathrm{P}$ $=0.95$ ).

\section{Secondary outcomes:}

Person-centred assessment and care delivered by staff who had received additional training had positive effects on self-reported mental health-related quality of life and psychosocial well-being.

\section{Primary outcomes:}

There was greater reduction in symptom burden (POS-S-MS) in the HSPC group compared to usual care $(P=0.047)$. Effect size was 0.20 at 3 months and 0.32 at 6 months. Changes in quality of life (SEIQoL-DW index) did not differ between the two groups.

\section{Secondary outcomes:}

There were no differences between the secondary patient (POS, HADS, FIM total score) and carer outcomes (ZBI) at three and six months. There were 22 serious adverse events in 20 patients, 15 events in 13 patients in the HSPC group (30\%) and 7 events in 7 patients in the control group (27\%; P $=0.78$ ). 
Table 16. Studies with qualitative components (Continued)

physician focus groups provided little experiential data.

\section{Slota 2014}

(linked to Wallen

2012) (cancer pa-

tients)
In Wallen 2012,

$n$ was unclear while Slota 2014 had 34 participants

Open-ended,
qualitative
questions on a
questionnaire.
Method of analy-
sis stated in
Wallen 2012 was
transcript-based
analysis while
thematic analy-
sis was stated in
Slota 2014

Findings:

Patients identified consistent communication, emotional support, and pain and symptom management as positive contributions delivered by the intervention. Consistent communication was described in terms of the team as a whole and their focus on individualising patients' pain and comfort needs. When describing emotional support or 'being there' participants emphasised the support and reassurance they felt knowing the Pain and Palliative Care Team was available across time. They saw team members as their advocates.

\section{Primary outcomes and \\ secondary outcomes:}

There was no difference between HSPC and control group. However, for those who remained on study for 12 months, the HSPC group performed better than their standard of care counterparts.

ACP:

APOS: African Palliative Care Outcome Scale

BIS:

$\mathrm{Cl}:$

CNS: Clinical Nurse Specialist

COPD:

CRQ: Chronic Respiratory Questionnaire

ENABLE II:

FIM:

GBP: Great British Pounds

GP: General Practitioner

HADS: Hospital Anxiety and Depression Scale

HF:

HIV:

HRQL: Health-Related Quality of Life

HRQoL:

HSPC:

n: number

HSPC: Hospital-based Specialist Palliative Care

ILD:

IQR: Interquartile range

IRR:

POS: Palliative Care Outcome Scale

POS-S-MS:

SD:

SE: Standard Error

SEIQoL-DW index: Schedule for the Evaluation of Individual Quality of Life-Direct Weighting index SF-36:

ZBI: Zarit Burden Inventory

\section{AP P E N D ICES}

\section{Appendix 1. MEDLINE search strategy}

1.exp Palliative Care/

The effectiveness and cost-effectiveness of hospital-based specialist palliative care for adults with advanced illness and their caregivers 
2 exp Terminal Care/

3 exp Terminally III/

4 palliat*.mp.

5 (terminal* ${ }^{*}$ adj5 (care or caring)).mp.

6 ((advanced or terminal) adj5 (ill* or disease $\left.\left.{ }^{\star}\right)\right) \cdot \mathrm{mp}$.

7 (end stage or end of life or last year of life or LYOL or life's end).mp.

8 or/1-7

9 (home adj5 (hospital or palliat*)).mp.

10 ((outreach or hospital at home or outpatient or out-patient or ambulatory or posthospital or post-hospital or consult*) adj2 (care or center $^{\star}$ or centre $^{\star}$ or interven ${ }^{\star}$ or management or model ${ }^{\star}$ or nurs ${ }^{\star}$ or program ${ }^{\star}$ or service $^{\star}$ or team ${ }^{\star}$ or therap $^{\star}$ or treat $\left.^{\star}\right)$ ).mp.

11 exp Outpatients/

12 exp Hospitals/

13 exp Inpatients/

14 ((hospital ${ }^{\star}$ or inpatient $\left.{ }^{\star}\right)$ adj2 (base* or care or center $^{\star}$ or centre ${ }^{\star}$ or interven* or management or model $^{\star}$ or nurs $^{\star}$ or program $^{\star}$ or service $^{\star}$ or team ${ }^{\star}$ or therap ${ }^{\star}$ or treat $\left.\left.{ }^{\star}\right)\right) . \mathrm{mp}$.

15 hospice*.mp.

16 or/9-15

178 and 16

18 (child $^{\star}$ or adolescent ${ }^{\star}$ or infant ${ }^{\star}$ or baby or babies or neonat ${ }^{\star}$ or juvenil ${ }^{\star}$ or pediatric ${ }^{\star}$ or paediatric ${ }^{\star}$ or young person* or young people or youth* or young adult*).ti.

\section{7 not 18}

20 randomized controlled trial.pt.

21 controlled clinical trial.pt.

22 randomized.ab.

23 placebo.ab.

24 randomly.ab.

25 trial.ab.

26 groups.ab.

27 (random $^{\star}$ or control ${ }^{\star}$ or intervention ${ }^{\star}$ or evaluat $\left.{ }^{\star}\right)$.tw.

28 ("before and after" or case control* or cohort study or quasi experiment* or time series).tw.

29 or $/ 20-28$

3019 and 29

31 exp budgets/ or exp "costs and cost analysis"/ or economics/ or exp economics, hospital/ or exp economics, medical/ or economics, nursing/ or exp "fees and charges"/ or exp resource allocation/ or value of life/

32 (cost* or economic $\left.{ }^{\star}\right)$.ti. or (cost* adj2 (effective* or utilit* or benefit ${ }^{\star}$ or minimi $\left.{ }^{\star}\right)$ ).ab. or economic model ${ }^{\star}$.tw. or (budget ${ }^{\star}$ or fee $^{\star}$ or financ $^{\star}$ or price ${ }^{\star}$ or pricing or resourc ${ }^{\star}$ allocat ${ }^{\star}$ or (value adj2 (monetary or money))).ti,ab.

3331 or 32

The effectiveness and cost-effectiveness of hospital-based specialist palliative care for adults with advanced illness and their caregivers 
3419 and 33

3530 or 34

36 (animals not (humans and animals)).sh.

3735 not 36

\section{Appendix 2. Embase search strategy}

1 exp palliative therapy/

2 exp terminal care/

3 exp terminally ill patient/

4 palliat* ${ }^{\star}$.tw.

5 (terminal* ${ }^{\star}$ adj5 (care or caring)).tw.

6 ((advanced or terminal) adj5 (ill* or disease $\left.\left.{ }^{\star}\right)\right) . t w$.

7 (end stage or end of life or last year of life or LYOL or life's end).tw.

8 or/1-7

9 (home adj5 (hospital or palliat*)).tw.

10 ((outreach or hospital at home or outpatient or out-patient or ambulatory or posthospital or post-hospital or consult ${ }^{\star}$ ) adj2 (base* or care or center ${ }^{\star}$ or centre or interven $^{\star}$ or management or model ${ }^{\star}$ or nurs ${ }^{\star}$ or program ${ }^{\star}$ or service or team $^{\star}$ or therap $^{\star}$ or treat $\left.\left.^{\star}\right)\right)$.tw.

11 exp outpatients/

12 or/9-11

13 hospice ${ }^{\star} . t w$.

1412 or 13

15 exp hospital/

16 exp hospital patient/

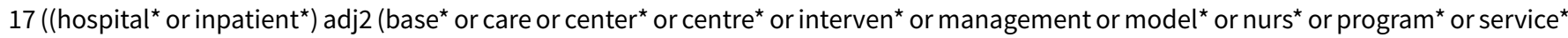
or team ${ }^{\star}$ or therap ${ }^{\star}$ or treat $\left.{ }^{\star}\right)$ ).tw.

18 or/15-17

1914 or 18

20 (child $^{\star}$ or adolescent ${ }^{\star}$ or infant ${ }^{\star}$ or baby or babies or neonat ${ }^{\star}$ or juvenil ${ }^{\star}$ or pediatric ${ }^{\star}$ or paediatric ${ }^{\star}$ or young person ${ }^{\star}$ or young people or youth* or young adult*).tw.

2119 not 20

22 random $\$ . t w$.

23 factorial\$s.tw.

24 crossover\$.tw.

25 cross over\$.tw.

26 cross-over\$.tw.

27 placebo\$.tw.

28 (doubl\$ adj blind\$).tw.

The effectiveness and cost-effectiveness of hospital-based specialist palliative care for adults with advanced illness and their caregivers 
29 (singl\$ adj blind\$).tw.

30 assign\$.tw.

31 allocat\$.tw.

32 volunteer\$.tw.

33 crossover procedure/

34 double-blind procedure.tw.

35 randomized controlled trial/

36 single blind procedure/

37 ("before and after" or case control* or cohort study or quasi experiment* or time series).tw.

38 or $/ 22-37$

398 and 21 and 38

40 exp budgets/ or exp "costs and cost analysis"/ or economics/ or exp economics, hospital/ or exp economics, medical/ or economics, nursing/ or exp "fees and charges"/ or exp resource allocation/ or value of life/

41 (cost $^{\star}$ or economic $\left.{ }^{\star}\right)$.ti. or (cost ${ }^{\star}$ adj2 (effective ${ }^{\star}$ or utilit* or benefit ${ }^{\star}$ or minimi $\left.{ }^{\star}\right)$ ).ab. or economic model ${ }^{\star}$.tw. or (budget ${ }^{\star}$ or fee ${ }^{\star}$ or financ ${ }^{\star}$ or price* or pricing or resourc* allocat ${ }^{\star}$ or (value adj2 (monetary or money))).ti,ab.

4240 or 41

438 and 21 and 42

4439 or 43

45 (animal/ or nonhuman/) not human/

4644 not 45

Appendix 3. PsycINFO search strategy

\begin{tabular}{|c|c|}
\hline 1 & exp Palliative Care/ \\
\hline 2 & exp Terminally Ill Patients/ \\
\hline 3 & palliat*.tw. \\
\hline 4 & (terminal ${ }^{*}$ adj5 (care or caring)).tw. \\
\hline 5 & ((advanced or terminal) adj5 (ill ${ }^{\star}$ or disease* $\left.)\right)$. tw. \\
\hline 6 & (end stage or end of life or last year of life or LYOL or life's end).tw. \\
\hline 7 & or/1-6 \\
\hline 8 & (home adj5 (hospital or palliat*)).tw. \\
\hline 9 & $\begin{array}{l}\text { ((outreach or hospital at home or outpatient or out-patient or ambulatory or posthospital or post- } \\
\left.\text { hospital or consult }{ }^{\star}\right) \text { adj2 }\left(\text { base }^{\star} \text { or care or center } \text { or centre }^{\star} \text { or interven* or management or mod- }\right. \\
\left.\text { el }^{\star} \text { or nurs } \text { or program }^{\star} \text { or service } \text { or team }^{\star} \text { or therap } \text { or }^{\star} \text { treat }^{\star}\right) \text { ).tw. }\end{array}$ \\
\hline 10 & exp OUTPATIENTS/ \\
\hline
\end{tabular}

The effectiveness and cost-effectiveness of hospital-based specialist palliative care for adults with advanced illness and their caregivers 
11

or/8-10

$$
12
$$

13

$$
14
$$

$$
15
$$

15

16

$$
17
$$

17

$$
21
$$$$
22
$$

23

$$
23
$$

25

26

26

27

28

29

30

31

32

32

33

34

35

36

36

37

11 or 12

13 or 17 exp HOSPICE/

exp HOSPITALS/

exp Hospitalized Patients/

((hospital ${ }^{\star}$ or inpatient $\left.{ }^{\star}\right)$ adj2 (base ${ }^{\star}$ or care or center ${ }^{\star}$ or centre $^{\star}$ or interven ${ }^{\star}$ or management or model $^{\star}$ or nurs ${ }^{\star}$ or program ${ }^{\star}$ or service ${ }^{\star}$ or team $^{\star}$ or therap ${ }^{\star}$ or treat $\left.^{\star}\right)$ ).tw.

or/14-16

(child $^{\star}$ or adolescent ${ }^{\star}$ or infant ${ }^{\star}$ or baby or babies or neonat ${ }^{\star}$ or juvenil ${ }^{\star}$ or pediatric ${ }^{\star}$ or paediatric $^{\star}$ or young person ${ }^{\star}$ or young people or youth ${ }^{\star}$ or young adult* or matern $\left.{ }^{\star}\right)$.tw.

18 not 19

exp Clinical Trials/

$\left(\right.$ randomis $^{\star}$ or randomiz $\left.{ }^{\star}\right) . t w$.

(random\$ adj3 (allocat\$ or assign\$)).tw.

((clinic\$ or control\$) adj trial\$).tw.

((singl\$ or doubl\$ or trebl\$ or tripl\$) adj3 (blind\$ or mask\$)).tw.

(crossover\$ or "cross over\$").tw.

exp Random Sampling/

exp Experiment Controls/

exp PLACEBO/

placebo\$.tw.

exp Program Evaluation/

exp Treatment Effectiveness Evaluation/

((effectiveness or evaluat\$) adj3 (stud\$ or research\$)).tw.

or/21-33

("before and after" or case control* or cohort study or quasi experiment* or time series).tw.

34 or 35

7 and 20 and 36

The effectiveness and cost-effectiveness of hospital-based specialist palliative care for adults with advanced illness and their caregivers 
$\left(\operatorname{cost}^{\star}\right.$ or economic $)$.ti. or (cost ${ }^{\star}$ adj2 (effective ${ }^{\star}$ or utilit ${ }^{\star}$ or benefit ${ }^{\star}$ or minimi $\left.\left.{ }^{\star}\right)\right)$.ab. or economic model ${ }^{\star}$.tw. or (budget ${ }^{\star}$ or fee ${ }^{\star}$ or financ ${ }^{\star}$ or price ${ }^{\star}$ or pricing or resourc ${ }^{\star}$ allocat $^{\star}$ or (value adj2 (monetary or money))).ti,ab.

\begin{tabular}{ll}
\hline 39 & exp BUDGETS/ \\
\hline 40 & exp health care costs/or exp "costs and cost analysis"/ \\
\hline 41 & exp Resource Allocation/ \\
\hline 42 & exp Health Care Economics/ \\
\hline 43 & or/38-42 \\
\hline 44 & 7 and 20 and 43 \\
\hline 45 & 37 or 44 \\
\hline 46 & limit 45 to human \\
\hline
\end{tabular}

\section{Appendix 4. CINAHL search strategy}

\begin{tabular}{|c|c|}
\hline S45 & S43 not S44 \\
\hline S44 & TI (animals not (humans and animals)) \\
\hline S43 & $\mathrm{S} 33$ or $\mathrm{S} 42$ \\
\hline S42 & $\mathrm{S} 8$ and $\mathrm{S} 21$ and $\mathrm{S} 41$ \\
\hline S41 & $\mathrm{S} 34$ or $\mathrm{S} 35$ or $\mathrm{S} 36$ or $\mathrm{S} 37$ or $\mathrm{S} 38$ or $\mathrm{S} 39$ or $\mathrm{S} 40$ \\
\hline S40 & MH economic value of life \\
\hline S39 & $\mathrm{MH}$ resource allocation \\
\hline S38 & MH fees and charges \\
\hline S37 & MH economics \\
\hline S36 & $\mathrm{MH}$ costs and cost analysis \\
\hline S35 & MH budgets \\
\hline S34 & 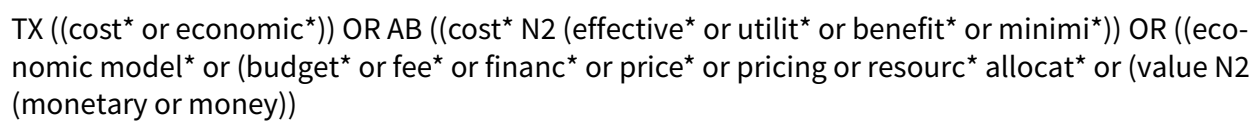 \\
\hline S33 & S8 and S21 and S32 \\
\hline S32 & S30 or S31 \\
\hline S31 & TX ("before and after" or case control* or cohort study or quasi experiment* or time series) \\
\hline
\end{tabular}

The effectiveness and cost-effectiveness of hospital-based specialist palliative care for adults with advanced illness and their caregivers 


\begin{tabular}{|c|c|}
\hline S30 & S22 or S23 or S24 or S25 or S26 or S27 or S28 or S29 \\
\hline S29 & TX (allocat* random*$\left.^{\star}\right)$ \\
\hline S28 & MH quantitative studies \\
\hline S27 & MH placebos \\
\hline S26 & TX placebo* \\
\hline S25 & TX (random* allocat*) \\
\hline S24 & MH random assignment \\
\hline S23 & TX (Randomi?ed control* trial $\left.^{\star}\right)$ \\
\hline $\mathrm{S} 22$ & $\begin{array}{l}\text { TX }\left(\text { singl }{ }^{\star} \text { blind }^{\star}\right) \text { or }\left(\text { doubl }^{\star} \text { blind }^{\star}\right) \text { or }\left(\text { tripl }^{\star} \text { blind }^{\star}\right) \text { or }\left(\text { trebl }^{\star} \text { blind }^{\star}\right) \text { or }\left(\text { trebl }^{\star} \text { mask }^{\star}\right) \text { or }(\text { tripl } \\
\left.\text { mask }^{\star}\right) \text { or }\left(\text { doubl }^{\star} \text { mask }^{\star}\right) \text { or }\left(\text { singl }^{\star} \text { mask }^{*}\right)\end{array}$ \\
\hline S21 & S19 not S20 \\
\hline
\end{tabular}

S20
TI (child* or adolescent ${ }^{\star}$ or infant ${ }^{\star}$ or baby or babies or neonat ${ }^{\star}$ or juvenil or pediatric $^{\star}$ or paedi-

\begin{tabular}{|c|c|}
\hline S19 & $\mathrm{S} 14$ or $\mathrm{S} 18$ \\
\hline S18 & $\mathrm{S} 15$ or $\mathrm{S} 16$ or $\mathrm{S} 17$ \\
\hline S17 & $\begin{array}{l}\left.\text { TX ((hospital }{ }^{\star} \text { or inpatient }{ }^{\star}\right) \text { N2 (base } \text { or care or center }^{\star} \text { or centre }{ }^{\star} \text { or interven }{ }^{\star} \text { or management or } \\
\left.\left.\text { model }^{\star} \text { or nurs } \text { or program }^{\star} \text { or service* } \text { or team }^{\star} \text { or therap } \text { or treat }^{\star}\right)\right)\end{array}$ \\
\hline S16 & MH inpatients \\
\hline S15 & MH hospitals \\
\hline S14 & $\mathrm{S} 12$ or S13 \\
\hline S13 & TX hospice* \\
\hline S12 & $\mathrm{S} 9$ or $\mathrm{S} 10$ or $\mathrm{S} 11$ \\
\hline S11 & MH outpatients \\
\hline S10 & $\begin{array}{l}\text { TX (outreach or hospital at home or outpatient or out-patient or ambulatory or posthospital or } \\
\text { post-hospital or consult }{ }^{\star} \text { ) and (base } \text { or care or center }^{\star} \text { or centre } \text { or interven }^{\star} \text { or management or } \\
\left.\text { model }^{\star} \text { or nurs } \text { or program }^{\star} \text { or service } \text { or team }^{\star} \text { or therap } \text { or treat }^{\star}\right) \text { ) }\end{array}$ \\
\hline S9 & TX home and (hospital or palliat*) \\
\hline S8 & S1 OR S2 OR S3 OR S4 OR S5 OR S6 OR S7 \\
\hline S7 & TX (end stage or end of life or last year of life or LYOL or life's end) \\
\hline S6 & TX ((advanced or terminal) N5 (ill* or disease $\left.\left.{ }^{\star}\right)\right)$ \\
\hline S5 & TX (terminal ${ }^{\star}$ N5 (care or caring)) \\
\hline
\end{tabular}

The effectiveness and cost-effectiveness of hospital-based specialist palliative care for adults with advanced illness and their caregivers 


S4 TX palliat*

\begin{tabular}{ll}
\hline S3 & MH terminally ill patients \\
\hline S2 & MH terminal care \\
\hline S1 & MH palliative care \\
\hline
\end{tabular}

\section{Appendix 5. Cochrane Library (CENTRAL, CDSR, DARE, HTA, NHS EDD) search strategy}

\#1 MeSH descriptor: [Palliative Care] explode all trees

\#2 MeSH descriptor: [Terminal Care] explode all trees

\#3 MeSH descriptor: [Terminally III] explode all trees

\#4 palliat*:ti,ab,kw

\#5 (terminal ${ }^{\star}$ near/5 (care or caring)):ti,ab,kw

\#6 ((advanced or terminal) near/5 (ill* or disease $\left.{ }^{\star}\right)$ ):ti,ab,kw

\#7 (end stage or end of life or last year of life or LYOL or life's end):ti,ab,kw

\#8 \#1 or \#2 or \#3 or \#4 or \#5 or \#6 or \#7

\#9 (home near/5 (hospital or palliat*)):ti,ab,kw

\#10 ((outreach or hospital at home or outpatient or out-patient or ambulatory or posthospital or post-hospital or consult ${ }^{\star}$ ) near/2 (base*

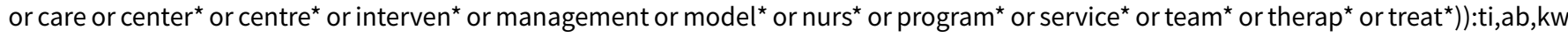

\#11 MeSH descriptor: [Outpatients] explode all trees

\#12 \#9 or \#10 or \#11

\#13 hospice*:ti,ab,kw

\#14 \#12 or \#13

\#15 MeSH descriptor: [Hospitals] explode all trees

\#16 MeSH descriptor: [Inpatients] explode all trees

\#17 ((hospital ${ }^{\star}$ or inpatient ${ }^{\star}$ ) near/2 (base or care or center $^{\star}$ or centre or interven $^{\star}$ or management or model or nurs $^{\star}$ or program ${ }^{\star}$ or

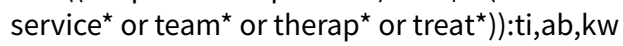

\#18 \#15 or \#16 or \#17

\#19 \#14 or \#18

$\# 20 \# 8$ and \#19

\#21 (child ${ }^{\star}$ or adolescent ${ }^{\star}$ or infant* ${ }^{\star}$ or baby or babies or neonat ${ }^{\star}$ or juvenil ${ }^{\star}$ or pediatric ${ }^{\star}$ or paediatric ${ }^{\star}$ or young person ${ }^{\star}$ or young people or youth ${ }^{\star}$ or young adult*):ti

\#22 (\#20 and not \#21)

\section{Appendix 6. CareSearch search strategy}

1. Inpatient

2. Hospital

3. \#1 OR \#2

The effectiveness and cost-effectiveness of hospital-based specialist palliative care for adults with advanced illness and their caregivers 
4. (((Palliative) OR Terminal) OR End stage) OR End of life

5. \#3 AND \#4

6. Outpatient

7. Outreach

8. Hospital at home

9. Ambulatory

10. Post-hospital

11. Consult

12. \#6 OR \#7 OR \#8 OR \#9 OR \#10 OR \#11

13. Hospice

14. 12 or 13

15. (((Palliative) OR Terminal) OR End stage) OR End of life

16. \#14 AND \#15

17. \#5 OR \#16

\section{Appendix 7. Items to be included in the data extraction form}

\section{Study details}

- publication details (author(s), year, journal);

- country of origin;

- verification of the study eligibility;

- aim/hypothesis;

- type of hospital.

\section{Study design and methods}

- study design;

- type of intervention and control (if used);

- inclusion/exclusion criteria;

- allocation sequence procedures;

- allocation concealment;

- type of blinding;

- details of blinding (including instances of blinding being compromised);

- data collection period;

- baseline measurement(s);

- number of follow-ups;

- time that follow-ups occurred;

- sample size (number in each group);

- sample size calculations;

- outcome measures used (differentiating primary and secondary);

- recruitment rate;

- method of analysis;

- method of managing missing data;

- study participant characteristics for patient and unpaid caregiver (e.g. age, sex, race, sexual orientation, diagnosis);

- selective reporting.

Intervention(s) and comparator(s)

- setting of intervention;

The effectiveness and cost-effectiveness of hospital-based specialist palliative care for adults with advanced illness and their caregivers

Copyright @ 2020 The Cochrane Collaboration. Published by John Wiley \& Sons, Ltd. 
- type of intervention;

- staff composition;

- staff training and experience;

- components of intervention;

- frequency of intervention;

- duration of intervention.

\section{Primary outcome}

- Patient health-related quality of life

- Patient symptom burden

\section{Secondary outcomes}

- Patient satisfaction with care;

- unpaid caregiver/family satisfaction with care;

- Achieving patient's preferred place of death;

- Achieving patient's preferred place of care;

- Patient mortality/survival;

- Pain;

- Patient anxiety and depression;

- Breathlessness;

- Adverse events in participants and unpaid caregivers;

- unpaid caregiver symptom control, specifically physical, psychological (e.g. anxiety and depression), social or spiritual domains, reported through validated assessment scales and burden, including emotional strain, burden, distress, mastery or positive aspects of caregiving; and

- Unpaid caregiver pre- and post-bereavement outcomes, reported using outcome scales of multidimensional caregiving experiences (strain, distress, positive appraisals, and family well-being), unpaid caregiver prolonged grief, multidimensional grief responses (despair, panic behaviour, blame and anger, detachment, disorganisation and personal growth), quality of life.

\section{Costs (resource use)}

- Resource use: institutional care services use, outpatient clinic services use, community care services use, unpaid caregiver's care, and medications and other resources;

- Costs and cost-effectiveness. 


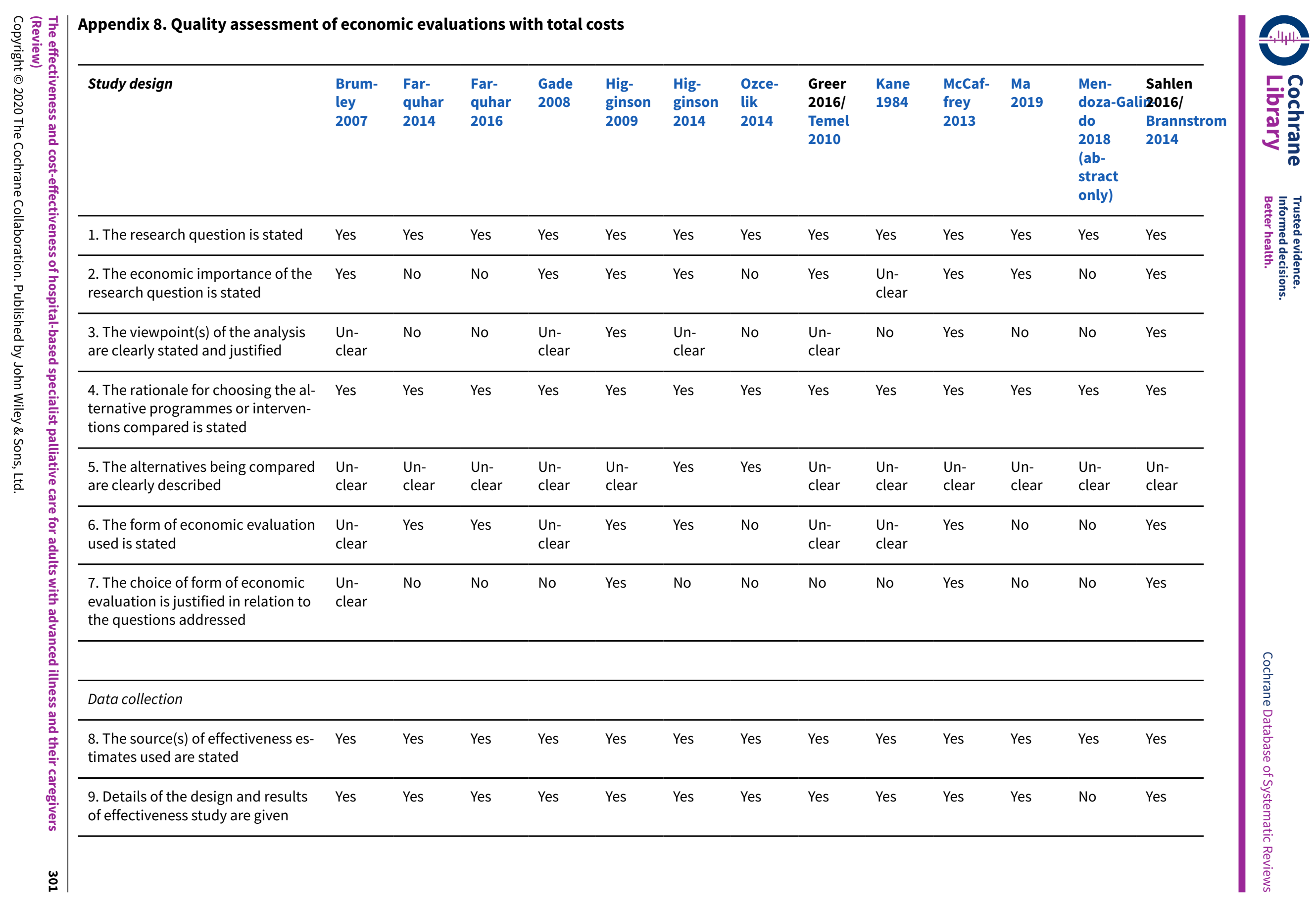




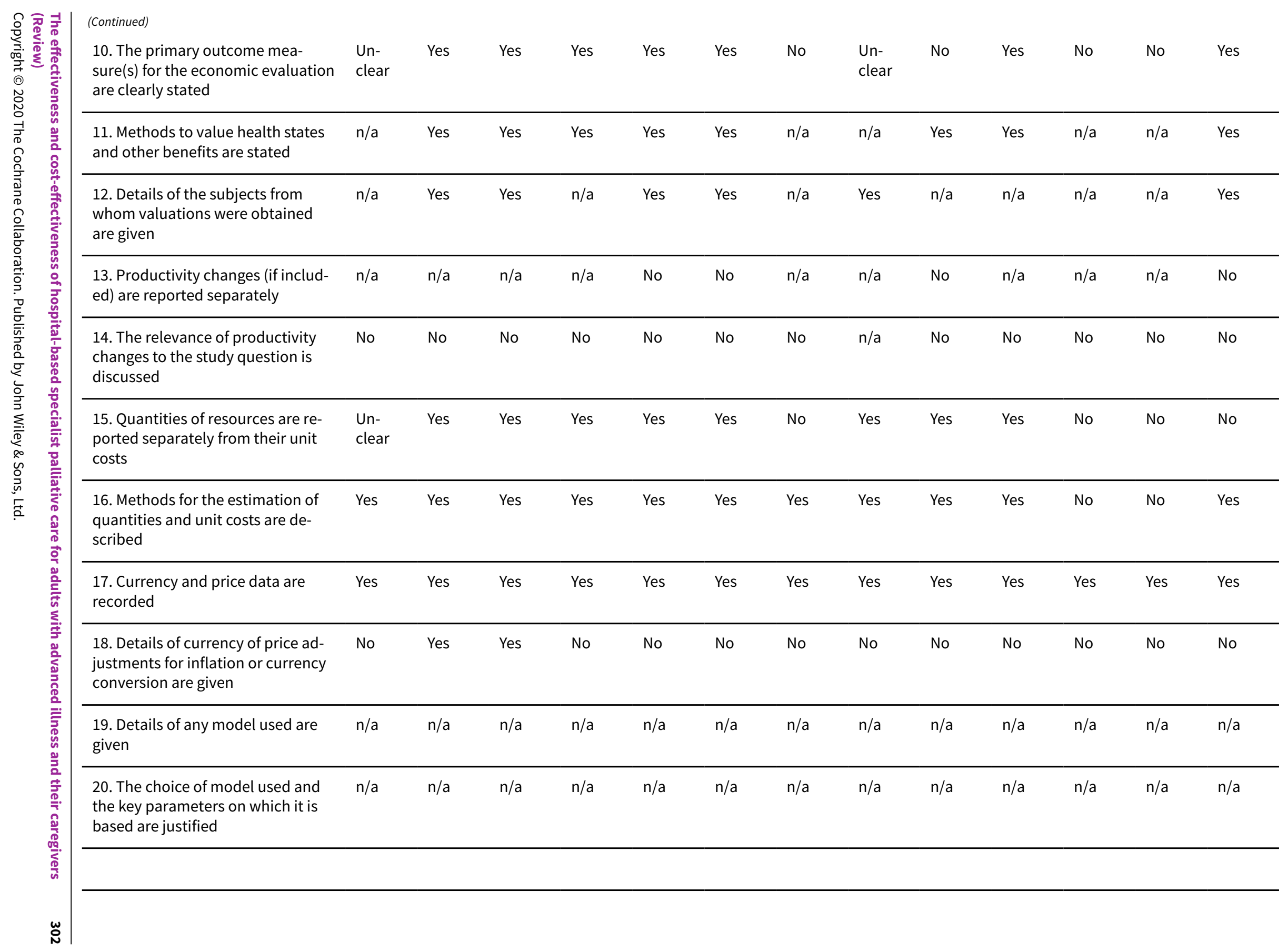




\begin{tabular}{|c|c|c|c|c|c|c|c|c|c|c|c|c|c|c|}
\hline 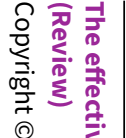 & $\begin{array}{l}\text { (Continued) } \\
\text { Analysis and interpretation of re- } \\
\text { sults }\end{array}$ & & & & & & & & & & & & & \\
\hline 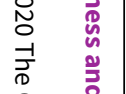 & $\begin{array}{l}\text { 21. Time horizon of costs and bene- } \\
\text { fits is stated }\end{array}$ & Yes & Yes & Yes & Yes & Yes & Yes & Yes & Yes & Yes & Yes & Yes & No & Yes \\
\hline $\begin{array}{l}0 \\
\vdots \\
0 \\
0 \\
0\end{array}$ & 22. The discount rate(s) is stated & $\mathrm{n} / \mathrm{a}$ & $\mathrm{n} / \mathrm{a}$ & $\mathrm{n} / \mathrm{a}$ & $\mathrm{n} / \mathrm{a}$ & $\mathrm{n} / \mathrm{a}$ & $\mathrm{n} / \mathrm{a}$ & $\mathrm{n} / \mathrm{a}$ & $\mathrm{n} / \mathrm{a}$ & $\mathrm{n} / \mathrm{a}$ & $\mathrm{n} / \mathrm{a}$ & $\mathrm{n} / \mathrm{a}$ & $\mathrm{n} / \mathrm{a}$ & $\mathrm{n} / \mathrm{a}$ \\
\hline 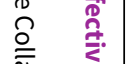 & 23. The choice of rate(s) is justified & $\mathrm{n} / \mathrm{a}$ & $\mathrm{n} / \mathrm{a}$ & $\mathrm{n} / \mathrm{a}$ & $\mathrm{n} / \mathrm{a}$ & $\mathrm{n} / \mathrm{a}$ & $\mathrm{n} / \mathrm{a}$ & $\mathrm{n} / \mathrm{a}$ & $\mathrm{n} / \mathrm{a}$ & $\mathrm{n} / \mathrm{a}$ & $\mathrm{n} / \mathrm{a}$ & $\mathrm{n} / \mathrm{a}$ & $\mathrm{n} / \mathrm{a}$ & $\mathrm{n} / \mathrm{a}$ \\
\hline 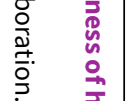 & $\begin{array}{l}\text { 24. An explanation is given if costs } \\
\text { or benefits are not discounted }\end{array}$ & No & No & No & $\mathrm{n} / \mathrm{a}$ & $n / a$ & $n / a$ & No & $n / a$ & $\mathrm{n} / \mathrm{a}$ & Yes & $\mathrm{n} / \mathrm{a}$ & $\mathrm{n} / \mathrm{a}$ & $\mathrm{n} / \mathrm{a}$ \\
\hline 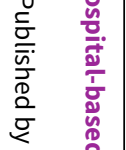 & $\begin{array}{l}\text { 25. Details of statistical tests and } \\
\text { confidence intervals are given for } \\
\text { stochastic data }\end{array}$ & $\begin{array}{l}\text { Un- } \\
\text { clear }\end{array}$ & No & $\begin{array}{l}\text { Un- } \\
\text { clear }\end{array}$ & Yes & Yes & Yes & No & No & No & Yes & No & No & Yes \\
\hline 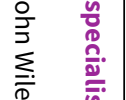 & $\begin{array}{l}\text { 26. The approach to sensitivity } \\
\text { analysis is given }\end{array}$ & $\mathrm{n} / \mathrm{a}$ & Yes & $\mathrm{n} / \mathrm{a}$ & $\mathrm{n} / \mathrm{a}$ & $\mathrm{n} / \mathrm{a}$ & $\mathrm{n} / \mathrm{a}$ & $\mathrm{n} / \mathrm{a}$ & No & Yes & Yes & $\mathrm{n} / \mathrm{a}$ & $\mathrm{n} / \mathrm{a}$ & Yes \\
\hline 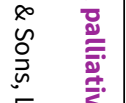 & $\begin{array}{l}27 . \text { The choice of variables for sen- } \\
\text { sitivity analysis is justified }\end{array}$ & $\mathrm{n} / \mathrm{a}$ & No & $\mathrm{n} / \mathrm{a}$ & $\mathrm{n} / \mathrm{a}$ & $\mathrm{n} / \mathrm{a}$ & $\mathrm{n} / \mathrm{a}$ & $\mathrm{n} / \mathrm{a}$ & $\mathrm{n} / \mathrm{a}$ & Yes & Yes & $\mathrm{n} / \mathrm{a}$ & $\mathrm{n} / \mathrm{a}$ & Yes \\
\hline 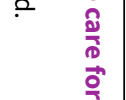 & $\begin{array}{l}\text { 28. The ranges over which the vari- } \\
\text { ables are varied are stated }\end{array}$ & $\mathrm{n} / \mathrm{a}$ & No & $\mathrm{n} / \mathrm{a}$ & $\mathrm{n} / \mathrm{a}$ & $\mathrm{n} / \mathrm{a}$ & $\mathrm{n} / \mathrm{a}$ & $\mathrm{n} / \mathrm{a}$ & $\mathrm{n} / \mathrm{a}$ & Yes & Yes & $\mathrm{n} / \mathrm{a}$ & $\mathrm{n} / \mathrm{a}$ & Yes \\
\hline 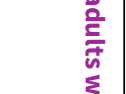 & $\begin{array}{l}\text { 29. Relevant alternatives are com- } \\
\text { pared }\end{array}$ & Yes & Yes & Yes & Yes & Yes & Yes & Yes & Yes & Yes & Yes & Yes & Yes & Yes \\
\hline 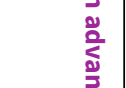 & $\begin{array}{l}\text { 30. Incremental analysis is report- } \\
\text { ed }\end{array}$ & Yes & Yes & Yes & No & Yes & No & No & No & Yes & Yes & No & No & $\begin{array}{l}\text { Un- } \\
\text { clear }\end{array}$ \\
\hline 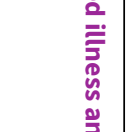 & $\begin{array}{l}\text { 31. Major outcomes are presented } \\
\text { in a disaggregated as well as aggre- } \\
\text { gated form }\end{array}$ & No & Yes & Yes & Yes & No & Yes & No & No & Yes & Yes & Yes & No & $\begin{array}{l}\text { Un- } \\
\text { clear }\end{array}$ \\
\hline 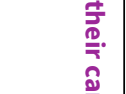 & $\begin{array}{l}\text { 32. The answer to the study ques- } \\
\text { tion is given }\end{array}$ & Yes & Yes & Yes & Yes & Yes & Yes & Yes & Yes & Yes & Yes & Yes & Yes & Yes \\
\hline 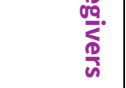 & $\begin{array}{l}\text { 33. Conclusions follow from the da- } \\
\text { ta reported }\end{array}$ & Yes & Yes & Yes & Yes & Yes & Yes & Yes & $\begin{array}{l}\text { Un- } \\
\text { clear }\end{array}$ & Yes & Yes & Yes & Yes & Yes \\
\hline
\end{tabular}




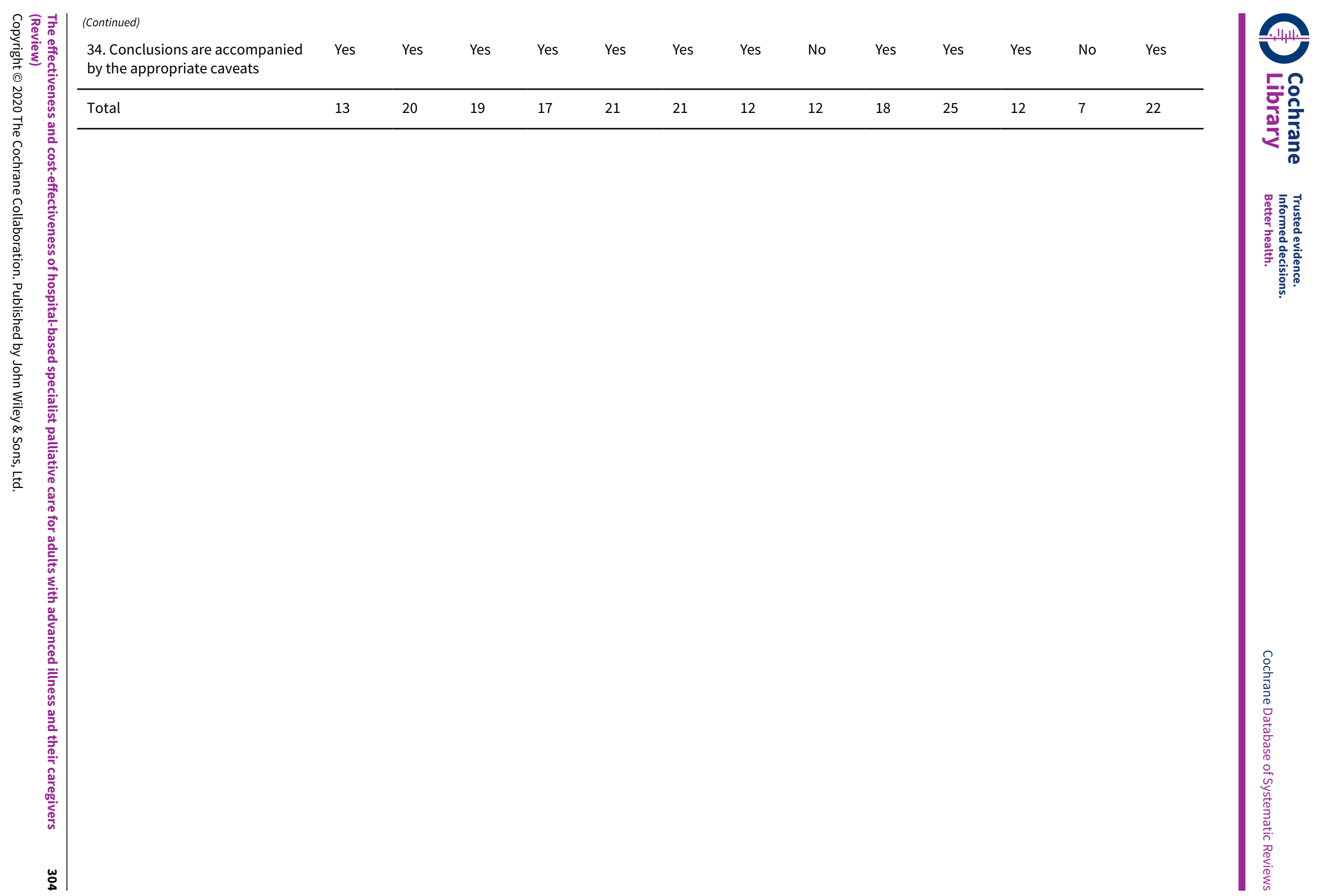


Notes:

n/a: not applicable 


\begin{tabular}{|c|c|c|c|c|c|c|c|c|c|c|c|c|c|c|c|}
\hline & Appendix 9. Assessment of meth & dologi & I qualit & of econ & mic stu & es using & he CHEC & list & & & & & & & \\
\hline 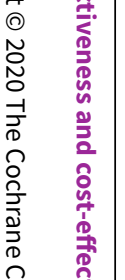 & $\begin{array}{l}\text { Consensus on Health Economic } \\
\text { Criteria (CHEC) list }\end{array}$ & $\begin{array}{l}\text { Brum- } \\
\text { ley } \\
2007\end{array}$ & $\begin{array}{l}\text { Far- } \\
\text { quhar } \\
2014\end{array}$ & $\begin{array}{l}\text { Far- } \\
\text { quhar } \\
2016\end{array}$ & $\begin{array}{l}\text { Gade } \\
2008\end{array}$ & $\begin{array}{l}\text { Hig- } \\
\text { ginson } \\
2009\end{array}$ & $\begin{array}{l}\text { Hig- } \\
\text { ginson } \\
2014\end{array}$ & $\begin{array}{l}\text { Ozce- } \\
\text { lik } \\
2014\end{array}$ & $\begin{array}{l}\text { Temel } \\
2010 / \\
\text { Greer } \\
2016\end{array}$ & $\begin{array}{l}\text { Kane } \\
1984\end{array}$ & $\begin{array}{l}\text { Ma } \\
2019\end{array}$ & $\begin{array}{l}\text { McCaf- } \\
\text { frey } \\
2013\end{array}$ & $\begin{array}{l}\text { Men- } \\
\text { doza } \\
\text { do } \\
2018 \\
\text { (ab- } \\
\text { strac } \\
\text { only) }\end{array}$ & $\begin{array}{l}\text { Brannstrom } \\
\text { li2014/ } \\
\text { Sahlen } \\
2016\end{array}$ & 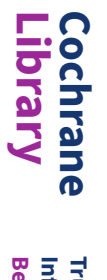 \\
\hline 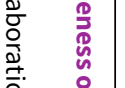 & $\begin{array}{l}\text { 1. Is the study population clearly } \\
\text { described? }\end{array}$ & Yes & Yes & Yes & Yes & Yes & Yes & Yes & Yes & Yes & Yes & Yes & Yes & Yes & 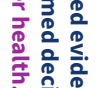 \\
\hline 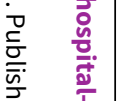 & $\begin{array}{l}\text { 2. Are competing alternatives } \\
\text { clearly described? }\end{array}$ & No & No & No & No & No & Yes & Yes & No & No & No & No & No & No & \\
\hline 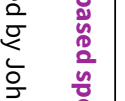 & $\begin{array}{l}\text { 3. Is a well-defined research ques- } \\
\text { tion posed in answerable form? }\end{array}$ & Yes & Yes & Yes & Yes & Yes & Yes & Yes & Yes & Yes & Yes & Yes & Yes & Yes & \\
\hline 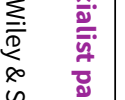 & $\begin{array}{l}\text { 4. Is the economic study design ap- } \\
\text { propriate to the stated objective? }\end{array}$ & No & Yes & Yes & Yes & Yes & Yes & Yes & Yes & Yes & Yes & Yes & Yes & No & \\
\hline 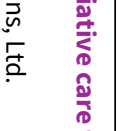 & $\begin{array}{l}\text { 5. Is the chosen time horizon ap- } \\
\text { propriate in order to include rele- } \\
\text { vant costs and consequences? }\end{array}$ & Yes & Yes & Yes & Yes & Yes & Yes & Yes & Yes & Yes & Yes & Yes & No & Yes & \\
\hline$\frac{\bar{z}}{\stackrel{\bar{c}}{\mathrm{c}}}$ & $\begin{array}{l}\text { 6. Is the actual perspective chosen } \\
\text { appropriate? }\end{array}$ & No & No & No & No & Yes & No & No & Yes & No & No & Yes & No & No & \\
\hline 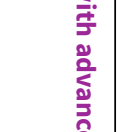 & $\begin{array}{l}\text { 7. Are all important and relevant } \\
\text { costs for each alternative identi- } \\
\text { fied? }\end{array}$ & Yes & Yes & Yes & Yes & Yes & No & Yes & No & Yes & No & Yes & Yes & No & \\
\hline 禀 & $\begin{array}{l}\text { 8. Are all costs measured appropri- } \\
\text { ately in physical units? }\end{array}$ & Yes & Yes & Yes & Yes & Yes & Yes & Yes & Yes & Yes & No & Yes & No & No & 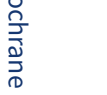 \\
\hline 흘 & 9. Are costs valued appropriately? & No & Yes & Yes & Yes & Yes & Yes & Yes & Yes & Yes & No & Yes & No & No & 离 \\
\hline 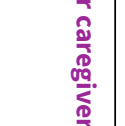 & $\begin{array}{l}\text { 10. Are all important and relevant } \\
\text { outcomes for each alternative } \\
\text { identified? }\end{array}$ & Yes & Yes & Yes & Yes & Yes & Yes & Yes & Yes & Yes & Yes & Yes & Yes & Yes & 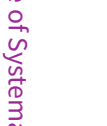 \\
\hline
\end{tabular}




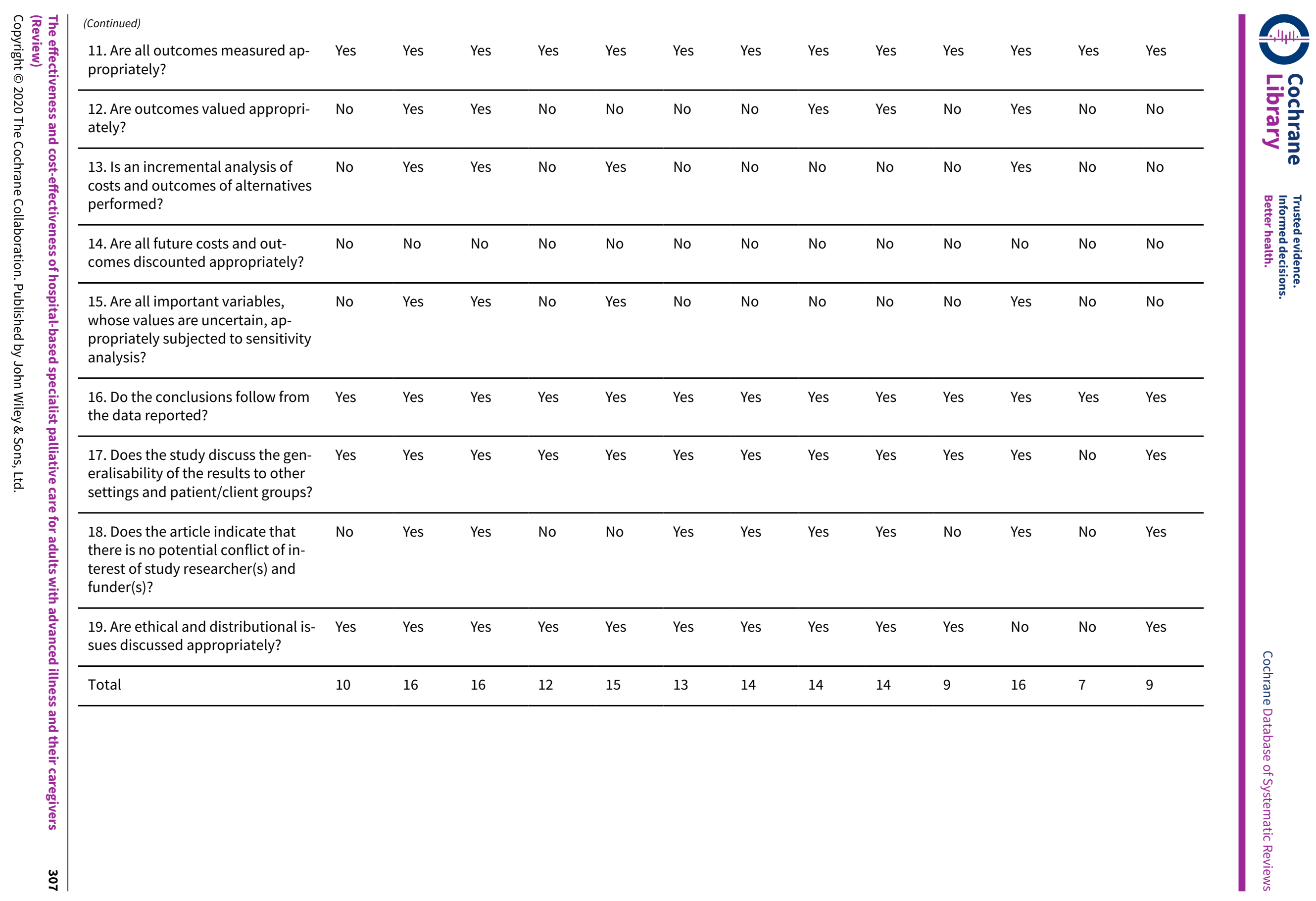




\section{H IS T O R Y}

Protocol first published: Issue 9, 2017

Review first published: Issue 9, 2020

\section{CONTRIBUTIONS OF AUTHORS}

SB won funding for the review from National Institute for Health Research Health Services and Delivery Research programme (Project Number 16/02/17) and was Chief Investigator on the grant. SB, DY, CJE, GG, CT, FEM, MC and IJH contributed to the writing of the 2017 protocol. AO carried out the searches. SB and AO screened the studies and extracted the data. All authors reviewed the final studies for inclusion. AO entered the data. AO and DY carried out the analyses. SB, AO, DY and IJH prepared the final text. II authors reviewed and contributed to the final draft.

\section{DECLARATIONS OF INTEREST}

SB: none known; SB is a Consultant in Palliative Medicine and manages patients with advanced life-threatening illness.

AO: none known.

DY: none known.

WG: none known.

GG: none known.

CT: none known.

MC: none known.

FEM: none known; FEM is a Consultant in Palliative Medicine and manages patients with advanced life-threatening illness.

CJE: none known; CJE is an Honorary Nurse Consultant and manages patients with advanced life-threatening illness.

IJH: none known; IJH is a Consultant in Palliative Medicine and manages patients with advanced life-threatening illness.

\section{SOURCES OF SUPPORT}

\section{Internal sources}

- Department of Palliative Care, Policy and Rehabilitation, Cicely Saunders Institute, King's College London, London, UK

- Institute of Psychiatry, King's College London, London, UK

\section{External sources}

- School of Nursing, Midwifery and Social Work, University of Manchester, Manchester, UK

- Amsterdam Institute of Social Science Research, University of Amsterdam, Amsterdam, Netherlands

- Regional Palliative Care Network, IRCCS AOU San Martino-IST, Genoa, Italy

- National Institute for Health Research (NIHR), UK

Earlier drafts of this review were completed by the MORECare project, which was funded by the NIHR and managed by the Medical Research Council as part of the Methodology Research Programme (project number: G0802654/1).

- The Atlantic Philanthropies and Cicely Saunders International, Other

The review was finalised through support from The Atlantic Philanthropies and Cicely Saunders International

- NIHR Health Services and Delivery Research (HS\&DR), UK

Ongoing work for this project has been funded by NIHR HS\&DR Project number: 16/02/17

\section{DIFFERENCES BETWEEN PROTOCOLAND REVIEW}

There are a number of differences between the published protocol (Bajwah 2017), and this review.

\section{Study design}

In the published protocol, we stated that we will include a number of study designs including randomised trials, non-randomised trials, controlled before-and-after studies, interrupted time series studies and repeated measures studies. Due to the expansion of our review

The effectiveness and cost-effectiveness of hospital-based specialist palliative care for adults with advanced illness and their caregivers 308 (Review)

Copyright (c) 2020 The Cochrane Collaboration. Published by John Wiley \& Sons, Ltd. 
from only inpatient specialist palliative care to include other models of HSPC (HSPC) and given that RCTs are the most rigorous study design, we refrained from analysing studies that were not RCTs in order to reduce heterogeneity and allow meta-analyses where possible. We initially wanted to minimise cross-contamination by including only cluster-randomised studies. However, our project advisory group suggested that both cluster and non-cluster-RCTs should be included to capture the breadth of evidence from RCTs that met our eligibility criteria.

\section{Intervention}

The published protocol was focussed on assessing the effectiveness and cost-effectiveness of inpatient specialist palliative care in acute hospitals for adults with advanced illness and their unpaid caregivers. However, we expanded the scope of our review from inpatient specialist palliative care to all models of HSPC, and the title has been amended to reflect this. Given that models of HSPC are evolving, we broadened the review to increase relevance for clinical practice and policy makers with the potential to aid the future development, funding and implementation of evidence-based HSPC. As a result of expanding the scope of our review to cover models of HSPC, we also expanded the scope of usual care to "inpatient or outpatient hospital care without specialist palliative care input at the point of entry into the study, community care or hospice care provided outside of the hospital setting".

In our protocol, we stated that the intervention should be administered by hospital staff who have completed specialist training in palliative care or who had obtained clinical competencies and professional characteristics required for the delivery of inpatient specialist palliative care through clinical experience. Experts in our project advisory group recommended that we include studies where the training of the palliative care team was unclear, with eligibility informed by activity of delivering specialist palliative care rather than level of specialist training. In order to capture this difference, we included studies where the training/clinical competence of the palliative care team was described as well as studies that simply stated the involvement of a palliative care team.

\section{Outcomes}

We changed our primary outcome from pain to two primary outcomes, patient HRQoL (previously, a secondary outcome) and patient symptom burden assessed using a composite measure of two or more symptoms (a new outcome we introduced following expert advice). The clinical experts on our project advisory group suggested that pain may not be an appropriate outcome for those with non-malignant conditions, where pain may be less prevalent compared to patients with cancer. Furthermore, the aim of palliative care is to improve quality of life, while also ensuring effective symptom management.

We have further provided clarity around the outcomes we presented in our protocol.

- We included number of home deaths in the review as a proxy for achieving patient preferred place of death, as people's preference is mostly to die at home (Gomes 2012).

- In our protocol, one of our secondary outcomes was patient's other symptoms (e.g. physical, psychological, social or spiritual domains). We specifically presented data on patient anxiety and patient depression for this outcome.

- Another secondary outcome in our protocol was satisfaction with care, which we reported as patient satisfaction with care and unpaid caregiver satisfaction with care in this review.

- We had unpaid caregiver symptom control (e.g. physical, psychological, social or spiritual domains) as an outcome in our protocol. In this review, we presented unpaid caregiver anxiety and caregiver depression for caregiver symptom control.

- For the caregiver pre- and post-bereavement outcome we reported in the protocol, we presented caregiver grief and caregiver quality of life.

- Although we presented achieving preferred place of care or death as one outcome in the protocol, we split it into two outcomes in the review: achieving patient preferred place of death and achieving patient preferred place of care.

- We added a new secondary outcome (breathlessness) to this review because of the recommendations we received from clinical experts in our project advisory group on its relevance as an appropriate outcome in non-malignant conditions.

Given the expansion of these outcomes, there has been a change in the order of the outcomes reported in this review compared to the protocol. Compared to our protocol, we now have two economic outcomes: resource use; costs and cost-effectiveness. Resource use encompasses institutional care services use, outpatient clinic services use, community care services use, unpaid caregiver care and medication and other resources. Where possible, we summarised data on cost and cost-effectiveness of HSPC.

\section{Data analysis and assessments}

We added early versus late palliative care as a subgroup analysis which was recommended for inclusion in our review by clinical experts because of its relevance to practice. Although we had initially specified that pain and other outcomes presented as binary data will be treated as binary outcomes in our published protocol, this was not possible as most studies presented their outcomes as continuous data. The only outcome where we were able to calculate an odds ratio and $95 \%$ confidence intervals in addition to standardised mean differences was patient depression.

We expanded our risk of bias (ROB) methods by carrying out separate assessments for all subjective outcomes (e.g. health-related quality of life) and all objective outcomes (e.g. mortality). Where studies did not include either subjective or objective outcomes, we left the domain

The effectiveness and cost-effectiveness of hospital-based specialist palliative care for adults with advanced illness and their caregivers 
that was not included blank. We added the domain 'Other bias (other sources of bias)' in the full review in order to assess whether groups were balanced at baseline and whether differences at baseline were adjusted for. We further expanded on the response options for 'size of study bias'. In particular, we assessed the following as unclear risk of bias under 'size of study bias': studies that had < 50 participants in one treatment arm and 50 to 199 participants in another treatment arm; and studies that had 50 to 199 participants in one treatment arm and $\geq 200$ participants in another treatment arm.

We had planned to use either a fixed-effects or random-effects model for meta-analysis. Due to the different models of HSPC in our review, we presented only random-effects models as we are estimating the average effect across HSPC rather than any single true effect. We had planned to estimate an intra-class correlation coefficient (ICC) where the authors of cluster-RCTs did not carry out adjustment or provide an ICC. However, we decided to use an estimate of ICC we obtained from a previous Cochrane Review in adjusting for clustering in McCorkle 2015. We contacted the authors of McCorkle 2015 for their ICC but at the time of publication they have not responded. In our protocol we stated that we would contact the original investigators for missing data and describe any strategy used for imputing missing data. We decided to only contact authors for missing data without carrying out imputations as this is the preferred method for dealing with missing data (Higgins 2011). We initially wanted to explore reasons for heterogeneity in sensitivity analysis. However, Cochrane editors recommended the use of subgroup analysis for assessing heterogeneity. Consequently, we explored heterogeneity using subgroup analysis, while we used sensitivity analysis to test the estimate we used in adjusting for clustering in the cluster-RCT. As we did not include nonrandomised studies, we did not have to pay particular attention to selection bias and reporting bias in such studies. We did not carry out a subgroup analysis assessing provision of single or few components of HSPC because very few studies provided a single component of HSPC. One of our subgroup analyses in the protocol was models of specialist palliative care. In our protocol, we have clarified this as models of HSPC because we expanded our review to include more models of HSPC.

Given that combining endpoint scores and change scores is not recommended when using standardised mean differences (SMDs) and also that Cochrane does not recommend pooling adjusted and unadjusted estimates together, we pooled studies presenting adjusted endpoint scores as our main meta-analysis, while we carried out sensitivity analyses with studies reporting unadjusted endpoint scores, adjusted change scores and unadjusted change scores. This is a change from our protocol based on advice from Cochrane editors.

In our protocol, we planned to include three 'Summary of Findings' tables: inpatient hospital specialist palliative care and usual care versus inpatient hospital care without any specialist palliative care input (e.g. oncological care only); inpatient hospital specialist palliative care and usual care versus community care (e.g. primary or specialist care provided in the patient's place of residence); and inpatient hospital specialist palliative care and usual care versus hospice care provided outside of the hospital setting. We decided to present only one 'Summary of Findings' (SoF) table, rather than three, for the comparison of HSPC (plus or minus usual care) versus usual care as experts in our project advisory group advised us this comparison alone would be the most informative for decision-makers. We expanded usual care to "inpatient or outpatient hospital care without specialist palliative care input at the point of entry into the study, community care or hospice care provided outside of the hospital setting". We presented results on both cost and cost-effectiveness in our SoF table as opposed to only cost-effectiveness in our protocol.

We initially stated that we would rate the strength of the evidence using a tool by Van Tulder 2003. However, we decided to use the GRADE approach in accordance with Cochrane standards.

\section{IN DEX TERMS}

\section{Medical Subject Headings (MeSH)}

Ambulatory Care [economics]; Bias; Caregivers [psychology] [^statistics \& numerical data]; Cost-Benefit Analysis; Family; Heart Failure [mortality] [therapy]; Home Care Services, Hospital-Based [ ${ }^{\star}$ economics]; Hospitalization [economics]; Neoplasms [mortality] [therapy]; Pain Management [statistics \& numerical data]; Palliative Care [*economics] [ ${ }^{*}$ methods]; Patient Satisfaction; Quality of Life; Randomized Controlled Trials as Topic; Symptom Assessment [statistics \& numerical data]; Terminal Care [ ${ }^{\star}$ economics] [*methods]

\section{MeSH check words}

Humans

The effectiveness and cost-effectiveness of hospital-based specialist palliative care for adults with advanced illness and their caregivers 